\title{
BIOMECHATRONICS: HARMONIZING MECHATRONIC SYSTEMS WITH HUMAN BEINGS
}

EDITED BY: Dingguo Zhang, Venketesh Dubey, Wenwei Yu and Kin Huat Low PUBLISHED IN: Frontiers in Neuroscience

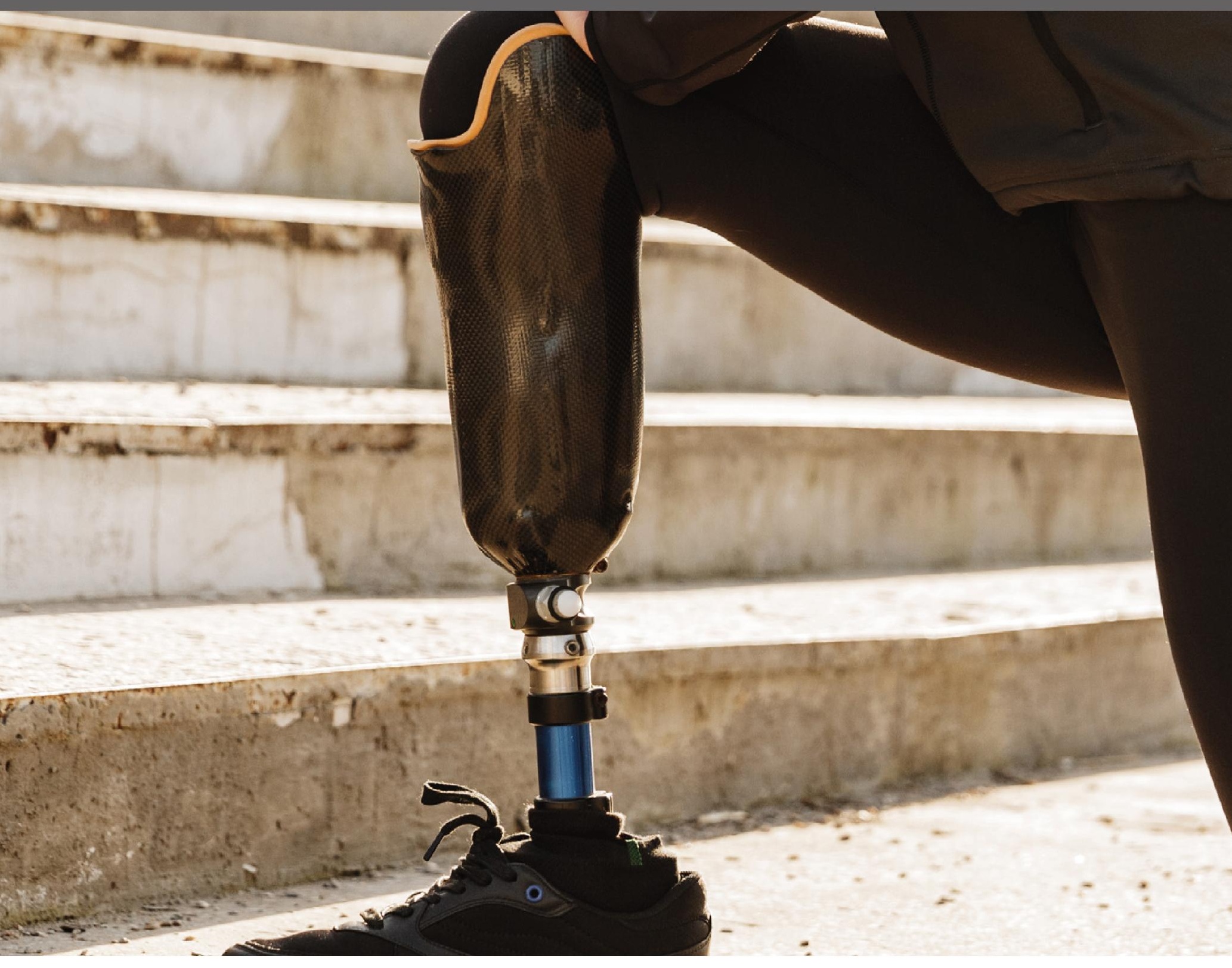

frontiers Research Topics 


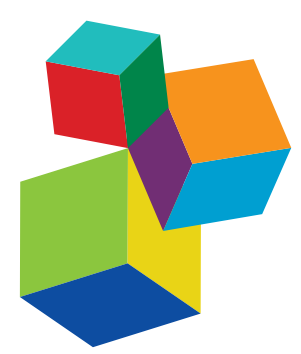

Frontiers Copyright Statement

C Copyright 2007-2019 Frontiers Media SA. All rights reserved.

All content included on this site, such as text, graphics, logos, button

icons, images, video/audio clips, downloads, data compilations and

software, is the property of or is

licensed to Frontiers Media SA

("Frontiers") or its licensees and/or subcontractors. The copyright in the text of individual articles is the property of their respective authors, subject to a license granted to Frontiers.

The compilation of articles constituting this e-book, wherever published, as well as the compilation of all other content on this site, is the exclusive property of Frontiers. For the

conditions for downloading and copying of e-books from Frontiers website, please see the Terms for Website Use. If purchasing Frontiers e-books from other websites or sources, the conditions of the website concerned apply.

Images and graphics not forming part of user-contributed materials may not be downloaded or copied without permission

Individual articles may be downloaded and reproduced in accordance with the principles of the CC-BY

licence subject to any copyright or other notices. They may not be re-sold as an e-book.

As author or other contributor you grant a CC-BY licence to others to reproduce your articles, including any graphics and third-party materials supplied by you, in accordance with the Conditions for Website Use and subject to any copyright notices which you include in connection with your articles and materials.

All copyright, and all rights therein, are protected by national and international copyright laws.

The above represents a summary only.

For the full conditions see the Conditions for Authors and the Conditions for Website Use.

ISSN 1664-8714

ISBN 978-2-88945-737-3

DOI $10.3389 / 978-2-88945-737-3$

\section{About Frontiers}

Frontiers is more than just an open-access publisher of scholarly articles: it is a pioneering approach to the world of academia, radically improving the way scholarly research is managed. The grand vision of Frontiers is a world where all people have an equal opportunity to seek, share and generate knowledge. Frontiers provides immediate and permanent online open access to all its publications, but this alone is not enough to realize our grand goals.

\section{Frontiers Journal Series}

The Frontiers Journal Series is a multi-tier and interdisciplinary set of open-access, online journals, promising a paradigm shift from the current review, selection and dissemination processes in academic publishing. All Frontiers journals are driven by researchers for researchers; therefore, they constitute a service to the scholarly community. At the same time, the Frontiers Journal Series operates on a revolutionary invention, the tiered publishing system, initially addressing specific communities of scholars, and gradually climbing up to broader public understanding, thus serving the interests of the lay society, too.

\section{Dedication to Quality}

Each Frontiers article is a landmark of the highest quality, thanks to genuinely collaborative interactions between authors and review editors, who include some of the world's best academicians. Research must be certified by peers before entering a stream of knowledge that may eventually reach the public - and shape society; therefore, Frontiers only applies the most rigorous and unbiased reviews.

Frontiers revolutionizes research publishing by freely delivering the most outstanding research, evaluated with no bias from both the academic and social point of view. By applying the most advanced information technologies, Frontiers is catapulting scholarly publishing into a new generation.

\section{What are Frontiers Research Topics?}

Frontiers Research Topics are very popular trademarks of the Frontiers Journals Series: they are collections of at least ten articles, all centered on a particular subject. With their unique mix of varied contributions from Original Research to Review Articles, Frontiers Research Topics unify the most influential researchers, the latest key findings and historical advances in a hot research area! Find out more on how to host your own Frontiers Research Topic or contribute to one as an author by contacting the Frontiers Editorial Office: researchtopics@frontiersin.org 


\section{BIOMECHATRONICS: HARMONIZING MECHATRONIC SYSTEMS WITH HUMAN BEINGS}

Topic Editors:

Dingguo Zhang, Shanghai Jiao Tong University, China

Venketesh Dubey, Bournemouth University, United Kingdom

Wenwei Yu, Chiba University, Japan

Kin Huat Low, Nanyang Technological University, Singapore

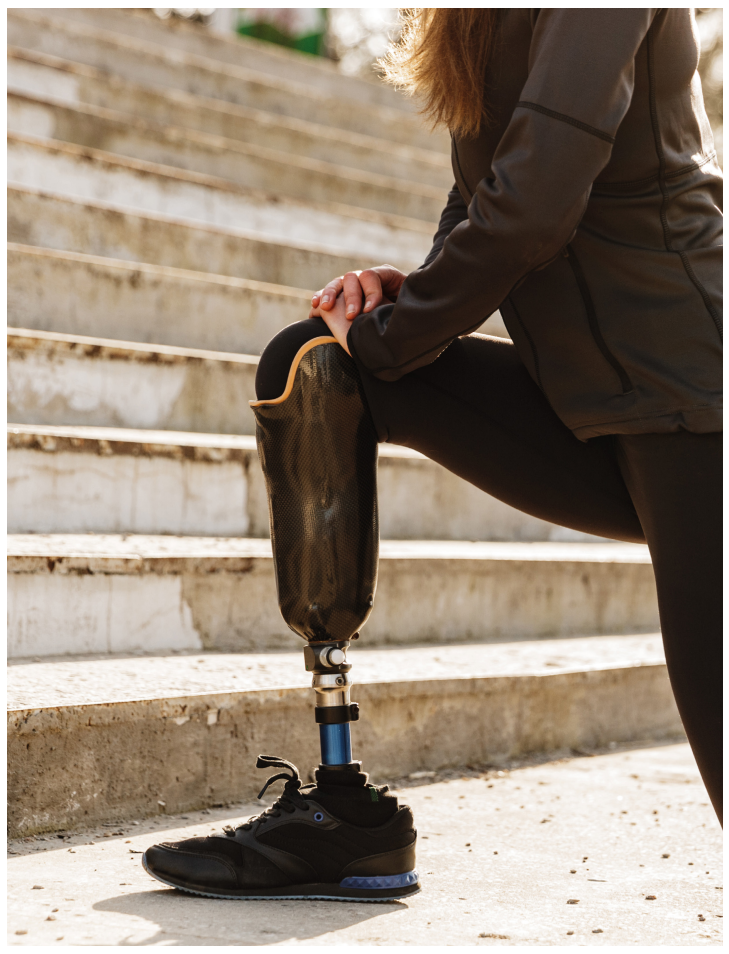

Image: Dean Drobot/Shutterstock.com

This eBook provides a comprehensive treatise on modern biomechatronic systems centred around human applications. A particular emphsis is given to exoskeleton designs for assistance and training with advanced interfaces in human-machine interaction. Some of these designs are validated with experimental results which the reader will find very informative as building-blocks for designing such systems. This eBook will be ideally suited to those researching in biomechatronic area with bio-feedback applications or those who are involved in high-end research on manmachine interfaces. This may also serve as a textbook for biomechatronic design at post-graduate level.

Citation: Zhang, D., Dubey, V., Yu, W., Low, K. H., eds. (2019). Biomechatronics: Harmonizing Mechatronic Systems with Human Beings. Lausanne: Frontiers Media. doi: 10.3389/978-2-88945-737-3 


\section{Table of Contents}

05 Editorial: Biomechatronics: Harmonizing Mechatronic Systems With Human Beings

Dingguo Zhang, Venketesh N. Dubey, Wenwei Yu and Kin Huat Low

\section{SECTION A}

NOVEL EXOSKELETONS FOR ASSISTANCE AND TRAINING

08 Design of a Soft Robotic Elbow Sleeve With Passive and Intent-Controlled Actuation

Tze Hui Koh, Nicholas Cheng, Hong Kai Yap and Chen-Hua Yeow

20 Design and Preliminary Feasibility Study of a Soft Robotic Glove for Hand Function Assistance in Stroke Survivors

Hong Kai Yap, Jeong Hoon Lim, Fatima Nasrallah and Chen-Hua Yeow

34 Design and Validation of Exoskeleton Actuated by Soft Modules Toward

Neurorehabilitation-Vision-Based Control for Precise Reaching Motion of Upper Limb

Victoria W. Oguntosin, Yoshiki Mori, Hyejong Kim, Slawomir J. Nasuto, Sadao Kawamura and Yoshikatsu Hayashi

54 Assessment of Motor Control During Three-Dimensional Movements Tracking With Position-Varying Gravity Compensation

Yao Huang, Qianqian Yang, Ying Chen and Rong Song

63 Development and Feasibility Assessment of a Rotational Orthosis for Walking With Arm Swing

Juan Fang, Qing Xie, Guo-Yuan Yang and Le Xie

\section{SECTION B}

\section{ADVANCED HUMAN-MACHINE INTERFACES IN BIOMECHATRONICS}

73 EMG-Based Continuous and Simultaneous Estimation of Arm Kinematics in Able-Bodied Individuals and Stroke Survivors

Jie Liu, Sang Hoon Kang, Dali Xu, Yupeng Ren, Song Joo Lee and

Li-Qun Zhang

84 Simultaneous and Continuous Estimation of Shoulder and Elbow Kinematics From Surface EMG Signals

Qin Zhang, Runfeng Liu, Wenbin Chen and Caihua Xiong

96 Grip Force and 3D Push-Pull Force Estimation Based on SEMG and GRNN Changcheng Wu, Hong Zeng, Aiguo Song and Baoguo Xu

111 sEMG Sensor Using Polypyrrole-Coated Nonwoven Fabric Sheet for Practical Control of Prosthetic Hand

Yinlai Jiang, Masami Togane, Baoliang Lu and Hiroshi Yokoi

123 Evolutionary Algorithm Based Feature Optimization for Multi-Channel EEG Classification

Yubo Wang and Kalyana C. Veluvolu 
137 Decoding Lower Limb Muscle Activity and Kinematics From Cortical

Neural Spike Trains During Monkey Performing Stand and Squat

Movements

Xuan Ma, Chaolin Ma, Jian Huang, Peng Zhang, Jiang Xu and Jiping He

153 A 3D Computational Model of Transcutaneous Electrical Nerve Stimulation for Estimating Aß Tactile Nerve Fiber Excitability

Kaihua Zhu, Liming Li, Xuyong Wei and Xiaohong Sui

166 Improving Challenge/Skill Ratio in a Multimodal Interface by Simultaneously Adapting Game Difficulty and Haptic Assistance Through Psychophysiological and Performance Feedback

Carlos Rodriguez-Guerrero, Kristel Knaepen, Juan C. Fraile-Marinero, Javier Perez-Turiel, Valentin Gonzalez-de-Garibay and Dirk Lefeber

181 Physiological Signal-Based Method for Measurement of Pain Intensity Yaqi Chu, Xingang Zhao, Jianda Han and Yang Su

\section{SECTION C}

\section{EXPERIMENTAL OUTCOMES AND VALIDATION}

194 Phase-II Clinical Validation of a Powered Exoskeleton for the Treatment of Elbow Spasticity

Simona Crea, Marco Cempini, Stefano Mazzoleni, Maria Chiara Carrozza, Federico Posteraro and Nicola Vitiello

204 On the Value of Estimating Human Arm Stiffness During Virtual Teleoperation With Robotic Manipulators

Jacopo Buzzi, Giancarlo Ferrigno, Joost M. Jansma and Elena De Momi

216 Generation of a Movement Scheme for Positive Training

Lin Liu, Le Xie, Yun-Yong Shi and Bing-Chen An

229 Virtual Normalization of Physical Impairment: A Pilot Study to Evaluate Motor Learning in Presence of Physical Impairment

Christopher Jarrett and Andrew McDaid

243 Analysis of Interrelationships Among Voluntary and Prosthetic Leg Joint Parameters Using Cyclograms

Farahiyah Jasni, Nur Azah Hamzaid, Nor Elleeiana Mohd Syah, Tze Y. Chung and Noor Azuan Abu Osman 


\title{
Editorial: Biomechatronics: Harmonizing Mechatronic Systems With Human Beings
}

\author{
Dingguo Zhang ${ }^{1 *}$, Venketesh N. Dubey ${ }^{2 *}$, Wenwei Yu ${ }^{3}$ and Kin Huat Low ${ }^{4}$ \\ ${ }^{1}$ School of Mechanical Engineering, Robotics Institute, Shanghai Jiao Tong University, Shanghai, China, ${ }^{2}$ Faculty of Science \\ and Technology, Bournemouth University, Poole, United Kingdom, ${ }^{3}$ Center for Frontier Medical Engineering, Chiba University, \\ Chiba, Japan, ${ }^{4}$ School of Mechanical and Aerospace Engineering, Nanyang Technological University, Singapore, Singapore
}

Keywords: biomechatronics, exoskeleton, prosthesis, rehabilitation robot, human-machine interface, brainmachine interface, electromyography, electroencephalography

\section{Editorial on the Research Topic}

Biomechatronics: Harmonizing Mechatronic Systems With Human Beings

There has been a growing body of research in the recent years on human-robot interactions, human-machine interfaces and intelligent devices that are centered around human application, however, these works by and large lacked in focus on how to harmonize the interactions between mechatronic systems and users in the loop. This is one of the key areas for evaluating the success of any mechatronic system implementation on human. The collection of papers in this volume is touching upon the frontiers of this research area as to how the efficacy of such biomechatronic systems could be evaluated and improved. There are a total of 19 papers looking into various aspects of human-machine interfaces (HMIs) using electromyography (EMG) and electroencephalography (EEG), tactile feedback, external devices such as exoskeletons and prosthetic devices for assistance and rehabilitation, novel techniques like machine learning and intelligent computation, and experimental evaluation or validation. The following paragraphs aim to give a glimpse of the contents presented in this eBook. Specifically, these are categorized under three distinct headings: (A) Novel exoskeletons for assistance and training, (B) Advanced human-machine interfaces in biomechatronics, and (C) Experimental outcomes and validation.

\section{A. NOVEL EXOSKELETONS FOR ASSISTANCE AND TRAINING}

There has been a surge of interests in implementing "soft robots" into every aspect of robotic assistance and training since it can provide compliance with inherent safety features to users. The paper by Koh et al. discussed a soft robotic elbow sleeve with full design features and functionality using elastomeric and fabric-based pneumatic actuators; what was more interesting was the intent based actuation control. The user intent was detected by surface electromyography (sEMG) on healthy subjects and the results showed that all participants were capable of achieving EMG control of the elbow sleeve. An extension of the above idea by the same group was explored by Yap et al. using soft robotic gloves with fabric-reinforced soft actuators, which targeted hand function assistance. They integrated elastic fabric with soft actuators to enhance the driven force for finger extension. A pilot test on stroke survivors showed improved grasping performance with the assistance of the soft glove. In a slightly different approach Oguntosin et al. have demonstrated functional properties of the exoskeleton actuated by soft modules. The exoskeleton has 3D printed parts with passive joints and utilizing the gravity compensation scheme to make it ultra-light. The exoskeleton was proposed for precise reaching control in neurorehabilitation for individuals with specific motor impairments. These papers highlighted the advantage of using soft modules for rehabilitation. 
In an unusual way Huang et al. have studied a cable based robot for assessment of motor control during 3D movements tracking with position varying gravity compensation. They have shown that there was a significant difference in control strategies with and without gravity compensation which might assist subjects in performing optimal movements during rehabilitation. Fang et al. have realized the fact that people swing their arms synchronously with leg movement in the normal gait. So they developed a rotational orthosis for walking with arm swing for gait training to provide coordinated interlimb performance. This could potentially improve gait rehabilitation outcomes of patients undergoing training; the device was tested for functional assessment with normal subjects and was reported to provide a stronger feeling of walking with arm swing than without.

\section{B. ADVANCED HUMAN-MACHINE INTERFACES IN BIOMECHATRONICS}

Advanced human-machine interfaces (HMIs) are the key to harmonizing mechatronic systems with human beings in the loop; many developed systems simply fail because they lack of good interfaces for interpreting user motion intention/states and providing tactile feedback in the systems. Liu et al. have developed a model for continuous and simultaneous decoding of multi-joint dynamic arm movements based on multichannel surface EMG signals. This was used to myoelectrically control exoskeletons for upper-limb rehabilitation which showed the potential for effortless control of impaired arms. On a similar approach Zhang et al. have used sEMG signals for simultaneous and continuous estimation of shoulder and elbow kinematics using principal/independent component analysis and artificial neural network for learning electromechanical association. This was done to achieve natural and intuitive human-machine interaction particularly for applications in exoskeletons, prostheses and other arm rehabilitation techniques. In an extension to the above approach $\mathrm{Wu}$ et al. developed grip force and three-dimensional push-pull force estimation based on sEMG and generalized regression neural network. This work was proposed to meet the requirements of force control of the intelligent prosthetic hand which employed EMG and grip force sensors to measure the output force of the human hand. Since all the above reported work employed EMG sensors, there must be a better way of acquiring stable signals. Jiang et al. have proposed a Polypyrrole-coated nonwoven fabric sheet for designing EMG sensors. The design can be customized for shape and size and can be sewn onto elastic band for close contact with skin for practical control of prosthetic hand. The sensors provided comparable results with conventional $\mathrm{Ag} / \mathrm{AgCl}$ electrodes typically used in EMG sensors.

In a slight depart from the EMG based HMIs using muscle signals, EEG based HMIs employ brain signals, which are well-known as brain-machine interfaces (BMIs). Some previous non-invasive BMIs adopted Fourier based methods for time-frequency decomposition for feature extraction of EEG. However, this technique was challenging for multichannel EEG signals. To improve performance of multi-channel
EEG classification, Wang and Veluvolu developed evolutionary algorithms for optimizing the features to reduce the overall dimensionality and preserve the class-related information. Their results show that the combination of Fourier based method with covariance matrix adaptation evolution strategy has the best overall performance. Compared with non-invasive BMIs based on scalp EEG, invasive BMIs use high-quality brain signals, which are crucial for advanced control of dexterous robotic arms or prosthetic hands. Ma et al. have conducted a trial with monkeys to decode lower limb muscle activity and kinematics from cortical neural spike recordings during stand/squat movements. They provided detailed analyses of recorded data and new insights into invasive BMIs that could be used for controlling complex biomechatronic systems.

To explore the mechanism of tactile sensation under transcutaneous electrical nerve stimulation, a 3D computational model was developed for estimating tactile nerve fiber excitability (Zhu et al.). The simulation showed comparable results with psychophysical experimental data with healthy subjects. This study has potential for providing tactile feedback for prosthesis and realizing bi-directional neural-machine interfaces in future. In order to improve challenge/skill ratio in a multi-modal interface for human-robot interaction, Rodriguez-Guerrero et al. have investigated simultaneous adaptation of game difficulty and haptic assistance through psychophysiological signals (heart rate, skin conductance level, and skin conductance response frequency) and performance feedback. They came up with a new matric (FlowIndex) to numerically quantify and visualize the challenge/skill relation which improved the balance between augmented performance and user satisfaction. Different from the traditional diagnosis based on the patient's self-description, Chu et al. have developed a method of pattern recognition to classify the pain intensity based on multiple physiological signals (blood volume pulse, electrocardiogram, and skin conductance level). This has implications for the patients with major cognitive and communicative impairments who are not able to describe their level of pain. The experimental results on healthy subjects showed that the method can provide objective and quantitative evaluation of pain intensity.

\section{EXPERIMENTAL OUTCOMES AND VALIDATION}

The level of harmonization between mechatronic systems and human beings could not be assessed without validating these devices under experiments with human subjects. There are several studies that focused on experimental trials to prove the efficacy of their devices. For example, Crea et al. have reported Phase II of clinical validation of a powered exoskeleton for the treatment of elbow spasticity. The rehabilitation outcomes from 17 post-stroke patients with robotic elbow exoskeleton showed that intensive early treatment prevented the occurrence of spasticity at a later stage. Buzzi et al. proposed a non-disruptive method to study the arm endpoint stiffness in a teleoperated robotic system. Based on a musculoskeletal model, they could use arm kinematics and muscular activation (sEMG) to estimate the 
endpoint stiffness. Here, they just focused on experimental study to investigate how different master devices and tasks influence the regulation of arm endpoint stiffness and its relation with task performance, hand speed and acceleration. This experimental study may further help to understand which characteristics and parameters play a fundamental role in compliant humanrobot interactions. In order to generate sufficient and accurate motion data for positive rehabilitation using exoskeletons, Liu et al. conducted experimental study based on their custommade Neo-Arm exoskeleton. At each joint of Neo-Arm, an angular displacement sensor was equipped through the axis of the rotation, by which the angular information could be captured. Their methods have been compared with the conventional method i.e., a camera-based (Vicon) system. The experiments were conducted on 8 healthy subjects performing a series of movements, including five actions and five hovering postures, and the results showed that the satisfactory data could be achieved with suitable precision for upper limb motion tasks without the need for platform based systems. Since motor learning is a critical component of the rehabilitation process, Jarrett and McDaid conducted a simulated pilot study with healthy subjects to evaluate motor learning in case of physical impairment. They developed a technique where simulated impairment is applied to constrain the motion, such that motor learning could be studied independently of physical impairment. Their tool could be used to get insight into underlying causes of motor learning deficits. This could be used to provide subjectspecific therapy. Jasni et al. have analyzed the interaction among the voluntary and mechanical joints and segments in users wearing lower-limb prosthesis/orthosis using cyclograms. The cyclogram model was developed using data from 20 healthy able-bodied subjects and 25 prosthesis and orthosis users (10 transtibial amputees, 5 transfemoral amputees, and 10 orthosis users).This cyclogram model enables quantitative judgment of the effect of changing a particular parameter in the prosthetic leg gait. This experimental study is particularly useful for understanding coordinated movement of several joints and limb segments wearing external devices.

So looking at the collection of papers in this research topic we are happy to endorse that this eBook provides a comprehensive appraisal of the latest developments in the field of biomechatronics including exoskeletons, prosthesis, and advanced human-machine interfaces and followed by experimental validation of some of the developed techniques. We hope that these papers will provide readers new insights into the aspects of harmonizing mechatronic systems with human beings.

\section{AUTHOR CONTRIBUTIONS}

DZ was invited to prepare this research topic. He invited VD, WY, and $\mathrm{KL}$ to be co-editors in it. They all contributed to soliciting papers, inviting guest-authors, revising their manuscripts, and handling revisions with invited reviewers. Specifically, DZ edited 12 papers, VD edited 2 papers, WY edited 4 papers, and KL edited 1 paper. VD drafted the editorial.

\section{FUNDING}

This work was supported by the National Natural Science Foundation of China (No. 51475292, No.61761166006), and was also supported by the EU International Research Staff Exchange Scheme.

Conflict of Interest Statement: The authors declare that the research was conducted in the absence of any commercial or financial relationships that could be construed as a potential conflict of interest.

Copyright (c) 2018 Zhang, Dubey, Yu and Low. This is an open-access article distributed under the terms of the Creative Commons Attribution License (CC BY). The use, distribution or reproduction in other forums is permitted, provided the original author(s) and the copyright owner(s) are credited and that the original publication in this journal is cited, in accordance with accepted academic practice. No use, distribution or reproduction is permitted which does not comply with these terms. 


\section{OPEN ACCESS}

Edited by:

Kin Huat Low,

Nanyang Technological University,

Singapore

Reviewed by:

Danilo Emilio De Rossi,

University of Pisa, Italy

Kyujin Cho,

Seoul National University, South Korea

*Correspondence:

Chen-Hua Yeow

rayeow@nus.edu.sg

Specialty section

This article was submitted to

Neural Technology,

a section of the journal

Frontiers in Neuroscience

Received: 19 December 2016

Accepted: 10 October 2017

Published: 25 October 2017

Citation:

Koh TH, Cheng N, Yap HK and Yeow C-H (2017) Design of a Soft

Robotic Elbow Sleeve with Passive and Intent-Controlled Actuation.

Front. Neurosci. 11:597.

doi: 10.3389/fnins.2017.00597

\section{Design of a Soft Robotic Elbow Sleeve with Passive and Intent-Controlled Actuation}

\author{
Tze Hui Koh ${ }^{1}$, Nicholas Cheng ${ }^{1}$, Hong Kai Yap ${ }^{1,2}$ and Chen-Hua Yeow ${ }^{1 *}$ \\ ${ }^{1}$ Evolution Innovation Laboratory, Department of Biomedical Engineering, National University of Singapore, Singapore, \\ Singapore, ${ }^{2}$ NUS Graduate School for Integrative Sciences and Engineering, National University of Singapore, Singapore, \\ Singapore
}

The provision of continuous passive, and intent-based assisted movements for neuromuscular training can be incorporated into a robotic elbow sleeve. The objective of this study is to propose the design and test the functionality of a soft robotic elbow sleeve in assisting flexion and extension of the elbow, both passively and using intent-based motion reinforcement. First, the elbow sleeve was developed, using elastomeric and fabric-based pneumatic actuators, which are soft and lightweight, in order to address issues of non-portability and poor alignment with joints that conventional robotic rehabilitation devices are faced with. Second, the control system was developed to allow for: (i) continuous passive actuation, in which the actuators will be activated in cycles, alternating between flexion and extension; and (ii) an intent-based actuation, in which user intent is detected by surface electromyography (sEMG) sensors attached to the biceps and triceps, and passed through a logic sequence to allow for flexion or extension of the elbow. Using this setup, the elbow sleeve was tested on six healthy subjects to assess the functionality of the device, in terms of the range of motion afforded by the device while in the continuous passive actuation. The results showed that the elbow sleeve is capable of achieving approximately $50 \%$ of the full range of motion of the elbow joint among all subjects. Next, further experiments were conducted to test the efficacy of the intent-based actuation on these healthy subjects. The results showed that all subjects were capable of achieving electromyography (EMG) control of the elbow sleeve. These preliminary results show that the elbow sleeve is capable of carrying out continuous passive and intent-based assisted movements. Further investigation of the clinical implementation of the elbow sleeve for the neuromuscular training of neurologically-impaired persons, such as stroke survivors, is needed.

Keywords: robotic, rehabilitation, elbow, stroke, assistive, electromyography-driven, wearable, soft-robotic

\section{INTRODUCTION}

According to the World Health Organization, every year approximately 15 million people around the world suffer from stroke (Rodrigo et al., 2013), and 70-80\% experience upper-extremity impairment (Hu et al., 2007). The upper extremity, which includes the shoulder, elbow, wrist and hand, is important for the spatial movement and manipulation of objects, as well as for gesturing. It is therefore essential that stroke survivors regain a certain level of functionality so that they are able to independently carry out the activities of daily living. 
Traditionally, stroke rehabilitation is done in the form of therapeutic intervention with the help of physiotherapists and occupational therapists, which, while effective, is timeconsuming and labor intensive ( $\mathrm{Hu}$ et al., 2007). To reduce the burden on caregivers and the need for oversight by trained therapists, robotic devices have been developed, which aim at supporting the patients in carrying out rehabilitation at their convenience and with minimal supervision. These robotic devices are typically devised to carry out: (1) continuous passive movements, and (2) active-assisted movements. Continuous passive movements are useful for reducing muscle tone to improve the mobility of the joint (Hu et al., 2009), while activeassisted movements help in recovering motor control through the positive shaping of the cortical reorganization following brain injuries such as stroke (Hesse et al., 2003). Current robotic rehabilitation devices include end-effector robots, such as the MIT Manus and MIME, and exoskeletons, such as the Myomo, ARMin, OrthoJacket and KIST Wearable Robotic Arm (Zhang et al., 2015). While these devices have demonstrated good efficacy in rehabilitation, they are costly, non-portable and rigid, which limits them to the clinical setting, and their rigidity also makes it difficult for them to be aligned with the patients' joints during rehabilitation (Oguntosin et al., 2015).

Soft robotics is an emerging area of robotics research resulting from the melding of materials chemistry and robotics, and aims to tackle the challenges faced by traditional robotics (Polygerinos et al., 2015b). As its name suggests, soft robotics consists of robots that are manufactured with flexible or elastomeric structural elements. In particular, soft pneumatic actuators are an important component used in soft wearable robots because they are lightweight, flexible and able to distribute pressure evenly along the joint (Oguntosin et al., 2015; Polygerinos et al., 2015a,b). Additionally, their response to actuation may be customized to a large degree to accomplish motions typically difficult with hard robotic devices, possibly overcoming alignment problems through the direct attachment of actuators to the limbs (In et al., 2015). Some current developments in soft robotics for rehabilitative purposes include a soft robotic glove for robotassisted hand therapy (Yap et al., 2015, 2016b; Yeo et al., 2016), as well as a soft robotic sock for robot-assisted ankle exercise (Low et al., 2016).

The provision of timely assistance upon detecting user-intent (Lenzi et al., 2012) is a common issue in the development of robotic devices for active-assisted rehabilitation. Current stateof-the-art devices such as the Myomo and OrthoJacket have builtin electromyography (EMG) capabilities that help to estimate the joint torque needed to perform movement (Benitez et al., 2013). Studies have shown that intention-based rehabilitation enhances the therapeutic effect, accelerating the speed with which functionality is recovered (Zhang and Zhou, 2012).

The above-mentioned developments open up possibilities in developing myoelectric-based lightweight wearable robotics, which have the potential to both restore normal function in patients affected by stroke, as well as to enhance the outcome of their neuro-muscular rehabilitation. In this paper, we present the design of a soft robotic elbow sleeve for enabling flexion and extension of the elbow both passively and intent-based motion reinforcement, which may be useful as a neuro-muscular training device. In the following sections, we discuss the methods for fabricating the actuators for the elbow sleeve, the design of the control system for the elbow sleeve, methods for mechanical characterization of the actuators, as well as a description of the range of motion study that was conducted to evaluate the functionality of the device. Finally, we present the results of the mechanical characterization and the range of motion study, and discuss their implications.

\section{MATERIALS AND METHODS}

\subsection{Design Overview}

The soft robotic elbow sleeve spans across the elbow joint, from below the biceps of the arm to the middle of the forearm (Figure 1), and comprises the following components: (1) two pneumatic actuators to individually effect elbow joint flexion and extension; (2) a programmable pump-valve control system to switch between modes of operation; (3) surface EMG sensors which are attached to the biceps and triceps; (4) an external pump for providing air supply to the actuators; and (5) hook and loop straps which secure the actuators to the arm.

The working principle of the soft robotic elbow sleeve is based on the controlled air pressurization of two separate pneumatic actuators working antagonistically with respect to one another. Mechanically, pressurization of the flexion actuator causes the elbow to flex, while pressurization of the extension actuator causes the elbow to extend. The two resulting configurations (flexed and extended) of the arm are shown in Figure 2. Electronic control for the mechanical action of the actuators allows for two distinct mode of operations of the soft robotic sleeve-passive rehabilitation and intent-based rehabilitation. This is accomplished through the valves, pressure sensor, microcontroller, which are packed within a custom-made control box, and two surface EMG sensors. The details of the actuators and the control system are given in the following sections.

\subsection{Actuator Design and Fabrication 2.2.1. Flexion Actuator}

The flexion actuator in the soft robotic sleeve is an elastomeric construct that bends upon an input air pressure. To obtain a desired angular configuration upon an input air pressure, a fiber-fabric-reinforced approach adapted from Polygerinos et al. (2015b) was adopted in the development of the flexion actuator. The molds for the flexion actuator body (which described a length of $24 \mathrm{~cm}$ and radius of $1 \mathrm{~cm}$ ) were first designed using a Computer-Aided Design software (Dassault Systèmes SOLIDWORKS Corp., USA) and 3D-printed with Acrylonitrile Butadiene Styrene (ABS). These molds were then used to fabricate the actuator body using a soft elastomeric material, Dragon Skin 20 (Figure 3A). The actuator body was then sealed with a flat piece of elastomeric layer (Dragon Skin 20), and a woven nylon strip (which acts as a strain-limiting layer) was glued to this flat surface to enable bending of the actuator (Figure 3B). Following that, monofilament thread (Nylon) was hand-wound around the actuator, with the thread pitch $(5 \mathrm{~mm})$ determined by the grooves imprinted onto the actuator (Figure 3C). This 


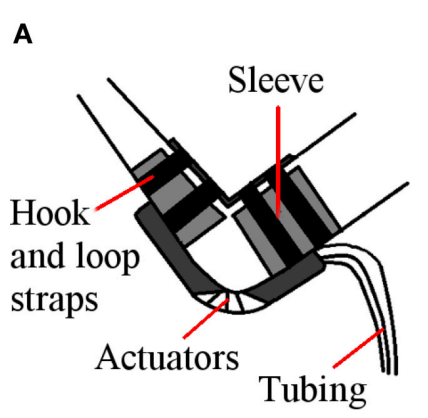

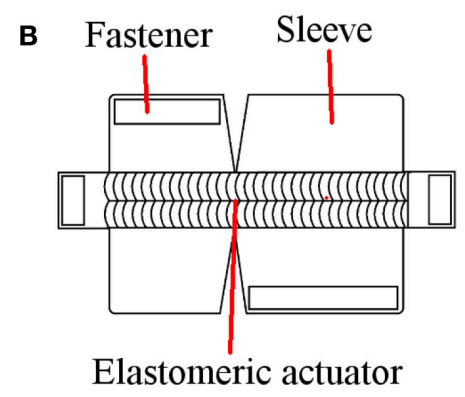

Elastomeric actuator

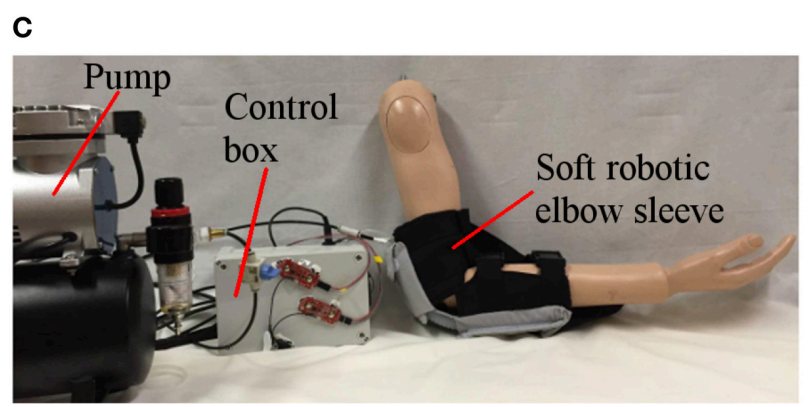

FIGURE 1 | Soft robotic elbow sleeve for stroke rehabilitation. (A) Illustration of the soft robotic sleeve. (B) Schematic of the constraining layer for the flexion actuator. (C) Fully assembled system.

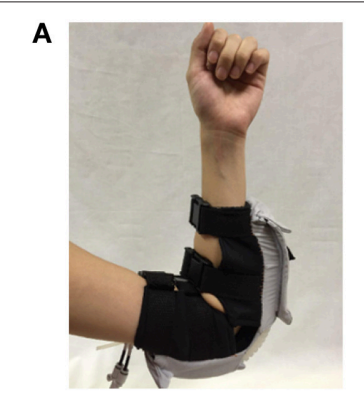

B

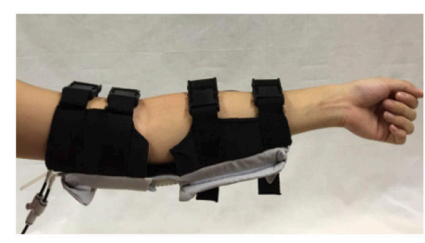

FIGURE 2 | Arm configurations during the use of the soft robotic elbow sleeve. (A) Flexed configuration. (B) Extended configuration.

serves to radially reinforce the actuator. Two of these flexion actuators were made and assembled for the elbow sleeve. They were wrapped with a Nylon textile where bending is not required, so as to allow for an angular configuration in bending (Figure 4).

Dragon Skin 20 was chosen as the elastomer for fabricating the actuator due to its ability to withstand higher pressure and provide higher force output at Shore A hardness of 20 as compared to other tested materials such as Dragon Skin 10. The length of the actuator $(24 \mathrm{~cm})$ was determined based on the distance of the center of gravity of the average human forearm from the elbow joint (approximately $41.7 \%$ from the elbow joint, which is $18.7 \mathrm{~cm}$, inclusive of hand) (Drillis et al., 1964) as well as the force that each actuator is able to produce, in order to ensure that sufficient torque can be generated to assist flexion. The Nylon monofilament thread that was wound around the actuator was introduced to limit radial expansion and thus improve the actuator's durability.

\subsubsection{Extension Actuator}

As the extension actuator is placed interior to the flexion actuator along the elbow sleeve, we cannot simply replicate the method used for the flexion actuator (elastomeric bending actuator) in developing the extension actuator as it would hinder the operation of the flexion actuator due to its sheer volume. An alternate design, following an inflatable beam model, was instead utilized, involving the use of the torque generated from the inflation of the actuator to extend the elbow.

Nylon ripstop sheets coated with thermoplastic polyurethane were heat sealed to form an air tight chamber with a single inlet, resulting in a fabric actuator with a length of $20 \mathrm{~cm}$. While deflated, the actuator does not take up much space, and provides little hindrance to the action of the flexion actuator (Figure 5A). Through inflation, the actuator is then able to act in a manner similar to that of a rigid beam and resist deflection (Sanan et al., 2009; Yap et al., 2016c) (Figure 5B).

\subsection{Actuator Characterization}

To evaluate the force-pressure relationship of the flexion and extension actuators, two experimental platforms were developed, as illustrated in Figure 6.

For the flexion actuator (Figure 6A), following a similar protocol introduced by Polygerinos et al. (Polygerinos et al., 2015b; Yap et al., 2016a), the actuator was constrained in a rigid fixture to minimize nonlinear effects, with the distal tip placed in contact with a force sensor. The pressure in the actuator was 
A

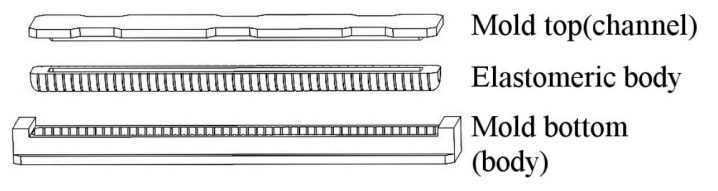

B

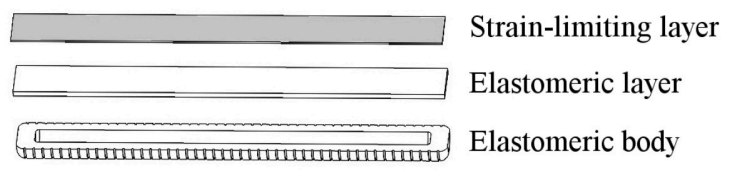

C Monofilament-
threaded assembly

FIGURE 3 | Key stages of actuator fabrication. (A) Molding of the actuator's elastomeric body (Dragon Skin 20) using a 3D-printed mold (ABS). (B) Application of a thin elastomeric layer (Dragon skin 20) together with strain limiting layer (Nylon). (C) Winding of a monofilament thread (Nylon) in a double-helical pattern to provide radial reinforcement.
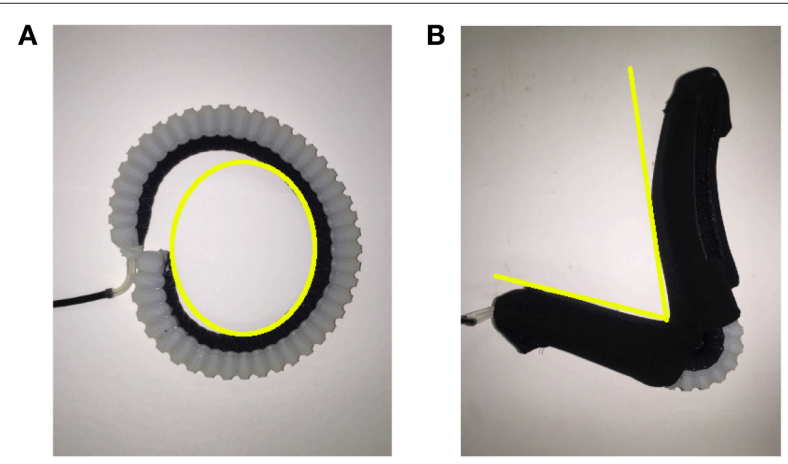

FIGURE 4 | Illustrating the effect of wrapping Nylon around the flexion actuator to acquire a desired angular configuration. (A) An unconstrained elastomeric actuator in flexed formation, with the yellow curve indicating a rounded curvature upon air pressure input. (B) A fabric constrained elastomeric actuator upon pressurization, with the yellow lines indicating the angular curvature achieved.
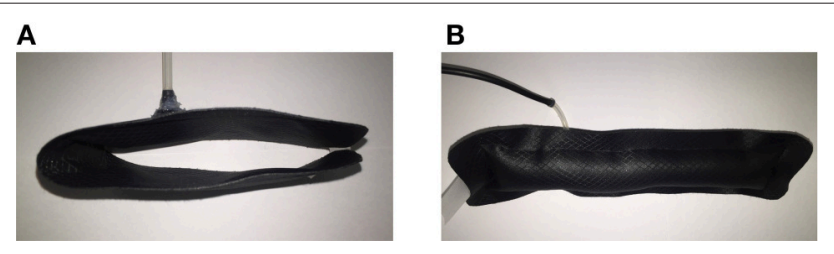

FIGURE 5 | Illustrating the two configurations of the extension actuator, with and without air pressurization. (A) The deflated nylon ripstop actuator in flexed configuration, which allows conformation to the flexion actuator. (B) The inflated nylon ripstop actuator in extended configuration, which acts as a rigid beam and resists bending deformation.

gradually increased as the force sensor recorded the bending force exerted by the tip. This enables the measurement of the maximum force generated by the tip of the actuator at each pressure.

For the extension actuator (Figure 6B), experimental deflection tests were conducted by fixing the actuator at one end with varying weights hung from the other end of the actuator. For each weight, the pressure was decreased to the point at which the actuator began to deflect. This setup is suitable for determining the relationship between pressure and load capacity. A similar mode of testing was utilized by Sanan et.al to find the relationship between load and deflection (Sanan et al., 2009).

\subsection{Control System}

The programmable pump-valve control system of the robotic elbow sleeve allows for switching between the two rehabilitation modes-passive and active modes-and uses a commercial pneumatic pump and valve for the supplying of air pressure for actuation, controlled by an Arduino Uno micro-controller. Two pneumatic pressure sensors are used to individually monitor the pressures within the flexion and extension actuators, and two surface EMG sensors are used for intent-detection by measuring EMG signals from the biceps and triceps. Feedback control of the system for both passive and EMG rehabilitation modes uses a closed-loop proportional-integral-derivative (PID) regulator, following the basic equation:

$$
u(t)=K_{p} e(t)+K_{i} \int_{0}^{t} e(t) \mathrm{d} t+K_{d} \frac{\mathrm{d} e(t)}{\mathrm{d} t}
$$

where the $u(t)$ is the control variable, in this case, the pressure value; $e(t)$ is the error value; $K_{p}, K_{i}$, and $K_{d}$ are the proportional, integral, and derivative gains, respectively; and $t$ is time. The controller uses pressure as an input and in turn regulates the duty cycle of the pulse-width-modulation (PWM) as a means to control the pressure according to a pre-set point. This also acts as a safety feature to ensure that the pressure within the actuators do not reach failure point. To streamline the process of switching between modes, calibrating the EMG sensors, and the processing of generated data, a Graphical User Interface (GUI) was specially developed in $\mathrm{C}++$ using the Processing Integrated Development Environment.

In the passive rehabilitation mode, the device is actuated in cycles, alternating between flexion and extension. This continuous repetitive movement was enabled by utilizing the internal timer of the micro-controller to track time. When the defined time interval was reached, the system would switch from flexion to extension and vice versa, and the timer would be reset.

Conversely, in active intent-based rehabilitation, the detection of user intent is achieved by the surface EMG sensors attached to the biceps and triceps, in which the EMG signals are passed 

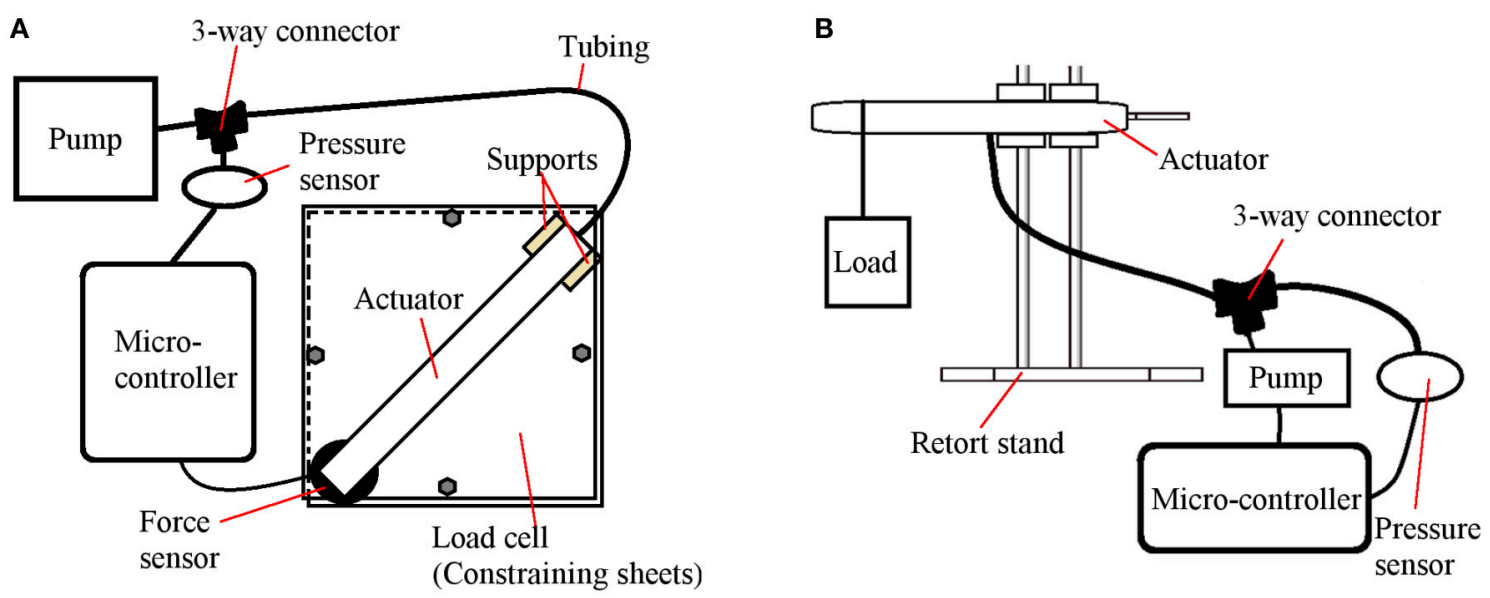

FIGURE 6 | Experimental platforms for the characterization of the actuators. (A) Flexion actuator experimental platform. (B) Extension actuator experimental platform.

through a logic sequence to allow for flexion or extension of the elbow. The EMG control scheme uses rectified, amplified and then integrated surface EMG signals captured from the biceps and triceps. Notably, the nature of the surface EMG signals captured is highly dependent on their spatial location from the muscle fibers, which is difficult to regulate with high precision. The strength of the EMG signals also typically changes with different subjects as well as different muscles (Halaki and Ginn, 2012). To rectify this, for comparison purposes, both signals from the biceps and triceps are normalized by first requesting maximum voluntary contraction (MVC) effort from the user in an initiation sequence. The MVC value obtained is then used as a reference point against which all muscle signals are compared to during the task. This allows assessment of the level of activity and improves the reproducibility of the data. The signals are subsequently passed through a series of conditions as shown in Figure 7 that cause the activation and deactivation of the actuators, based on a time-over-threshold algorithm. For example, the condition for flexing is met when the processed signal from the bicep crosses a percentage threshold for an interval of $20 \mathrm{~ms}$, while ensuring that the signal from the biceps is stronger than the signal from the triceps. A similar set of conditions exists for extension to occur. This method of control minimizes the occurrence of involuntary muscle contractions that may cause misinterpreted commands.

\subsection{Range of Motion Study}

Six healthy human subjects (20-25 years old), three males and three females, were enrolled in this study. Written and informed consent were obtained from all the subjects, with approval from the university's Institutional Review Board (NUS-IRB) prior to the experiment. All experimental trials were performed in a gait analysis laboratory.

Each trial comprises of three segments: (1) voluntary active elbow flexion and extension without the elbow sleeve; (2) use of the elbow sleeve in passive rehabilitation mode; and (3) use of the elbow sleeve in active rehabilitation mode.
In the first experimental segment, subjects were seated and requested to perform three vertical elbow flexions and extensions in succession, for a total of three trials, followed by three horizontal elbow flexions and extensions in succession for another three trials (Figure 8). All trials were started from an extended elbow configuration of the subjects (Figure 8C). This is representative of activities of daily living such as lifting an object (vertical motion) as well as wiping a table (horizontal motion).

In the second experimental segment, the subjects were then tasked to perform the trials in the first segment, with assistance provided by the soft robotic elbow sleeve in the absence of muscular signal input. After donning the soft robotic elbow sleeve, the passive mode of the elbow sleeve system was engaged, causing the subjects' arms to move in the same sequence of activities as in the first segment.

In the third experimental segment, the control mode of the elbow sleeve was switched to the active rehabilitation mode. Two surface EMG sensors (Myoware, Advancer Technologies, LLC) were mounted on the bicep and triceps muscle groups on the arm without the elbow sleeve. The subjects were then tasked with completing only horizontal elbow flexion using activation signals from the two muscle groups on the free arm to control the state of the elbow sleeve.

The Vicon motion capture system was used to track the arm positions of the subjects. Retroreflective markers were attached to each subjects' arm at positions shown in Figure 9, and their $3 \mathrm{D}$ coordinates were sampled at a frequency of $100 \mathrm{~Hz}$. At each sampling point, the resultant elbow flexion angle, formed between the shoulder-to-elbow segment and the elbow-to-wrist segment (as marked out by the retroreflective markers), was computed and recorded for analysis. As such, a fully extended elbow (no flexion) corresponded to a flexion angle of $0^{\circ}$. The range-of-motion (ROM) thus refers to the maximum angle of elbow flexion attainable by each subject.

For the recording of EMG signals from the muscles, the Delsys Trigno Wireless EMG system was used, with a sampling frequency of $1,000 \mathrm{~Hz}$. To minimize the effects of skin resistance, 


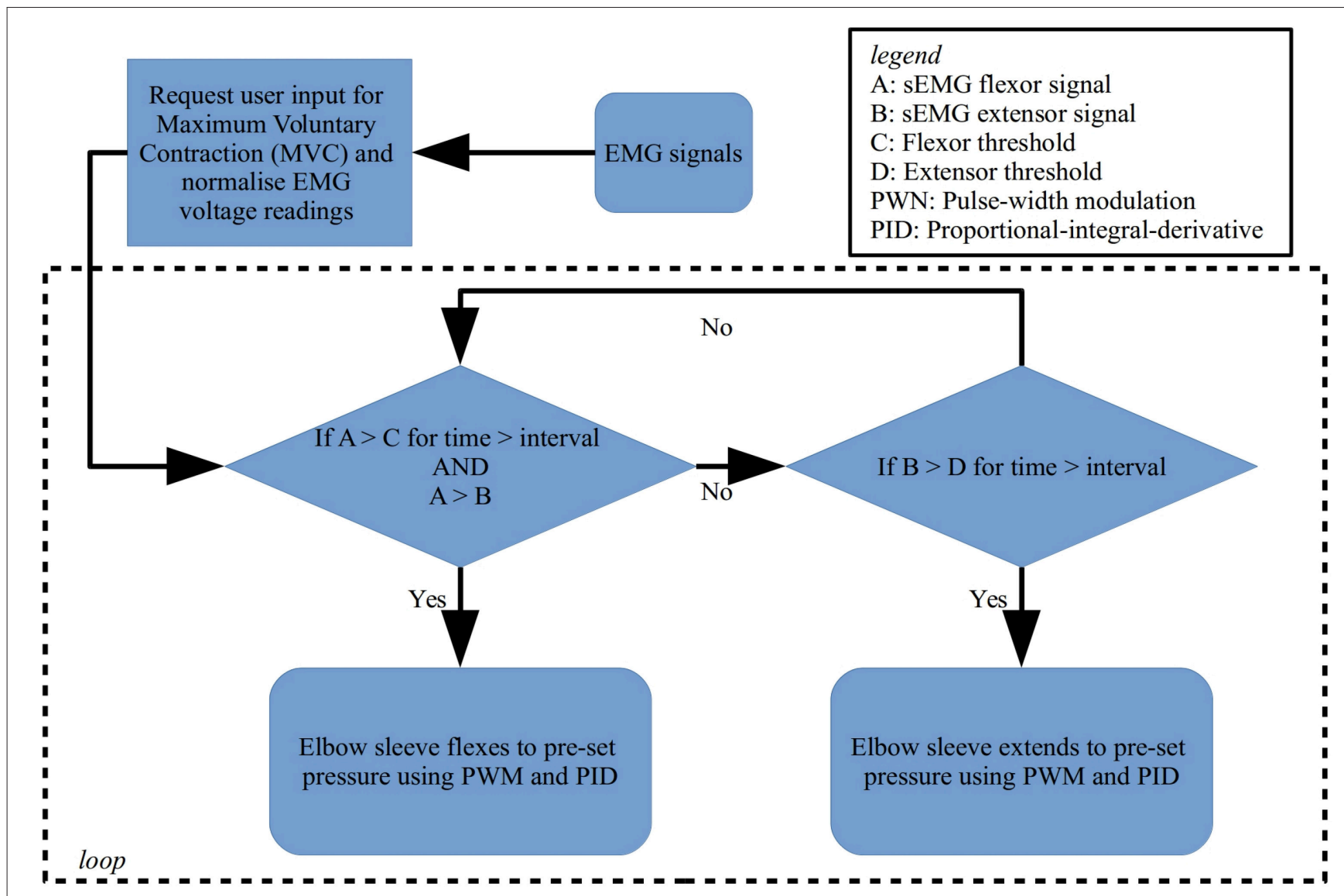

FIGURE 7 | Flowchart of the logic scheme used to detect user intent and control flexion and extension of the soft robotic elbow sleeve.

the area was swabbed with alcohol prior to the placement of the EMG sensors on the biceps and triceps. The inherent variability of the EMG signals necessitates post-processing of the recorded signals. In lieu of this the EMG signals were full-wave rectified, filtered with a second-order band pass Butterworth filter and normalized against the maximum muscle signal output during the first experimental segment.

\section{RESULTS}

\subsection{Actuator Characterization}

The experimental results of the bending force experiments conducted on the flexion actuators are presented in Figure 10A, showing a reproducible non-linear relationship between input pressure and tip force. The maximum force measured at a pressure of $350 \mathrm{kPa}$ was $67.4 \mathrm{~N}$, which is well above the sufficient $20 \mathrm{~N}$ required for the actuation of the elbow (data acquired from body segment data). A force higher than $20 \mathrm{~N}$ is necessary to compensate for the forces which are required to bend the actuator itself and ensure that sufficient bending force is supplied to the arm segments.

For the extension actuator, the pressure-force relationship showed a linear trend (Figure 10B). Linear regression was performed on the data to fit a calibration line for the elbow sleeve $\left(R^{2}=0.9951\right)$, which allows for an estimation of the forces produced at varying pressure magnitudes. At $80 \mathrm{kPa}$, the average deflective force of $16.5 \mathrm{~N}$ the actuator produced was capable of enabling full elbow extension.

\subsection{Control Characterization}

Figure 11 shows the control of pressure within the actuators for the passive rehabilitation mode of the elbow sleeve. The PID controller system was able to maintain pressure at $350 \mathrm{kPa}$ for the flexion actuator and $80 \mathrm{kPa}$ for the extension actuator with small variations of approximately $10 \mathrm{kPa}$. This is adequate for generating the necessary flexion and extension for the elbow joint in the range of motion study.

\subsection{Range of Motion Study}

\subsubsection{Efficacy of Soft Robotic Elbow Sleeve}

To quantify the efficacy of the elbow sleeve in assisting elbow flexion and extension, the elbow joint angles measured in the course of the first and second experimental segments were compared. The first experimental segment involved voluntary active elbow flexion and extension without the elbow sleeve while the second segment involved the use of the elbow sleeve in passive rehabilitation mode, flexing and extending a still elbow. 

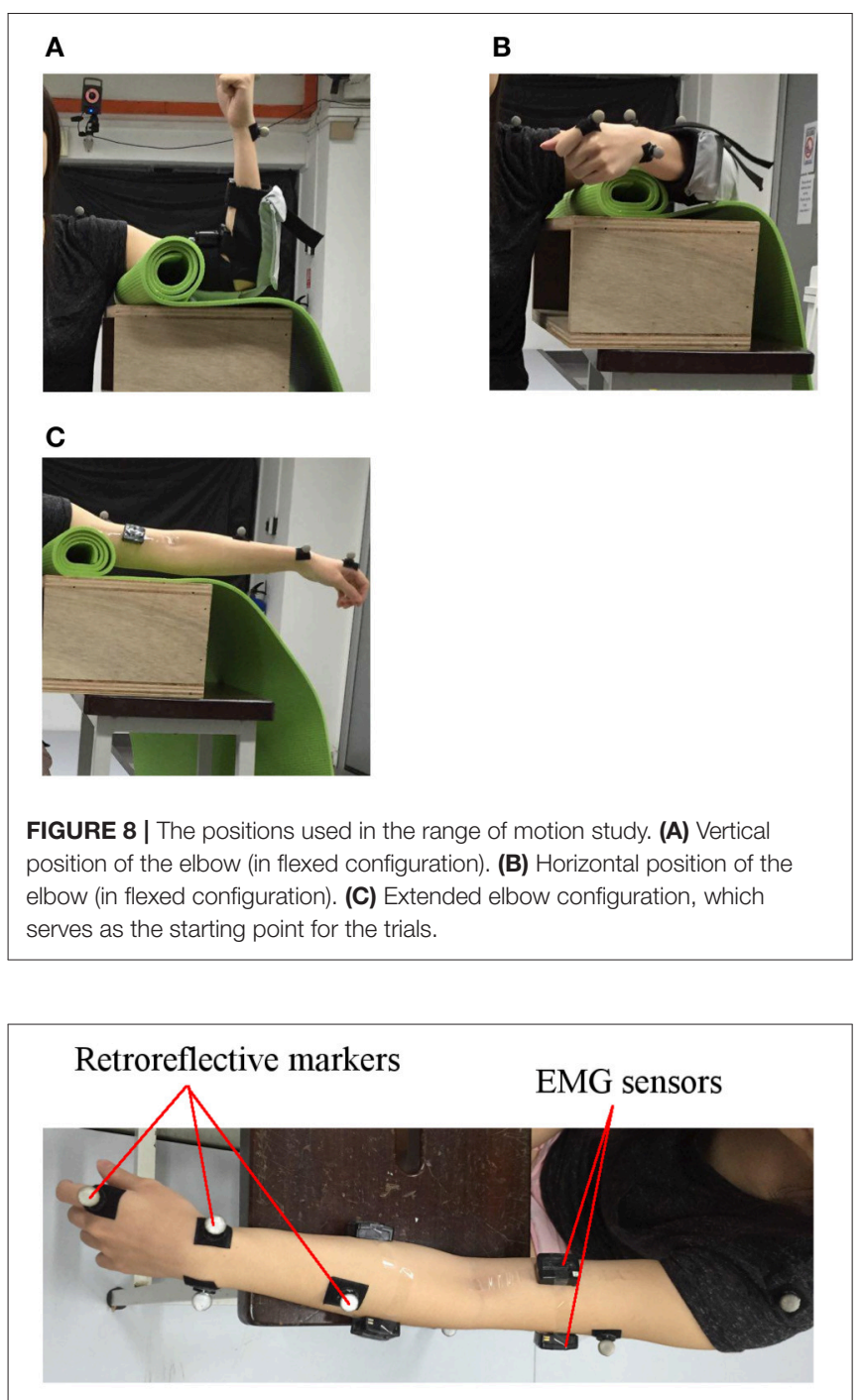

FIGURE 9 | Marker positions for real-time tracking of segment coordinates.

In each experimental segment, elbow flexion and extension were performed in two different directions, vertically and horizontally. As a representation, Figure 12A shows a single subject's elbow joint angle from three cycles of active elbow flexion and extension (experimental segment 1), while Figure 12B shows the same subject's elbow joint angle under passive contraction (experimental segment 2). Looking at the results from the different genders, for the female subjects, it was found that the average active maximum ROM was $127.8^{\circ}$ while the average passive maximum ROM was $69.3^{\circ}$. This translates to the soft robotic elbow sleeve's capability in achieving $54.2 \%$ of the active ROM (with standard deviation of $27.2^{\circ}$ ). In contrast, for male subjects, it was found that the average active maximum ROM was $132.1^{\circ}$ while the average passive maximum ROM was $59.7^{\circ}$. This meant that the robotic sleeve was able to achieve $45.2 \%$ of the active ROM (with standard deviation of $23.4^{\circ}$ ).

For the results in the horizontal direction, as a representation, Figure 12C shows a single subject's elbow joint angle from three cycles of active elbow flexion and extension (experimental segment 1), while Figure 12D shows the same subject's elbow joint angle under passive contraction (experimental segment 2). For the female subjects, it was found that the average active maximum $\mathrm{ROM}$ was $118.9^{\circ}$ while the average passive maximum ROM was $63.3^{\circ}$. This translates to the soft robotic elbow sleeve's capability in achieving $53.2 \%$ of the active ROM (with standard deviation of $15.6^{\circ}$ ). In contrast, for male subjects, it was found that the average active maximum $\mathrm{ROM}$ was $103.3^{\circ}$ while the average passive maximum ROM was $56.1^{\circ}$. This meant that the robotic sleeve was able to achieve $54.3 \%$ of the active ROM (with standard deviation of $9.7^{\circ}$ ).

The results for the different genders across the two experimental segments are summarized in Table 1. In all trials, extension of the elbow back to the starting point was achieved.

\subsubsection{Electromyography-Driven Active Control}

We reproduce here the results of the third segment of the ROM study, in which the control mode of the elbow sleeve was switched to the active rehabilitation mode, and the subjects were tasked with completing horizontal elbow flexion and extension cycles using only activation signals from the two muscle groups on the opposite arm (non-sleeved arm) to control the state of the elbow sleeve.

All the subjects were successful in controlling the state of the elbow sleeve with muscle effort from the target muscle groups detected by the surface EMG sensors. As a representation, Figure 13 shows the processed EMG data from the biceps and triceps of a single subject normalized to the threshold of $50 \%$, together with the change in elbow joint angle upon EMG activation by the user. It was observed that there is a tendency for co-contraction during extension (as observed by high EMG signals from both muscles). This was possibly due to the action of joint stabilization by both muscle groups, which did not affect the functionality of the elbow sleeve, for the algorithm continues to function so long as the threshold is met by the intended muscle.

\section{DISCUSSION}

In this paper, we presented the design and testing of a soft robotic elbow sleeve that could be used for passive and intentbased neuro-muscular training. The novelty of this design comes from the use of lightweight fiber-reinforced and fabric-based soft actuators for elbow moving, and this device represents the first of its kind in integrating both flexion and extension capabilities. The elbow sleeve's capability in enabling full extension is particularly useful for stroke patients. In studies assessing the motor impairment and activity limitations of stroke patients, it was found that spasticity, which is a velocity-dependent hyperexcitability of muscles to stretch, is a common complication encountered, at an estimate of $60 \%$ of post-stroke patients (Watkins et al., 2002; Sommerfeld et al., 2004). This is manifested in the form of a slightly flexed elbow with the forearm across the abdomen. Another common problem that arises in stroke is that of muscle contracture, which also leads to similar complications as spasticity. Typical physiotherapy treatments for such ailments include slow progressive elbow extensions, which 

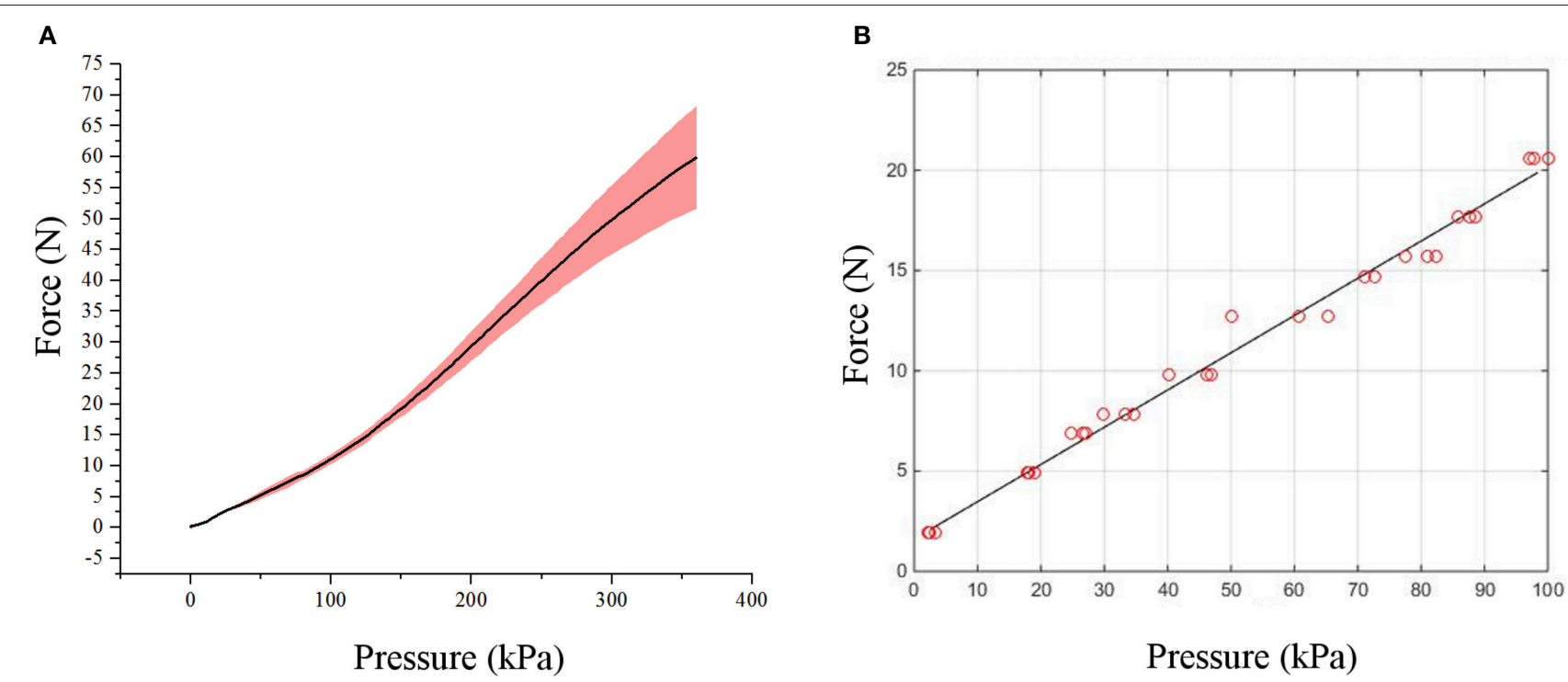

FIGURE 10 | Graphs showing the relationship between force and pressure for the actuators. (A) Graph of tip force vs. pressure of flexion actuator. (B) Graph of resistive force vs. pressure of extension actuator.

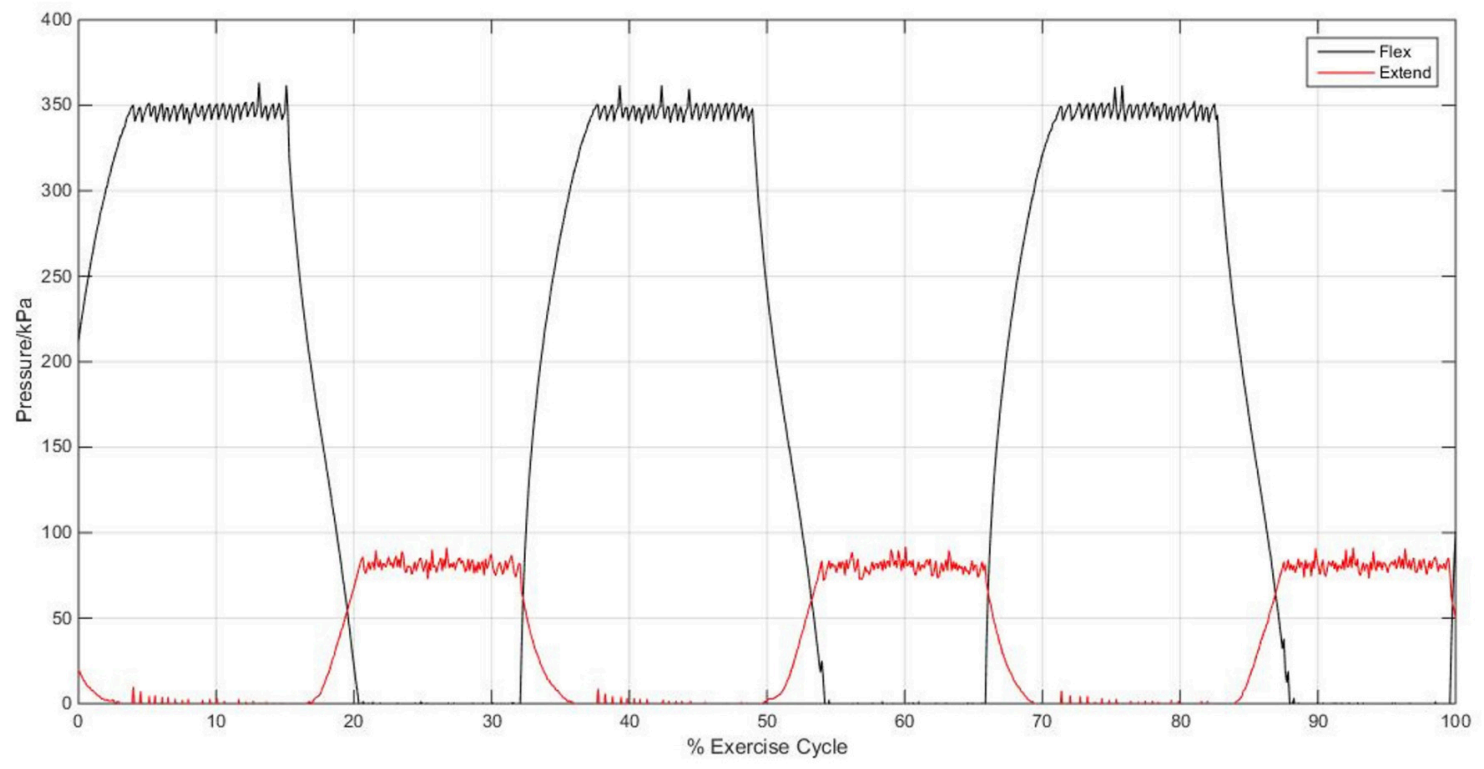

FIGURE 11 | Graph showing the functionality of the PID control of pressure within the actuators.

has seen reasonable success for ROM reestablishment (Bonutti et al., 1994). As such, this means of therapy can be potentially fulfilled by our elbow sleeve due to the inclusion of extension actuators.

An important design constraint-that of ensuring that extension actuators do not hinder the flexion actuatorsdetermined that each of the actuators has to be manufactured differently. In this regard, the flexion actuator is a fiber-reinforced silicone actuator coupled with nylon fabric to influence its bending behavior, whereas the extension actuator is a inflatable fabric actuator which provides resistive forces through a beam bending model. Both actuators show differences in their pressure-force relationships, with the flexion actuator exhibiting a nonlinear sigmoid-shaped curve, and the extension actuator showing a linear relationship. The non-linearity of the flexion actuator is largely due to the anisotropy that is incorporated within the flexion through fiber and fabric reinforcements, while the extension actuator displayed a linear pressure-force relationship in the absence of modifying reinforcements to the material. 


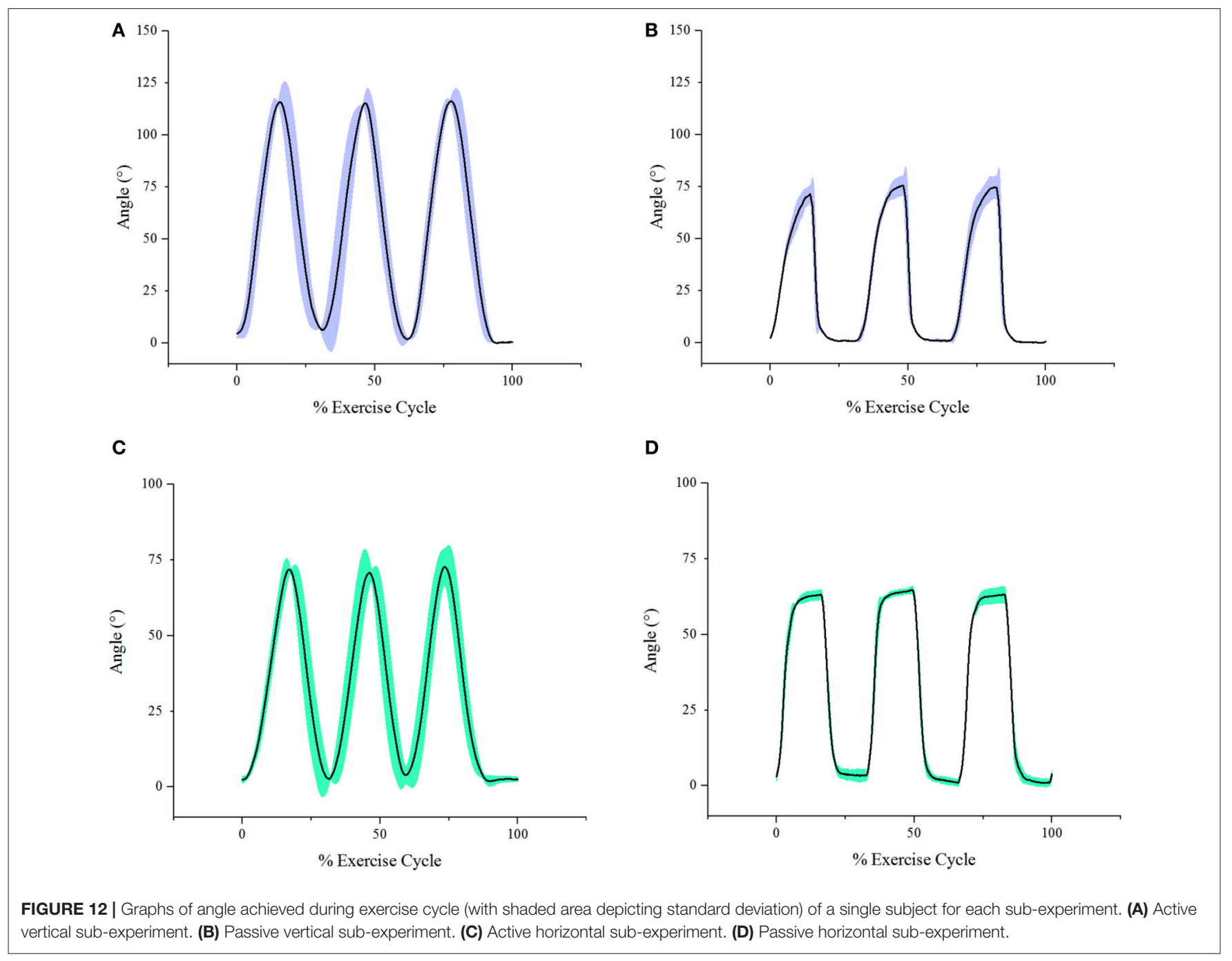

TABLE 1 | Summary of experimental results for segments 1 and 2.

\begin{tabular}{llcc}
\hline & Measurement & Average $\left(^{\circ}\right)$ & Standard deviation $\left(^{\circ}\right)$ \\
\hline Female & Active (vertical) & 127.8 & 6.7 \\
& Passive (vertical) & 69.3 & 27.2 \\
& Active (horizontal) & 117.9 & 15.0 \\
& Passive (horizontal) & 63.3 & 15.6 \\
\hline \multirow{2}{*}{ Male } & Active (vertical) & 132.1 & 15.05 \\
& Passive (vertical) & 59.7 & 23.4 \\
& Active (horizontal) & 103.3 & 24.1 \\
& Passive (horizontal) & 56.1 & 9.7 \\
\hline
\end{tabular}

In examining the control system of the elbow sleeve, while it is successful in generating and maintaining the necessary flexion and extension for the elbow joint in the ROM study, there remains an undesirable frequency switching which led to a slight oscillatory movement of the elbow sleeve during actuation. To overcome this, a dead-zone can possibly be introduced at the valve controller, creating boundaries around the pressure readings that allow the valves to remain at their current states until the change in pressure is sufficiently large, which is a suggested method by Guo et. al on modifications to improve the PID controller (Guo et al., 2002).

Preliminary studies were carried out on healthy subjects to quantify the basic functionality of the elbow sleeve. From our results across all the experiments, it was observed that while the extension actuators were able to ensure full extension of the elbow, the flexion actuators had difficulties in enabling full flexion of the elbow, achieving an average of approximately $50 \%$ of the full ROM across all subjects. As such, there is room for improvement with respect to the ROM achievable by the device. To improve the ROM, it is recommended that finite element modeling of the fiber-fabric-reinforced actuators be conducted to determine the maximum possible force output of these actuators. Once the full potential of the actuators is quantified in these computational models, feedback control loops may then be implemented to control the extent of actuation by monitoring the angle that the elbow is flexed to. Additionally, alternative 


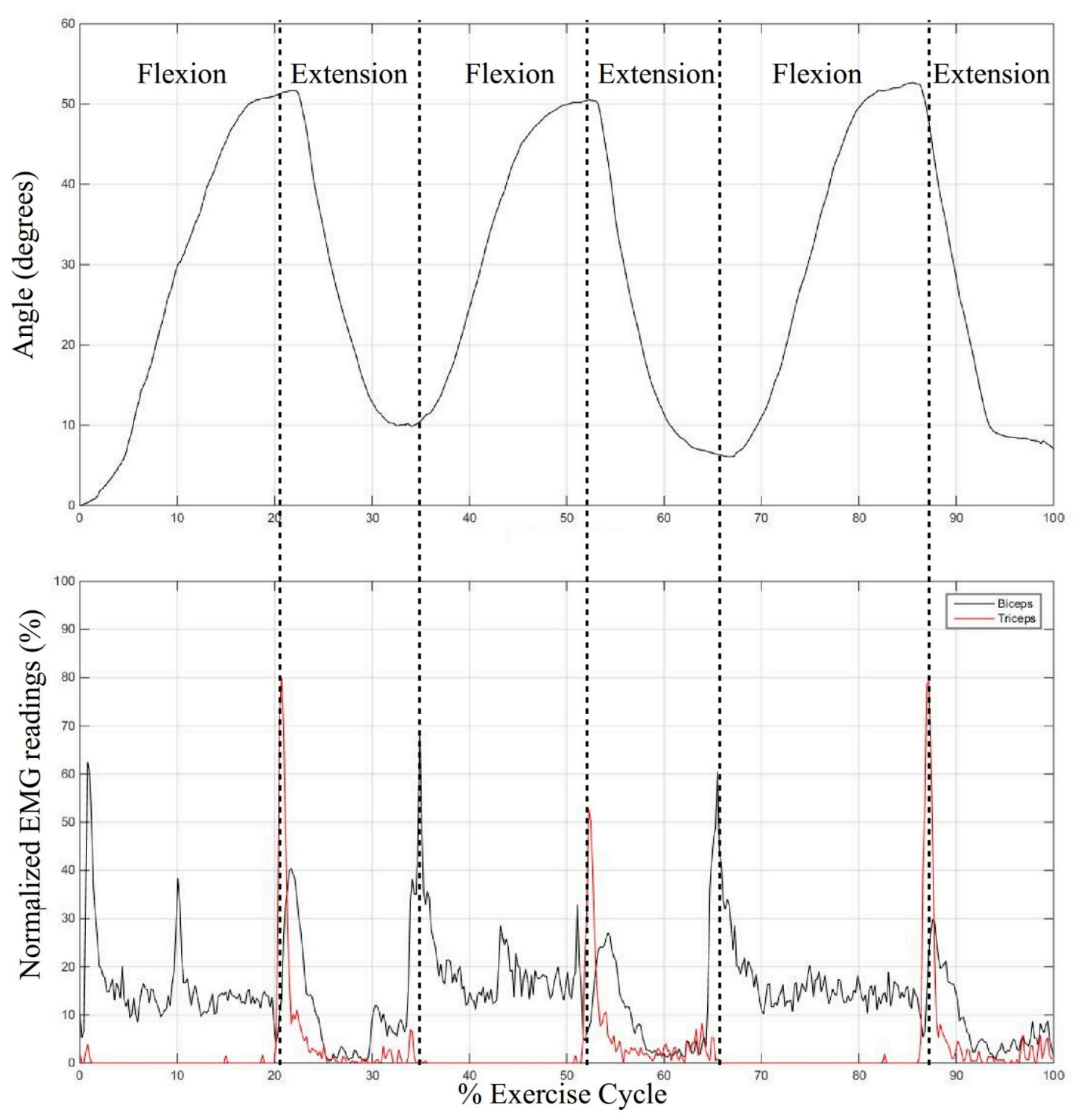

FIGURE 13 | Graph showing the angle of the elbow over the exercise cycle corresponding to the captured surface EMG signals over time from the biceps and triceps, in cyclic flexion and extension.

materials for actuator fabrication, or an increase in the number of flexion actuators on the device may also be considered.

In comparing the results gathered from each gender, it was observed that the average percentage ROM achieved from using the elbow sleeve was lower for the male subjects, which was expected due to male subjects having a generally higher segment mass as compared to female subjects. On a larger front, this analysis also suggests that there is a need to tailor the mechanical properties of the actuators to fit each individual's requirements.

In comparing the results gathered from elbow motion in the vertical and horizontal directions, it was observed that the variance in vertical motion is lower as compared to that in the horizontal direction of motion. This is possible due to the decreased effects of gravity acting in the horizontal direction as compared to the vertical direction, thereby making it easier to flex the elbow horizontally as compared to vertically. This set of results is useful in further optimizing the control system toward sensing and providing different bending forces suited for the mechanical task at hand.

The EMG mode of control was successfully implemented during the preliminary user studies. This indicates the functionality of the elbow sleeve as a means of providing active-assisted movements. Going forwards, by placing the surface EMG electrodes on the non-supported arm, bimanual 
practice may be enabled. The significance of this in stroke recovery is that the consensual operation of the non-affected upper limb may cause the stimulation of ipsilateral corticospinal projections to the paretic muscles, which is of relevance to hemiplegic recovery (Hesse et al., 2003). The logic scheme used for the EMG control is primarily based on an on-off algorithm, and a possible improvement to the system would be to integrate angular control into the device by modifying the algorithm. If bimanual control is maintained, two flex sensors calibrated to obtain angle of bending may be placed onto the back of the elbow. Through comparison of the angle bent by both elbows, a third PID regulator may be introduced to identify the error and adjust the supported elbow, using the non-supported elbow as the set-point angle. Another possible solution would be to record signals from more muscle groups. The array of signals and their amplitudes from these muscle groups would then provide a response pattern as type of fingerprint that may be used to identify different points of elbow actuation. These signals may be classified using either a fuzzy approach or an artificial neural network approach (Chan et al., 2000). In this manner, the surface EMG sensors may be placed on the supported arm to detect post-stroke using residual muscle signals.

Finally, the testing of the system was done with six healthy subjects. While this is sufficient for the purpose of demonstrating the functionality of the system in enabling robot-assisted flexion and extension of the elbow with and without intent detection,

\section{REFERENCES}

Benitez, L. M. V., Tabie, M., Will, N., Schmidt, S., Jordan, M., and Kirchner, E. A. (2013). Exoskeleton technology in rehabilitation: towards an EMG-based orthosis system for upper limb neuromotor rehabilitation. J. Robot. 2013, 1-13. doi: 10.1155/2013/610589

Bonutti, P., Windau, J., Ables, B., and Miller, B. (1994). Static progressive stretch to reestablish elbow range of motion. Clin. Orthop. Relat. Res. 303, 128-134. doi: 10.1097/00003086-199406000-00015

Chan, F., Yang, Y., Lam, F., Zhang, Y., and Parker, P. (2000). Fuzzy EMG classification for prosthesis control. IEEE Trans. Rehabil. Eng. 8, 305-311. doi: $10.1109 / 86.867872$

Drillis, R., Contini, R., and Bluestein, M. (1964). Body segment parameters: a survey of measurement techniques. Artif. Limbs 8, 44-66.

Guo, L., Hung, J. Y., and Nelms, R. (2002). "PID controller modifications to improve steady-state performance of digital controllers for buck and boost converters," in Applied Power Electronics Conference and Exposition, 2002. APEC 2002. Seventeenth Annual IEEE (Dallas, TX), 381-388.

Halaki, M., and Ginn, K. A. (2012). "Normalization of EMG signals: to normalize or not to normalize and what to normalize To?," in Computational Intelligence in Electromyography Analysis - A Perspective on Current Applications and Future Challenges, Ch. 7, ed G. R. Naik (Rijeka: InTech), 175-194.

Hesse, S., Schulte-Tigges, G., Konrad, M., Bardeleben, A., and Werner, C. (2003). Robot-assisted arm trainer for the passive and active practice of bilateral forearm and wrist movements in hemiparetic subjects. Arch. Phys. Med. Rehabil. 84, 915-920. doi: 10.1016/S0003-9993(02)04954-7

Hu, X., Tong, K., Song, R., Tsang, V., Leung, P., and Li, L. (2007). Variation of muscle coactivation patterns in chronic stroke during robot-assisted elbow training. Arch. Phys. Med. Rehabil. 88, 1022-1029. doi: 10.1016/j.apmr.2007.05.006

Hu, X., Tong, K., Song, R., Zheng, X., and Leung, W. (2009). A comparison between electromyography-driven robot and passive motion device on wrist clinically-relevant conclusions may not be reached as the system has to be tested on individuals with neurological impairments. A limitation of testing healthy subjects is such that, healthy subjects, in contrast with individuals with neurological impairments, have a tendency to activate their agonist muscles during assistive movement. In this study, we found one subject to exhibit biceps activation during passive mode rehabilitation. Moving forwards, the next step to this study would be to conduct clinical trials on stroke patients, who are the intended end-users of the elbow sleeve. These patients would present differing physical conditions (such as their EMG signal amplitudes) which would better elucidate the efficacy of the elbow sleeve, and the improvements that needed to be made.

\section{AUTHOR CONTRIBUTIONS}

TK and NC collected, processed and analyzed the data, drafted the manuscript and contributed equally to the work. TK, NC, HY, and CY designed the study. HY contributed to the interpretation of findings and drafted the manuscript. CY oversaw its coordination and helped to draft the manuscript. All authors read, edited and approved the final manuscript.

\section{FUNDING}

This work was supported in part by the Ministry of Education AcRF Tier 2 grant under Grant R-397-000-203-112.

rehabilitation for chronic stroke. Neurorehabil. Neural Repair 23, 837-846. doi: 10.1177/1545968309338191

In, H., Kang, B. B., and Sin, M. (2015). Exo-Glove: a wearable robot for the hand with a soft tendon routing system. IEEE Robot. Autom. Mag. 22, 97-105. doi: 10.1109/MRA.2014.2362863

Lenzi, T., De Rossi, S., Vitiello, N., and Carrozza, M. (2012). Intention-based EMG control for powered exoskeletons. IEEE Trans. Biomed. Eng. 59, 2180-2190. doi: 10.1109/TBME.2012.2198821

Low, F.-Z., Tan, H. H., Lim, J. H., and Yeow, C. H. (2016). Development of a soft pneumatic sock for robot-assisted ankle exercise. J. Med. Devices 10:014503. doi: $10.1115 / 1.4032616$

Oguntosin, V., Harwin, W. S., Kawamura, S., Nasuto, S. J., and Hayashi, Y. (2015). "Development of a wearable assistive soft robotic device for elbow rehabilitation," in 2015 IEEE International Conference on Rehabilitation Robotics (ICORR) (Singapore), 747-752.

Polygerinos, P., Galloway, K. C., and Sanan, S. (2015a). "EMG controlled soft robotic glove for assistance during activities of daily living," in 2015 IEEE International Conference on Rehabilitation Robotics (ICORR) (Singapore), 55-60.

Polygerinos, P., Wang, Z., and Overvelde, J. T. B. (2015b). Modeling of soft fiber-reinforced bending actuators. IEEE Trans. Robot. 31, 778-789. doi: 10.1109/TRO.2015.2428504

Rodrigo, R., Fernández-Gajardo, R., Gutiérrez, R., Matamala, J., Carrasco, R., Miranda-Merchak, A., et al. (2013). Oxidative stress and pathophysiology of ischemic stroke: novel therapeutic opportunities. CNS Neurol. Disord. Drug Targets 12, 698-714. doi: 10.2174/1871527311312050015

Sanan, S., Moidel, J. B., and Atkeson, C. G. (2009). "Robots with inflatable links," in Intelligent Robots and Systems, 4331-4336. doi: 10.1109/IROS.2009.5354151

Sommerfeld, D., Eek, E., Svensson, A., Holmqvist, L., and von Arbin, M. (2004). Spasticity after stroke: its occurrence and association with motor impairments and activity limitations. Stroke 35, 134-139. doi: 10.1161/01.STR.0000105386.05173.5E 
Watkins, C., Leathley, M., Gregson, J., Moore, A., Smith, T., and Sharma, A. (2002). Prevalence of spasticity post stroke. Clin. Rehabil. 16, 515-522. doi: 10.1191/0269215502cr512oa

Yap, H. K., Ang, W. K. B., Lim, J., Goh, J., and Yeow, C. H. (2016a). "A fabricregulated soft robotic glove with user intent detection using EMG and RFID for hand assistive application," in 2016 IEEE International Conference on Robotics and Automation (ICRA) (Stockholm), 3537-3542.

Yap, H. K., Kamaldin, N., Lim, J., Nasrallah, F., Goh, J., and Yeow, C. H. (2016b). A magnetic resonance compatible soft wearable robotic glove for hand rehabilitation and brain imaging. IEEE Trans. Neural Syst. Rehabil. Eng. 25, 782-793. doi: 10.1109/TNSRE.2016.2602941

Yap, H. K., Lim, J., Goh, J., and Yeow, C. H. (2016c). Design of a soft robotic glove for hand rehabilitation of stroke patients with clenched fist deformity using inflatable plastic actuators. ASME. J. Med. Devices. 10, 044504-1-6. doi: $10.1115 / 1.4033035$

Yap, H. K., Lim, J. H., Nasrallah, F., Goh, J. C. H., and Yeow, R. C. H. (2015). "A soft exoskeleton for hand assistive and rehabilitation application using pneumatic actuators with variable stiffness," in IEEE International Conference on Robotics and Automation, 4967-4971. doi: 10.1109/ICRA.2015. 7139889
Yeo, J. C., Yap, H. K., Xi, W., Wang, Z., Yeow, C. H., and Lim, C. T. (2016). Flexible and stretchable strain sensing actuator for wearable soft robotic applications. Adv. Mater. Technol. 1:1600018. doi: 10.1002/admt.201600018

Zhang, S., Guo, S., Gao, B., Hirata, H., and Ishihara, H. (2015). Design of a novel telerehabilitation system with a force-sensing mechanism. Sensors (Basel) 15, 11511-11527. doi: 10.3390/s150511511

Zhang, X., and Zhou, P. (2012). High-density myoelectric pattern recognition toward improved stroke rehabilitation. IEEE Trans. Biomed. Eng. 59, 16491657. doi: 10.1109/TBME.2012.2191551

Conflict of Interest Statement: The authors declare that the research was conducted in the absence of any commercial or financial relationships that could be construed as a potential conflict of interest.

Copyright (c) 2017 Koh, Cheng, Yap and Yeow. This is an open-access article distributed under the terms of the Creative Commons Attribution License (CC BY). The use, distribution or reproduction in other forums is permitted, provided the original author(s) or licensor are credited and that the original publication in this journal is cited, in accordance with accepted academic practice. No use, distribution or reproduction is permitted which does not comply with these terms. 


\section{OPEN ACCESS}

Edited by:

Venketesh N. Dubey,

Bournemouth University,

United Kingdom

Reviewed by:

Brent Winslow,

Design Interactive (United States),

United States

Victor Manuel Pulgar

Wake Forest School of Medicine,

United States

Arturo Forner-Cordero,

University of São Paulo, Brazil

Agnes Roby-Brami,

Institut National de la Santé et de la

Recherche Médicale, France

*Correspondence:

Chen-Hua Yeow

rayeow@nus.edu.sg

Specialty section:

This article was submitted to

Neural Technology,

a section of the journal

Frontiers in Neuroscience

Received: 31 March 2017 Accepted: 20 September 2017 Published: 09 October 2017

Citation:

Yap HK, Lim JH, Nasrallah F and Yeow C-H (2017) Design and Preliminary Feasibility Study of a Soft

Robotic Glove for Hand Function Assistance in Stroke Survivors.

Front. Neurosci. 11:547.

doi: 10.3389/fnins.2017.00547

\section{Design and Preliminary Feasibility Study of a Soft Robotic Glove for Hand Function Assistance in Stroke Survivors}

\author{
Hong Kai Yap ${ }^{1,2}$, Jeong Hoon Lim $^{3}$, Fatima Nasrallah ${ }^{4}$ and Chen-Hua Yeow ${ }^{1 *}$ \\ ${ }^{1}$ Department of Biomedical Engineering, National University of Singapore, Singapore, Singapore, ${ }^{2}$ NUS Graduate School for \\ Integrative Sciences and Engineering, National University of Singapore, Singapore, Singapore, ${ }^{3}$ Department of Medicine, \\ National University of Singapore, Singapore, Singapore, ${ }^{4}$ Queensland Brain Institute, University of Queensland, St. Lucia, \\ QLD, Australia
}

Various robotic exoskeletons have been proposed for hand function assistance during activities of daily living (ADL) of stroke survivors. However, traditional exoskeletons involve the use of complex rigid systems that impede the natural movement of joints, and thus reduce the wearability and cause discomfort to the user. The objective of this paper is to design and evaluate a soft robotic glove that is able to provide hand function assistance using fabric-reinforced soft pneumatic actuators. These actuators are made of silicone rubber which has an elastic modulus similar to human tissues. Thus, they are intrinsically soft and compliant. Upon air pressurization, they are able to support finger range of motion (ROM) and generate the desired actuation of the finger joints. In this work, the soft actuators were characterized in terms of their blocked tip force, normal and frictional grip force outputs. Combining the soft actuators and flexible textile materials, a soft robotic glove was developed for grasping assistance during ADL for stroke survivors. The glove was evaluated on five healthy participants for its assisted ROM and grip strength. Pilot test was performed in two stroke survivors to evaluate the efficacy of the glove in assisting functional grasping activities. Our results demonstrated that the actuators designed in this study could generate desired force output at a low air pressure. The glove had a high kinematic transparency and did not affect the active ROM of the finger joints when it was being worn by the participants. With the assistance of the glove, the participants were able to perform grasping actions with sufficient assisted ROM and grip strength, without any voluntary effort. Additionally, pilot test on stroke survivors demonstrated that the patient's grasping performance improved with the presence and assistance of the glove. Patient feedback questionnaires also showed high level of patient satisfaction and comfort. In conclusion, this paper has demonstrated the possibility of using soft wearable exoskeletons that are more wearable, lightweight, and suitable to be used on a daily basis for hand function assistance of stroke survivors during activities of daily living.

Keywords: soft robotic, hand exoskeleton, rehabilitation, activities of daily living, soft actuators 


\section{INTRODUCTION}

The ability to perform basic activities of daily living (ADL) impacts a person's quality of life and independence (Katz, 1983; Andersen et al., 2004). However, an individual's independence to perform ADLs is jeopardized due to hand motor impairments, which can be observed in patients with neurological disorders such as stroke. In order to improve hand motor functions in terms of strength and range of motion (ROM) (Kutner et al., 2010), stroke survivors undergo rehabilitation programs comprising repetitive practice of simulated ADL tasks (Michaelsen et al., 2006). Normally, patients undergo rehabilitation exercises in a specialized rehabilitation center under the guidance of physiotherapists or occupational therapists. However, due to increasing patient population, it is foreseen that there will be a shortage of physiotherapists to assist in the rehabilitative process. Thus, there will be comparatively less therapy time, which will eventually lead to a slower recovery process for the patients. Over the past decade, technological developments in robotics have facilitated the rehabilitative process and have shown potential to assist patients in their daily life (Maciejasz et al., 2014). One example of such a device is the hand exoskeleton, which is secured around the hand to guide and assist the movement of the encompassed joints. However, due to the complexity of the hand, designing a hand exoskeleton remains a challenging task.

Traditional hand exoskeletons involve the use of rigid linkagebased mechanisms. In this kind of mechanism, rigid components, such as linear actuators, rotary motors, racks, and pinions as well as rigid linkages are normally involved (Worsnopp et al., 2007; Rotella et al., 2009; Martinez et al., 2010). To assist hand movements that have high degrees of freedom (DOFs), traditional exoskeletons can be incorporated with a substantial number of actuators to achieve the requirement. However, this means that their application is limited due to the increasing bulkiness for higher DOFs. Therefore, these devices are normally restricted in clinical settings and not suitable for performing home therapy. Additionally, their rigidity, weight and constraint on the non-actuated DOFs of the joints pose complications. As a result, the level of comfort and safety of patients is reduced. In view of this, there is an apparent need for the development of exoskeletons that may be used in both clinical and home settings. A lightweight and wearable exoskeleton may allow patients to bring back home to continue daily therapy or to serve as an assistive device for the ADLs.

The development of wearable robotic exoskeletons serves to provide an alternative approach toward addressing this need. Instead of using rigid linkage as an interface between the hand and the actuators, wearable exoskeletons typically utilize flexible materials such as fabric (Sasaki et al., 2004; Yap et al., 2016a) and polymer (Kang et al., 2016), driven by compliant actuators such as cables (Sangwook et al., 2014; Xiloyannis et al., 2016) and soft inflatable actuators (Polygerinos et al., 2015d; Yap et al., 2016c). Therefore, they are more compliant and lightweight compared to the rigid linkage-based mechanism. Cable-driven based exoskeletons involve the use of cables that are connected to actuators in the form of electrical motors situated away from the hand (Nilsson et al., 2012; Ying and Agrawal, 2012; Sangwook et al., 2014; Varalta et al., 2014). By providing actuations on both dorsal and palmar sides of the hand, bi-directional cable-driven movements are possible (Kang et al., 2016). These cables mimic the capability of the tendons of the human hand and they are able to transmit the required pulling force to induce finger flexion and extension. However, the friction of the cable, derailment of the tendon, and inaccurate routing of the cable due to different hand dimensions can affect the efficiency of force transmission in the system.

On the other hand, examples of the soft inflatable actuators are McKibben type muscles (Feifei et al., 2006; Tadano et al., 2010), sheet-like rubber muscles (Sasaki et al., 2004; Kadowaki et al., 2011), and soft elastomeric actuators (Polygerinos et al., 2015b,c; Yap et al., 2015); amongst which, soft elastomeric actuators have drawn increasing research interest due to their high compliance (Martinez et al., 2013). This approach typically embeds pneumatic chamber networks in elastomeric constructs to achieve different desired motions with pressurized air or water (Martinez et al., 2012). Soft elastomeric actuators are highly customizable. They are able to achieve multiple DOFs and complex motions with a single input, such as fluid pressurization. The design of a wearable hand exoskeleton that utilizes soft elastomeric actuators is usually simple and does not require precise routing for actuation, compared to the cabledriven mechanism. Thus, the design reduces the possibility of misalignment and the setup time. These properties allow the development of hand exoskeletons that are more compliant and wearable, with the ability to provide safe human-robot interaction. Additionally, several studies have demonstrated that compactness and ease of use of an assistive device critically affect its user acceptance (Scherer et al., 2005, 2007). Thus, these exoskeletons provide a greater chance of user acceptance.

Table 1 summarizes the-state-of-art of soft robotic assistive glove driven by inflatable actuators. Several pioneer studies on inflatable assistive glove have been conducted by Sasaki et al. (2004); Kadowaki et al. (2011) and Polygerinos et al. (2015a,b,c). Sasaki et al. have developed a pneumatically actuated power assist glove that utilizes sheet-like curved rubber muscle for hand grasping applications. Polygerinos et al. have designed a hydraulically actuated grip glove that utilizes fiber-reinforced elastomeric actuators that can be mechanically programmed to generate complex motion paths similar to the kinematics of the human finger and thumb. Fiber reinforcement has been proved to be an effective method to constrain the undesired radial expansion of the actuators that does not contribute to effective motion during pressurization. However, this method limits the bending capability of the actuators (Figure S1); as a result, higher pressure is needed to achieve desired bending.

This paper presents the design and preliminary feasibility study of a soft robotic glove that utilizes fabric-reinforced soft pneumatic actuators. The intended use of the device is to support the functional tasks during ADLs, such as grasping, for stroke survivors. The objectives of this study were to characterize the soft actuators in terms of their force output and to evaluate the performance of the glove with healthy participants and stroke survivors. The glove was evaluated on five healthy participants 
TABLE 1 | Hand assistive exoskeletons driven by inflatable actuators.

\begin{tabular}{|c|c|c|c|c|}
\hline Device/references & $\begin{array}{l}\text { Actuators (all pneumatic-driven unless stated } \\
\text { otherwise) }\end{array}$ & Weight (g) & DOF & $\begin{array}{l}\text { Operating } \\
\text { pressure (kPa) }\end{array}$ \\
\hline Power-Assist Glove/Sasaki et al., 2004 & Sheet-like rubber muscles & N.A. & 1 & 500 \\
\hline Power-Assist Glove/Noritsugu et al., 2008 & Curved-type rubber artificial muscle & 120 & 1 & 500 \\
\hline Power-Assist Glove/Kadowaki et al., 2011 & Sheet-like rubber muscles & 135 & 4 & 100 \\
\hline Power-Assist Glove/Toya et al., 2011 & Bend-type rubber artificial muscles & 180 & 4 & 350 \\
\hline Yap et al., 2016c & Elastomeric actuators & 200 & 1 & 180 \\
\hline ExoGlove/Yap et al., 2015 & Elastomeric actuators with variable stiffness & 200 & 5 & 160 \\
\hline MR-Glove/Yap et al., 2017 & Fabric-reinforced actuators & 180 & 5 & 120 \\
\hline Grip Glove/Maeder-York et al., 2014; Polygerinos et al., 2015a,b,c & Fiber-reinforced actuators (Pneumatic or Hydraulic) & $<500$ & 5 & 345 \\
\hline Nordina et al., 2016 & Fiber-reinforced actuators & N.A. & 5 & 300 \\
\hline Haghshenas-Jaryani et al., 2016 & Bellow-type soft-rigid hybrid actuators & N.A. & 6 & 165 \\
\hline Exo-Glove PM/Yun et al., 2017 & Modular elastomeric actuators & N.A. & 5 & 300 \\
\hline Lobster-inspired Robotic Glove/Chen et al., 2017 & Soft actuators with rigid shells & 150 & 5 & 250 \\
\hline Kline et al., 2005 & Rigidizing air bladders & 100 & 1 & 34 \\
\hline PneuGlove/Connelly et al., 2010; Thielbar et al., 2014 & Rigidizing air bladders & N.A. & 5 & 69 \\
\hline Coffey et al., 2014 & Rigidizing air bladders & N.A. & 1 & 69 \\
\hline Yap et al., 2016b & Plastic rigidizing actuators & 150 & 5 & 100 \\
\hline
\end{tabular}

in order to determine the ROM of individual finger joints and grip strength achieved with the assistance of the glove. Pilot testing with two stroke survivors was conducted to evaluate the feasibility of the glove in providing grasping assistance for ADL tasks. We hypothesized that with the assistance of the glove, the grasping performance of stroke patients improved.

Specific contributions of this work are listed as follows:

(a) Presented fabric-reinforcement as an alternative method to reinforce soft actuators, which enhanced the bending capability and reduced the required operating pressure of the actuators,

(b) Utilized the inherence compliance of soft actuators and allowed the actuators to achieve multiple motions to support ROM of the human fingers,

(c) Integrated elastic fabric with soft actuators to enhance the extension force for finger extension,

(d) Designed and characterized a soft robotic glove using fabricreinforced soft actuators with the combination of textile materials, and

(e) Conducted pilot tests with stroke survivors to evaluate the feasibility of the glove in providing functional assistance for ADL tasks.

\section{DESIGN REQUIREMENTS AND RATIONALE}

The design requirements of the glove presented in this paper are similar to those presented by Polygerinos et al. (2015a,b,c) in terms of design considerations, force requirements, and control requirements. For design considerations, weight is the most important design criterion when designing a hand exoskeleton. Previous studies have identified the threshold for acceptable weight of device on the hand, which is in the range of 400-500 g (Aubin et al., 2013; Gasser and Goldfarb, 2015). Cable-driven, hydraulic, and pneumatic driven mechanisms are found to be suitable options to meet the criteria. To develop a fully portable system for practical use in home setting, reduction in the weight of the glove as well as the control system is required. The total weight of the control system should not exceed $3 \mathrm{~kg}$ (Polygerinos et al., 2015a,b,c). In this work, the criteria for the weight of the glove and control system are defined as: (a) the weight of the glove should be $<200 \mathrm{~g}$, and (b) the weight of the control system should be $<1.5 \mathrm{~kg}$.

Considering the weight requirement, hydraulic systems are not ideal for this application, as the requirement of a water reservoir for hydraulic control systems and actuation of the actuators with pressurized water will add extra weight to the hand. The second consideration is that the hand exoskeleton should allow fast setup time. Therefore, it is preferable for the hand exoskeleton to fit the hand anatomy rapidly without precise joint alignment. Compared to cable-driven mechanisms, soft pneumatic actuators are found to be more suitable as they allow rapid customization to different finger length. Additionally, they do not require precise joint alignment and cable routing for actuation as the attachment of the soft pneumatic actuators on the glove is usually simple. Therefore, in this work, pneumatic mechanisms were selected. Using pneumatic mechanism, Connelly et al. and Thielbar et al. have developed a pneumatically actuated glove, PneuGlove that is able to provide active extension assistance to each finger while allowing the wearer to flex the finger voluntarily (Connelly et al., 2010; Thielbar et al., 2014). The device consists of five air bladders on the palmar side of the glove. Inflation of the air bladders due to air pressurization created an extension force that extends the fingers. However, due to the placement of the air bladders on the palmar side, grasping activities such as palmar 
and pincer grasps were more difficult. Additionally, this device is limited to stroke survivors who are able to flex their fingers voluntarily.

In this work, the soft robotic glove is designed to provide functional grasping assistance for stroke survivors with muscle weakness and impairments in grasping by promoting finger flexion. While the stroke survivors still preserve the ability to modulate grip force within their limited force range, the grip release (i.e., hand opening) is normally prolonged (Lindberg et al., 2012). Therefore, the glove should assist with grip release by allowing passive finger extension via reinforced elastic components, similar to Saeboflex (Farrell et al., 2007) and HandSOME (Brokaw et al., 2011). The elastic components of these devices pull the fingers to the open hand state due to increased tension during finger flexion. Additionally, the glove should generate the grasping force required to manipulate and counteract the weight of the objects of daily living, which are typically below $1.07 \mathrm{~kg}$ (Smaby et al., 2004). Additionally, the actuators in the glove should be controlled individually in order to achieve different grasping configurations required in simulated ADL tasks, such as palmar grasp, pincer grasp, and tripod pinch. For the speed of actuation, the glove should reach full grasping motion in $<4 \mathrm{~s}$ during simulated ADL tasks and rehabilitation training.

For the actuators, we have recently developed a new type of soft fabric-reinforced pneumatic actuator with a corrugated top fabric layer (Yap et al., 2016a) that could minimize the excessive budging and provide better bending capability compared to fiber-reinforced soft actuators developed in previous studies (Polygerinos et al., 2015c,d). This corrugated top fabric layer allows a small initial radial expansion to initiate bending and then constrains further undesired radial expansion (Figure 1). The detailed comparison of the fiber-reinforced actuators and fabric-reinforced actuators can be found in the Supplementary Material.

Upon air pressurization, the top surface of the actuators expands due to the inflation of the embedded pneumatic chamber (Figure 1B). With inextensible layers attached at the bottom of the actuators, bending motion can be obtained due to asymmetrical strain along the length of the actuators. To support a complete hand closure, previous studies, including our group, have developed multi-segment soft actuators that are mechanically programmed to achieve bending and extending motions by controlling the placement of the inextensible bottom layers at different localities along the length of the actuators (Polygerinos et al., 2015b,c; Yap et al., 2017). The bending motion supports finger joint flexion while the extending motion offsets the increased distance due to skin stretching during finger flexion. Bending and extending segments can be pre-designed to conform to the finger anatomy of different patients. This feature demonstrates the high customizability of the soft actuators. However, as the hand dimension varies for different patients, pre-designing actuators with different lengths and controlling the placement of the bending and extending segments for each patient can be time consuming.

Therefore, in this work, we have designed the actuators without pre-programmed bending and extending motions. Instead of controlling the placement of the inextensible bottom layers to achieve bending and extending motions, a single elastic fabric with higher elastic modulus $(0.5 \mathrm{~N} / \mathrm{mm})$ than the silicone rubber is placed at the bottom of the actuators. With the elastic fabric, the actuators are able to achieve both bending and extending motions (Figure 1B). As the actuators are compliant, they are able to conform to the shape of the object during actuation. A bending motion is preferred at the

\section{A}

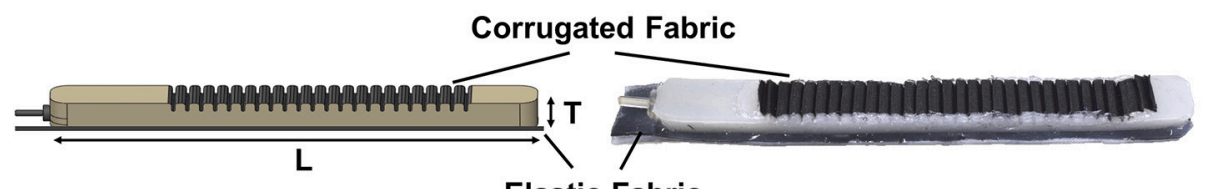

Elastic Fabric

B

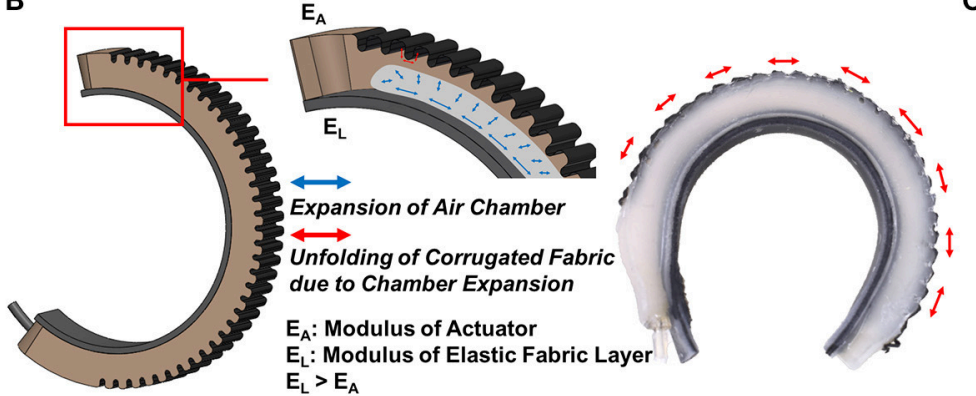

C

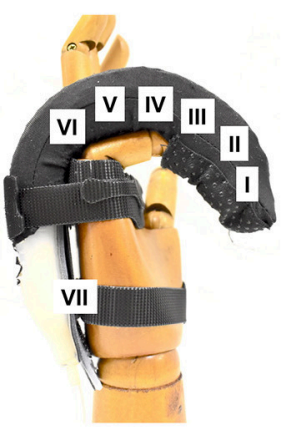

FIGURE 1 | (A) A fabric-reinforced soft actuators with a corrugated fabric layer and an elastic fabric later [Actuator thickness, $T=12 \mathrm{~mm}$, and length, $L=160 \mathrm{~mm}$ (Thumb), $170 \mathrm{~mm}$ (Little Finger), $180 \mathrm{~mm}$ (Index \& Ring Fingers), $185 \mathrm{~mm}$ (Middle Finger)]. (B) Upon air pressurization, the corrugated fabric layer unfolds and expands due to the inflation of the embedded pneumatic chamber. Radial budging is constrained when the corrugated fabric layer unfolds fully. The elastic fabric elongates during air pressurization and stores elastic energy. The actuator achieves bending and extending motions at the same time. (C) A bending motion is preferred at the finger joints (II, IV, VI). An extending motion is preferred over the bending motion at the finger segments (I, III, V) and the opisthenar (VII). 
location with lower impedance, i.e., the finger joints. At the finger segments that possess higher impedance, the bending motion of the actuators is limited and thus the extending motion is preferred (Figure 1C).

\section{MATERIALS AND METHODS}

\section{Actuator Fabrication}

A two-part 3D-printed mold is used to fabricate the actuators. A lower-part mold (chamber mold) is used to create a pneumatic chamber inside the actuators, which will inflate upon air pressurization. An upper-part mold (top layer mold) is used to impose a corrugated outer layer for the placement of fabric at the top of the actuators (Figure 1A). The detailed description of the fabrication process as well as the mold dimension can be found in the Supplementary Material.

\section{Actuator Characterization}

The actuators were characterized in terms of their blocked tip force and grip force upon pressurization. The blocked tip force

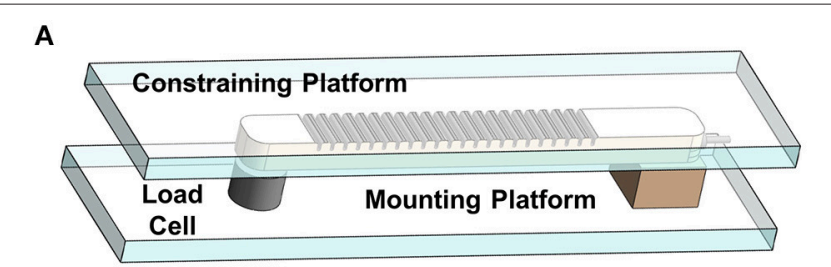

B
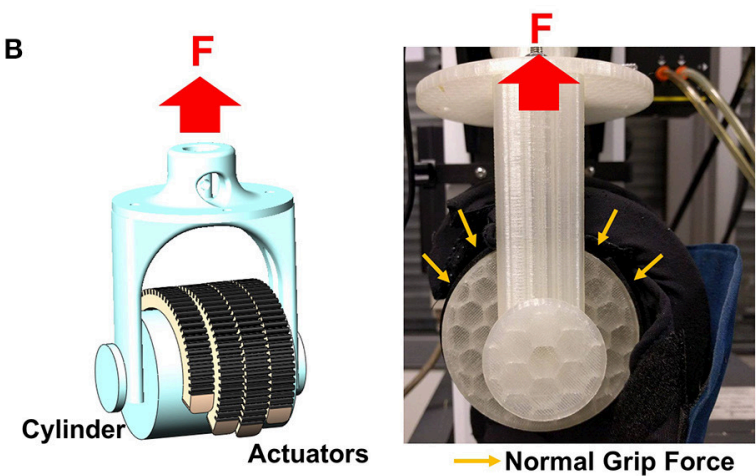

C

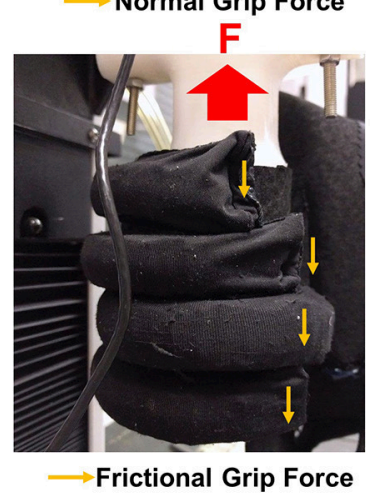

FIGURE 2 | Schematic of the force measurement system for (A) blocked tip force, (B) normal grip force, and (C) frictional grip force measurement. exerted by the actuator was measured over increasing pressures using a customized force measurement system (Figure 2A). The system consisted of a compression load cell (FC22, Measurement Specialties Inc., USA) and a mounting platform. The proximal end of the actuator was mounted on the platform and connected to the air source. The distal end of the actuator was in contact with the load cell. A constraining platform was positioned on top of the actuator. During pressurization, the actuator flexed and the tip of the actuator came into contact with the constraining platform, which constrained the height and the curvature of the actuator. This force measurement setup was similar to the setup presented in previous studies (Polygerinos et al., 2015c,d). Constraining the top surface of the actuator minimized the non-linear effects caused by the bending of the actuator when pressurized (Polygerinos et al., 2015d). Thus, this setup could measure the maximum blocked tip force generated by the actuator regardless of the bending angle. The pressure was increased from 0 to $120 \mathrm{kPa}$. The experiment was repeated three times. Additionally, a theoretical model was adapted from the model presented by Polygerinos et al. (2015d) and Wang et al. (2017) to predict the tip force output of the actuators at this configuration (Figure S4).

For hand exoskeleton application, the force that is of particular interest is the normal grip force (Figure 2B) generated by the gripping of the actuators on an object and the frictional grip force (Figure 2C) that counteracts the weight of that particular object to prevent it from falling. Both the normal and frictional grip forces were measured using a universal testing machine (Model 3345, Instron, MA, USA). Four actuators, which corresponded to four fingers, were pressurized to $120 \mathrm{kPa}$ to enclose and grasp a cylinder, which was of 50 and $75 \mathrm{~mm}$ in diameter, in two orientations (i.e., horizontal orientation to measure the normal grip force and vertical orientation to measure the frictional grip force). The cylinder was pulled upward by the Instron at a fixed velocity $(8 \mathrm{~mm} / \mathrm{s})$ until the cylinder was released from the actuators' grip. The setup was similar to the setup reported in previous literature (Galloway et al., 2016).

Both the normal and frictional grip forces were measured when they were resisting the upward motion of the cylinder. The normal grip force is the sum of the forces applied by four actuators normal to the cylinder surface. The measured force was that resisting the upward motion of the cylinder, which tried to pull the actuators straight from the default bending state (Figure 2B). The frictional grip force is the force exerted by the actuators as the cylinder moves and slides across them (Figure 2C). The experiment was repeated three times and the results were averaged.

\section{Integration and Evaluation of Soft Wearable Robotic Glove}

The overall structure of the soft robotic glove is a glove with five finger-actuator pockets attached on the dorsal side (Figure 3A). The actuators are integrated to the glove via the actuator pockets (Figure 3B). Open palm design is adopted for easy donning and doffing of the glove. The glove is secured to the wearer's hand via the finger pockets and a wrist strap. The finger pocket consists of 


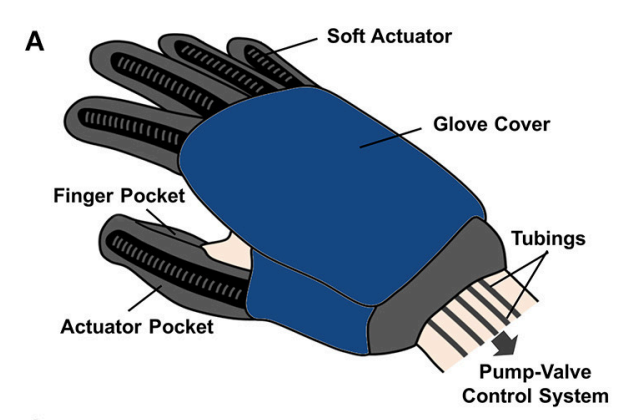

C

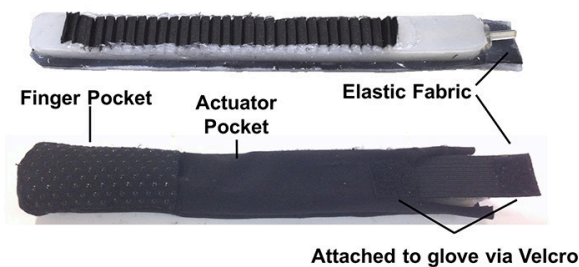

B

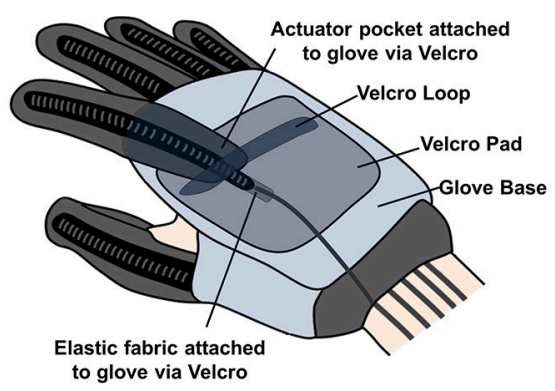

D

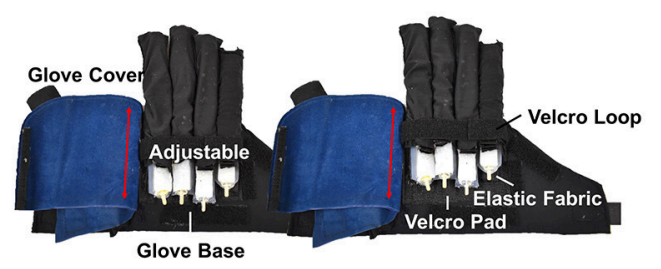

FIGURE 3 | (A) Outer view and (B) inner view of the soft robotic glove. (C) Actuators are inserted into actuator pockets. (D) The length of the finger-actuator pockets can be adjusted and attached to the base of the glove via Velcro.

anti-slip material to enhance the grip strength. To wear the glove, the wearer just needs to insert the fingers into the finger pockets and secure the glove to the wrist via the wrist strap. The glove base serves as a compliant interface between the actuators and the human hand, providing minimal mechanical impedance to the finger motion when it is being worn and ensuring kinematic transparency.

The actuators can be easily inserted into the actuator pockets, which are made from stretchable lycra fabrics (Figure 3C). The lycra fabrics serve as second constraining layers for the actuators, which further prevent over expansion of the actuators. Each actuator is isolated with respect to the others. The assistance of each finger can be achieved independently, which allows execution of different simulated ADL tasks. The length of the finger-actuator pockets can be adjusted and attached to the base of the glove via Velcro, in order to accommodate different finger lengths (Figure 3D). Both the Velcro loop and the glove cover constrain the bending movement of the actuators at the proximal part of the hand (Figure 3D).

The total weight of the glove is $\sim 180 \mathrm{~g}$, which is much lower than the design requirement. The thickness of the glove (including the actuators) and the width of each finger-actuator pocket is $<2 \mathrm{~cm}$. Additionally, as the actuators work under air pressure, inflation of the actuators does not add a significant amount of extra weight to the hand, as compared to hydraulically actuated actuators.

\section{Pump-Valve Control System}

To realize isolated control of each actuator, a pump-valve control system was assembled and integrated into a portable waist belt pack (Figure 4A). The control system consisted of a a microcontroller (Arduino Mega, Arduino), a miniature diaphragm pneumatic pump (D737-23-01, Parker, USA), five miniature solenoid valves (X-Valve, Parker, USA), and five air pressure sensors (MPX5500DP, Freescale, USA) (Figure 4B). The microcontroller regulated the measured air pressure $(\mathrm{P})$ to track the desired pressure $\left(\mathrm{P}_{\text {ref }}\right)$ and used pulse width modulation (PWM) to control the activation and deactivation of the valves and pump based on the readings of the pressure sensors. The control system could be powered by a $12 \mathrm{~V}$ rechargeable lithium polymer battery (DC12300, China). The total weight of the control system was $\sim 1.26 \mathrm{~kg}$, which was lighter than the control system $(3.3 \mathrm{~kg})$ presented by Polygerinos et al. (2015c).

A Proportional Integral Derivative (PID) control algorithm was used to ensure that the measured air pressure $(\mathrm{P})$ of the actuators was close to desired air pressure $\left(\mathrm{P}_{\text {ref }}\right)$. The PID control scheme was implemented on the microcontroller with a sampling frequency of $100 \mathrm{~Hz}$. The valves have a nominal response time of $20 \mathrm{~ms}$ and a PWM frequency of $50 \mathrm{~Hz}$ was used. The control parameters were selected as $\mathrm{K}_{\mathrm{p}}=10, \mathrm{~K}_{\mathrm{i}}=0.7$, and $\mathrm{K}_{\mathrm{d}}=0.1$. The desired pressure $\mathrm{P}_{\text {ref }}$ was set at $120 \mathrm{kPa}$, which was the pressure that corresponded to full finger flexion based on the results from ROM test. The actuator for the index finger was pressurized from 0 to $120 \mathrm{kPa}$. The control loop was tested in a step response experiment (Figure 4C). The closed-loop system had a rise time of $1.36 \mathrm{~s}$ and a settling time (5\%) of $1.63 \mathrm{~s}$. The closed-loop system bandwidth was found to be $0.342 \mathrm{~Hz}$ (Figure S5).

\section{Glove-Assisted Range of Motion}

Five healthy participants ( 3 males, 2 females, age: $26.2 \pm 2.4$ yr) were recruited in order to evaluate the performance of the glove in terms of its assisted-finger ROM and grip strength. All participants gave written informed consent in accordance with the Declaration of Helsinki. The study protocol was approved 

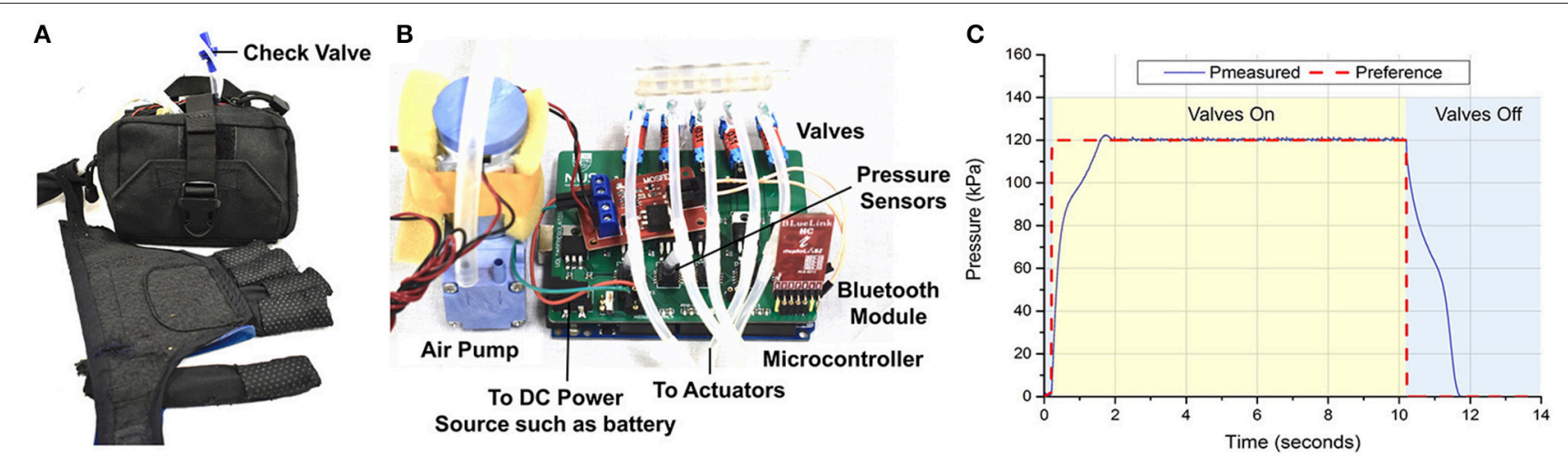

FIGURE 4 | (A) Pump-valve control system integrated into a waist belt pack. (B) Inner view of the control system. (C) Step response of the controller implemented on the pump-valve control system.

by the Institutional Review Board of the National University of Singapore.

Two sessions were conducted for each participant. In the first session, which consisted of three active trials, the participants were instructed to close and open their right hand with the presence of the glove. The length of the finger-actuator pockets was adjusted to fit the finger length of the specific subject. In the second session, which consisted of three glove-assisted trials, the participants were instructed to relax their muscles and not to exert any voluntary finger action. The hand closing and opening were assisted by the actuation of the soft actuators. EMG signals of the finger flexors and extensors were recorded during the trials to identify whether the subject was exerting voluntary control of the hand during glove-assisted trials.

The assisted-finger ROM and the EMG data were obtained through the test method reported in our previous study (Yap et al., 2016c). Additional details regarding the test method are reported in the "Test Method for Glove-assisted ROM" section of the Supplementary Material.

\section{Glove-Assisted Grip Strength}

A portable setup with a digital dynamometer (HF-50, China) was developed to measure the glove-assisted grip strength of the participants (Figure 5A). Upon pressurization to $120 \mathrm{kPa}$, the glove assisted participants with grasping a cylinder (50 and $75 \mathrm{~mm}$ in diameter) (Figure 5B). The participants were instructed to not exert voluntary force and hence, the grasping of the cylinder would be solely due to the force generated by the inflated actuators. The cylinder was tied to a Kevlar cable attached to the dynamometer. As the participants grasp and lift the cylinder upwards, the cylinder would be slowly released from the actuators' grip, when the tension in the cable became larger than the frictional force applied by the actuators (Figure 5C). This setup allowed the measurement of frictional grip force applied by the actuators, with the glove worn by the participants. The setup and the mechanism of the slip of the cylinder from the hand are similar to those reported in previous literature (Westling and Johansson, 1984; Johansson and Westling, 1988; In et al., 2017).
A

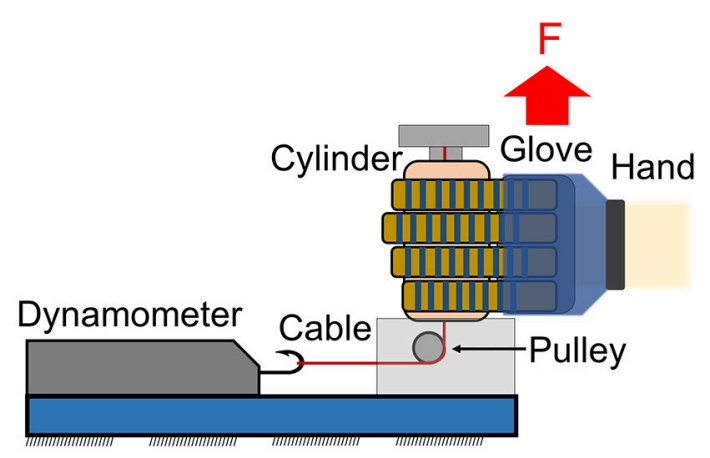

B

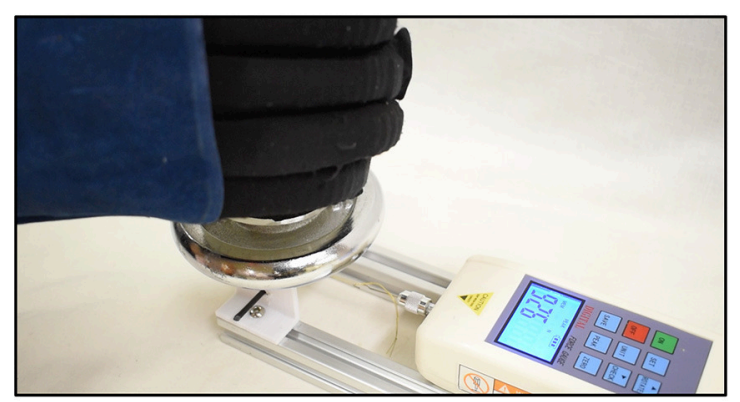

FIGURE 5 | (A) Schematic and (B) the real setup of a portable setup with dynamometer to measure the glove-assisted grip strength. Maximum frictional grip force was recorded before the cylinder released from the actuators' grip.

\section{Pilot Test with Stroke Survivors}

A pilot test that evaluated the feasibility of the glove in providing hand grasping assistance for ADL tasks was performed on two stroke survivors. All participants gave written informed consent in accordance with the Declaration of Helsinki. The study protocol was approved by the NHG Domain Specific Review Board. The subject demographics are shown in Table 2.

The patients were instructed to use their affected hand to grasp an empty water bottle (Weight: $20 \mathrm{~g}$, Diameter: $60 \mathrm{~mm}$ ) and a tin can (Weight: $454 \mathrm{~g}$, Diameter: $75 \mathrm{~mm}$ ) typically used in Jebsen 
TABLE 2 | Stroke patients' demographic data.

\begin{tabular}{lcllll}
\hline Sex (age) & Time post stroke (months) & Type of stroke & Affected side & Fugl-meyer motor assessment (Upper extremity) & Modified ashworth scale \\
\hline$F(50)$ & 13 & Infarct & Left & 4 & 1 \\
M (40) & 10 & Infarct & Left & 5 & $1+$
\end{tabular}

Hand Function Tests (Beebe and Lang, 2009). The same task was repeated having the patients wear the glove. The task was considered successful if the patient was able to grasp, lift, and put down the object. The participants were allowed to use their nonparetic hand to support their paretic forearm if they could not lift their paretic arm. The time taken to complete the tasks (grasp, lift, and put down the bottle or can) was recorded. The maximum time allowed to complete the task was $90 \mathrm{~s}$. The experiment was repeated three times for each object and the results were averaged across six trials for each patient in each condition (i.e., with and without glove assistance).

In this work, we employed a straightforward button control strategy to control the glove. A phone application with three virtual buttons, (namely grasping, pinching, and tripod pinching) was built to interface with the control system through wireless Bluetooth communication (Figure S7). The patients were instructed to click the virtual buttons using their non-paretic hand to activate the glove for grasping activities. To deactivate the glove, the patients just simply clicked the specific activated button again.

After the test, the patients' feedback on the device was obtained. The patients were instructed to fill in the UsefulnessSatisfaction-and-Ease-of-use questionnaire (USE) (Lund, 2001) and a questionnaire focusing on comfort level, desire to use, and desire to purchase the device. Both questionnaires use a seven-point Likert rating scale that focuses on the experience and feedback of the system.

\section{RESULTS}

\section{Blocked Tip Force Output}

The average values of the experimental force and the force obtained from a theoretical model were compared and shown in Figure 6. The force increased with increased pressure (Figure 6A). The soft actuators could generate a maximum tip force of $9.12 \mathrm{~N}$ at $120 \mathrm{kPa}$. Similarly, the force values obtained from the theoretical model increased with increased pressure, with a tip force value of $9.47 \mathrm{~N}$ at $120 \mathrm{kPa}$.

\section{Normal and Frictional Grip Force Output}

Figures 6B,C plotted the force (normal/frictional grip force)displacement of the cylinder (distance traveled by the cylinder from initial position) characteristics of the force measurement scenario. The average values of the normal and frictional grip force applied by the actuators are shown in Figures 6B,C. The results showed that four actuators could generate a normal grip force of up to $36.2 \pm 4.5 \mathrm{~N}$ (Diameter: $75 \mathrm{~mm}$ ) and $28.1 \pm 3.6 \mathrm{~N}$ (Diameter: $50 \mathrm{~mm}$ ) (Figure 6B). The actuators could generate a frictional grip force of up to $18.7 \pm 0.9 \mathrm{~N}$ (Diameter: $75 \mathrm{~mm}$ ) and $12.4 \pm 1.7 \mathrm{~N}$ (Diameter: $50 \mathrm{~mm}$ ) (Figure 6C). The normal and
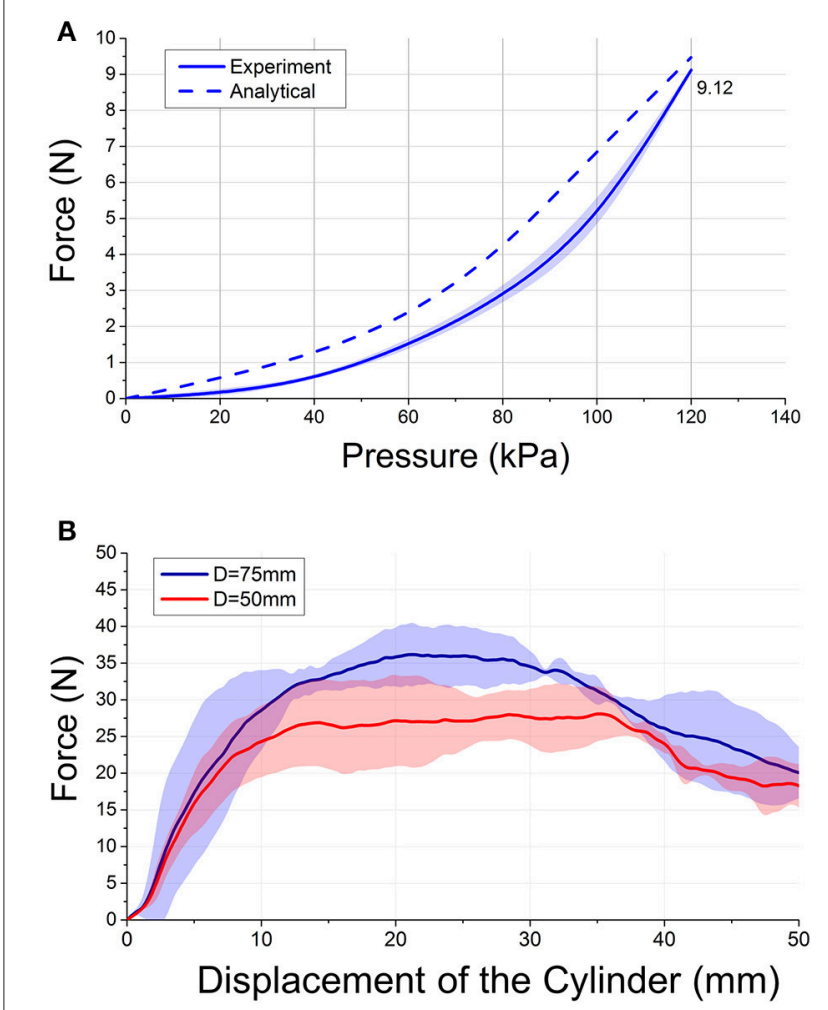

C

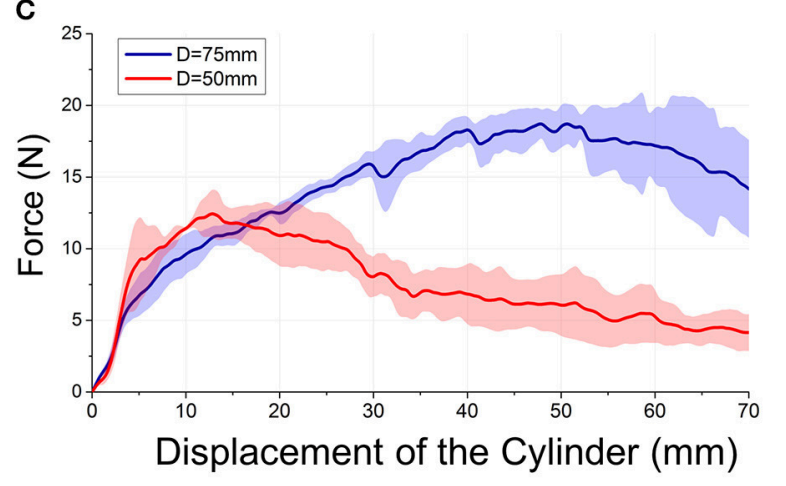

FIGURE 6 | (A) Experimental and theoretical blocked tip force values over increasing pressures. (B) Normal and (C) frictional grip force

output-displacement of the cylinder relationships of four actuators gripping cylinder with diameter $D=75 \mathrm{~mm}$ and $D=50 \mathrm{~mm}$ at a fixed pressure $(120 \mathrm{kPa})$.

frictional grip forces decreased with decreased cylinder diameter. As the objects of daily living do not weigh more than $1.5 \mathrm{~kg}$ (Matheus and Dollar, 2010), the frictional force was found to be sufficient to lift most of the objects. 


\section{Glove-Assisted Range of Motion}

During active trials, the maximum achievable joint angles were $82.3 \pm 6.8^{\circ}, 84.1 \pm 7.9^{\circ}$, and $44.8 \pm 4.4^{\circ}$ at MCP, PIP, and DIP joints. The sums of finger joint angles during active trials were measured to be $\sim 211^{\circ}$. During glove-assisted trials, the maximum achievable joint angles were $73.9 \pm 10.4^{\circ}, 79.9 \pm 4.2^{\circ}$, and $46.0 \pm 3.3^{\circ}$ at MCP, PIP, and DIP joints. The sums of finger joint angles were measured to be $\sim 200^{\circ}$ (Figure 7A). Statistical analysis was conducted using the non-parametric Wilcoxon's Signed-Rank tests. The tests showed no significant difference between active and glove-assisted trials ( $p=0.38$ for MCP joint, $P=0.32$ for PIP joint, and $P=1.25$ for DIP joint).

\section{Muscle Activation}

The muscle activation signals showed clear differences between active and glove-assisted trials (Figure 7B). During the glove-assisted trials, the amplitude of the normalized EMG signal confirmed that the participants exerted minimal muscle effort. Therefore, we concluded that the participants exerted

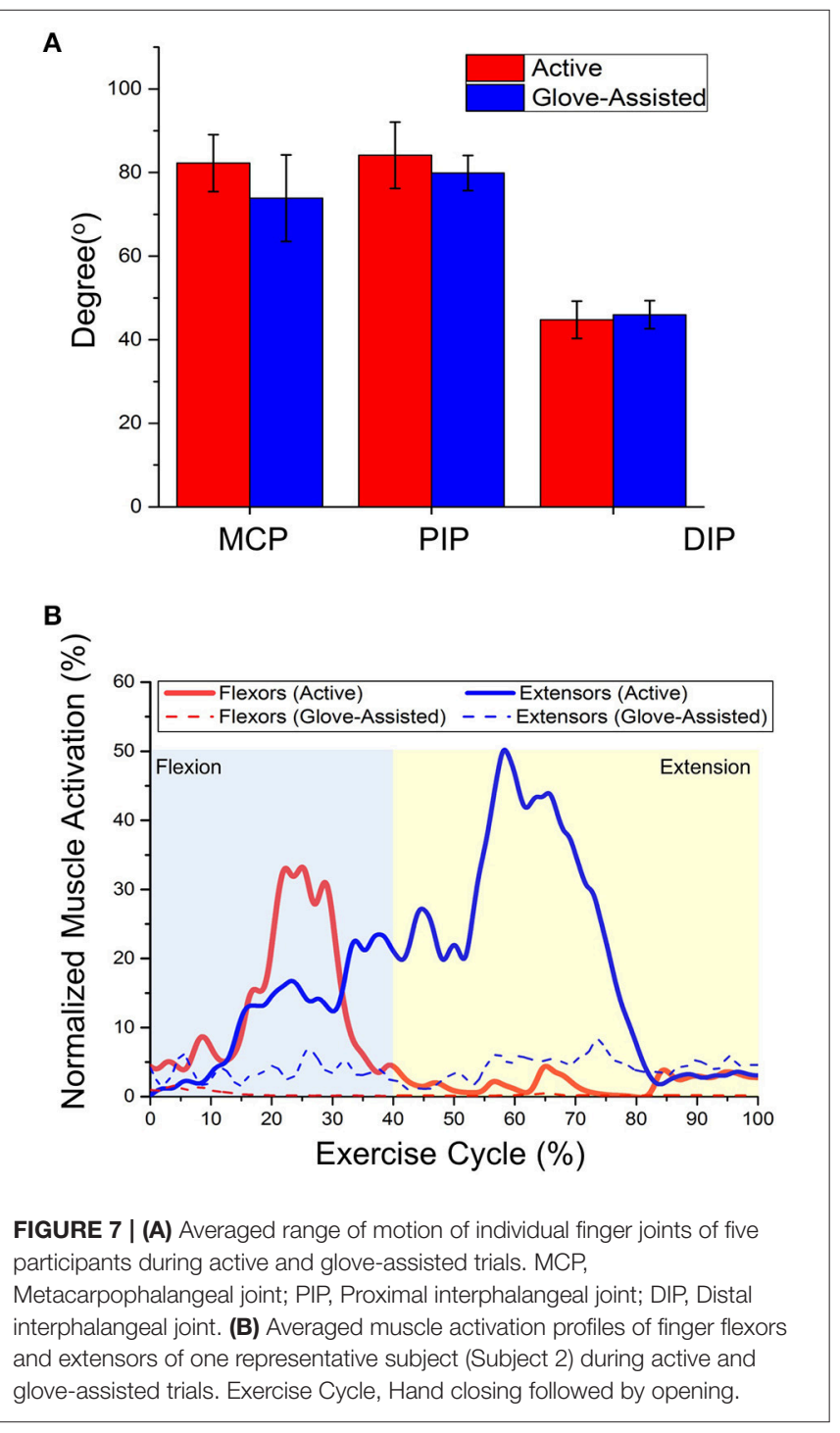

minimal voluntary movement during the glove-assisted trials. The hand closing and opening motions were completely assisted by the glove.

\section{Glove-Assisted Grip Strength}

When the glove was worn by the participants, the glove could generate a frictional grip force up to $8.4 \pm 1.8 \mathrm{~N}$ (Cylinder diameter: $75 \mathrm{~mm}$ ) and $5.8 \pm 1.7 \mathrm{~N}$ (Cylinder diameter: $50 \mathrm{~mm}$ ) to counteract the weight of an object. Statistical analysis was conducted using the Wilcoxon's Signed-Rank tests. The frictional grip force decreased with decreased cylinder diameter. Significant was concluded $(P=0.03)$.

\section{Simulated ADL Tasks}

Figure 8 demonstrates the ability of the glove to hold and lift a 454 g-weighted tin can in vertical (Figure 8A) and horizontal (Figure 8B) orientation. Additionally, we demonstrated that the glove was also able to assist with palmar grasp and pincer grasp that are required for simulated ADL tasks, such as grasping and pinching (Figures 8C,D).

\section{Pilot Test with Stroke Survivors}

Both patients were able to activate the glove using the phone application with virtual buttons (Figure 9). Without the assistance of the glove, patient S1 was able to finish the tasks in an average time of $9.0 \pm 1.4 \mathrm{~s}$ (Figure 9B). When patient S1 was grasping and lifting the water bottle, the patient could not grasp the bottle effectively and the bottle toppled. With the assistance of the glove, patient $\mathrm{S} 1$ could finish the tasks in an average time of $8.0 \pm 0.7 \mathrm{~s}$ (Figure 9C).

The hand of the patient S2 was slightly clenched. As a result, the patient could not finish the tasks within $90 \mathrm{~s}$ as the patient could not grasp both objects (Figure 9B). The patient could finish the tasks in an average time of $12.3 \pm 2.7 \mathrm{~s}$ with the assistance of the glove (Figure 9C).

Statistical analysis was conducted using the non-parametric tests. Due to poststroke heterogeneity and small sample size, Wilcoxon's Signed-Rank tests on individual participant data were used to determine each patient's improved time-taken to complete the task with the assistance of the glove. Significance was concluded on patient S2 $(p=0.02)$. Although significance was not concluded on patient S1 $(p=0.06)$, we could still observe that patient S1 could grasp the bottle more effectively with the assistance of the glove. Therefore, as hypothesized, the grasping performance of stroke patients improved with the assistance of the glove.

For patients' feedback, the mean and standard deviation (SD) of the questionnaire parameters are presented in Table 3. Generally, the patients reported scale of at least five out of seven-point Likert rating.

\section{DISCUSSION}

Compared to the fiber reinforced actuators developed for soft robotic glove application in previous studies, which operate at the pressure range of $275-375 \mathrm{kPa}$ (Polygerinos et al., 2015c), the fabric-reinforced soft actuators designed in this study required 

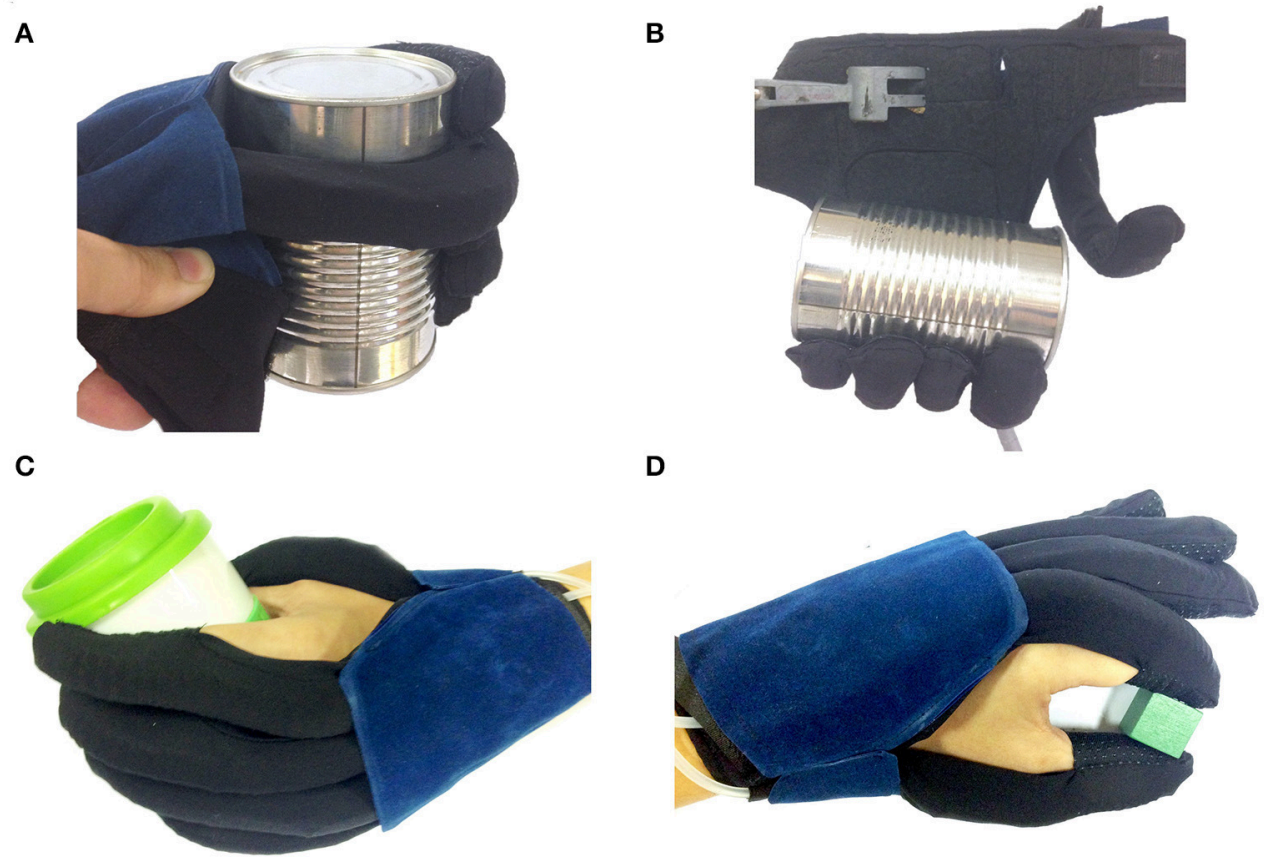

FIGURE 8 | The glove generated sufficient force to lift a 454 g-weighted can in the (A) vertical and (B) horizontal orientation. (C) Palmar grasp, (D) Pincer grasp achieved with the assistance of the glove.

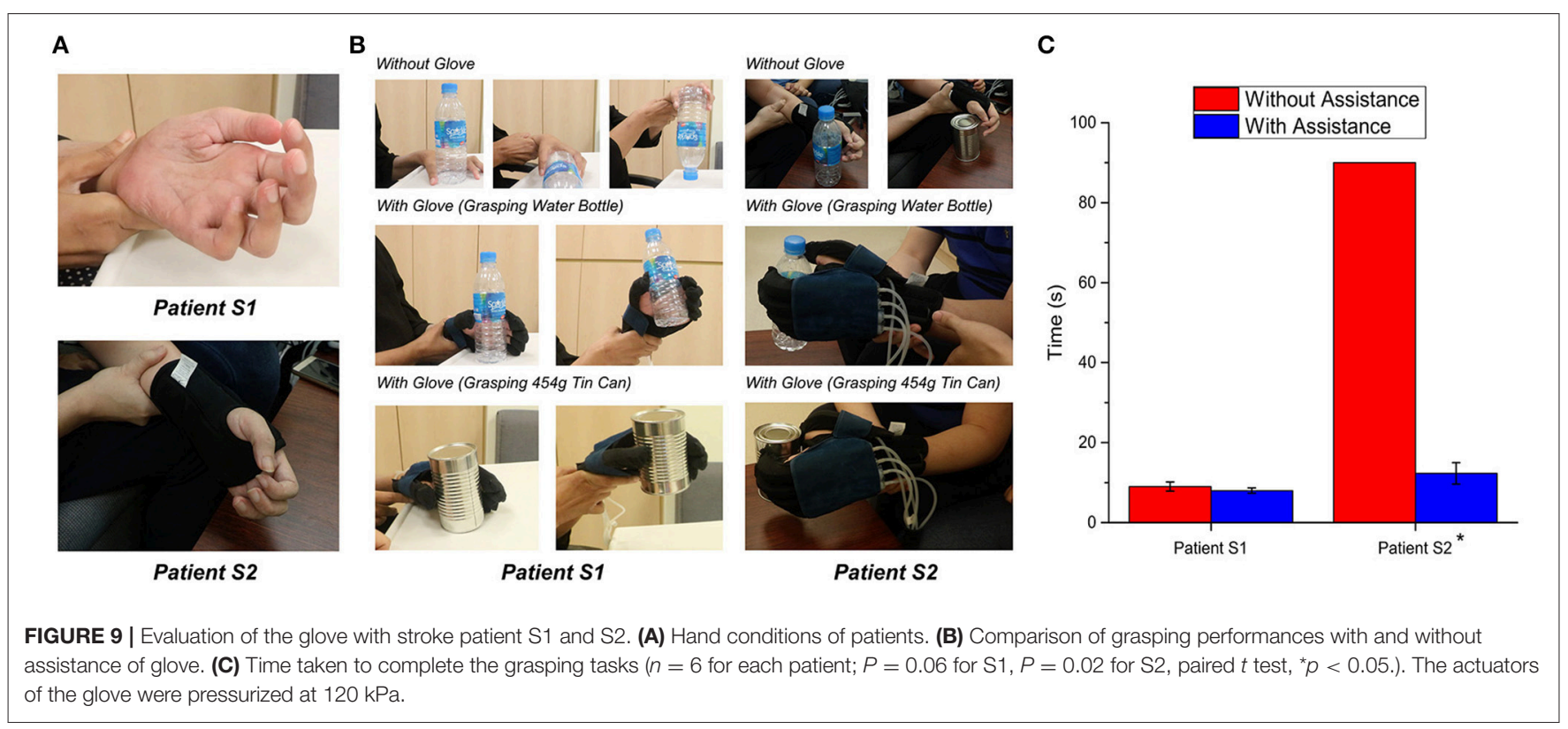

a lower range of operating pressures to achieve similar force output. With this advantage, we can select pumps and valves that operate at a lower pressure range and consume less power, which will lead to a more portable system.

Compared to the frictional grip force applied by the actuators when the glove was not worn (section Normal and Frictional Grip Force Output), the frictional grip force reduced when the glove was worn by the participants (section Muscle Activation). This might be due to the joint stiffness of the participants that imposed an opposing torque that the actuators needed to overcome during finger flexion. Nevertheless, the force generated was considered sufficient to grasp and achieve most of the ADLs (Matheus and Dollar, 2010; Polygerinos et al., 2015c). The frictional grip force is dependent on the object size, which can be observed from the grip force experiment. The experiment showed that the force output decreased with smaller cylinder diameter. This 
TABLE 3 | Questionnaire parameters and relative ratings of the USE questionnaire and a questionnaire focusing on comfort level, desire to use, and desire to purchase the device.

\begin{tabular}{lc}
\hline Parameters & Mean (SD) \\
\hline USE QUESTIONNAIRES & \\
Usefulness & $5.9 \pm 0.3$ \\
Ease of use & $6.4 \pm 0.4$ \\
Ease of learning & $6.6 \pm 0.2$ \\
Satisfaction & $6.6 \pm 0.5$ \\
PATIENTS' FEEDBACK & \\
Comfort level & $6.0 \pm 1.4$ \\
Desire to use & $6.5 \pm 0.7$ \\
Desire to purchase & $5.0 \pm 1.4$ \\
\hline
\end{tabular}

SD, standard deviation.

was because the force exerted by the actuators changed with the distance (i.e., the force decreased with increasing actuator bending angle). Additionally, the frictional grip force is also affected by the friction coefficient of the surface of the object and the anti-slip material of the finger pockets on the glove. The friction coefficient between the anti-slip material on the finger pocket and the surface of the cylinder could be estimated from the relationship between normal grip force and frictional grip force. In the measurement scenario described in this work, the coefficient was estimated at between 0.4 and 0.5 .

The average functional ROM of the hand, which are sufficient to perform more than $90 \%$ of daily functional activities, at MCP, PIP, and DIP are 61, 60, and $39^{\circ}$, respectively (Hume et al., 1990). Additionally, as the actuators designed in this study are able to extend to offset the increased distance due to skin stretching during finger flexion, the reported assisted ROM at MCP is higher than the assisted ROM reported in our previous study (Yap et al., 2016c). The EMG experiment confirmed that the participants exerted no voluntary effort during the glove-assisted trials and the hand grasping action was completely assisted by the glove. Therefore, our results demonstrated that the glove could achieve sufficient ROM and provide assistance with grasping motions typically found in ADL, in the absence of voluntary muscle control.

The choice of pneumatic over hydraulic allows the control system to be more portable and lightweight than the control system presented by Polygerinos et al. (2015b,c), as it does not require a water reservoir. However, the limitation of the pneumatic system is that it suffers from slow dynamics due to slow valve discharging speed and slow response times when depressurizing, as presented in previous related works on soft robotic gloves (Polygerinos et al., 2015c; Yap et al., 2017). In this work, the closed-loop system bandwidth of the control system was found to be $0.342 \mathrm{~Hz}$. The value $(0.342 \mathrm{~Hz})$ is similar to the bandwidth of the control system presented by Polygerinos et al. $(0.25 \mathrm{~Hz})$. Although the bandwidth was less than the common bandwidth of rigid linkage-based and cable-driven hand exoskeletons (Agarwal et al., 2015; Xiloyannis et al., 2016), it can be considered sufficient for patients to conduct functional tasks for ADLs. The valves used in current system could be replaced with valves with faster flow rate in the future to increase the bandwidth of the actuators.

The limitation of the previous soft actuator design is that the actuators apply forces to flex the finger upon air pressurization. Upon depressurization, the actuators assist finger extension passively with the elastic properties of the actuators and the elastic textile materials of the actuator pockets (Polygerinos et al., 2015b,c; Yap et al., 2016a, 2017). The extension force might not be enough for patients with hypertonicity and increased finger flexor tone. In this work, the bottom elastic fabric layer is able to further enhance the extension force required to bring the fingers to the open hand state. The elastic modulus of the elastic fabric $(0.45 \mathrm{~N} / \mathrm{mm})$ was chosen to match the elastic modulus of the elastic cords described in previous devices that allow the patients to flex their fingers voluntarily and provide assistance in the hand opening (Brokaw et al., 2011). The elastic cords are attached to the distal phalanx of each finger at the dorsal side. The tensions in the elastic cords increase with increasing finger flexion and pull the fingers to the open hand state. Assuming an offset distance of $10 \mathrm{~mm}$ due to skin stretching during finger flexion, the elastic fabric is able to provide an estimated $4.5 \mathrm{~N}$ of extension force to pull the fingers to the open hand state when the actuators are depressurized.

Pilot testing with two stroke survivors demonstrated the feasibility of the glove in providing functional grasp assistance for ADL. The glove is suitable for stroke survivors with a flaccid hand and without increased flexor tone (Modified Ashworth Scale $>2$ ). Without the assistance of the glove, the patients could not grasp cylindrical objects effectively, due to weak grasp strength and contracture. With the presence of the glove, the patients could then grasp the objects more effectively as the fingers were slightly more extended due to the elastic components of the glove. When the actuators were pressurized, the glove provided an additional grasping force to the patients' hand and assisted with the grasping activities. Therefore, the preliminary results showed that with the presence and assistance of the glove, the patients' grasping performance improved. The results of the USE questionnaire and the patients' feedback revealed that the patients were generally satisfied with the glove. Additionally, patients reported high level of comfort and ease of use. The parameter with the lowest rating is "Desire to purchase". The patients pointed out that they would purchase if the selling price was within their affordable range, which was between 500 and 1,000 USD. Otherwise, they would prefer rental. The most positive aspects that the patients pointed out included (1) The glove serves the purposes of mobilizing the finger joints and assisting their grasping activities and (2) The glove motivates the patient to do rehabilitation exercises. On the other hand, the most negative aspect is that the fabric of the glove should be able to be sanitized and washed easily for hygiene purpose.

\section{CONCLUSION}

This paper presented a soft robotic glove designed to assist stroke survivors with grasping tasks during their ADLs. Fabricreinforced actuators with a corrugated fabric layer and a 
reinforced elastic layer were developed and experimental results have shown that the actuators could support finger motions with desired force output at lower operating air pressure, compared to the required operating pressure of previously developed actuators. A control system was built to allow isolated control of each actuator and integrated into a portable waist belt back. Both the glove and the control system are more lightweight than previously developed glove systems. The glove was evaluated with five healthy participants in terms of the assisted ROM and grip strength. Our results demonstrated that the glove was able to achieve sufficient ROM and provided assistance with motions typically found in ADL, in the absence of voluntary muscle control. A pilot test on two stroke survivors with reduced hand function also demonstrated that the glove allowed the patients to perform functional grasping activities more effectively.

\section{FUTURE WORK}

In this work, the experiments on grip force, glove-assisted ROM, and pilot tests with stroke survivors were conducted at a fixed actuator pressure $(120 \mathrm{kPa})$. While the results from section Blocked Tip Force Output have shown that the actuator force increased with increased pressure, it is possible to conduct a more extensive study in the future by obtaining objective measurements, such as force, ROM, and grip strength, as a function of actuator pressure and presenting the time course of the pressure during each task. The force, ROM and grip strength can be controlled by adjusting the air pressure according to patient's condition. This feature will be further explored in subsequent work.

Future iterations of the glove will include sensor components, such as joint angle sensors and force sensors, which can provide joint angle and grasp force feedback. Joint angle feedback allows closed-loop position control and will enable the pumpvalve control system to identify whether the desired handfinger posture has been achieved. The grasp force feedback will permit us to examine the force output of the glove and to facilitate impedance control strategies (Marchal-Crespo and Reinkensmeyer, 2009).

While stroke survivors are the target patient population of the glove presented in this work, the glove can also potentially assist patients with incomplete spinal cord injuries or amyotrophic lateral sclerosis. Apart from serving as an assistive device for ADLs, the glove described in this work can potentially serve as a rehabilitative device, providing task-specific rehabilitation training. Several research groups working on hand exoskeletons have shown that incorporation of these devices into hand therapy can benefit the stroke survivors. Significant improvements in functional outcome have been observed with the combination of assist-as-needed control strategy and user intent detection strategy (Hu et al., 2013; Thielbar et al., 2014, 2017). For example, Theilbar et al. have developed a cable-driven robotic glove with the combination of voice and EMG to detect user intention.
Their study has shown that incorporation of the glove into the rehabilitation therapy has the potential for clinical use to improve hand function.

For stroke patients with increased flexor tone and spasticity (Modified Ashworth Scale $>2$ ), the passive extension mechanism might not have enough force to extend the fingers. For these patients, soft robotic glove that is capable of providing active flexion and extension will be preferable. Finally, a larger clinical study will be conducted in order to evaluate and improve the actuator and glove design, and to study the long-term efficacy of the glove-assisted intervention in rehabilitation.

\section{ETHICS STATEMENT}

This study was carried out in accordance with the recommendations of Institutional Review Board of the National University of Singapore (B-14-141) and the NHG Domain Specific Review Board (2015/00975) with written informed consent from all subjects. All subjects gave written informed consent in accordance with the Declaration of Helsinki. The protocol was approved by the Institutional Review Board of the National University of Singapore (B-14-141) and the NHG Domain Specific Review Board (2015/00975).

\section{AUTHOR CONTRIBUTIONS}

HY, JL, FN, and CY designed the study. HY collected, processed and analyzed the data and drafted the manuscript. JL, FN, and CY contributed to the interpretation of findings. CY oversaw its coordination and helped to draft the manuscript. All authors read, edited and approved the final manuscript.

\section{FUNDING}

This work was funded by the A*STAR Medtech Innovation Grant (R397-000-260-305) and the National University Health System (NUHS)-Clinical Imaging Research Center (CIRC) Seed Funding Grant (R-172-000-323-511).

\section{ACKNOWLEDGMENTS}

The authors wish to thank all the participants and their family members who volunteered to take part to the experiment. The authors would like to express their appreciation NUS Graduate School for Integrative Sciences and Engineering for providing scholarship support to HY.

\section{SUPPLEMENTARY MATERIAL}

The Supplementary Material for this article can be found online at: https://www.frontiersin.org/articles/10.3389/fnins. 2017.00547/full\#supplementary-material 


\section{REFERENCES}

Agarwal, P., Fox, J., Yun, Y., O'malley, M. K., and Deshpande, A. D. (2015). An index finger exoskeleton with series elastic actuation for rehabilitation: design, control and performance characterization. Int. J. Rob. Res. 34, 1747-1772. doi: $10.1177 / 0278364915598388$

Andersen, C. K., Wittrup-Jensen, K. U., Lolk, A., Andersen, K., and Kragh-Sørensen, P. (2004). Ability to perform activities of daily living is the main factor affecting quality of life in patients with dementia. Health Qual. Life Outcomes 2, 52-52. doi: 10.1186/14777525-2-52

Aubin, P. M., Sallum, H., Walsh, C., Stirling, L., and Correia, A. (2013). A pediatric robotic thumb exoskeleton for at-home rehabilitation: the Isolated Orthosis for Thumb Actuation (IOTA). IEEE Int. Conf. Rehabil. Robot. 2013:6650500. doi: 10.1109/ICORR.2013.6650500

Beebe, J. A., and Lang, C. E. (2009). Relationships and responsiveness of six upper extremity function tests during the first six months of recovery after stroke. $J$. Neurol. Phys. Ther. 33, 96-103. doi: 10.1097/NPT.0b013e3181a33638

Brokaw, E. B., Black, I., Holley, R. J., and Lum, P. S. (2011). Hand Spring Operated Movement Enhancer (HandSOME): a portable, passive hand exoskeleton for stroke rehabilitation. IEEE Trans. Neural Syst. Rehabil. Eng. 19, 391-399. doi: 10.1109/TNSRE.2011.2157705

Chen, Y., Le, S., Tan, Q. C., Lau, O., Wan, F., and Song, C. (2017). A lobsterinspired robotic glove for hand rehabilitation. IEEE Int. Conf. Robot. Automat. 2017, 4782-4787. doi: 10.1109/ICRA.2017.7989556

Coffey, A. L., Leamy, D. J., and Ward, T. E. (2014). A novel BCI-controlled pneumatic glove system for home-based neurorehabilitation. Conf. Proc. IEEE Eng. Med. Biol. Soc. 2014, 3622-3625. doi: 10.1109/EMBC.2014.6944407

Connelly, L., Jia, Y., Toro, M. L., Stoykov, M. E., Kenyon, R. V., and Kamper, D. G. (2010). A pneumatic glove and immersive virtual reality environment for hand rehabilitative training after stroke. IEEE Trans. Neural Syst. Rehabil. Eng. 18, 551-559. doi: 10.1109/TNSRE.2010.2047588

Farrell, J. F., Hoffman, H. B., Snyder, J. L., Giuliani, C. A., and Bohannon, R. W. (2007). Orthotic aided training of the paretic upper limb in chronic stroke: results of a phase 1 trial. NeuroRehabilitation 22, 99-103.

Feifei, Z., Dohta, S., Akagi, T., and Matsushita, H. (2006). Development of a bending actuator using a rubber artificial muscle and its application to a robot hand. SICE-ICASE Int. Joint Conf. 2006, 381-384. doi: 10.1109/SICE.2006.315260

Galloway, K. C., Becker, K. P., Phillips, B., Kirby, J., Licht, S., Tchernov, D., et al. (2016). Soft robotic grippers for biological sampling on deep reefs. Soft Robot. 3, 23-33. doi: 10.1089/soro.2015.0019

Gasser, B. W., and Goldfarb, M. (2015). Design and performance characterization of a hand orthosis prototype to aid activities of daily living in a poststroke population. Conf. Proc. IEEE Eng. Med. Biol. Soc. 2015, 3877-3880. doi: 10.1109/EMBC.2015.7319240

Haghshenas-Jaryani, M., Carrigan, W., Nothnagle, C., and Wijesundara, M. B. J. (2016). Sensorized soft robotic glove for continuous passive motion therapy. IEEE Int. Conf. Biomed. Robot. Biomechatronics 2016, 815-820. doi: 10.1109/BIOROB.2016.7523728

Hu, X. L., Tong, K. Y., Wei, X. J., Rong, W., Susanto, E. A., and Ho, S. K. (2013). The effects of post-stroke upper-limb training with an electromyography (EMG)-driven hand robot. J. Electromyogr. Kinesiol. 23, 1065-1074. doi: 10.1016/j.jelekin.2013.07.007

Hume, M. C., Gellman, H., McKellop, H., and Brumfield, R. H. Jr. (1990). Functional range of motion of the joints of the hand. J. Hand Surg. Am. 15, 240-243. doi: 10.1016/0363-5023(90)90102-W

In, H., Kang B. B., and Cho, K. J. (2017). "Pinching performance of a spinal cord injured patient with exo-glove with respect to the tendon route design," in Converging Clinical and Engineering Research on Neurorehabilitation II. Biosystems \& Biorobotics, Vol. 15, eds J. Ibáñez, J. González-Vargas, J. Azorín, M. Akay, and J. Pons (Cham: Springer). doi: 10.1007/978-3-319-46669-9_77

Johansson, R. S., and Westling, G. (1988). Programmed and triggered actions to rapid load changes during precision grip. Exp. Brain Res. 71, 72-86. doi: 10.1007/BF00247523

Kadowaki, Y., Noritsugu, T., Takaiwa, M., Sasaki, D., and Kato, M. (2011). Development of soft power-assist glove and control based on human intent. J. Robot. Mechatron. 23, 281-291. doi: 10.20965/jrm.2011.p0281
Kang, B. B., Lee, H., In, H., Jeong, U., Chung, J., and Cho, K.-J. (2016). Development of a polymer-based tendon-driven wearable robotic hand. IEEE Int. Conf. Robot. Automat. 2016, 3750-3755. doi: 10.1109/ICRA.2016. 7487562

Katz, S. (1983). Assessing self-maintenance: activities of daily living, mobility, and instrumental activities of daily living. J. Am. Geriatr. Soc. 31, 721-727. doi: 10.1111/j.1532-5415.1983.tb03391.x

Kline, T., Kamper, D., and Schmit, B. (2005). Control system for pneumatically controlled glove to assist in grasp activities. Int. Conf. Rehabil. Robot. 2005, 78-81. doi: 10.1109/ICORR.2005.1501056

Kutner, N. G., Zhang, R., Butler, A. J., Wolf, S. L., and Alberts, J. L. (2010). Qualityof-life change associated with robotic-assisted therapy to improve hand motor function in patients with subacute stroke: a randomized clinical trial. Phys. Ther. 90, 493-504. doi: 10.2522/ptj.20090160

Lindberg, P. G., Roche, N., Robertson, J., Roby-Brami, A., Bussel, B., and Maier, M. A. (2012). Affected and unaffected quantitative aspects of grip force control in hemiparetic patients after stroke. Brain Res. 1452, 96-107. doi: 10.1016/j.brainres.2012.03.007

Lund, A. M. (2001). Measuring usability with the USE questionnaire. Usabil. Interface 8, 3-6.

Maciejasz, P., Eschweiler, J., Gerlach-Hahn, K., Jansen-Troy, A., and Leonhardt, S. (2014). A survey on robotic devices for upper limb rehabilitation. J. Neuroeng. Rehabil. 11:3. doi: 10.1186/1743-0003-11-3

Maeder-York, P., Clites, T., Boggs, E., Neff, R., Polygerinos, P., Holland, D., et al. (2014). Biologically Inspired soft robot for thumb rehabilitation. J. Med. Dev. 8, 020933-020933-3. doi: 10.1115/1.4027031

Marchal-Crespo, L., and Reinkensmeyer, D. (2009). Review of control strategies for robotic movement training after neurologic injury. J. Neuroeng. Rehabil. 6:20. doi: 10.1186/1743-0003-6-20

Martinez, L. A., Olaloye, O. O., Talarico, M. V., Shah, S. M., Arends, R. J., and Busha, B. F. (2010). A power-assisted exoskeleton optimized for pinching and grasping motions. IEEE 36th Annu. Northeast Bioeng. Conf. 2010, 1-2. doi: 10.1109/NEBC.2010.5458232

Martinez, R. V., Branch, J. L., Fish, C. R., Jin, L., Shepherd, R. F., Nunes, R. M. D., et al. (2013). Robotic tentacles with three-dimensional mobility based on flexible elastomers. Adv. Mater. 25, 205-212. doi: 10.1002/adma.201203002

Martinez, R. V., Fish, C. R., Chen, X., and Whitesides, G. M. (2012). Elastomeric origami: programmable paper-elastomer composites as pneumatic actuators. Adv. Funct. Mater. 22, 1376-1384. doi: 10.1002/adfm.201102978

Matheus, K., and Dollar, A. M. (2010). Benchmarking grasping and manipulation: properties of the objects of daily living. IEEE/RSJ Int. Conf. Intell. Robot. Syst. 2010, 5020-5027. doi: 10.1109/IROS.2010.5649517

Michaelsen, S. M., Dannenbaum, R., and Levin, M. F. (2006). Task-specific training with trunk restraint on arm recovery in stroke: randomized control trial. Stroke 37, 186-192. doi: 10.1161/01.STR.0000196940.20446.c9

Nilsson, M., Ingvast, J., Wikander, J., and Von Holst, H. (2012). The Soft Extra Muscle system for improving the grasping capability in neurological rehabilitation. IEEE EMBS Conf. Biomed. Eng. Sci. 2012, 412-417. doi: 10.1109/IECBES.2012.6498090

Nordina, I., Faudzia, A., Kamarudina, M., Dewic, D. E. O., Rehmana, T., Razif, M., et al. (2016). Grip force measurement of soft-actuated finger exoskeleton. J. Teknol. 78, 25-30. doi: 10.11113/jt.v78.9268

Noritsugu, T., Takaiwa, M., and Sasaki, D. (2008). Power assist wear driven with pneumatic rubber artificial muscles. Int. Conf. Mechatron. Mach. Vision Pract. 2008, 539-544. doi: 10.1109/MMVIP.2008.4749589

Polygerinos, P., Galloway, K. C., Sanan, S., Herman, M., and Walsh, C. J. (2015a). EMG controlled soft robotic glove for assistance during activities of daily living. IEEE Int. Conf. Rehabil. Robot. 2015, 55-60. doi: 10.1109/ICORR.2015.7281175

Polygerinos, P., Galloway, K. C., Savage, E., Herman, M., Donnell, K. O., and Walsh, C. J. (2015b). Soft robotic glove for hand rehabilitation and task specific training. IEEE Int. Conf. Robot. Automat. 2015, 2913-2919. doi: 10.1109/ICRA.2015.7139597

Polygerinos, P., Wang, Z., Galloway, K. C., Wood, R. J., and Walsh, C. J. (2015c). Soft robotic glove for combined assistance and at-home rehabilitation. Rob. Auton. Syst. 73, 135-143. doi: 10.1016/j.robot.2014.08.014

Polygerinos, P., Wang, Z., Overvelde, J. T. B., Galloway, K. C., Wood, R. J., Bertoldi, K., et al. (2015d). Modeling of soft fiber-reinforced bending actuators. IEEE Trans. Robot. 31, 778-789. doi: 10.1109/TRO.2015.2428504 
Rotella, M. F., Reuther, K. E., Hofmann, C. L., Hage, E. B., and Busha, B. F. (2009). An orthotic hand-assistive exoskeleton for actuated pinch and grasp. IEEE 36th Annu. Northeast Bioeng. Conf. 2009, 1-2. doi: 10.1109/NEBC.2009.4967693

Sangwook, L., Landers, K. A., and Hyung-Soon, P. (2014). Development of a Biomimetic Hand Exotendon Device (BiomHED) for restoration of functional hand movement post-stroke. IEEE Trans. Neural Syst. Rehabil. Eng. 22, 886-898. doi: 10.1109/TNSRE.2014.2298362

Sasaki, D., Noritsugu, T., Takaiwa, M., and Yamamoto, H. (2004). Wearable power assist device for hand grasping using pneumatic artificial rubber muscle. IEEE Int. Workshop Robot Hum. Interact. Commun. 2004, 655-660. doi: 10.1109/ROMAN.2004.1374840

Scherer, M. J., Sax, C., Vanbiervliet, A., Cushman, L. A., and Scherer, J. V. (2005). Predictors of assistive technology use: the importance of personal and psychosocial factors. Disabil. Rehabil. 27, 1321-1331. doi: 10.1080/09638280500164800

Scherer, M., Jutai, J., Fuhrer, M., Demers, L., and Deruyter, F. (2007). A framework for modelling the selection of assistive technology devices (ATDs). Disabil. Rehabil. Assist. Technol. 2, 1-8. doi: 10.1080/17483100600845414

Smaby, N., Johanson, M. E., Baker, B., Kenney, D. E., Murray, W. M., and Hentz, V. R. (2004). Identification of key pinch forces required to complete functional tasks. J. Rehabil. Res. Dev. 41, 215-224. doi: 10.1682/JRRD.2004.02.0215

Tadano, K., Akai, M., Kadota, K., and Kawashima, K. (2010). Development of grip amplified glove using bi-articular mechanism with pneumatic artificial rubber muscle. IEEE Int. Conf. Robot. Autom. 2010, 2363-2368. doi: 10.1109/ROBOT.2010.5509393

Thielbar, K. O., Lord, T. J., Fischer, H. C., Lazzaro, E. C., Barth, K. C., Stoykov, M. E., et al. (2014). Training finger individuation with a mechatronic-virtual reality system leads to improved fine motor control post-stroke. J. Neuroeng. Rehabil. 11, 1-11. doi: 10.1186/1743-0003-11-171

Thielbar, K. O., Triandafilou, K. M., Fischer, H. C., O’toole, J. M., Corrigan, M. L., Ochoa, J. M., et al. (2017). Benefits of using a voice and EMG-Driven actuated glove to support occupational therapy for stroke survivors. IEEE Trans. Neural Syst. Rehabil. Eng. 25, 297-305. doi: 10.1109/TNSRE.2016.2569070

Toya, K., Miyagawa, T., and Kubota, Y. (2011). Power-assist glove operated by predicting the grasping mode. JSDD 5, 94-108. doi: 10.1299/jsdd.5.94

Varalta, V., Picelli, A., Fonte, C., Montemezzi, G., La Marchina, E., and Smania, N. (2014). Effects of contralesional robot-assisted hand training in patients with unilateral spatial neglect following stroke: a case series study. J. Neuroeng. Rehabil. 11:160. doi: 10.1186/1743-0003-11-160

Wang, Z., Polygerinos, P., Overvelde, J., Galloway, K., Bertoldi, K., and Walsh, C. (2017). Interaction forces of soft fiber reinforced bending actuators. IEEE/ASME Trans. Mechatron. 22, 717-727. doi: 10.1109/TMECH.2016.2638468

Westling, G., and Johansson, R. S. (1984). Factors influencing the force control during precision grip. Exp. Brain Res. 53, 277-284. doi: 10.1007/BF00238156
Worsnopp, T. T., Peshkin, M. A., Colgate, J. E., and Kamper, D. G. (2007) An actuated finger exoskeleton for hand rehabilitation following stroke. IEEE Int. Conf. Rehabil. Robot. 2007, 896-901. doi: 10.1109/ICORR.2007. 4428530

Xiloyannis, M., Cappello, L., Dinh Binh, K., Shih-Cheng, Y., and Masia, L. (2016). Modelling and design of a synergy-based actuator for a tendon-driven soft robotic glove. IEEE Int. Conf. Biomed. Robot. Biomechatron. 2016, 1213-1219. doi: 10.1109/BIOROB.2016.7523796

Yap, H. K., Ang, B. W. K., Lim, J. H., Goh, J. C. H., and Yeow, R. C. H. (2016a). A fabric-regulated soft robotic glove with user intent detection using EMG and RFID for hand assistive application. IEEE Int. Conf. Robot. Automat. 2016, 3537-3542. doi: 10.1109/ICRA.2016.7487535

Yap, H. K., Kamaldin, N., Lim, J. H., Nasrallah, F., Goh, J. C. H., and Yeow, C.-H (2017). A magnetic resonance compatible soft wearable robotic glove for hand rehabilitation and brain imaging. IEEE Trans. Neural Syst. Rehabil. Eng. 25, 782-793. doi: 10.1109/TNSRE.2016.2602941

Yap, H. K., Lim, J. H., Goh, J. C. H., and Yeow, C.-H. (2016b). Design of a soft robotic glove for hand rehabilitation of stroke patients with clenched fist deformity using inflatable plastic actuators. J. Med. Dev. 10:044504. doi: 10.1115/1.4033035

Yap, H. K., Lim, J. H., Nasrallah, F., Cho Hong Goh, J., and Yeow, C. H. (2016c). Characterisation and evaluation of soft elastomeric actuators for hand assistive and rehabilitation applications. J. Med. Eng. Technol. 40, 199-209. doi: 10.3109/03091902.2016.1161853

Yap, H. K., Lim, J. H., Nasrallah, F., Goh, J. C. H., and Yeow, R. C. H. (2015). A soft exoskeleton for hand assistive and rehabilitation application using pneumatic actuators with variable stiffness. IEEE Int. Conf. Robot. Automat. 2015, 4967-4972. doi: 10.1109/ICRA.2015.7139889

Ying, M., and Agrawal, S. K. (2012). Design of a Cable-Driven Arm Exoskeleton (CAREX) for neural rehabilitation. IEEE Trans. Robot. 28, 922-931. doi: 10.1109/TRO.2012.2189496

Yun, S. S., Kang, B. B., and Cho, K. J. (2017). Exo-Glove PM: an easily customizable modularized pneumatic assistive glove. IEEE Robot. Automat Lett. 2, 1725-1732. doi: 10.1109/LRA.2017.2678545

Conflict of Interest Statement: The authors declare that the research was conducted in the absence of any commercial or financial relationships that could be construed as a potential conflict of interest.

Copyright (C) 2017 Yap, Lim, Nasrallah and Yeow. This is an open-access article distributed under the terms of the Creative Commons Attribution License (CC BY). The use, distribution or reproduction in other forums is permitted, provided the original author(s) or licensor are credited and that the original publication in this journal is cited, in accordance with accepted academic practice. No use, distribution or reproduction is permitted which does not comply with these terms. 
OPEN ACCESS

Edited by:

Dingguo Zhang,

Shanghai Jiao Tong University, China

Reviewed by:

Yi Sun,

National University of Singapore,

Singapore

Min Li,

Xi'an Jiaotong University, China

*Correspondence:

Yoshikatsu Hayash

y.hayashi@reading.ac.uk

Specialty section: This article was submitted to

Neural Technology, a section of the journal

Frontiers in Neuroscience

Received: 16 February 2017 Accepted: 07 June 2017

Published: 07 July 2017

Citation:

Oguntosin WW, Mori Y, Kim H, Nasuto SJ, Kawamura S and

Hayashi Y (2017) Design and

Validation of Exoskeleton Actuated by

Soft Modules toward

Neurorehabilitation-Vision-Based Control for Precise Reaching Motion of Upper Limb. Front. Neurosci. 11:352. doi: 10.3389/fnins.2017.00352

\section{Design and Validation of Exoskeleton Actuated by Soft Modules toward Neurorehabilitation-Vision-Based Control for Precise Reaching Motion of Upper Limb}

\author{
Victoria W. Oguntosin ${ }^{1}$, Yoshiki Mori ${ }^{2}$, Hyejong Kim ${ }^{2}$, Slawomir J. Nasuto ${ }^{1}$, \\ Sadao Kawamura ${ }^{2}$ and Yoshikatsu Hayashi ${ }^{1 *}$ \\ ${ }^{1}$ Brain Embodiment Lab, Biomedical Engineering, School of Biological Sciences, University of Reading, Reading, United \\ Kingdom, ${ }^{2}$ Department of Robotics, Ritsumeikan University, Shiga, Japan
}

We demonstrated the design, production, and functional properties of the Exoskeleton Actuated by the Soft Modules (EAsoftM). Integrating the 3D printed exoskeleton with passive joints to compensate gravity and with active joints to rotate the shoulder and elbow joints resulted in ultra-light system that could assist planar reaching motion by using the vision-based control law. The EAsoftM can support the reaching motion with compliance realized by the soft materials and pneumatic actuation. In addition, the visionbased control law has been proposed for the precise control over the target reaching motion within the millimeter scale. Aiming at rehabilitation exercise for individuals, typically soft actuators have been developed for relatively small motions, such as grasping motion, and one of the challenges has been to extend their use for a wider range reaching motion. The proposed EAsoftM presented one possible solution for this challenge by transmitting the torque effectively along the anatomically aligned with a human body exoskeleton. The proposed integrated systems will be an ideal solution for neurorehabilitation where affordable, wearable, and portable systems are required to be customized for individuals with specific motor impairments.

Keywords: 3D printed exoskeleton, soft actuators, compliant assistance, active and passive mechanisms, gravity compensation, reaching motion

\section{INTRODUCTION}

Although an increased effort has been placed on the recovery process of patients following a stroke, with recent advances in technology for monitoring the brain functions, lack of human resources in therapeutic training of patients implies that patients generally may not reach their full recovery potential when discharged from hospital following initial rehabilitation (Loureiro and Harwin, 2007). Neurological illness often manifests in clinical condition resulting in weakness of the muscles controlling the elbow and rehabilitation devices are used in order to help in recovery of muscular power (Maciejasz et al., 2014). In stroke patients, damage to the motor-, and somatosensory cortices, makes it difficult to control and move the arm, forearm, and fingers. These patients often use assistive devices and therapy to encourage them to use their damaged limb so that the 
re-growth of a neural circuitry can take place that would eventually aid recovery through neuro-plasticity (Jack et al., 2001). Therefore, there is a growing interest in using robotic devices to deliver effective assistance. The aim of the robotic assistance is to provide a set of intensive and repetitive therapies to enhance the motor recovery of patients, decreasing the amount of work of a therapist. It has been found that it is important for a patient to undergo continuous exercise for successful rehabilitation (Heo et al., 2012) which robots are able to provide.

Typically, rehabilitation methods are either highly human intensive or involve attachment of rigid robotic system controlling the upper limb. Robot-based rehabilitation has been shown to have positive effects by reducing impairment (Loureiro et al., 2014). Robots provide an acceptable performance measurement test and control of the amount of exercise delivered to the subject (Kutner et al., 2014). Hard robotic structures are often based on joints connected by rigid links; this makes them heavy, with expensive and complicated control. In addition, these robotic systems are less compliant than the joints they actuate making it difficult to be directly attached to the human body unless through the use of specialized end effectors (Krebs et al., 2000), thereby mitigating their use for personal use by patients. To overcome these challenges, safe human robotic interactions, and inexpensive design can be achieved through a soft wearable robotic device (Tondu and Lopez, 2000; Tsagarakis and Caldwell, 2000).

A novel approach to rehabilitation is making use of inexpensive and soft actuators. Soft actuators are usually actuated with compressed air or SMAs (Shape Memory Alloy) (Chiu et al., 2013). SMA actuation is accomplished by embedding the trained alloy into the silicone rubber and using electric current to produce heat to deform the robot to the trained shape. Soft robots can be realized with the use of McKibben artificial muscles to produce a compliant motion similar to the skeletal muscle (Tsagarakis and Caldwell, 2000) and are actuated using a pneumatic source (Takagi et al., 2009).

Another novel approach uses embedded pneumatic networks of channels in elastomers (Ilievski et al., 2011) to achieve bending and crawling motions (Ilievski et al., 2011). Soft rehabilitation robots have been developed to generate assistive force for grasping (Shepherd et al., 2011) and gait rehabilitation (Shepherd et al., 2011). They make use of air channels embedded in elastomers to perform bending motions similar to the motion of the fingers. Other robots composed of silicone rubber for angular displacement (Sun et al., 2013) and as a 2D (Marchese et al., 2014) or 3D (Martinez et al., 2013) manipulators have been developed. An upper limb orthosis called Orthojacket actuated by pneumatics and designed for elbow rehabilitation has been developed (Schulz et al., 2011).

There have been a lot of research in developing and implementation of robotic devices for upper limb rehabilitation especially index and finger rehabilitation in the form of exoskeletons (Chiri et al., 2012; Maciejasz et al., 2014). These hand exoskeletons make use of cables (Dovat et al., 2008) and rubber (Polygerinos et al., 2015) for actuation purposes. Virtual reality has been used together with these hand exoskeletons to further aid in recovery (Ueki et al., 2012). Target tracking have also been incorporated in order to increase motivation and cooperation from the user as well as individualize exercise difficulty depending on strength the user (Jack et al., 2001).

In traditional, robotic rehabilitation systems interacting with the patient using a single distal attachment point on the forearm by means of an orthosis [for example, MIT-MANUS (Krebs et al., 2004)], exercises are defined in the XYZ Cartesian space relative to the robots single end-effector attachment point, and the assistance magnitude is modulated using impedance/admittance control schemes in the robot task-space. To support comfortably upper limb flexion and extension, WREX, Wilmington Robotic EXoskeleton, was designed at A. I. DuPont Hospital for Children to provide assistance for the wide range of natural motion in daily settings (Sanchez et al., 2006; Tariq Rahman et al., 2006). The JAECO WREX is a light weight exoskeleton with two links and four degrees of motion that approximates normal human anatomy. It incorporates elastic band elevation assists for both the shoulder and elbow to eliminate influence of gravity on the extremity (Sanchez et al., 2006; Tariq Rahman et al., 2006). The unique design of the shoulder and elbow joints allows for a significant improvement in the available range of motion when compared to other assistive devices.

A new type of wearable robots called soft exoskeletons or exosuits is becoming more popular, aiming at therapeutic intervention of upper limb by mirroring the motion of the other limb (Panasonic, 2005). Removing the external metal frame, soft exoskeletons become compliant, much lighter, and could be significantly less expensive than rigid frame wearable robots. However, soft exoskeleton's main drawback is the same as their main strength: they have no external rigid frame to support the body parts and transfer force effectively from actuator to some area of the body. We think that the effective transfer of the force should take into account the anatomical structure of limbs and joints, requiring the solid structure of the assistive device in order not to add unnecessary strain on the body parts that might damage patient joints, muscles, or tendons.

As a unique approach to a lightweight and flexible robotic structure, inflatable structures have been developed for the robotic arm as an attractive alternative method of imbuing robots with the desired lightness. Kim et al. developed the robotic arm that comprised of the inflatable links, air bag actuators, and ABS joint structures (Kim et al., 2015). Also, the novel control schemes have been applied on the basis of visual feedback control, which do not require the precise inverse kinematics for the position control (Nishida and Kawamura, 2012).

In our study, aiming at the reaching motion of the upper limb, we developed and validated the Exoskeleton Actuated by Soft Modules (EASoftM) to fulfill the four conditions; (1) A few degrees of freedom, (2) Precise motion control, (3) Wearable assistive robots, (4) Compliant assistance. We proposed the exoskeleton aligned with the anatomical structure, actuated by the soft modules located at the joint position, and the soft actuators realize the compliant motion based on pneumatic actuation and visco-elastic properties of soft materials such as plastic and rubber.

From the patient perspective, the ultra-light EASoftM offers upper limb assistance, i.e., it can assist the reaching motion 
of the upper limb, supporting the movement of the elbow and shoulder, and can record trajectories of hand reaching a target based on the visual feedback control. It also has high compliance and high safety integrity from the perspective of the patient, because it is entirely composed of rubber and soft modules, and attached to the body via Velcro straps. We first describe the design specification of the EASoftM with the scene setting (Sections 2.1 and 2.2), and secondly describe the detailed kinematics and the vision-based control schemes (Sections 3.13.3). After explaining the production of the plastic modules (Section 3.4), their torque characteristics and reliability were presented (Section 4). In the Result section (Section 5.1), a series of experiments is described for the motion of one and two degrees of freedom, first tested by using the weight. Finally, we presented the participant experiments, demonstrating the closedloop reaching motion on precise position control (Section 5.2). The General discussion is given in Section 6 .

As a contribution to neurorehabilitation, the EASoftM can be integrated with the Brain Computer Interface, for example, for detecting the motor command generation by online electroencephalogram (EEG) analysis, in order for the EASoftM to guide the hand to a target position in a rehabilitation therapy. The advantage of using EASoftM is that it does not include any motors which generate the elecro-magnetic waves which inevitably influence the electric signals from brain activity.

\section{DESIGN AND SPECIFICATIONS}

\subsection{Scene Setting for Rehabilitation}

To minimize detrimental effects of technology on the rehabilitation process, some of the technical requirements present in the design of most rehabilitation robots take into consideration the ergonomics of the system and its ability to cope with a variety of patient demographic and anthropomorphic parameters. An imperative requirement in the design of the human machine interface is that it should mimic behavior of the human therapist. That is, it should be compliant when assisting movement, provide full support within the patients passive range of motion and nourish the patient's confidence and motivation levels through goal-oriented motion with informative biofeedback.

Reaching motion is one of the most critical behaviors in our daily life at home; at the table, we reach and grasp a cup to drink, and in the kitchen we reach for the spice jars or pots. Thus, it is important to assist the motion for reaching purposes. required system characteristics can be decomposed into the following;

1. Attachable to chairs: A whole unit should be easy to be attached to a chair by the mount base.

2. Gravity compensation: An equilibrium point of the upper limb should be shifted to match the desired work space for reaching motion (Sanchez et al., 2006; Tariq Rahman et al., 2006).

3. Identification of the target: A target position should be programmed to be fed into the control law.

4. Guidance of the wrist toward the target: Active joints should provide appropriate torque to rotate the shoulder and elbow to assist the reaching motion.

5. Allowance for small deviation from the planned trajectories: Passive joints should should accommodate small spontaneous motion with compliance, and soft modules should provide compliance naturally by contained air and soft material.

We aim at rehabilitation for people with neuromuscular weakness such as muscle disease, cerebral palsy, spinal cord injury, and stroke, that effect the spontaneous motion of upper limbs. Also, given a proper balance between passive and active joints, the proposed system should provide an appropriate active assistance in guiding the hand to a target position. The integrated system should act as a functional aid in activities of daily living ideally at home, serving as a cost effective exercise/therapy device for people recovering from stroke.

\subsection{Design Specifications}

All of the considerations in Section 2.1 lead to five main specifications to be fulfilled by the Exoskeleton Actuated by Soft Modules which can assist the reaching motion;

1. Gravity compensation using exoskeleton: For any actuator, it is challenging to lift up the upper limb against gravity. Thus, the passive joints should be applied to shift the equilibrium position of upper limb (Sanchez et al., 2006; Tariq Rahman et al., 2006).

2. Degrees of freedom: Two passive joints should shift the equilibrium plane of the upper limb, and two active joints should guide the wrist to the target position.

3. Viscoelastic property for passive motion: The assistance by the exoskeleton should be controlled compliantly by viscoelastic properties of air and materials used in the actuators.

4. Structural transparency for active motion: Structural transparency should be such that the mass, viscosity and elasticity of the robotic arm are so small that patients performing spontaneous movement does not feel resistance in interactions with the robotic arm.

5. Vision-based control law: Control law should not require the encoders to decrease the total weight, and to increase the precision in reaching a target, the control law should not rely on the precise inverse kinematics of the exoskeleton.

\section{DESIGN, MATERIAL, AND METHODS}

Fulfilling the design specifications in the Section 2.2 in order to assist comfortable flexion and extension of the upper limb, we mainly concentrated on three characteristics; (1) A few degrees of freedom; anatomically structured exoskeleton with the parallel links for gravity compensation, (2) Compliant assistance: soft modules to actuate the elbow and shoulder joints, and (3) Precise motion control: visual control loop to guide the hand to the target at high precision.

\subsection{Kinematic Structure of Exoskeleton Actuated by Soft Modules (EASoftM)}

The overall structure of the proposed EASoftM is given in Figure 1 with a schematic representation of a participant, 


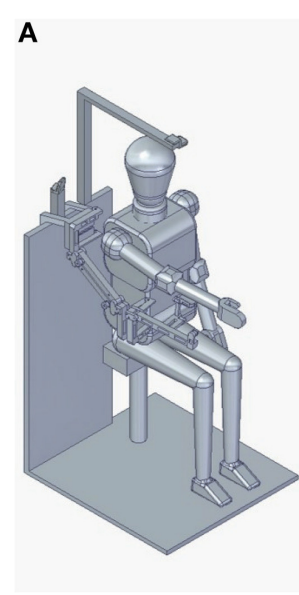

D
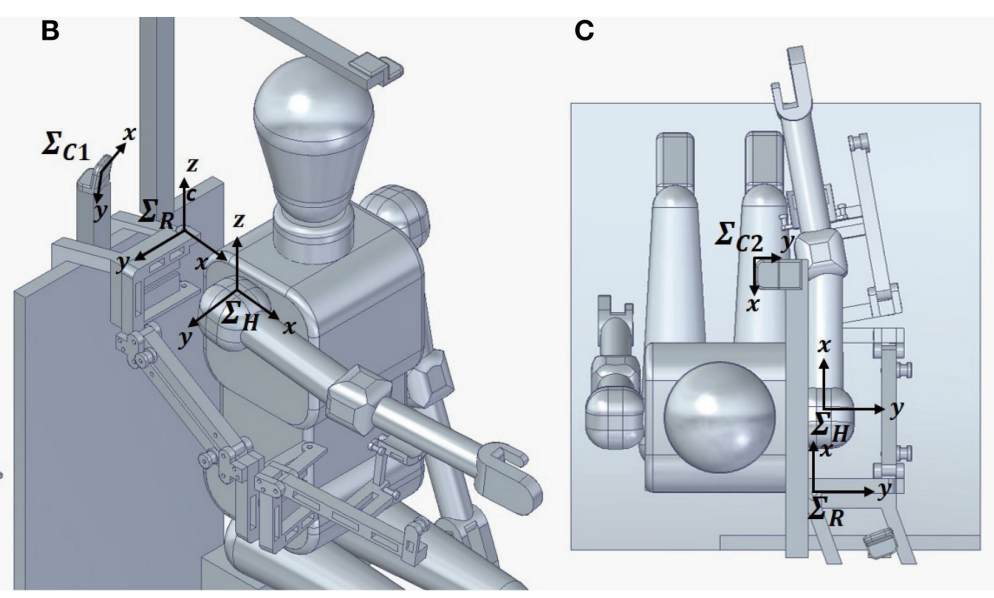

E

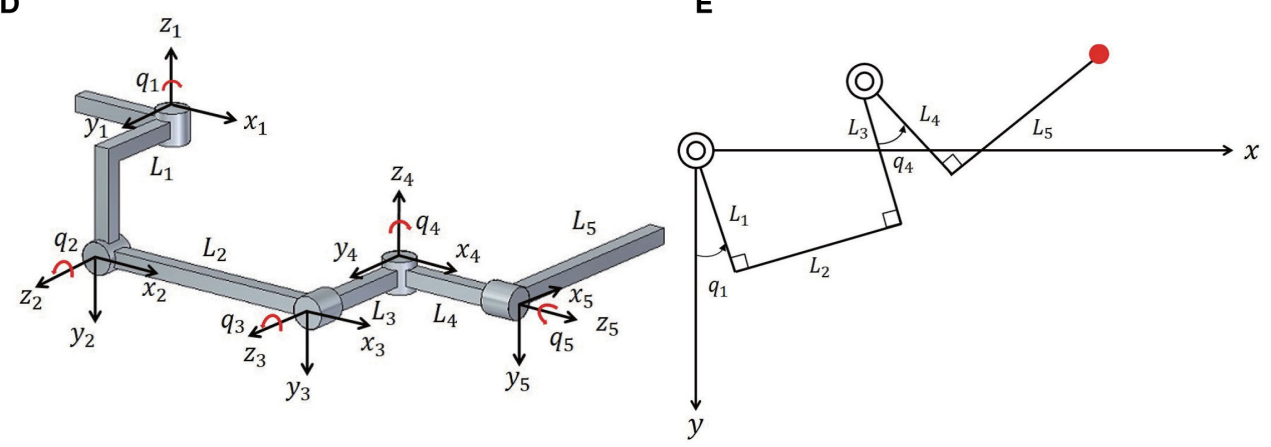

FIGURE 1 | Kinematic structure of Exoskeleton Actuated by Soft Modules (EASoftM) (A) Overall structure of the proposed EASoftM with a participant. (B) Close-up picture of upper body. $\sum_{R}$ represents the base coordinate of the robot, $\sum_{C 1}$ the camera coordinate used in the vision-based control law, and $\sum_{C 2}$ the camera coordinate to record the trajectories of the end-effector. The exoskeleton has two parallel links and four degrees of motion that approximates normal human anatomy of upper limb. (C) Overall structure of the proposed EASoftM (view from top). The attachment to the upper limb was set up at the end of exoskeleton to support the wrist of a participant. (D) Denavit-Hartenberg parameters of exoskeleton structure. The soft modules are attached to the joints $\left(q_{1}\right.$ and $\left.q_{4}\right)$ to rotate the joints, and the rubber bands are attached to the parallel structure of link $L_{2}$ and $L_{5}$ to fix the joint angles of $q_{2}$ and $q_{5}$ within a certain range, resulting in compensation of the gravity. (E) Kinematic structure of exoskeleton in 2D work plane. As the passive joints $\left(q_{2}\right.$ and $\left.q_{5}\right)$ lift up the upper limb,the active joints rotates shoulder and elbow $\left(q_{1}\right.$ and $q_{4}$ ) to make a reaching motion possible within this $2 \mathrm{D}$ plane.

TABLE 1 | DH parameters for the kinematic structure.

\begin{tabular}{ccccc}
\hline $\boldsymbol{i}$ & $\boldsymbol{a}_{\boldsymbol{i}-\mathbf{1}}$ & $\boldsymbol{\alpha}_{\boldsymbol{i}-\mathbf{1}}$ & $\boldsymbol{d}_{\boldsymbol{i}}$ & $\boldsymbol{\theta}_{\boldsymbol{i}}$ \\
\hline 1 & 0 & 0 & 0 & $q_{1}$ \\
2 & 0 & $-\frac{\pi}{2}$ & $L_{1}$ & $q_{2}$ \\
3 & $L_{2}$ & 0 & 0 & $q_{3}$ \\
4 & 0 & $\frac{\pi}{2}$ & $-L_{3}$ & $q_{4}$ \\
5 & 0 & $-\frac{\pi}{2}$ & $L_{4}$ & $q_{5}$ \\
$\mathrm{E}$ & $L_{5}$ & 0 & 0 & 0 \\
\hline
\end{tabular}

where $\sum_{R}$ represents the base coordinate of the robot, $\sum_{C 1}$ the camera coordinate used in the vision-based control law, and $\sum_{C 2}$ the camera coordinate to record the trajectories of the end-effector. The exoskeleton has two parallel links and four degrees of motion that approximate normal human anatomy of the upper limb. The attachment to the upper limb was set up at Robot link $L_{5}$ to support the wrist of a participant.
TABLE 2 | Length of links of exoskeleton.

\begin{tabular}{ccccc}
\hline $\boldsymbol{L}_{1}[\mathrm{~mm}]$ & $\boldsymbol{L}_{2}[\mathrm{~mm}]$ & $\boldsymbol{L}_{3}[\mathrm{~mm}]$ & $\boldsymbol{L}_{4}[\mathrm{~mm}]$ & $\boldsymbol{L}_{5}[\mathrm{~mm}]$ \\
\hline 100 & 210 & 100 & 100 & 210 \\
\hline
\end{tabular}

The kinematic structure exoskeleton is given in Figure 1D with DH (Denavit-Hartenberg) parameters in Table 1 (Denavit and Hartenberg, 1955). The length of the links is provided in Table 2. Four joints were represented by $q$ where $q_{1}$ and $q_{4}$ were active joints and $q_{2}=-q_{3}$ (parallel link) and $q_{5}$ were passive joints.

Before actuation of the soft modules, these two passive joints, $q_{2}$ and $q_{5}$ were adjusted by the elastic property of the rubber band in order to shift the equilibrium point of upper limb by compensating the gravity. Two soft modules were attached to $q_{1}$ and $q_{4}$ of the exoskeleton to actuate pneumatically the rotation of joints.

By combining the passive and active joints, we can predetermine the $2 \mathrm{D}$ work plane, in which two active joints 
guide the wrist to the target position. The soft actuators made of polyethylene were attached to individual joints of exoskeleton, providing the visco-elastic property in guidance of motion. All the parts were printed by the $3 \mathrm{D}$ printer using $\mathrm{ABS}$ plastic. The ultra-light polyethylene modules were used as ideal material for designable and wearable soft robotic modules.

\subsection{Control Schemes of Exoskeleton Actuated by Soft Modules (EASoftM)}

This study used a control scheme which exploited direct visualmotor loops in the feedback control to actuate robotic arms (Nishida and Kawamura, 2012). Based on the transformations from visual space to actuator space, the proposed control scheme will allow a robot to execute visually directed reaching motion with its end-effector without reference to its precise kinematics and necessity to calculate the inverse kinematics. The block diagram of the control law is given in Figure 2.

Specifically, we apply the Proportional and Integral (PI) feedback control laws to the relative distance between the target and the end effector of the exoskeleton in the $2 \mathrm{D}$ work plane defined in Figure 1E. First, we calculate the Jacobian of the exoskeleton in this 2D plane (Figure 1E), which is used in the control law. In this $2 \mathrm{D}$ work plane, the position of the endeffector is given by

$$
\begin{aligned}
\mathbf{X}= & {\left[\begin{array}{l}
x \\
y
\end{array}\right] } \\
= & {\left[\begin{array}{c}
\left(L_{1}-L_{3}\right) \sin q_{1}+L_{2} \cos q_{1}+L_{4} \sin \left(q_{1}+q_{4}\right) \\
+L_{5} \cos \left(q_{1}+q_{4}\right) \\
\left(L_{1}-L_{3}\right) \cos q_{1}-L_{2} \sin q_{1}+L_{4} \cos \left(q_{1}+q_{4}\right) \\
+L_{5} \sin \left(q_{1}+q_{4}\right)
\end{array}\right] }
\end{aligned}
$$

Thus, the Jacobian, $\mathbf{J}_{(\mathbf{q})}$ is given in the $2 \mathrm{D}$ plane by $\dot{\mathbf{X}}=\mathbf{J}_{(\mathbf{q})} \dot{\mathbf{q}}$, where $\dot{\mathbf{q}}=\left[\begin{array}{ll}\dot{\mathbf{q}}_{1} & \dot{\mathbf{q}}_{4}\end{array}\right]^{\mathrm{T}} \cdot \mathbf{J}_{(\mathbf{q})}$ is given by

$$
\begin{gathered}
\mathbf{J}_{(\mathbf{q})}=\left[\begin{array}{ll}
a_{11} & a_{12} \\
a_{21} & a_{22}
\end{array}\right], \\
a_{11}=\left(L_{1}-L_{3}\right) \cos q_{1}-L_{2} \sin q_{1}+L_{4} \cos \left(q_{1}+q_{4}\right) \\
\\
\quad-L_{5} \sin \left(q_{1}+q_{4}\right) \\
a_{12}=L_{4} \cos \left(q_{1}+q_{4}\right)-L_{5} \sin \left(q_{1}+q_{4}\right) \\
a_{21}=-\left(L_{1}-L_{3}\right) \sin q_{1}-L_{2} \cos q_{1}-L_{4} \sin \left(q_{1}+q_{4}\right) \\
\\
\quad+L_{5} \cos \left(q_{1}+q_{4}\right) \\
a_{12}=-L_{4} \sin \left(q_{1}+q_{4}\right)+L_{5} \cos \left(q_{1}+q_{4}\right)
\end{gathered}
$$

Generally speaking, a PI control law for the target torque is given by

$$
\tau_{\mathbf{d}}(\mathbf{t})=\mathbf{K}_{\mathbf{P}} \mathbf{J}_{(\mathbf{q})}^{\mathrm{T}} \Delta \mathbf{X}+\int_{\mathbf{0}}^{\mathbf{t}} \mathbf{K}_{\mathbf{I}} \mathbf{J}_{(\mathbf{q})}^{\mathrm{T}} \Delta \mathbf{X} \mathbf{d} \delta
$$

with $\tau_{\mathbf{d}}(\mathbf{t})$ : Target torque, $\mathbf{J}_{(\mathbf{q})}^{\mathrm{T}}$ : Transposed Jacobian, $\mathbf{K}_{\mathbf{P}}$ : Partial gain parameter, $\Delta \mathbf{X}$ : Relative distance between the target and the end-effector in the robot coordinate, $\Sigma_{R}, \mathbf{K}_{\mathrm{I}}$ : Integral gain parameter.

Here, we need to perform the coordinate transformation from the camera coordinate, $\Sigma_{C 1}$, to the robotic coordinate, $\Sigma_{R}$ (Figure 1). Thus, $\Delta \mathbf{X}$ is calculated in the following way;

$$
\Delta \mathrm{X}=\mathrm{C}_{1} \mathrm{~W}_{1} \Delta \mathrm{U}_{1}
$$

where $\mathbf{C}_{\mathbf{1}}$ is the coordinate direction transformation matrix, $\mathbf{W}_{\mathbf{1}}$ is the coordinate rotation transformation matrix, and the relative distance between the target and the end-effector, $\Delta \mathbf{U}_{\mathbf{1}}$ is represented by

$$
\Delta \mathbf{U}_{\mathbf{1}}=\left[\begin{array}{c}
u_{d 1}-u_{1} \\
v_{d 1}-v_{1}
\end{array}\right]
$$

where $u_{d 1}$ : Pixel value of the target on $\mathrm{x}$-axis in $\Sigma_{R}, v_{d 1}$ : Pixel value of the target on y-axis in $\Sigma_{R}, u_{1}$ : Pixel value of the eneffector on x-axis in $\Sigma_{R}, v_{1}$ : Pixel value of the en-effector on y-axis in $\Sigma_{R}$.

In this study the $\mathbf{W}_{\mathbf{1}}$ is given by

$$
\mathbf{W}_{\mathbf{1}}=\left[\begin{array}{cc}
\cos \left(\theta_{y 1}\right) & 0 \\
\sin \left(\theta_{x 1}\right) \sin \left(\theta_{y 1}\right) & \cos \left(\theta_{x 1}\right)
\end{array}\right]=\left[\begin{array}{cc}
0.87 & 0 \\
0.43 & 0.50
\end{array}\right] \text {, }
$$

with $\theta_{x 1}=60$ [deg]: Angle between $\mathrm{x}$-axis of $\Sigma_{R}$ and $\sum_{C 1}$, $\theta_{y 1}=30$ [deg]: Angle between $x$-axis of $\Sigma_{R}$ and $\sum_{C 1}$.

However, $\mathbf{W}_{\mathbf{1}}$ is always accompanied by the error from calibration, thus, we approximate $\mathbf{W}_{\mathbf{1}}$ to be unit matrix.

$\mathrm{C}_{\mathbf{1}}$ is given by

$$
\mathbf{C}_{1}=\left[\begin{array}{cc}
0 & -1 \\
-1 & 0
\end{array}\right]
$$

Using the coordinate transformation matrix and the video image obtained from the camera, and from Equation (3), we apply the following control scheme to calculate the target torque;

$$
\tau_{\mathbf{d}}(\mathbf{t})=\mathbf{K}_{\mathrm{P}} \mathbf{J}_{(\mathbf{q d})}{ }^{\mathrm{T}} \mathbf{C}_{\mathbf{1}} \Delta \mathbf{U}_{1}+\int_{0}^{\mathrm{t}} \mathbf{K}_{\mathrm{I}} \mathbf{J}_{(\mathbf{q d})}{ }^{\mathrm{T}} \mathbf{C}_{\mathbf{1}} \Delta \mathbf{U}_{\mathbf{1}} \mathbf{d} \delta
$$

where $\mathbf{J}_{(\mathbf{q d})}{ }^{\mathbf{T}}$ is a transposed Jacobian (Equation 2) which is approximated by the one close to the target position and kept constant throughout the reaching motion. $\mathbf{K}_{\mathbf{P}}$ is a gain factor for proportional control, and $\mathrm{K}_{\mathbf{I}}$ for integral control.

As a next step, let us calculate the control law based on the robotic coordinate, $\Sigma_{R}$. Using the coordinate transformation from the camera to the work space coordinate system, Equations (4) and (8) becomes

$$
\tau_{\mathbf{d}}(\mathbf{t})=\mathbf{K}_{\mathbf{P}} \tilde{\mathbf{J}}^{\mathbf{T}} \Delta \mathbf{X}+\int_{0}^{t} \mathbf{K}_{\mathbf{I}} \tilde{\mathbf{J}}^{\mathbf{T}} \Delta \mathbf{X} \mathbf{d} \delta
$$

where $\tilde{\mathbf{J}}^{\mathbf{T}}$ has been modified as follows,

$$
\tilde{\mathbf{J}}^{\mathrm{T}}=\mathrm{J}_{(\mathbf{q} \mathbf{d})}{ }^{\mathrm{T}} \mathrm{C}_{1} \mathrm{~W}_{1}{ }^{-1} \mathrm{C}_{1}{ }^{-1}
$$




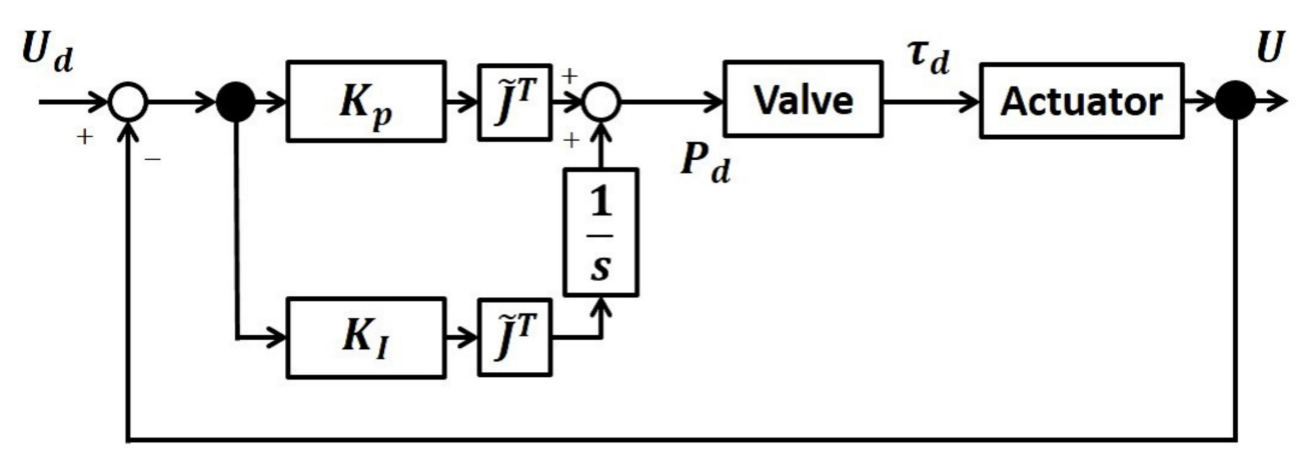

FIGURE 2 | Block Diagram of the control law. Based on the direct transformations from visual space to actuator space, the proposed control scheme will allow a robot to execute visually directed reaching motion with its end-effector without reference to its precise kinematics and necessity to calculate the inverse kinematics.

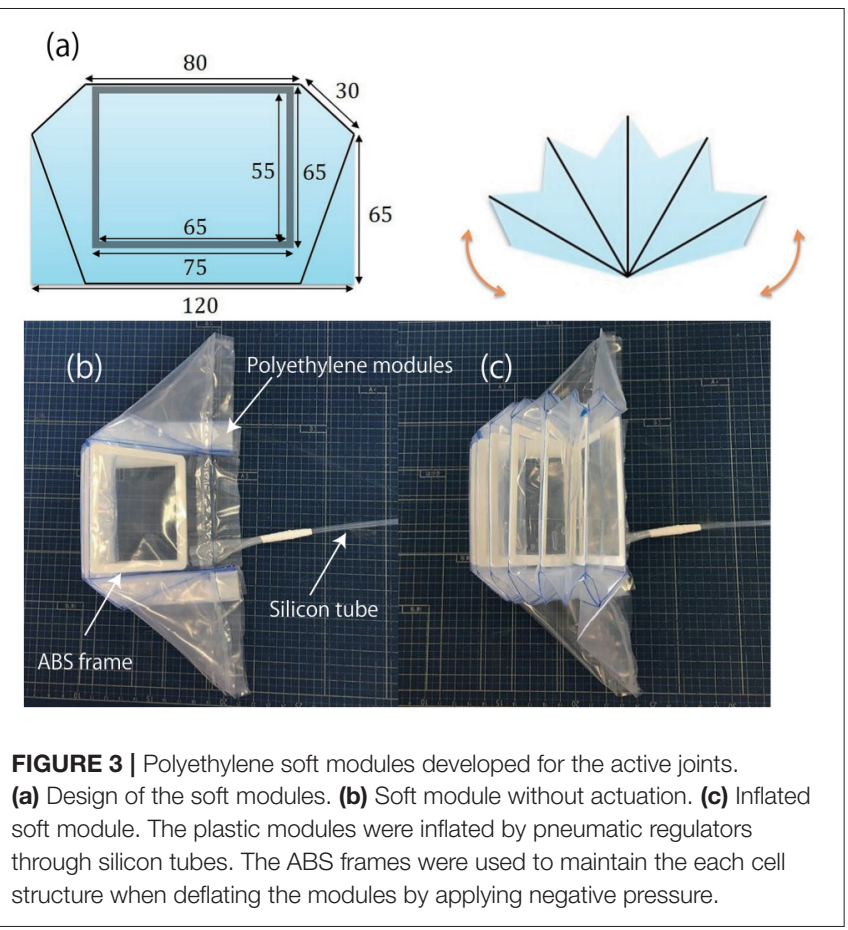

Note here that $\tilde{\mathbf{J}}^{\mathbf{T}}$ is the approximated Jacobian, which contains errors. However, the convergence of $\Delta \mathbf{X}$ is guaranteed as long as there is not so much difference between $\tilde{\mathbf{J}}^{\mathbf{T}}$ and $\mathbf{J}_{(\mathbf{q d})}{ }^{\mathbf{T}}$ (Nishida and Kawamura, 2012). Thus, the proposed control scheme here does not require any encoders to measure the angle of the joints, contributing to the ultra-light weight of the exoskeleton.

In order to output the target pressure to control the soft modules, it is necessary to convert the target torque to the target pressure, which require the detail modeling and the non-linear mapping. This relation should be generally given by $\mathbf{P}_{\mathbf{d}}=\mathbf{B}_{(\mathbf{q})} \tau_{\mathbf{d}}$. However, in this work, we simply related the desired torque with the desired pressure by $\mathbf{P}_{\mathbf{d}}=\mathbf{B}_{\mathbf{0}} \tau_{\mathbf{d}}$. Note here, that this simple relation was made possible due to the positive and negative

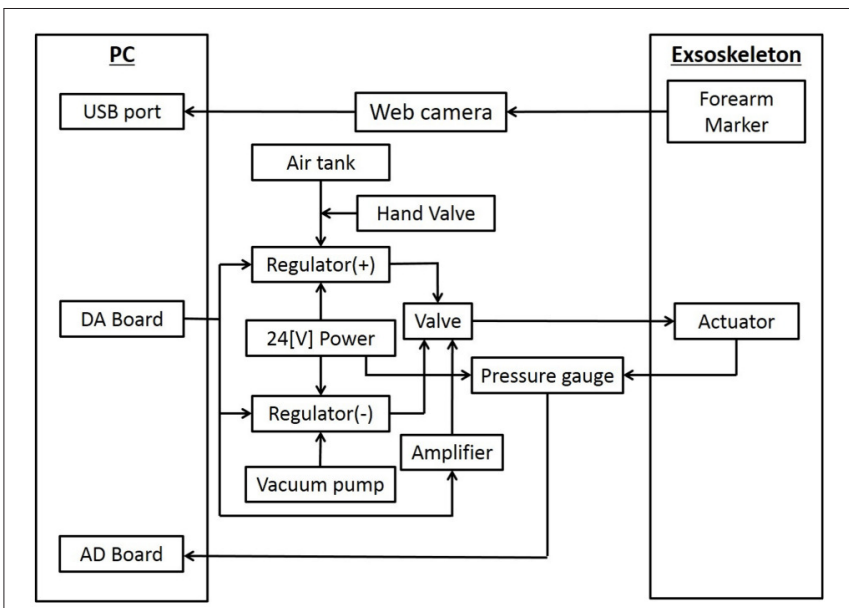

FIGURE 4 | Control Diagram. The vision-based control law was used to minimize the relative position between the target and the tracer. The pressure sensors were placed, and AD board was used to transmit the signals to the PC. The positive pressure regulator and the negative pressure regulator was connected to each soft actuator through the flow regulator valve which switched the pressure between positive and negative. The positive pressure regulator was connected to the tank with the compressor and the negative pressure tank to the vacuum pump. pressure actuation. We set $B_{0}=1$, and only use $\mathbf{K}_{\mathbf{P}}$ and $\mathbf{K}_{\mathbf{I}}$ as the gain parameters to be tuned. The gain parameters will be determined empirically for each experimental condition. The schematic summary of the control scheme is given in Figure 2. As experimental results, we will show the trajectories of the end-effector in the scale of $[\mathrm{mm}]$, using $\Delta \mathbf{U}$.

\subsection{Camera Coordinate to Record the Trajectories of the End-Effector}

In this section, we explain how we set up the camera coordinate to record the trajectories of the end-effector in Figure 1. While performing the experiments, another camera located above the $2 \mathrm{D}$ work plane records the trajectories of the end-effector. Based on the robot coordinate, $\Sigma_{R}$, the relative distance between the 
target and the end-effector, $\Delta \mathbf{X}$ is given by

$$
\Delta \mathrm{X}=\mathrm{C}_{2} \mathbf{W}_{2} \Delta \mathbf{U}_{2}
$$

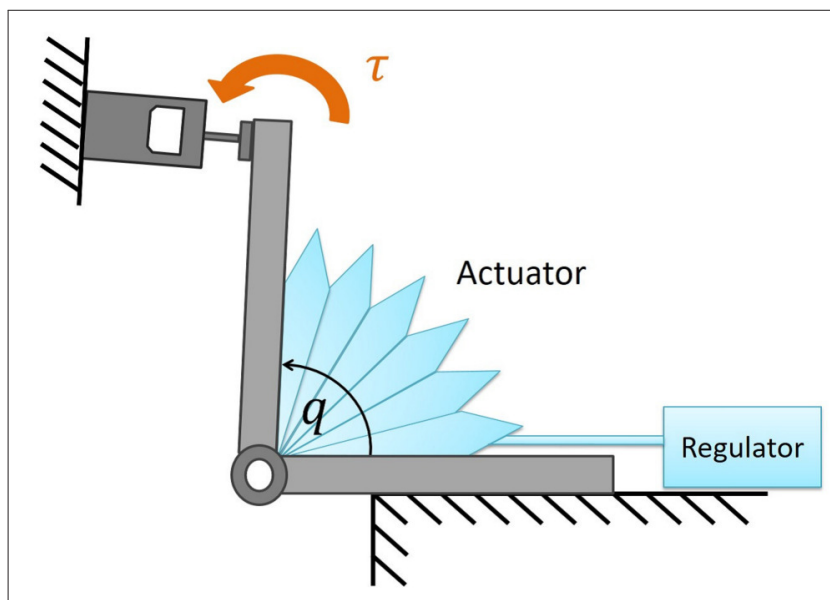

FIGURE 5 | Experimental setup to measure the torque produced by the soft actuator. By changing the angle of the joint, the torque was measured as a function of angle. where $\mathbf{C}_{2}$ : Coordinate direction transformation matrix from $\Sigma_{C 2}$ to $\Sigma_{R}, \mathbf{W}_{2}$ : Coordinate rotation transformation matrix from $\Sigma_{C 2}$ to $\Sigma_{R}$.

$\Delta \mathbf{U}_{2}$ is the relative distance between the target position and the end-effector based on $\Sigma_{C 2}$ in Figure 1 given by

$$
\Delta \mathbf{U}_{2}=\left[\begin{array}{l}
u_{d 2}-u_{2} \\
v_{d 2}-v_{2}
\end{array}\right]
$$

with $u_{d 2}$ : Pixel value of the target position on x-axis, $v_{d 2}$ : Pixel value of the target position on $y$-axis, $u_{2}$ : Pixel value of the endeffector position on $\mathrm{x}$-axis, $v_{2}$ : Pixel value of the end-effector position on $y$-axis.

In this study, $\mathbf{W}_{\mathbf{2}}$ is calculated by setting the camera on $\theta_{x 2}=0$ [deg], $\theta_{y 2}=0$ [deg].

$$
\mathbf{W}_{\mathbf{2}}=\left[\begin{array}{cc}
\cos \left(\theta_{y 2}\right) & 0 \\
\sin \left(\theta_{x 2}\right) \sin \left(\theta_{y 2}\right) & \cos \left(\theta_{x 2}\right)
\end{array}\right]=\left[\begin{array}{ll}
1 & 0 \\
0 & 1
\end{array}\right],
$$

with $\theta_{x 2}$ : Angle between $\mathrm{x}$-axis of $\Sigma_{R}$ and $\Sigma_{C 2}, \theta_{y 2}$ : Angle between y-axis of $\Sigma_{R}$ and $\Sigma_{C 2}$.

And $\mathbf{C}_{2}$ is given by

$$
\mathbf{C}_{2}=\left[\begin{array}{cc}
-1 & 0 \\
0 & 1
\end{array}\right]
$$
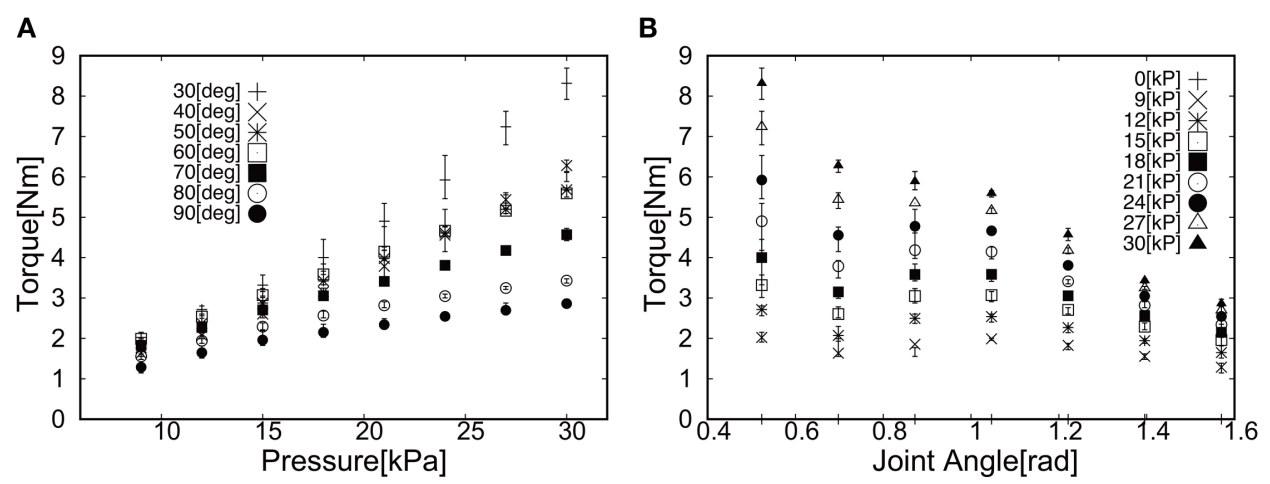

FIGURE 6 | Torque as a function of (A) Pressure and (B) Joint angle when applied positive pressure.
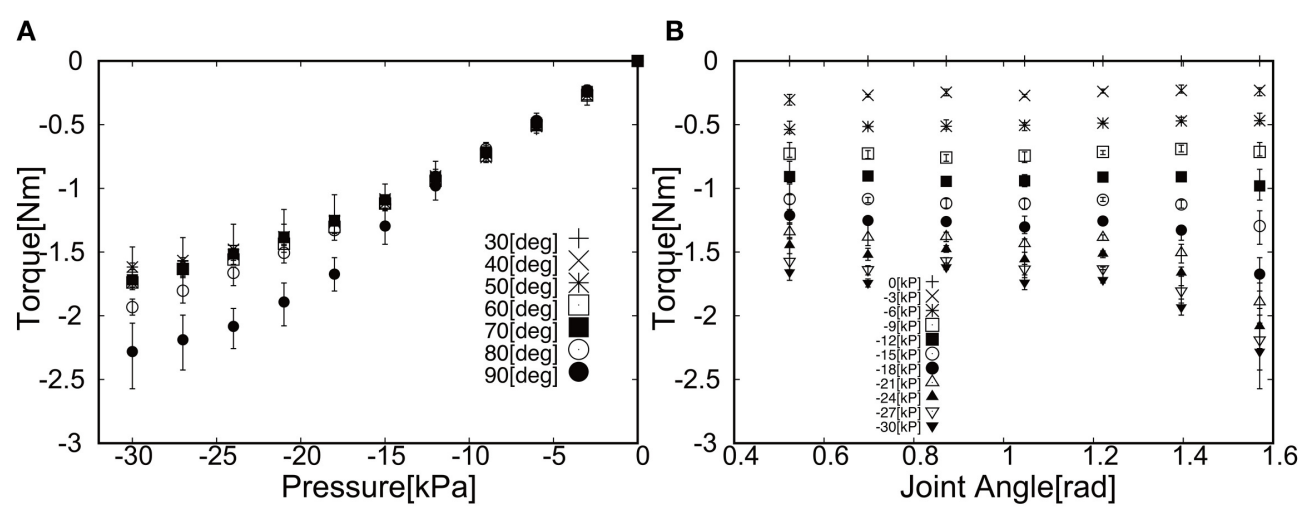

FIGURE 7 | Torque as a function of (A) Pressure and (B) Joint angle when applied negative pressure. 
A

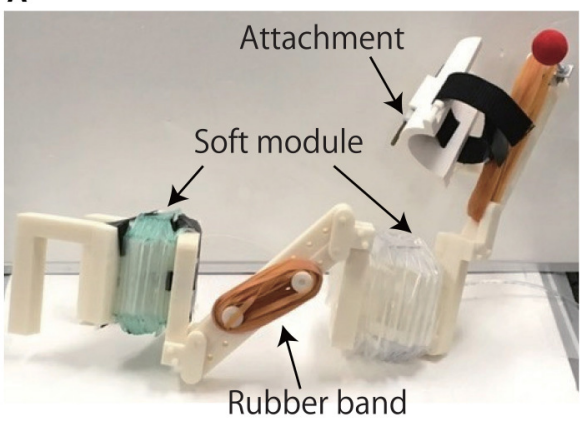

C

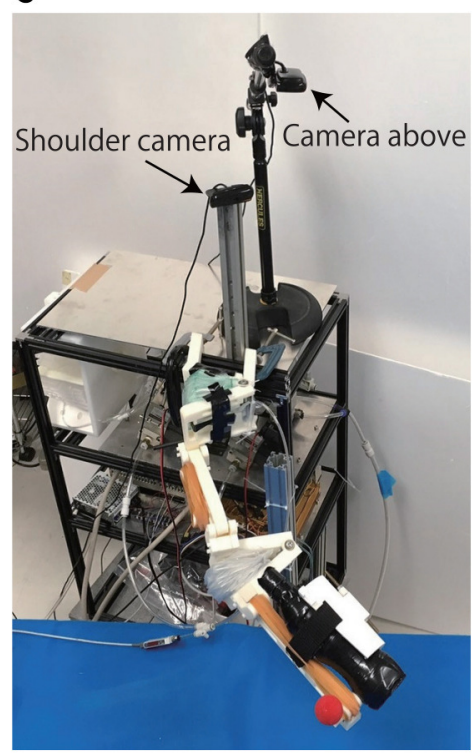

B

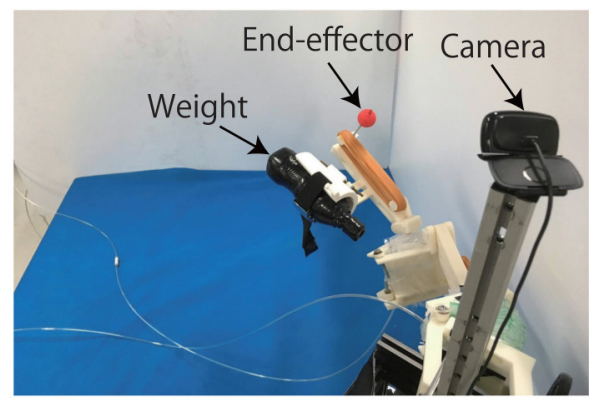

D

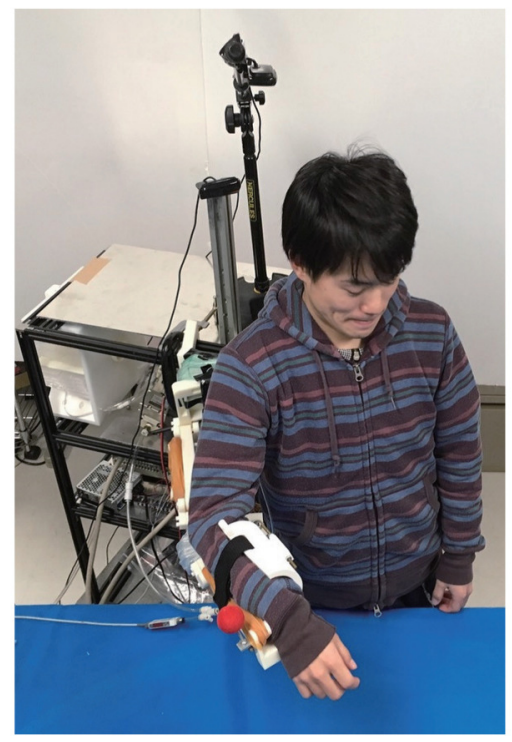

FIGURE 8 | Produced Exoskeleton Actuated by Soft Modules and experimental setups. (A) Produced exoskeleton structure with passive and active joints. (B) Experimental setup to test the functional properties of the system. The end-effector was attached to the end-point of the links, and the weight of 1.0 [Kg] was attached to the attachment. The camera was located on the shoulder position to apply the visual feedback control. (C) Experimental setup of the exoskeleton with the weight on the attachment. (D) Experimental setup with the participant (with permission from the participant).

\subsection{Production of Soft Actuators and Exoskeleton}

The polyethylene soft modules developed for the active joints were designed to be attached to the joints $\left(q_{1}\right.$ and $q_{4}$ in Figure 1D), and have multi-cells with one end glued together by thermal adhesion (Figure 3). The ABS frames were used to maintain the structure of each cell, when deflating the modules by applying negative pressure.

The inflatable actuator was pressurized by pneumatic actuators through silicon tubes, and as the individual cells start to push against each other, the whole module produces the torque in the fan-shaped manner as a function of pressure. Also, the modules could produce negative torque by being deflated. The pressure could be as low as $-30[\mathrm{kPa}]$ and range up to $30[\mathrm{kPa}]$. This range of pressure was sufficient to actuate the exoskeleton carrying the weight of upper limb of participants.

The forces generated by the soft modules are distributed along the entire length of $L_{1}, L_{3}$, and $L_{4}$, providing the torque to rotate the shoulder and elbow joint respectively as in Figure 1E.
Note here, that reaching motion can be only realized in the two dimensional plane defined by the kinematic structure in Figure 1E. The ABS parts of exoskeleton were printed by a $3 \mathrm{D}$ printer. The parts for the parallel link structures were assembled, using the ABS made bolts.

\subsubsection{Development of Control Circuits}

The control diagram is given in Figure 4. We used a USB camera (logicool HD WEBCAM C525) located above the shoulder of exoskeleton for the visual feedback control to calculate the relative distance between the target and the end-effector. The marker was attached to the forearm to represent the hand position. The visual processing was programmed in $\mathrm{C}++$ (Microsoft Visual Studio 2013). The control law in Equation (8) outputs the desired pressure to actuate the inflatable plastic modules. The desired pressure was transmitted to the regulator which controlled the valves of the air flow via DA board.

In order to measure the pressure at the vicinity of soft modules, the pressure sensors (SMC ISE10-M5-C) were placed, 

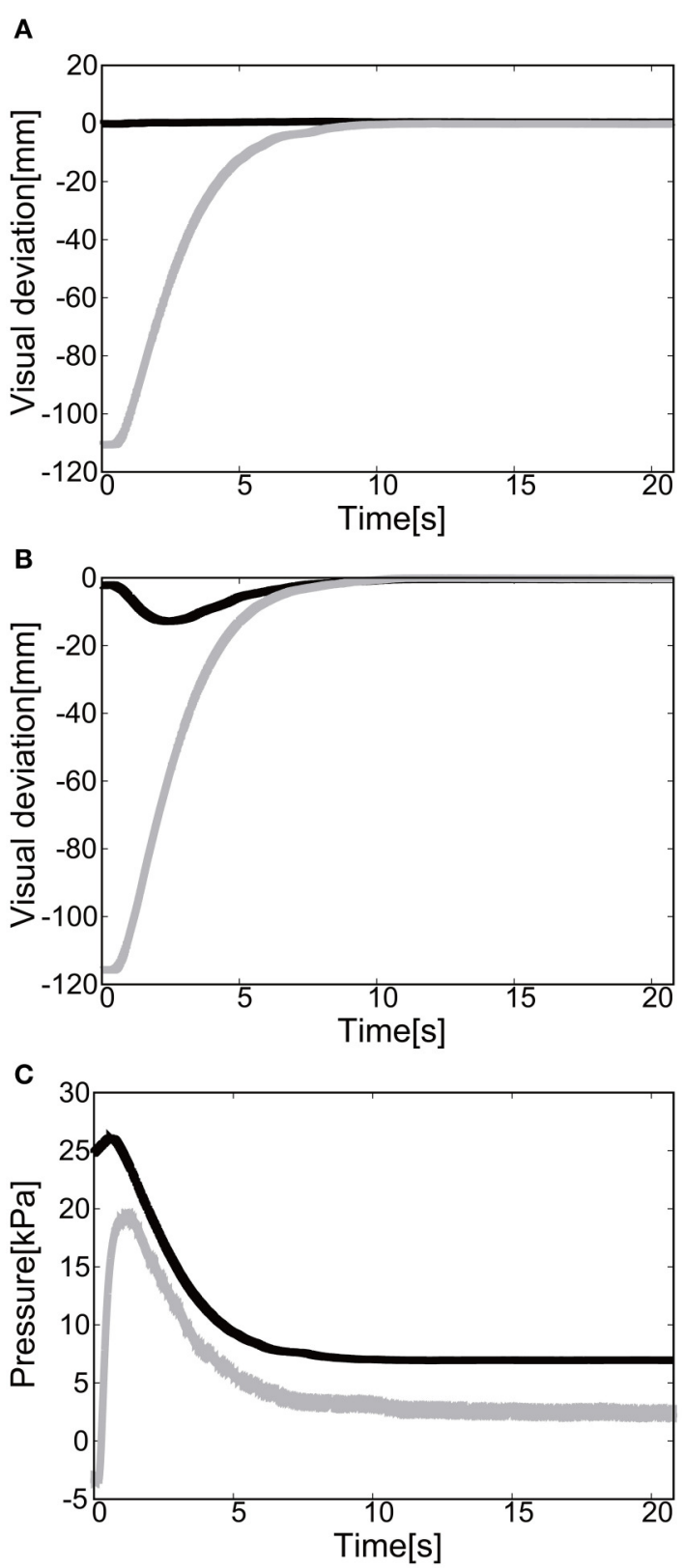

FIGURE 9 | Flexion motion (positive pressure actuation) of the elbow of the Exoskeleton Actuated by Soft Modules. (A) Visual deviation $\left(\Delta U_{1}\right) x(t)$ (Black line) and $y(t)$ (Gray line) as a function of time, using the camera on the shoulder. (B) Visual deviation $\left(\Delta U_{1}\right) x(t)$ (Black line) and $y(t)$ (Gray line) as a function of time, using the camera from above. (C) The pressure as a function of time. The black line represents the target pressure, and the gray line represents the measured pressure.

and AD board (Contec AI-1616LI-PE) was used to transmit the signals to the PC. The positive pressure regulator (SMC ITV2050212BS) and the negative pressure regulator (SMC ITV20290212BS5) was connected to each soft actuator through the flow regulator valve (SMC VEF3121-1) which switched the pressure between positive and negative. The positive pressure regulator was connected to the tank with the compressor (IWATA
TFP04B-10 C) and the negative pressure tank to the vacuum pump (IWATA SCROLL VACUUM PUMP).

\section{TORQUE CHARACTERISTICS OF SOFT ACTUATORS}

The torque characteristics of soft actuators, torque as a function of the joint angle, was investigated using the experimental setup shown in Figure 5. We performed the torque measurement of the soft modules in the following procedure;

1. Fix the angle of the arm in Figure 5.

2. Pressurize the soft module in Figure 5.

3. Measure the force in the force gauge after $5 \mathrm{~s}$ from the moment when the force exhibited its highest peak.

4. Go back to the procedure 1 for the next angle.

Thus, in Figures 6, 7 we measured the torque produced by the soft module at the equilibrium state by fixing the angle of the joint in Figure 5. We characterized the functional properties of the soft modules. The torque was measured as a function of (1) pressure in the range from -30 to $30[\mathrm{kPa}]$ at the incremental step of $3.0[\mathrm{kPa}]$; and (2) an angle when the soft module was actuating the rotational joint with two rigid arms (Figure 5).

Figure 6 shows the torque as a function of (a) Pressure and (b) Joint angle. The general trend can be observed; (1) the torque increased as a function of pressure and (2) the torque decreased as a function of angle. For the case of applying the negative pressure to the soft module, Figure 7 shows the torque as a function of (a) Pressure and (b) Joint angle. The general trend can be noted; (1) the torque increased as a function of pressure and (2) the torque increased as a function of an angle.

We expect that the largest hysteresis when switching the pressure from positive to negative, as it takes a certain time for the air flow in pipes and plastic modules to relax to the steady state. However, we aim at the upper limb assistance in slow motion, thus, air flow would be equilibrated throughout the actuation.

\section{FUNCTIONAL PROPERTIES OF THE EXOSKELETON ACTUATED BY SOFT MODULES (EAsoftM)}

The developed Exoskeleton Actuated by Soft Modules is shown in Figure 8. The rubber bands were attached to the parallel structures of the exoskeleton in order to compensate the gravity, and the soft modules were attached in-between two plastic plates on shoulder and elbow joints. The total weight of the EASoftM was 967 [g], composed of assembled 3D printed arm (724 [g]), soft module (31 [g]), and attachment (181 [g]). The end-effector was attached to the end-point of the links, and the camera was located on the shoulder position to apply the visual feedback control in Figure 1.

In the following sections, we validated the functional properties of the EASoftM, first by putting the weight of 1.0 $[\mathrm{kg}]$ on the attachment of the exoskeleton arm, and secondly, by 
A

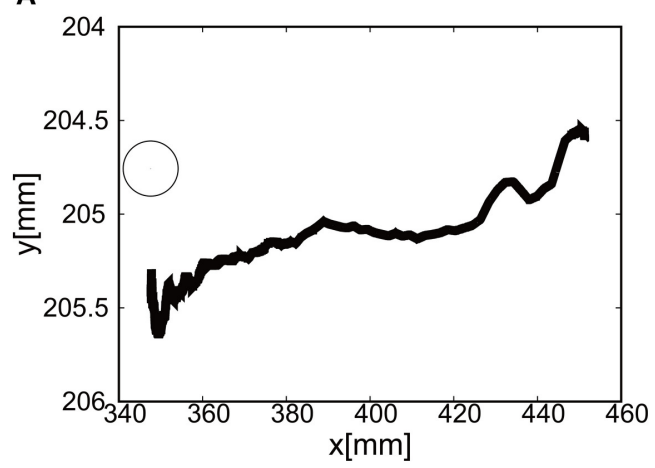

B

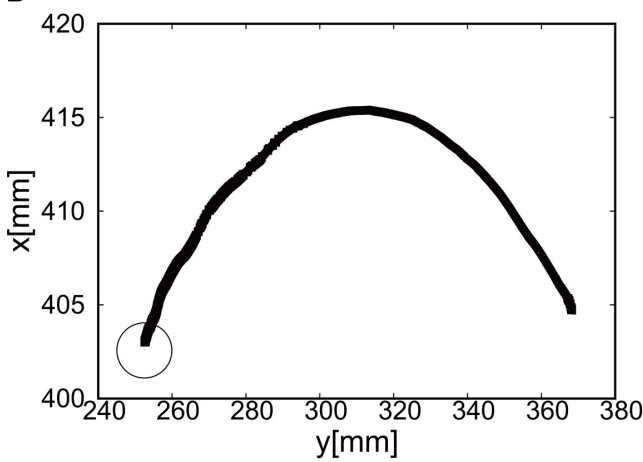

FIGURE 10 | Trajectories of the end-effector in $\mathrm{x}$-y plane (positive pressure actuation), using (A) the camera on the shoulder and (B) the camera from above. The target is denoted by the circle.

performing the participant experiments as shown in Figure 8D. In both experiments, the relative distance between the endeffector and target, and trajectories in the $2 \mathrm{D}$ camera frames were measured as a function of time as well as the pressure applied to the individual soft modules.

\subsection{Functional Properties of the Exoskeleton Actuated by Soft Modules (EASoftM) under the Weight Load}

In this section, we demonstrated the functional properties of EASoftM with the weight load of $1.0[\mathrm{~kg}]$ attached to the exoskeleton arm. To validate the motion of the EASoftM for positive and negative pressure actuation, we characterized the motion of the exoskeleton for flexion and extension, respectively, from one degree of freedom (elbow) to two degrees of freedom (elbow and shoulder). Finally, we performed more complex reaching motion which combined the extension and flexion by switching target positions.

\subsubsection{D Elbow Motion Using the Elbow Joint of the Exoskeleton Actuated by Soft Modules (EASoftM)}

5.1.1.1. Flexion motion toward the target (positive pressure actuation)

For the 1D rotation of the elbow of the EASoftM, the gain parameters were set to $k_{p 1}=k_{p 2}=2.0$ and $k_{i 1}=k_{i 2}=$ 0.2 in Equation (8). Figures 9A,B shows the visual deviation (relative distance between the target and the end-effector, $\Delta \mathbf{U}_{\mathbf{1}}$ in Equation 5) of $x(t)$ and $y(t)$ as a function of time, using the camera on the shoulder. Note here, that this visual deviation was input to the control law in Equation (8) whereas Figure 9B shows the visual deviation (relative distance between the target and the end-effector, $\Delta \mathbf{U}_{2}$ in Equation 12), using the camera from above to record the trajectories of the end-effector. It takes about $12 \mathrm{~s}$ to reach the target set at $x=y=0$.

Figure 9C shows the pressure of the soft module as a function of time. Since the relative distance between the target and the tracer is highest at the initial position of the tracer, the pressure profile shows the slow convergence to the target pressure, reflecting the slow pneumatic actuation of the soft modules. Due to the loss of the pressure through the pipelines, the deviation between the target pressure and the measure pressure remained.

The final visual deviation was $\Delta x=0.44[\mathrm{~mm}]$ and $\Delta y=-0.20[\mathrm{~mm}]$ in Figure 10A, demonstrating the substantial accuracy for reaching motion. This accuracy also proves that the visual-based control as represented in Equation (8) can work efficiently with the approximated kinematics. The trajectory of the tracer attached to the attachment of the exoskeleton shows the circular trajectory as a result of the motion of the elbow rotation in Figure 10B.

\subsubsection{Extension motion to the target (negative pressure actuation)}

To actuate the soft modules by the negative pressure, the gain parameters were set to $k_{p 1}=k_{p 2}=2.5$ and $k_{i 1}=k_{i 2}=0.2$ in Equation (8). We could successfully show that extension of the elbow of the EMSoftM was possible by applying the negative pressure as shown in Figure 11. The convergence was shown to the target position (Figures 11A,B) and to the target pressure (Figure 11C). The ABS frame inserted to each plastic cell worked to prevent the collapse of the structure of the cells when deflating air from them. Figure 12 shows the trajectories of the endeffector, demonstrating the extension of the exoskeleton. The final visual deviation was $\Delta x=0.30[\mathrm{~mm}]$ and $\Delta y=1.65[\mathrm{~mm}]$ in Figure 12A.

\subsubsection{D Motion Using the Elbow and the Shoulder Joints of the Exoskeleton Actuated by Soft Modules (EASoftM)}

As a next step, to validate the $2 \mathrm{D}$ motion using the elbow and the shoulder rotation, we performed the experiments using the weight on the attachment under following conditions; (1) Flexion motion to the target, (2) Extension motion to the target, (3) Triangular motion with three targets.

\subsubsection{Flexion motion to the target (positive pressure actuation)}

The gain parameters were set to $k_{p 1}=2.0, k_{p 2}=2.0$ for the elbow joint, and $k_{p 1}=0.2, k_{p 2}=0.2$ for the shoulder in Equation (8). For the case of $2 \mathrm{D}$ motion using the elbow and shoulder joints 

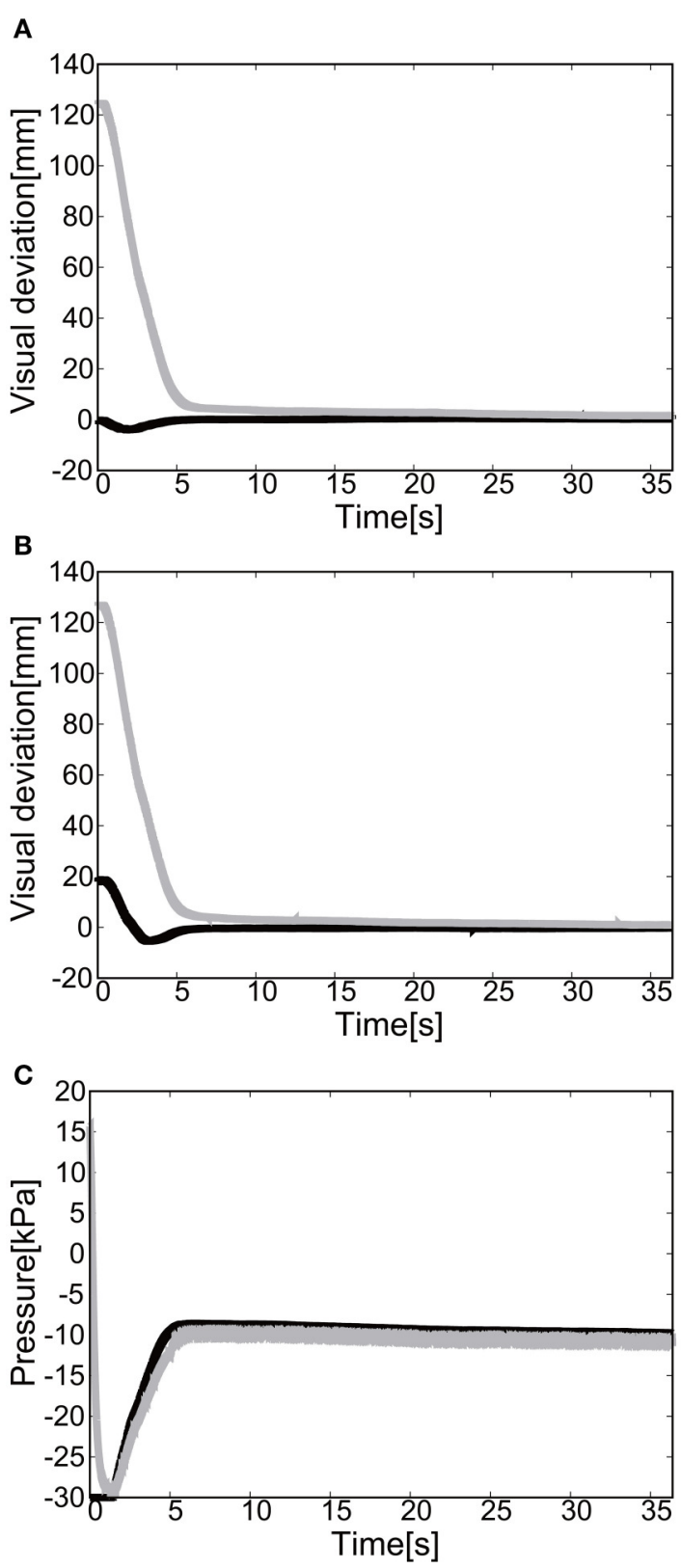

FIGURE 11 | Extension motion (negative pressure actuation) of the elbow of the Exoskeleton Actuated by Soft Modules. (A) Visual deviation $\left(\Delta U_{1}\right) x(t)$ (Black line) and $y(t)$ (Gray line) as a function of time, using the camera on the shoulder. (B) Visual deviation $\left(\Delta \mathrm{U}_{2}\right) x(t)$ (Black line) and $y(t)$ (Gray line) as a function of time, using the camera from above. (C) The pressure as a function of time. The black line represents the target pressure, and the gray line represents the measured pressure.

of the EASoftM, it was shown that the reaching motion was also possible as shown in Figures 13A,B.

The drop in pressure around $14 \mathrm{~s}$ in Figures 13C,D was due to the change of torque generated by the posture transformation and the gravitational force, as the exoskeleton arm flexed. The non-linear nature of this drop should come from the kinematics of the exoskeleton arm, which is highly non-linear itself. The flow regulator valve controls the constant air flows from the positive and negative regulator, and the pressure within a range of -5.0 and $5.0[\mathrm{kPa}]$ is outside the precision of the air flow/pressure regulators. However, due to the friction between the ABS frames, this low pressure range will not result in any substantial torque generation. Since the relative distance between the target and the tracer is highest at the initial position of the tracer, the pressure profile shows the slow convergence reflecting the slow pneumatic actuation of the soft modules. The trajectories of the end-effector are shown in Figure 14.

The relative distance to the target was achieved to be $\Delta x=$ $0.65[\mathrm{~mm}]$ and $\Delta y=-1.75[\mathrm{~mm}]$. This precision is surprising as the control laws in Equation (8) only used the visual information of the relative distance between the target and the end-effector as input to the control law. Note here, that the kinematics of the exoskeleton is only provided as approximation and is kept constant throughout the reaching motion. In the classical control theory, once a target has been defined in the robots work space using its sensors, the necessary final pose to intercept the object must be calculated using some form of inverse kinematics: the explicit transformation of work space coordinates into joint angles. However, the inverse kinematics is very sensitive to error, thus, the visual-based control should be appropriate for control of soft modules.

\subsubsection{Extension motion to the target (negative pressure actuation)}

The gain parameters were set to $k_{p 1}=2.0, k_{p 2}=2.0$ for the elbow joint, and $k_{p 1}=0.2, k_{p 2}=0.2$ for the shoulder joint in Equation (8). It was shown that the extension motion is also possible when applying the negative pressure to achieve the flexion motion as shown in Figures 15A,B. Due to the integral control accumulating the visual deviation and the friction between the ABS frames, there were, around 20 and $35 \mathrm{~s}$, two steep changes in values of the measured pressure in Figures 15C,D. Note here that the pressure range between -5.0 and $5.0[\mathrm{kPa}]$ is out of the controllable range of the flow/pressure regulators in Figure 4. The measured negative pressure in the flow regulation valve is attributed to the constant deflation of the air from the negative pressure regulator. Figure $\mathbf{1 6}$ shows the trajectories of the end-effector. The relative distance to the target was achieved to be $\Delta x=-0.93[\mathrm{~mm}]$ and $\Delta y=-1.57[\mathrm{~mm}]$.

\subsubsection{Reaching motion with three target positions}

We placed three targets to allow the end-effector to demonstrate the closed loop motion, i.e., the end-effector should come back to its initial place. The target was switched to another target when the relative distance between the target and the end-effector reaches $<5.0[\mathrm{~mm}]$. The gain parameters were set to $k_{p 1}=$ $2.0, k_{p 2}=2.0$ for the elbow joint, and $k_{p 1}=0.2, k_{p 2}=0.2$ for the shoulder joint in Equation (8).

Figures 17A,B show the position of the target, $x(t)$ and $y(t)$, using the camera on the shoulder. We can clearly observe that the end-point reaches the first target, the second target, and goes back to the initial position where the third target was located. Figure $\mathbf{1 8}$ shows the target and measured 

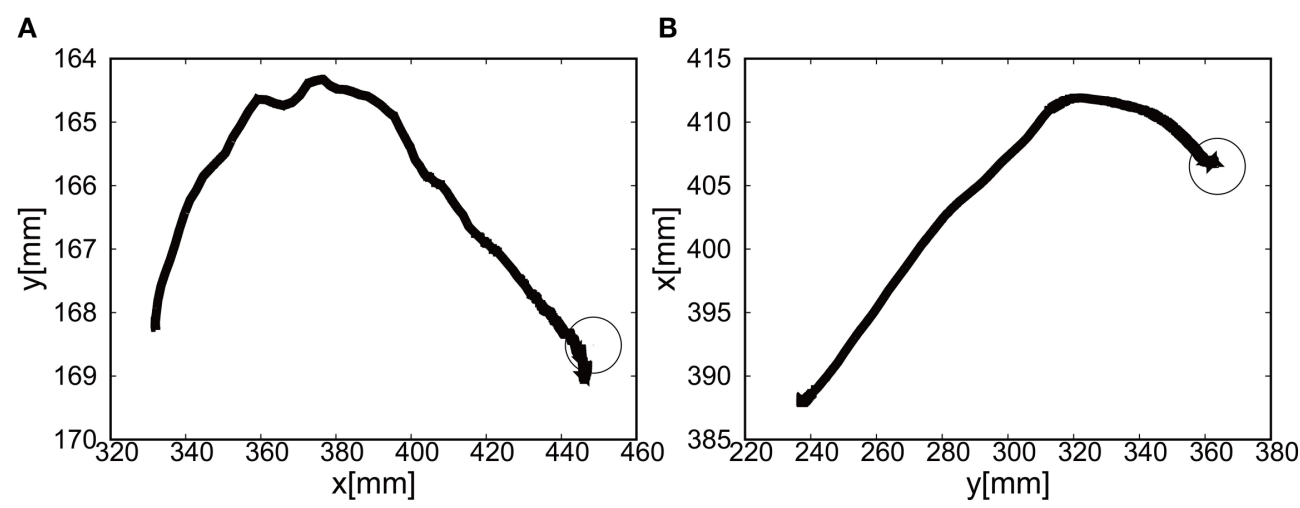

FIGURE 12 | Trajectories of the end-effector in $x-y$ plane (negative pressure actuation), using (A) the camera on the shoulder and (B) the camera from above. The target is denoted by the circle.
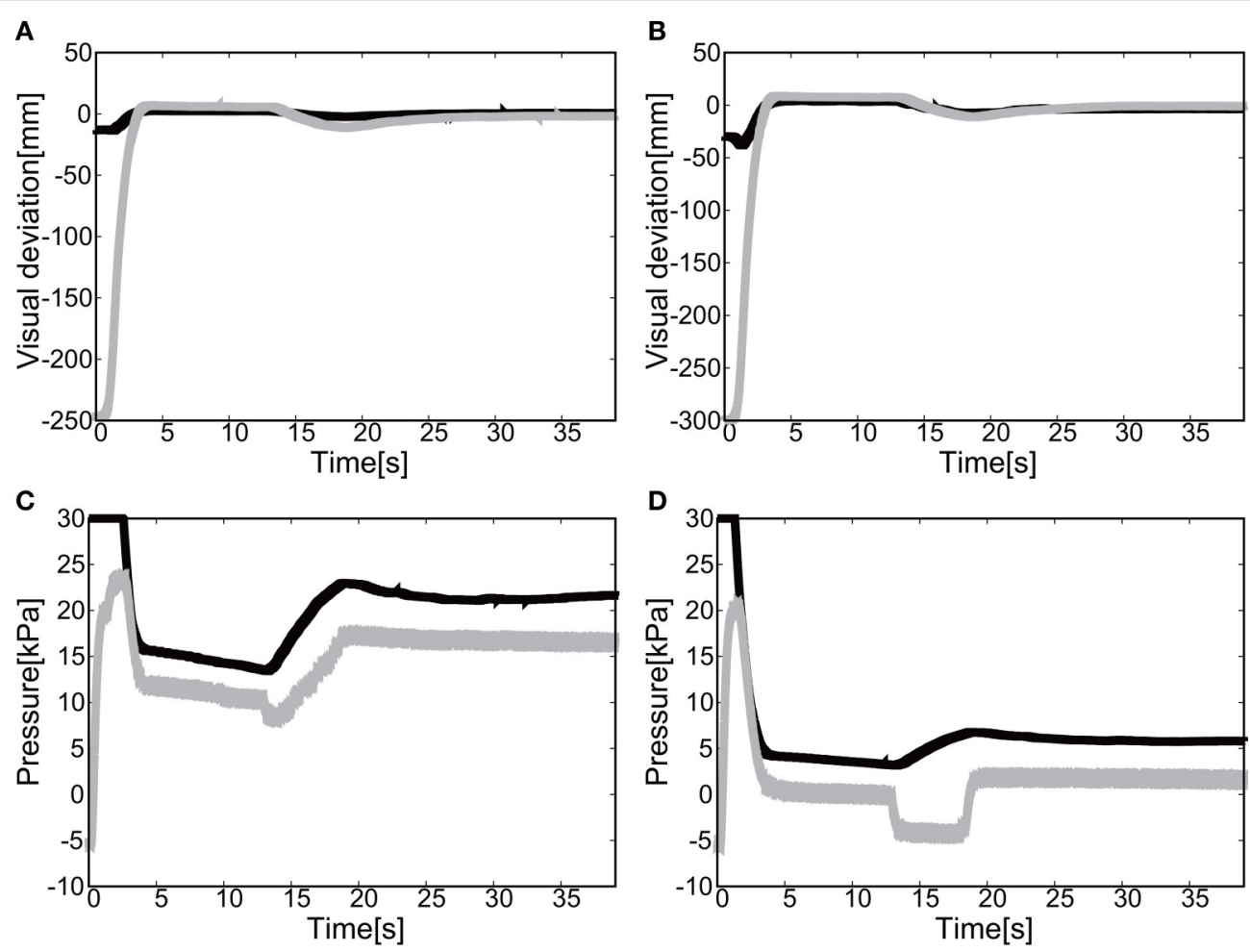

FIGURE 13 | Flexion motion (positive pressure actuation) of the elbow and the shoulder of the Exoskeleton Actuated by Soft Modules. (A) Visual deviation $\left(\Delta U_{1}\right) x(t)$ (Black line) and $y(t)$ (Gray line) as a function of time, using the camera on the shoulder. (B) Visual deviation $\left(\Delta \mathrm{U}_{1}\right) x(t)$ (Black line) and $y(t)$ (Gray line) as a function of time, using the camera from above. (C) The pressure of the shoulder module as a function of time. The black line represents the target pressure, and the gray line represents the measured pressure. (D) The pressure of the elbow module as a function of time. The black line represents the target pressure, and the gray line represents the measured pressure.

pressure as a function of time for the shoulder (a) and the elbow (b) joint. When switching the target position, there were corresponding jumps in the target pressure, but note here that the measured pressure followed a smooth curve from the positive to negative pressure around $7 \mathrm{~s}$ in Figures 18A,B. It is a promising feature of the soft module used to actuate the exoskeleton to demonstrate the smooth transition for switching the sign of the pressure, leading to the smooth transformation of motion from flexion to extension.

For the case of reaching motion by switching three targets sequentially, it was shown that the reaching motion could demonstrate the closed loop motion as shown in Figure 19, even though there was some overshoot in the relative distance and 
A

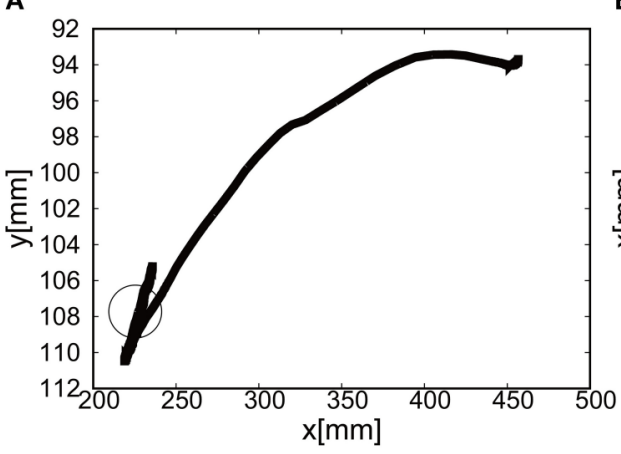

B

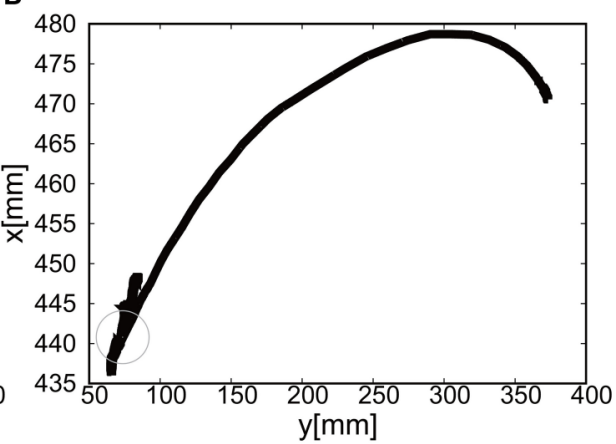

FIGURE 14 | Trajectories of the end-effector (positive pressure actuation) in x-y plane, using (A) the camera on the shoulder and (B) the camera from above. The target is denoted by the circle.

A

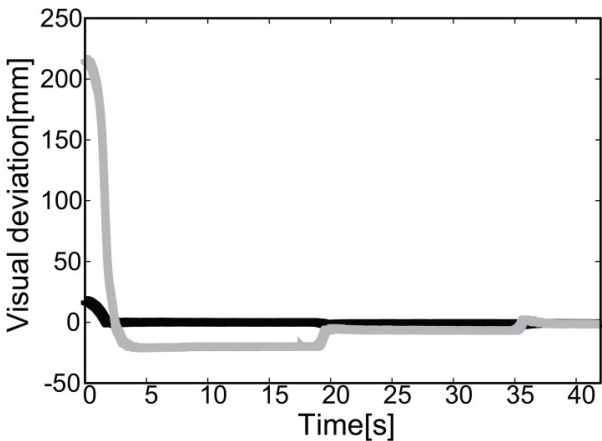

C

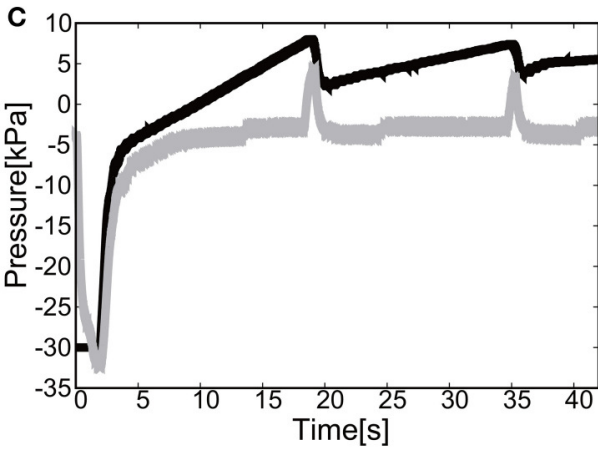

B
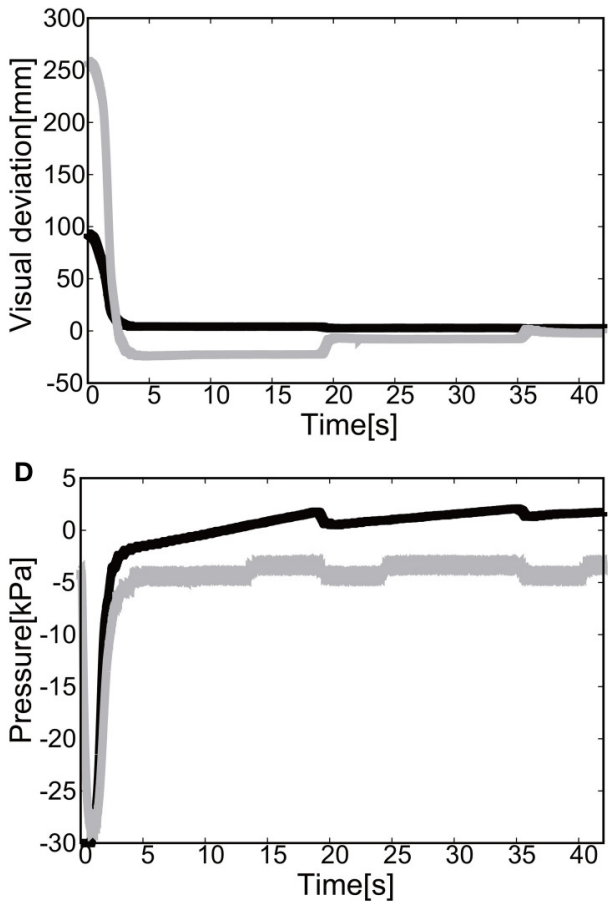

FIGURE 15 | Extension motion (negative pressure actuation) of the elbow and the shoulder of the Exoskeleton Actuated by Soft Modules. (A) Visual deviation $\left(\Delta \mathrm{U}_{1}\right)$ $x(t)$ (Black line) and $y(t)$ (Gray line) as a function of time, using the camera on the shoulder. (B) Visual deviation $\left(\Delta \mathrm{U}_{2}\right) x(t)($ Black line) and $y(t)$ (Gray line) as a function of time, using the camera from above. (C) The pressure of the shoulder module as a function of time. The black line represents the target pressure, and the gray line represents the measured pressure. (D) The pressure of the elbow module as a function of time. The black line represents the target pressure, and the gray line represents the measured pressure.

the measured pressure as a function of time when switching the targets.

\subsection{Functional Properties of the Exoskeleton Actuated by Soft Modules (EASoftM) Validated by Participant Experiments}

To evaluate the functionality of EASoftM in providing assistance with a wide range of reaching motion and precision to reach a target, we performed the experiments with one participant. The ethical approval was permitted by the ethical committee of Ritsumeikan University (BKC-medical-2016-051). Figure 8D shows the Exoskeleton system attached to the wrist of the participant. The equilibrium point of the upper limb was shifted to match the desired work space appropriate for reaching motion. The visual process using the camera on the shoulder could successfully monitor the position of the tracer to apply the visual control law (Equation 8). 

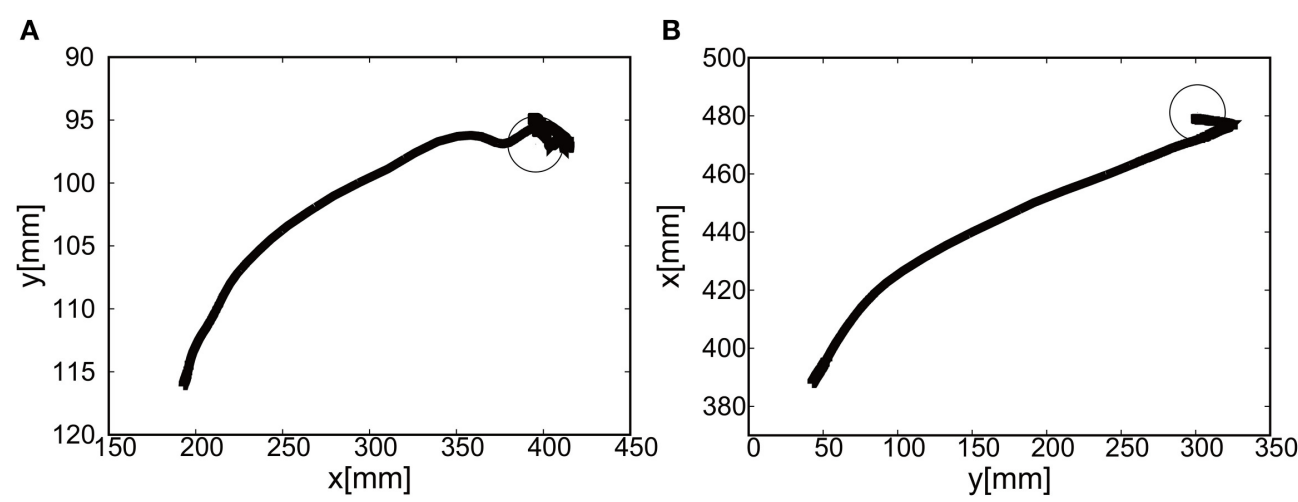

FIGURE 16 | Trajectories of the end-effector in $\mathrm{x}$-y plane (negative pressure actuation), using (A) the camera on the shoulder and (B) the camera from above. The target is denoted by the circle.
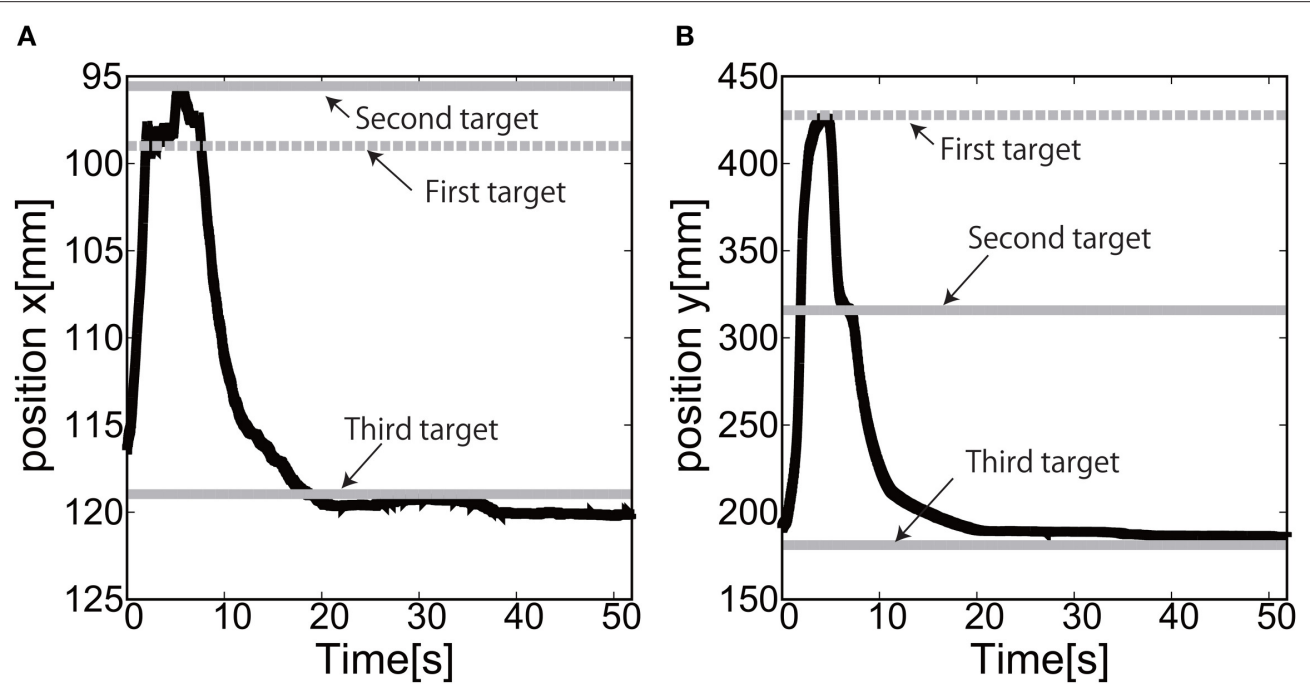

FIGURE 17 | Reaching motion using the elbow and shoulder joint of the Exoskeleton Actuated by Soft Modules, using the camera on the shoulder. (A) $x(t)$ as a function of time. (B) $y(t)$ as a function of time.

\subsubsection{Flexion Motion to the Target (Positive Pressure Actuation)}

The gain parameters were set to $k_{p 1}=2.0, k_{p 2}=2.0$ for the elbow joint, and $k_{p 1}=0.2, k_{p 2}=0.2$ for the shoulder in Equation (8). For the case of $2 \mathrm{D}$ motion using the elbow and shoulder joints of the EASoftM, it was shown that the reaching motion is also possible, as shown in Figures 20A,B.

The sudden rise in pressure around $8 \mathrm{~s}$ in Figures 20C,D was due to the configuration changes of the soft modules under the friction. However, this type of fluctuation within the low pressure range does not produce enough torque to affect the rotation of the joints Since the relative distance between the target and the tracer is highest at the initial position of the tracer, the pressure profile shows the slow convergence reflecting the slow pneumatic actuation of the soft modules. The trajectories of the end-effector are shown in Figure 21. As the exoskeleton itself was designed based on the anatomical nature of human body, the two link motion in parallel to the human upper limb was smooth. The relative distance to the target was achieved to be $\Delta x=-0.89[\mathrm{~mm}]$ and $\Delta y=2.66[\mathrm{~mm}]$.

\subsubsection{Extension Motion to the Target (Negative Pressure Actuation)}

The gain parameters were set to $k_{p 1}=2.0, k_{p 2}=2.0$ for the elbow joint, and $k_{p 1}=0.2, k_{p 2}=0.2$ for the shoulder joint in Equation (8). It was shown that the extension motion is also possible when applying the negative pressure to achieve the flexion motion as shown in Figures 22A,B. Figures 22C,D show the pressure of the shoulder and elbow module respectively as a function of time. After $3 \mathrm{~s}$ from the initial targets, their pressure converged to the target pressure. Figure 23 shows the trajectories of the end-effector. The relative distance to the target was achieved to be $\Delta x=10.12[\mathrm{~mm}]$ and $\Delta y=9.92[\mathrm{~mm}]$. 

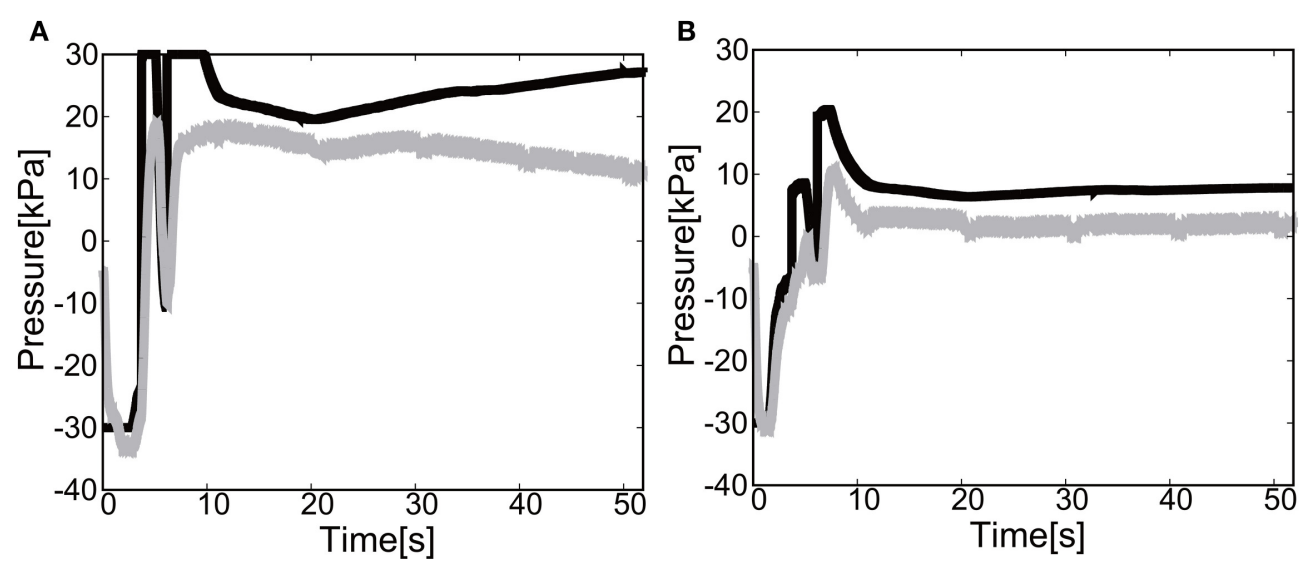

FIGURE 18 | Reaching motion using the elbow and shoulder joint of the Exoskeleton Actuated by Soft Modules, using the camera on the shoulder. The black line represents the target pressure, and the gray line represents the measured pressure. (A) The pressure of the shoulder module as a function of time. (B) The pressure of the elbow module as a function of time.
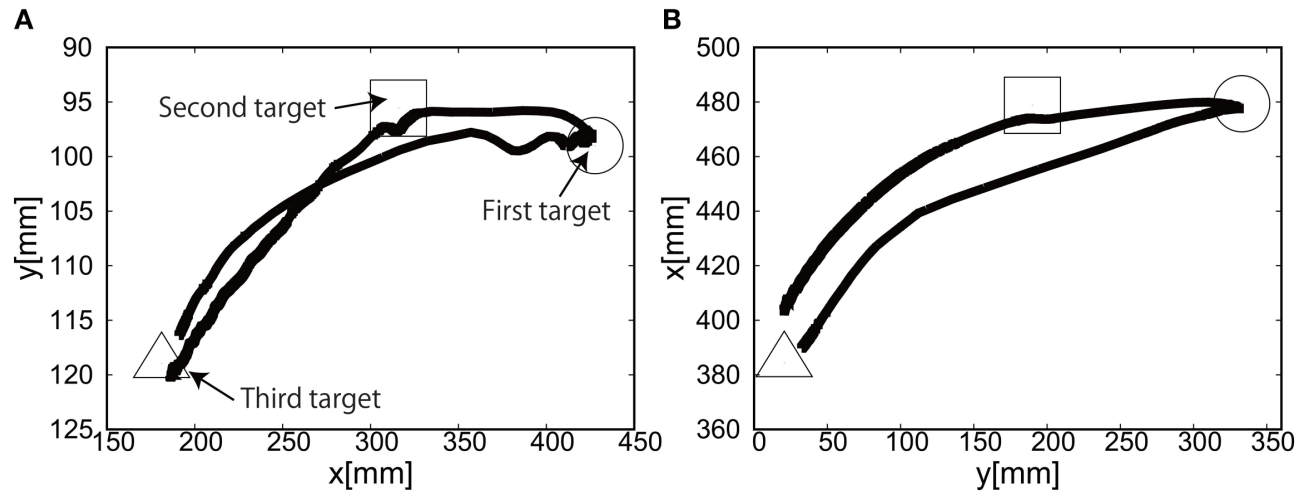

FIGURE 19 | Trajectories of the end-effector in $x-y$ plane, using (A) the camera on the shoulder and (B) the camera from above. The first target is denoted by circle, the second target by square, and the third target by triangle.

\subsubsection{Reaching Motion with Three Target Positions}

By switching three targets sequentially, we demonstrated the closed loop motion, i.e., the end-effector should come back to its initial place. The target was switched to another target when the relative distance between the target and the end-effector reached $<5.0[\mathrm{~mm}]$. The gain parameters were set to $k_{p 1}=2.20, k_{p 2}=2.20$ for the elbow joint, and $k_{p 1}=0.50, k_{p 2}=0.50$ for the shoulder joint in Equation (8).

Figures 24A,B show the position of the target, $x(t)$ and $y(t)$, using the camera on the shoulder. We can clearly observe that the end-point reaches the first target, the second target, and goes back to the initial position where the third target was located. Figure 25 shows the target and measured pressure as a function of time for the shoulder (a) and the elbow (b) joint. When switching the target position, there were corresponding jumps in the target pressure. Overall, it is a promising feature of the soft module used to actuate the exoskeleton to demonstrate the smooth transition in switching the sign of the pressure, leading to the smooth transition from flexion to extension.

For the case of reaching motion, by switching three targets sequentially it was shown that the EASoftM could demonstrate the closed loop motion as shown in Figure 26, even though there were some overshoots in the relative distance and the measured pressure as a function of time when switching the targets. Note here, that the straight trajectories can be realized by tuning the transformation matrix from visual to work space in Equation (8) (Henry Eberle and Hayashi, 2017).

\section{GENERAL DISCUSSION}

Therapeutic intervention using the assistive robots has been proven to be effective when including the following design specification (Loureiro et al., 2014);

1. A few degrees of freedom; Motion in daily life requires the coordinated joint motion. Thus, the assistive robots are expected to support a set of joints in upper or lower limbs. 

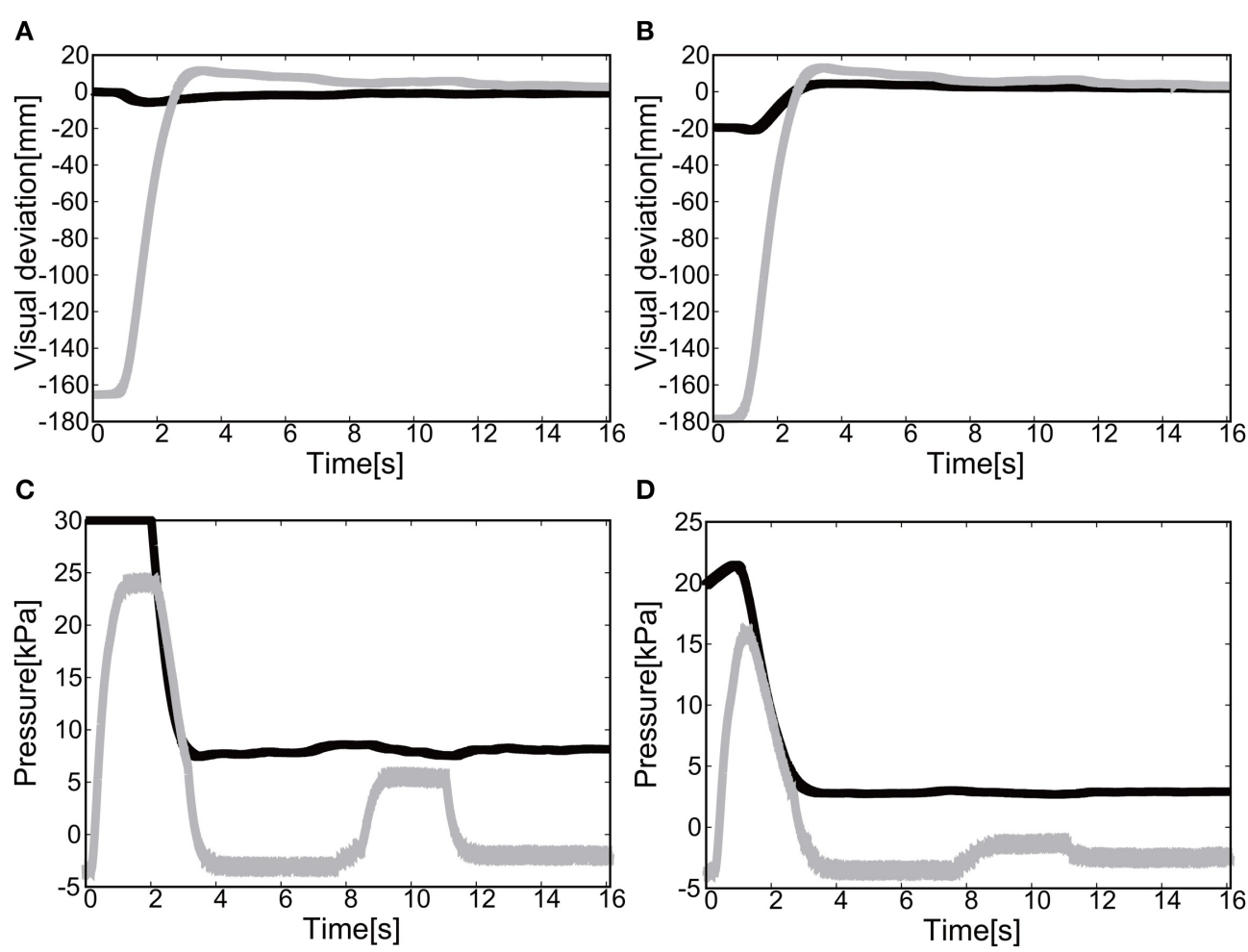

FIGURE 20 | Flexion motion (positive pressure actuation) of the elbow and the shoulder of the Exoskeleton with the participant. (A) Visual deviation $\left(\Delta U_{1}\right) x(t)(B l a c k$ line) and $y(t)$ (Gray line) as a function of time, using the camera on the shoulder. (B) Visual deviation $\left(\Delta \mathrm{U}_{1}\right) x(t)$ (Black line) and $y(t)$ (Gray line) as a function of time, using the camera from above. (C) The pressure of the shoulder module as a function of time. The black line represents the target pressure, and the gray line represents the measured pressure. (D) The pressure of the elbow module as a function of time. The black line represents the target pressure, and the gray line represents the measured pressure.
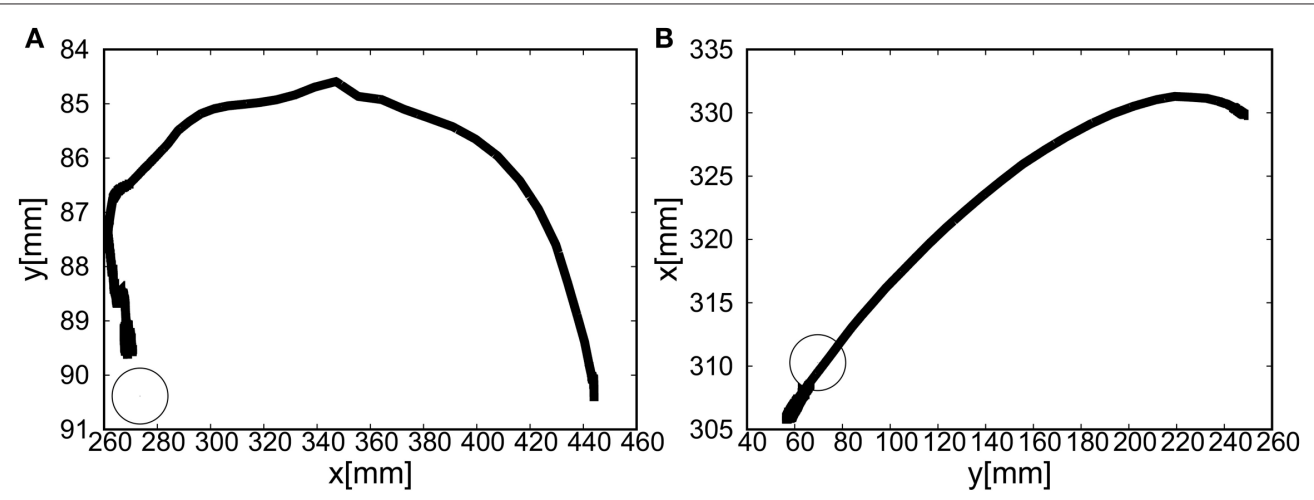

FIGURE 21 | Trajectories of the end-effector (positive pressure actuation) in $\mathrm{x}$-y plane (with the participant), using (A) the camera on the shoulder and (B) the camera from above. The target is denoted by the circle.

2. Precise motion control: The precision at the reaching point is required, as well as the precision in trajectories and certain dynamical aspects (target velocity and acceleration).

3. Wearable assistive robots in daily life: Cost-effective and ultra-light solutions should be developed for wearable assistive robots which support the motion in daily life.
4. Compliant assistance: Muscle contraction provides compliance in joint dynamics. Thus, assistance should also be compliant.

To realize the precise motion with a few degrees of freedom, the conventional methods would suggest the industrial type robot such as metal exoskeleton which is a hard, rigid structure and heavy (Krebs et al., 2000), not fulfilling the condition 3. A new 

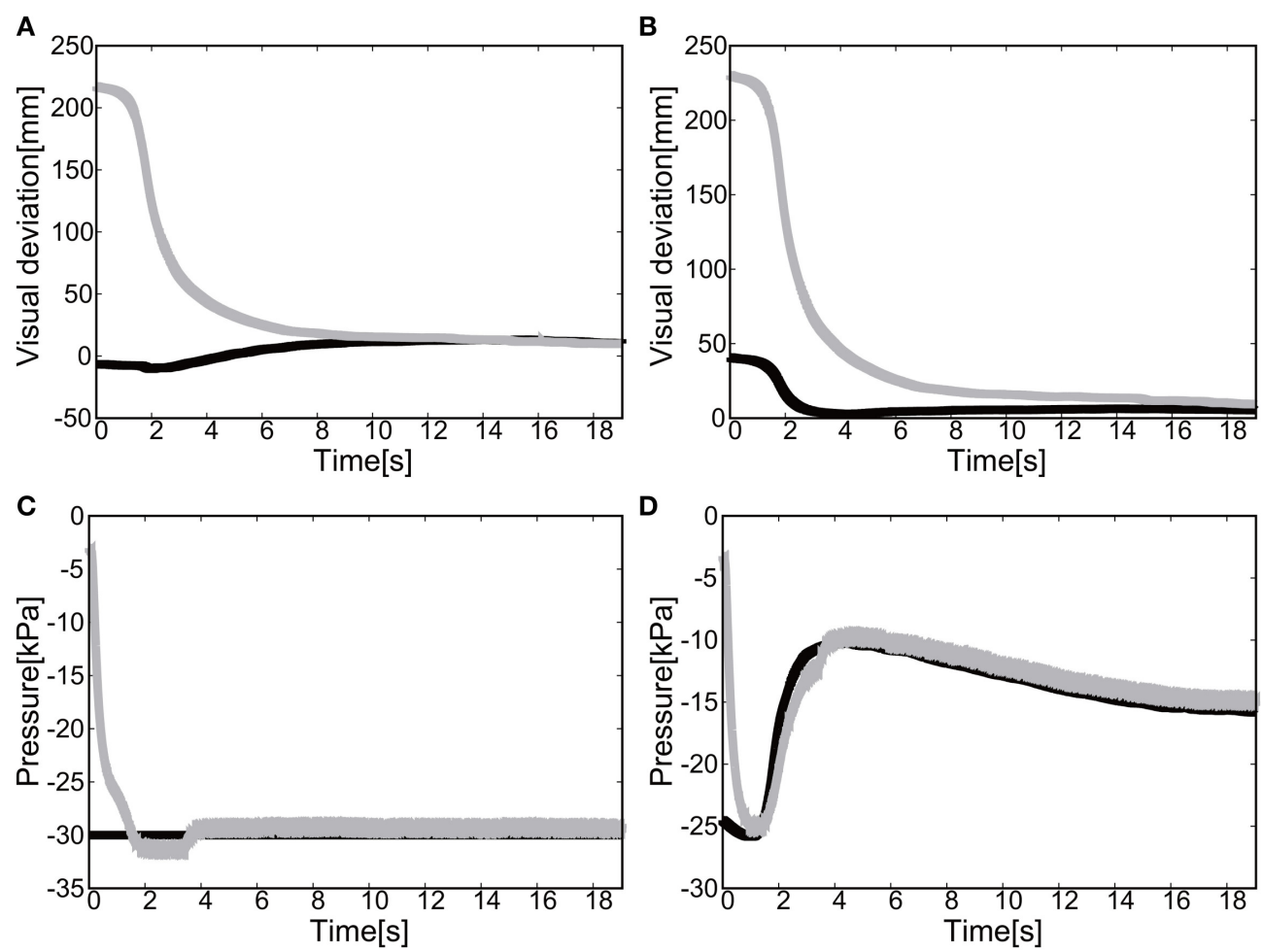

FIGURE 22 | Extension motion (negative pressure actuation) of the elbow and the shoulder of the Exoskeleton with the participant. (A) Visual deviation $\left(\Delta \mathrm{U}_{1}\right) x(t)$ (Black line) and $y(t)$ (Gray line) as a function of time, using the camera on the shoulder. (B) Visual deviation $\left(\Delta \mathrm{U}_{2}\right) x(t)$ (Black line) and $y(t)$ (Gray line) as a function of time, using the camera from above. (C) The pressure of the shoulder module as a function of time. The black line represents the target pressure, and the gray line represents the measured pressure. (D) The pressure of the elbow module as a function of time. The black line represents the target pressure, and the gray line represents the measured pressure.
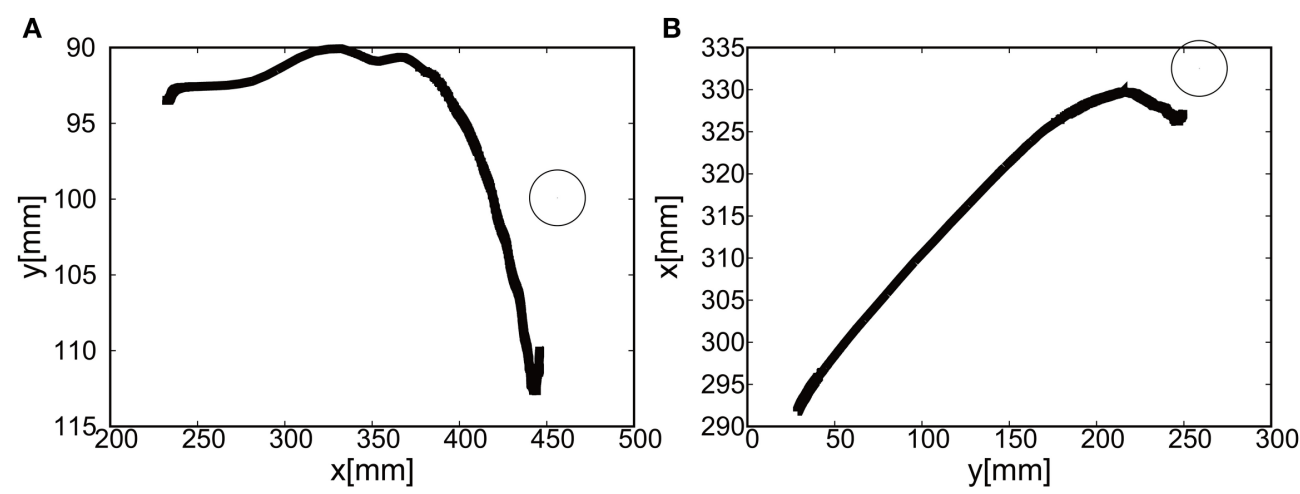

FIGURE 23 | Trajectories of the end-effector (negative pressure actuation) in $\mathrm{x}$-y plane (with the participant), using (A) the camera on the shoulder and (B) the camera from above. The target is denoted by the circle.

type of a wearable robot called soft exoskeletons or exosuits is an interesting alternative. Removing the external metal frame, soft exoskeletons become compliant, much lighter, and could be significantly less expensive than rigid frame wearable robots (Panasonic, 2005). However, soft exoskeletons main drawback is the same as their main strength: they have no external rigid frame to support the body parts and transfer force effectively from actuator to some area of body.
We think that the effective transfer of the force should take into account the anatomical structure of limbs and joints, requiring the solid structure of the assistive device. Elderly and disabled people already have weakened bones and muscles, so if the force is transmitted in the wrong manner, adding unnecessary strain to their body parts, it might lead to joint degeneration, damage their muscles or tendons. Thus, in our study, aiming at the reaching motion of upper limb, we developed 

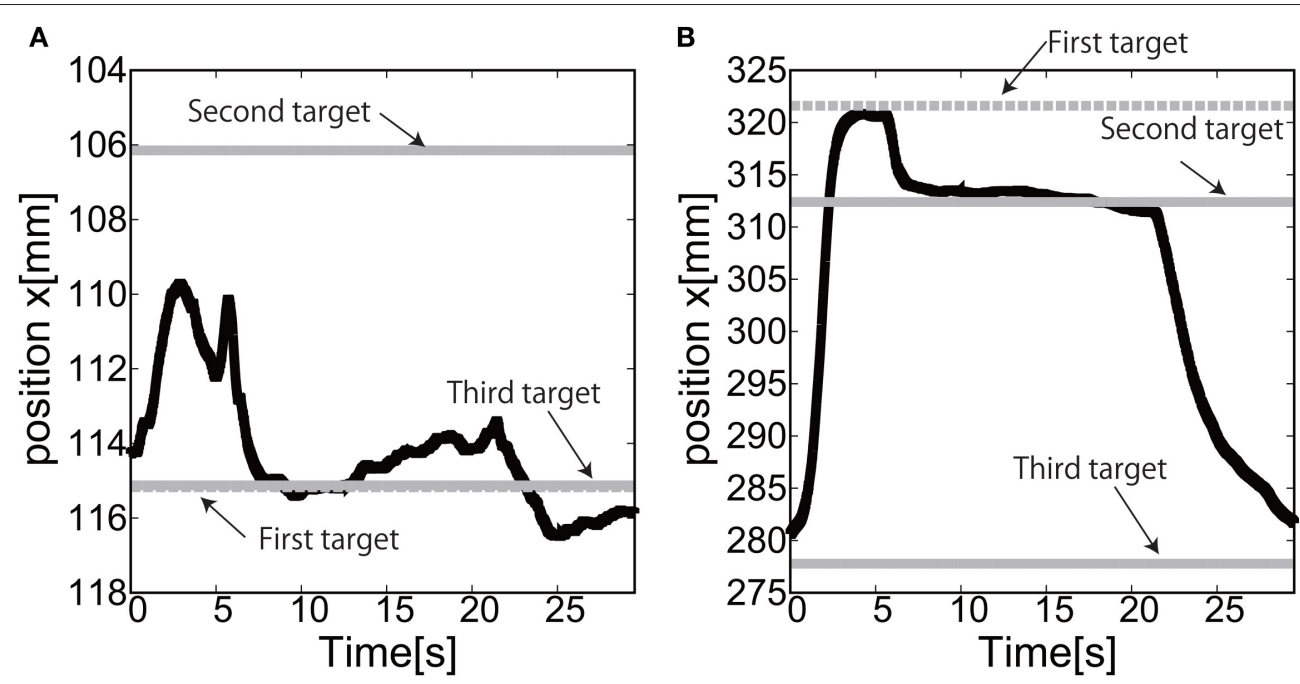

FIGURE 24 | Reaching motion using the elbow and shoulder joint of the Exoskeleton, using the camera on the shoulder. (A) $x(t)$ as a function of time. (B) $y(t)$ as a function of time.
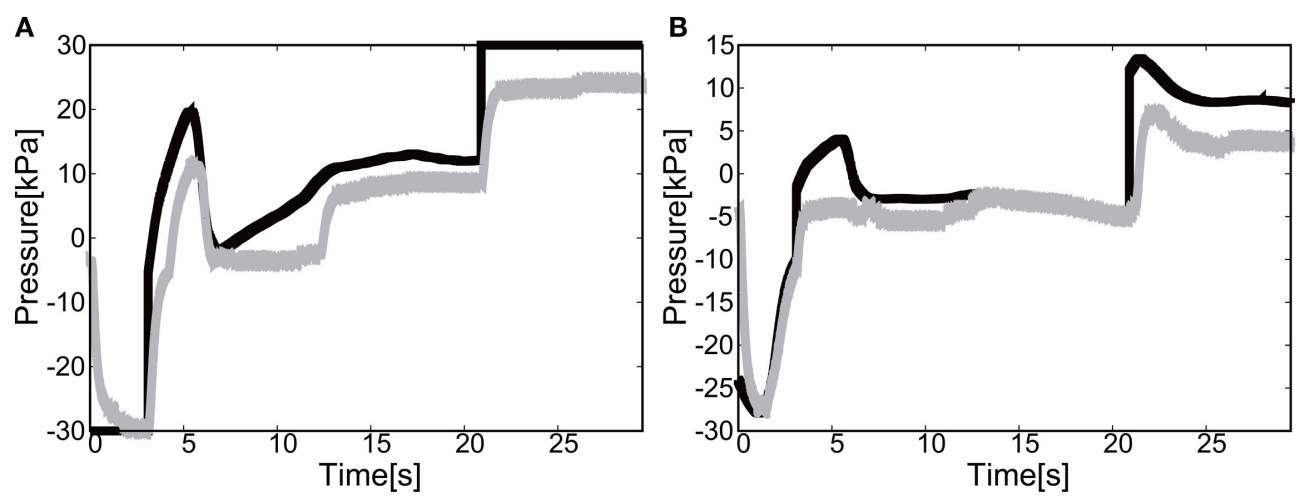

FIGURE 25 | Reaching motion using the elbow and shoulder joint of the Exoskeleton, using the camera on the shoulder. The black line represents the target pressure, and the gray line represents the measured pressure. (A) The pressure of the shoulder module as a function of time. (B) The pressure of the elbow module as a function of time.

A

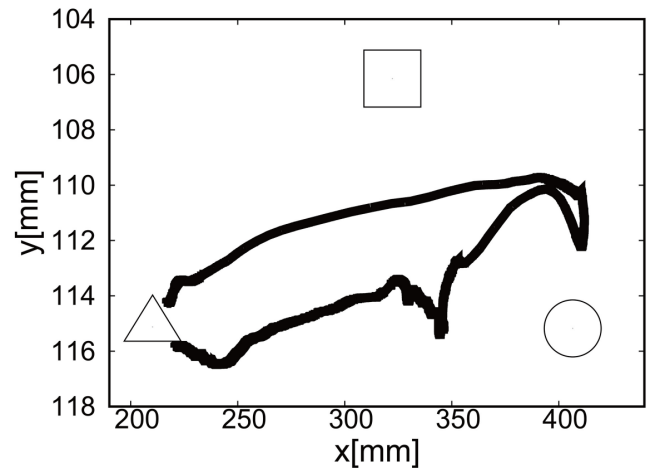

B

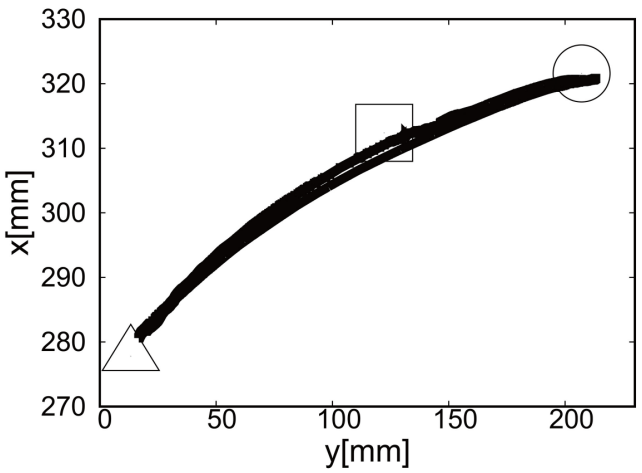

FIGURE 26 | Trajectories of the end-effector in $x$-y plane, using (A) the camera on the shoulder and (B) the camera from above. The first target is denoted by circle, the second target by square, and the third target by triangle. 
and validated the exoskeleton actuated by soft modules to fulfill these four conditions. We proposed the exoskeleton aligned with the anatomical structure actuated by the soft modules located at the joint positions, and these soft actuators realized the compliant motion based on pneumatic actuation and viscoelastic properties of soft materials such as plastic and rubber. As far as we are aware of, this proposed structure demonstrated the first possibility of integrating the rigid exoskeleton and the soft actuators to assist the reaching motion, as well as the vision-based control law showing its effective functionality in guiding the hand of the participant to the target.

This vision-based control has a certain advantage when the exoskeleton is used in the daily life, i.e. the visual analysis can be easily extended to detect the target such as cups and pens in the work space, and it does not require any encoders and a set of cables transmitting the signals, which add technical complexity to the integrated system. Thus, closing the control loop only by visual information is a flexible solution that can easily integrate low level motion control with higher level intelligent control. More specifically, since we aim at slow reaching motion, the necessary torque mainly will be used to compensate the gravity. Although, it is in principle possible to use the active actuator for this purpose, however, it would be hard to produce the wearable device aligned with the anatomical structure, as it would increase the number of actuators required. Thus, in our study we used the passive actuator for gravity compensation to decrease the weight for wearable purposes. As a next step, we developed the pneumatic actuators made of plastic sheets, which has good tensile strength and is light, and could produce substantial torque for our purposes under 1 atmospheric pressure. Conventionally, antagonist actuator is necessary to actuate the single joint, resulting in more weight and higher number of tubes. In our study, we could successfully demonstrate that the positive and negative pressure can be applied subsequently to produce the torque to increase and decrease the angle of joints. The low pressure actuation contributed to the smooth transition of the pressure as shown in Figures 6, 7 .

Most of the assistive robots were made of rigid links and motors which used metallic materials. To realize the compliant motion, the mechanical structure required additional component such as spring, which also added more weight onto the whole structure (Loureiro et al., 2011). Also, considering the assistive soft robotics, though the pneumatic actuators are light weighted, they were used with the metal links and pulleys, thus, the total weight tend to increase, making them inappropriate for the wearable solution (for example, see Schulz et al., 2011). Our proposed exoskeleton aimed at the ultra-light solution, using the $3 \mathrm{D}$ printed links and the soft modules (plastic sheets) for actuation, and reducing the number of actuations by employing

\section{REFERENCES}

Chiri, A., Vitiello, N., Giovacchini, F., Roccella, S., Vecchi, F., and Carrozza, M. C. (2012). Mechatronic design and characterization of the index finger module of a hand exoskeleton for post-stroke rehabilitation. the negative pressure actuation and gravity compensation. In addition, despite of the challenges imposed by such choice of hardware components materials, we did not lose the precision of position control since the vision-based control was employed.

The further work is planned to make a whole system portable and wearable. This can be achieved by the pneumatic actuators, the regulators and the power supplies being mounted in a waist belt pack so that the participants can freely move around. Also, aiming at the wearable and portable system, we can use the small motors to pump in the air, and the simple electric circuits to control the pressure of the soft modules (Oguntosin et al., 2015). By placing those electric components in the waist belt, the electromagnetic waves should not interfere with the EEG measurement. In the near future, we will integrate the robotic system with an eye tracking system to transmit the intention of the participant identifying the desired targets, as a natural extension of the vision-based control schemes toward the daily exercise. As a mid-term plan, we will integrate the proposed robotic system with Brain Computer Interface to estimate the user's motion intention in order to trigger the assistive motion (Hayashi et al., 2012; Kondo et al., 2015), as the system discussed in this article provides an optimal solution for the measurement of the EEG signals, since its construction made of plastic (metal-free structure and actuation) in principle does not produce any electromagnetic waves.

\section{ETHICS STATEMENT}

This study was carried out in accordance with the recommendations of Ritsumeikan medical ethical guidelines and Ritsumeikan Ethical Committee with written informed consent from all subjects (BKC-medical-2016-051). All subjects gave written informed consent in accordance with the Declaration of Helsinki. The protocol was approved by the Ritsumeikan Ethical Committee.

\section{AUTHOR CONTRIBUTIONS}

VO and YM substantially contributed to the development of the systems, data acquisition, and analysis. HK contributed to the performance of experiments, and the interpretation of the results. $\mathrm{SN}, \mathrm{SK}$, and $\mathrm{YH}$ contributed to the design of the work and overall discussion. All of us contributed to the writing of the paper.

\section{ACKNOWLEDGMENTS}

This work is partially supported by the School of Biological Sciences, University of Reading and by the Department of Robotics, Ritsumeikan University. 740, 408-415. doi: 10.4028/www.scientific.net/AMR.740.408 
Denavit, J., and Hartenberg, R. S. (1955). A kinematic notation for lower-pair mechanisms based on matrices. Trans. ASME J. Appl. Mech. 23, 215-221.

Dovat, L., Lambercy, O., Gassert, R., Maeder, T., Milner, T., Leong, T. C., et al. (2008). Handcare: a cable-actuated rehabilitation system to train hand function after stroke. IEEE Trans. Neural Syst. Rehabil. Eng. 16, 582-591. doi: 10.1109/TNSRE.2008.2010347

Hayashi, Y., Nagai, K., Ito, K., Nasuto S. J., Loureiro, R., and Harwin, W. S. (2012). "A feasible study of EEG-driven assistive robotic system for stroke rehabilitation," in 4th Conference on Biomedical Robotics and Biomechatronics (BioRob 2012) (Rome), 1733-1739. doi: 10.1109/BioRob.2012.6290919

Henry Eberle, S. J. N., and Hayashi, Y. (2017). Integration of visual and joint information to enable linear reaching motions. Sci. Rep. 7:40869. doi: $10.1038 /$ srep40869

Heo, P., Gu, G. M., Lee, S.-J., Rhee, K., and Kim, J. (2012). Current hand exoskeleton technologies for rehabilitation and assistive engineering. Int. J. Precis. Eng. Manuf. 13, 807-824. doi: 10.1007/s12541-012-0107-2

Krebs, H. I., Ferraro, M., Buerger, S. P., Newbery, M. J., Makiyama, A., Sandmann, M., et al. (2004). Rehabilitation robotics: pilot trial of a spatial extension for mit-manus. J. Neuroeng. Rehabil. 1:5. doi: 10.1186/1743-0003-1-5

Ilievski, F., Mazzeo, A. D., Shepherd, R. F., Chen, X., and Whitesides, G. M. (2011). Soft robotics for chemists. Angew. Chem. Int. Ed. Engl. 50, 1890-1895. doi: 10.1002/anie.201006464

Jack, D., Boian, R., Merians, A. S., Tremaine, M., Burdea, G. C., Adamovich, S. V., et al. (2001). Virtual reality-enhanced stroke rehabilitation. IEEE Trans. Neural Syst. Rehabil. Eng. 9, 308-318. doi: 10.1109/7333.948460

Kim, H. J., Tanaka, Y., Kawamura, A., Kawamura, S., and Nishioka, Y. (2015). "Improvement of position accuracy for inflatable robotic arm using visual feedback control method," in 2015 IEEE International Conference on Advanced Intelligent Mechatronics (AIM) (Busan), 767-772. doi: 10.1109/AIM.2015.7222630

Kondo, T., Saeki, M., Hayashi, Y., Nakayashiki, K., and Takata, Y. (2015). Effect of instructive visual stimuli on neurofeedback training for motor imagery-based braincomputer interface. Hum. Mov. Sci. 43, 239-249. doi: 10.1016/j.humov.2014.08.014

Krebs, H., Volpe, B., Aisen, M., and Hogan, N. (2000). Increasing productivity and quality of care: robot-aided neuro-rehabilitation. J. Rehabil. Res. Dev. 37, 493-504.

Kutner, N., Zhang, R., Butler, A., Wolf, S., and Alberts, J. (2014). Quality-oflife change associated with robotic-assisted therapy to improve hand motor function in patients with subacute stroke: a randomized clinical trial. Phys. Ther. 90, 493-504. doi: 10.2522/ptj.20090160

Loureiro, R. C., Harwin, W. S., Nagai, K., and Johnson, M. (2011). Advances in upper limb stroke rehabilitation: a technology push. Med. Biol. Eng. Comput. 49, 1103-1118. doi: 10.1007/s11517-011-0797-0

Loureiro, R. C. V., H. W., Lamperd, R., and Collin, C. (2014). Evaluation of reach and grasp robot-assisted therapy suggests similar functional recovery patterns on proximal and distal arm segments in sub-acute hemiplegia. IEEE Trans. Neural Syst. Rehabil. Eng. 22, 593-602. doi: 10.1109/TNSRE.2013.2265263

Loureiro, R., and Harwin, W. (2007). "Reach \& grasp therapy: design and control of a 9-DOF robotic neuro-rehabilitation system," in IEEE 10th International Conference on Rehabilitation Robotics (ICORR) (Noordwijk), 757-763. doi: 10.1109/icorr.2007.4428510

Maciejasz, P., Eschweiler, J., Gerlach-Hahn, K., Jansen-Troy, A., and Leonhardt, S. (2014). A survey on robotic devices for upper limb rehabilitation. J. NeuroEng. Rehabil. 11:3. doi: 10.1186/1743-0003-11-3

Marchese, A., Komorowski, K., Onal, C., and Rus, D. (2014). "Design and control of a soft and continuously deformable $2 \mathrm{D}$ robotic manipulation system," in 2014 IEEE International Conference on Robotics and Automation (ICRA) (Hong Kong), 2189-2196. doi: 10.1109/ICRA.2014.6907161

Martinez, R. V., Branch, J. L., Fish, C. R., Jin, L., Shepherd, R. F., Nunes, R. M. D., et al. (2013). Robotic tentacles with three-dimensional mobility based on flexible elastomers. Adv. Mater. 25, 205-212. doi: 10.1002/adma.201 203002

Nishida, R., and Kawamura, S. (2012). "A new feedback robot control method based on position/image sensor integration," in 2012 IEEE/RSJ International Conference on Intelligent Robots and Systems (Vilamoura-Algarve), 5012-5017. doi: 10.1109/IROS.2012.6385785

Oguntosin, V., Harwin, W. S., Kawamura, S., Nasuto, S. J., and Hayashi, Y. (2015). "Development of a wearable assistive soft robotic device for elbow rehabilitation," in 2015 IEEE International Conference on Rehabilitation Robotics (ICORR) (Singapore), 747-752. doi: 10.1109/ICORR.2015.7281291

Panasonic (2005). Realive Power Assist Device. Available online at: http:// panasonic.co.jp/corp/news/official.data/data.dir/en060925-6/en060925-6.html

Polygerinos, P., Galloway, K. C., Sanan, S., Herman, M., and Walsh, C. J. (2015). "EMG controlled soft robotic glove for assistance during activities of daily living," in 2015 IEEE International Conference on Rehabilitation Robotics (ICORR) (Singapore), 55-60. doi: 10.1109/ICORR.2015.7281175

Sanchez, R. J., Liu, J., Rao, S., Shah, P., Smith, R., Rahman, T., et al. (2006). Automating arm movement training following severe stroke: functional exercises with quantitative feedback in a gravity-reduced environment. IEEE Trans. Neural Syst. Rehabil. Eng. 14, 378-389. doi: 10.1109/TNSRE.2006. 881553

Schulz, S., Schmitz, B., Wiegand, R., Pylatiuk, C., and Reischl, M. (2011). "The hybrid fluidic driven upper limb orthosis-orthojacket," in Proceedings of the MyoElectric Controls/Powered Prosthetics Symposium Fredericton (New Brunswick).

Shepherd, R., Ilievski, F., Choi, W., Morin, S., Stokes, A., Mazzeo, A., et al. (2011). Multi-gait soft robot. Proc. Natl. Acad. Sci. U.S.A. 108, 20400-20403. doi: 10.1073/pnas.1116564108

Sun, Y., Song, Y. S., and Paik, J. (2013). "Characterization of silicone rubber based soft pneumatic actuators," in 2013 IEEE/RSJ International Conference on Intelligent Robots and Systems (IROS) (Tokyo), 4446-4453. doi: 10.1109/IROS.2013.6696995

Takagi, M., Iwata, K., Takahashi, Y., Ichiroh Yamamoto, S., Koyama, H., and Komeda, T. (2009). "Development of a grip aid system using air cylinders," in 2009 IEEE International Conference on Robotics and Automation (Kobe), 2312-2317. doi: 10.1109/ROBOT.2009.5152246

Rahman, T., Sample, W., Jayakumar, S., King, M. M., Wee, J. Y., Seliktar, R. et al. (2006). Passive exoskeletons for assisting limb movement. J. Rehabil. Res. Dev. 43, 583-590. doi: 10.1682/JRRD.2005.04.0070

Tondu, B., and Lopez, P. (2000). Modeling and control of McKibben artificial muscle robot actuators. IEEE Control Syst. 20, 15-38. doi: 10.1109/37.833638

Tsagarakis, N., and Caldwell, D. (2000). "Improved modelling and assessment of pneumatic muscle actuators," in IEEE International Conference on Robotics and Automation (ICRA), Vol. 4 (San Francisco, CA), 3641-3646. doi: 10.1109/robot.2000.845299

Ueki, S., Kawasaki, H., Ito, S., Nishimoto, Y., Abe, M., Aoki, T., et al. (2012). Development of a hand-assist robot with multi-degrees-offreedom for rehabilitation therapy. IEEE ASME Trans. Mech. 17, 136-146. doi: 10.1109/TMECH.2010.2090353

Conflict of Interest Statement: The authors declare that the research was conducted in the absence of any commercial or financial relationships that could be construed as a potential conflict of interest.

Copyright (c) 2017 Oguntosin, Mori, Kim, Nasuto, Kawamura and Hayashi. This is an open-access article distributed under the terms of the Creative Commons Attribution License (CC BY). The use, distribution or reproduction in other forums is permitted, provided the original author(s) or licensor are credited and that the original publication in this journal is cited, in accordance with accepted academic practice. No use, distribution or reproduction is permitted which does not comply with these terms. 
OPEN ACCESS

Edited by:

Dingguo Zhang,

Shanghai Jiao Tong University, China

Reviewed by:

Steven Weidong Su,

University of Technology, Sydney,

Australia

Qinghua Huang,

South China University of Technology,

China

Wei Zhou,

Xiamen University, China

*Correspondence:

Rong Song

songrong@mail.sysu.edu.cn

${ }^{\dagger}$ These authors have contributed equally to this work

Specialty section

This article was submitted to

Neural Technology,

a section of the journal

Frontiers in Neuroscience

Received: 31 December 2016

Accepted: 20 April 2017

Published: 16 May 2017

Citation:

Huang $Y$, Yang $Q$, Chen $Y$ and Song $R$

(2017) Assessment of Motor Control during Three-Dimensional Movements

Tracking with Position-Varying Gravity Compensation.

Front. Neurosci. 11:253 doi: 10.3389/fnins.2017.00253

\section{Assessment of Motor Control during Three-Dimensional Movements Tracking with Position-Varying Gravity Compensation}

\author{
Yao Huang ${ }^{1,2 \dagger}$, Qianqian Yang ${ }^{1,2 \dagger}$, Ying Chen ${ }^{1,2}$ and Rong Song ${ }^{1,2 *}$ \\ 1 Key Laboratory of Sensing Technology and Biomedical Instrument of Guangdong Province, School of Engineering, Sun \\ Yat-sen University, Guangzhou, China, ${ }^{2}$ Guangdong Provincial Engineering and Technology Center of Advanced and \\ Portable Medical Devices, School of Engineering, Sun Yat-sen University, Guangzhou, China
}

Active movements are important in the rehabilitation training for patients with neurological motor disorders, while weight of upper limb impedes movements due to muscles weakness. The objective of this study is to develop a position-varying gravity compensation strategy for a cable-based rehabilitation robot. The control strategy can estimate real-time gravity torque according to position feedback. Then, the performance of this control strategy was compared with the other two kinds of gravity compensation strategies (i.e., without compensation and with fixed compensation) during movements tracking. Seven healthy subjects were invited to conduct tracking tasks along four different directions (i.e., upward, forward, leftward, and rightward). The performance of movements with different compensation strategies was compared in terms of root mean square error (RMSE) between target and actual moving trajectories, normalized jerk score (NJS), mean velocity ratio (MVR) of main motion direction, and the activation of six muscles. The results showed that there were significant effects in control strategies in all four directions with the RMSE and NJS values in the following order: without compensation > fixed compensation > position-varying compensation and MVR values in the following order: without compensation $<$ fixed compensation $<$ position-varying compensation $(p<0.05)$. Comparing with movements without compensation in all four directions, the activation of muscles during movements with position-varying compensation showed significant reductions, except the activations of triceps and in forward and leftward movements, the activations of upper trapezius and middle parts of deltoid in upward movements and the activations of posterior parts of deltoid in all four directions $(p<0.05)$. Therefore, with position-varying gravity compensation, the upper limb cable-based rehabilitation robotic system might assist subjects to perform movements with higher quality and improve the participation of robot-aided rehabilitation training. Further studies are needed to explore the effectiveness and clinic application across pathologies.

Keywords: gravity compensation, upper limb rehabilitation, cable-based rehabilitation robotics, arm tracking, muscle activation 


\section{INTRODUCTION}

Since the upper limb dysfunctions after stroke seriously affect daily lives, it is essential for patients to restore the affected motor functions through rehabilitation training. Post-stroke reaching movements is affected by gravity weight of upper limb due to the weakness of those anti-gravity muscles (Beer et al., 2007). Position control was adopted in traditional robots (Lahouar et al., 2009; Yuan et al., 2014) and rehabilitation robots (Lum et al., 2002; Staubli et al., 2009), to follow predetermined trajectories. Although, position control in rehabilitation robots could counteract the effect of gravity weight of upper limb, it focused on control accuracy and ignores patients' voluntary participation in task execution, and its effectiveness in rehabilitation therapy needed further improvement. For patients who suffer from muscle weakness, gravity support showed promising results in minimizing the gravity-induced interference for tasks execution (van Elk et al., 2005; Cheng et al., 2015; Runnalls et al., 2015).

Gravity compensation can be provided by support devices (Herder et al., 2006; Kloosterman et al., 2010) or robotics (Kahn et al., 2006; Ball et al., 2007; Ladenheim et al., 2013), and can be generally grouped into three categories. Firstly, fixed or manually adjusted compensation were commonly used to counteract the gravity weight of upper limb (Nef et al., 2007; Stopforth, 2013; Lenzo et al., 2015). Similarly, a fixed external vertical force was applied to an upper limb robot by a motorized vertical cabling system for gravity compensation (Ball et al., 2007). Secondly, passive compensations by flexible force from elastic materials were chosen (De Luca et al., 2005; Stienen et al., 2009). As an assistive arm exoskeleton, T-WREX provided gravity compensation from the elastic bands whose number could be adjusted at different arm support levels (Housman et al., 2009). Arm orthosises with springs was designed to passively compensate arm weight, and could help patients with little moving ability to reach and grasp (Herder et al., 2006; Kramer et al., 2007). Freebal, a device with gravity compensation at different levels from cables connected to springs, could facilitate upper limb movements tracking in the horizontal and vertical planes (Prange et al., 2009; Kloosterman et al., 2010; Coscia et al., 2014). Thirdly, position-varying compensations can be provided according to human physical characteristics, since gravity torque of upper limb is highly coupled with the dynamics of the limbs and dependent on the postures and positions of moving limbs. Hsu et al. proposed an active control strategy to estimate the subject's movement intention and the control strategy included a gravity compensation term modified by the upper limb dynamics (Hsu et al., 2012). Cheng et al. developed a two degrees of freedom (2-DOF) compliant beam which could compensate the torques on each joint based on the dynamic of the upper limb (Cheng et al., 2015). A torque-angle model containing a gravity compensation term was proposed by Lin et al. to evaluate motion quality of adhesive capsulitis patients (Lin et al., 2014).

Since the gravity torque is highly dependent on positions of upper and fore arm during movements, fixed or varying elastic compensation strategies did not consider the position-coupling effect. Although, Hsu et al. and Li et al. included compensation strategies according to human physical characteristics, both their strategies were combined with other control terms (Hsu et al., 2012; Li et al., 2015) which might affect voluntary participation. Gravity compensation should provide suitable assistance to subjects without affecting voluntary participation, but few previous studies about compensation strategy considered both varying position of upper limb and voluntary participation. Meanwhile, how varying position affects movement performance and muscle activations is rarely reported.

The objective of this study is to develop a position-varying gravity compensation strategy for a cable-based rehabilitation robot. A gravity torque estimation model according to position feedback is proposed for estimating real-time upper limb gravity torque. Then, the performance of this strategy is compared with the other two kinds of gravity compensation strategies (i.e., without compensation and with fixed compensation) during four different directions of man-machine cooperation movements tracking. Root mean square error (RMSE) between target and actual moving trajectories, normalized jerk score (NJS), mean velocity ratio (MVR) of main motion direction, and the mean activation of six muscles are used for assessing different compensation strategies with a cable-based rehabilitation robot.

\section{MATERIALS AND METHODS}

\section{Participants}

Seven healthy men (mean age: $23.7 \pm 1.1$ yrs., mean weight: $63.5 \pm 9.3 \mathrm{~kg}$, mean height: $172.5 \pm 4.5 \mathrm{~cm}$ ) were recruited in this study. All the subjects were able to lift their right arm against gravity, and had no musculoskeletal or neurological problems. All subjects provided their written informed consent prior to participating in this study. All experimental procedures were approved by the human ethic committee of the First Affiliated Hospital of Sun Yat-sen University.

\section{Experimental Apparatus}

A cable-based rehabilitation robotic system was recruited for providing assistance to counteract the influence of gravity during multi-joints upper limb movements (Figure 1). The robotic system consisted of a mechanical part, a motion capture system with four cameras (OptiTrack, NaturalPoint, USA), a sixchannel surface electromyographic (EMG) signals amplifier, a 16bit analog-to-digital data acquisition card (PXI-6229, National Instruments, USA), and a personal computer. The mechanical part composed of a cubic base frame made of aluminum links, a splint, three cables, and three motors (DM1B-045G, Yokogawa, Japan) with three servo drivers (UB1DG3, Yokogawa, Japan; Yang et al., 2016). The splint was controlled by three motors through three bundled cables, resulting in 3 degrees of freedom (DOF). The group of motors could apply force on the split and assist the user performing movements in a 3-dimensional (3D) space. Three markers were attached at the dorsal centers of three joints (i.e., wrist, elbow, and shoulder), respectively, to record actual positions by the motion capture system. The sampling rate of the motion capturing was set at $100 \mathrm{~Hz}$, and raw position data were filtered with a second-order Butterworth filter with a cutoff frequency of $6 \mathrm{~Hz}$. Bi-polar surface EMG of six superficial muscles of the upper extremity [i.e., biceps (BIC), triceps (TRI), 


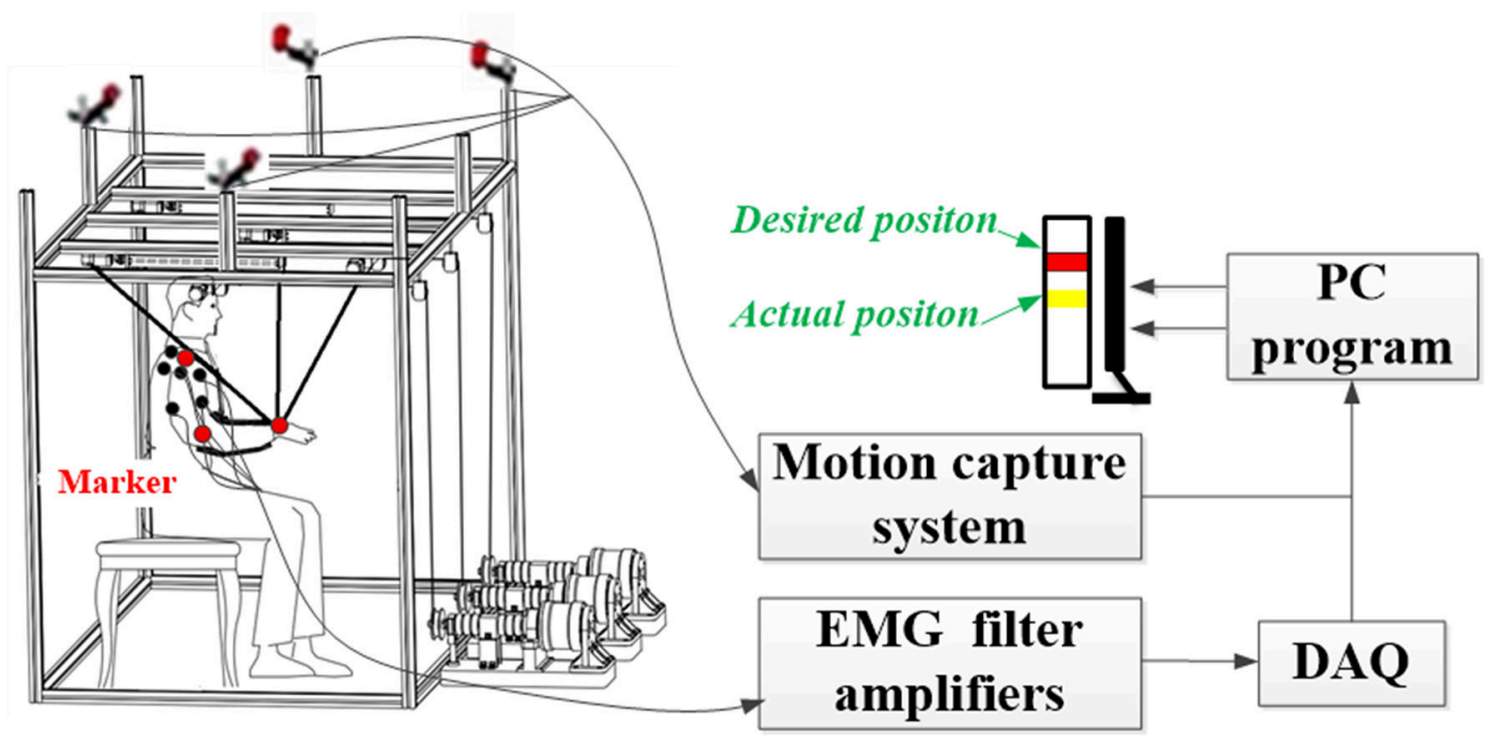

FIGURE 1 | Architecture of the cable-based rehabilitation robot.

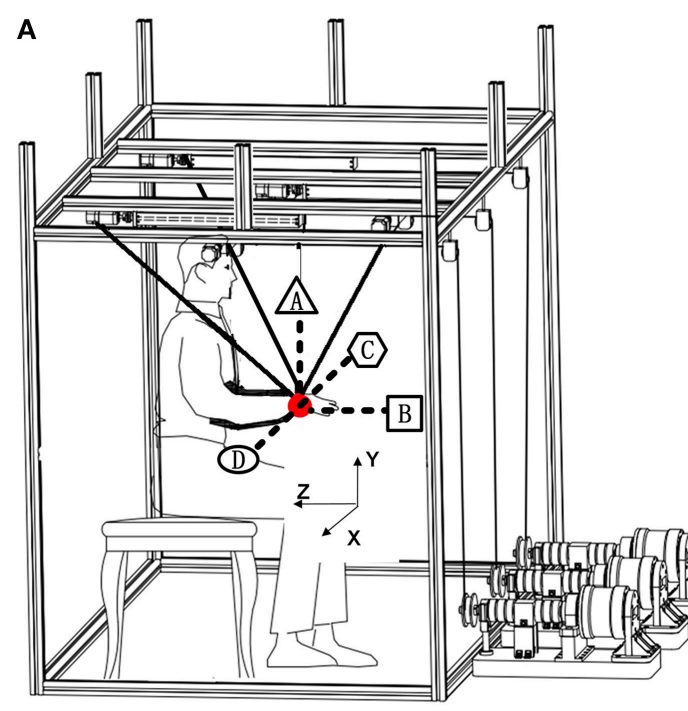

B

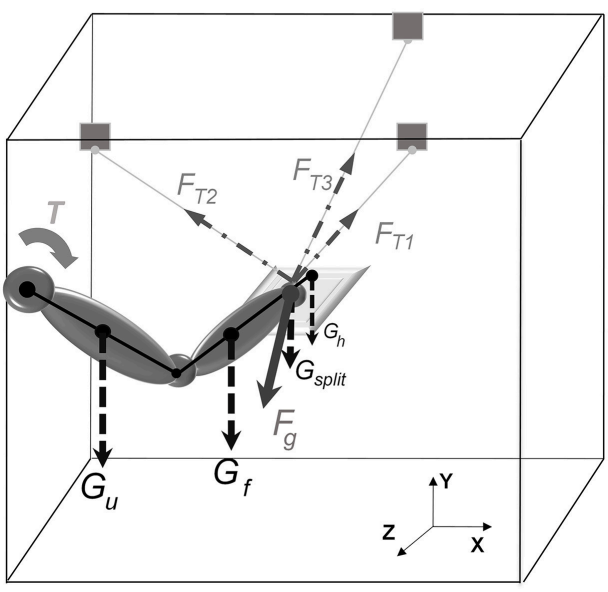

FIGURE 2 | (A) The tasks directions and (B) static force model.

anterior (DA), middle (DM), posterior (DP) parts of deltoid, and upper trapezius (TRA)] were recorded by attaching electrodes on the subject's skin. The raw EMG data from the six muscles were converted by the data acquisition card. The EMG signals were recorded at $1,000 \mathrm{~Hz}$, amplified with a gain of 5,000, and band-filtered by a 4 th-order Butterworth filter with a band of $10-400 \mathrm{~Hz}$.

\section{Experimental Procedure}

Before experiment, subjects should be seated in a chair and their trunks were strapped by soft fabrics to minimize the trunk movements. The initial posture of upper limb positioned at: the elbow flexion was at $90^{\circ}$, the forearm pronation was at $90^{\circ}$, the upper arm hung down vertically and was close to the trunk, the wrist was extended and the fingers were closed. A computer screen was placed in front of the subject for providing real-time visual feedback of the target and actual wrist positions. A yellow slide block represented the user's wrist showed the actual wrist position, and a red sliding block represented the target position. As shown in Figure 2A, each subject went through four kinds of multi-joint tracking tasks with the right arm [i.e., move upward (A)/forward (B)/leftward (C)/rightward (D) to the place $0.2 \mathrm{~m}$ away from the initial point along a straight line]. During one movement series, subjects are asked to finish three movement 
sessions including three kinds of gravity compensation strategies. And each session required the execution of six consecutive laps.

\section{Gravity Compensation Strategies}

The position-varying gravity compensation strategy was based on real-time estimation of upper limb gravity torque. A gravity torque estimation model was built to estimate the gravity torque from the joint positions and moments of the shoulder, elbow, and wrist:

$$
\boldsymbol{T}=\boldsymbol{G}_{\boldsymbol{u}} L_{u s h}+\boldsymbol{G}_{\boldsymbol{f}} L_{f s h}+\boldsymbol{G}_{\boldsymbol{h}} L_{h s h}
$$

where $\boldsymbol{T}$ was the gravity torque of upper limbs about shoulder, $\boldsymbol{G}_{\boldsymbol{h}}, \boldsymbol{G}_{\boldsymbol{f}}, \boldsymbol{G}_{\boldsymbol{u}}$ were the gravity of hand, forearm and upper arm, respectively, and $L_{h s h}, L_{f s h}, L_{u s h}$ were the moment arms from limb centroids of hand, forearm and upper arm to centroid of shoulder joint, respectively.

The equivalent force to the wrist can be calculated from the gravity torque as Equation (2):

$$
F_{g}=T / L_{s h w}
$$

where $\boldsymbol{F}_{\boldsymbol{g}}$ here was the equivalent force of the arm, $L_{s h w}$ was moment arm from limb centroids of shoulder to centroid of wrist joint.

The dynamic characteristics of the robotic system can be analyzed based on the static force model when the splint moved slowly and stably (Figure 2B). The resultant compensation force $\left(F_{\text {OUT }}\right)$ then can be expressed as Equation (3)

$$
\boldsymbol{F}_{\text {OUT }}=-\left(\boldsymbol{F}_{g}+\boldsymbol{G}_{\text {split }}\right)
$$

where $\boldsymbol{G}_{\text {split }}$ was the gravity of the split.

Orientations of the three cables should be considered when driving the motors and controlling the split. The cable-based structure required all cables kept in tension, and the tensile forces needed during arm movements can be presented as Equation (4)

$$
F_{T}=J^{-1} F_{O U T}
$$

where $\boldsymbol{F}_{T}=\left[\boldsymbol{F}_{T \mathbf{1}}, \boldsymbol{F}_{T \mathbf{2}}, \boldsymbol{F}_{T \mathbf{3}}\right]^{T}$ was the tensile force matrix, and $J=\left[\mathbf{u}_{1}, \boldsymbol{u}_{2}, \boldsymbol{u}_{3}\right]$ was the unit vector matrix of the mechanism structure.

There were the other two kinds of gravity compensation strategies (i.e., without compensation and with fixed compensation). The fixed compensation strategy provided a fixed gravity torque calculated when the wrist was in the initial place. When the strategy without compensation was applied, subjects were asked to finish movements without the assistance of the cable-based robot. The weight of the split is $2 \mathrm{~kg}$, thus when finishing movements without assistance, subjects were required to carry an extra $2 \mathrm{~kg}$ weight to guarantee the same experiment condition and to simulate the patients with arm weakness.
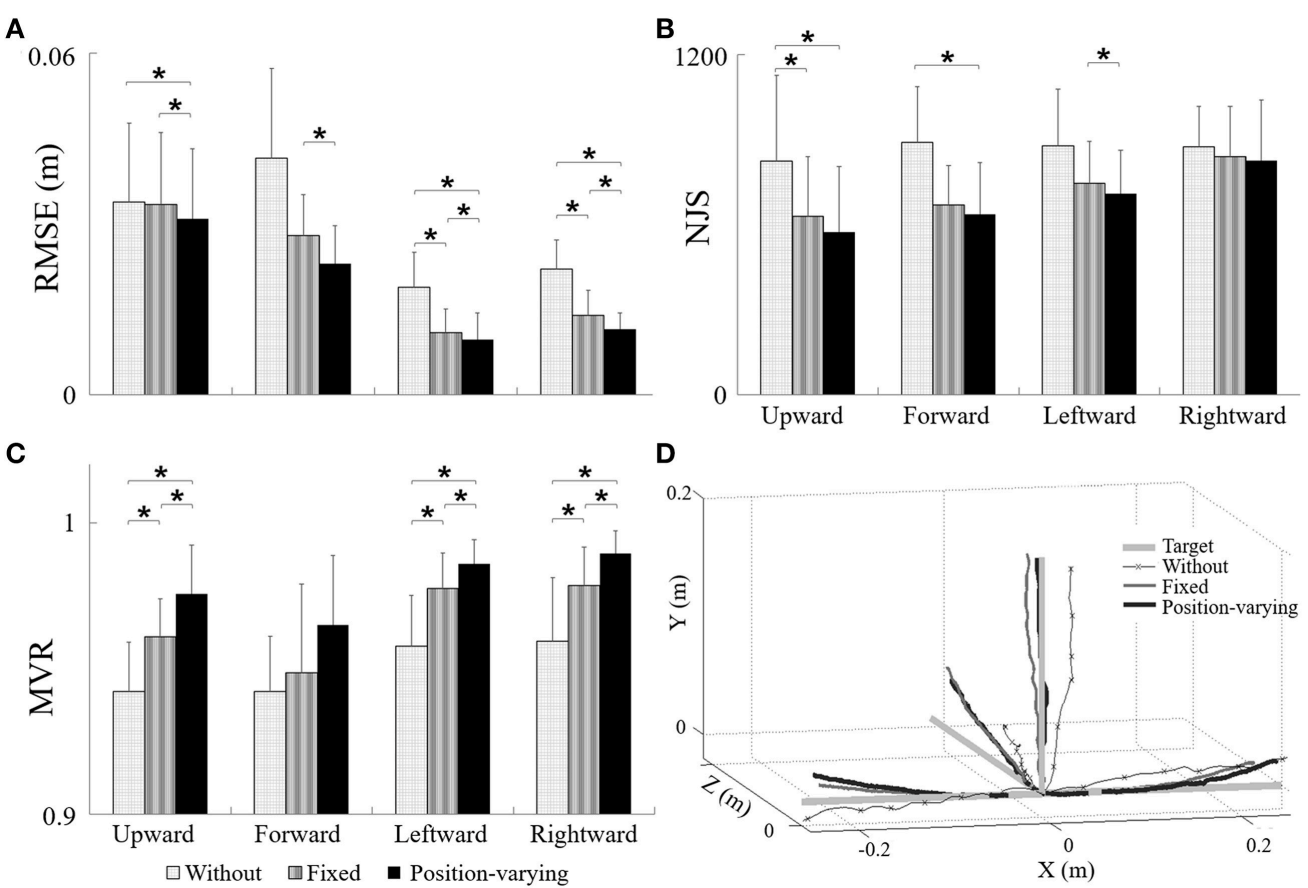

FIGURE 3 | The performance of (A) RMSE, (B) NJS and (C) MVR during movements with different gravity compensation strategies, (D) the target, and actual trajectories during movements tracking with different gravity compensation strategies. *Significant difference was found between two kinds of gravity compensation strategies $(p<0.05)$. 


\section{Data Analysis}

RMSE between the target trajectory and the actual trajectory of wrist was used to evaluate movement accuracy.

$\operatorname{RMSE}=\sqrt{\sum_{i=1}^{N}\left(\left(X_{a_{i}}-X_{t_{i}}\right)^{2}+\left(Y_{a_{i}}-Y_{t_{i}}\right)^{2}+\left(Z_{a_{i}}-Z_{t_{i}}\right)^{2}\right) / N}$

Where $i$ refers to the sampling point, $X_{a}, Y_{a_{i}}, Z_{a_{i}}$ refer to the actual values of $3 \mathrm{D}$ coordinates and $X_{t_{i}}, Y_{t_{i}}, Z_{t_{i}}$ refer to the target values of $3 \mathrm{D}$ coordinates.

In order to evaluate the control abilities of arm, NJS of the actual trajectory during movement, was adopted to represent movement smoothness in 3D space (Hogan and Sternad, 2009).

$$
\mathrm{NJS}=\sqrt{\frac{1}{2} \times \frac{T^{5}}{D^{2}} \times \int \dddot{s}^{2}(t)^{2} d t}
$$

Where $t$ refers to the actual time, $s(t)$ refers to the position at the time of $t$, and $T, D$ refer to the duration time, distance during the movement, respectively.

The MVR in each main motion direction is proposed for quantifying the relative velocity deviation in desired direction during movements tracking. It was assessed for movement efficiency, and was calculated as:

$$
M V R=\sum_{i}^{N}\left(V_{m_{i}} / \sqrt{V_{x_{i}}^{2}+V_{y_{i}}^{2}+V_{z_{i}}^{2}}\right) / N
$$

Where $i$ refers to the sampling point, $V_{m_{i}}$ refers to the velocity in the main motion direction at the point of $i$, and $V_{x_{i}}, V_{y_{i}}, V_{z_{i}}$ refer to the velocity in $\mathrm{X}, \mathrm{Y}, \mathrm{Z}$ direction at the point of $i$, respectively. $\mathrm{N}$ refers to the total sampling points.

Envelopes of EMG signals were obtained after a full-wave rectification and a low-pass filter at $20 \mathrm{~Hz}$. The muscle activation was calculated for each muscle and mean muscle activation of one muscle was the mean value during the whole sampling time in one tracking movement.

Two-way ANOVA (Analysis of Variance) as a statistical model was applied to test the main effect of compensation strategy (without, with fixed, and with position-varying gravity compensation), tracking direction (upward, forward, leftward, and rightward) and the interaction effect of these two factors on the RMSE, NJS, MVR, and mean muscle activation values. In the following, post-hoc Tukey tests as multiple comparisons were performed to test the difference on RMSE, NJS, and MVR. Paired $t$-tests were then utilized to compare RMSE, NJS, MVR, and mean muscle activation among the three compensation strategies in each direction. The significance level for all statistical tests was set at 0.05 . All statistical analysis was conducted with SPSS version 22.0 (SPSS Inc., Chicago, IL).

\section{RESULTS}

Figures 3A-C displayed RMSE (Figure 3A), NJS (Figure 3B), and MVR (Figure 3C) during movements tracking with different gravity compensation strategies. The results of ANOVA tests were shown in Table 1. As shown in Table 1, both compensation strategy and movement direction showed notable influences on RMSE $(P<0.05)$, NJS $(P<0.05)$, and $\operatorname{MVR}(P<$ 0.05). No significant interaction between compensation strategy and movement direction was found. The results showed that there were significant effects of compensation strategies in ) RMSE and NJS values represented by the following order: without compensation $>$ fixed compensation $>$ positionvarying compensation, and MVR values represented by the following order: without compensation $<$ fixed compensation $<$ position-varying compensation. According to the posthoc analysis, RMSE during upward and forward movements were remarkably higher than those during the leftward and rightward movements while MVR during upward and forward movements were significantly smaller than those during the leftward and rightward movements. Additionally, there was a significant decrease in NJS during upward movements compared with those during rightward movements. Based on paired $t$-test, RMSE of position-varying compensation strategy reduced significantly in leftward and rightward movements when comparing with those of without and fixed compensation strategy. In upward and forward movements, significant difference between the RMSE values of the position-varying and fixed compensation strategies were found. As for NJS, the significant difference between the position-varying and without compensation strategies during upward and forward movements and the significant difference between the position-varying and

\begin{tabular}{|c|c|c|c|c|}
\hline \multicolumn{2}{|c|}{$F$-value } & \multicolumn{2}{|c|}{ Main effects } & \multirow{3}{*}{$\begin{array}{l}\begin{array}{l}\text { Interaction } \\
\text { effect }\end{array} \\
\text { Compensation } \\
\text { Method } \times \text { Target } \\
\text { direction } \\
(\mathrm{DOF}=6)\end{array}$} \\
\hline \multirow{2}{*}{\multicolumn{2}{|c|}{$\begin{array}{l}\text { Outcome } \\
\text { measures }\end{array}$}} & $\begin{array}{l}\text { Compensation } \\
\text { Method }\end{array}$ & Target direction & \\
\hline & & $(\mathrm{DOF}=2)$ & $(\mathrm{DOF}=3)$ & \\
\hline RMSE & & $\begin{array}{l}9.823 \\
(P=0.000)^{*}\end{array}$ & $\begin{array}{l}25.986 \\
(P=0.000)^{\star}\end{array}$ & $\begin{array}{l}1.032 \\
(P>0.050)\end{array}$ \\
\hline NJS & & $\begin{array}{l}6.625 \\
(P=0.002)^{*}\end{array}$ & $\begin{array}{l}2.872 \\
(P=0.042)^{*}\end{array}$ & $\begin{array}{l}0.495 \\
(P>0.050)\end{array}$ \\
\hline MVR & & $\begin{array}{l}18.483 \\
(P=0.000)^{*}\end{array}$ & $\begin{array}{l}8.793 \\
(P=0.000)^{\star}\end{array}$ & $\begin{array}{l}0.276 \\
(P>0.050)\end{array}$ \\
\hline \multirow[t]{6}{*}{$\begin{array}{l}\text { Muscle } \\
\text { activation }\end{array}$} & $\mathrm{BIC}$ & $\begin{array}{l}54.228 \\
(P=0.000)^{*}\end{array}$ & $\begin{array}{l}0.091 \\
(P>0.050)\end{array}$ & $\begin{array}{l}0.842 \\
(P>0.050)\end{array}$ \\
\hline & TRI & $\begin{array}{l}4.415 \\
(P=0.020)^{*}\end{array}$ & $\begin{array}{l}0.928 \\
(P>0.050)\end{array}$ & $\begin{array}{l}0.362 \\
(P>0.050)\end{array}$ \\
\hline & DA & $\begin{array}{l}49.943 \\
(P=0.000)^{*}\end{array}$ & $\begin{array}{l}7.628 \\
(P=0.000)^{*}\end{array}$ & $\begin{array}{l}1.622 \\
(P>0.050)\end{array}$ \\
\hline & DM & $\begin{array}{l}19.882 \\
(P=0.000)^{*}\end{array}$ & $\begin{array}{l}4.197 \\
(P=0.009)^{\star}\end{array}$ & $\begin{array}{l}1.201 \\
(P>0.050)\end{array}$ \\
\hline & $\mathrm{DP}$ & $\begin{array}{l}1.031 \\
(P>0.050)\end{array}$ & $\begin{array}{l}2.594 \\
(P>0.050)\end{array}$ & $\begin{array}{l}0.664 \\
(P>0.050)\end{array}$ \\
\hline & TRA & $\begin{array}{l}18.705 \\
(P=0.000)^{*}\end{array}$ & $\begin{array}{l}3.963 \\
(P=0.011)^{\star}\end{array}$ & $\begin{array}{l}0.429 \\
(P>0.050)\end{array}$ \\
\hline
\end{tabular}

TABLE 1 | The results for all factors involved in ANOVA tests.

*Indicated significant difference $(P<0.05)$. 
without compensation strategies during upward movement were found. All the MVR values with fixed and with position-varying gravity compensation were larger than those without gravity compensation. Comparisons of MVR between each two of three gravity compensation strategies showed significant difference in three directions: upward, leftward, and rightward. The actual trajectories in four movements from a same subject were plotted in Figure 3D.

Figure 4 represented EMG envelope of the six muscles for one subject during the movements tracking with three different compensation strategies. The results indicated that when the subject moved upward, leftward, and rightward with gravity compensation, BIC, DA, DM, and TRA were less activated while he moved rightward, BIC and DA were less activated. Two-way ANOVA showed that effect of compensation strategy on mean muscle activation of BIC, TRI, DA, DM, and TRA $(P<0.05)$ together with effect of direction on mean muscle activation of DA, DM, and TRA were significant $(P<0.05)$. No significant interaction between compensation strategy and movement direction was found. In Figure 5, mean muscle activation of per muscle during four direction movements with different gravity compensation strategies were displayed. Compared with movements without compensation, the mean activation of some muscles during movements with position-varying compensation

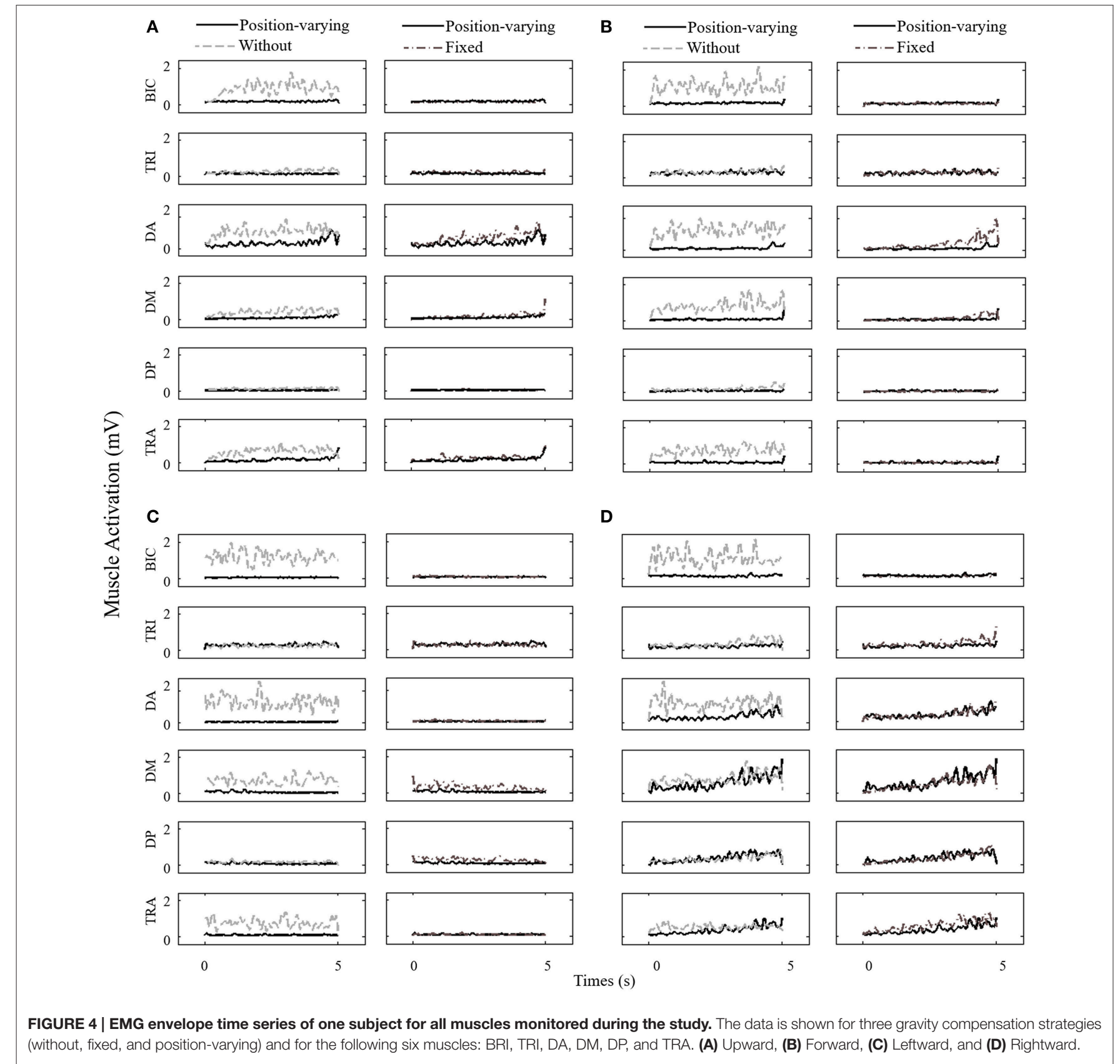




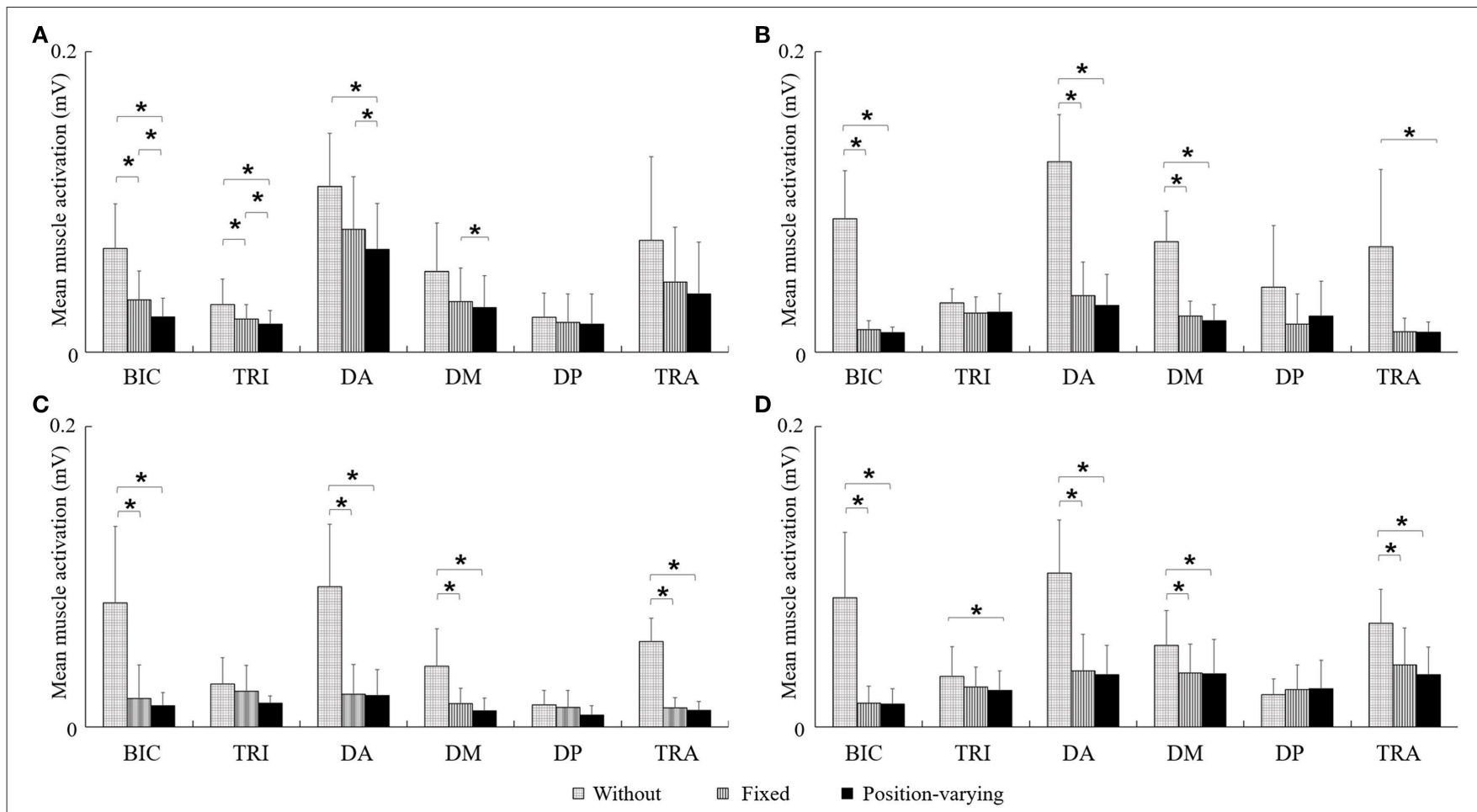

FIGURE 5 | The mean activation of six muscles during four direction movements with different gravity compensation strategies. * Significant difference was found between two kinds of gravity compensation strategies $(P<0.05)$. (A) Upward, (B) Forward, (C) Leftward, and (D) Rightward.

showed significant reductions [i.e., BIC and DA in all four directions, the mean activation of DM and TRA in three directions (forward, leftward, and rightward), and the mean activation of TRI in both upward and rightward]. The results revealed that the activation of BIC, TRI, DA, and DM with position-varying gravity compensation showed a significant decrease compared with fixed compensation during the upward movements.

\section{DISCUSSION}

The purpose of this study was to compare three different gravity compensation strategies by a cable-based robotic system. Kinematics parameters in terms of RMSE, NJS, and MVR together with mean muscle activations were applied to evaluate their movement performance. RMSE can reflect the general tracking accuracy, and is determined by sensory perception, motor planning and execution (Seppänen et al., 2013). The lower RMSE values with position-varying gravity compensation reflected improved tracking accuracy, which was in agreement with previous studies (Grimm et al., 2016). NJS reflected movement smoothness and was generally utilized to evaluate the arm control abilities (Adamovich et al., 2009). The significant decrease in NJS with compensation than without compensation were also reported by Coscia et al. who found significant difference in normalized jerk between without and with gravity compensation during arm reaching movements (Coscia et al., 2014). MVR is utilized to quantify the relative velocity deviation in desired direction during movements tracking. A similar parameter, normalized angular velocity ratio, was proposed by Kim et al. for assessing locomotion precision (Kim et al., 2014). The higher MVR values with position-varying gravity compensation indicated that subjects could more voluntarily participate in the desired movements. The effects of direction on movement kinematics were significant which was in accordance with previous studies in sagittal (Papaxanthis et al., 1998), frontal (d'Avella et al., 2008), or horizontal plane (Beer et al., 2004). Both RMSE and MVR could describe movement accuracy from different points of view, and higher RMSE during upward and forward movements were corresponding to lower MVR. There explanation of the result might be that, the variation of gravity torques during upward and forward movement might be larger.

There were significant reductions in main contributed muscle activations with position-varying and with fixed gravity compensation when compared with without compensation. Previous studies also found that BIC, DA, and TRA were the mainly contributed muscles for gravity support of upper limb, and the activations of these muscles also decreased significantly during movements in horizontal plane (Sabatini, 2002; Prange et al., 2009) and frontal plane (Kloosterman et al., 2010; Coscia et al., 2014). In addition, McCrea et al. proposed that DM was recruited if the primary anti-gravity muscles were not capable during upper limb movements (McCrea et al., 2005). The findings of this study were in accordance with the above-mentioned studies. Although, upper limb muscles activations were dependent on direction (Hughes et al., 2009), 
activations of DP and TRI were not affected by direction in this study. It might be explained by that they were not the main contributed muscles indicated by in low activations in the four directions. As reported, subjects could maintain satisfy dynamic criteria during movements such as optimization of motor command (Nakano et al., 1999) or energy expenditure (Soechting et al., 1995) by appropriately activating arm muscles. The reduction of the mean muscle activation due to gravity compensation indicated that subjects could put more focus on target-directed movements, and result in a larger range of motion or more repetitions of movements. Previous studies also reported subjects had larger range of motion on the horizontal panel with gravity compensation (Housman et al., 2009; Wang and Dounskaia, 2012). Furthermore, muscle activation is related to muscle forces(Lloyd and Besier, 2003), therefore, lower activation of muscles might reflect the robot is able to share the gravity loading, and muscle forces mainly focus on desired movement execution.

This study found that position-varying gravity compensation provided by the cable-based rehabilitation robot could improve man-machine cooperation movements in $3 \mathrm{D}$ working space in terms of RMSE, NJS, and MVR, which was the first explorative investigation on the position-varying gravity compensation to our knowledge. Moreover, since the position-varying gravity compensation strategy could reduce activations of anti-gravity muscles, it could assist patients with muscle weakness to perform rehabilitation training in clinic. The advantages of the positionvarying compensation strategy are located on two aspects: firstly, the assistance is provided to counteract the effect of gravity; secondly, the assistance does not let the training in the passive way, and voluntary residual motor efforts can be focused on target-directed task. The reorganization in brains of subjects after stroke can be facilitated by active rehabilitation training (Cauraugh et al., 2000). In the future, more parameters should

\section{REFERENCES}

Adamovich, S. V., Fluet, G. G., Merians, A. S., Mathai, A., and Qiu, Q. (2009). Incorporating haptic effects into three-dimensional virtual environments to train the hemiparetic upper extremity. IEEE Trans. Neural Syst. Rehabil. Eng. 17, 512-520. doi: 10.1109/TNSRE.2009.2028830

Ball, S. J., Brown, I. E., and Scott, S. H. (2007). "MEDARM: a rehabilitation robot with 5DOF at the shoulder complex," in 2007 IEEE/ASME International Conference on Advanced Intelligent Mechatronics (Zurich), 1-6.

Beer, R. F., Dewald, J. P., Dawson, M. L., and Rymer, W. Z. (2004). Targetdependent differences between free and constrained arm movements in chronic hemiparesis. Exp. Brain Res. 156, 458-470. doi: 10.1007/s00221-003-1807-8

Beer, R. F., Ellis, M. D., Holubar, B. G., and Dewald, J. P. (2007). Impact of gravity loading on post-stroke reaching and its relationship to weakness. Muscle Nerve 36, 242-250. doi: 10.1002/mus.20817

Cauraugh, J., Light, K., Kim, S., Thigpen, M., and Behrman, A. (2000). Chronic motor dysfunction after stroke: recovering wrist and finger extension by electromyography-triggered neuromuscular stimulation. Stroke 31, 1360-1364. doi: 10.1161/01.str.31.6.1360

Cheng, Z., Foong, S., Sun, D., and Tan, U. X. (2015). “Towards a multi-DOF passive balancing mechanism for upper limbs," in 2015 IEEE International Conference on Rehabilitation Robotics (ICORR) (Singapore), 508-513.

Coscia, M., Cheung, V. C., Tropea, P., Koenig, A., Monaco, V., Bennis, C., et al. (2014). The effect of arm weight support on upper limb be adopted to clarify performance of the position-varying gravity compensation, and patients should be employed to explore the clinical effectiveness.

\section{CONCLUSION}

The present study firstly explored the effect of position-varying gravity compensation strategy provided by a cable-based robot on kinematics and muscle activations in comparison with the other two gravity compensation strategies. The improvement in kinematics and less activated anti-gravity muscles with the position-varying gravity compensation indicated its potential in robot-aided rehabilitation therapy. More studies across pathologies with gender-matched subjects are needed to validate whether training with position-varying gravity compensation is clinically feasible and effective.

\section{AUTHOR CONTRIBUTIONS}

YH and RS conceived and designed the study. YH and QY performed the experiments. YH and QY wrote the paper. RS reviewed and edited the manuscript. YH and QY contributed to the work equally and should be regarded as co-first authors. YC made a contribution to experiments. All authors had read and approved the manuscript.

\section{FUNDING}

The project was supported by the National Natural Science foundation of China (Grant No. 61273359 and 91520201), the Guangdong Science and Technology Planning Project (Grant No. 2014B090901056 and 2015B020214003) and the Guangzhou Research Collaborative Innovation Projects (Grant No. 201604020108). muscle synergies during reaching movements. J. Neuroeng. Rehabil. 11:22. doi: 10.1186/1743-0003-11-22

d'Avella, A., Fernandez, L., Portone, A., and Lacquaniti, F. (2008). Modulation of phasic and tonic muscle synergies with reaching direction and speed. $J$. Neurophysiol. 100, 1433-1454. doi: 10.1152/jn.01377.2007

De Luca, A., Siciliano, B., and Zollo, L. (2005). PD control with online gravity compensation for robots with elastic joints: theory and experiments. Automatica 41, 1809-1819. doi: 10.1016/j.automatica.2005. 05.009

Grimm, F., Naros, G., and Gharabaghi, A. (2016). Compensation or restoration: closed-loop feedback of movement quality for assisted reach-to-grasp exercises with a multi-joint arm exoskeleton. Front. Neurosci. 10:280. doi: 10.3389/fnins.2016.00280

Herder, J. L., Vrijlandt, N., Antonides, T., Cloosterman, M., and Mastenbroek, P. L. (2006). Principle and design of a mobile arm support for people with muscular weakness. J. Rehabil. Res. Dev. 43, 591-604. doi: 10.1682/JRRD.2006. 05.0044

Hogan, N., and Sternad, D. (2009). Sensitivity of smoothness measures to movement duration, amplitude, and arrests. J. Mot. Behav. 41, 529-534. doi: 10.3200/35-09-004-RC

Housman, S. J., Scott, K. M., and Reinkensmeyer, D. J. (2009). A randomized controlled trial of gravity-supported, computer-enhanced arm exercise for individuals with severe hemiparesis. Neurorehabil. Neural Repair 23, 505-514. doi: $10.1177 / 1545968308331148$ 
Hsu, L. C., Wang, W. W., Lee, G. D., Liao, Y. W., Fu, L. C., and Lai, J. S. (2012). “A gravity compensation-based upper limb rehabilitation robot," in 2012 American Control Conference (ACC) (Montreal, QC), 4819-4824.

Hughes, A. M., Freeman, C. T., Burridge, J. H., Chappell, P. H., Lewin, P. L., Pickering, R. M., et al. (2009). Shoulder and elbow muscle activity during fully supported trajectory tracking in neurologically intact older people. J. Electromyogr. Kinesiol. 19, 1025-1034. doi: 10.1016/j.jelekin.2008. 09.015

Kahn, L. E., Zygman, M. L., Rymer, W. Z., and Reinkensmeyer, D. J. (2006), Robot-assisted reaching exercise promotes arm movement recovery in chronic hemiparetic stroke: a randomized controlled pilot study. J. Neuroeng. Rehabil. 3:12. doi: $10.1186 / 1743-0003-3-12$

Kim, S. C., Kim, J. Y., Lee, H. N., Lee, H. H., Kwon, J. H., Kim, N. B., et al. (2014). A quantitative analysis of gait patterns in vestibular neuritis patients using gyroscope sensor and a continuous walking protocol. J. Neuroeng. Rehabil. 11:58. doi: 10.1186/1743-0003-11-58

Kloosterman, M. G., Snoek, G. J., Kouwenhoven, M., Nene, A. V., and Jannink, M. J. (2010). Influence of gravity compensation on kinematics and muscle activation patterns during reach and retrieval in subjects with cervical spinal cord injury: an explorative study. J. Rehabil. Res. Dev. 47, 617-628. doi: 10.1682/JRRD.2010.02.0014

Kramer, G., Romer, G. R. B. E., and Stuyt, H. J. A. (2007). "Design of a Dynamic Arm Support (DAS) for gravity compensation," in 2007 IEEE 10th International Conference on Rehabilitation Robotics (Noordwijk), 1042-1048.

Ladenheim, B., Altenburger, P., Cardinal, R., Monterroso, L., Dierks, T., Mast, J., et al. (2013). The effect of random or sequential presentation of targets during robot-assisted therapy on children. Neurorehabilitation 33, 25-31. doi: $10.3233 /$ nre-130924

Lahouar, S., Ottaviano, E., Zeghoul, S., Romdhane, L., and Ceccarelli, M. (2009). Collision free path-planning for cable-driven parallel robots. Robot. Auton. Syst. 57, 1083-1093. doi: 10.1016/j.robot.2009.07.006

Lenzo, B., Fontana, M., Marcheschi, S., Salsedo, F., Frisoli, A., and Bergamasco, M. (2015). Trackhold: a novel passive arm-support device. J. Mech. Robot. 8, 021007. doi: 10.1115/1.4031716

Li, Z., Milutinovic, D., and Rosen, J. (2015). Spatial map of synthesized criteria for the redundancy resolution of human arm movements. IEEE Trans. Neural Syst. Rehabil. Eng. 23, 1020-1030. doi: 10.1109/TNSRE.2014.2382105

Lin, C. H., Lien, W. M., Wang, W. W., Chen, S. H., Lo, C. H., Lin, S. Y., et al. (2014). "NTUH-II robot arm with dynamic torque gain adjustment method for frozen shoulder rehabilitation," in 2014 IEEE/RSJ International Conference on Intelligent Robots and Systems (Chicago, IL), 3555-3560.

Lloyd, D. G., and Besier, T. F. (2003). An EMG-driven musculoskeletal model to estimate muscle forces and knee joint moments in vivo. J. Biomech. 36, 765-776. doi: 10.1016/S0021-9290(03)00010-1

Lum, P. S., Burgar, C. G., Shor, P. C., Majmundar, M., and Van der Loos, M. (2002). Robot-assisted movement training compared with conventional therapy techniques for the rehabilitation of upper-limb motor function after stroke. Arch. Phys. Med. Rehabil. 83, 952-959. doi: 10.1053/apmr.2001. 33101

McCrea, P. H., Eng, J. J., and Hodgson, A. J. (2005). Saturated muscle activation contributes to compensatory reaching strategies after stroke. J. Neurophysiol. 94, 2999-3008. doi: 10.1152/jn.00732.2004

Nakano, E., Imamizu, H., Osu, R., Uno, Y., Gomi, H., Yoshioka, T., et al. (1999). Quantitative examinations of internal representations for arm trajectory planning: minimum commanded torque change model. J. Neurophysiol. 81, $2140-2155$.
Nef, T., Mihelj, M., and Riener, R. (2007). ARMin: a robot for patientcooperative arm therapy. Med. Biol. Eng. Comput. 45, 887-900. doi: 10.1007/s11517-007-0226-6

Papaxanthis, C., Pozzo, T., and Stapley, P. (1998). Effects of movement direction upon kinematic characteristics of vertical arm pointing movements in man. Neurosci. Lett. 253, 103-106. doi: 10.1016/S0304-3940(98)00604-1

Prange, G. B., Kallenberg, L. A., Jannink, M. J., Stienen, A. H., van der Kooij, H., Ijzerman, M. J., et al. (2009). Influence of gravity compensation on muscle activity during reach and retrieval in healthy elderly. J. Electromyogr. Kinesiol. 19, e40-e49. doi: 10.1016/j.jelekin.2007.08.001

Runnalls, K. D., Anson, G., and Byblow, W. D. (2015). Partial weight support of the arm affects corticomotor selectivity of biceps brachii. J. Neuroeng. Rehabil. 12, 94. doi: 10.1186/s12984-015-0085-6

Sabatini, A. M. (2002). Identification of neuromuscular synergies in natural upperarm movements. Biol. Cybern. 86, 253-262. doi: 10.1007/s00422-001-0297-7

Seppänen, T. M., Alho, O.-P., and Seppänen, T. (2013). Reducing the airflow waveform distortions from breathing style and body position with improved calibration of respiratory effort belts. Biomed. Eng. Online 12:97. doi: 10.1186/1475-925X-12-97

Soechting, J. F., Buneo, C. A., Herrmann, U., and Flanders, M. (1995). Moving effortlessly in three dimensions: does Donders' law apply to arm movement? J. Neurosci. 15, 6271-6280.

Staubli, P., Nef, T., Klamroth-Marganska, V., and Riener, R. (2009). Effects of intensive arm training with the rehabilitation robot ARMin II in chronic stroke patients: four single-cases. J. Neuroeng. Rehabil. 6:46. doi: 10.1186/1743-0003-6-46

Stienen, A. H. A., Hekman, E. E. G., Prange, G. B., Jannink, M. J. A., Aalsma, A. M. M., van der Helm, F. C. T., et al. (2009). Dampace: design of an exoskeleton for force-coordination training in upper-extremity rehabilitation. J. Med. Device 3, 031003. doi: $10.1115 / 1.3191727$

Stopforth, R. (2013). Control investigation of a customizable/adjustable exoskeleton upper-limb. Ind. Robot 40, 132-142. doi: 10.1108/014399113 11297739

van Elk, M. G., Driessen, B. J. F., Dorrepaal, M., van der Werff, J. J., van der Meche, E. G., and Aulbers, A. P. (2005). "A motorized gravity compensation mechanism used for active rehabilitation of upper limbs," in 9th International Conference on Rehabilitation Robotics, ICORR (Chicago, IL), 152-155.

Wang, W., and Dounskaia, N. (2012). Load emphasizes muscle effort minimization during selection of arm movement direction. J. Neuroeng. Rehabil. 9:70. doi: 10.1186/1743-0003-9-70

Yang, J., Su, H., Li, Z., Ao, D., and Song, R. (2016). Adaptive control with a fuzzy tuner for cable-based rehabilitation robot. Int. J. Control Autom. Syst. 14, 865-875. doi: 10.1007/s12555-015-0049-4

Yuan, P., Wang, Q., Shi, Z., Wang, T., Wang, C., Chen, D., et al. (2014). A microadjusting attitude mechanism for autonomous drilling robot end-effector. Sci. China Inform. Sci. 57, 1-12. doi: 10.1007/s11432-014-5190-9

Conflict of Interest Statement: The authors declare that the research was conducted in the absence of any commercial or financial relationships that could be construed as a potential conflict of interest.

Copyright (๑) 2017 Huang, Yang, Chen and Song. This is an open-access article distributed under the terms of the Creative Commons Attribution License (CC BY). The use, distribution or reproduction in other forums is permitted, provided the original author(s) or licensor are credited and that the original publication in this journal is cited, in accordance with accepted academic practice. No use, distribution or reproduction is permitted which does not comply with these terms. 


\title{
Development and Feasibility Assessment of a Rotational Orthosis for Walking with Arm Swing
}

\author{
Juan Fang ${ }^{1,2}$, Qing Xie ${ }^{3}$, Guo-Yuan Yang ${ }^{2}$ and Le Xie ${ }^{2,4 *}$ \\ 1 Jiangsu Key Laboratory of Advanced Food Manufacturing, Equipment and Technology, Jiangnan University, Wuxi City, \\ China, ${ }^{2}$ The Joint Lab of the Institute of Rehabilitation Centre and Chejing Robotics Technology (Shanghai) Co., Ltd., Med-X \\ Research Institute, Shanghai Jiao Tong University, Shanghai, China, ${ }^{3}$ Department of Rehabilitation Medicine of Ruijin \\ Hospital, School of Medicine, Shanghai Jiao Tong University, Shanghai, China, ${ }^{4}$ School of Material Science and Engineering, \\ Shanghai Jiao Tong University, Shanghai, China
}

\section{OPEN ACCESS}

Edited by:

Venketesh N. Dubey, Bournemouth University, UK

Reviewed by:

Brent Winslow,

Design Interactive, USA

Hans-Eckhardt Schaefer,

University of Stuttgart, Germany

*Correspondence:

Le Xie

lexie@sjtu.edu.cn

Specialty section:

This article was submitted to

Neural Technology,

a section of the journal

Frontiers in Neuroscience

Received: 01 October 2016

Accepted: 16 January 2017

Published: 01 February 2017

Citation:

Fang J, Xie Q, Yang G-Y and Xie L (2017) Development and Feasibility Assessment of a Rotational Orthosis

for Walking with Arm Swing

Front. Neurosci. 11:32.

doi: 10.3389/fnins.2017.00032
Interlimb neural coupling might underlie human bipedal locomotion, which is reflected in the fact that people swing their arms synchronously with leg movement in normal gait. Therefore, arm swing should be included in gait training to provide coordinated interlimb performance. The present study aimed to develop a Rotational Orthosis for Walking with Arm Swing (ROWAS), and evaluate its feasibility from the perspectives of implementation, acceptability and responsiveness. We developed the mechanical structures of the ROWAS system in SolidWorks, and implemented the concept in a prototype. Normal gait data were used as the reference performance of the shoulder, hip, knee and ankle joints of the prototype. The ROWAS prototype was tested for function assessment and further evaluated using five able-bodied subjects for user feedback. The ROWAS prototype produced coordinated performance in the upper and lower limbs, with joint profiles similar to those occurring in normal gait. The subjects reported a stronger feeling of walking with arm swing than without. The ROWAS system was deemed feasible according to the formal assessment criteria.

Keywords: interlimb neural coupling, arm swing, normal gait, coordinated movement, rehabilitation robotics

\section{INTRODUCTION}

People swing their arms synchronously with leg movement during walking due to interlimb neural linkage, in addition to mechanical factors. Although the arms have no direct function for propulsion (Barbeau et al., 1987), people normally swing their arms so as to improve gait stability (Behrman and Harkema, 2000; Bovonsunthonchai et al., 2012) and energy efficiency (Dietz, 2002; Collins et al., 2009). Apart from such behavioral relevance, many phenomena imply that arm swing during walking is a neural-coordinated motor output. Rhythmic muscle activity was observed in the constrained arms during walking overground (Eke-Okoro et al., 1997), which implies the existence of neural coupling between the upper and lower limbs. Furthermore, adding mass to one ankle induced adaptive changes in both arms, in addition to changes in EMG from the leg muscles (Donker et al., 2002). This resulted in a coordinated movement pattern similar to that seen in unloaded normal gait. Studies of walking on a split-belt treadmill with different speed ratios between the legs resulted in coordinated locomotion in the legs and arms (Dietz et al., 2001). Short accelerations or decelerations randomly applied to the right leg during treadmill walking produced EMG response in the bilateral arm muscles, in addition to that in the right leg (Dietz et al., 2001). Interlimb neural interaction thus appears to be an underlying neural mechanism of human bipedal locomotion. 
The theory of interlimb neural coupling brings new requirements for gait rehabilitation robotics. Interlimb modulation is active during walking, but not in standing or sitting (Dietz et al., 2001; Zehr et al., 2012). The neural interaction between the upper and lower limbs is maintained in patients with injury to central nervous system (Visintin and Barbeau, 1994; Stephenson et al., 2010). The task-specific practice strategy suggests gait restoration robotic systems should provide locomotion-like movements to improve gait control and functional ability (Harkema, 2001). Judging from the implication of interlimb neural coupling and the fact of arm swing during walking, it was suggested that gait training after neurological injury should incorporate simultaneous upper limb and lower limb rhythmic exercise to take advantage of neural coupling (Ferris et al., 2006).

In spite of the existence of many types of rehabilitation robots, there is no system which activates both the upper and lower limbs in the same way as during walking. Over the last few decades many types of rehabilitation systems have emerged, including systems for gait restoration (Díaz et al., 2011) or for upper limb rehabilitation (Lum et al., 2005). Several lowerlimb exoskeletons are commercially available to assist walking restoration, such as the Lokomat (Hidler et al., 2008) and the G-EO (Hesse et al., 2010) systems. They induce upright walking movement at variable speeds in the lower limbs. The arms often hold horizontal fixed bars to support the body. There are also several systems, such as the Armeo (Nef et al., 2006) and GENTLE/s (Loureiro et al., 2003), for those who have functional impairments in the upper limbs. The users often practice various arm movements in a sitting position. To the best of the authors' knowledge, there is no gait orthosis which incorporates arm swing.

Based on these limitations, a new rehabilitation system was to be developed in the present work. As the early initiation of gait rehabilitation is generally deemed important (Fang et al., 2011), the requirements of the proposed system included:

(1) to allow the users to practice walking at the early post-injury stage;

(2) to mimic the ground reaction forces on the foot which occur during walking;

(3) to activate the upper limbs synchronously with the lower limb movement.

The aim of this work was to develop and evaluate the feasibility of a Rotational Orthosis for Walking with Arm Swing (ROWAS). The formal criteria for feasibility assessment were (Bowen et al., 2009): (i) implementation-was the system technically implementable? (ii) acceptability-was the system acceptable to the users? and (iii) responsiveness-was there a measurable movement that was close to the target joint trajectories?

\section{METHODS}

The starting point for this work was a gait analysis experiment to determine the target joint trajectories for the ROWAS system. After the ROWAS was developed and implemented as a prototype, a feasibility assessment was conducted using the formal feasibility criteria of implementation, acceptability and responsiveness.

\section{Gait Analysis Experiment}

In order to provide reference data for the design of a system actuating both the upper and lower limbs, a gait experiment was performed using a Vicon motion analysis system (Oxford Metrics Ltd., Oxford, UK) in Ruijin Hospital, Shanghai Institute of Orthopaedics and Traumatology, China. Ethical approval was obtained from the Ethics Committee at the Med-X Research Institute, Shanghai Jiao Tong University, Shanghai, China. Twenty-four able-bodied subjects were recruited and they provided written informed consent prior to participation.

The experimental set-up consisted of a ten-camera Vicon MX13 motion capture system (Vicon Peak, Oxford, UK). Thirtynine reflective markers of $14 \mathrm{~mm}$ diameter were affixed to specific anatomical landmarks (Plug-In Gait Marker Set, Vicon Peak, Oxford, UK) to record the segmental trajectories with a sampling frequency of $100 \mathrm{~Hz}$ (Kadaba et al., 1990). The details of this gait experiment can be found in our previous paper (Fang et al., 2016).

The angle trajectories of the shoulder, hip, knee and ankle joints within a gait cycle during overground walking were analyzed. The duration of one gait cycle was normalized to $100 \%$, with heel strikes at 0 and $100 \%$. The joint data were visually observed to remove outliers, and smoothed with the loess or rloess Matlab functions. The joint data from the overground walking experiment provided reference information of the joint profiles for the ROWAS system.

\section{Development of the ROWAS System}

Based on the design requirements, the ROWAS system was implemented as a rotational bed linking frames for the upper and lower limbs. To achieve the required functional features, modules for a retractable wheel-set, a ground-simulation plate and a foot platform were further developed. A 3D CAD design software tool (SolidWorks, Version 2016, Dassault Systèmes SolidWorks Corporation, Massachusetts, the USA) was used to develop the structure of the ROWAS system.

\section{Rotational Bed Frame}

In order to provide rehabilitation to users who are in an early post-injury phase, a rotational bed frame was developed, which allows practice in various positions. Depending on their physical situation, users can initiate walking training in a lying position (Figure 1A). Then as the rehabilitation progresses, the users can gradually be tilted up until in an upright position (Figure 1B).

We adopted a linear actuator MD60 from the Moteck Electric Corporation to rotate the bed up. As Figure 2 shows, the linear actuator allows the bed plate to be: (i) in a lying position when it is retracted to the shortest length $d$; (ii) in an upright position when it is extended to its longest length $p$. The following equations 

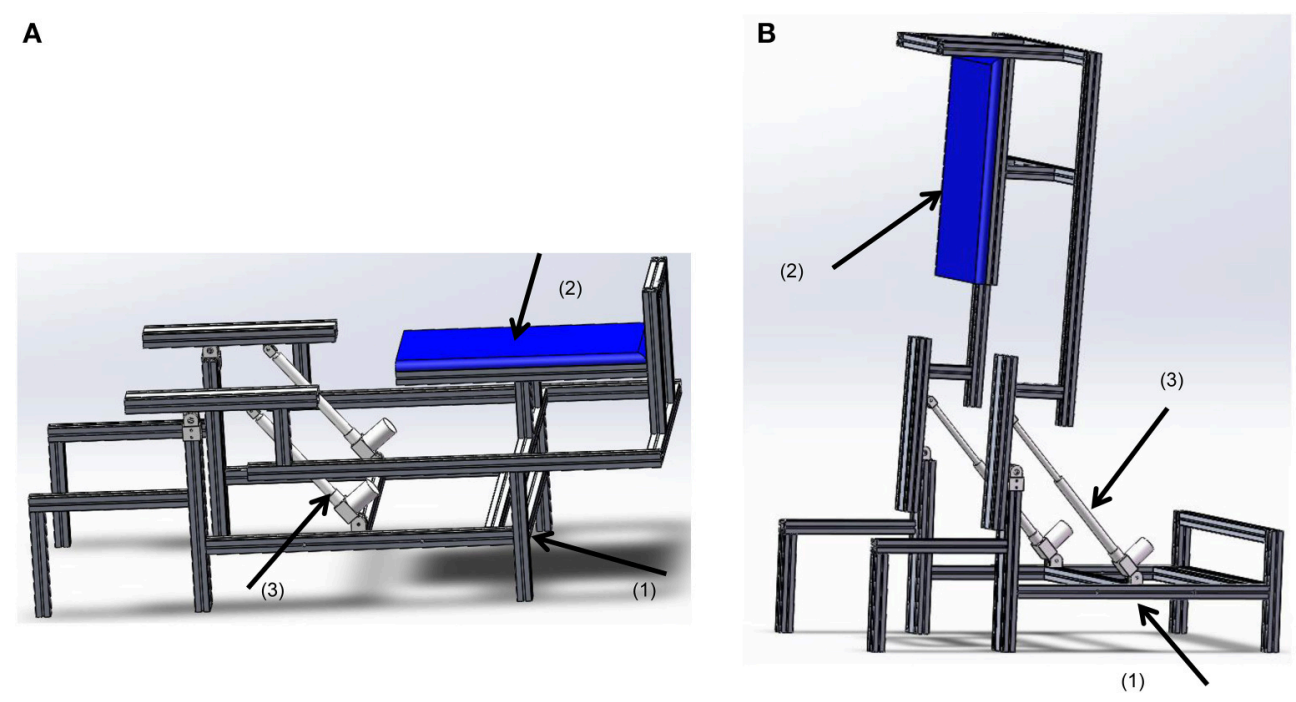

FIGURE 1 | The rotational bed. (1) The bed base, (2) the bed plate, and (3) the linear actuator MD60. (A) In a lying position, (B) in an upright position.

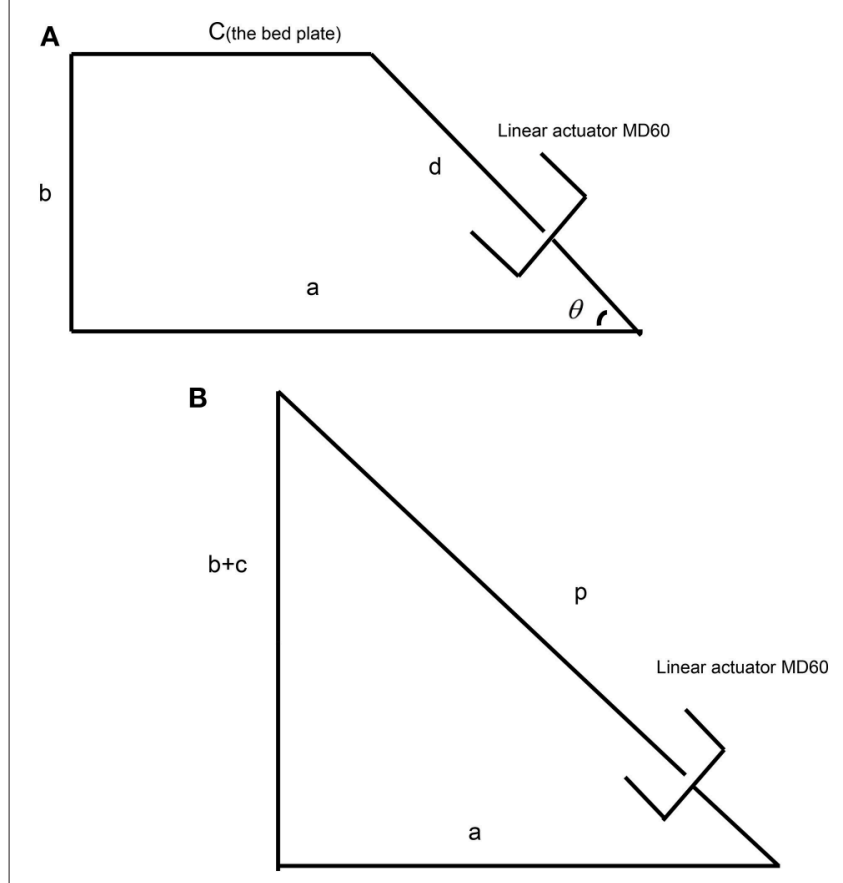

FIGURE 2 | The setup of the linear actuator for the simplified rotational bed. (A) The actuator in the retracted state, $(\mathbf{B})$ the actuator in the extended state.

describe the device geometry:

$$
\begin{aligned}
d^{2} & =(a-c)^{2}+b^{2} \\
p^{2} & =(b+c)^{2}+a^{2} \\
\theta & =\arctan \frac{b}{a-c}
\end{aligned}
$$

We assumed the original height of the bed was $400 \mathrm{~mm}$, i.e., $b=$ 400. To secure an acceptable efficiency of the actuator, the angle of the actuator and the horizontal level $\theta$ should be larger than $45^{\circ}$. For the chosen linear actuator noted above, stroke $=300$ $\mathrm{mm} ; d=530 \mathrm{~mm} ; p=830 \mathrm{~mm}$; thus meeting these requirements. Using Equations (1) and (2), $a=560.2 \mathrm{~mm}$ and $c=212.5 \mathrm{~mm}$. In this case, the angle between the linear actuator and the horizontal level $\theta$ was about $50^{\circ}$, which satisfies the above requirement.

\section{Retractable Wheel Support}

For ease of translation, a wheel-set was employed at the bottom of the bed frame. However, during gait training, the system should be supported by a stable base. Thus, a retractable wheel support was designed with the requirements: (i) to support the system when the system was to be moved; (ii) to allow the system to be supported by the bed leg when the system is in use for rehabilitation training.

The wheel-set was attached by four parallel bars, which are lifted by a linear actuator. When the system is used for rehabilitation training, the wheel-set is retracted under the bed (Figure 3A) and the system is supported by the bed legs. When pushed by the linear actuator, the wheel-set was extended to the lowest position (Figure 3B), and the system is lifted up and supported by the wheels. The geometry for this linear actuator is similar to Equations (1)-(3). We also chose an MD21 from Moteck Electric Corporation, but with a stroke of $150 \mathrm{~mm}$.

\section{The Ground-Simulation Plate and Foot Platform}

We designed a plate to support the leg during simulation of the stance phase. According to our systematic study on toe trajectories (Fang et al., 2016), it was found that the toe trajectory relative to the hip joint is curved. Therefore, the groundsimulation plate (Figure 4) was designed to have two ends taking a curved shape. 

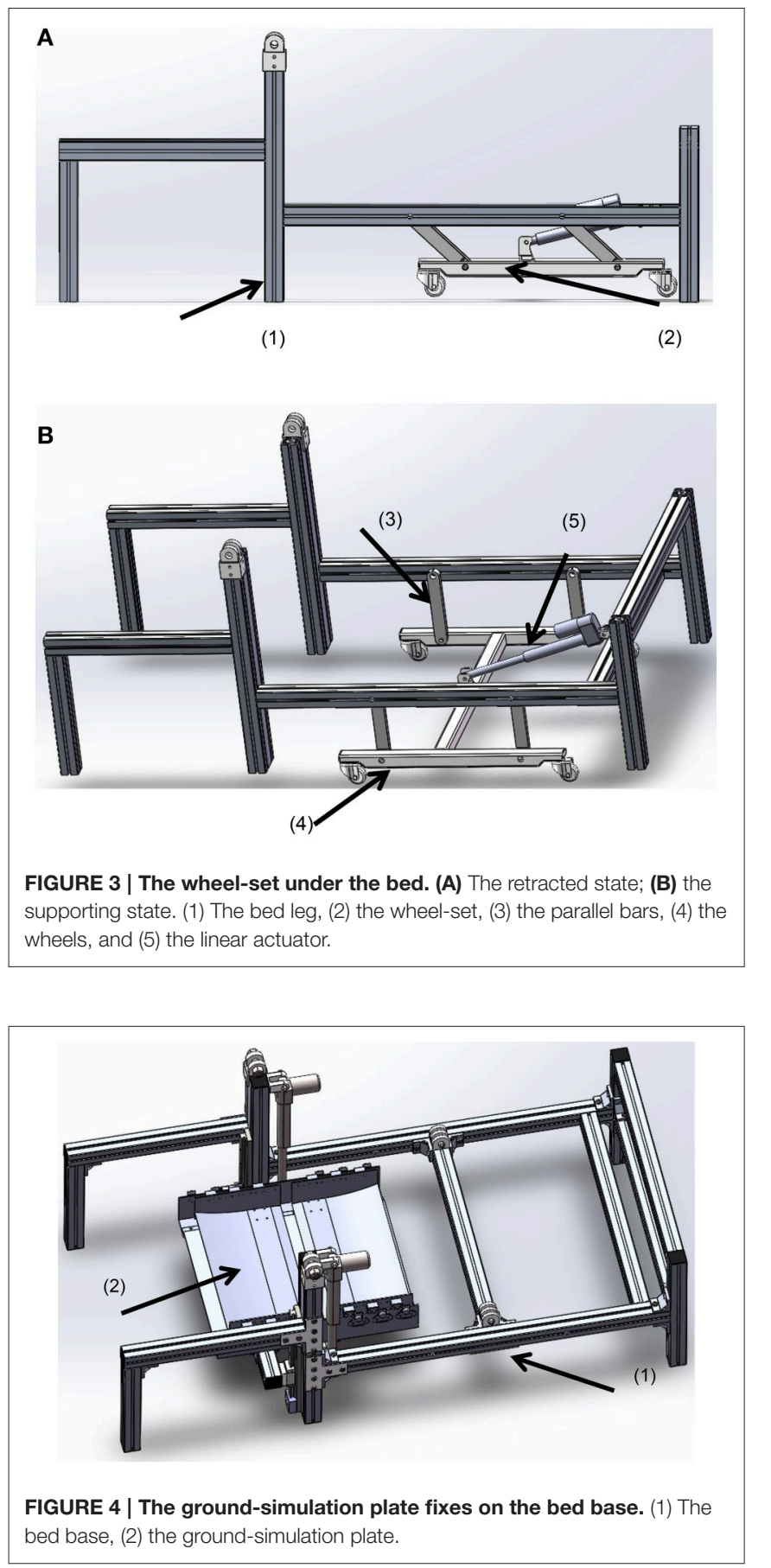

In order to enable the subject to walk on the groundsimulation plate, we further designed a shoe platform (Figure 5) to support the foot and simulate the ground reaction force. With six wheels at the bottom, the shoe can roll on the ground-simulation plate. Two air bags were fixed on the shoe sole to simulate the events of heel-strike and toe-off. The springs between the foot base and the wheels were to provide space between the shoe platform and the ground-simulation plate.

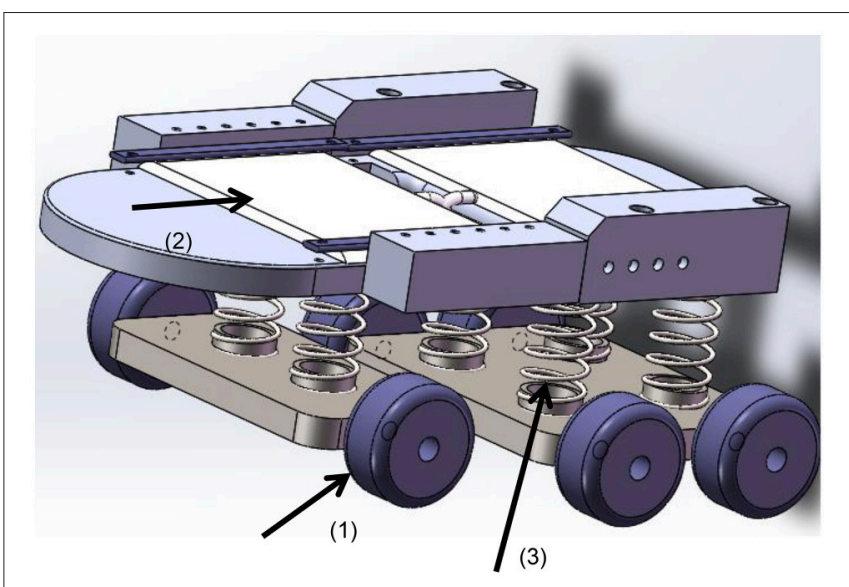

FIGURE 5 | The shoe platform. (1) Wheels, (2) air bag, (3) springs.

\section{Joint Drives}

For ease of control, we mounted the drives for the shoulder, knee, and ankle joints bilaterally at the sides (Figure 6). In order to get the upper limbs, especially the forearms, swing naturally as in normal walking, the hip joints require free space on each lateral side. Thus, we positioned the hip drives between the legs, as shown in Figure 6B. The drive for the right hip was mounted pointing to the joint center, while the drive for the left hip was mounted behind the left joint and the power was transmitted to the joint by a synchronous belt.

The rigs for the upper and lower limbs were designed adjustable in length to align the mechanical joints with the human joints. Size adjustment was implemented using a sliding nut that could be locked at any position on the frame by a clamp. The rigs for the lower limbs were covered with plates, which could support the leg during the transfer of the patient to the system.

\section{The Whole ROWAS System}

With a wheel set support at the bottom, the system finally included two leg rigs and two upper limbs rigs which were fixed on a rotational bed (Figure 7). Secured by the body-weight support system, the users lie upon the bed. After their arms and legs are fixed to the upper and lower limb frames, the bed is tilted to a position where the users feel comfortable. Then the user starts walking training with arm swing. If the users can be tilted to an upright position, the shoe platforms roll on the ground simulation plate. When the system needs to be moved, the wheel-set can be lowered.

The ROWAS prototype was manufactured as illustrated (Figure 8). Using strut profiles (Bosch Rexroth group), the bed frame was manufactured. The other structures were manufactured using aluminum alloy. The support frame and the joints of the ROWAS system had to be strong enough to transfer the necessary forces and torques to support a subject. To ensure this, the exoskeleton should be capable of developing the joint torques of a person of $135 \mathrm{~kg}$ 
A
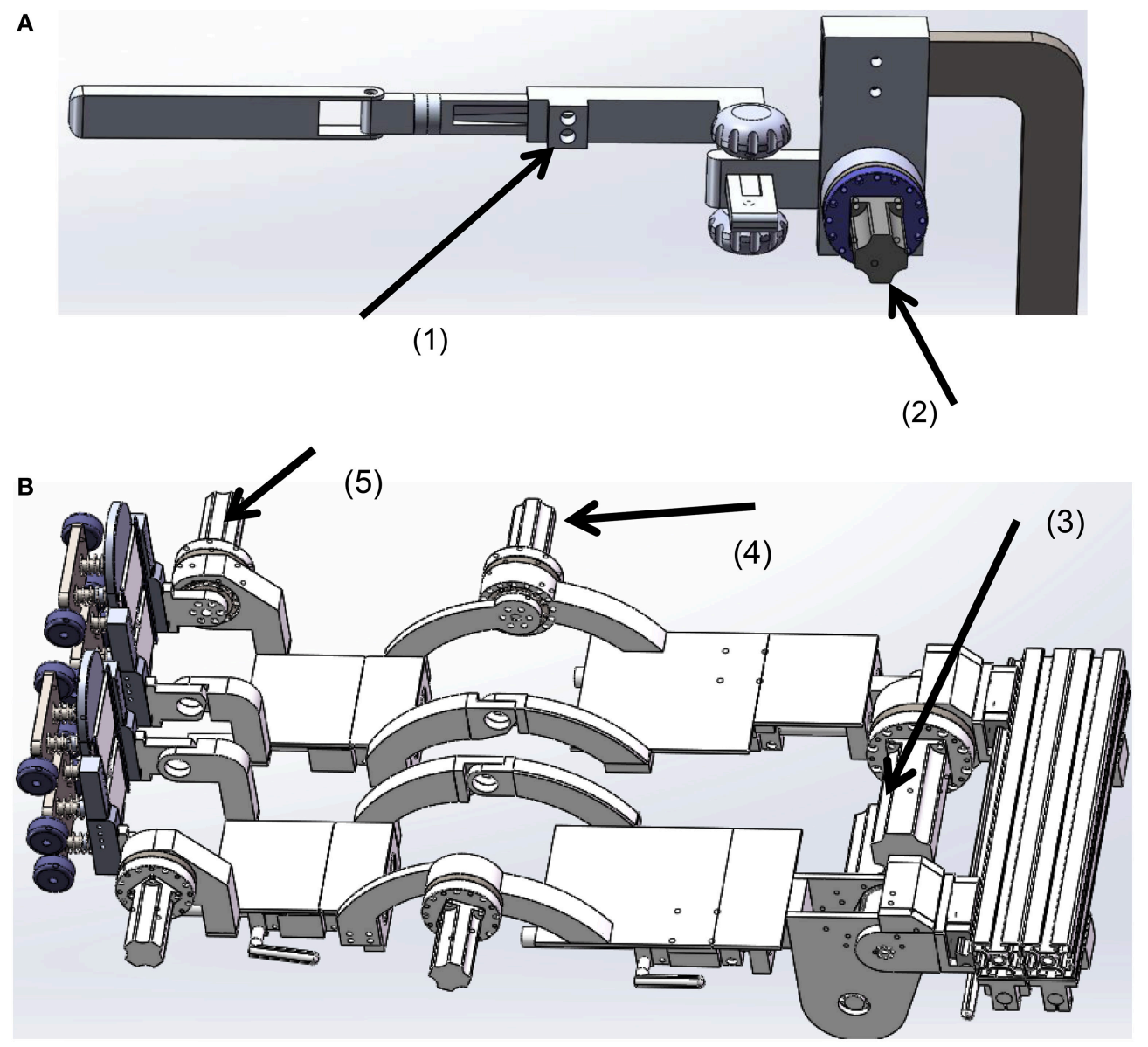

FIGURE 6 | The joint drives. (A) The frame for the left upper limb, (B) the frame for the lower limbs. (1) The sliding nut with a clamp, (2) the shoulder drive, (3) the hip drive, (4) the knee drive, (5) the ankle drive.

walking at various speeds in various postures. For supine and upright walking, the maximal loads were derived from data presented in the literature (Fang et al., 2014a). The motors we selected were Yaskawa AC motors with harmonic gearboxes. The drive components for the two hip joints were a combination of a 400W AC motor (SGM7J-04AFC6S+SGD7S2R8A00A) and a gearbox with ratio of 160 (LCSG-25-160), while the drive assemblies for the shoulder, knee and ankle joints were a combination of a 100W AC motor (SGM7J01AFC6S+SGD7S-R90A00A) and a gearbox with ratio of 120 (LCSG-17-120).

An eight-axis Googol motion controller (GTS-800-PVVB-PCI, GoogolTech Limited) was adopted to control the joint motors. The joint performance data from the overground experiment was the target movement for motors. To obtain satisfactory movement, the time course of each joint angle was fit using Fourier series functions. The fitted reference movements were sent to the corresponding motors via $\mathrm{C}++$ software. Then the PID control algorithm imbedded in the Googol motion controller was employed to move the ROWAS prototype according to the target joint profiles.

\section{Experimental Evaluation of the ROWAS Prototype}

Experimental evaluation had two subtests: a test on the prototype to assess only the system function, and tests on able-bodied subjects to collect user feedback on the prototype. As a preliminary trial on this novel training modality, we recruited five able-bodied subjects to evaluate the performance (Table 1). Ethical approval was obtained from the Ethics Committee at the Med-X Research Institute, Shanghai Jiao Tong University, Shanghai, China. Subjects provided written informed consent prior to participation.

\section{Experimental Procedure}

During the experiment, the system produced walking at a cadence of 30 steps $/ \mathrm{min}$. In the preliminary test on the prototype only, the bed frame was rotated up at a speed of $1.5^{\circ} / \mathrm{s}$ to $75^{\circ}$ relative to the ground. Then the system moved the frames for the upper and lower limbs. During the tests on the able-bodied subjects, we firstly collected their anthropometric data (Table 1). We then asked them to lie on the bed and secured them with the body-weight support system. After fixing their arms and lower 


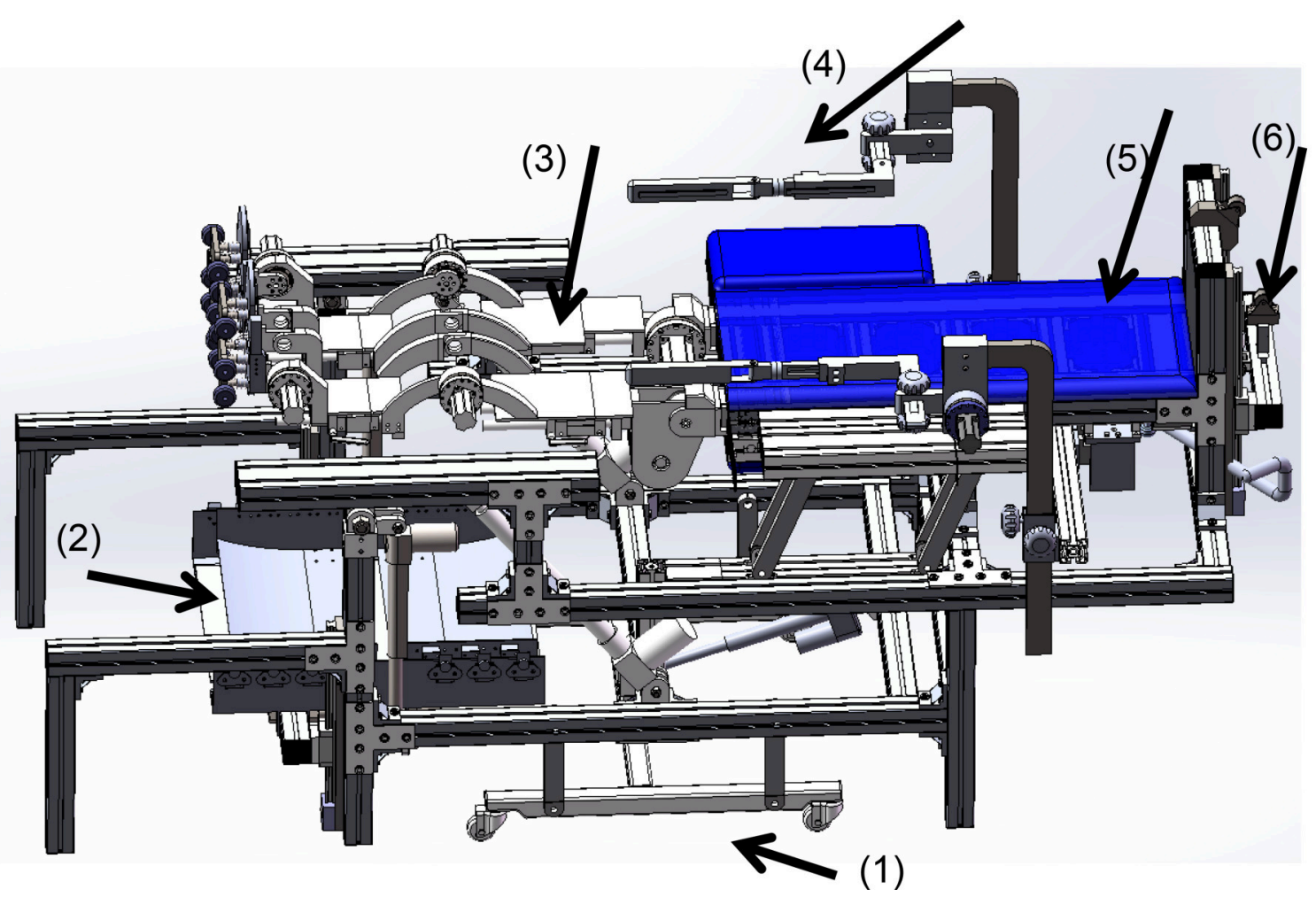

FIGURE 7 | Schematic presentation of the ROWAS system. (1) The retractable wheel support (extended state), (2) the ground-simulation plate, (3) the rigs for the lower limbs, (4) the rigs for the upper limbs, (5) the rotational bed plate and (6) the body-weight support system.

A

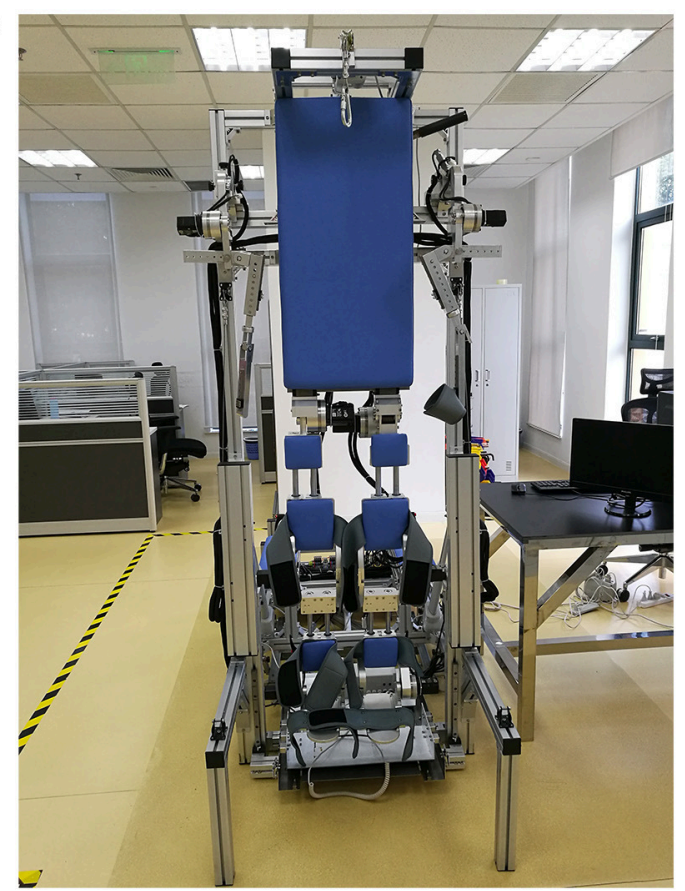

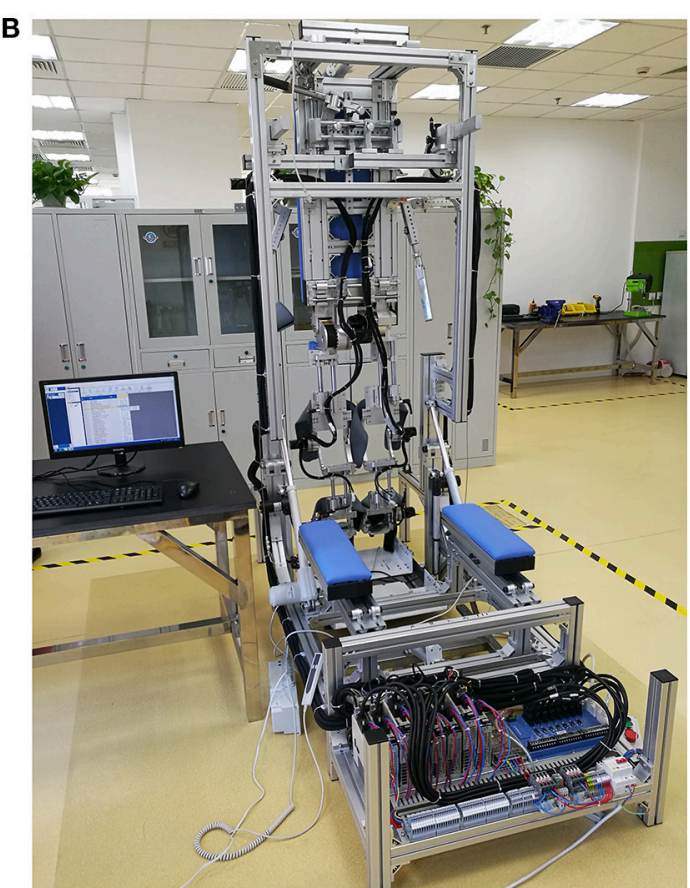

FIGURE 8 | The ROWAS prototype. (A) The front view, (B) the back view. 
TABLE 1 | Subject information.

\begin{tabular}{|c|c|c|c|c|c|c|}
\hline Subject & Age (years) & Sex & Body mass (kg) & Height (m) & Thigh length (mm) & Shank length (mm) \\
\hline S1 & 32 & $\mathrm{~F}$ & 55 & 1.60 & 390 & 350 \\
\hline S2 & 28 & $\mathrm{M}$ & 78 & 1.78 & 430 & 410 \\
\hline S3 & 28 & $\mathrm{M}$ & 60 & 1.70 & 410 & 370 \\
\hline S4 & 23 & $\mathrm{M}$ & 71 & 1.72 & 440 & 390 \\
\hline S5 & 29 & $\mathrm{M}$ & 66.7 & 1.72 & 430 & 380 \\
\hline Mean \pm SD & $28 \pm 3.2$ & NA & $66.1 \pm 9.0$ & $1.70 \pm 0.065$ & $420 \pm 20$ & $380 \pm 22.4$ \\
\hline
\end{tabular}

limbs with Velcro straps, we rotated the bed to a position which was $75^{\circ}$ relative to the ground. Finally we actuated the joint motors. The subjects tried to totally relax and follow the motion that the prototype produced. The test on each subject included two sessions: (i) the prototype actuated both the upper and lower limbs; (ii) it only moved the lower limbs, while the upper limb frames were left unactuated. Please see the Supplementary Video to see the performance of a representative subject during the test. The prototype produced walking for $45 \mathrm{~s}$ in each session. The joint performance was recorded via encoders. After the test, all the subjects were asked the questions below so as to collect their feedback:

(1) Did you have any sense of insecurity during the process of bed rotation, walking with arm swing or walking without arm movement?

(2) Did you feel any discomfort when using the system? If yes, please describe it.

(3) Which type of motion did you feel was more like walking: walking with arm swing or walking without arm movement?

(4) Do you have any suggestions for system improvement?

\section{Data Analysis}

To compare the joint movements produced by the ROWAS prototype with those recorded during overground walking, three parameters were analyzed: difference of ROM $\left(\mathrm{R}_{\text {error }}\right)$, phase shift $\left(\mathrm{T}_{\text {error }}\right)$ and root-mean-square error $\left(\mathrm{RMS}_{\text {error }}\right)$ for the angle performance (Fang et al., 2011, 2014b). The ROM of the hip joint from the overground walking experiment and the ROWAS test were $\mathrm{R}_{\mathrm{OH}}$ and $\mathrm{R}_{\mathrm{RH}}$, respectively, while the onsets of maximal hip extension in the overground walking experiment and the ROWAS test within one gait cycle $\mathrm{T}$ were $\mathrm{T}_{\mathrm{OH}}$ and $\mathrm{T}_{\mathrm{RH}}$. The angle amplitudes of the hip joint from the ROWAS and the overground walking were $\mathrm{A}_{\mathrm{OH}}$ and $A_{R H}$. The differences of the ROM, phase and RMS regarding the hip joint $\left(\mathrm{R}_{\text {errorH }}, \mathrm{T}_{\text {errorH }}\right.$ and $\left.\mathrm{RMS}_{\text {errorH }}\right)$ between overground walking and the ROWAS test were obtained from Eqs (4-6). A similar procedure was adopted to compare the ROM difference, phase shift and RMS difference of the shoulder ( $\mathrm{R}_{\text {errors}}, \mathrm{T}_{\text {errors, }} \mathrm{RMS}_{\text {errors }}$ ), knee ( $\mathrm{R}_{\text {errork }}, \mathrm{T}_{\text {errork, }}$ $\left.\mathrm{RMS}_{\text {errorK }}\right)$ and ankle $\left(\mathrm{R}_{\text {errorA }}, \mathrm{T}_{\text {errorA }}, \mathrm{RMS}_{\text {errorA }}\right)$ angle profiles from overground walking and the experiment. In order to obtain the phase shift of the shoulder and knee joints, the onsets of the maximal shoulder extension and knee flexion were analyzed. Thus,

$$
\begin{aligned}
R_{\text {errorH }} & =\frac{R_{R H}-R_{O H}}{R_{O H}} \times 100 \% 0 \\
T_{\text {errorH }} & =\frac{T_{R H}-T_{O H}}{T} \times 100 \% 0 \\
R M S_{\text {errorH }} & =\frac{\sqrt{\frac{1}{N} \sum_{i=1}^{N}\left(A_{R H}-A_{O H}\right)^{2}}}{R_{O H}} \times 100 \% 0
\end{aligned}
$$

\section{Criteria for Feasibility Assessment}

The criteria employed for assessment of the feasibility of the ROWAS system were (Bowen et al., 2009): (i) implementation-was the system technically implementable? (ii) acceptability - was the system acceptable to the users? and (iii) responsiveness-was there a measurable movement that was close to the target joint trajectories? The ROWAS was considered to have satisfied responsiveness criteria if $\mathrm{R}_{\text {error }}, \mathrm{T}_{\text {error, }}$, and $\mathrm{RMS}_{\text {error }}$ were less than $10 \%$.

\section{RESULTS}

When the system was in a horizontal position, the dimension of the whole system was $2145 \mathrm{~mm}$ in length and $850 \mathrm{~mm}$ in width. The bed surface was $755 \mathrm{~mm}$ above the ground. It could be used by subjects with a height between 1.50 and $1.85 \mathrm{~m}$. When the system was tilted up to be $90^{\circ}$ relative to the ground (vertical), it was $2345 \mathrm{~mm}$ in height.

The target performance of the ROWAS system was taken from the gait experiment as described above. The joint angles of a representative subject with a height of $1.72 \mathrm{~m}$ were selected for illustration (the dotted lines in Figure 9). The fit data from the calculated Fourier functions served as the final reference values for the prototype (the dashed lines in Figure 9).

In the preliminary test on the prototype only, we applied a PID controller to actuate the joints. We observed that the parameters $\mathrm{kp}=2$ (proportional gain), $\mathrm{ki}=0$ (integral parameter) and $\mathrm{kd}=0$ (derivative parameter) provided acceptable results (the solid lines in Figure 9). During the test on able-bodied subjects, we applied the same PID parameters. The angle performance from the all subjects was close to that of preliminary tests on the prototype without users (dash-dot lines with shading in Figure 9). Compared to overground walking, the joint angle 

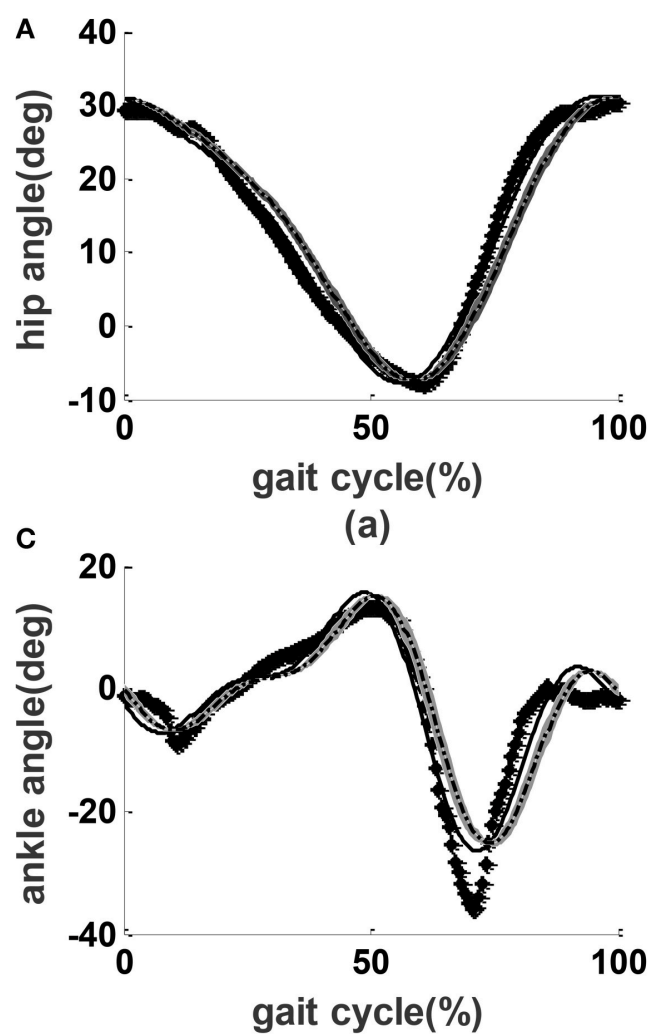

(c)

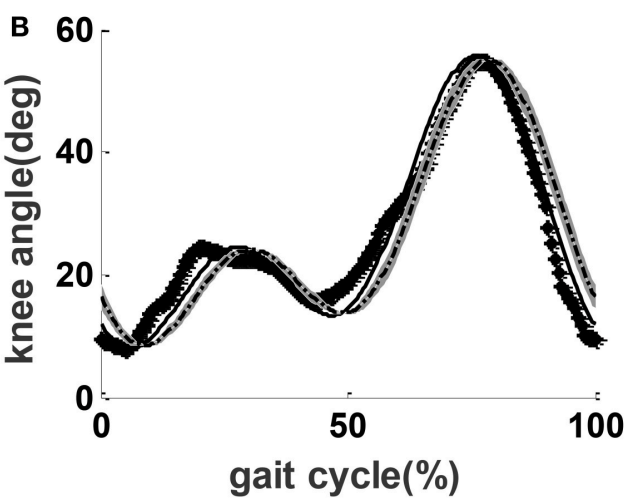

(b)

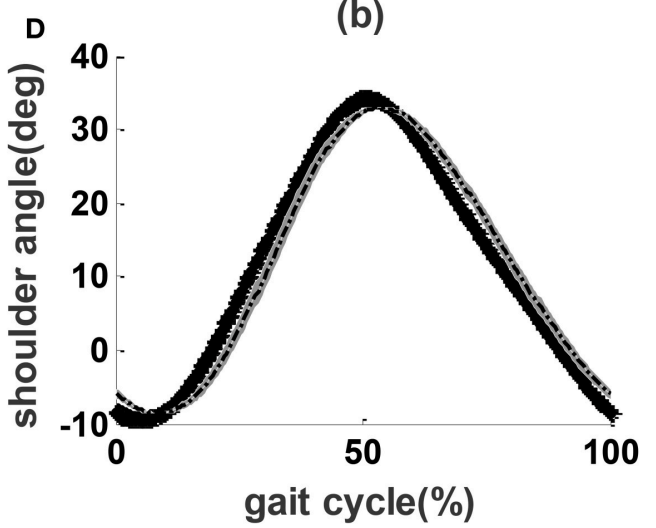

(d)

FIGURE 9 | Joint performance. Lines with diamonds: measured experimental values; dashed lines: reference values; solid lines: the encoder readings from the prototype without users; dash-dot lines with shading: the mean \pm SD performance from the prototype with users. (A) Hip angles, (B) knee angles, (C) ankle angles, (D) shoulder angles.

performance had a phase shift of about 3.5\%, mean ROM errors of less than $8 \%$, and RMS errors of less than $9 \%$ (Table 2).

Feedback obtained from the subjects showed that S2, S4, and S5 had no sense of insecurity. S1 and S3 reported a sense of insecurity when tilted up, but not during walking sessions. All users reported a stronger sense of walking when the arms were actuated compared to when they were not. S2 and S4 said that the medial sides of both knee joints were somewhat uncomfortable. S1, S2, and S4 reported that the arm movement was not comfortable. S1 thought the step length should be smaller.

\section{DISCUSSION}

The aim of this work was to develop and evaluate the feasibility of the prototype ROWAS system. Feasibility assessment, as suggested by Bowen et al. (2009) and Saengsuwan et al. (2015), considered the formal criteria of implementation, acceptability and responsiveness.

The results demonstrated that the gait system with arm swing was technically implementable. Developed via computer modeling, ROWAS was implemented as a prototype. The main features were: (i) the system included a rotational bed which
TABLE 2 | The difference of the ROM error (\%), the phase shift (\%) and RMS error (\%) between the ROWAS and overground data of all five subjects.

\begin{tabular}{lccc}
\hline Joints & $\begin{array}{c}\text { ROM deviation } \\
\left(\boldsymbol{R}_{\text {error }}\right)\end{array}$ & $\begin{array}{c}\text { Phase shift } \\
\left(\boldsymbol{T}_{\text {error }}\right)\end{array}$ & $\begin{array}{c}\text { RMS error } \\
\left(\boldsymbol{R M S S}_{\text {error }}\right)\end{array}$ \\
\hline Shoulder & 5.23 & -2.19 & 1.75 \\
Hip & 3.33 & 0.27 & 3.65 \\
Knee & 7.93 & -0.28 & 6.32 \\
Ankle & 6.79 & 3.49 & 8.87 \\
\hline
\end{tabular}

allowed the potential user to try various positions from supine to upright walking; (ii) the main support area was flat, such as the trunk support plate and the support plate for the lower limbs, which facilitates easy transfer of the users from their wheelchair to the system; (iii) the shoe platform produced ground reaction forces on the foot sole; and (vi) synchronous movement between the upper and lower limbs. Inclusion of arm swing could be simply implemented by adding the upper limb frames with drives. The challenge was that the arm swing function restricted the mounting options for the hip joint actuators. For ease of control, it was desirable for the drives to directly actuate the joints. However, arm swing required free space on both sides, 
especially around the hip joint area. Therefore, we put the hip drives between the legs. Furthermore, the ROWAS system was easy to move due to a retractable wheel support. The ROWAS prototype met the design requirements and is an innovative system.

The ROWAS system was tested regarding the acceptability criterion by the able-bodied subjects. Currently available gait orthoses normally have the arms fixed using a horizontal level bar for balance (Lum et al., 2005; Díaz et al., 2011). Recent research on interlimb neural coupling suggests that the inclusion of arm swing is helpful for gait restoration (Ferris et al., 2006). Therefore, the novel ROWAS system was developed with the inclusion of arm swing, and we were interested in testing whether this new training modality was accepted or not. Lying on the bed, and further secured by the body-weight support system, the subjects in the preliminary tests did not report any sense of insecurity during the walking session. After experiencing passive walking with and without arm swing in ROWAS, all the subjects reported a stronger sense of walking when arm movement was activated.

Regarding the responsiveness criterion, the test measurements proved that the prototype produced accurate responses close to the target profiles, i.e., a walking-like coordinated movement among the shoulder, hip, knee and ankle joints of both sides. Our study was motivated due to the absence of a gait system with arm swing. Although there are tricycles which produce movements in both the upper and lower limbs (Hunt et al., 2012), users are in a sitting position, with the limbs moving in a circular path. Thus, the movement profiles are quite different from those occurring during normal gait. The measured joint trajectories from our system demonstrated that a synchronized movement was produced among the upper and lower limbs, with a maximal phase shift of about $3.5 \%$. Compared to overground normal walking, the mean ROM error of less than $8 \%$ and a maximal RMS error of $9 \%$ demonstrated that a natural walking style was generated in ROWAS. It was thus demonstrated that the ROWAS prototype was feasible regarding the responsiveness feasibility criterion.

Our study and the ROWAS prototype have some limitations. As a feasibility study, we only recruited five subjects. More subjects are desirable to obtain a more detailed evaluation of the prototype. Nevertheless, these subjects provided useful feedback on how to improve the ROWAS prototype for general application. S2 and S4 reported pressure around the knee joint. This was because the knee joint of the frame was not properly aligned with their knee joint. To address this limitation, we plan to add one more adjusting mechanism for the knee joint alignment. Three out of five subjects reported that the arm movement felt uncomfortable. This was because the shoulder joint was actuated in the sagittal plane only. We therefore plan to use a spherical joint to obtain more degrees of freedom. The ROWAS prototype had a fixed hip joint height, which was 1250 $\mathrm{mm}$ above the ground when the bed was in an upright position. People with different heights were aligned with the system via the hip joint. Therefore, shorter subjects (S1 and S3) had a sense of being lifted too high and had a sense of insecurity when tilted up. This insecurity resolved after the subjects got used to the height and it did not disturb their walking session. To solve this problem, the bed base could be improved to have an adjustable height. Another limitation was the fixed ROMs of the upper and lower limbs. Because of this, shorter users such as S1 thought the step length was too large. A variable walking style should be implemented.

Future work should prepare a modified ROWAS system for clinical tests on patients from target populations with neurological impairments. The height of the bed base should be reduced so that the patients can be easily transferred to the system from their wheel chair. To provide clinical testing, we need to design a better body-weight support system which secures them, but without discomfort in the bottom area. Finally, the control algorithms need to be extended to allow compliant movement, and also to allow potential subjects, especially hemiplegic patients, to use their healthy side to control the impaired side. EMG responses should be investigated when patients use the system, as this will allow further investigation of interlimb neural coupling.

\section{CONCLUSIONS}

In conclusion, we designed, manufactured and tested a ROWAS. During testing with five able-bodied subjects, the system could produce coordinated joint performance in the upper and lower limbs which was similar to normal overground gait. This system was deemed feasible, as far as the formal implementation, acceptability and responsiveness criteria were concerned.

\section{AUTHOR CONTRIBUTIONS}

JF mainly developed this project, designed the system, manufactured and tested the system, analyzed the data and wrote the paper; QX helped develop the concept of this project and modified the manuscript; GY developed this project, supervised the experiments and analyzed the data and checked the paper; LX developed this project, helped to develop the design concepts, analyzed the data and checked the paper.

\section{ACKNOWLEDGMENTS}

This work was supported by grants from the National Natural Science Foundation of China (JF: Grant No. 81401856, LX: Grant Nos. 61672341 and 61190124), Science and Technology Commission of Shanghai Municipality (LX: Grant No. 14441900800) and from the youth research funding scheme of Jiangnan University (JF: Grant No. JUSRP115A12). The authors acknowledge the staff of the Chejing Robotics Technology (Shanghai) Co., Ltd. for their support in manufacturing the prototype; and we particularly thank the mechanical research group for suggestions on the development of the ROWAS system.

\section{SUPPLEMENTARY MATERIAL}

The Supplementary Material for this article can be found online at: http://journal.frontiersin.org/article/10.3389/fnins. 2017.00032/full\#supplementary-material 


\section{REFERENCES}

Barbeau, H., Wainberg, M., and Finch, L. (1987). Description and application of a system for locomotor rehabilitation. Med. Biol. Eng. Comput. 25, 341-344. doi: $10.1007 / \mathrm{BF} 02447435$

Behrman, A. L., and Harkema, S. J. (2000). Locomotor training after human spinal cord injury: a series of case studies. Phys. Ther. 80, 688-700.

Bovonsunthonchai, S., Hiengkaew, V., Vachalathiti, R., Vongsirinavarat, M., and Tretriluxana, J. (2012). Effect of speed on the upper and contralateral lower limb coordination during gait in individuals with stroke. Kaohsiung J. Med. Sci. 28, 667-672. doi: 10.1016/j.kjms.2012.04.036

Bowen, D. J., Kreuter, M., Spring, B., Cofta-Woerpel, L., Linnan, L., Weiner, D., et al. (2009). How we design feasibility studies. Am. J. Prev. Med. 36, 452-457. doi: 10.1016/j.amepre.2009.02.002

Collins, S. H., Adamczyk, P. G., and Kuo, A. D. (2009). Dynamic arm swinging in human walking. Proc. R. Soc. B Biol. Sci. 76, 3679-3688. doi: 10.1098/rspb.2009.0664

Díaz, I., Gil, J. J., and Sánchez, E. (2011). Lower-limb robotic rehabilitation: literature review and challenges. J. Rob. 2011, 1-11. doi: 10.1155/2011/759764

Dietz, V. (2002). Do human bipeds use quadrupedal coordination? Trends Neurosci. 25, 462-467. doi: 10.1016/S0166-2236(02)02229-4

Dietz, V., Fouad, K., and Bastiaanse, C. M. (2001). Neuronal coordination of arm and leg movements during human locomotion. Eur. J. Neurosci. 14, 1906-1914. doi: 10.1046/j.0953-816x.2001.01813.x

Donker, S. F., Mulder, T., Nienhuis, B., and Duysens, J. (2002). Adaptations in arm movements for added mass to wrist or ankle during walking. Exp. Brain Res. 146, 26-31. doi: 10.1007/s00221-002-1145-2

Eke-Okoro, S. T., Gregoric, M., and Larsson, L. E. (1997). Alterations in gait resulting from deliberate changes of arm-swing amplitude and phase. Clin. Biomech. 12, 516-521. doi: 10.1016/S0268-0033(97)00050-8

Fang, J., Galen, S., Vuckovic, A., Conway, B. A., and Hunt, K. J. (2014a). Kinetic analysis of supine stepping for early rehabilitation of walking. Proc. Inst. Mech. Eng. H. 228, 456-464. doi: 10.1177/0954411913518031

Fang, J., Gollee, H., Galen, S., Allan, D. B., Conway, B. A., Vuckovic, A. (2011). Kinematic modelling of a robotic gait device for early rehabilitation of walking. Proc. Inst. Mech. Eng. H. 225, 1177-1187. doi: 10.1177/0954411911424976

Fang, J., Hunt, K. J., Xie, L., and Yang, G.-Y. (2016). Modelling of the toe trajectory during normal gait using circle-fit approximation. Med. Biol. Eng. Comput. 54, 1481-1489. doi: 10.1007/s11517-015-1414-4

Fang, J., Vuckovic, A., Galen, S., Conway, B. A., and Hunt, K. J. (2014b). Mechanical stimulation of the foot sole in a supine position for ground reaction force simulation. J. Neuroeng. Rehabil. 159, 1-13. doi: 10.1186/1743-0003-11-159

Ferris, D. P., Huang, H. J., and Kao, P.-C. (2006). Moving the arms to activate the legs. Exerc. Sport Sci. Rev. 34, 113-120. doi: 10.1249/00003677200607000-00005

Harkema, S. J. (2001). Neural plasticity after human spinal cord injury: application of locomotor training to the rehabilitation of walking. Neuroscientist 7, 455-468. doi: 10.1177/107385840100700514
Hesse, S., Waldner, A., and Tomelleri, C. (2010). Innovative gait robot for the repetitive practice of floor walking and stair climbing up and down in stroke patients. J. Neuroeng. Rehabil. 7:30. doi: 10.1186/17430003-7-30

Hidler, J., Wisman, W., and Neckel, N. (2008). Kinematic trajectories while walking within the Lokomat robotic gait-orthosis. Clin. Biomech. 23, 1251-1259. doi: 10.1016/j.clinbiomech.2008.08.004

Hunt, K. J., Fang, J., Saengsuwan, J., Grob, M., and Laubacher, M. (2012). On the efficiency of FES cycling: a framework and systematic review. Technol. Health Care 20, 395-422. doi: 10.3233/THC-2012-0689

Kadaba, M. P., Ramakrishnan, H. K., and Wootten, M. E. (1990). Measurement of lower extremity kinematics during level walking. J. Orthop. Res. 8, 383-392. doi: 10.1002/jor.1100080310

Loureiro, R., Amirabdollahian, F., Topping, M., Driessen, B., and Harwin, W. (2003). Upper limb robot mediated stroke therapyGENTLE/s approach. Auton. Robots. 15, 35-51. doi: 10.1023/A:10244367 32030

Lum, P. S., Burgar, C. G., Van der Loos, M., Shor, P. C., Majmundar, M., and Yap, R. (eds.). (2005). "The MIME robotic system for upper-limb neuro-rehabilitation: results from a clinical trial in subacute stroke," in 9th International Conference on Rehabilitation Robotics, 2005 ICORR 2005 (Chicago, IL).

Nef, T., Mihelj, M., Colombo, G., and Riener, R. (2006). "ARMin-robot for rehabilitation of the upper extremities," in Proceedings of IEEE International Conference on Robotics and Automation (Orlando, FL), 3152-3157.

Saengsuwan, J., Huber, C., Schreiber, J., Schuster-Amft, C., Nef, T., and Hunt, K. J. (2015). Feasibility of cardiopulmonary exercise testing and training using a robotics-assisted tilt table in dependent-ambulatory stroke patients. $J$. Neuroeng. Rehabil. 12, 1-10. doi: 10.1186/s12984-015-0078-5

Stephenson, J. L., De Serres, S. J., and Lamontagne, A. (2010). The effect of arm movements on the lower limb during gait after a stroke. Gait Posture 31, 109-115. doi: 10.1016/j.gaitpost.2009.09.008

Visintin, M., and Barbeau, H. (1994). The effects of parallel bars, body weight support and speed on the modulation of the locomotor pattern of spastic paretic gait. A preliminary communication. Paraplegia 32, 540-553. doi: $10.1038 /$ sc. 1994.86

Zehr, E. P., Loadman, P. M., and Hundza, S. R. (2012). Neural control of rhythmic arm cycling after stroke. J. Neurophysiol. 108, 891-905. doi: 10.1152/jn.01152.2011

Conflict of Interest Statement: The authors declare that the research was conducted in the absence of any commercial or financial relationships that could be construed as a potential conflict of interest.

Copyright (C) 2017 Fang, Xie, Yang and Xie. This is an open-access article distributed under the terms of the Creative Commons Attribution License (CC BY). The use, distribution or reproduction in other forums is permitted, provided the original author(s) or licensor are credited and that the original publication in this journal is cited, in accordance with accepted academic practice. No use, distribution or reproduction is permitted which does not comply with these terms. 


\title{
EMG-Based Continuous and Simultaneous Estimation of Arm Kinematics in Able-Bodied Individuals and Stroke Survivors
}

\author{
Jie Liu ${ }^{1}$, Sang Hoon Kang ${ }^{2}$, Dali Xu ${ }^{1}$, Yupeng Ren ${ }^{1}$, Song Joo Lee ${ }^{3}$ and Li-Qun Zhang ${ }^{4,5 *}$ \\ ${ }^{1}$ Sensory Motor Performance Program, Rehabilitation Institute of Chicago, Chicago, IL, United States, ${ }^{2}$ School of \\ Mechanical, Aerospace, and Nuclear Engineering, Ulsan National Institute of Science and Technology, Ulsan, South Korea, \\ ${ }^{3}$ Center for Bionics, Biomedical Research Institute, Korea Institute of Science and Technology, University of Science and \\ Technology, Seoul, South Korea, ${ }^{4}$ Department of Physical Therapy and Rehabilitation Science and Department of \\ Orthopaedics, University of Maryland, Baltimore, MD, United States, ${ }^{5}$ Department of Bioengineering, University of Maryland, \\ College Park, MD, United States
}

OPEN ACCESS

Edited by:

loan Opris,

Leonard M. Miller School of Medicine,

United States

Reviewed by:

Domenico Caputo,

Sapienza Università di Roma, Italy Giovanni Mirabella,

Sapienza Università di Roma, Italy

${ }^{*}$ Correspondence:

Li-Qun Zhang

zhangl@umd.edu

Specialty section:

This article was submitted to

Neural Technology,

a section of the journal

Frontiers in Neuroscience

Received: 14 November 2016

Accepted: 14 August 2017

Published: 25 August 2017

Citation:

Liu J, Kang SH, Xu D, Ren Y, Lee SJ and Zhang L-Q (2017) EMG-Based

Continuous and Simultaneous Estimation of Arm Kinematics in Able-Bodied Individuals and Stroke

Survivors. Front. Neurosci. 11:480. doi: 10.3389/fnins.2017.00480
Among the potential biological signals for human-machine interactions (brain, nerve, and muscle signals), electromyography (EMG) widely used in clinical setting can be obtained non-invasively as motor commands to control movements. The aim of this study was to develop a model for continuous and simultaneous decoding of multi-joint dynamic arm movements based on multi-channel surface EMG signals crossing the joints, leading to application of myoelectrically controlled exoskeleton robots for upper-limb rehabilitation. Twenty subjects were recruited for this study including 10 stroke subjects and 10 able-bodied subjects. The subjects performed free arm reaching movements in the horizontal plane with an exoskeleton robot. The shoulder, elbow and wrist movements and surface EMG signals from six muscles crossing the three joints were recorded. A non-linear autoregressive exogenous (NARX) model was developed to continuously decode the shoulder, elbow and wrist movements based solely on the EMG signals. The shoulder, elbow and wrist movements were decoded accurately based only on the EMG inputs in all the subjects, with the variance accounted for (VAF) > 98\% for all three joints. The proposed approach is capable of simultaneously and continuously decoding multi-joint movements of the human arm by taking into account the non-linear mappings between the muscle EMGs and joint movements, which may provide less effortful control of robotic exoskeletons for rehabilitation training of individuals with neurological disorders and arm impairment.

Keywords: electromyogram (EMG), non-linear autoregressive exogenous model, continuous decoding, exoskeleton robot, computational neuroscience

\section{INTRODUCTION}

Rehabilitation robots have been developing rapidly and used for therapeutic training of patients with neurological disorders, including stroke, cerebral palsy, and spinal cord injury (Dipietro et al., 2005; Krebs et al., 2008; Song et al., 2008; Marchal-Crespo and Reinkensmeyer, 2009; Pons, 2010; Frisoli et al., 2012; Heo et al., 2012; Reinkensmeyer and Boninger, 2012; Zariffa et al., 2012; Ren et al., 2013). Advances have been made to build more practical and functional upper-limb powered robotic exoskeleton devices (Nef et al., 2007; Perry et al., 2007; Gupta et al., 2008; Kim et al., 2012; Mao and Agrawal, 2012; Ren et al., 2013; Shao et al., 2014). The advances in powered 
exoskeletons imply great promise to allow neurologically impaired patients to perform versatile activities, therefore helping restore strength and flexibility of their limbs. In contrast, relatively less attention has been given to providing less effortful control of exoskeleton robots. Previous studies have shown that motor commands are generated by the combination of a small number of muscle synergies, which allows the coordinated recruitment of groups of muscles with specific amplitude balances (Jiang et al., 2010; Overduin et al., 2015; d'Avella, 2016). Muscle synergies can be used to predict the movement of multiple degrees of freedom (DOFs; Ison and Artemiadis, 2014; Jiang et al., 2014a). Controlling a multiple DOFs robotic device requires sophisticated techniques for identification of various movements from recorded electromyography (EMG) signals (Fleischer and Hommel, 2008; Lo et al., 2010; Peerdeman et al., 2011; Scheme and Englehart, 2011; Fougner et al., 2012). A neural control interface is crucial to providing accurate, natural and less effortful control of powered exoskeletons (Kiguchi and Hayashi, 2012; Lenzi et al., 2012).

Among the potential biological signals for human-machine interaction (brain, nerve, and muscle signals), EMG, the ensemble electrical activity of a muscle may be the only experimentally non-invasive record of the motor commands to the muscles that enables routine clinical applications. EMG is generated by the neural activation from the brain and spinal cord and therefore contains substantial movementrelated information. It is worth noting that EMG signals do not necessarily reflect the overall computations carried on by the motor system. In fact, they are unlikely to catch neural signals related to a key executive function for shaping motor behavior, i.e., the ability of cancelling pending movements (Mirabella, 2014; Mirabella and Lebedev, 2017). However, in practice EMG signals could enable efficient control of robotic exoskeletons by extracting those motor commands that reach the muscles. Inhibitory control is fundamental for achieving a proper behavioral flexibility due to the fact events cannot be fully predicted practically. In many instances, preplanned actions must be aborted to avoid catastrophic consequences. Often suppression of a planned action occurs within the central nervous system, and thus the related neural activity does not reach the muscles. It is not by chance that brain-machine interfaces enacting inhibitory control have proposed to reproduce goaldirected behaviors in a more naturalistic way recently (Mirabella, 2012; Mirabella and Lebedev, 2017).

Use of EMG in decoding motor commands is one of the most robust and accurate interfaces for controlling robotic devices (Farina and Aszmann, 2014). As a non-invasive measurement containing rich motor control information, surface EMG is an important input for the control of powered robotic devices (Parker et al., 2006; Pons, 2010). As a result, surface EMG is increasingly recognized as one of the important control signals for assistive or rehabilitative devices in robot-aided therapy (Song et al., 2008; Hincapie and Kirsch, 2009; Marchal-Crespo and Reinkensmeyer, 2009; Jiang et al., 2010; Smith and Brown, 2011). Myoelectric control is a promising approach for controlling the multiple DOFs of multifunctional dexterous exoskeletons (Fleischer and Hommel, 2008). However, a major challenge in myoelectric control is to provide simultaneous and proportional control signals for robotic devices with multiple DOFs (Jiang et al., 2010; Fougner et al., 2012). To facilitate a less effortful myoelectric control paradigm, myoelectric controllers should provide proportional control of multiple DOFs simultaneously. This has been addressed in a few recent studies (Ameri et al., 2014a; Farmer et al., 2014; Fougner et al., 2014; Hahne et al., 2014; Ngeo et al., 2014). To provide simultaneous, independent and proportional control of multiple DOFs, various linear and non-linear estimators have been used, including artificial neural networks (Koike and Kawato, 1995; Cheron et al., 1996; Au and Kirsch, 2000; Shrirao et al., 2009; Pulliam et al., 2011; Jiang et al., 2012; Zhang et al., 2012; Ameri et al., 2014b; Farmer et al., 2014; Ngeo et al., 2014), regression techniques (Chen et al., 2013; Ameri et al., 2014a; Hahne et al., 2014), and state-space models (Artemiadis and Kyriakopoulos, 2010, 2011; Pan et al., 2014). Recent research has shown that continuous decoding plays increasingly important role in myoelectric control (Ameri et al., 2014a; Farmer et al., 2014; Fougner et al., 2014; Hahne et al., 2014; Ngeo et al., 2014).

Previous studies have shown that artificial neural networks, being a widely used supervised non-linear approach, outperformed linear regression (a supervised linear approach) and non-negative matrix factorization (a linear unsupervised method) in the EMG decoding paradigm (Hahne et al., 2014; Jiang et al., 2014b). It was possible to predict wrist joint angle instead of forces from EMGs with artificial neural networks when the subject was performing free dynamic movements (Jiang et al., 2012). In particular, a non-linear autoregressive exogenous (NARX) model was utilized to continuously map the kinematics of a transtibial prosthesis and EMG activity to estimate the prosthetic ankle angle in transtibial amputees in a recent study (Farmer et al., 2014). However, few investigators have been able to draw on any systematic research into decoding dynamic multi-joint arm movements. Most studies on EMG decoding have only focused on the estimation of kinematics of the leg and fingers. The purpose of this study was to develop a novel multi-input multi-output decoding method based on the NARX neural network model and predict dynamic multi-joint arm movements simultaneously based only on multichannel EMG inputs, which can potentially be used to achieve user-friendly, less effortful myoelectric control of robot-aided multi-joint movements.

\section{METHODS}

\section{Subjects}

Twenty subjects were recruited for this study including 10 stroke subjects (Table 1) and 10 with no neurological disorder ( 5 males and 5 females, age: $46.1 \pm 14.6$ year). All subjects gave written informed consent, which was approved by the Institutional Review Board of Northwestern University. A screening examination and clinical assessment to determine the eligibility for each stroke subject were performed by a physical therapist. Inclusion criteria for participation of the study include: (a) age between 21 and 65 years old; (b) experience of stroke, 
TABLE 1 | Physical Characteristics of Subjects.

\begin{tabular}{lcccccc}
\hline Subject no. & Age & Sex & Duration & Paretic & F-M (UE) & MSS \\
\hline 1 & 56 & M & 6 & R & 36 & 49.02 \\
2 & 58 & M & 1 & R & 38 & 54.01 \\
3 & 59 & M & 9 & R & 22 & 23.00 \\
4 & 59 & M & 26 & L & 16 & 14.67 \\
5 & 59 & M & 11 & R & 46 & 60.67 \\
6 & 63 & F & 2 & L & 48 & 66.02 \\
7 & 54 & F & 2 & R & 21 & 28.66 \\
8 & 47 & F & 4 & L & 22 & 19.35 \\
9 & 45 & M & 10 & L & 30 & 43.33 \\
10 & 55 & F & 6 & R & 50 & 50.69 \\
\hline
\end{tabular}

Duration: years since the onset of stroke. Paretic: the side of hemiparesis. F-M (UE): the Fugl-Meyer assessment scale of the paretic upper extremity (total score: 66). MSS: the motor status scale measures shoulder, elbow (maximum score $=40$ ), wrist, hand, and finger movements (maximum score $=42$ ).

initial onset $>6$ months; (c) medically stable with clearance to participate.

\section{Experimental Procedure}

An upper limb exoskeleton robot, called IntelliArm and capable of controlling the shoulder, elbow and wrist individually or simultaneously in the horizontal plane (Ren et al., 2013), was used in the study to conduct supported free arm reaching and movement, as commonly used in stroke rehabilitation (Lo et al., 2010). The subject was seated with the upper arm, forearm, and hand strapped to the IntelliArm through the corresponding braces (Figure 1). The subject's shoulder horizontal adductionabduction, elbow flexion-extension and wrist flexion-extension axes were aligned with the corresponding mechanical axes of the IntelliArm robot (Ren et al., 2013). The IntelliArm robot was made back-drivable under the internal model based impedance control (IMBIC; Kang et al., 2009), thus the subject could move the arm voluntarily with little resistance from the robot. Six wireless pre-amplified, single differential surface EMG electrodes (Trigno, Delsys Inc; Boston, Massachusetts) were used to record EMG signals from the anterior deltoid, posterior deltoid, biceps brachii, long head of the triceps brachii, flexor carpi radialis, and extensor carpi radialis muscles. The subject was instructed to move their arm voluntarily in the horizontal plane with large concurrent movements across the shoulder, elbow, and wrist joints at the subject's self-selected comfortable speeds ( $<70$ degrees/second), as the speed and movement duration are more likely to vary in real-world settings. The motors used to control the motion of the robot in other planes were locked in this study. The movement range of each of the joints was about $90^{\circ}$. Relative long single trial instead of a few short time trials were used for data recording when movements are freely repeated several times in order to capture both the commonality and the variability of the EMG and motion properties. Thus, the subject performed a single movement trial over a span of about $3 \mathrm{~min}$ in this study. Previous studies have used a similar approach for modeling functional relationships between EMG and limb kinematics
(Cheron et al., 1996, 2003). The reliability of an experimental study could be affected by several factors such as motivation or boredom. For example, during series of trials, the second one is likely better than the first because subjects intend to improve their performance or because they may benefit from the learning experience of the first one. By contrast, a decreased performance between the first trial and the following trial could possibly be explained by lack of motivation or fatigue (Hopkins, 2000).

\section{Data Collection and Signal Processing}

The surface EMG signals were sampled at $1,000 \mathrm{~Hz}$ by using the Trigno EMG system, rectified, and then low-pass filtered with a six-order Butterworth filter (cutoff frequency $=4 \mathrm{~Hz}$ ) to obtain the linear envelope (LE). Multi-joint position signals from the IntelliArm robot were sampled at $1,000 \mathrm{~Hz}$ and synchronized to the EMG data through a trigger signal sent to the aforementioned IntelliArm and EMG data acquisition systems simultaneously. The trigger was implemented as a push button switch. The resultant EMG LE and position signals were then down sampled to $20 \mathrm{~Hz}$ for subsequent data analysis. Figure 2 illustrates the representative EMG and kinematic data recorded during a typical experimental trial.

\section{NARX Model}

The multi-joint dynamic relationship between the six arm muscles and the shoulder, elbow and wrist movements was characterized by an AutoRegressive with eXogeneous inputs (NARX) model for discrete-time non-linear systems (Leontaritis and Billings, 1985; Billings, 2013) and is represented as

$$
\begin{aligned}
y(t) & =f\left[y(t-1), \ldots, y\left(t-n_{y}\right), x(t-1), \ldots, x\left(t-n_{x}\right)\right] \\
& +e(t)(t)(t-1) t-n x
\end{aligned}
$$

where $x(t)$ and $y(t)$ are the input and output of the model at discrete time step $t$, respectively. While the input order $\mathrm{n} \_\mathrm{x}$ $\geq 1$, the output order $\mathrm{n} \_\mathrm{y} \geq 1$, and $\mathrm{n} \_\mathrm{x} \leq \mathrm{n} \_\mathrm{y}$ (Leontaritis and Billings, 1985; Billings, 2013). $\mathrm{f}(\cdot)$ is a non-linear function, which can be approximated by a standard multilayer perceptron (MLP) neural network. The resulting connectionist architecture is called a NARX recurrent neural network, which has been used in modeling non-linear dynamic systems (Chen et al., 1990; Lin et al., 1996, 1997; Menezes and Barreto, 2008; Farmer et al., 2014).

Assuming that $\mathrm{e}(\mathrm{t})$ has zero mean and finite variance, the minimized mean-square error (MSE) predictor for the NARX model is approximated by

$$
\begin{aligned}
\hat{y}(t)= & y(t)-e(t)=f\left[y(t-1), \ldots, y\left(t-n_{y}\right), x(t-1),\right. \\
& \left.\ldots, x\left(t-n_{x}\right)\right]
\end{aligned}
$$

The NARX model has a delay structure, creating embedded memory within the neural network. The MSE one-step predictor is a non-linear function of a finite number of past outputs and past inputs. 


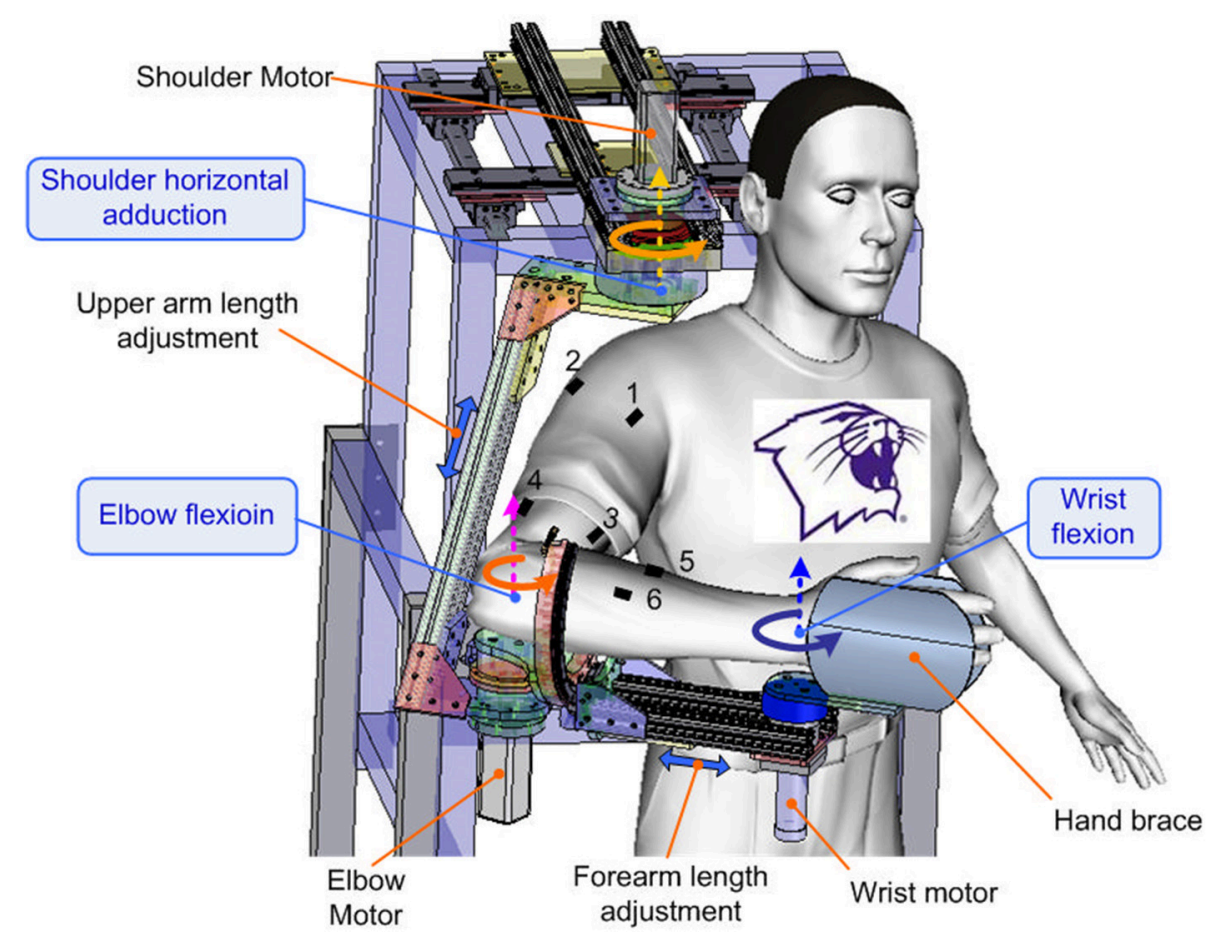

FIGURE 1 | Experimental setup with the subject performing self-paced multi-joint movements with the upper limb exoskeleton robot. The dashed-line arrows mark the axis shoulder horizontal adduction-abduction, elbow flexion-extension, and wrist flexion-extension axes. The double-headed arrows indicate the arm adjustment of the robot. The other degrees of freedom of the robot have been omitted from the figure for clarity. EMG electrodes 1 to 6 were used to record muscle EMGs from the anterior deltoid, posterior deltoid, biceps brachii, triceps long head, flexor carpi radialis, and extensor carpi radialis muscles of the dominant arm of the subject, respectively.

In contrast to other recurrent networks, NARX neural networks have feedback from the outputs rather than from the hidden states (Siegelmann et al., 1997). As a result, NARX neural networks are able to learn more effectively, converge faster and exhibit better generalizations (Lin et al., 1996, 1997). In addition, the NARX neural networks are computationally as powerful as Turing machines (Siegelmann and Sontag, 1991, 1995), which means that theoretically any problem can be modeled and solved using a NARX neural network. Thus, we used a NARX neural network to predict dynamic arm movements by modeling the non-linear dynamic relationship between the multiple muscle activities and arm kinematics.

\section{NARX Neural Network Structure}

The NARX network model used in this study was developed by using the Neural Network Toolbox in Matlab (Mathworks, Natick, MA), which consisted of an input layer containing the sixchannel EMG LE signals recorded from the arm, a hidden layer containing three neurons, and a linear output layer containing three outputs corresponding to the shoulder, elbow, and wrist angular positions (Figure 3).

To continuously decode the multi-joint positions simultaneously from the EMG LE signals, the following
NARX network model was used:

$$
\begin{aligned}
U_{n}(t)= & f_{1}\left(\sum_{i=1}^{N_{x}} \sum_{k=0}^{n_{x}} A_{n i}(k) X_{i}(t-m-k)\right. \\
& \left.-\sum_{j=1}^{N_{y}} \sum_{k=0}^{n_{y}} B_{\mathrm{nj}}(k) Y_{j}(t-m-k)+b_{1 \mathrm{n}}\right) \\
& n=1,2,3 \\
Y_{j}(t)= & f_{2}\left(\sum_{n=1}^{3} C_{\mathrm{jn}}(t) U_{n}(t)+b_{2 \mathrm{j}}\right), \quad j=1,2,3
\end{aligned}
$$

where $U_{n}(t)$ represents output of one of the non-linear nodes of the hidden layer, $Y_{j}(t)$ is the shoulder, elbow or wrist angular position predicted by the NARX network model at time $t ; N_{x}$ (=6) is the number of EMG LE signals; $n_{x}(=2)$ is the number of past input time lags used in the NARX model; $N_{y}(=3)$ is the number of joint angles, $n_{y}(=2)$ is the number of past output time lags used in the NARX model; $m(=2)$ is the number of time lags, which specifies the embedding delay $(\tau=\mathrm{m} \Delta \mathrm{t})$ and correspond to the prediction interval; $X_{i}(t-m-k)$ is the ith EMG LE at time lag $m+k ; Y_{j}(t-m-k)$ is a joint angle predicted by the NARX model at time lag $m+k ; A_{n i}, B_{\mathrm{nj}}$ and $C_{\text {jn }}$ are the weight vectors. $b_{1 \mathrm{n}}$ and $b_{2 \mathrm{j}}$ are the bias weights. $f_{1}$ and $f_{2}$ are the sigmoid function and linear function, respectively.

The NARX neural network model has 51 parameters in total, which are estimated in training the neural network offline by using a supervised learning procedure to minimize the errors 

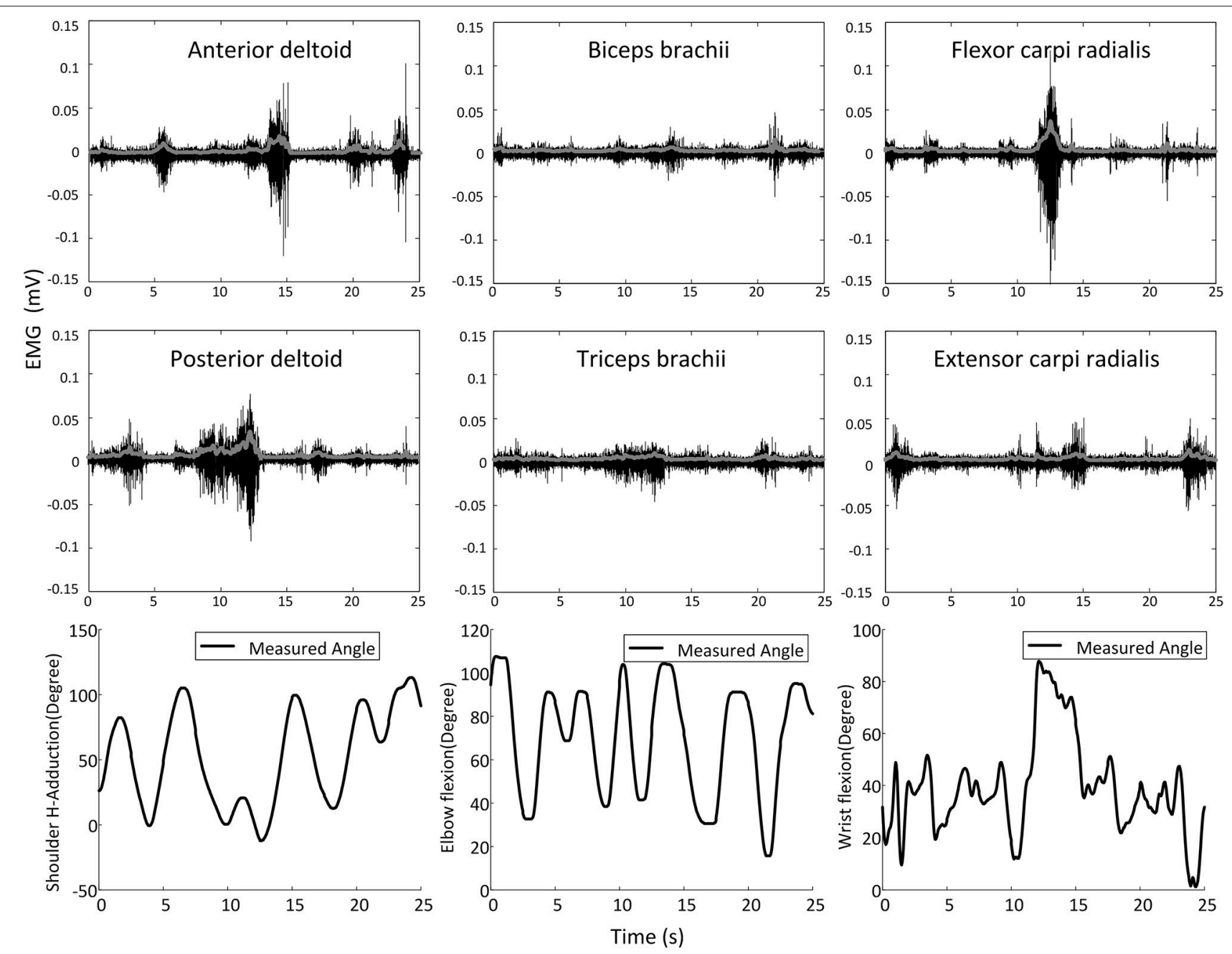

FIGURE 2 | Representative shoulder, elbow, and wrist movements and the corresponding surface EMG signals (raw signals are in black and the corresponding linear envelope profiles are in gray) recorded in a movement trial on one subject.

between the NARX network model outputs and experimentally measured angles.

\section{Performance Measure}

For each subject, a five-fold cross-validation procedure was used to evaluate the performance. The EMG and arm joint kinematic data were divided into five segments with equal length. Each time, four out of five segments of data were used as the training set and the remaining segment was used as the validation set (training: $80 \%$, validation: $20 \%$ ). The procedure was repeated with each of five segments used as the validation set once. To evaluate the performance of the trained decoder, the variance accounted for (VAF) was calculated between the measured and decoded joint angles based on the fresh data (Kearney and Hunter, 1990; Fagg et al., 2009).

One-way ANOVA with repeated measures was utilized to compare the decoding performance across the multiple joints. The group factor had three levels, corresponding to the shoulder, elbow, and wrist joints. The null hypothesis was that there was no difference between the decoding performance of the shoulder, elbow and wrist joints. Multiple comparisons with Bonferroni correction were conducted to compare pairwise differences between the joints.

\section{EXPERIMENTAL RESULTS}

\section{Decoding Performance and the Effect of Prediction Interval}

The effect of the length of prediction interval on the decoding performance was evaluated as suggested by previous research (Farmer et al., 2014). The relationship between decoding accuracy and the prediction interval was plotted for the shoulder, elbow, and wrist joint angles predicted based on only six muscle EMG signals, which shows that the decoding performance varies with the length of prediction interval (Figure 4). An increase of window length from 50 to 100 


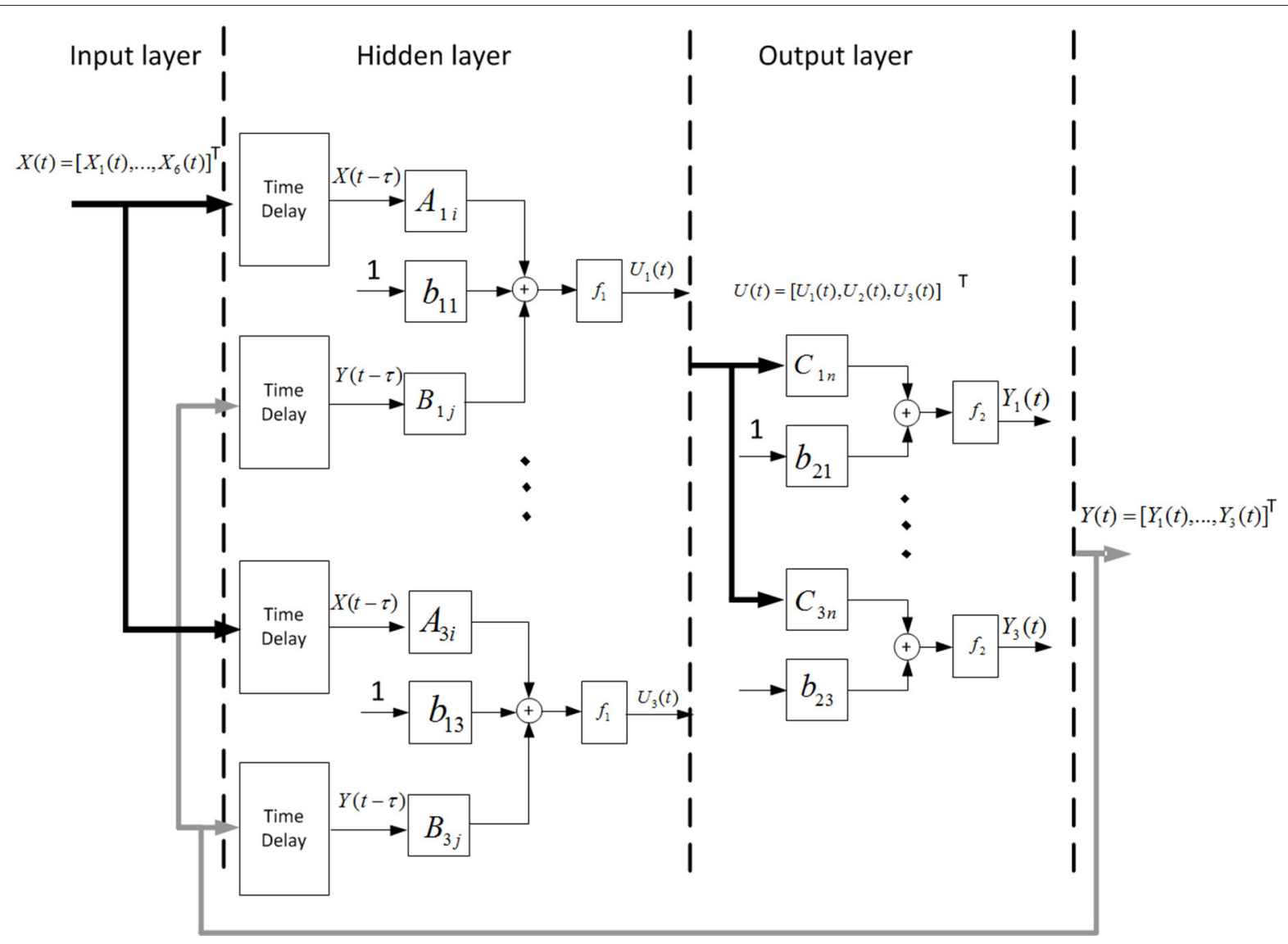

FIGURE 3 | Schematic of the NARX neural network. Measured EMG LE signals and past estimates of the shoulder, elbow, and wrist angles were weighted and fed back via tapped delay lines to the hidden layer consisted of three nodes. Outputs of the hidden layer were weighted and linearly combined to provide continuous estimates of the outputs (shoulder, elbow, and wrist joint angles) over time.

ms led to consistent improvement in decoding performance across the joints, as indicated by the higher VAF. A further increase of the prediction interval to $300 \mathrm{~ms}$ was accompanied with a slight decrease in the decoding performance. For each prediction interval, no significant differences were observed between different joints [One-way repeated measures ANOVA, $F_{(2,15)}=0.16, P=0.85$, partial eta squared $\left.\eta_{p}^{2}=0.02\right]$. Averaged across all the able-bodied subjects, the prediction interval of $100 \mathrm{~ms}$ yielded the best performance with the highest average VAF, which was used in the subsequent analysis for decoding.

\section{Position Decoding Results}

The offline decoding performance of NARX neural network was assessed in terms of its performance to decode the shoulder, elbow and wrist joint positions during active movements using only the six muscle EMG LEs as inputs. High performance in position decoding was achieved for all subjects by using the NARX model, which has a hidden layer containing three neurons, and a linear output layer containing the shoulder, elbow and wrist angle outputs with the prediction interval of $100 \mathrm{~ms}$. Joint angle predictions made using the NARX neural network model based on only the multiple EMG inputs matched very closely with the measured shoulder, elbow, and wrist positions (Figure 5).

Very close matches between the decoded and measured joint angles were achieved for all 10 able-bodied subjects, as shown in Figure 6. The ANOVA showed a significant difference among the decoding performances for the shoulder, elbow and wrist joints [One-way ANOVA, $F_{(2,18)}=5.61, P=0.0092$, partial eta squared $\left.\eta_{p}{ }^{2}=0.29\right]$. The decoding accuracy for the wrist was lower than that achieved for the shoulder and elbow (Bonferroni test, $P<$ 0.05).

Similarly, we have further tested the feasibility of the NARX decoder in 10 stroke subjects showing that the decoded and measured joint angles were close for all 10 stroke subjects who had various levels of functional impairment (Motor status scale 14.67-66.02, Fugl-Meyer score 16-50), as shown in Figure 7. The ANOVA showed a significant difference among the decoding performances for the shoulder, elbow and wrist joints [Oneway ANOVA, $F_{(2,18)}=7.31, P=0.0029$, partial eta squared $\left.\eta_{p}{ }^{2}=0.35\right]$. The decoding accuracy for the wrist was lower than that achieved for the shoulder and elbow (Bonferroni test, $P<0.05)$. 


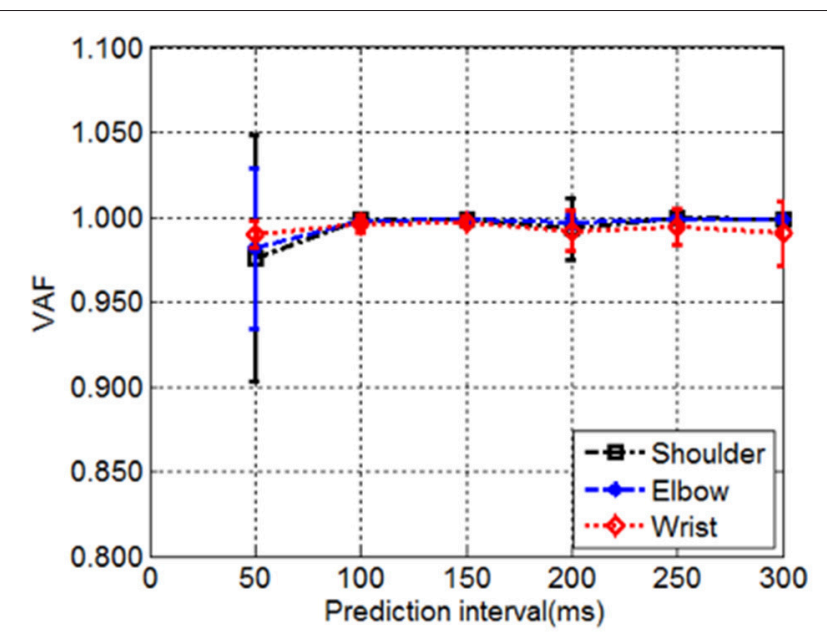

FIGURE 4 | The decoding performance demonstrated by the VAF as a function of prediction interval. The VAF between measured and decoded shoulder, elbow and wrist joint angles over the tested prediction intervals were averaged across all subjects. The vertical bars gave the corresponding standard deviations.

\section{DISCUSSION AND CONCLUSION}

The study investigated decoding multi-joint dynamic arm movements based solely on surface EMG signals from muscles crossing the joints by extracting those motor commands that reach the muscles. This study contributes to rapidly growing research on myoelectric control of limb movements by demonstrating continuous decoding of arm kinematics using surface EMG signals based on the NARX model. In the current study, taking into account the non-linear dynamical relationship between the muscle EMGs and arm movements, we successfully estimated shoulder, elbow and wrist joint movements using only the six EMG signals by adopting time delays and feedback stream into an artificial neural network. These results are consistent with recent studies (Jiang et al., 2012; Farmer et al., 2014; Hahne et al., 2014; Ngeo et al., 2014) in which proportional and simultaneous estimation of limb kinematics was achieved toward myoelectric control, and suggest the use of EMG as a robust and accurate interface for robot control (Farina and Aszmann, 2014). These findings further support the approach to decoding dynamic limb movements using surface EMG signals by taking advantage of artificial neural networks.

Motivated by the fact that NARX neural networks have a recurrent dynamic nature and are computationally equivalent to Turing machines (Siegelmann and Sontag, 1991, 1995), we assessed the NARX networks for continuous decoding of multi-joint dynamic arm movements using surface EMG signals. The performance of the decoder was evaluated by offline analysis using EMG signals from the six muscles and kinematic data measured by the robot during dynamic multijoint arm movements in the horizontal plane. The decoding accuracies in terms of VAF, were above $98 \%$ for the shoulder, elbow and the wrist joints possibly due to the fact that the
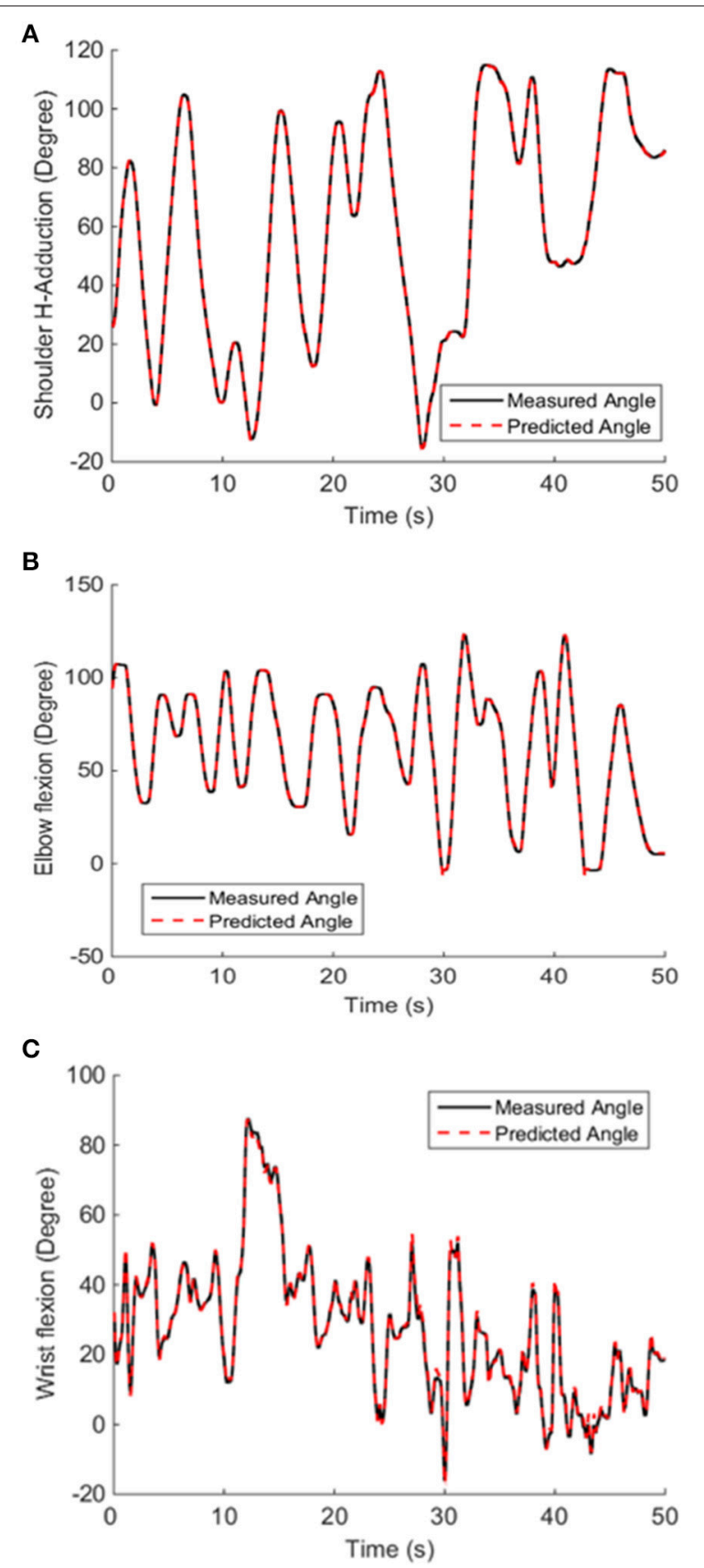

FIGURE 5 | Decoded and measured shoulder (A), elbow (B), and wrist (C) joint angles from a representative subject. The NARX neural network accurately predicted actual kinematics with the prediction interval of $100 \mathrm{~ms}$. The VAF of the predicted shoulder, elbow and wrist position calculated from fresh validation data (50 s long) were 0.9999, 0.999, and 0.9977, respectively.

noise of EMG recordings was suppressed by low-pass filtering the EMG signals with a six-order Butterworth filter (cutoff frequency $=4 \mathrm{~Hz}$ ). The decoding performance of the wrist was slightly inferior to that obtained for the shoulder and elbow, possibly because the kinematic data collected from the wrist joint were noisy due to the relatively large noise caused by 


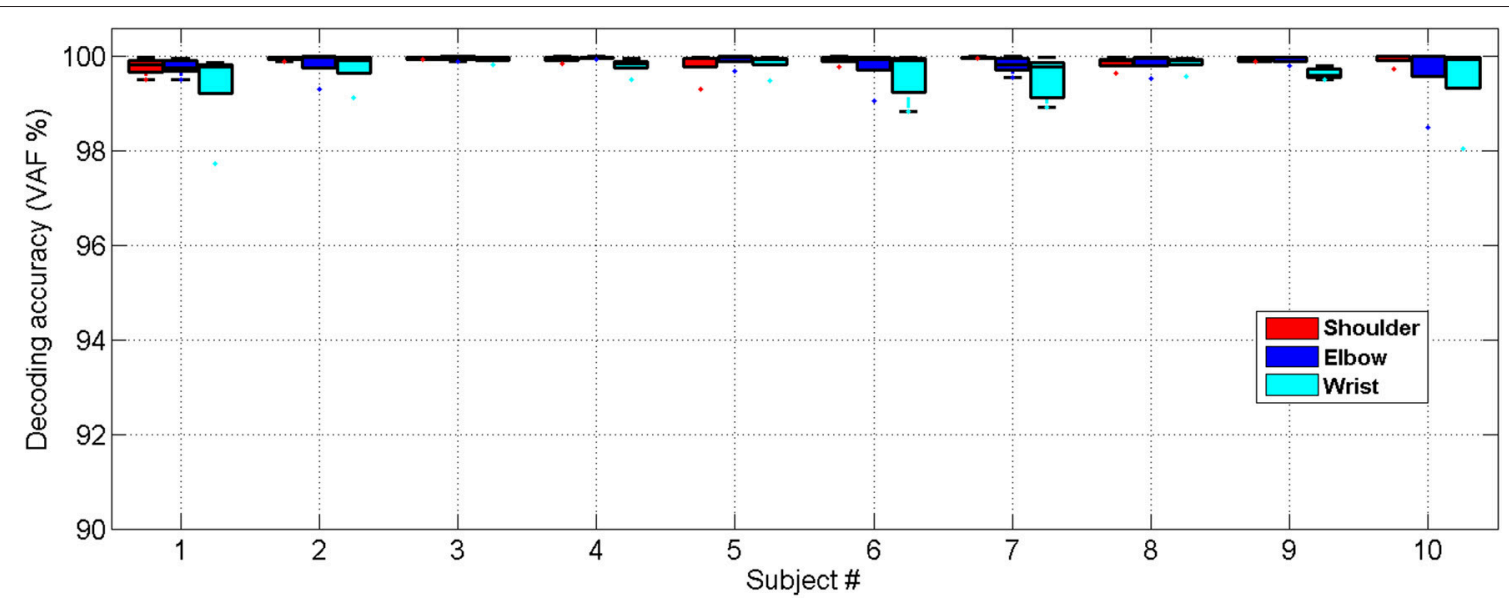

FIGURE 6 | Cross-validated position decoding performance characterized by the VAF over all able-bodied subjects.

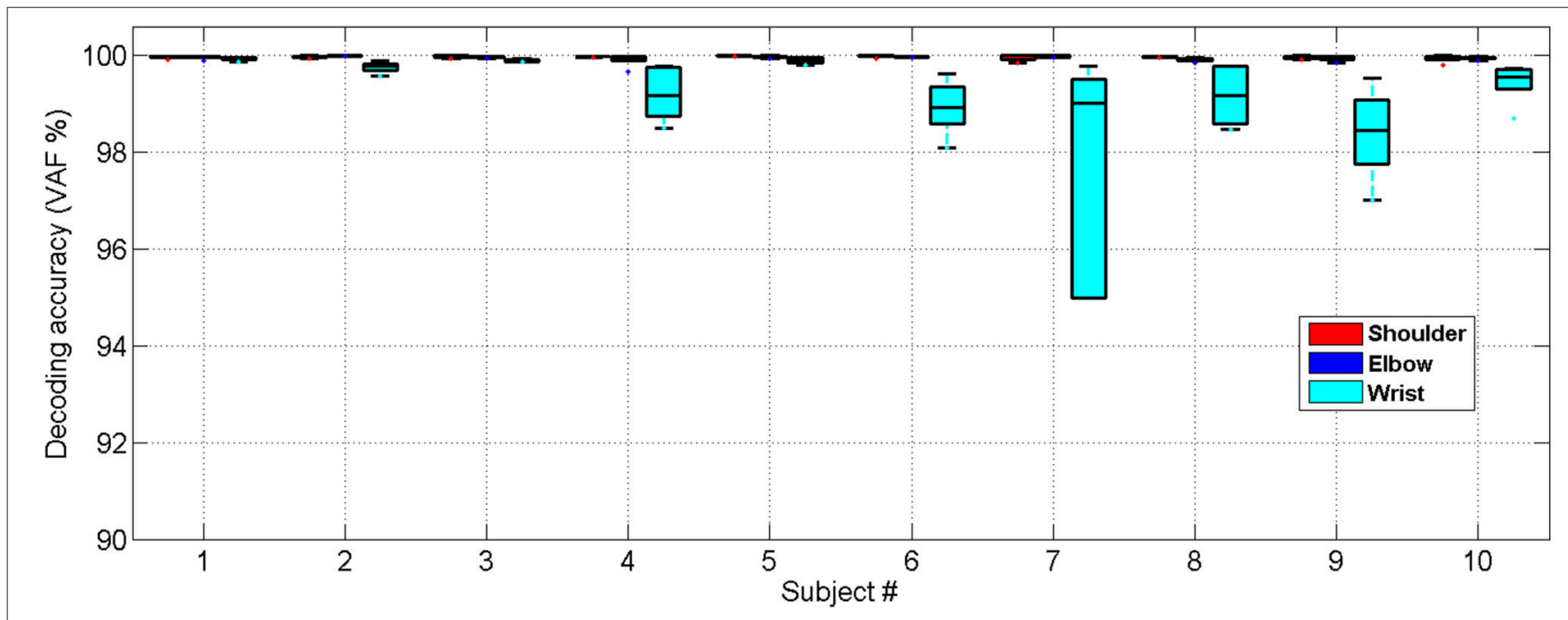

FIGURE 7 | Cross-validated position decoding performance characterized by the VAF over all subjects post stroke.

the Harmonic Drive gear compared to the torque generated by subjects during the voluntary wrist movement under impedance control. This finding is in agreement with that reported by Farmer et al. who showed a NARX model could continuously estimate the prosthetic ankle angle in transtibial amputees using the EMGs recorded from the residual lower limb (Farmer et al., 2014). Furthermore, the method is able to continuously decode movements of multiple joint simultaneously instead of a single joint movement.

Previous studies with able-bodied individuals showed promising results, which need to be interpreted with caution, as the findings might not be directly transferable to neurologically impaired patients, because voluntary surface EMG signals might be contaminated by spontaneous motor activity in studies involving neurological impairments. For instance, when recording EMG signals from paretic muscles of stroke or spinal cord injury patients, abnormal hyper-excitable motor unit discharges may induce spontaneous spurious spikes, compromising the voluntary EMG signals (McKay et al., 2011). Previous studies with incomplete spinal cord injury have shown that the resulting involuntary background spikes can contaminate surface EMG recordings thus result in inappropriate interpretation of the signals (Liu et al., 2014a,b). Therefore, we have further tested the feasibility of the NARX decoder in 10 stroke subjects to evaluate the current method on neurologically impaired patients, we observed that the high decoding accuracy (VAF $>98 \%$ for all three joints) was consistent across different stroke subjects who had various levels of functional impairment (Motor status scale 14.67-66.02, Fugl-Meyer score 16-50). It was observed that the stroke subjects have more variable decoding results compared to the healthy subjects with respect to the wrist joint angle, In contrast, the healthy subjects have more variable decoding results for the shoulder and elbow joints, This result may be explained by 
the fact that the healthy subjects have bigger motion ranges, high speeds and velocity changes. These stroke subjects might have different clinical features, because they are very likely of having undergone very different degree of brain plasticity. We observed that nothing would change by removing patients with more than 10 years since the onset of stroke. However, with a small sample size, caution must be applied, as the findings might not be extrapolated to all patients with different clinical features.

Note that unlike the time delay neural network (TDNN) approach for predicting joint-angle trajectories based on EMG signals during arm movements (Au and Kirsch, 2000; Pulliam et al., 2011; Smith and Brown, 2011), the NARX network makes full use of the model output feedback and has powerful modeling, learning and generalization capacities (Chen et al., 1990; Lin et al., 1996, 1997; Menezes and Barreto, 2008; Farmer et al., 2014). The presence of direct feedback from the output gives strong decoding power to the NARX model. More specifically, the NARX model makes use of the AR model's implicit characterization of the limb kinematics vis-à-vis the feedback. In contrast, the absence of such a feedback in the TDNN model makes it less powerful in decoding. Thus, the NARX network outperforms conventional neural network based decoders, such as the TDNN network (Menezes and Barreto, 2008) qualitatively. Despite this, we acknowledge that future systematic comparison studies on different decoding methods are needed.

EMG signals have been used to control joint angular velocity indirectly by commanding the stiffness equilibrium angle ( $\mathrm{Ha}$ et al., 2011). However, from a practical point of view, models characterizing multiple muscles controlling human joints are rather complex even for a single joint due to the complex nature of neuromusculoskeletal systems, and usually they require exhaustive, time-consuming offline analysis of neuromusculoskeletal system identification problem (Koo and Mak, 2005). Thus, using such models for real-time decoding is difficult and their real-time application remains to be demonstrated, especially for applications to neurologically impaired cases.

Despite these promising results, questions remain. A limitation is that the high accuracy may be based on the fact that the subjects was training in performing a simple and repetitive arm movement and the arm movements were restricted to a horizontal plane with arm-weight-supported, as opposed to 3-D arm movement. Horizontal plane arm movements have been widely studied and commonly used in rehabilitation of patients post stroke (Hollerbach and Flash, 1982; Lo et al., 2010). It could be argued that the positive results were due to the repeated

\section{REFERENCES}

Ameri, A., Kamavuako, E., Scheme, E., Englehart, K., and Parker, P. (2014a). Support vector regression for improved realtime, simultaneous myoelectric control. IEEE Trans. Neural Syst. Rehabil. Eng. 22, 1198-1209. doi: 10.1109/TNSRE.2014.23 23576 simplified movements in 2-D space. Still, further research should be done to investigate different arm movements in 3-D space without arm-weight-support. Furthermore, although the IntelliArm robot was controlled to be backdrivable, it might still induce some external loading to the subject's arm during the multi-joint arm movements, which might affect the EMG signals and the myoelectric control behavior of human arm movement. Everyday tasks involve behaviors uncertainty originating from a large variety of sources, which in some circumstances might require to suppress preplanned actions (Mirabella, 2014). However, in this study the presence of behaviors uncertainty was very limited if not completely absent. Therefore, it was not possible to examine the EMG signal variability in the presence of unpredictable changes. This is an important issue for future research.

In summary, the proposed approach is capable of continuously decoding multiple joint movements of the human arm simultaneously by taking into account the non-linear mappings between the multi-joint arm kinematics and multiple muscle EMGs, which may help improve myoelectric control of robotic exoskeletons and robot-guided arm rehabilitation in neurological disorders.

\section{ETHICS STATEMENT}

Twenty subjects were recruited for this study including ten stroke subjects (Table 1) and ten with no neurological disorder (5 males and 5 females, age: $46.1 \pm 14.6$ year). This study was carried out in accordance with the recommendations of guidelines from the Collaborative Institutional Training Initiative (TICI) program, and the Institutional Review Board of Northwestern University. All subjects gave written informed consent, which was approved by the Institutional Review Board of Northwestern University.

\section{AUTHOR CONTRIBUTIONS}

Conceived and designed the experiments: JL and LZ. Performed the experiments and data acquisitions: JL, SK, SL, DX, and YR. Analyzed and the data interpretation: JL, DX, YR, and LZ. Contributed reagents/materials/analysis tools: JL, SK, DX, YR, and SL. Wrote the paper: JL and LZ. Final approval of the version to be published: JL and LZ.

\section{FUNDING}

This work was supported in part by the National Institute on Disability, Independent Living and Rehabilitation Research, National Science Foundation, and DGIST. position-based training paradigms. IEEE Trans. Biomed. Eng. 61, 279-287. doi: 10.1109/TBME.2013.2281595

Artemiadis, P. K., and Kyriakopoulos, K. J. (2010). EMG-based control of a Robot arm using low-dimensional embeddings. IEEE Trans. Rob. 26, 393-398. doi: 10.1109/TRO.2009.2039378 
Artemiadis, P. K., and Kyriakopoulos, K. J. (2011). A Switching Regime Model for the EMG-Based Control of a Robot Arm. IEEE Trans. Syst. Man Cybern. B Cybern. 41, 53-63. doi: 10.1109/TSMCB.2010.2045120

Au, A. T. C., and Kirsch, R. F. (2000). EMG-Based prediction of shoulder and elbow kinematics in able-bodied and spinal cord injured individuals. IEEE Trans. Rehabil. Eng. 8, 471-480. doi: 10.1109/86.895950

Billings, S. A. (ed.). (2013). "Models for linear and nonlinear systems," in Nonlinear System Identification, (John Wiley \& Sons, Ltd.), 17-59.

Chen, S., Billings, S. A., and Grant, P. M. (1990). Nonlinear-System identification using neural networks. Int. J. Control 51, 1191-1214. doi: $10.1080 / 00207179008934126$

Chen, Y., Zhao, X. G., and Han, J. D. (2013). Hierarchical projection regression for online estimation of elbow joint angle using EMG signals. Neural Comput. Appl. 23, 1129-1138. doi: 10.1007/s00521-012-1045-8

Cheron, G., Draye, J. P., Bourgeios, M., and Libert, G. (1996). A dynamic neural network identification of electromyography and arm trajectory relationship during complex movements. IEEE Trans. Biomed. Eng. 43, 552-558. doi: 10.1109/10.488803

Cheron, G., Leurs, F., Bengoetxea, A., Draye, J. P., Destree, M., and Dan, B. (2003). A dynamic recurrent neural network for multiple muscles electromyographic mapping to elevation angles of the lower limb in human locomotion. $J$. Neurosci. Methods 129, 95-104. doi: 10.1016/S0165-0270(03)00167-5

d'Avella, A. (2016). Modularity for motor control and motor learning. Adv. Exp. Med. Biol. 957, 3-19. doi: 10.1007/978-3-319-47313-0_1

Dipietro, L., Ferraro, M., Palazzolo, J. J., Krebs, H. I., Volpe, B. T., and Hogan, N. (2005). Customized interactive robotic treatment for stroke: EMG-triggered therapy. IEEE Trans. Neural Syst. Rehabil. Eng. 13, 325-334. doi: 10.1109/TNSRE.2005.850423

Fagg, A. H., Ojakangas, G. W., Miller, L. E., and Hatsopoulos, N. G. (2009). Kinetic trajectory decoding using motor cortical ensembles. IEEE Trans. Neural Syst. Rehabil. Eng. 17, 487-496. doi: 10.1109/TNSRE.2009.2029313

Farina, D., and Aszmann, O. (2014). Bionic limbs: clinical reality and academic promises. Sci. Transl. Med. 6:257ps212. doi: 10.1126/scitranslmed.3010453

Farmer, S., Silver-Thorn, B., Voglewede, P., and Beardsley, S. A. (2014). Within-socket myoelectric prediction of continuous ankle kinematics for control of a powered transtibial prosthesis. J. Neural Eng. 11:056027. doi: 10.1088/1741-2560/11/5/056027

Fleischer, C., and Hommel, G. (2008). A human-exoskeleton interface utilizing electromyography. IEEE Trans. Robot. 24, 872-882. doi: $10.1109 /$ TRO.2008.926860

Fougner, A. L., Stavdahl, O., and Kyberd, P. J. (2014). System training and assessment in simultaneous proportional myoelectric prosthesis control. J. Neuroeng. Rehabil. 11:75. doi: 10.1186/1743-0003-11-75

Fougner, A., Stavdahl, O., Kyberd, P. J., Losier, Y. G., and Parker, P. A. (2012). Control of upper limb prostheses: terminology and proportional myoelectric Control-A review. IEEE Trans. Neural Syst. Rehabil. Eng. 20, 663-677. doi: 10.1109/TNSRE.2012.2196711

Frisoli, A., Procopio, C., Chisari, C., Creatini, I., Bonfiglio, L., Bergamasco, M., et al. (2012). Positive effects of robotic exoskeleton training of upper limb reaching movements after stroke. J. Neuroeng. Rehabil. 9:36. doi: 10.1186/1743-0003-9-36

Gupta, A., O’Malley, M. K., Patoglu, V., and Burgar, C. (2008). Design, control and performance of RiceWrist: a force feedback wrist exoskeleton for rehabilitation and training. Int. J. Robot. Res. 27, 233-251. doi: 10.1177/02783649070 84261

Ha, K. H., Varol, H. A., and Goldfarb, M. (2011). Volitional control of a prosthetic knee using surface electromyography. IEEE Trans. Biomed. Eng. 58, 144-151. doi: 10.1109/TBME.2010.2070840

Hahne, J. M., Biessmann, F., Jiang, N., Rehbaum, H., Farina, D., Meinecke, F. C., et al. (2014). Linear and nonlinear regression techniques for simultaneous and proportional myoelectric control. IEEE Trans. Neural Syst. Rehabil. Eng. 22, 269-279. doi: 10.1109/TNSRE.2014.2305520

Heo, P., Gu, G. M., Lee, S. J., Rhee, K., and Kim, J. (2012). Current hand exoskeleton technologies for rehabilitation and assistive engineering. Int. J. Precision Eng. Manuf. 13, 807-824. doi: 10.1007/s12541-012-0107-2

Hincapie, J. G., and Kirsch, R. F. (2009). Feasibility of EMG-Based neural network controller for an upper extremity neuroprosthesis. IEEE Trans. Neural Syst. Rehabil. Eng. 17, 80-90. doi: 10.1109/TNSRE.2008.2010480
Hollerbach, M. J., and Flash, T. (1982). Dynamic interactions between limb segments during planar arm movement. Biol. Cybern. 44, 67-77. doi: $10.1007 / \mathrm{BF} 00353957$

Hopkins, W. G. (2000). Measures of reliability in sports medicine and science. Sports Med. 30, 1-15. doi: 10.2165/00007256-200030010-00001

Ison, M., and Artemiadis, P. (2014). The role of muscle synergies in myoelectric control: trends and challenges for simultaneous multifunction control. J. Neural Eng. 11:051001. doi: 10.1088/1741-2560/11/5/051001

Jiang, N., Falla, D., d'Avella, A., Graimann, B., and Farina, D. (2010). Myoelectric control in neurorehabilitation. Crit. Rev. Biomed. Eng. 38, 381-391. doi: 10.1615/CritRevBiomedEng.v38.i4.30

Jiang, N., Rehbaum, H., Vujaklija, I., Graimann, B., and Farina, D. (2014a), Intuitive, online, simultaneous, and proportional myoelectric control over two degrees-of-freedom in upper limb amputees. IEEE Trans. Neural Syst. Rehabil. Eng. 22, 501-510. doi: 10.1109/TNSRE.2013.2278411

Jiang, N., Vest-Nielsen, J. L. G., Muceli, S., and Farina, D. (2012). EMG-based simultaneous and proportional estimation of wrist/hand kinematics in uni-lateral trans-radial amputees. J. Neuroeng. Rehabil. 9:42. doi: $10.1186 / 1743-0003-9-42$

Jiang, N., Vujaklija, I., Rehbaum, H., Graimann, B., and Farina, D. (2014b). Is accurate mapping of EMG signals on kinematics needed for precise online myoelectric control? IEEE Trans. Neural Syst. Rehabil. Eng. 22, 549-558. doi: 10.1109/TNSRE. 2013.2287383

Kang, S. H., Jin, M., and Chang, P. H. (2009). A solution to the accuracy/robustness dilemma in impedance control. IEEE/ASME Trans. Mechatronics 14, 282-294. doi: 10.1109/TMECH.2008.2005524

Kearney, R. E., and Hunter, I. W. (1990). System identification of human joint dynamics. Crit. Rev. Biomed. Eng. 18, 55-87.

Kiguchi, K., and Hayashi, Y. (2012). An EMG-Based control for an upper-limb power-assist exoskeleton robot. IEEE Trans. Syst. Man Cybern. B Cybern. 42, 1064-1071. doi: 10.1109/TSMCB.2012.2185843

Kim, H., Miller, L. M., Byl, N., Abrams, G. M., and Rosen, J. (2012). Redundancy resolution of the human arm and an upper limb exoskeleton. IEEE Trans. Biomed. Eng. 59, 1770-1779. doi: 10.1109/TBME.2012.2194489

Koike, Y., and Kawato, M. (1995). Estimation of dynamic joint torques and trajectory formation from surface electromyography signals using a neuralnetwork model. Biol. Cybern. 73, 291-300. doi: 10.1007/BF00199465

Koo, T. K. K., and Mak, A. F. T. (2005). Feasibility of using EMG driven neuromusculoskeletal model for prediction of dynamic movement of the elbow. J. Electromyogr. Kinesiol. 15, 12-26. doi: 10.1016/j.jelekin.2004.06.007

Krebs, H. I., Dipietro, L., Levy-Tzedek, S., Fasoli, S. E., Rykman-Berland, A., Zipse, J., et al. (2008). A paradigm shift for rehabilitation robotics. IEEE Eng. Med. Biol. Mag. 27, 61-70. doi: 10.1109/MEMB.2008.919498

Lenzi, T., De Rossi, S. M., Vitiello, N., and Carrozza, M. C. (2012). Intentionbased EMG control for powered exoskeletons. IEEE Trans. Biomed. Eng. 59, 2180-2190. doi: 10.1109/TBME.2012.2198821

Leontaritis, I. J., and Billings, S. A. (1985). Input output parametric models for nonlinear systems .1. Deterministic non-linear systems. Int. J. Control 41, 303-328. doi: $10.1080 / 0020718508961129$

Lin, T. N., Giles, C. L., Horne, B. G., and Kung, S. Y. (1997). A delay damage model selection algorithm for NARX neural networks. IEEE Trans. Signal Process. 45, 2719-2730. doi: $10.1109 / 78.650098$

Lin, T. N., Horne, B. G., Tino, P., and Giles, C. L. (1996). Learning long-term dependencies in NARX recurrent neural networks. IEEE Trans. Neural Netw. 7, 1329-1338. doi: 10.1109/72.548162

Liu, J., Ying, D. W., and Zhou, P. (2014a). Wiener filtering of surface EMG with a priori SNR estimation toward myoelectric control for neurological injury patients. Med. Eng. Phys. 36, 1711-1715. doi: 10.1016/j.medengphy.2014.09.008

Liu, J., Ying, D. W., Zev Rymer, W., and Zhou, P. (2014b). Subspace based adaptive denoising of surface EMG from neurological injury patients. J. Neural Eng. 11:056025. doi: 10.1088/1741-2560/11/5/056025

Lo, A. C., Guarino, P. D., Richards, L. G., Haselkorn, J. K., Wittenberg, G. F., Federman, D. G., et al. (2010). Robot-assisted therapy for longterm upper-limb impairment after stroke. N. Engl. J. Med. 362, 1772-1783. doi: 10.1056/NEJMoa0911341

Mao, Y., and Agrawal, S. K. (2012). Design of a Cable-Driven Arm Exoskeleton (CAREX) for Neural Rehabilitation. IEEE Trans. Robot. 28, 922-931. doi: 10.1109/TRO.2012.2189496 
Marchal-Crespo, L., and Reinkensmeyer, D. J. (2009). Review of control strategies for robotic movement training after neurologic injury. J. Neuroeng. Rehabil. 6:20. doi: 10.1186/1743-0003-6-20

McKay, W. B., Ovechkin, A. V., Vitaz, T. W., de Paleville, D. G. L. T., and Harkema, S. J. (2011). Long-lasting involuntary motor activity after spinal cord injury. Spinal Cord 49, 87-93. doi: 10.1038/sc.2010.73

Menezes, J. M. P., and Barreto, G. A. (2008). Long-term time series prediction with the NARX network: an empirical evaluation. Neurocomputing 71, 3335-3343. doi: 10.1016/j.neucom.2008.01.030

Mirabella, G. (2012). Volitional inhibition and brain-machine interfaces: a mandatory wedding. Front. Neuroeng. 5:20. doi: 10.3389/fneng.2012.00020

Mirabella, G. (2014). Should I stay or should I go? Conceptual underpinnings of goal-directed actions. Front. Syst. Neurosci. 8:206. doi: 10.3389/fnsys.2014.00206

Mirabella, G., and Lebedev, M. A. (2017). Interfacing to the brain's motor decisions. J. Neurophysiol. 117, 1305-1319. doi: 10.1152/jn.00051.2016

Nef, T., Mihelj, M., and Riener, R. (2007). ARMin: a robot for patientcooperative arm therapy. Med. Biol. Eng. Comput. 45, 887-900. doi: 10.1007/s11517-007-0226-6

Ngeo, J. G., Tamei, T., and Shibata, T. (2014). Continuous and simultaneous estimation of finger kinematics using inputs from an EMG-to-muscle activation model. J. Neuroeng. Rehabil. 11:122. doi: 10.1186/1743-0003-11-122

Overduin, S. A., d'Avella, A., Roh, J., Carmena, J. M., and Bizzi, E. (2015). Representation of muscle synergies in the primate brain. J. Neurosci. 35, 12615-12624. doi: 10.1523/JNEUROSCI.4302-14.2015

Pan, L., Zhang, D., Liu, J., Sheng, X., and Zhu, X. (2014). Continuous estimation of finger joint angles under different static wrist motions from surface EMG signals. Biomed. Signal Process. Control 14, 265-271. doi: $10.1016 /$ j.bspc.2014.08.004

Parker, P., Englehart, K., and Hudgins, B. (2006). Myoelectric signal processing for control of powered limb prostheses. J. Electromyogr. Kinesiol. 16, 541-548. doi: 10.1016/j.jelekin.2006.08.006

Peerdeman, B., Boere, D., Witteveen, H., in't Veld, R. H., Hermens, H., Stramigioli, S., et al. (2011). Myoelectric forearm prostheses: state of the art from a user-centered perspective. J. Rehabil. Res. Dev. 48, 719-737. doi: 10.1682/JRRD.2010.08.0161

Perry, J. C., Rosen, J., and Bums, S. (2007). Upper-limb powered exoskeleton design. IEEE/ASME Trans. Mechatronics 12, 408-417. doi: 10.1109/TMECH.2007.901934

Pons, J. L. (2010). Rehabilitation exoskeletal robotics the promise of an emerging field. IEEE Eng. Med. Biol. Mag. 29, 57-63. doi: 10.1109/MEMB.2010.936548

Pulliam, C. L., Lambrecht, J. M., and Kirsch, R. F. (2011). Electromyogram-based neural network control of transhumeral prostheses. J. Rehabil. Res. Dev. 48, 739-754. doi: 10.1682/JRRD.2010.12.0237

Reinkensmeyer, D. J., and Boninger, M. L. (2012). Technologies and combination therapies for enhancing movement training for people with a disability. $J$. Neuroeng. Rehabil. 9:17. doi: 10.1186/1743-0003-9-17
Ren, Y., Kang, S. H., Park, H.-S., Wu, Y.-N., and Zhang, L.-Q. (2013). Developing a multi-joint upper limb exoskeleton robot for diagnosis, therapy and outcome evaluation in neurorehabilitation. IEEE Trans. Neural Syst. Rehabil. Eng. 21, 490-499. doi: 10.1109/TNSRE.2012.2225073

Scheme, E., and Englehart, K. (2011). Electromyogram pattern recognition for control of powered upper-limb prostheses: state of the art and challenges for clinical use. J. Rehabil. Res. Dev. 48, 643-659. doi: 10.1682/JRRD.2010.0 9.0177

Shao, Z. F., Tang, X. Q., and Yi, W. M. (2014). Optimal Design of a 3-DOF CableDriven Upper Arm Exoskeleton. Adv. Mech. Eng. doi: 10.1155/2014/157096

Shrirao, N. A., Reddy, N. P., and Kosuri, D. R. (2009). Neural network committees for finger joint angle estimation from surface EMG signals. Biomed. Eng. Online 8:2. doi: 10.1186/1475-925X-8-2

Siegelmann, H. T., and Sontag, E. D. (1991). Turing computability with neural nets. Appl. Math. Lett. 4, 77-80. doi: 10.1016/0893-9659(91)90080-F

Siegelmann, H. T., and Sontag, E. D. (1995). On the computational power of neural nets. J. Comp. Syst. Sci. 50, 132-150. doi: 10.1006/jcss.1995.1013

Siegelmann, H. T., Horne, B. G., and Giles, C. L. (1997). Computational capabilities of recurrent NARX neural networks. IEEE Trans. Syst. Man Cybern. B Cybern. 27, 208-215. doi: 10.1109/3477.558801

Smith, A., and Brown, E. E. (2011). Myoelectric control techniques for a rehabilitation robot. Appl. Bionics Biomech. 8, 21-37. doi: 10.1155/2011/143016

Song, R., Tong, K. Y., Hu, X. L., and Li, L. (2008). Assistive control system using continuous myoelectric signal in robot-aided arm training for patients after stroke. IEEE Trans. Neural Syst. Rehabil. Eng. 16, 371-379. doi: 10.1109/TNSRE.2008.926707

Zariffa, J., Kapadia, N., Kramer, J. L. K., Taylor, P., Alizadeh-Meghrazi, M., Zivanovic, V., et al. (2012). Feasibility and efficacy of upper limb robotic rehabilitation in a subacute cervical spinal cord injury population. Spinal Cord 50, 220-226. doi: 10.1038/sc.2011.104

Zhang, F., Li, P. F., Hou, Z. G., Lu, Z., Chen, Y. X., Li, Q. L., et al. (2012). sEMGbased continuous estimation of joint angles of human legs by using BP neural network. Neurocomputing 78, 139-148. doi: 10.1016/j.neucom.2011.05.033

Conflict of Interest Statement: LZ and YR hold equity positions in Rehabtek LLC, which is involved in developing the rehabilitation robot used in this study.

The other authors declare that the research was conducted in the absence of any commercial or financial relationships that could be construed as a potential conflict of interest.

Copyright (c) 2017 Liu, Kang, Xu, Ren, Lee and Zhang. This is an open-access article distributed under the terms of the Creative Commons Attribution License (CC BY). The use, distribution or reproduction in other forums is permitted, provided the original author(s) or licensor are credited and that the original publication in this journal is cited, in accordance with accepted academic practice. No use, distribution or reproduction is permitted which does not comply with these terms. 


\section{OPEN ACCESS}

Edited by:

Dingguo Zhang,

Shanghai Jiao Tong University, China

Reviewed by:

Lizhi Pan,

North Carolina State University,

United States

Zhong Yin,

University of Shanghai for Science and

Technology, China

Zhaojie Ju,

University of Portsmouth,

United Kingdom

*Correspondence:

Qin Zhang

gin.zhang@hust.edu.cn

Wenbin Chen

wbchen@hust.edu.cn

Caihua Xiong

chxiong@hust.edu.cn

Specialty section:

This article was submitted to

Neural Technology,

a section of the journal

Frontiers in Neuroscience

Received: 19 January 2017 Accepted: 01 May 2017

Published: 30 May 2017

Citation:

Zhang Q, Liu R, Chen W and Xiong C (2017) Simultaneous and Continuous

Estimation of Shoulder and Elbow Kinematics from Surface EMG

Signals. Front. Neurosci. 11:280.

doi: 10.3389/fnins.2017.00280

\section{Simultaneous and Continuous Estimation of Shoulder and Elbow Kinematics from Surface EMG Signals}

\author{
Qin Zhang *, Runfeng Liu, Wenbin Chen * and Caihua Xiong * \\ The State Key Laboratory of Digital Manufacturing Equipment and Technology, Institute of Rehabilitation and Medical \\ Robotics, Huazhong University of Science and Technology, Wuhan, China
}

In this paper, we present a simultaneous and continuous kinematics estimation method for multiple DoFs across shoulder and elbow joint. Although simultaneous and continuous kinematics estimation from surface electromyography (EMG) is a feasible way to achieve natural and intuitive human-machine interaction, few works investigated multi-DoF estimation across the significant joints of upper limb, shoulder and elbow joints. This paper evaluates the feasibility to estimate 4-DoF kinematics at shoulder and elbow during coordinated arm movements. Considering the potential applications of this method in exoskeleton, prosthetics and other arm rehabilitation techniques, the estimation performance is presented with different muscle activity decomposition and learning strategies. Principle component analysis (PCA) and independent component analysis (ICA) are respectively employed for EMG mode decomposition with artificial neural network (ANN) for learning the electromechanical association. Four joint angles across shoulder and elbow are simultaneously and continuously estimated from EMG in four coordinated arm movements. By using ICA (PCA) and single ANN, the average estimation accuracy $91.12 \%(90.23 \%)$ is obtained in 70 -s intra-cross validation and $87.00 \%(86.30 \%)$ is obtained in 2 -min inter-cross validation. This result suggests it is feasible and effective to use ICA (PCA) with single ANN for multi-joint kinematics estimation in variant application conditions.

Keywords: principle component analysis (PCA), independent component analysis (ICA), artificial neural network (ANN), simultaneous and continuous motion estimation, myoelectric control

\section{INTRODUCTION}

Surface Electromyography (EMG) signal represents ample electric information of muscle contractions innervated by neural signals. It has been used as a viable and effective neural interface in interpreting human's movement intent into control command especially for various robotic systems (Farina et al., 2014; Maciejasz et al., 2014). Traditionally, the predefined motion patterns are classified from EMG features through the trained motion classifier (Kuiken et al., 2009; Chen et al., 2013; Ju and Liu, 2014). As long as a predefined motion pattern is selected, the robot will be driven along a predefined trajectory although the user would like change their movement intent. For multiDoF coordinated motions, motion patterns of each DoF are respectively classified and sequentially employed to drive the robot to achieve the intended coordinated movement. Such motion decoding 
style based on pattern-classification technology is different from the natural neuromuscular control strategy where the human's intent (represented by EMG) is consecutively mapped to the human's motion (Farina et al., 2014). Therefore, simultaneous and continuous motion estimation has been proposed as an alternative manner to achieve natural and intuitive motor control.

Comparing with the motion pattern classification, the simultaneous and continuous motion estimation provides concurrent motion information (such as joint angle, muscle force) rather than motion trigger command together with the EMG recording. With simultaneous and continuous motion intent decoding method, the estimates of the motion information can be simultaneously obtained which is more efficient especially for coordinated motion estimation with multiple DoFs involved. This procedure continuously proceeds with the persisted recording and interpreting of the EMG signals. Although the continuous motion estimation of one or two DoFs at one joint (such as elbow Zhang et al., 2013; Han et al., 2015, wrist and/or hand Jiang et al., 2012; Muceli and Farina, 2012 or finger Ngeo et al., 2014; Pan et al., 2014) has drawn a lot of attention, there is few research on continuous estimation of multiple DoFs across shoulder and elbow joint (Au and Kirsch, 2000; Artemiadis and Kyriakopoulos, 2010, 2011). Thus, the prosthetics (Miller et al., 2008), exoskeletons (Kiguchi et al., 2008; Kiguchi and Hayashi, 2012) and other assistive devices for upper limb rehabilitation (Vogel et al., 2013; Jang et al., 2016) cannot benefit from the continuous motion estimation technology. This paper investigates the feasibility of the simultaneous and continuous estimation of 4-DoF kinematics across shoulder and elbow from EMG signals during coordinated arm movements.

Due to the nonlinearity of the mapping between the EMG and the human motions as well as the non-stationarity of the EMG signals over time, the performance of myoelectrical control heavily depends on the accuracy and robustness of the human motion estimation. To achieve this purpose, various research efforts were placed on exploring the correlation between EMG and kinematics or dynamics during different physiological or application conditions as well as developing feasible and effective EMG feature extraction and estimation methods to obtain desired kinematic and/or dynamic information. However, due to the structural and functional heterogeneity of muscles and the inherent stochastic nature of EMG signals, methodologies of motion estimation from EMG have been researched with various kinematic or dynamic constraints (Staudenmann et al., 2010; Zhang et al., 2011, 2013; Clancy et al., 2012; Li et al., 2014). Most estimation methods are developed based on various machine learning algorithms, such as linear-regression (LR), non-negative matrix factorization (NMF) and artificial neural network (ANN). In the context of multi-DoF estimation, a time-delayed artificial neural network was applied to predict the shoulder and elbow joint angles with average root-mean-square (RMS) errors of $20^{\circ}$ on able-bodied subjects during 30-s arm movements (Au and Kirsch, 2000). By using principle component analysis (PCA) for both the EMG and motion data decomposition, the shoulder and elbow motions were estimated in the low-dimensional space by a state-space decoding model (Artemiadis and Kyriakopoulos,
2010, 2011). Although the estimation accuracy was not explicitly reported, it was sufficient for the tele-operation of a robotic arm in the 3-D Cartesian space (Artemiadis and Kyriakopoulos, 2010). However, the non-stationarity characteristics of the EMG signals were still troublesome (Artemiadis, 2012), so that the decoding model had to be switched in the course of motion completion according to the muscle contraction states of each muscle involved in order to compensate the motion estimation deviation over time in 3-min arm movements (Artemiadis and Kyriakopoulos, 2011).

Apart from the nonlinearity and non-stationarity, subjectspecific and crosstalk problems have always companied with the application of EMG whose characteristics apt to be influenced by both physiological and nonphysiological factors during dynamic movements (Beck et al., 2006; Artemiadis, 2012). Thus, it was difficult to find consistent patterns among subjects regarding the motor control strategies (Beck et al., 2006). Even for single subject, since the concentric and eccentric muscle contractions adopted different motor control strategies, the joint angle had different effects on related EMG signals (Qi et al., 2011). Therefore, when shoulder and elbow coordinate to generate large-range arm motions, the EMG-based simultaneous and continuous estimation of the involved joint kinematics is complicated. This paper attempts to simultaneously and continuously estimate three shoulder angles and one elbow angle without switching the motion estimation model, which is validated with up to 2-min arm motions on six subjects.

This work proposes an alternative method to simultaneously and continuously estimate the joint angles from EMG signals during multi-joint coordinated arm movements. With respect to the previous works, EMG-driven estimation of 4 DoFs across 2 joints (shoulder and elbow) is investigated in this work during coordinated arm movements which are involved in daily activities. The estimation performance was evaluated by multiple motion repetitions up to $2 \mathrm{~min}$ in order to evaluate the robustness of the proposed methods to time-variant characteristics of EMG. During all the estimation processes, only one estimation model is learned and used for motion estimation for single subject without the model switching during the course of motion completion.

\section{METHODS}

The coordinated upper limb movements, such as reaching, pointing, exercising and manipulating, play important roles in human daily life. Although the three main joints of upper limb, shoulder, elbow and wrist, represent distinct kinematic and dynamic properties, their coordination determines the completion of human intended movement. Correspondingly, each muscle activating these joints has different spatial and temporal pattern. Wherein, the shoulder and elbow joints confer the wide range of motion for upper limb, so the coordination of the 3 DoFs at shoulder (flexion/extension, abduction/adduction and pronation/supination) and $1 \mathrm{DoF}$ at elbow (flexion/extension) were investigated in this work with the wrist joint being excluded for simplification as did in Artemiadis and Kyriakopoulos (2010) and Artemiadis and Kyriakopoulos 
(2011). In our opinion, it is more meaningful to estimate shoulder and elbow DoFs simultaneously and continuously as there are more variabilities during a wide range of their motion comparing with wrist. The pattern recognition-based humanmachine interface (HMI) cannot represent such instantaneous variations except static and discrete motion pattern. This study was approved by the local ethical committee of Huazhong University of Science and Technology, and all subjects signed informed consent forms.

\subsection{Experiment Setup}

Six able-bodied subjects (all male, $23 \pm 1$ year-old, $62 \pm 4.5$ $\mathrm{kg}$ ) participated in this study. All of them are right-handed dominant and have no reported neuromuscular disorders of their upper limb. They were ever instructed to practise the intended movements until they feel comfortable to the experimental setup.

\subsubsection{EMG Recordings}

Surface EMG signals were acquired by a commercial EMG acquisition system (Me6000, Mega Electronics Ltd, Kuopio, Finland). The configuration of the EMG recording is shown in Figure 1. Eight predominant muscles activating the four shoulder and elbow DoFs were selected to be the muscle of test, that is, biceps, triceps, deltoid (anterior), pectoralis major (clavicular head), deltoid (middle), deltoid (posterior), trapezius, and teres major muscles. Eight channel of bipolar differential amplifier were carefully placed on these muscles according to both the anatomy and hand touch experience. The active EMG electrodes of each channel were positioned at the muscle belly along the muscle fiber direction with the reference electrode orthogonal to the midline of the active electrodes according to the recommendation of Me6000. The skin underneath the electrodes was cleaned with alcohol patch to reduce the resistance between

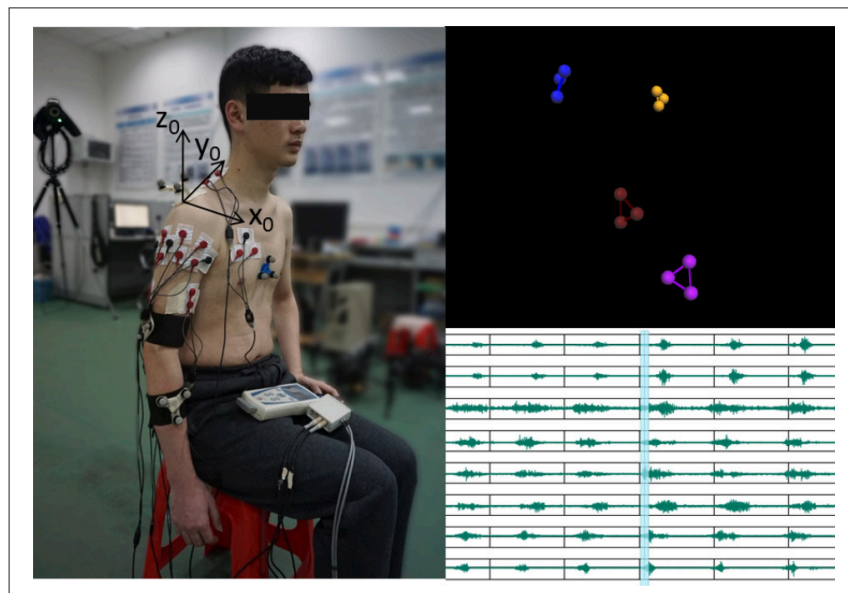

FIGURE 1 | Illustration of the experiment setup. The configuration of the EMG electrodes and reflective marker clusters is shown on the left plot with the bony landmarks being removed. Correspondingly, the motion recording marker clusters and EMG traces are respectively shown on the top right and bottom right. (The subject in the present image provided written informed consent for the publication of this image.) the skin and the electrodes. The EMG signals were amplified (gain 305) and sampled at $1 \mathrm{KHz}$.

\subsubsection{Motion Recordings}

A 6-camera motion capture system (Vicon F20-MX3, Vicon Motion Systems Ltd, Oxford, UK) was used to record the motions of the upper limb. The Euler angles were calculated from the reflexible marker positions as proposed previously (Chen et al., 2010). During the dynamic motion recording, only four marker clusters were needed (as shown in Figure 1). The recording marker clusters were attached respectively at the flat part of the acromion, the lateral upper arm just below the insertion of the deltoid, the dorsal surface of the distal forearm. The reference marker cluster was located at the flat part of sternum close to the jugular notch. Before the dynamic motion recording, seven bony landmarks were added to specific positions together with the marker clusters according to the recommendation on definitions of joint coordinate systems ( $\mathrm{Wu}$ et al., 2005). The position relationship between the bony landmarks and the marker clusters was established in a static recording trial. The position of the bony landmarks could then be reconstructed by the marker clusters and their relationship after the bony landmarks were removed. The advantage of such process, reconstructing the bony landmarks from the rigid marker clusters rather than directly recording the bony landmark positions, is to eliminate the recording errors due to the relative bone movement across specific joints. The motion recording was sampled at $50 \mathrm{~Hz}$ and synchronized with the EMG recording through the motion capture system.

\subsection{Experimental Protocol}

During the experiment, the subjects were quietly seated in the chair with their torso keeping upright and their right hand keeping relaxing. Considering both the functional tasks of upper limb (van Andel et al., 2008) and the present experimental setup (for example, some movements having serious occlusion problems of the reflexive markers during the course of movement have to be excluded), four motions shown in Figure 2 were designed to obtain sufficient range of motion of the right upper limb. All the motions were initiated when the right arm was

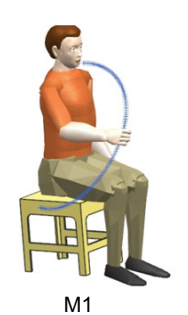

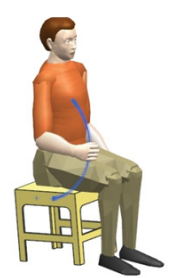

M2

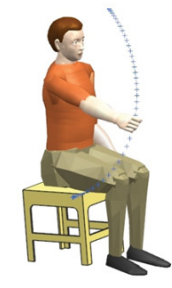

M3

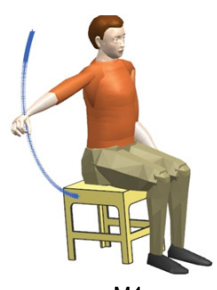

M4
FIGURE 2 | Illustration of the movements and their trajectories involved in this study. All these movements were resulted from the coordination of multiple muscles and multiple joints. Each movement was initiated from the lowest position and returned to the initial position after the completion of the movement. 
freely hanging and close to the torso. The traces in Figure 2 indicated the movement trajectory from the initial position to the destination position in a single trip of each motion. Motion M1 simulated delivering water to drink. In motion M2, the subjects were asked to touch their right shoulder with their right hand in the sagittal plane. In motion M3 (M4), the subjects were asked to lift their right arm in the sagittal (coronal) plane as they can. After the completion of each movement, the right arm returned to the initial position. As most daily-life movements of human are quasi-rhythmic after sufficient learning and training except when unexpected events happen (Churchland et al., 2012; Kandel et al., 2012), these four arm movements are freely repeated several times in order to capture both the commonality and the variability of the EMG and motion properties.

In order to collect sufficient experimental data and catch most variabilities during the experiments, we designed two separate motion sessions with each session consisting of fifteen repetitions of the motion (see Figure 3) for each motion of each subject. The muscles were allowed to relax shortly (around $3 \mathrm{~s}$ ) before initiating the next motion repetition. Around 5-min rest was allowed before the start of the second test session. All the motions were performed naturally without any kinematic or dynamic constraints of the right arm. The exact duration of the single motion completion and the rest between adjacent motion repetitions were controlled by the subjects themselves as they felt comfortably. Such experimental setup without strict kinematic constraints was also suitable to verify the feasibility of the proposed joint angle estimation method in practical myoelectric control scenario.

\subsection{Data Processing}

With the above EMG and motion recording strategies, both of the EMG and motion data were saved in a computer and treated off-line in Matlab (The MathWorks, Version 7.10.0.499, 64-bit, 2010). The purpose of data processing is to extract suitable signal features, including EMG and motion features, for estimation

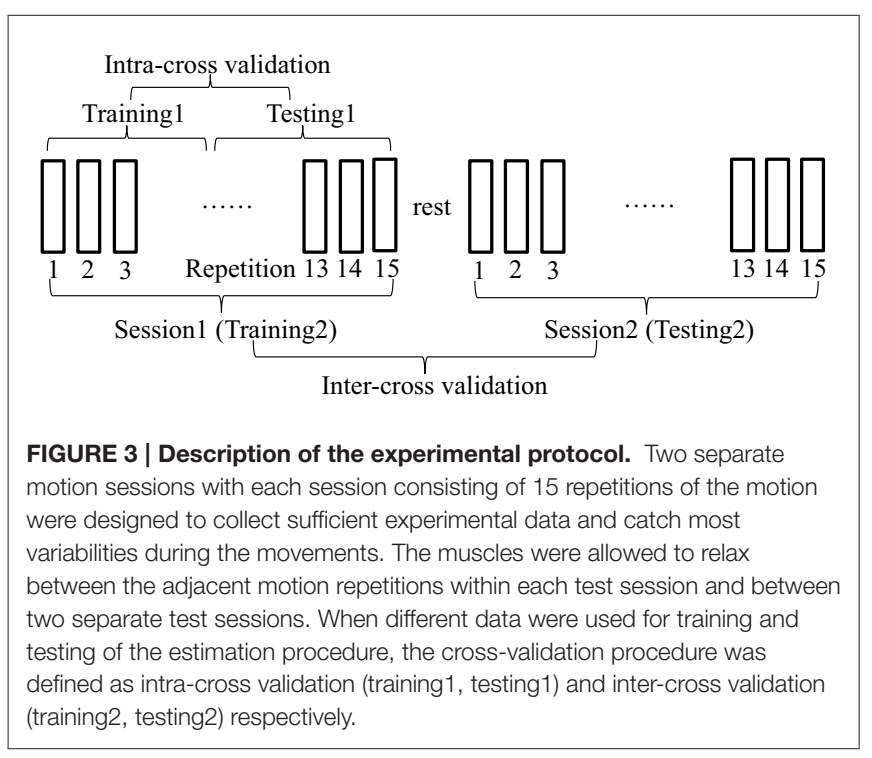

model learning. In addition, the test data used for cross validation were also processed as did for the training data.

As we know, the muscle synergies and motion synergies contribute to reducing the complexity of neural control in the redundant human motor system. Mathematics techniques, such as PCA, can be used to parse the complex data into a small number of components, for example, only two synergies were needed to account for more than $96 \%$ of the activation patterns of eleven human arm muscles during free arm motions (Artemiadis and Kyriakopoulos, 2011). Independent component analysis (ICA) was also effective to extract statistically independent muscle activity source signal from their combinations from multi-channel EMG (Nakamura et al., 2004; Garcia et al., 2005) and high-density EMG signals. ICA has been considered superior to PCA in improving force estimation accuracy from EMG (Staudenmann et al., 2007). Inspired by these previous works, two mode decomposition methods, PCA and ICA, were respectively conducted following band-pass filtering (6th Butterworth with $10-400 \mathrm{~Hz}$ cut-off frequency) of the EMG signals.

The multi-channel of EMG recordings are usually noisy and have super-Gaussian or Gaussian distributions in different activation levels (Nazarpour et al., 2013). PCA is selected to process the EMG signals as it is especially efficient in dimension reduction when the signals are highly correlated. With the PCA processing, the recorded EMG signals can be on one hand transformed to the low-dimensional principal components (PCs) with noise free. On the other hand, the dependence among different recording channels can be broken resulting in uncorrelated signals. As for the ICA algorithm, it works under the hypothesis that the original signals are linearly mixed and have non-Gaussian distribution, so that it is reasonable to perform PCA as a preprocessing for ICA to discard the irrelevant structures among the EMG signals and satisfy the hypothesis of ICA algorithm. From now on, whenever we mentioned ICA, it means ICA was conducted after preprocessing of PCA while PCA means only PCA was conducted. The detailed algorithm of PCA and ICA can be found in Johnson and Wichern (2007) and Hyvarinen et al. (2001).

Noth that, the feature vector obtained by the training data with PCA was saved for the testing data to be projected in the same low dimensional space as did on the training data. With ICA, the estimation of unmixing matrix can be obtained through different ways, such as the approximations of negentropy, the minimization of mutual information and the maximum likelihood estimation. We achieved the estimation of unmixing matrix through negative entropy maximization due to its advantages such as fast convergency and robustness (Hyvarinen et al., 2001). Similarly, the unmixing matrix obtained with the training data was kept for the testing data to be unmixed in the same way as did on the training data.

After the PCs (ICs) of the recorded EMG signals were induced from PCA (ICA), the EMG feature set, including mean absolute value (MAV), root mean square, waveform length, zero crossing, variance, median frequency and mean frequency were further calculated in a $40 \mathrm{~ms}$ analysis window with $50 \%$ overlapping. Although such feature set was applied or partly applied in previous works (Artemiadis and Kyriakopoulos, 2011; Jiang et al., 
2012), we did not find consistent improvement in estimation quality when using the combined feature set comparing with using only MAV feature. Therefore, we only present the analysis and results by using only MAV as the EMG feature in the following description.

For the motion data, 2-order butterworth filter with cutoff frequency of $6 \mathrm{~Hz}$ was applied firstly to the recorded reflective marker position data. The three joint angles at shoulder and one joint angle at elbow were then calculated from the marker position data according to the method proposed previously (Chen et al., 2010). The 4 DoFs are defined as shoulder abduction/adduction (SAA), shoulder flexion/extension (SFE), shoulder pronation/supination (SPS) and elbow flexion/extension (EFE). Although the joint motion dimension can be reduced from original 4-fold into 2-fold representing $98 \%$ of the total variance as did in Artemiadis and Kyriakopoulos (2010), since we found this processing did not contribute in reducing the complexity or improving the estimation accuracy significantly, the original 4 joint angles were directly used as the estimation target rather than the low-dimensional 2 components. In Figure 4, the 4 DoFs are respectively rectified and normalized by their maximum value to represent in the same range of $[0,1]$. As we expect, for the same DoF, it differs over time during different movement repetitions. Among all the DoFs, their correlation differs during different movement repetitions as well, which reveals clear evidence of redundancy in achieving the same movement with different activating strategies of multiple DoFs. In addition, the behavior of single DoF and the correlation among all the DoFs are both different in different subjects for the same movement. Because of these variabilities, simultaneous and continuous estimation of
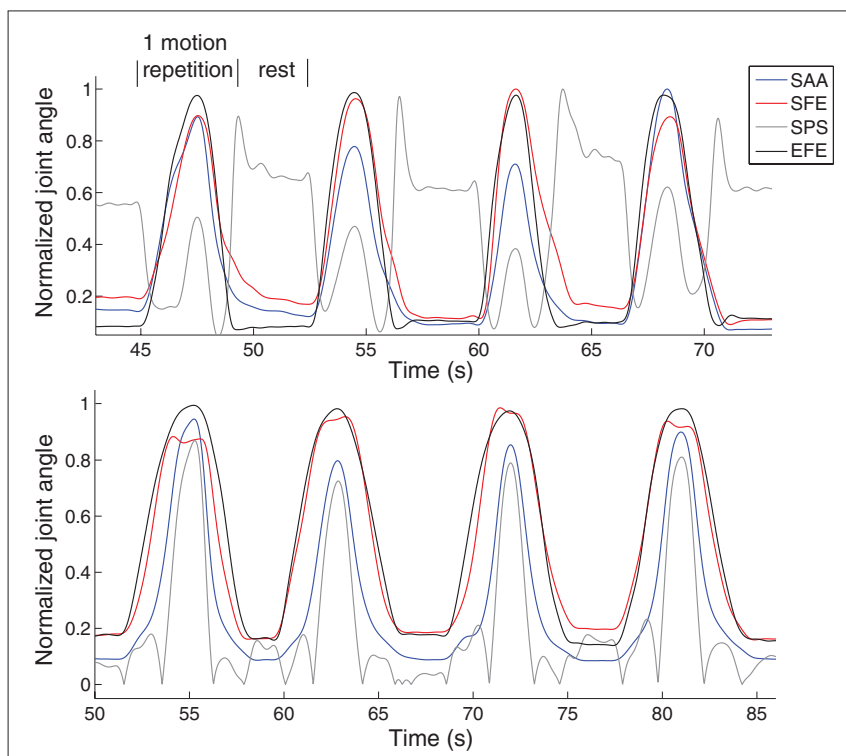

FIGURE 4 | Examples of 4 motion repetitions of M1 in two subjects. It is clear to find evidence of redundancy in achieving the same movement with different activating strategies of multiple DoFs in the same subject. The behavior of single DoF and the correlation among all the DoFs are both different in different subjects for the same movement. the joint angles is important to interpret instantaneous neural variations for natural and accurate myoelectric control.

Until now, we obtained both the EMG features and joint angle features to be the input and output respectively of the motion estimation model.

\subsection{Joint Angle Estimation with ANN}

Multi-layer perceptron (MLP), a feedforward ANN model, was used to learn the mapping from the low-dimensional EMG feature to the shoulder and elbow joint angles. The multiple MLPs (mMLPs) has been popularly used for training where one MLP outputs one joint angle in order to obtain accurate estimation for each joint angle (Jiang et al., 2012; Muceli and Farina, 2012). In addition to the separate MLP training for each joint angle estimation, we attempted to use single MLP (sMLP) for concurrent estimation of all the joint angles. The sMLP was then assessed to see if it could be used instead of mMLPs with comparable estimation accuracy and compact training procedure. In each MLP training neural network, there were three hidden layers with five neurons in each layer. The neurons in the hidden layers and output layer had a tangent sigmoid transfer function and a Linear transfer function respectively. The MLP was trained through the Leverberg-Marquardt back-propagation algorithm. All these network training parameters were fixed during the training and testing processes.

The training and testing of the SMLP strategy are illustrated in Figure 5. In the training phase, the EMG features and joint angles were fed into the MLP, the correlation between them was learned and kept for the following testing phase. In the testing phase, the trained MLP outputs the joint angle estimates in the presence of new EMG feature data. The performance index $R^{2}$ is calculated from the measured and estimated joint angles through Equation (1). Note that, in the feature extraction procedure, either PCA or ICA was applied before the calculation of MAV of EMG.

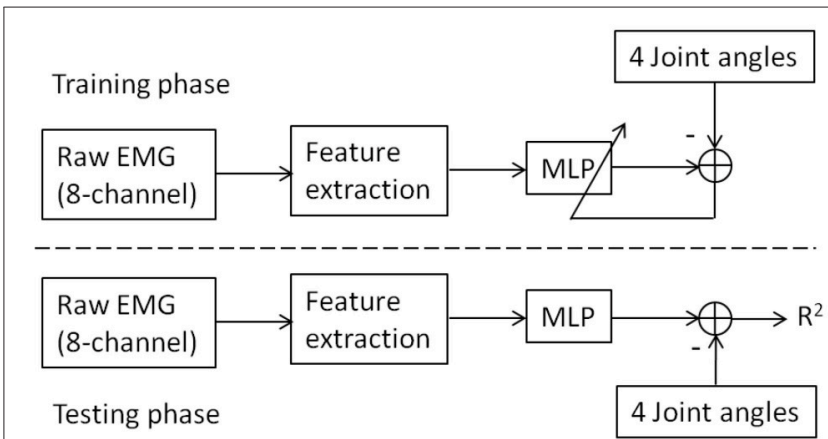

FIGURE 5 | The illustration of joint angle estimation from EMG through sMLP network. In the training phase, the MLP were learned by the differences between the measured joint angles and the MLP output. In the testing phase, the trained MLP outputs the joint angle estimates in the presence of new EMG feature data. The performance index $R^{2}$ is calculated from the measured and estimated joint angles through Equation (1). In the feature extraction, the raw EMG signals were sequentially processed with band-pass filtering, PCA or ICA decomposition and MAV calculation. 


\subsection{Performance Index}

The estimation performance can be evaluated by different indices, such as RMS error, correlation coefficient (CC) and coefficient of determination $R^{2}$. In order to compare the performance of the proposed method with other works, global $R^{2}$ was calculated across all the joint angles as below:

$$
R^{2}=1-\frac{\sum_{i=1}^{D} \sum_{t=0}^{N}\left(\widehat{y_{i}(t)}-y_{i}(t)\right)^{2}}{\sum_{i=1}^{D} \sum_{t=0}^{N}\left(y_{i}(t)-\overline{y_{i}(t)}\right)^{2}}
$$

where $y_{i}(t), \overline{y_{i}(t)}$ and $\widehat{y_{i}(t)}$ are respectively the real joint angle calculated from motion capture system, its average over time and its estimates from only EMG signals at time instant $t$ for the ith DoF. $N$ is the number of samples of each joint angle. $D$ is the number of DoF involved in the study.

All the estimations were performed on the experimental data recorded from six able-bodied subjects. In a word, the human motion estimation process consists of two phases: training and testing. In the traditional estimation method, $k$-fold cross validation ( $k=4$ or 5$)$ was popularly applied, where one out of the $k$ repetitions of each movement was used as testing set and the rest repetitions as training set (Jiang et al., 2012; Muceli and Farina, 2012). In comparison, we applied leave-p-out strategy for the cross-validation where $p$ motion repetitions were used for testing with the rest for training. As the variabilities over time likely deviate the estimation result, this strategy is helpful to evaluate the possibility of the trained model in estimating joint angle in continued movement repetitions. If the training and testing data coming from the same test session, we call such traditional cross-validation process as intra-cross validation. In addition, we proposed to conduct inter-cross validation to reveal more convincing results where the training and testing data were coming from two test sessions for each motion in each subject (see Figure 3). Comparing with the intra-cross validation, the inter-cross validation is closer to the practical online condition in myoelectric control, where the testing was performed online following the offline training.

\section{RESULTS}

The purpose of the current work is to propose a simultaneous and continuous joint angle estimation method for multi-joint coordinated upper limb myoelectric control. In the course of joint angle estimation, several factors, such as signal feature extraction method, time-variant properties of EMG and MLP training/testing strategy, may have unexpected influence on the estimation accuracy. Any failure on the estimation course may result in the failure or high error in the joint angle estimation. Therefore, the estimation performance was evaluated in several aspects.

1) EMG Mode Decomposition: The estimation performance with ICA and PCA decomposition was compared, suggesting their contribution and advantages for the estimation quality. Original 8-channel raw signals were reduced to 3 5 -manifold for different subjects representing $95 \%$ of the total variances by PCA processing. When the ICA algorithm was conducted, the uncorrelated principal components obtained by PCA was treated as the mixed signals.

2) Training Strategy: We compared to training strategies in this paper, that is, four joint angles were derived from four MLP networks individually (mMLPs) or from one MLP together (sMLP). Their feasibility and difference were presented and compared by the statistic analysis of the estimation results.

3) EMG Non-stationarity: As nonstationary EMG property was always a trouble in EMG relevant works, including myoelectric control, the estimation performance of the proposed methods was tested in short-term and middleterm as well, suggesting its feasibility for middle-term use. Either in the intra-cross validation or the inter-cross validation, we divided the testing data into two equivalent parts. The estimation of the first half was called short-term estimation with the last half corresponding to the middle-term estimation.

4) Cross-Validation: In the intra-cross validation procedure, all the training and testing data were selected from the same test session with half for training and the other half for testing as shown in Figure 3. In this work, around seven motion repetitions were used for testing which can catch more variabilities during the experiments. In the inter-cross validation, the data in session 1 were used for training with the data in session 2 for testing. In total, there were fifteen motion repetitions being used for testing. The estimation accuracy was the average of the estimates of multiple motion repetitions which was more practical and convincing to represent the overall estimation quality of the proposed methods.

\subsection{Multi-DoF Motion Estimation during Intra-Cross Validation}

An example of the joint angle estimation during intra-cross validation is presented in Figure 6. The blue lines indicate the joint angles calculated from motion capture system which are considered as the real joint angles. The red lines indicate the joint angle estimates from the EMG and MLP (trained with the training datasets). The estimation was obtained by SMLP and the EMG was processed by ICA. The global estimation accuracy is $94.6 \%$ represented by $R^{2}$. In such arm movement, multiple muscles and multiple joints were involved to achieve the coordinated muscle contractions and joint movements. Although the motion velocities and the trajectories are both different among different motion repetitions as shown in Figure 4, all the joint angles can be still estimated in the rest, initial concentric and final eccentric motion phase, indicating the feasibility and accuracy of the joint angle estimation method from EMG even in the presence of some kinematic variabilities.

Although the estimates of joint angles can be directly used as the control commands for the robot control, the spacial trajectory of the end-effector is also what we concern. The trajectory must be natural and smooth, otherwise, the joint coordination may be problematic in practice. Therefore, we evaluated the 4 joint angle estimation performance not only by comparing the estimated 


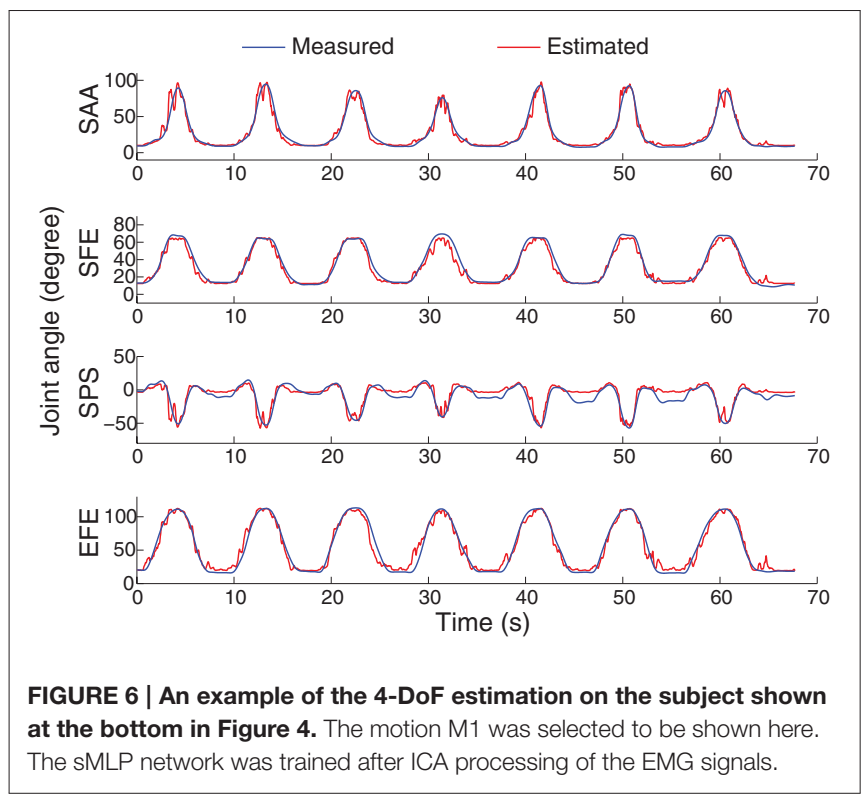

joint angles with the measured angles, but also by comparing the trajectories of the end-effector driven by the estimated angles and measured angles. The forward kinematics analysis was conducted using the measured and estimated joint angles respectively to compare the measured and estimated trajectories of the endeffector. As the wrist joint was excluded in this work, the center of the wrist joint was considered as the end-effector with the wrist joint being fixed. Figure 7 shows the motion trajectory of the end-effector constructed by the measured joint angles (blue) and the estimated joint angles (red) in Figure 6. In order to show the movement trajectory intuitively, the original coordinate is set at $\left(x_{0}, y_{0}, z_{0}\right)$ as shown in Figure 1. In this motion (M1), both the shoulder and elbow joint move in a large range of motion. The determination of $R^{2}$ of the trajectory matching is $94.58 \%$. The estimation error mainly exists at the beginning of the movement. Comparatively, the midline and the destination of the trajectory calculated from the estimated joint angles matches the recorded quite well.

\subsubsection{Performance Comparison with Different EMG Features and Training Strategies}

PCA and ICA were both considered necessary and useful in EMG mode decomposition and represented their suitability in EMGbased joint angle or force estimation (Staudenmann et al., 2007; Artemiadis and Kyriakopoulos, 2010). We intended to evaluate their contribution to the motion estimation performance. Thus, the motion estimation accuracies were calculated and compared between PCA and ICA processing of the EMG signals in this study. For the neural network training, previous works applied mMLPs to yield one angle from each MLP. To simply the training strategy, we compared the estimation performance with sMLP and mMLPs. Finally, sMLP and mMLPs grouping with PCA and ICA algorithm respectively were used for neural network training. All the estimation accuracies of each motion across all the subjects were averaged and summarized in Figure 8. We find that the sMLP is sufficient to yield comparable even better estimation accuracy with respect to the MMLPs either with PCA or ICA for EMG mode decomposition. In addition, the training complexity and computation time is superior when training by sMLP. For the comparison between ICA and PCA, both of them can give high estimation accuracy for each motion. If we average the estimation accuracies across all the motions, the four combinations, that is, ICA+mMLPs, ICA+sMLP, PCA+mMLPs and PCA+sMLP, have respectively yielded $89.23,91.12,88.7$, and $90.23 \%$ estimation accuracy comparing with the measured joint angles. By conducting two-way ANOVA test between the two factors, EMG decomposition method (ICA/PCA) and training strategy (sMLP/mMLPs), the $p$-values for the ICA/PCA, 0.5919, the sMLP/mMLPs, 0.1409 , and the interaction between the two factors, 0.8225 , indicating all these combinations get similar motion estimation performance and no significant interactions between the two factors. Considering the potential versatility of the ICA and the simple configuration of the SMLP, in the following study, if we did not explain especially, the EMG was processed by ICA and the sMLP was chosen as the training strategy.

\subsubsection{Performance Comparison between Short-Term and Middle-Term Estimation}

Nonstationary properties of EMG is a troublesome effect for myoelectric control. For example, the estimation accuracy decreased from around $30 \mathrm{~s}$ without model switching strategy in a free arm movement (Artemiadis and Kyriakopoulos, 2011). The inherent cause may come from the complex electrochemical behaviors of the skeletal muscles. The robustness of the estimation method to the time-related factors was evaluated by comparing the estimation accuracies in the short-term and middle-term application. If any stage of the estimation process, including feature extraction and training, can not overcome the effects of the time-variant factors, the estimation quality may deteriorate over time. In Figure 9, the estimation performance in short-term and middle-term was summarized in the condition of intra-cross validation. The global estimation accuracy is the averaged $R^{2}$ when all the test data were used for estimation. When the first half of the testing data were estimated, the short-term accuracy was calculated, whereas the last half of the testing data corresponding to middle-term estimation. The performance across the estimation duration was not statistically significant $(p=0.9676)$, indicating the estimation performance is similar during the 70-s arm movements.

\subsection{Multi-DoF Motion Estimation during Inter-Cross Validation}

In intra-cross validation, part of data from the same motion session was used for training with the other part for testing. In practice, when the training phase finished, a new motion session starts and new EMG signals may result in corresponding estimates. Therefore, we also tested the motion estimation using EMG signals from two different sessions for training and testing respectively, which was more practical from the muscle states and estimation conduction procedure. An example of 

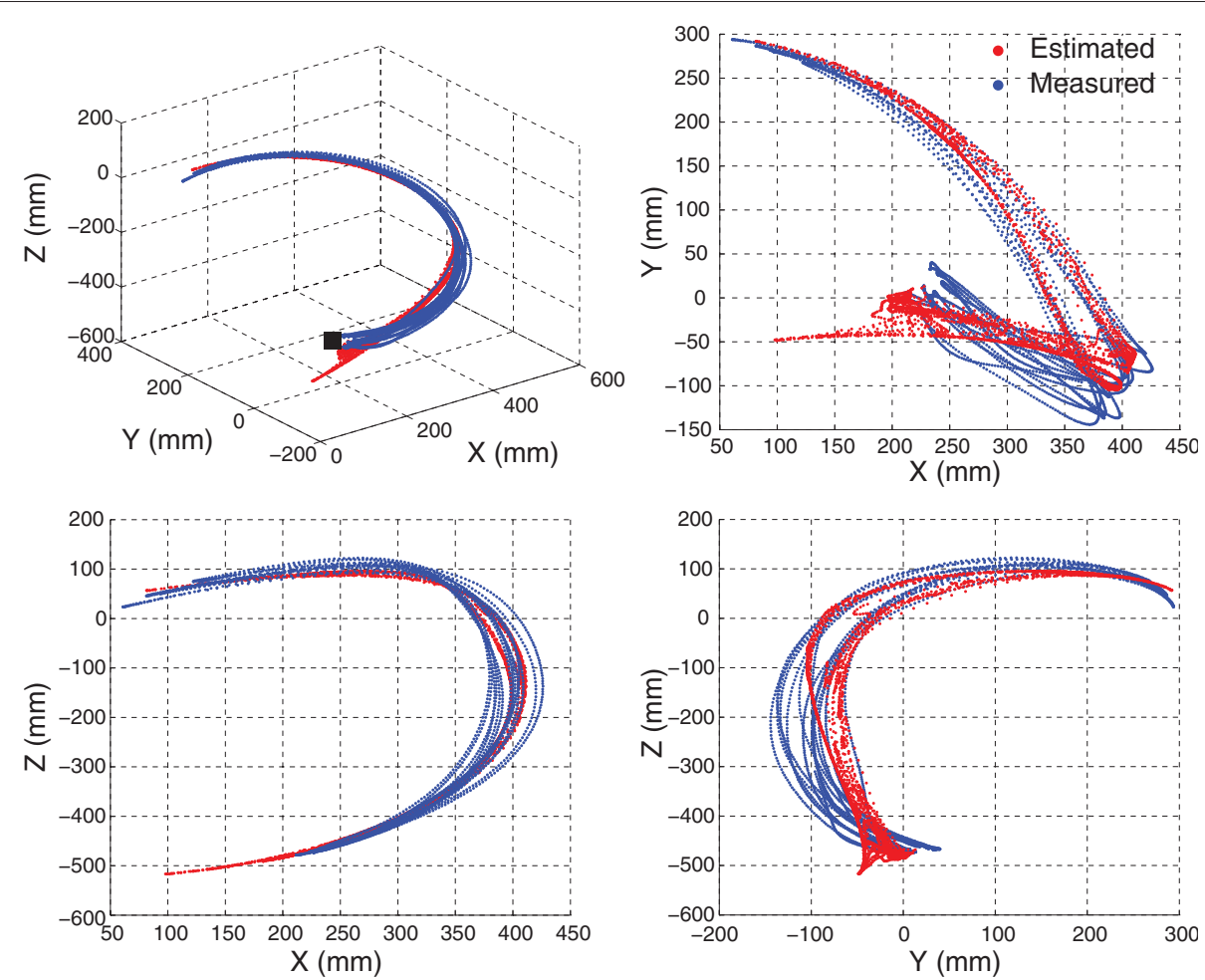

FIGURE 7| An example of the movement trajectory of the end-effector constructed by the measured joint angles (blue) and the joint angle estimates (red). The motion M1 with seven repetitions, the same as shown in Figure 6, was shown here. The movement trajectory in 3-D space is shown on the left top with the others being the movement trajectory projection in 2-D space. The initiation position of the movement is indicated by a black solid square.

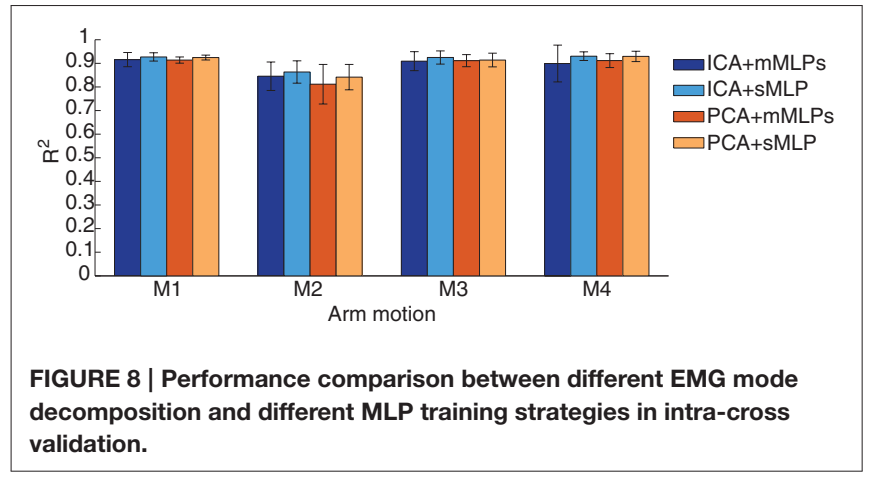

the joint angle estimation during the inter-cross validation is shown in Figure 10. The estimation accuracy is $98.95 \%$ for the motion M4 of this subject in the 2-min estimation. In this motion, the subjects were asked to lift their right arm in the coronal plane. Due to no kinematic or dynamic constraints being exerted, the subject could perform this motion as they felt comfortably. For example, they may flex their elbow to compensate the uncomfortability during the motion. In this motion, the 4 joint angles were ranged quite differently, while the joint angle estimates were quite close to the measured angles. The corresponding motion trajectory estimation accuracy is 91.38\% (shown in Figure 11). Although the practical initiation positions and trajectories (blue) in different motion repetitions

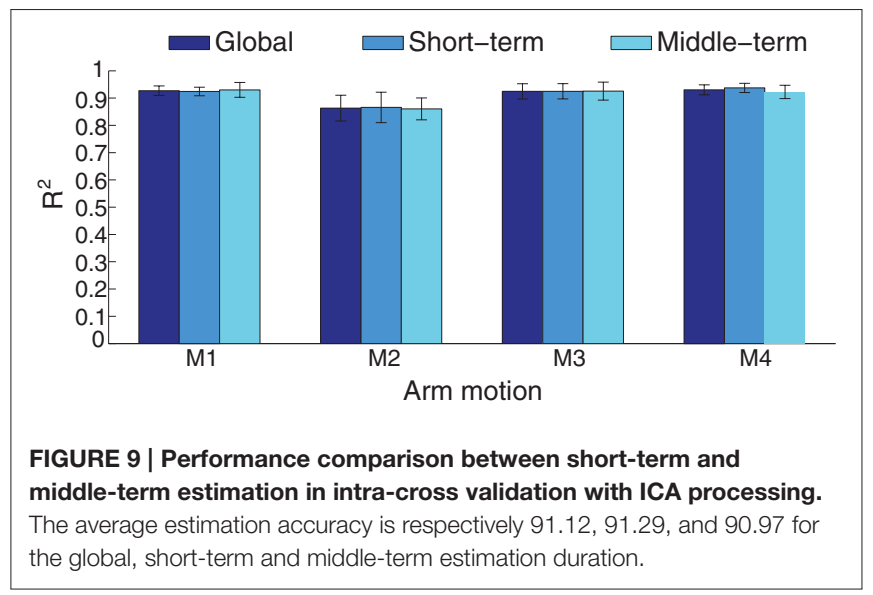

are scattering in each plane, the constructed estimate of the motion trajectories (red) are still smooth and accurate in reaching destination.

The estimation comparison between ICA and PCA in this inter-cross validation is shown in Figure 12. The average estimation accuracy with ICA and PCA of four motions across six subjects is 87 and $86.3 \%$ respectively. The estimation performance seems similar in total with ICA or PCA for EMG mode decomposition in this inter-cross validation $(p=$ 0.8531), except for the motion M2 where ICA presents apparent 

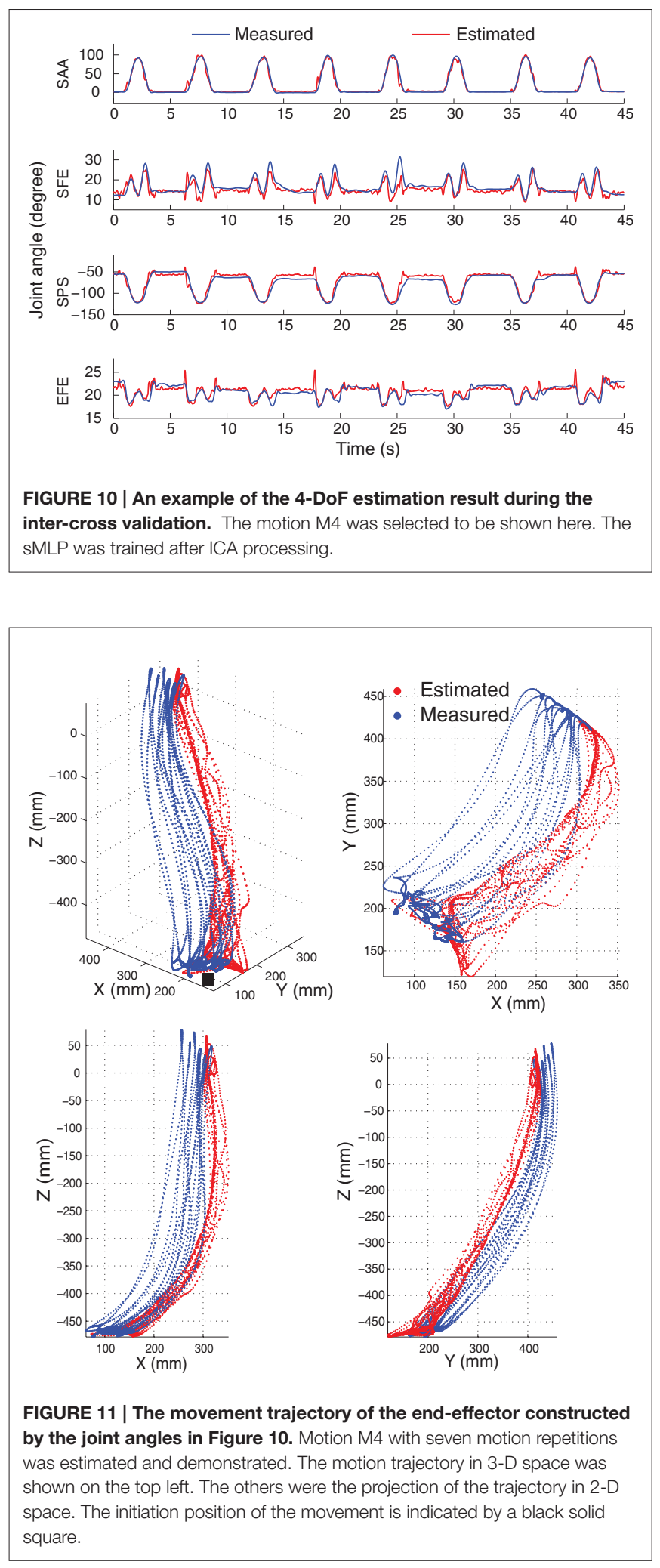

advantages comparing with PCA. With respect to the intra-cross validation where $91.12 \%(90.23 \%)$ estimation accuracies were presented in Figure 8, the estimation in the inter-cross validation

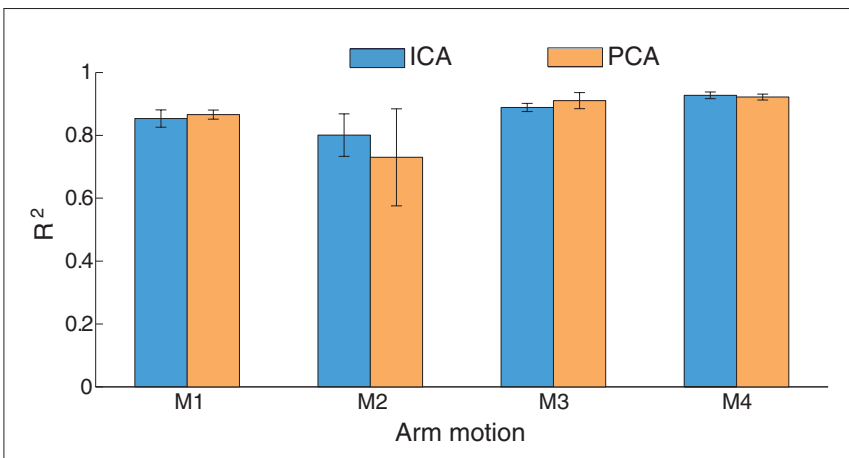

FIGURE 12 | Statistics results $\left(R^{2}\right)$ between ICA and PCA in inter-cross validation. The results were averaged across six subjects.

is a little worse. However, considering the practical condition of myoelectric control and the estimation accuracies of the previous works as described in the introduction, this estimation performance is quite satisfactory. Moreover, the proposed method also works well during 2-min estimation process in the inter-cross validation. The average estimation accuracy was summarized in Figure $\mathbf{1 3}$ during middle-term estimation with ICA. There are also no significant differences among the global, short-term and middle-term estimation performance ( $p$ $=0.8870$ ).

\section{DISCUSSION}

This paper investigated the feasibility of simultaneous and continuous motion estimation from EMG during arm movements involving coordinated activation of four DoFs across shoulder and elbow joints. In comparison to single joint (such as wrist or elbow joint), the coordination of shoulder and elbow joints offer more contribution for the upper limb to reach objects in a large range of motion. Thus, the simultaneous and continuous estimation of shoulder and elbow motion is important and challenging due to the complex activation of multiple muscles and multiple DoFs (Prilutsky et al., 2011). Although only four arm motions were involved in this work, all of them were functional movements popularly in daily life and achieved by activating all the four DoFs under the condition of no kinematic or dynamic constraints. It is especially meaningful in rehabilitation robotics systems where functional movements are practically important for the users (Takahashi et al., 2008). Moreover, such rehabilitation robot is usually able to provide functional but few movements due to the tradeoff between the complexity of mechanical configuration and the complexity of control system (Maciejasz et al., 2014).

Myoelectric control based on simultaneous and continuous motion estimation has been considered superior to that based on pattern recognition as it provides continuous motion (joint angle) estimates as long as the EMG signals are available. In this work, the proposed method either with PCA or ICA for EMG mode decomposition yields accurate joint angle estimation of the 4 DoFs at shoulder and elbow joints. The estimation performance was not only evaluated by intra-cross validation and inter-cross 


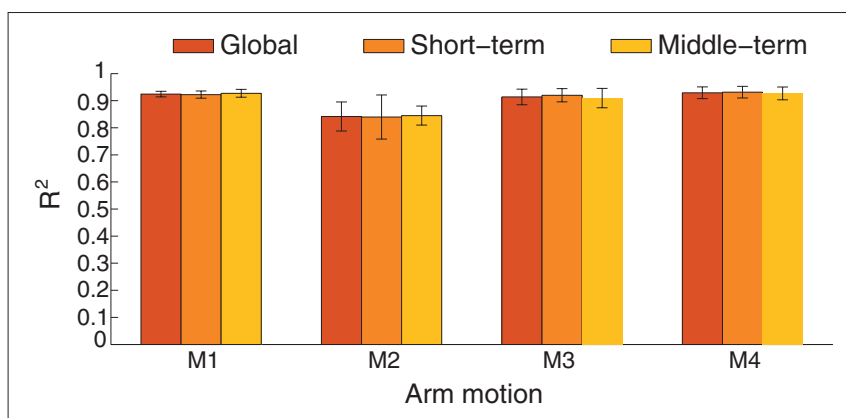

FIGURE 13 | The joint angle estimation performance is compared between short-term and middle-term estimation in inter-cross validation across six subjects.

validation, but also evaluated in short-term and middle-term condition. In intra-cross validation, ICA and PCA with SMLP training respectively gives 91.12 and $90.23 \%$ estimation accuracy on average over four arm motions across six subjects. In intercross validation, the corresponding estimation accuracy is 87.14 and $86.30 \%$ respectively. This estimation accuracy is high in the context of simultaneous and continuous estimation of multiple DoFs. In addition, the estimation model was not needed to be switched even during 2-min movement, which is relatively stable and efficient for online myoelectric control.

In this preliminary study, only able-bodied subjects were involved. In this situation, the method can be directly used for tele-operation of assistive robots as in Artemiadis and Kyriakopoulos (2011) and any mechatronics device as long as we would like to control it by our movement intent. This method can be also applied for myoelectrical control of exoskeletons of upper limb during the rehabilitation stage of muscle force enhancement. Furthermore, this method is quite promising to be applied for arm prosthetics control benefiting from more motor information provided by targeted muscle reinnervation (TMR) which has been proved able to use EMG decoding to regain intuitive and simultaneous functional control (Miller et al., 2008; Kuiken et al., 2009; Hargrove et al., 2013). In the proposed method, PCA and ICA both provided satisfactory estimation accuracies in the proposed simultaneous motion estimation, while the latter is a little better due to its capacity in further separating the source muscle activities following muscle synergies extraction in the surface EMG recording scenario. Moreover, ICA is expected to be more advantageous comparing with PCA in the presence of more muscle activity cross-talks through highdensity EMG recordings or multiple EMG recordings from the targeted muscles after TMR surgery.

The accurate and stable estimation of multiple DoFs from muscle activities can not only contribute to practical myoelectric control in various robotic systems, but also reveal the inherent correlation between the muscle activities and the coordinated joints through suitable feature extraction and correlation learning methods. Such correlation may be different among different motion tasks, yet determinable for the same motion task even multiple joints and multiple muscles were involved to obtain the coordinated motions.

\section{CONCLUSIONS AND PERSPECTIVES}

EMG signals have been popularly used to decode human motion intent for myoelectric control. Traditional motion estimation method uses pattern recognition to provide binary control commands which can only move the robot in a predefined pattern. Simultaneous and continuous motion estimation from EMG has been proposed to provide more natural and accurate motion control command with respect to pattern-recognition based estimation. However, few works have achieved significant progress in simultaneous and continuous estimation of multijoint coordinated motions. In this work, we proposed a simultaneous and continuous estimation method which can accurately estimate 4 joint angles across two joints for coordinated upper limb motions only from multi-channel EMG signals. EMG mode decomposition through PCA and ICA both revealed their effectivities and feasibilities to obtain accurate joint angle estimation with ICA a litter better. A sMLP network was proposed to estimate the joint angles from the EMG signals after short training. The experimental data from six able-bodied subjects were used to train the neural network and validate the joint angle estimation performance. By comparing the estimated joint angles with the calculated joint angles from the motion capture system, we can find the joint angle estimation accuracy is quite high which indicates that the inherent correlation between the muscle activities and joint angles during complex coordinated motion tasks is feasible to be explored through the proposed mode decomposition and training methods. As the application of EMG decoding in the assistive robot teleoperation (Artemiadis, 2012) and the robotic leg control after a TMR surgery (Kuiken et al., 2009; Hargrove et al., 2013), the accurate arm motion estimation is promising to be applied to control the assistive robot, prosthetic arm or any mechatronics system that we expect to control by our movement intent. In the early future, the proposed method will be further researched on myoelectric control of an exoskeleton our team developed (Liu et al., 2013) and increasing the motion of range of upper limb.

\section{AUTHOR CONTRIBUTIONS}

QZ: Conceived this study. QZ and RL: Performed the experiments and collect the data. WC: Calculated the joint angle from position data. QZ and CX: Proposed the EMG signal processing method. QZ and RL: Analyzed the data and implemented the motion estimation. QZ: Wrote the manuscript. All the co-authors helped revising the manuscript and agreed with the present version.

\section{ACKNOWLEDGMENTS}

This work was partly supported by the National Natural Science Foundation of China (Grant No. 51305148, 91648203, 51335004), the International Science and Technology Cooperation Program of China (Grant No. 2016YFE0113600), and the Natural Science Foundation of Hubei Province (Grant No.2015CFA004). 


\section{REFERENCES}

Artemiadis, P. K., and Kyriakopoulos, K. J. (2010). EMG-based control of a robot arm using low-dimensional embeddings. IEEE Trans. Robot. 26, 393-398. doi: 10.1109/TRO.2009.2039378

Artemiadis, P. K., and Kyriakopoulos, K. J. (2011). A switching regime model for the EMG-based control of a robot arm. IEEE Trans. Syst. Man Cybern. B Cybern. 41, 53-63. doi: 10.1109/TSMCB.2010.2045120

Artemiadis, P. K. (2012). EMG-based robot control interfaces: past, present and future. Adv. Robot. Autom. 1:e107. doi: 10.4172/2168-9695.1000e107

$\mathrm{Au}, \mathrm{A}$., and Kirsch, R. (2000). EMG-based prediction of shoulder and elbow kinematics in able-bodied and spinal cord injured individuals. IEEE Trans. Rehabil. Eng. 8, 471-480. doi: 10.1109/86.895950

Beck, T., Housh, T., Johnson, G., Cramer, J., Weir, J. P., Coburn, J. W., et al. (2006). Electromyographic instantaneous amplitude and instantaneous mean power frequency patterns across a range of motion during a concentric isokinetic muscle action of biceps brachii. J. Electromyogr. Kinesiol. 16, 531-539. doi: 10.1016/j.jelekin.2005.10.002

Chen, W., Xiong, C., Huang X., Sun, R., and Xiong, Y. (2010). Kinematic analysis and dexterity evaluation of upper extremity in activities of daily living. Gait Posture 32, 475-481. doi: 10.1016/j.gaitpost.2010.07.005

Chen, X. P., Zhang, D. G., and Zhu, X. Y. (2013). Application of a selfenhancing classification method to electromyography pattern recognition for multifunctional prosthesis control. J. Neuroeng. Rehabil. 10:44. doi: 10.1186/1743-0003-10-44

Churchland, M., Cunningham, J., Kaufman, M., Foster, J., Nuyujukian, P., Ryu, S., et al. (2012). Neural population dynamics during reaching. Nature 487, 51-56. doi: 10.1038/nature11129

Clancy, E., Liu, L., Liu, P., and Zandt Moyer, D. (2012). Identification of constant-posture EMGCtorque relationship about the elbow using nonlinear dynamic models. IEEE Trans. Biomed. Eng. 59, 205-212. doi: 10.1109/TBME.2011.2170423

Farina D., Jiang N., Rehbaum, H., Holobar A., Graimann B., and Dietl, H., (2014). The Extraction of neural information from the surface EMG for the control of upper-Limb prostheses: emerging avenues and challenges. IEEE Trans. Neural Syst. Rehabil. Eng. 22, 797-809. doi: 10.1109/TNSRE.2014.2305111

Garcia, G., Okuno, R., and Akazawa, K. (2005). A decomposition algorithm for surface electrode-array electromyogram: a noninvasive, three-step approach to analyze surface EMG signals. IEEE Eng. Med. Biol. Magazine 24, 63-72. doi: 10.1109/MEMB.2005.1463398

Han, J., Ding, Q., Xiong, A. B., and Zhao, X. G. (2015). A state-space EMG model for the estimation of continuous joint movement. IEEE Trans. Indust. Electr. 62, 4267-4275. doi: 10.1109/TIE.2014.2387337

Hargrove, L., Simon, A., Young, A., Lipschutz, R., Finucane, S., Smith, D., et al. (2013). Robotic leg ontrol with EMG decoding in amputee with nerve transfers. New Engl. J. Med. 369:13. doi: 10.1056/NEJMoa1300126

Hyvarinen, A., Karhunen, J., and Oja, E. (2001). Independent Component Analysis. Hoboken, NJ: John Wiley and Sons, Inc.

Jang, G., Kim, J., Lee, S., and Choi, Y. (2016). EMG-based continuous control scheme with simple classifier for electric-powered wheelchair. IEEE Trans. Indust. Electr. 63, 3695-3705. doi: 10.1109/TIE.2016.2522385

Jiang, N., Vest-Nielsen, J., Muceli, S., and Farina, D. (2012). EMG-based simultaneous and proportional estimation of wrist/hand kinematics in uni-lateral trans-radial amputees. J. Neuroeng. Rehabil. 9:42. doi: $10.1186 / 1743-0003-9-42$

Johnson, R., and Wichern, D. (2007). Applied Multivariate Statistical Analysis, 6th Edn. New York, NY: Pearson.

Ju, Z. J., and Liu, H. H. (2014). Human hand motion analysis with multisensory information. IEEE/ASME Trans. Mechatr. 19, 456-466. doi: 10.1109/TMECH.2013.2240312

Kandel, E., Schwartz, J., Jessell, T., Siegelbaum, S., and Hudspeth, A. (2012). Principles of Neural Science, 5 th Edn. New York, NY: McGraw-Hill Professional.

Kiguchi, K., and Hayashi, Y. (2012). An EMG-based control for an upper-limb power-assist exoskeleton robot. IEEE Trans. Syst. Man Cybern. B Cybern. 42, 1064-1071. doi: 10.1109/TSMCB.2012.2185843

Kiguchi, K., Rahman, M., Sasaki, M., and Teramoto, K. (2008). Development of a 3DOF mobile exoskeleton robot for human upper-limb motion assist. Robot. Auton. Syst. 56, 678-691. doi: 10.1016/j.robot.2007.11.007
Kuiken, T. A., Li, G. L., Lock, B. A., Lipschutz, R. D., Miller, L. A., Kathy A., et al. (2009). Targeted muscle reinnervation for real-time myoelectric control of multifunction artificial arms. JAMA 301, 619-628. doi: 10.1001/jama.2009.116

Li, Z., Hayashibe, M., Fattal, C., and Guiraud, D. (2014). Muscle fatigue tracking with evoked EMG via recurrent neural network: toward personalized neuroprosthetics. IEEE Comput. Intell. Magazine 9, 38-46. doi: 10.1109/MCI.2014.2307224

Liu, K., and Xiong, C. (2013). "A novel 10-DoF exoskeleton rehabilitation robot based on the postural synergies of upper extremity movements," in Proceedings of the International Conference on Intelligent Robotics and Applications, Sep 25-28 (Busan), 362-372.

Maciejasz, P., Eschweiler, J., Gerlach-Hahn, K., Jansen-Troy, A., and Leonhardt, S. (2014). A survey on robotic devices for upper limb rehabilitation. J. Neuroeng. Rehabil. 11:3. doi: 10.1186/1743-0003-11-3

Miller, L., Lipschutz, R., Stubblefield, K., Lock, B., Huang, H., and Williams, T. (2008). Control of a six degree of freedom prosthetic Arm after targeted muscle reinnervation surgery. Arch. Phys. Med. Rehabil. 89, 2057-2065. doi: 10.1016/j.apmr.2008.05.016

Muceli, S., and Farina, D. (2012). Simultaneous and proportional estimation of hand kinematics from EMG during mirrored movements at multiple degrees-of freedom. IEEE Trans. Neural Syst. Rehabil. Eng. 20, 371-378. doi: 10.1109/TNSRE.2011.2178039

Nakamura, H., Yoshida, M., Kotani, M., Akazawa, K., and Moritani, T. (2004). The application of independent component analysis to the multi-channel surface electromyographic signals for separation of motor unit action potential trains: part Iłmeasuring techniques. J. Electromyogr. Kinesiol. 14, 423-432. doi: 10.1016/j.jelekin.2004.01.004

Nazarpour, K., Al-Timemy, A., Bugmann, G., and Jackson, A. (2013). A note on the probability distribution function of the surface electromyogram. Brain Res. Bull. 90, 88-91. doi: 10.1016/j.brainresbull.2012.09.012

Ngeo, J., Tamei, T., and Shibata, T. (2014). Continuous and simultaneous estimation of finger kinematics using inputs from an EMG-to-muscle activation model. J. Neuroeng. Rehabil. 11:122. doi: 10.1186/1743-0003-11-122

Pan, L., Zhang, D., Liu, J., Sheng, X., and Zhu, X. (2014). Continuous estimation of finger joint angles under different staticwrist motions from surface EMG signals. Biomed. Signal Process. Control 14, 265-271. doi: 10.1016/j.bspc.2014.08.004

Prilutsky, B., Ashley, D., VanHiel, L., Harley, L., Tidwell, J., and Backus, D. (2011). Motor control and motor edundancy in the upper extremity: implications for neurorehabilitation. Top Spinal Cord Injury Rehabil. 17, 7-15. doi: 10.1310/sci1701-07

Qi, L., Wakeling, J., and Ferguson-Pell, M. (2011). Spectral properties of electromyographic and mechanomyographic signals during dynamic concentric and eccentric contractions of the human biceps brachii muscle. J. Electromyogr. Kinesiol. 21, 1056-1063. doi: 10.1016/j.jelekin.2011.08.011

Staudenmann, D., Daffertshofer, A., Kingma, I., Stegeman, D., and van Dieen, J. (2007). Independent component analysis of high-density electromyography in muscle force estimation. IEEE Trans. Biomed. Eng. 54, 751-754. doi: 10.1109/TBME.2006.889202

Staudenmann, D., Roeleveld, K., Stegeman, D. F., and van Dieen, J. H. (2010). Methodological aspects of SEMG recordings for force estimation-a tutorial and review. J. Electromyogr. Kinesiol. 20, 375-387. doi: 10.1016/j.jelekin.2009.08.005

Takahashi, C., Yeghiaian, L., Le, V., Motiwala, R., and Cramer, S. (2008). Robot-based hand motor therapy after stroke. Brain 131, 425-437. doi: 10.1093/brain/awm311

van Andel, C., Wolterbeek, N., Doorenbosch, C. A., Veeger, D. H., and Harlaar, J. (2008). Complete 3D kinematics of upper extremity functional tasks. Gait Posture 27, 120-127. doi: 10.1016/j.gaitpost.2007.03.002

Vogel, J., Bayer, J., and Smagt, P. (2013). "Continuous robot control using surface electromyography of atrophic muscles," in Proceedings of 2013 IEEE/RSJ International Conference on Intelligent Robots and Systems (IROS), Nov. 3-7 (Tokyo), 845-850.

Wu, G., van der Helm, F., Veeger, H. E. J., Makhsous, M., Van Roy, P., Anglin, C., et al. (2005). ISB recommendation on definitions of joint coordinate systems of various joints for the reporting of human joint motionłPart II: shoulder, elbow, wrist and hand. J. Biomech. 38, 981-992. doi: 10.1016/j.jbiomech.2004.05.042

Zhang, Q., Hayashibe, M., and Azevedo-Coste, C. (2013). Evoked electromyography-based closed-loop torque control in functional 
electrical stimulation. IEEE Trans. Biomed. Eng. 60, 2299-2307. doi: 10.1109/TBME.2013.2253777

Zhang, Q., Hayashibe, M., Fraisse, P., and Guiraud, D. (2011). FES-induced torque prediction with evoked EMG sensing for muscle fatigue tracking. IEEE/ASME Trans. Mechatr. 16, 816-826. doi: 10.1109/TMECH.2011.21 60809

Zhang, Q., Hosoda, R., and Venture G. (2013). "Human joint motion estimation for electromyography (EMG)-based dynamic motion control," in Proceedings of the 35th International Conference of the IEEE Engineering in Medcine and Biology Society (EMBC) July 3-7 (Osaka), 21-24.
Conflict of Interest Statement: The authors declare that the research was conducted in the absence of any commercial or financial relationships that could be construed as a potential conflict of interest.

Copyright (c) 2017 Zhang, Liu, Chen and Xiong. This is an open-access article distributed under the terms of the Creative Commons Attribution License (CC BY). The use, distribution or reproduction in other forums is permitted, provided the original author(s) or licensor are credited and that the original publication in this journal is cited, in accordance with accepted academic practice. No use, distribution or reproduction is permitted which does not comply with these terms. 


\title{
Grip Force and 3D Push-Pull Force Estimation Based on SEMG and GRNN
}

\author{
Changcheng $W^{1,2}$, Hong Zeng ${ }^{1}$, Aiguo Song ${ }^{1 *}$ and Baoguo $X u^{1}$ \\ ${ }^{1}$ School of Instrument Science and Engineering, Southeast University, Nanjing, China, ${ }^{2}$ College of Automation Engineering, \\ Nanjing University of Aeronautics and Astronautics, Nanjing, China
}

OPEN ACCESS

Edited by:

Dingguo Zhang,

Shanghai Jiao Tong University, China

Reviewed by:

Tjeerd W. Boonstra,

University of New South Wales,

Australia

Lizhi Pan,

North Carolina State University,

United States

*Correspondence:

Aiguo Song

a.g.song@seu.edu.cn

Specialty section:

This article was submitted to

Neural Technology,

a section of the journal

Frontiers in Neuroscience

Received: 31 December 2016

Accepted: 01 June 2017

Published: 30 June 2017

Citation:

Wu C, Zeng $H$, Song A and Xu B (2017) Grip Force and 3D Push-Pull

Force Estimation Based on SEMG and

GRNN. Front. Neurosci. 11:343.

doi: 10.3389/fnins.2017.00343
The estimation of the grip force and the 3D push-pull force (push and pull force in the three dimension space) from the electromyogram (EMG) signal is of great importance in the dexterous control of the EMG prosthetic hand. In this paper, an action force estimation method which is based on the eight channels of the surface EMG (sEMG) and the Generalized Regression Neural Network (GRNN) is proposed to meet the requirements of the force control of the intelligent EMG prosthetic hand. Firstly, the experimental platform, the acquisition of the sEMG, the feature extraction of the sEMG and the construction of GRNN are described. Then, the multi-channels of the sEMG when the hand is moving are captured by the EMG sensors attached on eight different positions of the arm skin surface. Meanwhile, a grip force sensor and a three dimension force sensor are adopted to measure the output force of the human's hand. The characteristic matrix of the sEMG and the force signals are used to construct the GRNN. The mean absolute value and the root mean square of the estimation errors, the correlation coefficients between the actual force and the estimated force are employed to assess the accuracy of the estimation. Analysis of variance (ANOVA) is also employed to test the difference of the force estimation. The experiments are implemented to verify the effectiveness of the proposed estimation method and the results show that the output force of the human's hand can be correctly estimated by using SEMG and GRNN method.

Keywords: grip force, 3D push-pull force, force estimation, EMG, GRNN

\section{INTRODUCTION}

Prosthetic hand is a kind of human-machine interface. The upper limb amputees can recover some hand function with the help of the prosthetic hand. In recent years, many kinds of prosthetic hands have been investigated to meet the requirements of the amputees (Davidson, 2002; Zaini and Ahmad, 2011; Maat et al., 2017). Among these hands, the prosthetic hand based on the EMG has received lots of attention due to its simple operation and that it is in accordance with the operation habits of the natural hand. Figure 1 shows a typical block diagram of the EMG prosthetic hand. Firstly, the EMG signals are captured from the amputee's remaining arm by the EMG sensors on the skin surface. Then, the motion recognition unit outputs the user's motion intention based on analysis of the captured EMG signals. In the motion recognition unit, the features extracted from EMG signals are used to identify the user's motion intention. The controller sends the control commands to the prosthetic hand according to the user's motion intention which has been recognized in the motion recognition unit. In such manner, the prosthetic hand can be 


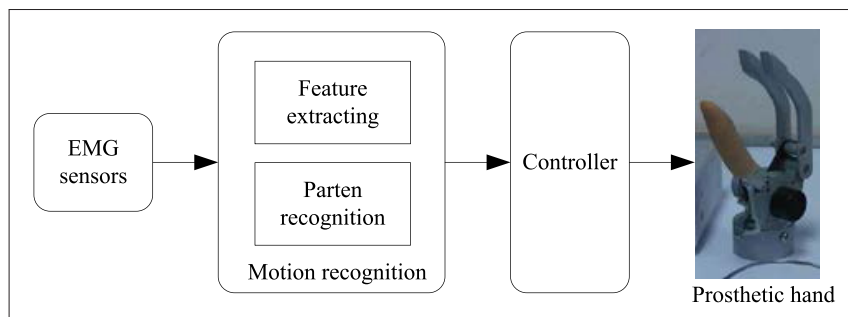

FIGURE 1 | A typical control mode of the EMG prosthetic hand.

controlled by the amputee's EMG signals. The motion recognition unit plays an important role in this control mode. Whether the prosthetic hand movement is in line with the user's intention is directly decided by the motion recognition unit. As a matter of fact, many scholars have made a lot of investigations in this area (Zhang et al., 2013; Xie et al., 2015; Hofmann et al., 2016; Segil et al., 2016).

The hand movement is described by type, speed, force, and amplitude of the action, and etc. And all these are important in the control of the prosthetic hand, especially type and force. Therefore, the main tasks of the motion recognition are type classification and force estimation of the action.

In the process of type classification of the action, plenty of research work has been done in the past decades (Li et al., 2010; Hioki and Kawasaki, 2012; Ngeo et al., 2013; He et al., 2015; Pan et al., 2015). Nishikawa et al. used two channels of the EMG signals and the real-time learning method to discriminate a maximum 10 forearm motions including 4 wrist motions and 6 hand motions (Nishikawa et al., 1999). Ten different features extracted from four channels of the EMG signals are used to classify the eight hand motions in Al Omari et al. (2014). And the authors reported that an accuracy of $94 \%$ was achieved by using LDA (Linear Discriminant Analysis) based on the combination of wavelength, Willson amplitude, and root mean square. $\mathrm{Wu}$ proposed an EMG self-learning recognition method to reduce the effects of the individual difference on the EMG motion recognition (Wu et al., 2015). Ju and Liu used multiple sensors to analyze the human hand motions and reported that there exist significant relationships between the muscle signals and the finger trajectories as well as between the muscle signals and the contact force (Ju and Liu, 2014). To improve the performance of the EMG prosthetic hand, some scholars attempted to obtain the continuous motor variables such as the limb positions and force of the action. Ngeo used a multi-output convolved Gaussian Process to estimate the finger joint kinematics from the EMG signals (Ngeo et al., 2014).

The other part of the hand movement intention, force of hand movement (grip force, push force, pull force, etc.), is also important in the control of the prosthetic hand, especially in dexterous manipulation. There are mainly two kinds of force of the action output from the human hand. One is the grip force and the other is the push force and pull force in three dimensional space (we call it 3D force). In the process of grip force estimation, the most common method is detecting the strength of the muscle contraction from the EMG signals and then mapping the measured strength to the expecting grip force. During the bilateral grasping task, Kamavuako et al. investigated the use of the EMG features in order to estimate the grip force on the ipsilateral and contralateral hand (Kamavuako et al., 2012). And the author also reported that one channel of EMG signals measured from the flexor digitorum profundus can be used to represent the grip force within the entire range of force (Kamavuako et al., 2013). Castellini et al. used five channels of the EMG signals and the SVM (Support Vector Machine) to achieve the grip force estimation and their estimation error is $<7 \%$ (Castellini et al., 2009). In 3D force estimation, Nielsen et al. used the artificial neural network to estimate the isometric force in multiple degrees of freedom from the wrist (Nielsen et al., 2011). Hashemi et al. researched the EMG based on force estimation in dynamic contractions by using the parallel cascade identification modeling (Hashemi et al., 2012, 2015).

Although, force estimation of the action is also important in the control of the prosthetic hand, the amount of the research work in this area is apparently less than the work in type classification of the action. Actually, not only the grip force but also the 3D force plays an important role in the control of the dexterous prosthetic hand. For the prosthetic hand with movable wrist, the $3 \mathrm{D}$ push/pull force is important in the process of moving objects. The estimate of 3D push/pull force can be used to control the rotation of the wrist.

In this paper, we present a force estimation method for the purpose of estimating the force of hand movement comprehensively. Firstly, we set up the experimental platform which is used to capture the EMG signals and force of the action. The eight channels of the EMG signals are captured from the eight different positions on the arm skin surface. The grip force and the $3 \mathrm{D}$ force are captured by a grip force sensor and a three dimensional force sensor, respectively. Secondly, the time domain feature extraction method is employed to extract the features from the captured EMG signals. Then, the generalized regression neural network (GRNN) is employed to estimate force of the action which includes the grip force and the 3D force, by using the extracted EMG features and the captured force of action. At last, experiments are implemented to verify the effectiveness of the proposed force estimation method of the action.

The rest of the paper is organized as follows. Section "Experimental Platform" introduces the experimental platform in detail. The detailed process of force estimation of the action is described in Section "Force Estimation of Hand Movement." The experiments are implemented in Section "Experiment and Result," and the Conclusions are in Section "Conclusion."

\section{EXPERIMENTAL PLATFORM}

An experiment platform as shown in Figure 2 is set up in this section. The experimental platform consists of several EMG sensors, a three dimensional force sensor, a data collector, a computer, and the application software.

The EMG sensors and the $3 \mathrm{D}$ force sensor are respectively used to capture the surface EMG signals generated on the arm 

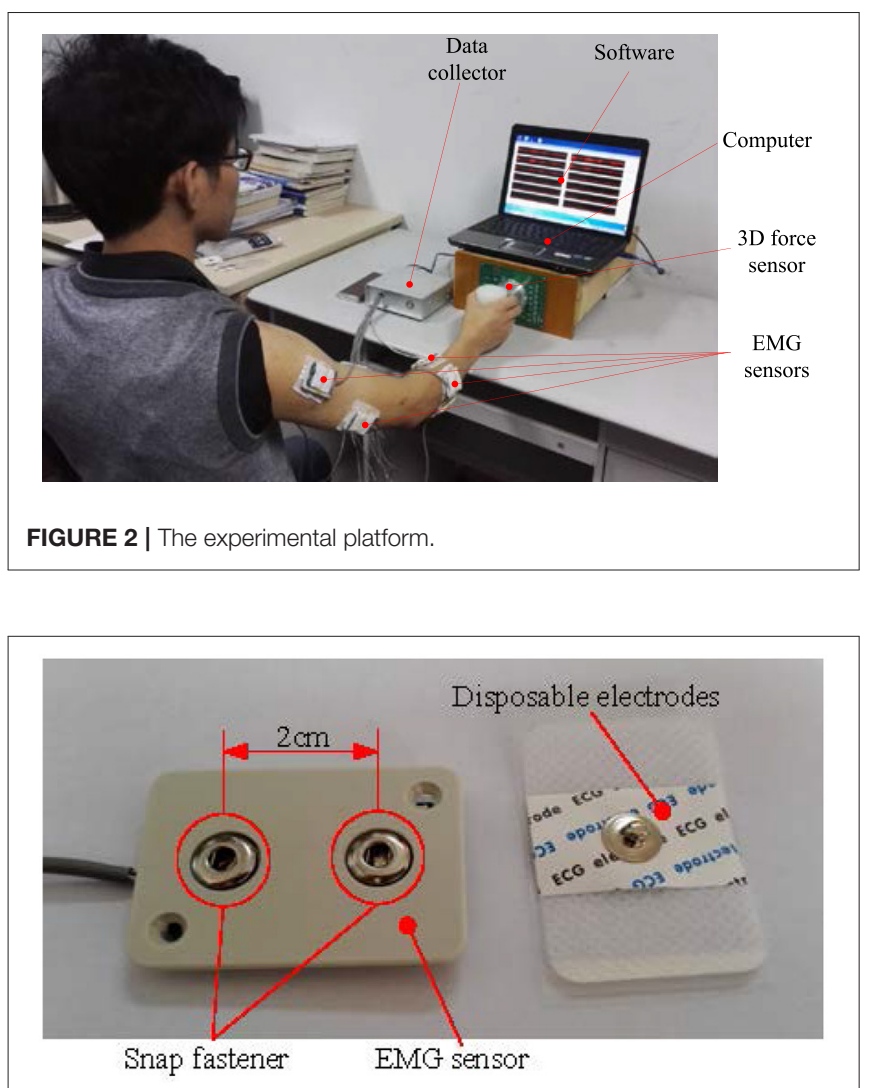

FIGURE 3 | The EMG sensor and disposable electrode.

skin surface and the force signals output by the hand while the hand is moving and grasping. The EMG signals and the force signals are digitalized in the data collector. The data collector has a USB interface via which the data can be transmitted between the data collector and computer.

\section{EMG Sensor}

The EMG signal is a kind of weak signal. To meet the requirements of EMG signal measurement, we design a surface EMG sensor shown in Figure 3. The sensor's pass-band is 10$500 \mathrm{~Hz}$ and the voltage gain is about 1,000. The disposable moisture $\mathrm{Ag} / \mathrm{AgCl}$ electrodes are used in the sensor. There are two snap-fasteners in the EMG sensor. The distance between these two snap-fasteners is $2 \mathrm{~cm}$. And the snap-fastener is made of conductive metal. There is also a snap-fastener in the disposable electrode. The snap-fastener in the disposable electrode should be buckled into the snap-fastener in the EMG sensor.

There is a conditioning circuit in the EMG sensor. The structure diagram of the conditioning circuit is shown in Figure 4. The voltage gains of the two amplifiers, A1 and A2, are set as 15 and 40, respectively. The high-pass filter (cut-off frequency: $10 \mathrm{~Hz}$ ) is used to remove the direct current component from the signal. The low-pass filter (cut-off frequency: $500 \mathrm{~Hz}$ ) is used to eliminate the high-frequency noises. The voltage gains of these two filters are all about 1.3. The notch filter is used to
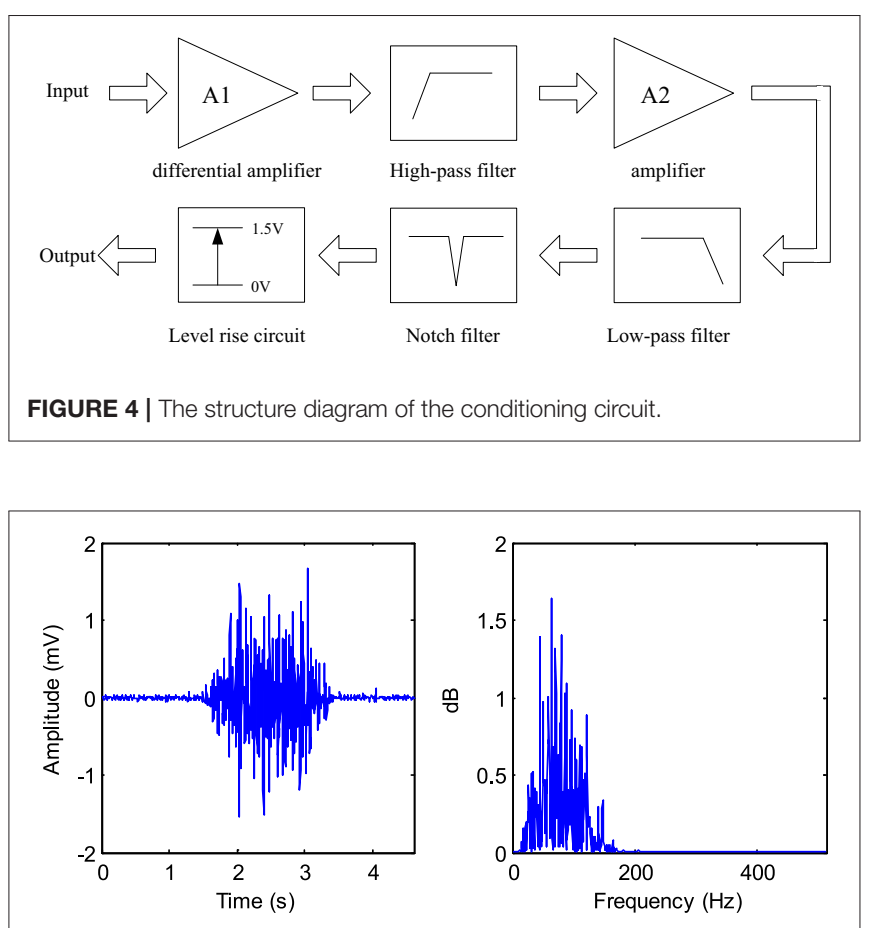

FIGURE 5 | A typical EMG signal and its power spectrum.

reduce the $50 \mathrm{~Hz}$ power-line interference. Since the input range of the data collector is from 0 to $3 \mathrm{~V}$, we design the level-rising circuit to make the voltage of the signals $>0 \mathrm{~V}$. Figure 5 shows a typical signal measured by the EMG sensor.

\section{Grip Force Sensor}

Figure 6 shows the grip force sensor and its manipulation sketch. The grip force sensor mainly contains a grasping mechanism (GM), an elastic beam, a signal conditioning circuit, and a sensor shell. There is a connecting rod between the elastic beam and the grasping mechanism. The elastic beam is fixed in the sensor shell. Grasping the GM with force will lead to the deformation of the elastic beam. There are two strain gauges, $\mathrm{sg}_{1}$ and $\mathrm{sg}_{2}$, attached on the surface of the elastic beam. These two strain gauges are used to measure the deformation of the elastic beam. According to the knowledge of the mechanics of the material, we know that there exists a relationship between the deformation of the beam and the force applied to the beam. Therefore, we can measure the grip force by detecting the deformation of the strain gauges. The widely used measurement circuit, Wheatstone bridge, is employed to measure the deformation of the strain gauges. And the output signals of the Wheatstone bridge are amplified by a differential amplifier.

The calibration is necessary for the reason that the relationship between the force applied to the elastic beam and the deformation of the beam is non-linear and there are some other factors which may influence the measurement accuracy. After the calibration experiment, the accuracy of the designed grip force sensor is $0.1 \mathrm{~N}$. 


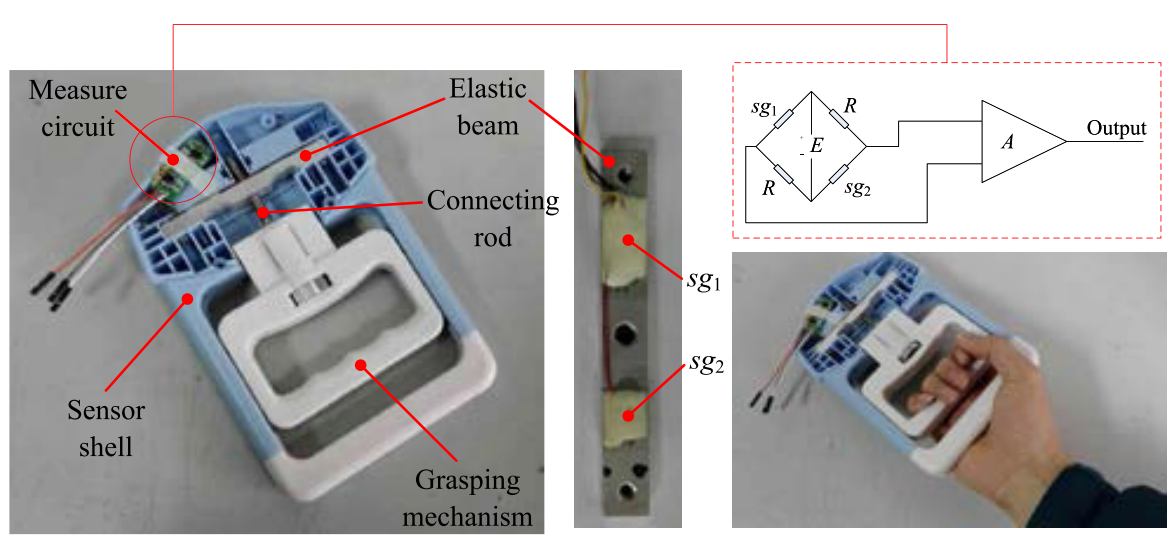

FIGURE 6 | The grip force sensor and its manipulation sketch.
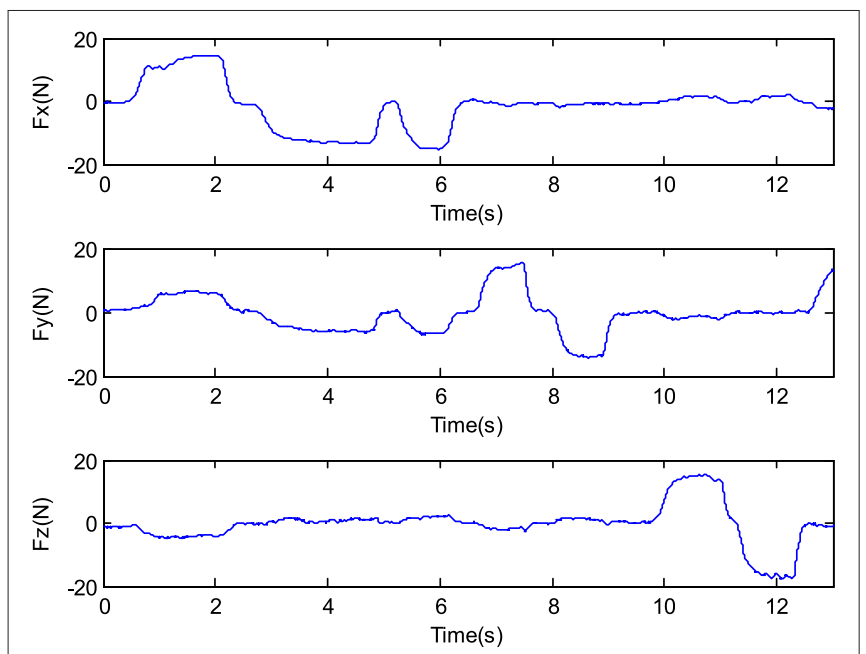

FIGURE 7 | The force signals measured by the 3D force sensor.

\section{D Force Sensor}

A three dimension (3D) force sensor (measurement range: $-30 \mathrm{~N} \sim 30 \mathrm{~N}$, accuracy: 2\%FS), which is designed by the Robot Sensor and Control Lab in Southeast University, is introduced to measure the force signal (Ma and Song, 2012; Ma et al., 2013). Figure 7 shows the signals measured by the $3 \mathrm{D}$ force sensor while the force is applied to the sensor optionally. The value $>0$ indicates that the direction of the force is in forward direction, and the value $<0$ indicates that the direction of force is in reverse direction.

As shown in Figure 8, the 3D force sensor is fixed on a vertical placed flat. And a cylindrical handle is installed at the end of the sensor. Holding the handle, human hand can apply force to the sensor in different directions.

\section{Data Collector}

Analogy signals captured by the sensors can be digitalized by the data collector. The designed data collector can realize analogy

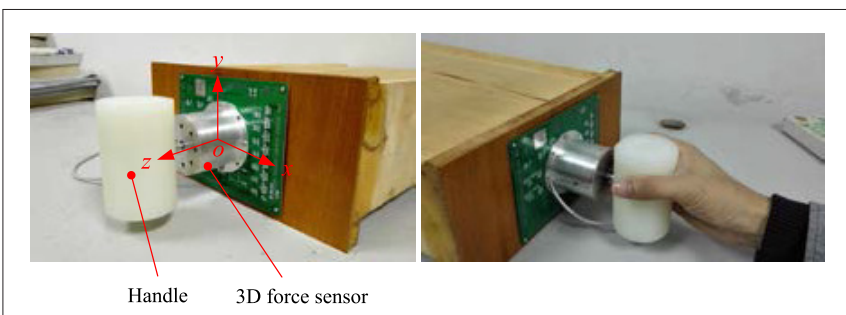

FIGURE 8 | The 3D force sensor and its manipulation sketch.

signal sampling in 16 channels simultaneously. The AD (analogy to digital) converters in the data collector are $10 \mathrm{bit}$. The sampling rate of the data collector can be set from 200 to $2000 \mathrm{~Hz}$ per channel. The voltage range of the input signals is from 0 to $3 \mathrm{~V}$. The data collector has a USB interface through which data can be exchanged between the data collector and computer.

\section{Application Software}

The application software running on the computer is used to display the EMG signal waves and force signal waves in real time. And the data of these signals can be recorded when needed. In addition, the application software has the function of changing the data sampling rate by means of sending commands to the data collector.

\section{FORCE ESTIMATION OF HAND MOVEMENT}

The relation between force and EMG signal is varied with many factors, such as force value, speed, path, etc. (Orizio et al., 2010; Kamavuako and Rosenvang, 2012). In order to study the relationship between the EMG signals and the force signals output by the hand and to estimate the force based on the EMG signal, we designed the signal processing diagram as shown in Figure 9. 


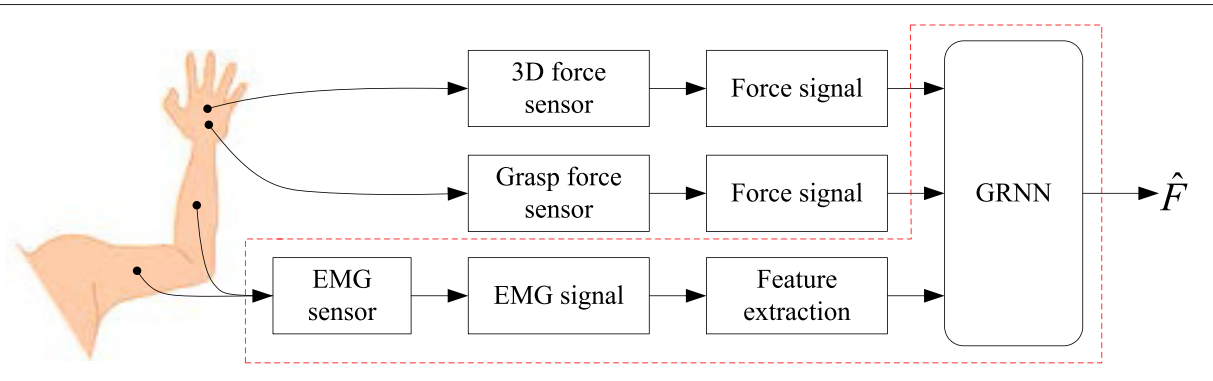

FIGURE 9 | The diagram of the force estimation.

Since the EMG signal is a kind of complex non-linear signal and the hand movement is completed by the cooperation of many muscles, it is difficult to describe the relationship between the EMG signals and the force output by the hand by using the linear model.

Under the assumption that there is a non-linear function, $\phi$, which can describe the relationship between the EMG signals and the force output by the hand, the force output by the hand can be estimated by measuring the EMG signals on the arm skin surface.

$$
F=\phi(X)
$$

where, $\phi$ is the non-linear function, $X$ are the EMG signals measured from the arm skin surface, $F$ is the force output by the hand.

In this paper, the GRNN is employed to describe the nonlinear relationship between the EMG signals and the force for its good non-linear mapping capability and high degree of parallel processing information capacity.

\section{EMG Signal Acquisition and Feature Extraction}

In this paper, eight different positions on the arm skin surface are selected to acquire the EMG signals. The distribution of the EMG sensors and the serial number of the EMG sensors are shown in Figure 10 and Table 1.

EMG signal captured by the data collector is a time series signal which can describe the characteristics of the hand movement after necessary preprocessing and feature extraction.

The feature extraction method of EMG signals is usually the time domain method, frequency domain method, and time-frequency domain method. The time domain method has the advantages of less computation comparing with the other two methods. The usual time domain feature extraction methods are mean absolute value (MAV), variance (VAR), zeros crossings (ZC), and Willison Amplitude (WA). Table 2 shows the mathematical equation of each introduced feature. All features are extracted by using Matlab.

Figure 11 shows a typical EMG signal and its time domain feature.

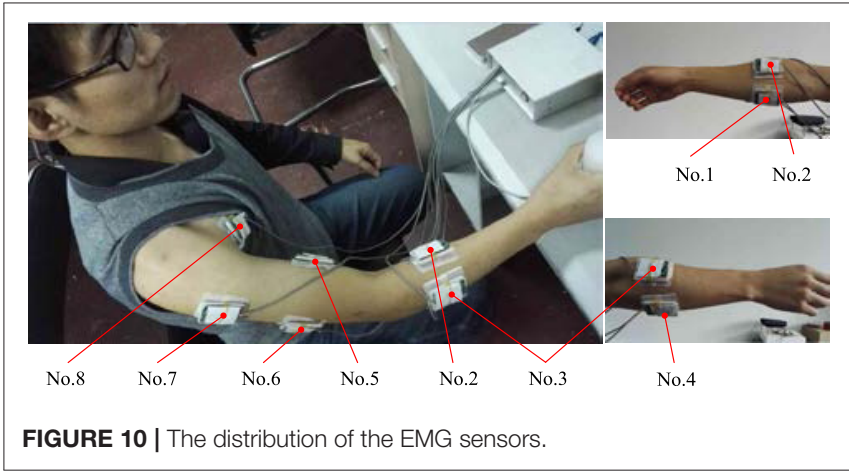

TABLE 1 | The distribution location and the serial number of the EMG sensors.

\begin{tabular}{ll}
\hline EMG sensor no. & Measuring site \\
\hline 1 & Lateral brachial wrist flexo \\
2 & Brachioradialis \\
3 & Extensor digitorum \\
4 & Extensor carpi ulnaris muscle \\
5 & Biceps \\
6 & Triceps \\
7 & Deltoid \\
8 & Shoulder capsulorrhaphy \\
\hline
\end{tabular}

\section{The Generalized Regression Neural Network}

The GRNN was proposed by Donald F. Specht in 1991. It is a kind of RBF (Radial Basis Function) neural network. It has the advantage of non-linear mapping, great robustness, and a high degree of parallel processing information capacity. The performance and the learning speed of the GRNN are better than the general neural network (Altiparmak et al., 2009).

The GRNN has a network structure of 4 layers as shown in Figure 12. These four layers are input layer, pattern layer, sum layer, and output layer, respectively (Altiparmak et al., 2009).

$X=\left[x_{1}, x_{2}, \ldots, x_{m}\right]^{T}$ is the input vector of the GRNN, and $Y=\left[y_{1}, y_{2}, \ldots, y_{l}\right]^{T}$ is the output of the GRNN. The number of the neuron in pattern layer is as the same as the number of the training samples. Each neuron in pattern layer corresponds to a 
TABLE 2 | The EMG features in time domain and mathematical equation.

\begin{tabular}{|c|c|}
\hline Feature & Mathematical equation \\
\hline MAV & $M A V_{i}=\frac{1}{N} \sum_{j=i-N+1}^{1}\left|x_{j}\right|$ \\
\hline VAR & $V A R_{i}=\frac{1}{N} \sum_{j=i-N+1}^{1} x_{j}^{2}$ \\
\hline ZC & $\begin{array}{l}Z C_{i}=\sum_{j=i-N+1}^{I} \operatorname{sgn}\left(x_{j} x_{j-1}\right) \\
\operatorname{sgn}(x)= \begin{cases}1, & x>0 \\
0, & x \leq 0\end{cases} \end{array}$ \\
\hline WA & $\begin{array}{l}W A_{i}=\sum_{j=i-N+2} f\left(x_{i}-x_{i-1}\right) \\
f(x)= \begin{cases}1, & |x|>\text { threadhold } \\
0, & \text { others }\end{cases} \end{array}$ \\
\hline
\end{tabular}

Where, $x_{j}$ is the jth sample data, $N$ is the length of the sliding window.
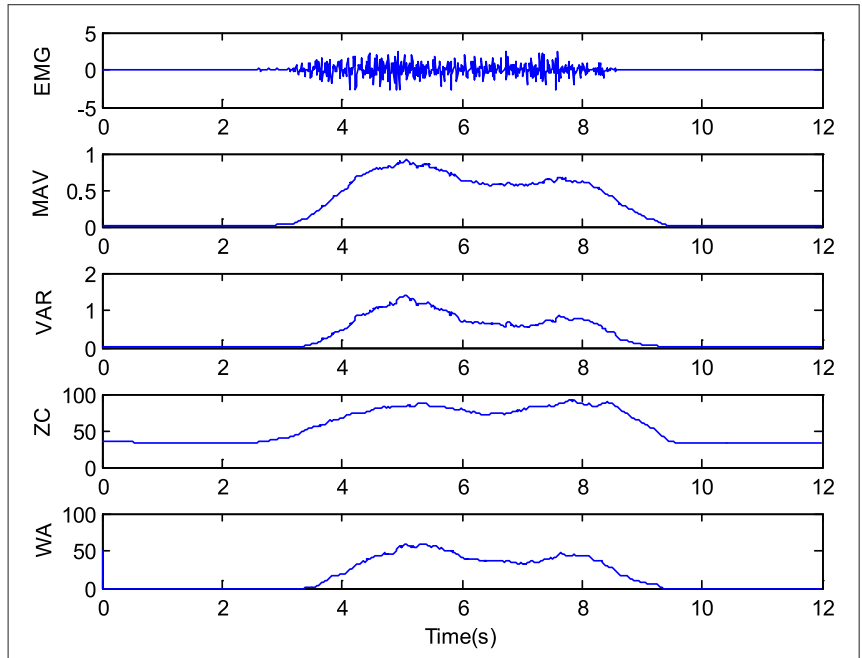

FIGURE 11 | A typical EMG signal and its time domain features.

training sample data. The transfer function of the $i$ th neuron in pattern layer is:

$$
P_{i}=\exp \left[\frac{\left(X-X_{i}\right)^{T}\left(X-X_{i}\right)}{2 \sigma^{2}}\right], i=1,2, \ldots, n
$$

where, $X$ is the input of the GRNN, $X_{i}$ is the learning sample corresponding to $i$ th neuron, $\sigma$ is the smoothing parameter.

The neuron in sum layer can be divided into two classes. One class corresponds to Equation (3), and the number is only one. The other class corresponds to Equation (4).

$$
\begin{aligned}
S_{D} & =\sum_{i=1}^{n} P_{i} \\
S_{N j} & =\sum_{i=1}^{n} y_{i j} P_{i}, j=1,2, \ldots, L
\end{aligned}
$$

where, $y_{i j}$ is $j$ th element in $Y_{i}, Y_{i}$ is the $i$ th output sample. $L$ is the dimension of the output vector.

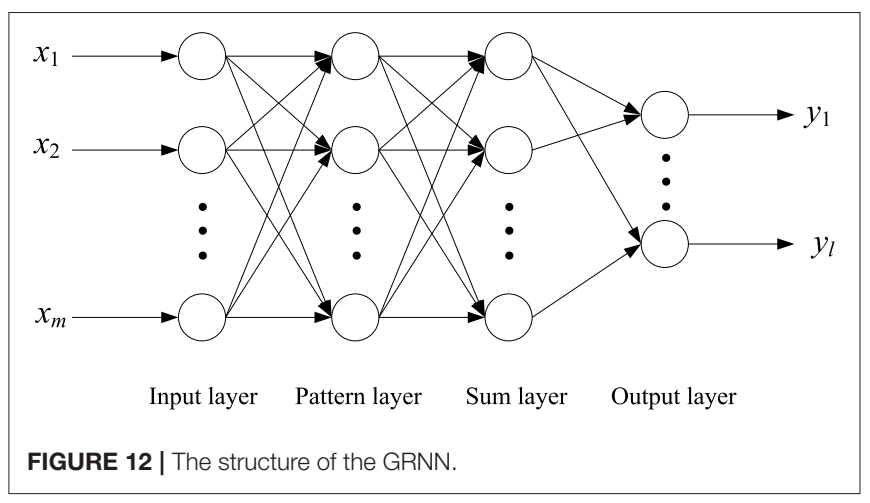

The output of the GRNN is defined as:

$$
y_{i}=\frac{S_{N i}}{S_{D}} i=1,2, \ldots, L
$$

To improve the performance of the GRNN in our study, the samples should be distributed as uniformly as possible within a certain range.

\section{Force Estimation of Hand Movement}

In this paper, the GRNN is employed to study the relationship between the EMG signals and the force output by the hand. As shown in Figure 9, the force estimation can be divided into two steps. One is offline training of the GRNN, and the other is the online force estimation. In the prosthetic hand based on EMG, the GRNN is offline trained by collecting the EMG signals and the force signals. The trained GRNN is used to estimate force of the action in real time. It is that mapping the extracted EMG features into the force of hand movement by using the trained GRNN.

The software in the computer records the eight channels of the EMG signals $(X)$, the grip force signals $\left(F_{g}\right)$, and the 3D force signals $\left(F_{3 D}\right)$ in real time.

$$
\begin{gathered}
X=\left[\begin{array}{c}
X_{1} \\
X_{2} \\
\vdots \\
X_{n}
\end{array}\right]=\left[\begin{array}{cccc}
x_{11} & x_{12} & \cdots & x_{1 m} \\
x_{21} & x_{22} & \cdots & x_{2 m} \\
\vdots & \vdots & \vdots & \vdots \\
x_{n 1} & x_{n 2} & \cdots & x_{n m}
\end{array}\right] \\
F_{3 D}=\left[\begin{array}{c}
F_{x} \\
F_{y} \\
F_{z}
\end{array}\right]=\left[\begin{array}{llll}
f_{x 1} & f_{x 2} & \cdots & f_{x m} \\
f_{y 1} & f_{y 2} & \cdots & f_{y m} \\
f_{z 1} & f_{z 2} & \cdots & f_{z m}
\end{array}\right] \\
F_{g}=\left[\begin{array}{llll}
f_{g 1} & f_{g 2} & \cdots & f_{g m}
\end{array}\right]
\end{gathered}
$$

where, $X_{1} \sim X_{8}$ are the eight channels of the EMG signals captured by the eight EMG sensors, respectively, $x_{i j}$ is the $j t h$ sample data captured by the ith EMG sensor. $F_{x}, F_{y}$, and $F_{z}$ are the force signal in $x, y$, and $z$ direction measured by the $3 \mathrm{D}$ force sensor, respectively; $f_{x i}, f_{y i}, f_{z i}$ are the ith sample data in $x, y$, and $z$ direction, respectively; $f_{g i}$ is the $i t h$ sample data captured by the grip force sensor and $m$ is the number of the sampling.

Time domain feature extraction methods described in Table 2 are used in the processing of the EMG signals. And we can get the 
feature matrixes: $X F_{M A V}, X F_{V A R}, X F_{Z C}$, and $X F_{W A}$.

$$
\begin{gathered}
X F_{M A V}=\left[\begin{array}{c}
X_{1 M A V} \\
X_{2 M A V} \\
\vdots \\
X_{8 M A V}
\end{array}\right] X F_{V A R}=\left[\begin{array}{c}
X_{1 V A R} \\
X_{2 V A R} \\
\vdots \\
X_{8 V A R}
\end{array}\right] \\
X F_{Z C}=\left[\begin{array}{c}
X_{1 Z C} \\
X_{2 Z C} \\
\vdots \\
X_{8 Z C}
\end{array}\right] X F_{W A}=\left[\begin{array}{c}
X_{1 W A} \\
X_{2 W A} \\
\vdots \\
X_{8 W A}
\end{array}\right]
\end{gathered}
$$

Combining the feature matrixes and the force signals, we can get a new matrix $S_{a m}$.

$$
S_{a m}=\left[\begin{array}{c}
X F \\
F
\end{array}\right]
$$

where, $X F$ is one of the $X F_{M A V}, X F_{V A R}, X F_{Z C}$, and $X F_{W A} . F$ is one of the $F_{3 D}$ and $F_{g}$.

In the offline training step of the GRNN, the $k$-fold cross validation is introduced to evaluate the performance of the GRNN.

(1) The $S_{a m}$ is split into $k$ equal-size groups of size $m / k$.

(2) One of the groups is used as validation group in order to test the performance of the GRNN; the rest $k-1$ groups are used in training process.

(3) The cross validation process is repeated $k$ times with different selected groups with the mean accuracy.

In the $k$-fold cross validation method, $k$ is set to two in the paper.

The mean absolute value of the estimation error $(M A V E)$, the root mean square (RMS) of the estimation error and the correlation coefficient $(\rho)$ between $F$ and $\tilde{F}$ are introduced to evaluate the accuracy of the estimation results. Take the force in direction $x$ for an example:

$$
\begin{array}{r}
M A V E_{x}=\frac{\sum_{i=1}^{N} a b s\left(\tilde{f}_{x i}-f_{x i}\right)}{N} \\
R M S_{x}=\sqrt{\frac{\sum_{i=1}^{N}\left(\tilde{f}_{x i}-f_{x i}\right)^{2}}{N-1}} \\
\rho_{\tilde{f}_{x}, f_{x}}=\frac{\operatorname{cov}\left(\tilde{f}_{x}, f_{x}\right)}{\sigma_{\tilde{f}_{x}} \sigma_{f_{x}}}=\frac{E\left[\left(\tilde{f}_{x}-\mu_{\tilde{f}_{x}}\right)\left(f_{x}-\mu_{f_{x}}\right)\right]}{\sigma_{\tilde{f}_{x}} \sigma_{f_{x}}}
\end{array}
$$

where, $\tilde{f}_{x i}$ is the estimate of force in direction $x ; f_{x i}$ is the force in direction $x$ measured by the 3D force sensor; $N$ is the length of the data.

A greater $M A V E$ indicates that the estimation result is poorer. A greater $R M S$ indicates that the estimation result has a larger fluctuation. And a greater $\rho$ indicates that the estimated result has a higher similarity with the force measured by the force sensor.
In grip force estimation experiments, One-way ANOVA is conducted to compare the performance of different features In One-way ANOVA, the factor is EMG feature (MVA, VAR, ZC, WA). In 3D force estimation experiments, Twoway ANOVA is conducted. The factors of the Two-way ANOVA are force direction $(x, y, z)$ and EMG feature (MVA, VAR, ZC, WA). Tukey comparison is performed when significance is detected. The significance level for all tests is set at 0.05 .

\section{EXPERIMENT AND RESULT}

To verify the effectiveness of the proposed method in this paper, the experiment was implemented.

Six right hand-dominant healthy subjects without any neuromuscular disorders ( 4 males, 2 females, and aged between 21 and 28) were chosen as the participants the experiment. And the participants were informed consent prior to study participation.

Before the experiment, the locations of the EMG sensors on the arm skin surface were cleaned by medical alcohol. Then, the electrodes were buckled into the EMG sensors and the EMG sensors were attached on the arm skin surface. The locations of the EMG sensors are shown in Table 1 and Figure 10.

During the experiments, the EMG signals and force signals of the action were displayed as waveforms in the computer screen and recorded by the software. The data sampling rate of the data collector was set as $1 \mathrm{kHz}$ per-channel.
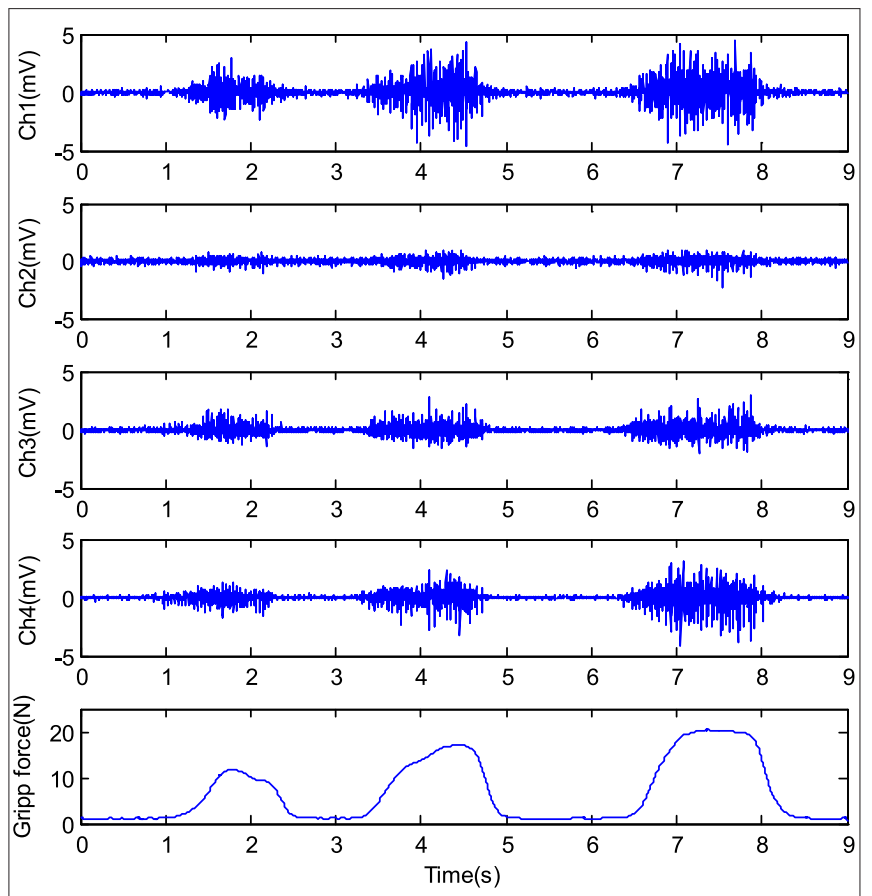

FIGURE 13 | The EMG signals and the corresponding grip force. 
In the experiments, the data captured from different individuals are used to train the GRNN, respectively.

\section{The Grip Force Estimation Experiment}

Firstly, the grip force estimation experiment is implemented. During the grip force estimation experiment, the subjects are asked to grasp the grip force sensor several times with different grip force. During the grasping, the subjects should not move their arms. The duration of the grasping is about 1-2 s. The maximum grip force is $<30 \mathrm{~N}$.

In the experiment, four channels of the EMG signals were selected to estimate the grip force. Figure 13 shows the EMG signals and the grip force while the subject 1 was grasping the grip sensor three times continuously with different grip force.

Four kinds of EMG time domain features which were extracted from the same raw EMG signals were used to train the GRNN, respectively. And the trained GRNNs were used to estimate the grip force, respectively.
The experiment results of the subject 1 are shown in Figure 14. It shows that the results of the WAV and VAR are better than the results of $\mathrm{ZC}$ and WA. When the grip force increases from 0 to $8 \mathrm{~N}$, the results of all features show a good estimation effect. When the grip force is larger than $10 \mathrm{~N}$, the estimation accuracy of ZC and WA decreases, especially when the force is larger than $15 \mathrm{~N}$. In the results of $\mathrm{ZC}$, the fluctuation may appear in the estimation results when the grip force is larger than $15 \mathrm{~N}$. And when the grip force is larger than $15 \mathrm{~N}$, the estimation accuracy is decline sharply in the result of ZC. When the grip force is larger than $20 \mathrm{~N}$, the estimation accuracy is also decline in the result of WA. In general, the estimate of force by using these four features and the actual force have the similar trend.

The results of all six subjects are shown in Table 3. The average values of the six subjects' results are calculated and shown in Table 4 and Figure 15. The variation range of the all subjects' results is also shown in the form of vertical red line, as shown in Figure 15. It shows that the result of MVA is better than the other features'. The result of ZC has a larger fluctuation than that of the other three features.
A

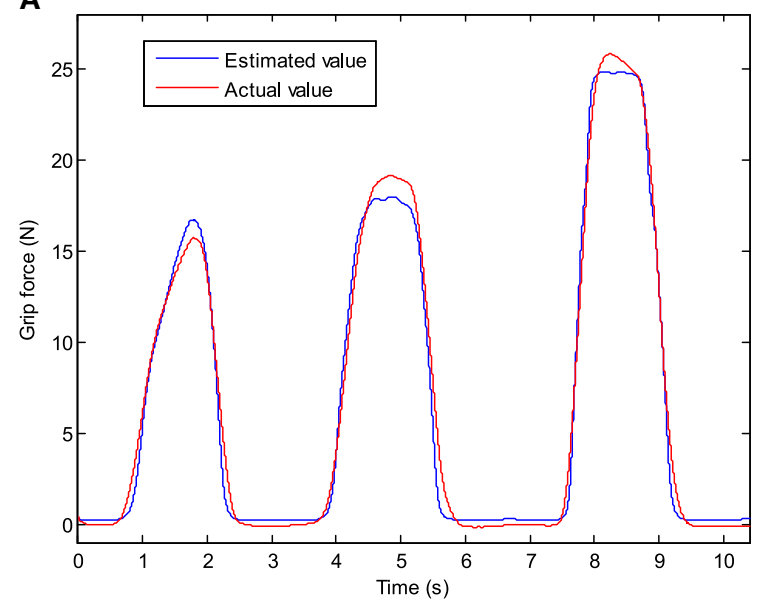

C

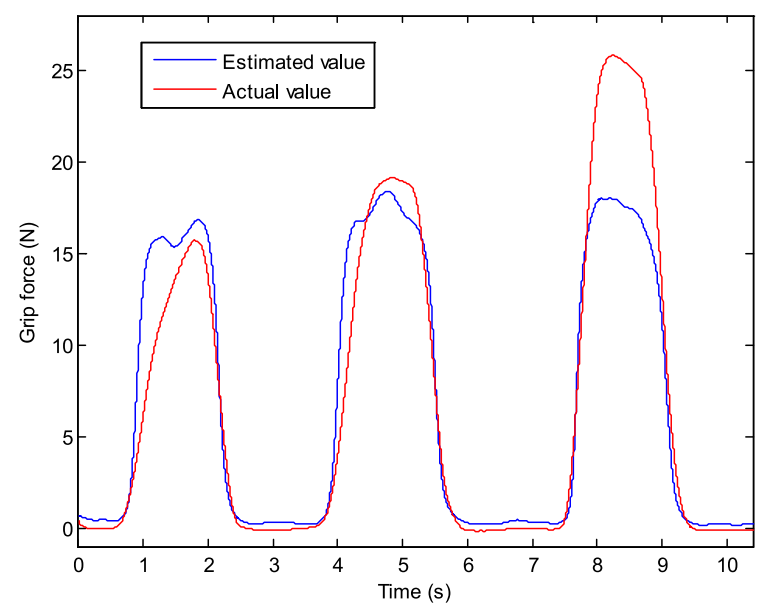

B

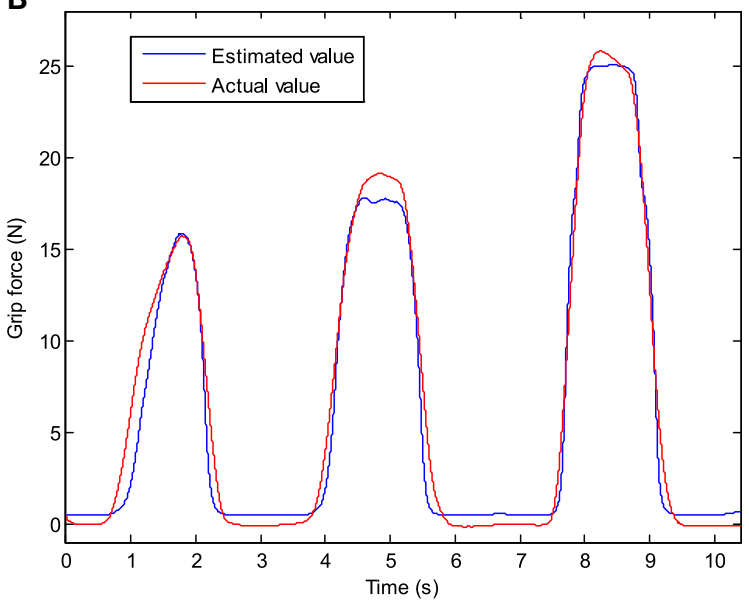

D

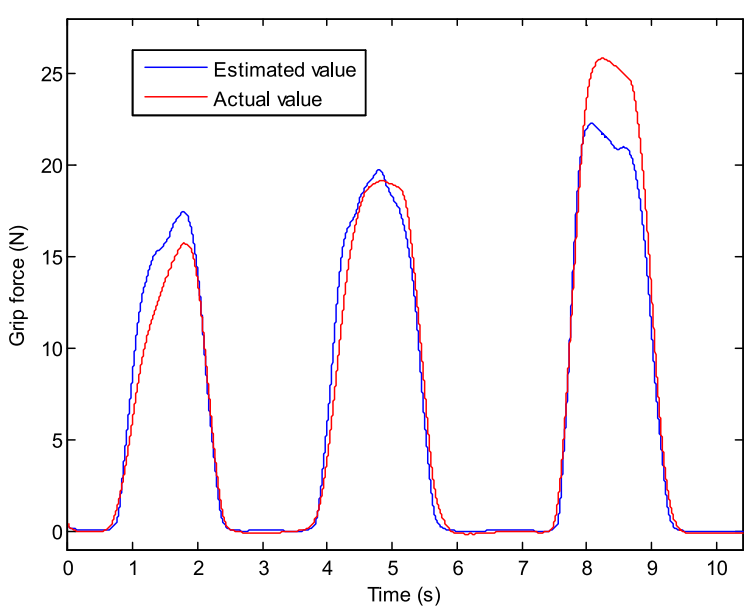

FIGURE 14 | The experiment results of the grip force estimation. (A) Feature = MAV. (B) Feature $=$ VAR. (C) Feature $=$ ZC. (D) Feature $=$ WA. 
TABLE 3 | The results of the grip force estimation experiments.

\begin{tabular}{|c|c|c|c|c|}
\hline & & \multicolumn{3}{|c|}{ Grip force } \\
\hline & & MAVE (N) & $R M S(N)$ & $\rho(\%)$ \\
\hline \multirow[t]{4}{*}{ Subject 1} & MAV & 0.69 & 0.89 & 99.46 \\
\hline & VAR & 1.12 & 1.36 & 98.75 \\
\hline & ZC & 1.87 & 2.85 & 94.72 \\
\hline & WA & 1.06 & 1.61 & 98.27 \\
\hline \multirow[t]{4}{*}{ Subject 2} & MAV & 0.55 & 0.73 & 99.62 \\
\hline & VAR & 1.07 & 1.36 & 98.64 \\
\hline & ZC & 1.70 & 2.76 & 94.79 \\
\hline & WA & 0.84 & 1.27 & 98.85 \\
\hline \multirow[t]{4}{*}{ Subject 3} & MAV & 0.63 & 0.79 & 99.58 \\
\hline & VAR & 1.03 & 1.28 & 98.91 \\
\hline & ZC & 1.82 & 2.82 & 94.89 \\
\hline & WA & 1.06 & 1.53 & 98.42 \\
\hline \multirow[t]{4}{*}{ Subject 4} & MAV & 0.63 & 0.82 & 99.38 \\
\hline & VAR & 1.10 & 1.41 & 98.20 \\
\hline & ZC & 1.43 & 2.49 & 94.64 \\
\hline & WA & 0.77 & 1.28 & 98.56 \\
\hline \multirow[t]{4}{*}{ Subject 5} & MAV & 0.62 & 0.77 & 99.56 \\
\hline & VAR & 1.12 & 1.40 & 98.51 \\
\hline & ZC & 1.83 & 3.03 & 93.13 \\
\hline & WA & 0.97 & 1.53 & 98.29 \\
\hline \multirow[t]{4}{*}{ Subject 6} & MAV & 0.54 & 0.79 & 99.56 \\
\hline & VAR & 0.85 & 1.24 & 98.89 \\
\hline & ZC & 1.44 & 2.63 & 95.08 \\
\hline & WA & 0.84 & 1.44 & 98.53 \\
\hline
\end{tabular}

TABLE 4 | The average results of the grip force estimation experiments across all the subjects.

\begin{tabular}{lccc}
\hline & \multicolumn{3}{c}{ Grip force } \\
\cline { 2 - 4 } & MAVE $(\boldsymbol{N})$ & $\boldsymbol{R M S}(\boldsymbol{N})$ & $\boldsymbol{\rho}(\%)$ \\
\hline MAV & 0.61 & 0.80 & 99.53 \\
VAR & 1.05 & 1.34 & 98.65 \\
ZC & 1.68 & 2.76 & 94.54 \\
WA & 0.92 & 1.44 & 98.49 \\
\hline
\end{tabular}

The results of One-way ANOVA and Tukey HSD are shown in Tables 5-8. Results in Table 5 indicate that there are significant differences $(p<0.05)$ among four features. The results of the Tukey HSD show that there are significant differences between each two features except VAR and WA. Four features can be divided into three subsets.

The above results indicate that the result of the WAV is better than the other three features' results by all three evaluation index. The result of the VAR and the result of the WA are similar. Among all the results, the ZC get the

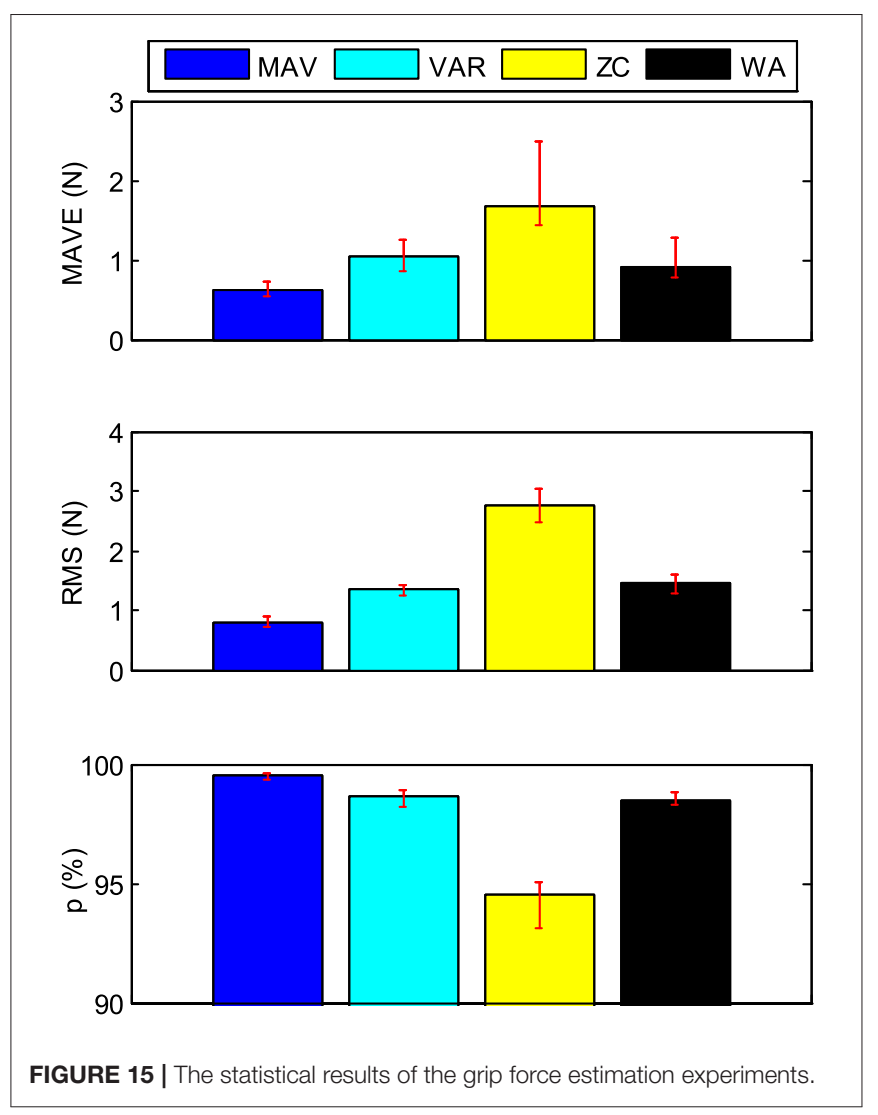

TABLE 5 | The results of the One-way ANOVA.

\begin{tabular}{llcllll}
\hline & & $\begin{array}{c}\text { Sum of } \\
\text { squares }\end{array}$ & df & $\begin{array}{c}\text { Mean } \\
\text { square }\end{array}$ & $\boldsymbol{F}$ & Sig. \\
\hline MAVE & Between groups & 3.646 & 3 & 1.215 & 70.554 & 0.000 \\
& Within groups & 0.345 & 20 & 0.017 & & \\
& Total & 3.990 & 23 & & & \\
\hline \multirow{2}{*}{ RMS } & Between groups & 12.519 & 3 & 4.173 & 268.170 & 0.000 \\
& Within groups & 0.311 & 20 & 0.016 & & \\
& Total & 12.831 & 23 & & & \\
\hline \multirow{2}{*}{$\rho$} & Between groups & 88.753 & 3 & 29.584 & 188.823 & 0.000 \\
& Within groups & 3.134 & 20 & 0.157 & & \\
& Total & 91.886 & 23 & & & \\
\hline
\end{tabular}

TABLE 6 | The results of the Tukey HSD for MAVE.

\begin{tabular}{lcccc}
\hline \multicolumn{5}{c}{ MAVE } \\
\hline Feature & $\boldsymbol{N}$ & \multicolumn{3}{c}{ Subset for alpha $=\mathbf{0 . 0 5}$} \\
\cline { 3 - 5 } & & $\mathbf{1}$ & $\mathbf{2}$ & $\mathbf{3}$ \\
\hline MAV & 6 & 0.6100 & & \\
WA & 6 & & 0.9233 & \\
VAR & 6 & & 1.0483 & 1.6817 \\
ZC & 6 & & & 1.000 \\
Sig. & & 1.000 & 0.375 & \\
\hline
\end{tabular}


worst one. The results also show that all of these four features can realize the estimation of the grip force in an acceptable performance.

\section{The 3D Force Estimation Experiment}

The $3 \mathrm{D}$ force is the representative of the force output by the human hand in three-dimensional space, which can be measured

TABLE 7 | The results of the Tukey HSD for RMS.

\begin{tabular}{llccc}
\hline \multicolumn{5}{c}{$\boldsymbol{R M S}$} \\
\hline Feature & $\boldsymbol{N}$ & \multicolumn{3}{c}{ Subset for alpha $=\mathbf{0 . 0 5}$} \\
\cline { 3 - 5 } & & $\mathbf{1}$ & $\mathbf{2}$ & $\mathbf{3}$ \\
\hline MAV & 6 & 0.7983 & & \\
VAR & 6 & & 1.3417 & \\
WA & 6 & & 1.4433 & 2.7633 \\
ZC & 6 & & & 1.000 \\
Sig. & & 1.000 & 0.507 & \\
\hline
\end{tabular}

by $3 \mathrm{D}$ force sensor. During the $3 \mathrm{D}$ force estimation experiment, the subjects were asked to grasp the handle which was set at the end of the 3D force sensor, and to apply force to the handle in $x$, $y$, and $z$ direction. The duration of the subjects applying force to each direction is about $1 \mathrm{~s}$. The maximum force is $<20 \mathrm{~N}$.

In this experiment, eight channels of the EMG signals were selected to estimate the grip force. Figure 16 shows the EMG

TABLE 8 | The results of the Tukey HSD for $\rho$.

\begin{tabular}{lcccc}
\hline \multicolumn{5}{c}{$\boldsymbol{\rho}$} \\
\hline Feature & $\boldsymbol{N}$ & \multicolumn{3}{c}{ Subset for alpha $=\mathbf{0 . 0 5}$} \\
\cline { 3 - 5 } & & $\mathbf{1}$ & $\mathbf{2}$ & $\mathbf{3}$ \\
\hline ZC & 6 & 94.5417 & & \\
WA & 6 & & 98.4867 & \\
VAR & 6 & & 98.6500 & 99.5267 \\
MAV & 6 & & & 1.000 \\
Sig. & & 1.000 & 0.890 & \\
\hline
\end{tabular}
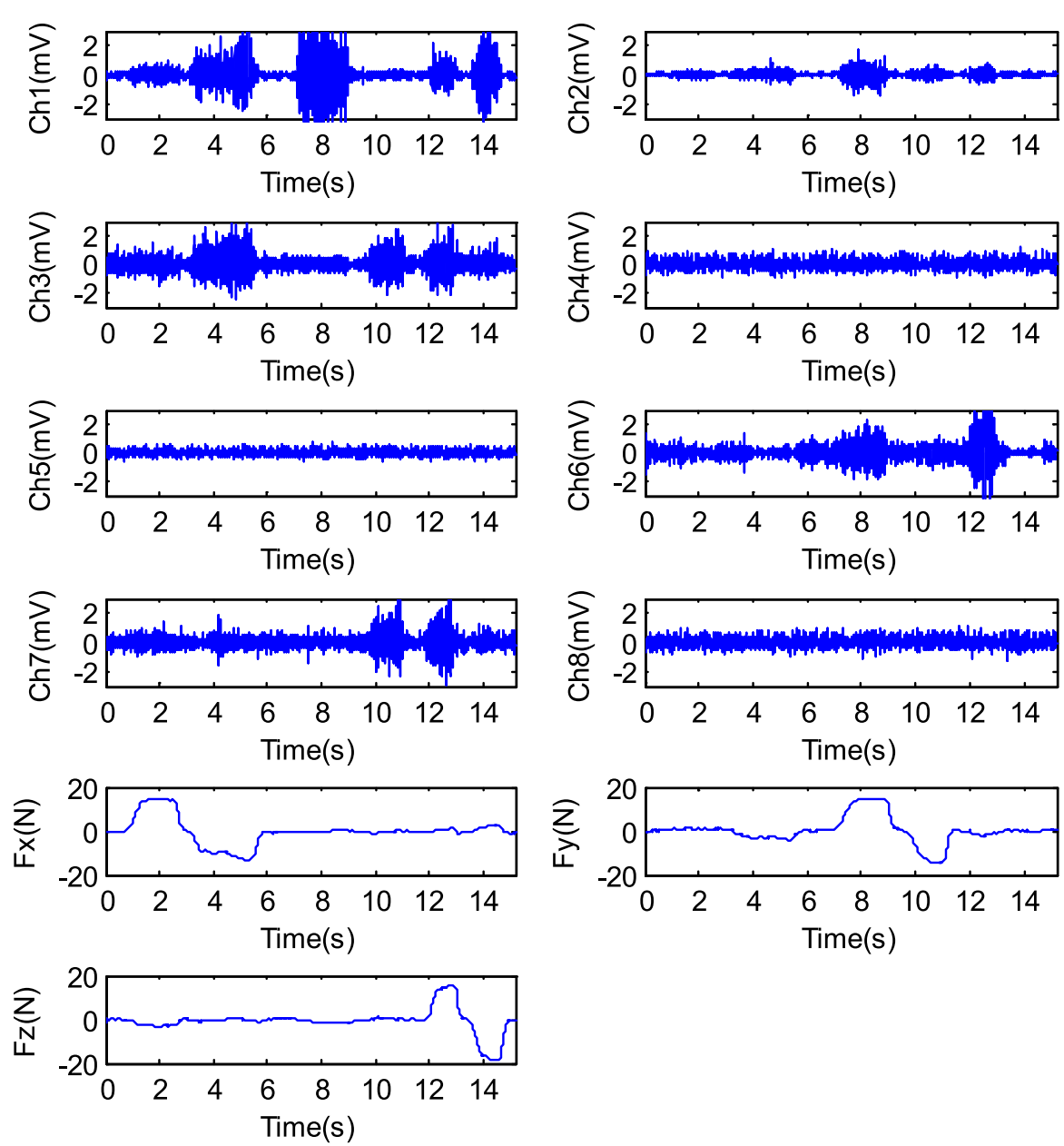

FIGURE 16 | The EMG signals and force signals output by the hand. 
A
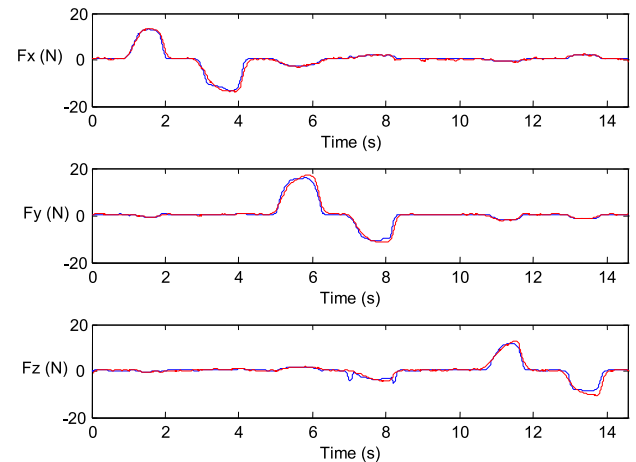

C
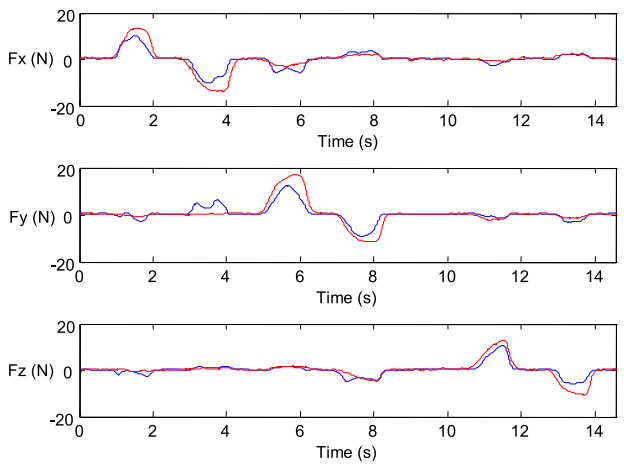

B
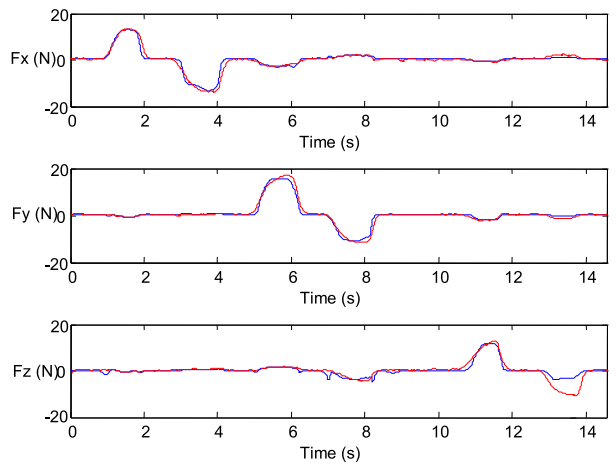

D
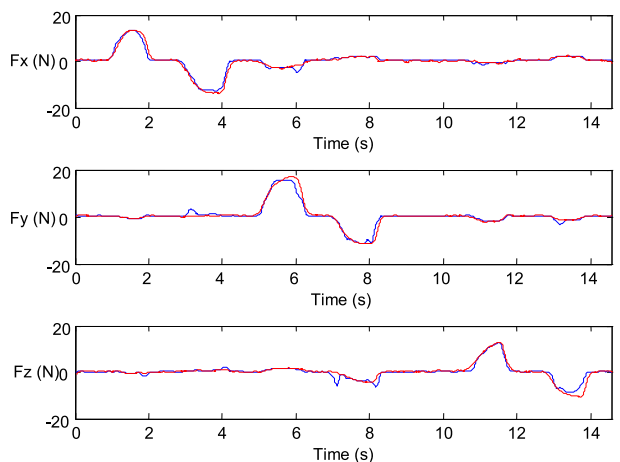

FIGURE 17 | The experimental results of the 3D force estimation. (A) Feature $=$ WAV. (B) Feature $=V A R$. (C) Feature $=Z C$. (D) Feature $=$ WA.
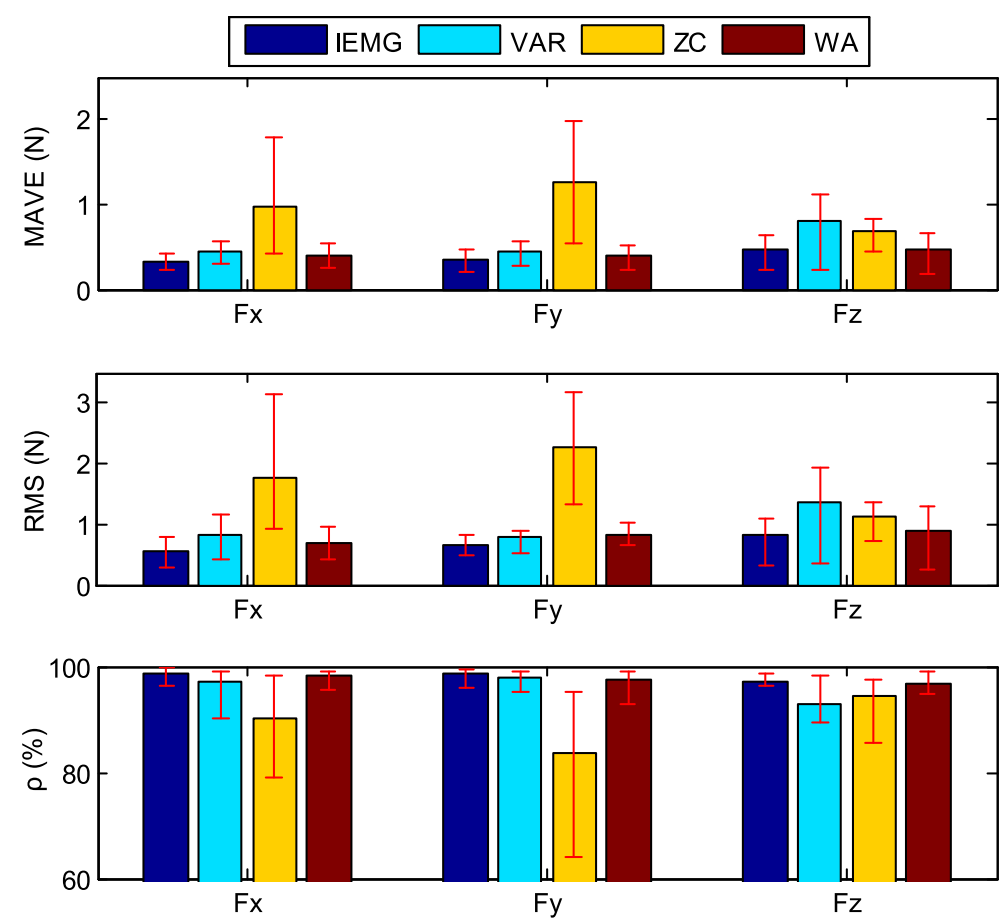

FIGURE 18 | The statistical results of the 3D force estimation experiments. 
TABLE 9 | The results of the 3D force estimation experiments.

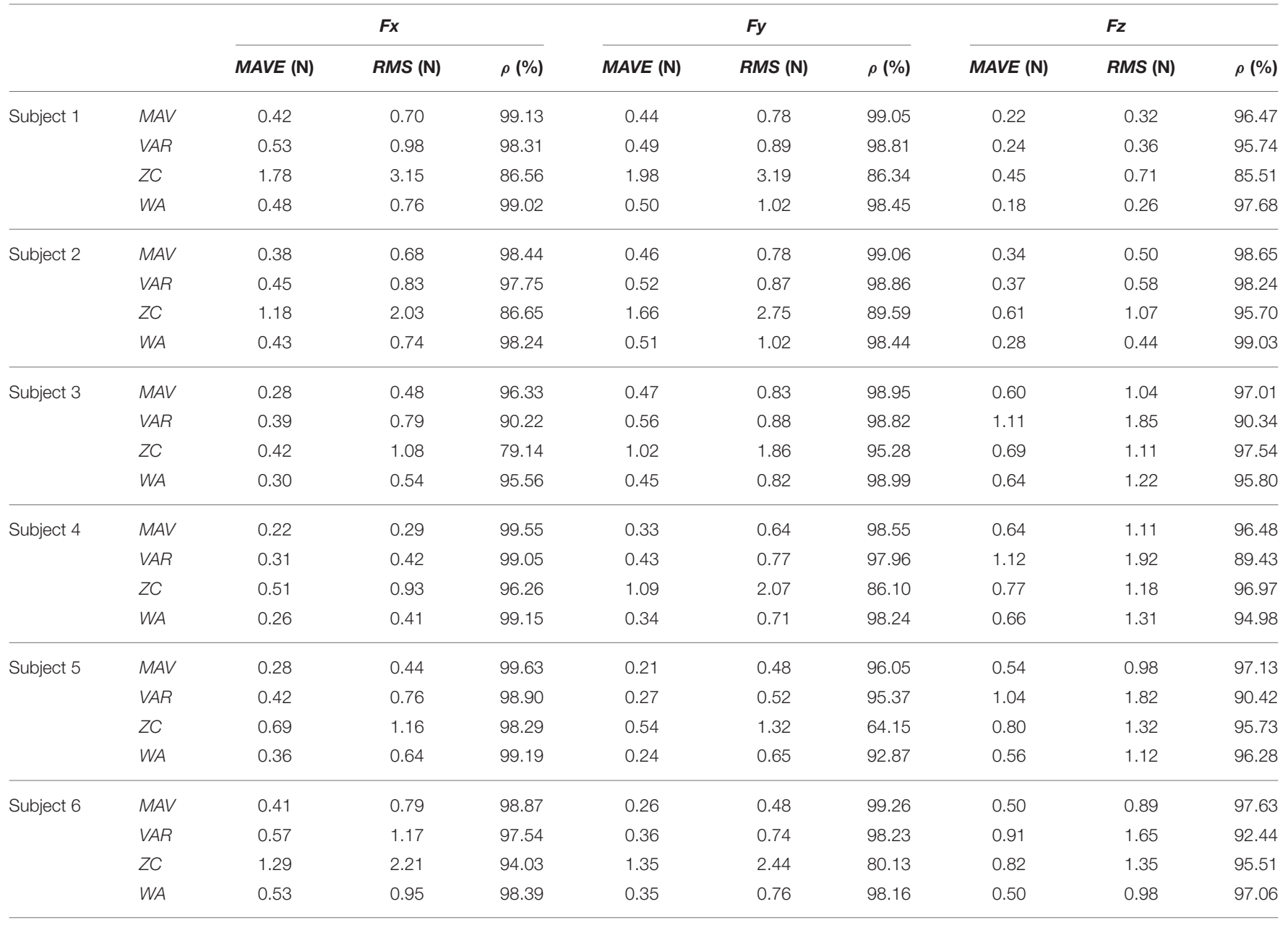

TABLE 10 | The average results of the 3D force estimation experiments across all the subjects.

\begin{tabular}{|c|c|c|c|c|c|c|c|c|c|}
\hline & \multicolumn{3}{|c|}{$F x$} & \multicolumn{3}{|c|}{$F y$} & \multicolumn{3}{|c|}{$F z$} \\
\hline VAR & 0.45 & 0.83 & 96.96 & 0.44 & 0.78 & 98.01 & 0.80 & 1.36 & 92.77 \\
\hline$Z C$ & 0.98 & 1.76 & 90.16 & 1.27 & 2.27 & 83.60 & 0.69 & 1.12 & 94.49 \\
\hline WA & 0.39 & 0.67 & 98.26 & 0.40 & 0.83 & 97.53 & 0.47 & 0.89 & 96.81 \\
\hline
\end{tabular}

TABLE 11 | The results of the Two-way ANOVA for MAVE.

\begin{tabular}{lrrrrr}
\hline Source & $\begin{array}{c}\text { Type III Sum } \\
\text { of squares }\end{array}$ & $\boldsymbol{d}$ ff & Mean square & $\boldsymbol{F}$ & Sig. \\
\hline Corrected model & 4.099 & 5 & 0.820 & 9.471 & 0.000 \\
Intercept & 24.863 & 1 & 24.863 & 287.250 & 0.000 \\
Force direction & 0.093 & 2 & 0.047 & 0.538 & 0.586 \\
EMG_Feature & 4.006 & 3 & 1.335 & 15.426 & 0.000 \\
Error & 5.713 & 66 & 0.087 & & \\
Total & 34.675 & 72 & & & \\
Corrected total & 9.811 & 71 & & & \\
\hline
\end{tabular}

TABLE 12 | The results of the Two-way ANOVA for RMS.

\begin{tabular}{lccccc}
\hline Source & $\begin{array}{c}\text { Type III sum } \\
\text { of squares }\end{array}$ & df & Mean square & $\boldsymbol{F}$ & Sig. \\
\hline Corrected model & 12.135 & 5 & 2.427 & 9.820 & 0.000 \\
Intercept & 78.730 & 1 & 78.730 & 318.566 & 0.000 \\
Force direction & 0.392 & 2 & 0.196 & 0.794 & 0.456 \\
EMG_Feature & 11.743 & 3 & 3.914 & 15.838 & 0.000 \\
Error & 16.311 & 66 & 0.247 & & \\
Total & 107.177 & 72 & & & \\
Corrected total & 28.446 & 71 & & & \\
\hline
\end{tabular}


signals and the $3 \mathrm{D}$ force while subject 1 applied force to the $3 \mathrm{D}$ force sensor.

The same as the grip force estimation experiment, four kinds of EMG time domain features which were extracted from the same raw EMG signals were used to train the GRNN, respectively. And the trained GRNNs were used to estimate the 3D force, respectively.

The experimental results of the subject 1 are shown in Figure 17. In Figure 17, the red line represents the actual force and the blue line represents the estimated force. The result of WAV has the best performance among the results of all these four features. And the result of $\mathrm{ZC}$ is the worst. When applying force to $z$-direction, which means pushing the $3 \mathrm{D}$ force sensor, the estimation results are poor by using the features of VAR and ZC. On the whole, the 3D force estimation results are acceptable.

The results from all six subjects are shown in Tables 9, 10. The average values and the variation range of the six subjects' results are shown in Figure $\mathbf{1 8}$ indicated by bar graph and vertical red line, respectively. It shows that the result of MVA is better than the other features'. The result of ZC has a larger fluctuation than that of the other three features. In the $z$ direction, the result of VAR also has a lager fluctuation. And in the $z$-direction, the result of VAR is worst by all three evaluation index.

The results of Two-way ANOVA and Tukey HSD are shown in Tables 11-13. The results of the Tukey HSD are shown in Tables 13-16. The results of Two-way ANOVA indicate that there are significant differences $(p<0.05)$ among four features. For all three evaluation index, the effects of force direction are not significant $(p>0.456)$. The results of Tukey HSD show that the effects of MAV, VAR, and

TABLE 13 | The results of the Two-way ANOVA for $\rho$.

\begin{tabular}{lccccc}
\hline Source & $\begin{array}{c}\text { Type III sum } \\
\text { of squares }\end{array}$ & $\boldsymbol{d f}$ & Mean square & $\boldsymbol{F}$ & Sig. \\
\hline Corrected model & 893.973 & 5 & 178.795 & 7.522 & 0.000 \\
Intercept & $653,163.541$ & 1 & $653,163.541$ & $27,478.191$ & 0.000 \\
Force direction & 31.084 & 2 & 15.542 & 0.654 & 0.523 \\
EMG_Feature & 862.888 & 3 & 287.629 & 12.100 & 0.000 \\
Error & 1568.837 & 66 & 23.770 & & \\
Total & $655,626.351$ & 72 & & & \\
Corrected total & 2462.809 & 71 & & & \\
\hline
\end{tabular}

TABLE 14 | The results of the Tukey HSD for MAVE.

\begin{tabular}{lccc}
\hline EMG_Feature & $\boldsymbol{N}$ & \multicolumn{2}{c}{ Subset } \\
\cline { 3 - 4 } & & $\mathbf{1}$ & $\mathbf{2}$ \\
\hline MAV & 18 & 0.3889 & \\
WA & 18 & 0.4206 & \\
VAR & 18 & 0.5606 & 0.9806 \\
ZC & 18 & & 1.000 \\
Sig. & & 0.306 & \\
\hline
\end{tabular}

WA are similar. Four features can be divided into two subsets.

The above results indicate that all of these four features can achieve the estimation of the $3 \mathrm{D}$ force successfully. The result of MAV is best. But compare with VAR and WA, there is no significant difference. The result of $\mathrm{ZC}$ is worst.

\section{CONCLUSION}

In order to meet the requirements of the dexterous control of the prosthetic hand, the paper proposes a force estimation method of hand movement based on the sEMG and GRNN. The estimated force of hand movement includes the grip force and the 3D force. An experimental platform is set up to measure the multi-channels of the sEMG signals, the grip force and the 3D push-pull force. Based on this platform, the sEMG on the arm skin surface and force of the action output by the hand can be measured synchronously. The widely used time domain feature extraction methods are employed to pre-process the sEMG signals. Then the extracted EMG features are mapped to force of the hand movement by using GRNN.

The experiments are implemented to verify the effectiveness of the proposed force estimation method of hand movement. And the results show that the proposed method can realize the force estimation of hand movement with an acceptable performance under the condition that grip force is $<30 \mathrm{~N}$ and the $3 \mathrm{D}$ push-pull force is $<20 \mathrm{~N}$. In grip force estimation, the result of MVA is best. In $3 \mathrm{D}$ force estimation, the result of MAV is also best, but compare with VAR and WA, there is no significant difference. The performance of $\mathrm{ZC}$ is worst both in grip estimation and $3 \mathrm{D}$ force estimation.

TABLE 15 | The results of the Tukey HSD for RMS.

\begin{tabular}{llll}
\hline EMG_Feature & $\boldsymbol{N}$ & \multicolumn{2}{c}{ Subset } \\
\cline { 3 - 4 } & & $\mathbf{1}$ & $\mathbf{2}$ \\
\hline MAV & 18 & 0.6783 & \\
WA & 18 & 0.7972 & \\
VAR & 18 & 0.9889 & \\
ZC & 18 & & 1.7183 \\
Sig. & & 0.249 & 1.000 \\
\hline
\end{tabular}

TABLE 16 | The results of the Tukey HSD for $\rho$.

\begin{tabular}{lccc}
\hline EMG_Feature & $\boldsymbol{N}$ & \multicolumn{2}{c}{ Subset } \\
\cline { 3 - 4 } & & $\mathbf{1}$ & $\mathbf{2}$ \\
\hline ZC & 18 & 89.4156 & \\
VAR & 18 & & 95.9128 \\
WA & 18 & & 97.5294 \\
MAV & 18 & & 98.1244 \\
Sig. & & 1.000 & 0.528 \\
\hline
\end{tabular}


For the future work, we will research the estimation of the parameters of the kinematics and dynamics based on the sEMG for the purpose of further improving the performance of the dexterous manipulation of the EMG prostheses.

\section{ETHICS STATEMENT}

The study is exempt from ethics approval. We put up a petition in Chinese for sEMG clinical measurement to the Nanjing Tongren Hospital (http://tongren.ecaihr.com/). The relevant organization of the Nanjing Tongren Hospital consider our clinical measurement without ethical problem. All the subjects were gave written, informed consent. The permission was granted by the subject to reproduce their likeness in Figures 2, 10.

\section{REFERENCES}

Al Omari, F., Hui, J., Mei, C., and Liu, G. (2014). Pattern recognition of eight hand motions using feature extraction of forearm EMG signal. Proc. Natl. Acad. Sci. India Sect. A Phys. Sci. 84, 473-480. doi: 10.1007/s40010-0140148-2

Altiparmak, F., Dengiz, B., and Smith, A. E. (2009). A general neural network model for estimating telecommunications network reliability. IEEE Trans. Reliab. 58, 2-9. doi: 10.1109/TR.2008.2011854

Castellini, C., Gruppioni, E., Davalli, A., and Sandini, G. (2009). Fine detection of grasp force and posture by amputees via surface electromyography. J. Physiol. (Lond). 103, 255-262. doi: 10.1016/j.jphysparis.2009.08.008

Davidson, J. (2002). A survey of the satisfaction of upper limb amputees with their prostheses, their lifestyles, and their abilities. J. Hand Ther. 15, 62-70. doi: 10.1053/hanthe.2002.v15.01562

Hashemi, J., Morin, E., Mousavi, P., and Hashtrudi-Zaad, K. (2015). Enhanced dynamic EMG-force estimation through calibration and PCI modeling. IEEE Trans. Neural Syst. Rehab. Eng. 23, 41-50. doi: 10.1109/TNSRE.2014. 2325713

Hashemi, J., Morin, E., Mousavi, P., Mountjoy, K., and Hashtrudi-Zaad, K. (2012). EMG-force modeling using parallel cascade identification. J. Electromyogr. Kinesiol. 22, 469-477. doi: 10.1016/j.jelekin.2011.10.012

He, J., Zhang, D., Sheng, X., Li, S., and Zhu, X. (2015). Invariant surface EMG feature against varying contraction level for myoelectric control based on muscle coordination. IEEE J. Biomed. Health Inform. 19, 874-882. doi: 10.1109/JBHI.2014.2330356

Hioki, M., and Kawasaki, H. (2012). Estimation of finger joint angles from sEMG using a neural network including time delay factor and recurrent structure. ISRN Rehab. 2012:604314. doi: 10.5402/2012/604314

Hofmann, D., Jiang, N., Vujaklija, I., and Farina, D. (2016). Bayesian filtering of surface EMG for accurate simultaneous and proportional prosthetic control. IEEE Trans. Neural Syst. Rehab. Eng. 24, 1333-1341. doi: 10.1109/TNSRE.2015.2501979

Ju, Z., and Liu, H. (2014). Human hand motion analysis with multisensory information. IEEE ASME Trans. Mechatr. 19, 456-466. doi: 10.1109/TMECH.2013.2240312

Kamavuako, E. N., Farina, D., Yoshida, K., and Jensen, W. (2012). Estimation of grasping force from features of intramuscular EMG signals with mirrored bilateral training. Ann. Biomed. Eng. 40, 648-656. doi: 10.1007/s10439-011-0438-7

Kamavuako, E. N., and Rosenvang, J. C. (2012). Hysteresis in the electromyography-force relationship: toward an optimal model for the estimation of force. Muscle Nerve 46, 755-758. doi: 10.1002/mus. 23393

\section{AUTHOR CONTRIBUTIONS}

CW: Project design; Experimental study; Writing paper. HZ: Data analysis. AS: Project design. BX: Data analysis.

\section{ACKNOWLEDGMENTS}

This paper is supported by the National Science Foundation of China under Grant No. 91648206, 61325018, 61403080, 61673105, 61673114. Jiangsu Technology Development Project under Grant No. BE2014132. Jiangsu Natural Science Foundation under Grant No. BK20140641. Young Teacher Startup Foundation of Nanjing University of Aeronautics and Astronautics under Grant No. 56YAH17027.

Kamavuako, E. N., Rosenvang, J. C., Bøg, M. F., Smidstrup, A., Erkocevic, E., Niemeier, M. J., et al. (2013). Influence of the feature space on the estimation of hand grasping force from intramuscular EMG. Biomed. Signal Process. Control 8, 1-5. doi: 10.1016/j.bspc.2012.05.002

Li, G., Schultz, A. E., and Kuiken, T. A. (2010). Quantifying pattern recognitionbased myoelectric control of multifunctional transradial prostheses. IEEE Trans. Neural Syst. Rehab. Eng. 18, 185-192. doi: 10.1109/TNSRE.2009.2039619 Ma, J., and Song, A. (2012). Development of a novel two-axis force sensor for Chinese massage robot. Appl. Mech. Mater. 103, 299-304. doi: 10.4028/www. scientific.net/AMM.103.299

Ma, J., Song, A., and Pan, D. (2013). Dynamic compensation for two-axis robot wrist force sensors. J. Sens. 2013:357396. doi: 10.1155/2013/357396

Maat, B., Smit, G., Plettenburg, D., and Breedveld, P. (2017). Passive prosthetic hands and tools: a literature review. Prosthet. Orthot. Int. doi: 10.1177/0309364617691622. [Epub ahead of print].

Ngeo, J., Tamei, T., and Shibata, T. (2014). Estimation of continuous multiDOF finger joint kinematics from surface EMG using a multi-output gaussian process. IEEE Int. Conf. Eng. Med. Biol. Soc. 2014, 3537-3540. doi: $10.1109 / \mathrm{embc} .2014 .6944386$

Ngeo, J., Tamei, T., Shibata, T., Orlando, M. F., Behera, L., Saxena, A., et al. (2013). Control of an optimal finger exoskeleton based on continuous joint angle estimation from EMG signals. Conf. Proc. IEEE Eng. Med. Biol. Soc. 2013, 338-341. doi: 10.1109/embc.2013.6609506

Nielsen, J. L. G., Holmgaard, S., Jiang, N., Englehart, K. B., Farina, D., and Parker, P. A. (2011). Simultaneous and proportional force estimation for multifunction myoelectric prostheses using mirrored bilateral training. IEEE Trans. Biomed. Eng. 58, 681-688. doi: 10.1109/TBME.2010.2068298

Nishikawa, D., Yu, W., Yokoi, H., and Kakazu, Y. (1999). EMG prosthetic hand controller using real-time learning method. IEEE Int. Conf. Syst. Man Cybernet. 1, 153-158. doi: 10.1109/icsmc.1999.814077

Orizio, C., Baruzzi, E., Gaffurini, P., Diemont, B., and Gobbo, M. (2010). Electromyogram and force fluctuation during different linearly varying isometric motor tasks. J. Electromyogr. Kinesiol. 20, 732-741. doi: 10.1016/j.jelekin.2010.03.005

Pan, L., Zhang, D., Sheng, X., and Zhu, X. (2015). Improving myoelectric control for amputees through transcranial direct current stimulation. IEEE Trans. Biomed. Eng. 62, 1927-1936. doi: 10.1109/TBME.2015.2407491

Segil, J. L., Huddle, S. A., and Weir, R. F. F. (2016). Functional assessment of a myoelectric postural controller and multi-functional prosthetic hand by persons with trans-radial limb loss. IEEE Trans. Neural Syst. Rehab. Eng. 25, 618-627. doi: 10.1109/tnsre.2016.2586846

Wu, C., Song, A., Ling, Y., Wang, N., and Tian, L. (2015). A control strategy with tactile perception feedback for EMG prosthetic hand. J. Sens. 2015:869175. doi: $10.1155 / 2015 / 869175$ 
Xie, H., Huang, H., Wu, J., and Liu, L. (2015). A comparative study of surface EMG classification by fuzzy relevance vector machine and fuzzy support vector machine. Physiol. Meas. 36, 191-206. doi: 10.1088/0967-3334/36/2/191

Zaini, M. H. M., and Ahmad, S. A. (2011). "Surgical and non-surgical prosthetic hands control: a review," in IEEE Symposium on Industrial Electronics and Applications (ISIEA) (Langkawi), 634-637.

Zhang, Z., Wong, C., and Yang, G. Z. (2013). "Forearm functional movement recognition using spare channel surface electromyography," in IEEE International Conference on Body Sensor Networks (Cambridge, MA), 1-6. doi: $10.1109 /$ bsn.2013.6575507
Conflict of Interest Statement: The authors declare that the research was conducted in the absence of any commercial or financial relationships that could be construed as a potential conflict of interest.

Copyright $\odot 2017 \mathrm{Wu}$, Zeng, Song and Xu. This is an open-access article distributed under the terms of the Creative Commons Attribution License (CC BY). The use, distribution or reproduction in other forums is permitted, provided the original author(s) or licensor are credited and that the original publication in this journal is cited, in accordance with accepted academic practice. No use, distribution or reproduction is permitted which does not comply with these terms. 


\section{OPEN ACCESS}

Edited by:

Dingguo Zhang

Shanghai Jiao Tong University, China

Reviewed by:

Yinfeng Fang,

University of Portsmouth, UK

Jie Shang,

Ningbo Institute of Industrial

Technology (CAS), China

*Correspondence:

Yinlai Jiang

jiang@hi.mce.uec.ac.jp

Specialty section:

This article was submitted to

Neural Technology,

a section of the journal

Frontiers in Neuroscience

Received: 19 October 2016

Accepted: 17 January 2017

Published: 06 February 2017

Citation:

Jiang Y, Togane M, Lu B and Yokoi H (2017) sEMG Sensor Using

Polypyrrole-Coated Nonwoven Fabric

Sheet for Practical Control of

Prosthetic Hand.

Front. Neurosci. 11:33.

doi: 10.3389/fnins.2017.00033

\section{sEMG Sensor Using Polypyrrole-Coated Nonwoven Fabric Sheet for Practical Control of Prosthetic Hand}

\author{
Yinlai Jiang ${ }^{1 *}$, Masami Togane ${ }^{2}$, Baoliang $\mathrm{Lu}^{3}$ and Hiroshi Yokoi ${ }^{1,2}$ \\ ${ }^{1}$ Brain Science Inspired Life Support Research Center, University of Electro-Communications, Tokyo, Japan, ${ }^{2}$ Department of \\ Mechanical Engineering and Intelligent Systems, University of Electro-Communications, Tokyo, Japan, ${ }^{3}$ Department of \\ Computer Science and Engineering, Shanghai Jiao Tong University, Shanghai, China
}

One of the greatest challenges of using a myoelectric prosthetic hand in daily life is to conveniently measure stable myoelectric signals. This study proposes a novel surface electromyography (sEMG) sensor using polypyrrole-coated nonwoven fabric sheet as electrodes (PPy electrodes) to allow people with disabilities to control prosthetic limbs. The PPy electrodes are sewn on an elastic band to guarantee close contact with the skin and thus reduce the contact electrical impedance between the electrodes and the skin. The sensor is highly customizable to fit the size and the shape of the stump so that people with disabilities can attach the sensor by themselves. The performance of the proposed sensor was investigated experimentally by comparing measurements of $\mathrm{Ag} / \mathrm{AgCl}$ electrodes with electrolytic gel and the sEMG from the same muscle fibers. The high correlation coefficient $(0.87)$ between the two types of sensors suggests the effectiveness of the proposed sensor. Another experiment of sEMG pattern recognition to control myoelectric prosthetic hands showed that the PPy electrodes are as effective as $\mathrm{Ag} / \mathrm{AgCl}$ electrodes for measuring sEMG signals for practical myoelectric control. We also investigated the relation between the myoelectric signals' signal-to-noise ratio and the source impedances by simultaneously measuring the source impedances and the myoelectric signals with a switching circuit. The results showed that differences in both the norm and the phase of the source impedance greatly affect the common mode noise in the signal.

Keywords: surface electromyography, myoelectric control, prosthetic hand, conductive polymer, electrode

\section{INTRODUCTION}

Robotic prosthetic hands have benefitted from significant improvements due to the quick advance of robotics and biomedical engineering in the last decade. The prosthetic hand is expected to restore the lost hand's function through hardware that can operate like a human hand and an interface that can transfer the user's motion intention to the hardware. In regard to the hardware, many dexterous prosthetic hands with multiple degrees of freedom (DoFs), such as the Vincent hand by Vincent Systems, the iLimb hand by Touch Bionics, the Bebionic hand by RSL Steeper, and the Michelangelo hand by Otto Bock, have been developed and are commercially available (Belter et al., 2013). In regard to the human interface, electromyography (EMG) signals associated with 
residual muscle contraction, from which the user's motion volition is recognized according to the remnant muscle activity, have been studied (Asghari Oskoei and Hu, 2007). Studies on the classification of EMG signals have reported remarkable results with advanced algorithms based on machine learning methods such as nonnegative matrix factorization, linear regression, linear discriminant analysis, support vector machines, and artificial neural networks (ANN), which have been proposed to identify as many patterns as possible from limited EMG sources and to deal with the unsteadiness, nonlinearity, individual difference, and low reproducibility of EMG signals (Al-Timemy et al., 2013; Young et al., 2013; Amsüss et al., 2014; Hahne et al., 2014; Jiang et al., 2014b; Earley et al., 2016).

However, despite the significant improvement of the mechanics and the EMG analysis methods, most of the currently commercially available myoelectric prosthetic hands used by amputees are still controlled by very simple algorithms that process the surface electromyogram (sEMG) to control $1^{\circ} \mathrm{C}$ of freedom (DoF) at a time. Threshold-level control, which generates a command to control one motion with one EMG source according the signal amplitude, is still the most widely used method (Dosen et al., 2010; Young et al., 2012). The main problem for applying advanced algorithms to control dexterous prosthetic hands in real life is the difficulty of measuring stable myoelectric signals. There are many factors, such as electrode shift (Young et al., 2012; Pan et al., 2015), noises in the signals (Chowdhury et al., 2013), and fatigue that causes the change of EMG (Beck et al., 2014), for the fault of applying advanced algorithms. Some studies turn to multi-modal sensing to overcome the difficulties of myoelectric control (Fang et al., 2015). The difficulty to measure useful signals with a high signalto-noise $(\mathrm{S} / \mathrm{N})$ ratio using the current measurement methods is one of the main reasons for keeping amputees away from the advanced prosthetic hands. Most of the algorithms reported are tested in labs with only high-quality signals which are unavailable for used by people in their daily lives. Furthermore, the shape, size, and site of the residual limbs are different from one amputee to another, especially for young amputees whose residual limbs are still changing with their growth (Vasluian et al., 2013). Commercially available sEMG sensors allow little customization to adapt to these various and variable conditions. In real-life applications, the differences caused by analysis algorithms are less than those caused by measurement methods, since the quality of the EMG signal determines the usability and the stability of the myoelectric control.

A sEMG sensor is an electrochemical transducer that detects biopotentials by using electrodes placed on the skin. Since the measured potentials have small amplitudes, $<30 \mathrm{mV}$ when measured on the muscle fiber and $<1 \mathrm{mV}$ when measured on the skin surface, the potentials are susceptible to electromagnetic interference. Consequently, circuits that amplify the signals and filter external noise are usually required. A traditional way to measure EMGs for myoelectric control is differential amplification, in which the electrodes and the amplifier are the pre-amplifier and are the most important factors affecting the $\mathrm{S} / \mathrm{N}$ ratio. $\mathrm{Ag} / \mathrm{AgCl}$ electrodes, so-called "wet electrodes" utilizing an electrolytic gel to form a conductive path between skin and electrode, are currently the most widely used in hospitals and laboratories. Despite the fact that the low electrode-skin impedance of $\mathrm{Ag} / \mathrm{AgCl}$ electrodes contributes to a high $\mathrm{S} / \mathrm{N}$ ratio, dry electrodes made from metals, e.g., MyoBock 13E20050 electrodes (Otto Bock HealthCare $\mathrm{GmbH}$ ) made of pure titanium, are always adopted in real-life myoelectric control, since wet $\mathrm{Ag} / \mathrm{AgCl}$ electrodes are disposable and not suited for long-term measurement. Electrodes reported recently include implantable electrodes (Merrill et al., 2011), invasive fine wire electrodes (Smith et al., 2016), low invasive percutaneous implant (Hahne et al., 2016), noninvasive metal electrodes with special curved microneedle array-based structures (Kim et al., 2015), and high-density multichannel electrodes (Popovic-Maneski et al., 2013). The implantable and invasive electrodes are suitable for measuring high-quality signals for dexterous control and diagnosis in hospitals, but the risk of infection makes them difficult for long-term everyday use. Most of the noninvasive electrodes made of metals have a fixed shape and size and are not customizable.

The objective of this study is therefore to develop usable and customizable sEMG sensors for practical control of a prosthetic hand in daily life. In preliminary studies, we developed both multi-DoF prosthetic hands with an interactive-wire driven mechanism (Seki et al., 2013; Morishita et al., 2014) and two DoF prosthetic hands simplified for quick practical use (Jiang et al., 2014a). An fMRI study with amputees showed the adaptation process in the brain for control of the prosthetic hand (Kato et al., 2009). However, for daily use of the prosthetic hand by a person with a disability, the number of recognizable sEMG patterns is limited by the usability and stability of the sEMG electrodes. This study introduces a sEMG electrode made of polypyrrole-coated nonwoven fabric sheet (PPy electrode) that can be easily customized to any person and can be worn onehanded for stable measurement of sEMG signals. Preliminary results on the PPy electrodes, including the manufacturing method, and basic performance for the control of myoelectric prosthetic hands, were reported in conference proceedings (Jiang et al., 2015). This study further tested the PPy electrodes by amputees and compared them with traditional disposable wet $\mathrm{Ag} / \mathrm{AgCl}$ electrodes. Furthermore, we investigated the relation between the $\mathrm{S} / \mathrm{N}$ ratio and the source impedances when using the PPy electrodes by simultaneous measurement of the source impedances and sEMG signals. The results showed that source impedances can serve as a useful reference when manufacturing the PPy electrodes. The possibility of improving the $\mathrm{S} / \mathrm{N}$ ratio by balancing the source impedances was also indicated.

\section{MATERIALS AND METHODS}

\section{Development of the sEMG Sensor Electrode Material}

A sEMG sensor using polypyrrole-coated nonwoven fabric sheet (Japan Vilene Company, Ltd) as electrodes (hereinafter referred to as PPy electrodes) is proposed for people with disabilities to control prosthetic limbs in daily life. The material is shown in Figure 1. The base material is polyethylene terephthalate and nylon, coated with polypyrrole, a kind of conducting 

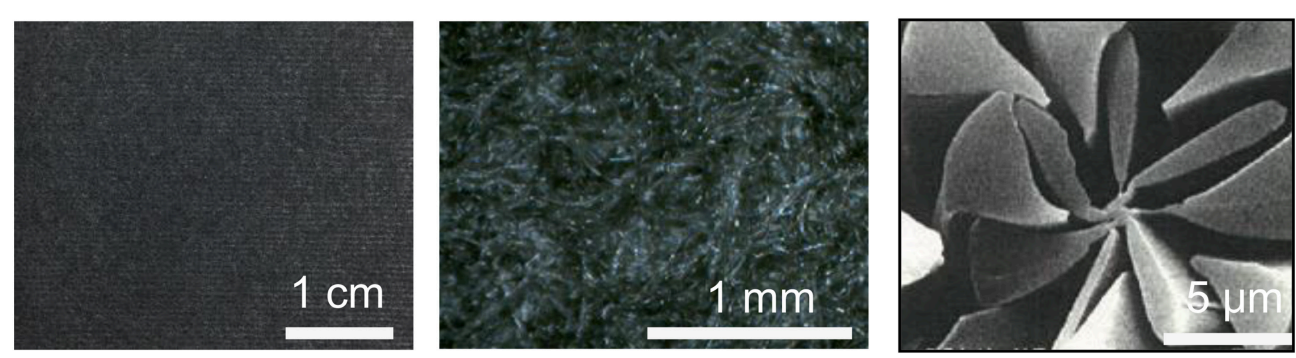

FIGURE 1 | Polypyrrole-coated nonwoven fabric sheet.

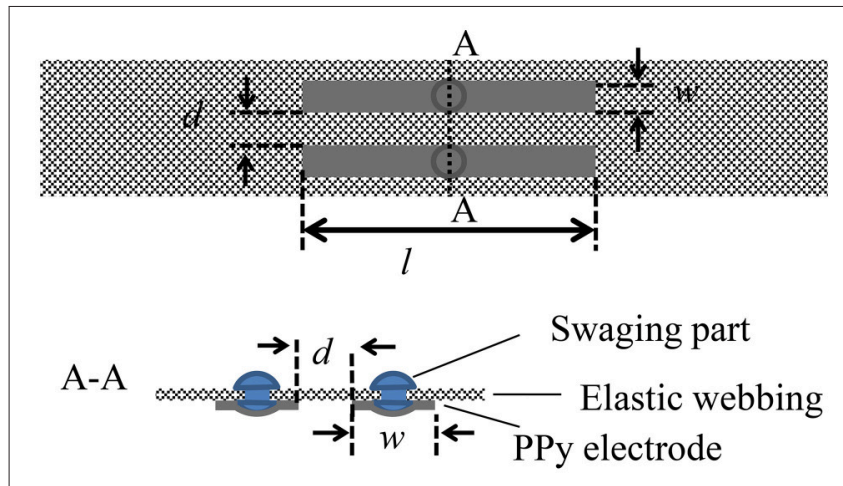

FIGURE 2 | Structural design of PPy electrode.

polymer. The thickness of the sheet is $0.5 \mathrm{~mm}$ and the surface resistivity is $<1 \times 10^{5} \Omega /$ sq. Compared with poly(3,4ethylenedioxythiophene)-poly(styrenesulfonate) (PEDOT-PSS), which has been reported for electrodes measuring bioelectrical signals (Yamato et al., 1995; Richardson-Burns et al., 2007; Lang et al., 2009; Tsukada et al., 2012; Isik et al., 2015; Nagamine et al., 2016; Pani et al., 2016; Papaiordanidou et al., 2016), the conductivity of polypyrrole is lower, but it is more stable in an ambient environment that may be humid and wet due to skin sweating with a lower watersolubility and a lower hygroscopicity (Lee et al., 2001; Jang et al., 2004; Xu et al., 2006; Lang et al., 2009; Elschner et al., 2010).

\section{Structural Design of the Sensor Band}

PPy electrodes are easily customized to fit the size and the shape of the residual parts of an amputee. To attach PPy electrodes to the stump and to keep them on the skin, the PPy electrodes are sewn on an elastic band, as shown in Figure 2. Length 1 and width $w$ of the electrodes, and distance $d$ between pairs of electrodes can be adjusted to match a specific user. The elasticity of the band leads to close contact to the skin, and thus reduces the skin-electrode impedance and enables stable sEMG measurement with a high signal-to-noise ratio. Stainless swaging parts are used to transduce the electrical potentials from the PPy electrodes to the circuit of amplifier and filter.

\section{Trial Use}

Myoelectric control of a prosthetic hand with PPy electrodes was tested by a 14-year-old girl with a congenital right upper limb deficiency and a 36-year-old man with a right upper limb amputation that happened 5 years earlier. All the experimental protocols of this study were approved by the ethics board of the University of Electro-Communications [No. 10006(4)]. All subjects were fully informed about the procedures, risk, and benefits of the study, and written informed consent was obtained from all subjects before the study.

Figure 3 shows the 3-channel sEMG sensor band with six recording electrodes for differential amplification and a common ground electrode. The potentials detected by the PPy electrodes were amplified and filtered by the circuit shown in Figure 3. The circuit was connected to the electrodes as closely as possible to ensure the electrical contact avoided noise. The circuit consisted of a preamplifier using an instrument amplifier AD620 (Analog Devices, USA) with a gain of $40 \mathrm{~dB}$, a band pass filter with a 1$1000 \mathrm{~Hz}$ bandwidth, a $50 \mathrm{~Hz}$ notch filter, and a second amplifier. The output voltage range of the circuit was $(-9 v, 9 v)$.

The subjects wearing the sensor band tried to control a prosthetic hand developed in a preliminary study (Jiang et al., 2014a). The prosthetic hand consisted of a lightweight robotic hand with two motors to realize power grasp, precision grasp, and lateral grasp, a socket to mount the hand to the residual forearm, and a controller (SH72544R, Renesas Electronics Corporation, Japan) that performed EMG signal processing, pattern recognition, and motor control. The sEMG sensor band was worn on the stump covered by the socket. Figure 4 shows that the stumps of the subjects are quite different. They were able to one-handed attach the sensor bands customized for them by themselves. With the sensor bands, they tried to control the myoelectric prosthetic hand to perform some daily task, e.g., picking up a cup or building blocks, as shown in the figure.

\section{COMPARISON WITH WET AG/AGCL ELECTRODES}

The trial by the subjects illustrated the practicability of the sensor band. To further verify the performance and characteristics of the PPy electrodes, these electrodes were compared with traditional wet $\mathrm{Ag} / \mathrm{AgCl}$ electrodes in two experiments: one to investigate the correlation of the measured sEMG signals and the other to 


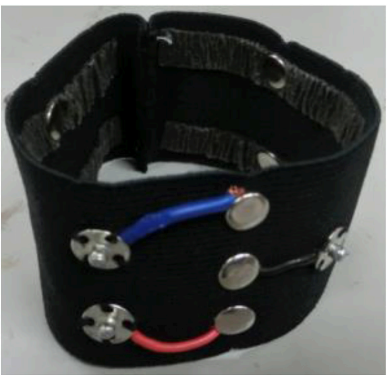

Sensor band

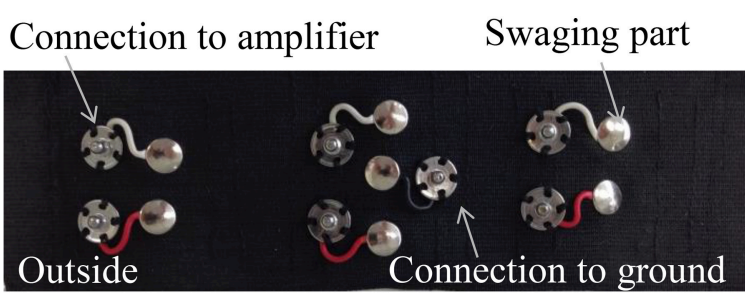

Channel 1

Channel 2

Channel 3

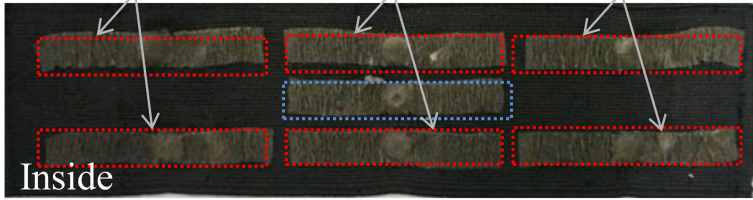

Recording electrode

Common ground electrode

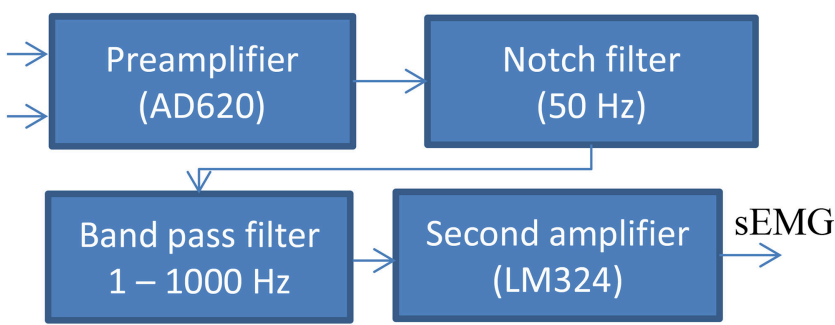

Amplifier and filter circuit

FIGURE 3 | Sensor band composed of PPy electrodes.
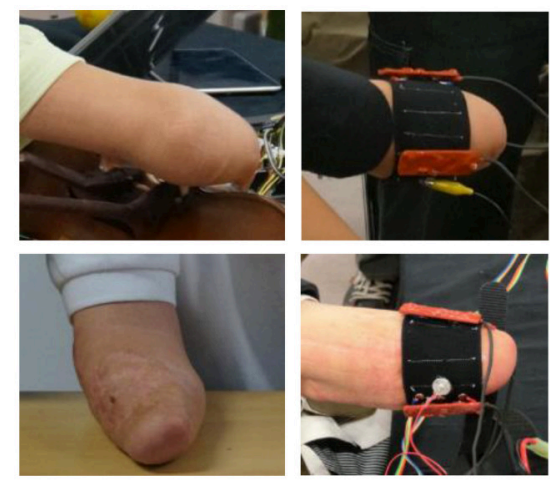

FIGURE 4 | Controlling prosthetic hand with the proposed PPy electrodes.

compare the accuracy of sEMG signal pattern recognition for myoelectric control.

\section{Analysis of Signal Correlation}

To investigate the quality of the signals measured by the proposed sensor, we carried out simultaneous measurement of sEMG

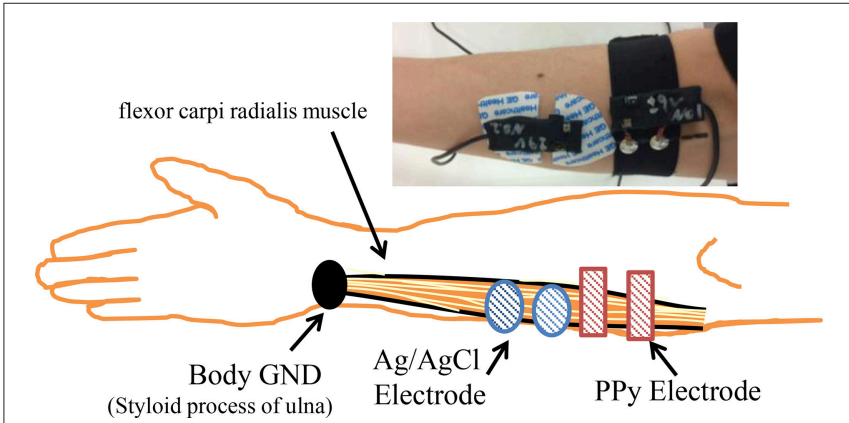

FIGURE 5 | Measurement settings of the electrodes.

with the PPy electrodes and the widely used wet electrodes, the $\mathrm{Ag} / \mathrm{AgCl}$ electrodes with electrolytic gel (GE Healthcare, Japan) from the same muscle fibers. The measurement settings are shown in Figure 5. Four able-bodied subjects participated in the first experiment. Each subject was measured three times. The sampling rate was $1600 \mathrm{~Hz}$ and the quantization bit length was 16. The correlation coefficient of the root mean square (RMS) of the signals measured by the two kinds of electrodes and the FFT results were calculated and compared. 


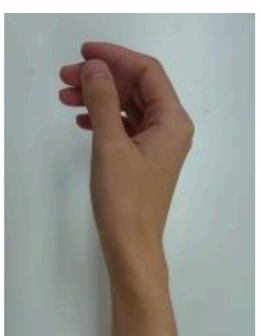

Relax

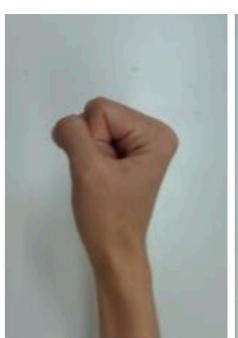

Close

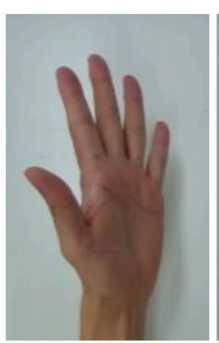

Open

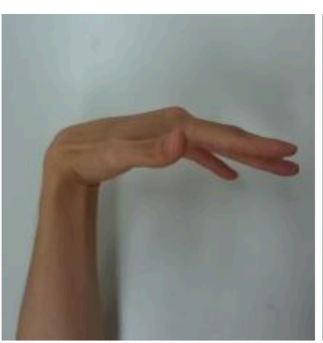

Flexion

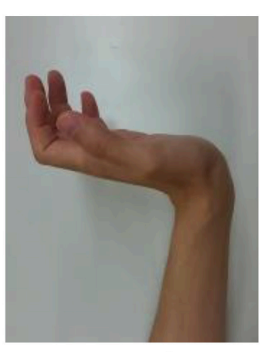

Extension
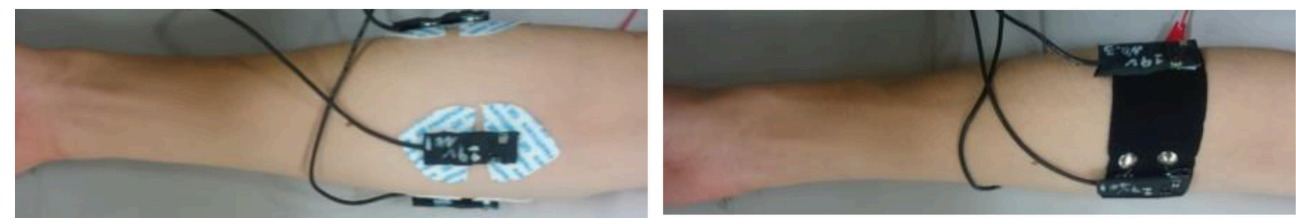

FIGURE 6 | Tasks of the experiment and position of the electrodes.

\section{Pattern Recognition for Myoelectric Control}

To test the practicality of the PPy electrode, a pattern recognition experiment was conducted. In this second experiment, the sEMG signals were used for classification of multiple hand and wrist movements. Figure 6 shows the five basic motions selected for myoelectric control.

The 3-channel sEMG sensor band with the same structure as shown in Figure 3 was worn below the elbow, and the wet $\mathrm{Ag} / \mathrm{AgCl}$ electrodes for comparison were adjusted to the same positions (Figure 6). The basic architecture of the pattern recognition algorithm was developed in the preceding study (Jiang et al., 2014a). The data were sampled at $1600 \mathrm{kHz}$. After A/D conversion, the SMG data were segmented into $128 \mathrm{~ms}$ epochs every $10 \mathrm{~ms}$ and FFT was performed on each epoch. The power spectrum was smoothed by the moving average. The powers of frequencies $31,55,78,102,148,195,258$, and 320 $\mathrm{Hz}$ were extracted as features for input into the artificial neural network (ANN). The ANN is a 3-layer feed-forward neural network with 24 nodes in the input layer, 32 nodes in the hidden layer, and 5 nodes in the output layer.

Six able-bodied subjects participated in the experiment. The ANN was trained beforehand for each subject with a back propagation algorithm. The subjects then performed each of the five tasks for $2 \mathrm{~s}$, during which the sEMG was classified 200 times. Each task was performed three times by each subject. The ratio of correct recognition to total recognition was used as the metric to compare the effectiveness of the electrodes.

\section{Results}

Examples of the RMS of the signals are shown in Figure 7. Although the amplitude of the signals measured by the wet $\mathrm{Ag} / \mathrm{AgCl}$ electrodes was slightly larger, the wave shape of the signals is quite similar, with respect to the PPy electrodes. The correlation coefficients of the 12 tests are listed in Table 1. Individual differences were significant, with Subjects 1 and 2

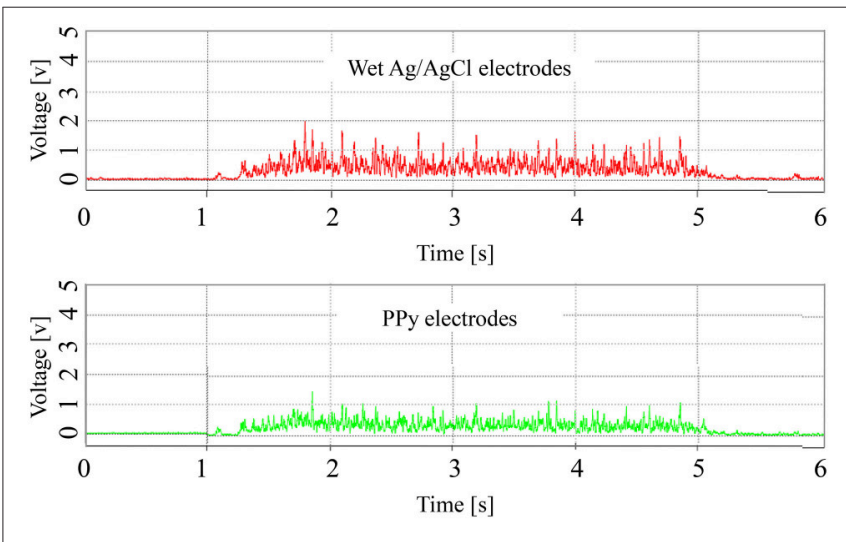

FIGURE 7 | Representative RMS of sEMG signals.

being lower and Subjects 3 and 4 being higher, but the average correlation coefficients of the 4 subjects were all above 0.82 . The overall average correlation coefficient of the 4 subjects was 0.87 $(S D=0.065, n=12)$. Examples of the FFT results are shown in Figure 8. The power spectra of the signals measured with the two kinds of electrodes were very similar in the low-frequency area under $150 \mathrm{~Hz}$. In the high-frequency area over $150 \mathrm{~Hz}$, the power of the signals measured with the PPy electrodes was higher than that with the wet $\mathrm{Ag} / \mathrm{AgCl}$ electrodes.

The results of the pattern recognition are listed in Table 2. Of these tasks, Relax was recognized most accurately at nearly $100 \%$. The other four tasks were recognized with similar accuracies around $85 \%$. The recognition accuracies of each subject were different but consistent, with both sets of accuracies with the different electrodes being relatively lower or higher for each subject. Although the recognition accuracy was quite different according to the subject and the task, no consistent difference was observed in the accuracy with respect to the electrode types. The mean recognition accuracies for each subject showed little 
TABLE 1 | Correlation coefficients of the 12 tests.

\begin{tabular}{|c|c|c|c|c|c|c|c|c|c|c|c|}
\hline \multicolumn{3}{|c|}{ Subject 1} & \multicolumn{3}{|c|}{ Subject 2} & \multicolumn{3}{|c|}{ Subject 3} & \multicolumn{3}{|c|}{ Subject 4} \\
\hline 0.82 & 0.83 & 0.87 & 0.76 & 0.87 & 0.83 & 0.83 & 0.85 & 0.88 & 0.97 & 0.96 & 0.96 \\
\hline
\end{tabular}

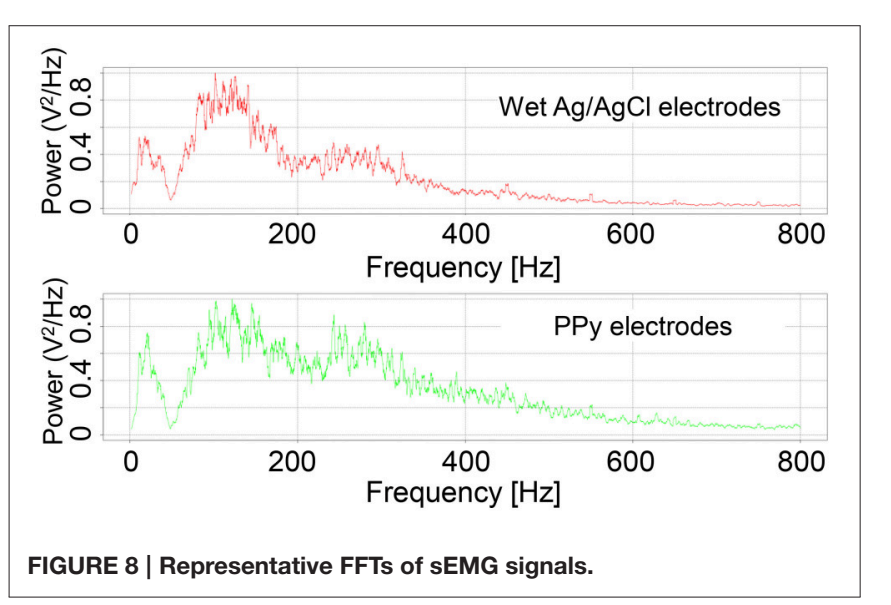

difference between the types. The accuracy difference was due much more to the subject difference than to the electrode types.

\section{Discussion}

PPy nonwoven sheet was adopted as the material for constructing sEMG electrodes that address the problem of customization to improve the usability and practicality of myoelectric control. The size and shape of the sheet are easily adjusted to amputees whose stumps have different residual muscles. The PPy electrodes were compared with the widely used wet $\mathrm{Ag} / \mathrm{AgCl}$ electrodes to investigate the signal quality and to confirm their feasibility for myoelectric control of multi-DoF prosthetic hands. The simultaneous measurements with the two types of electrodes suggest that signal quality was quite similar and the difference for myoelectric control was negligible. The displacement of electrodes and electrode material were two considerable reasons for the difference in the correlation coefficient. The displacements in the longitudinal direction with respect to the muscle fibers may affect the EMG characteristics (Young et al., 2012). It was hard to place the electrodes onto the skin strictly along the muscle fiber. Interface capacitance existed between skin and the dry PPy electrodes without utilize an electrolyte, as in the gel of the wet $\mathrm{Ag} / \mathrm{AgCl}$ electrodes. The different electrical property of the electrode materials might contribute to the difference in the sEMG signals.

From the results of the general FFT and the conventional ANN with a simple structure implementable on a microprocessor, the average recognition accuracy of the PPy electrodes for 5 classes of movement was nearly 90\%, which was comparable to the average accuracy of the wet $\mathrm{Ag} / \mathrm{AgCl}$ electrodes. These experimental results show that the proposed PPy electrodes, which are highly usable and customizable, are as effective as the wet electrodes for myoelectric control. Besides the conditions of the subject, the performance of EMG based motion classification mainly depends on measurement, feature extraction, and classification. Many methods have been reported for the analysis and classification of sEMG signals (Chowdhury et al., 2013). The methods, with their advantages and disadvantages, lead to different classification results which might further elucidate the characteristics of the measurement method. We adopted the general FFT and the conventional ANN in this study to show the performance of the PPy electrodes for the practical control of prosthetic hand. Future work will include other methods to further investigate the characteristics of the proposed PPy electrodes.

\section{RELATION BETWEEN SEMG QUALITY AND SOURCE IMPEDANCE}

The electrodes act as a transducer by converting ionic current within the tissue to electrical current to the amplifier. The source impedance, including the tissue impedance, the skinelectrode impedance, and the electrode impedance, is one of the most important factors affecting the $\mathrm{S} / \mathrm{N}$ ratio. The mismatch of source impedance decreases the common mode rejection ratio (CMRR), which degrades the $\mathrm{S} / \mathrm{N}$ ratio of the signal (Degen and Jackel, 2008). The proposed PPy electrodes can be easily cut into different shape and size with different conductivity and skin-electrode impedance. The balance of source impedances may be a criterion in preparation of the PPy electrodes. An experiment was therefore conducted to investigate the relation between sEMG quality and source impedance.

\section{Analysis of Signal Correlation}

The experimental system for simultaneous measurement of the sEMG and the source impedance is shown in Figure 9. Sources 1 and 2 are the origins of EMG signals. $Z_{1}$ and $Z_{2}$ indicate the combination of tissue impedance, skin-electrode impedance, and electrode impedance. $R_{1}$ and $R_{2}$ are variable resistances $(0-200 \mathrm{k} \Omega$ ) to enable manual adjustment of the source impedances, since the source impedances to the differential amplifier include variable resistances. By manually adjusting $\mathrm{R}_{1}$ and $R_{2}$ to increase the imbalance of the source impedances, the CMRR declines, thus causing a decrease in the $\mathrm{S} / \mathrm{N}$ ratio. The photo at the top right of Figure 9 shows the variable resistances installed before the amplification circuit. An LCR meter (ZM 2371, NF Corporation, Japan) was used to measure the magnitude and phase angle of the source impedances at $300 \mathrm{~Hz}$ and 1 Vrms. Switches 1 and 2, controlled by Arduino Uno (Arduino, Italy), switch the measurement of source impedance 1 and source impedance 2 with the LCR meter. It is important to note that the source impedance exhibits 
TABLE 2 | Results of pattern recognition.

\begin{tabular}{|c|c|c|c|c|c|c|c|c|c|c|c|c|c|c|}
\hline \multirow[t]{2}{*}{ Task } & \multicolumn{2}{|c|}{ Subject A } & \multicolumn{2}{|c|}{ Subject B } & \multicolumn{2}{|c|}{ Subject C } & \multicolumn{2}{|c|}{ Subject D } & \multicolumn{2}{|c|}{ Subject E } & \multicolumn{2}{|c|}{ Subject F } & \multicolumn{2}{|c|}{ Mean } \\
\hline & Wet* $^{\star}$ & $\mathbf{P P y}^{\star}$ & Wet & PPy & Wet & PPy & Wet & PPy & Wet & PPy & Wet & PPy & Wet & PPy \\
\hline Relax & 100 & 100 & 99.3 & 100 & 99.5 & 100 & 99.7 & 99.8 & 100 & 99.2 & 99.8 & 100 & 99.7 & 99.8 \\
\hline Close & 87.2 & 89.2 & 86.7 & 82.5 & 98.2 & 90.8 & 86.3 & 85.7 & 88.8 & 90.2 & 89.2 & 87.2 & 89.4 & 87.6 \\
\hline Open & 78.3 & 93.5 & 87.7 & 83.5 & 82.8 & 83.2 & 82.1 & 84.5 & 86.2 & 87.9 & 87.5 & 74.3 & 84.1 & 84.5 \\
\hline Flexion & 87.8 & 74.7 & 90.0 & 86.5 & 93.7 & 96.5 & 85.4 & 87.2 & 93.5 & 86.7 & 77.7 & 82.8 & 88.0 & 85.7 \\
\hline Extension & 89.7 & 91.5 & 76.8 & 69.2 & 83.2 & 89.7 & 79.2 & 78.8 & 85.7 & 89.4 & 85.5 & 88.3 & 83.3 & 84.5 \\
\hline Mean & 88.6 & 89.8 & 88.1 & 84.3 & 91.5 & 92.0 & 86.5 & 87.2 & 90.8 & 90.7 & 87.9 & 86.5 & 88.9 & 88.4 \\
\hline
\end{tabular}

*wet, wet Ag/AgCl electrodes; PPy, PPy electrodes.

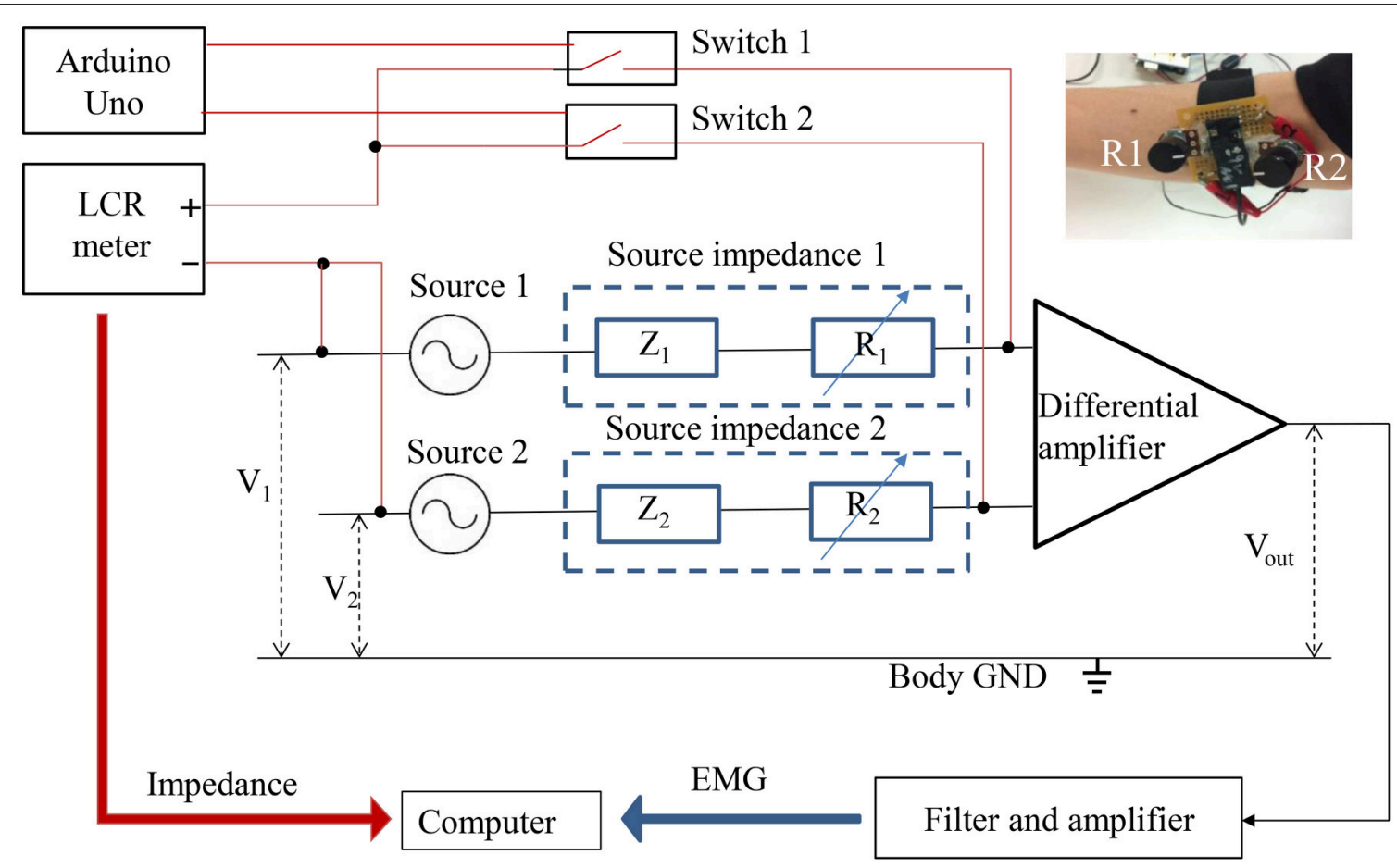

FIGURE 9 | Simultaneous measurements of EMG and impedance. The red line indicates the impedance measurement, and the black line indicates the EMG measurement.

a number of amplitude- and frequency-dependent nonlinear effects (Dorgan and Reilly, 1999). The source impedances at $300 \mathrm{~Hz}$ and $1 \mathrm{Vrms}$ were measured as representative values, since measurements at multiple frequencies would cost time and decrease the simultaneity between impedances and the sEMG. During the impedance measurement, the 1 Vrms input signal was also amplified, which caused amplifier saturation. The saturation started with the start and ended with the end of the input signal. The output of the differential amplifier during the impedance measurement was therefore excluded from the sEMG analysis.

The sEMG of an able-bodied subject doing flexion was measured by placing the PPy electrodes above the digitorum profundus muscle. To investigate the relation between the source impedance balance and the $\mathrm{S} / \mathrm{N}$ ratio, we adjusted the noise level by tuning $R_{1}$ and $R_{2}$ during the rest state, and then measured the sEMG and the source impedances at the noise level. The three noise levels are illustrated in Figure 10. Levels 1,2 , and 3 show little noise, noise in the range of $(-6 \mathrm{v}$, $6 \mathrm{v})$, and noise in the range of $(-9 \mathrm{v}, 9 \mathrm{v})$, respectively. At each level, the sEMG and source impedances were measured ten times. The differences of the magnitude and phase angle were used as an index of source impedance imbalance, and the FFT of the sEMG signals were calculated to analyze the noise.

\section{Results}

Table 3 shows the source impedance imbalance at the three noise levels. Both the magnitude differences and the phase angle differences are significant. At noise level 1, the imbalance of 
magnitude is around $1500 \Omega$ and the phase angles are nearly the same. To increase the noise to levels 2 and 3, the imbalance was increased in magnitude to around 8000 and 20,000 $\Omega$, and the phase angle was increased to around $10 \mathrm{deg}$ and 30 deg, respectively. Accordingly, the SD of the imbalances in the magnitude and phase angle also increased with the increase of noise level.

The power spectra of the measured sEMG signals at different noise levels are shown in Figure 11. The noise mainly came from the power line radiation $(50 \mathrm{~Hz})$ and its harmonics, and the measurement frequency $(300 \mathrm{~Hz})$ of the LCR meter. At noise level 1 , the noise was mainly caused by the LCR meter at $300 \mathrm{~Hz}$. With the decline of the CMRR due to the imbalance of source impedances, more noise was mixed in with the sEMG signals. Especially at the frequencies of 100,200 , and $300 \mathrm{~Hz}$, the power of the noise was much higher than that of the sEMG signal.

\section{Discussion}

An important factor of the dry electrodes is their ability to maintain electrical contact with skin, since the electrical contact affects the electrode-skin impedance and the source impedance balance. The dry electrodes do not utilize an electrolyte, as in the gel of the wet $\mathrm{Ag} / \mathrm{AgCl}$ electrodes. High interface capacitance therefore exists between the skin and the electrode, so that the source impedances are larger when using dry electrodes than those when using wet electrodes. A comparison study (Searle and

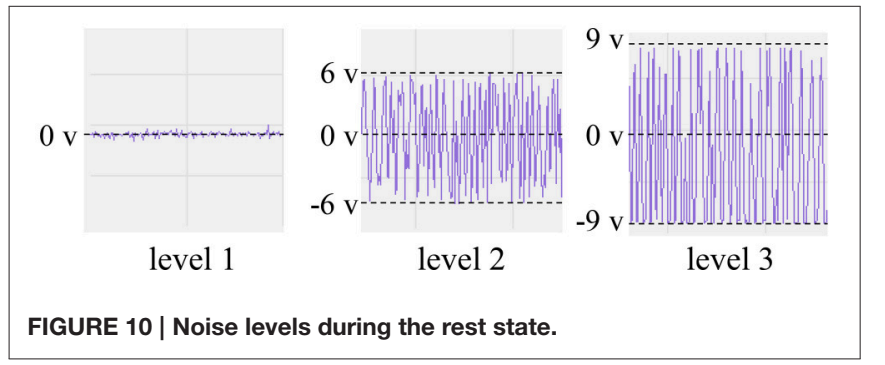

Kirkup, 2000) investigated the contact impedances, the resistance to electrical interference, and the movement artifacts of the dry and wet electrodes. However, the relation between contact impedances and $\mathrm{S} / \mathrm{N}$ ratio was not quantitatively measured.

In this study, the simultaneous measurement of the source impedance balance and the sEMG by using the switching circuit suggest that the imbalances in the magnitude and phase angle of the source impedances were the reason for the increase of noise level. By adjusting the variable resistances to unbalance the source impedances, the CMRR of the differential amplifier declines, and thus the common mode noises, caused by harmonics of the power line radiation and the measurement signal, were also amplified. Conversely, if the source impedances are balanced, the $\mathrm{S} / \mathrm{N}$ ratio can be improved. Source impedances, rather than the shape and the size of the electrodes, are important for sEMG measurement. Therefore, two electrodes connected to the same amplifier do not have to have the same shape and size as long as the source impedances are close. The proposed flexible PPy electrodes provide high freedom of shape and size adjustment to fit a specific amputee.

\section{GENERAL DISCUSSION}

We developed a novel PPy electrode for the convenient measurement of sEMG signals. The conductive nonwoven fabric sheet is easily cut and sewn to fit an individual. Currently, most amputees need to be checked as to whether they are suitable to use a myoelectric prosthetic hand at hospitals or other medical institutions. Before amputees get myoelectric prosthetic hands, which are custom-made according to the prescription by the prosthesis therapist, they are required to participate in training up to 2 months in order to generate a stable sEMG signal. This process decreases the accessibility of myoelectric prosthetic hands, especially for those who are poor and living in undeveloped areas. The goals of our current study and our preliminary studies (Jiang et al., 2014a, 2015) are to

TABLE 3 | Magnitude and phase angel differences at the three noise levels (Impedances were measured at $300 \mathrm{~Hz}$ and $1 \mathrm{Vrms}$ ).

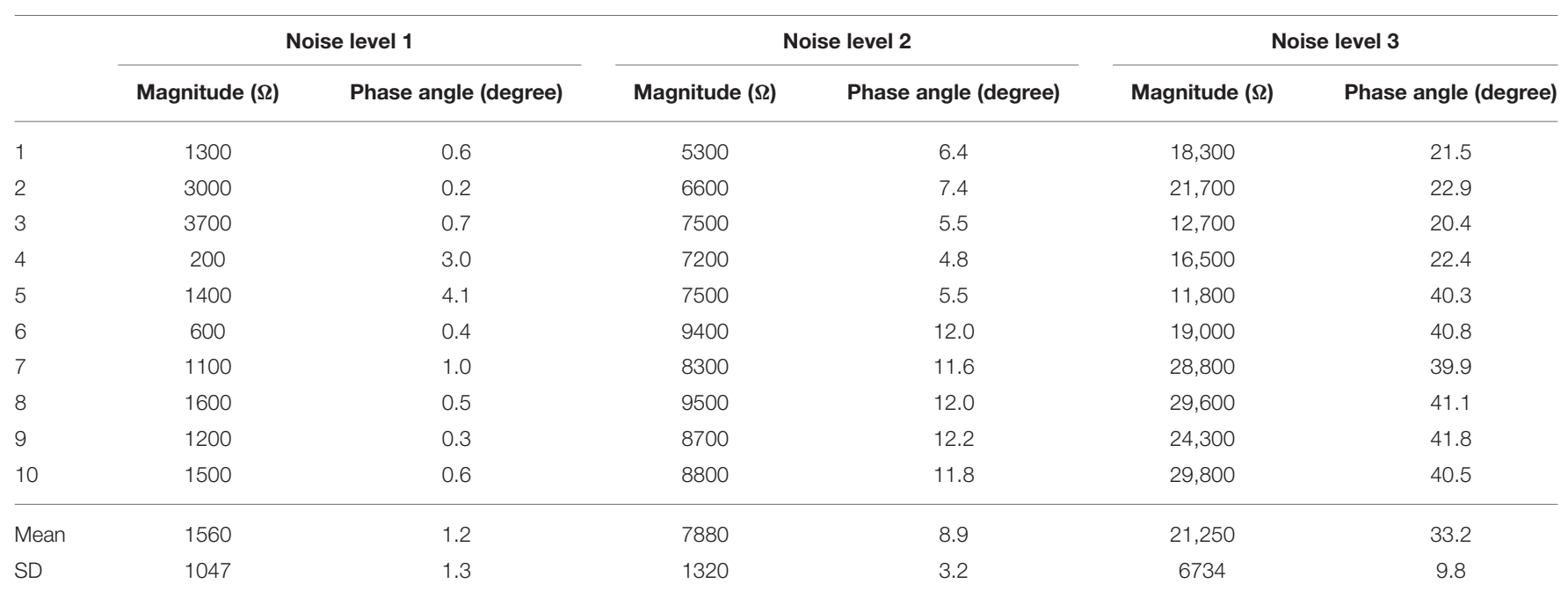




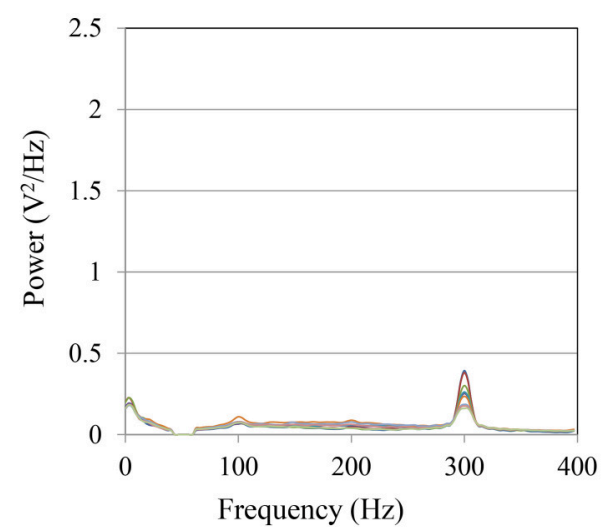

noise level 1

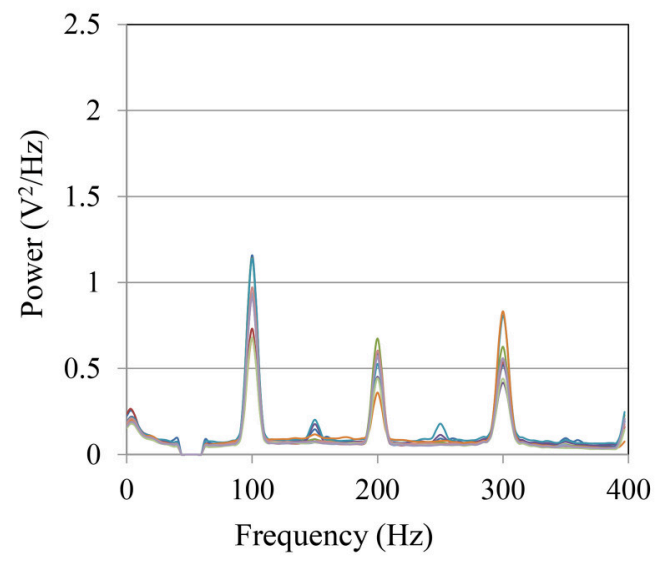

noise level 2

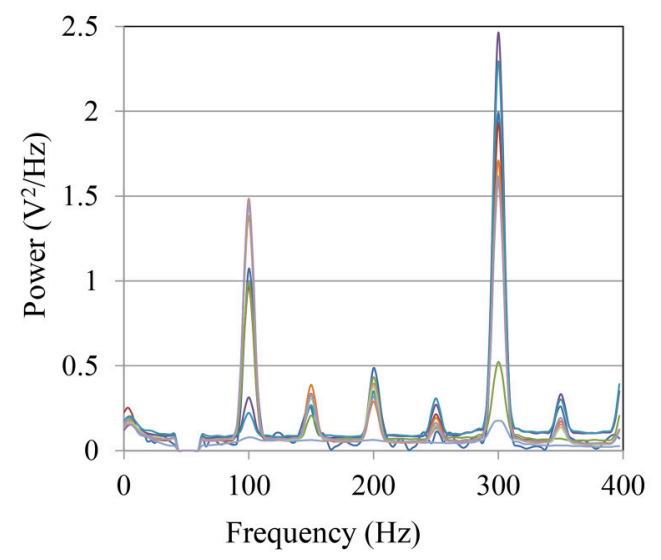

noise level 3

FIGURE 11 | Power spectra of sEMG signals at noise levels 1, 2, and 3.

develop a low-cost and highly usable myoelectric prosthetic hand system in order to increase its accessibility, both economically and technically. In order to provide the technology and the opportunities necessary for people with disabilities to maximize their quality of life by using prosthetic hands, the whole system including the sEMG measurement should be conveniently adaptable to any person, since each person represents different conditions. Usability is one of the main factors that affects prosthesis acceptance or rejection (Biddiss and Chau, 2007; Vasluian et al., 2013). The trial use experiment illustrated that the PPy electrodes provide a convenient and customizable measurement method for amputees. The comparison with wet $\mathrm{Ag} / \mathrm{AgCl}$ electrodes suggest that the PPy electrodes are usable and capable of recording sEMG signals for myoelectric control.

To address this challenge of sEMG measurement, many kinds of electrode construction using pure or mixed materials such as stainless steel, $\mathrm{Si} 3 \mathrm{~N} 4, \mathrm{Ag} / \mathrm{AgCl}$, and $\mathrm{Au}$ have been proposed (Searle and Kirkup, 2000). Recently, conductive polymers, especially PEDOT-PSS, have been widely studied as promising bioelectrode materials (Richardson-Burns et al., 2007; Lang et al., 2009; Tsukada et al., 2012; Isik et al., 2015; Nagamine et al., 2016; Pani et al., 2016; Papaiordanidou et al., 2016). PEDOT-PSS has highly electroconductive and biocompatible characteristics that meet the requirements for biomedical electrode materials (Takamatsu et al., 2015; Pani et al., 2016). However, because it is more water-soluble and hygroscopic, PEDOT-PSS exfoliates from the substrates and absorbs water in wet conditions, which will reduce its mechanical strength and electrical characteristics (Elschner et al., 2010). Although the conductivity is lower, polypyrrole is more stable than PEDOT-PSS in the sEMG measurement environment, in which the electrode may become wet with sweat. The low conductivity can be compensated to some extent by the use of active electrodes (where buffering and amplification are performed at the electrode site) and high-quality amplifiers. The experimental comparison with wet $\mathrm{Ag} / \mathrm{AgCl}$ electrodes suggests that although the conductivity of the PPy electrodes is much less than that of the wet $\mathrm{Ag} / \mathrm{AgCl}$ electrodes, the signal quality was quite similar and negligible for myoelectric control.

One major cause of the noise in the sEMG is the common mode interference that is mixed in the signal when the CMRR declines due to the skin-electrode impedance change (Yokus and Jur, 2016). The switched simultaneous measurements of the sEMG and source impedances suggest the importance of source impedance balance for signal quality. Theoretically, any conductive material has the potential to become an electrode if the amplifier is ideal, with infinitely great input impedance. Since such amplifiers do not exist, most of the studies focus on the electrical property in order to develop electrodes with skin-electrode impedance that is as low as possible (Hahne et al., 2016). However, for a practical use such as myoelectric control, a tradeoff exists between reducing the contact impedance and improving the usability and customizability. The electromechanical stability of the contact between sEMG electrodes and skin directly affects the quality of the signal (Roy et al., 2007), but the stability is hard to guarantee when using metallic electrodes to control a prosthetic hand in a real-life environment, since impedance changes can be induced by motion (Cömert and Hyttinen, 2014). 
With the improvement in electronic devices, more flexible conductive materials with relatively low conductivity can be alternatives to developing practical surface bioelectric electrodes. The simultaneous measurements of the source impedance balance and the sEMG also show the possibility of improving the $\mathrm{S} / \mathrm{N}$ ratio by balancing the source impedances. If the source impedances can be monitored and adjusted with variable resistances to decrease the impedance differences, the CMRR of the differential amplifier could be improved to exclude the common mode interferences.

In preparation of the PPy electrodes, source impedance and interelectrode distance (Young et al., 2012) are two important factors to determine their shape and size. Desirable characteristics of electrode-skin impedance are a low value of the impedance and a balance of values between the electrodes. Small electrodes with less contact area may lead to high electrodeskin impedance and the difference of electrode-skin impedance may cause source impedance unbalance. High density electrodes are often adopted in scientific studies to obtain fine EMG characteristics or to improve the robustness by redundancy. However, in practical control of prosthetic hands, most of which have $<5$ DoFs currently (Belter et al., 2013), three effective channels of sEMG signal are enough. Another non-negligible factor is the conditions of the amputees (i.e., the number and distribution of residual muscles). The flexible PPy electrodes can be easily cut into the shape and size that fit a specific amputee. Metal electrodes also can be customized into different shapes and sizes. But metallic processing is not as easy as cutting a fabric sheet. And metal electrodes are not flexible to fit closely to the body.

In sum, the PPy electrodes investigated here showed their usability and effectiveness in sEMG recording for myoelectric control of prosthetic hands. Nevertheless, because the trial use by amputees and the experiments were carried out over a short period of time $(<1$ day for the trial use and 1 week for the experiments), the durability needs to be further validated. The stability of conductive polymers including PPy and PEDOT:PSS is different with respect to dopants, synthesis methods, environment, and usage in applications (Yamato et al., 1995; Cui and Martin, 2003; Jang et al., 2004; Khodagholy et al., 2011; Andreoli et al., 2013). To the best of our knowledge, there is no studies comparing PPy and PEDOT:PSS in the form of fabric sheet. Since PPy is stable under wet and humid conditions, the durability is expected to be better than that of electrodes made of PEDOT:PSS. Furthermore, the properties of the nonwoven fabric sheet, including the surface texture and the geometric configuration, need to be further investigated. The performance of the PPy electrodes may be improved by optimizing their properties.

\section{CONCLUSION AND OUTLOOK}

We have proposed a sEMG sensor using PPy electrodes for practical myoelectric control of prosthetic hands in daily life. The flexible electrodes are highly customizable to fit the size and shape of different stumps. The PPy electrodes enable highly usable and effective sEMG measurements in real-life settings. By sewing the electrodes on elastic webbing, it is easy to construct a band that can be put on one-handed to record multiple channel sEMG signals. Experiments comparing PPy electrodes with wet $\mathrm{Ag} / \mathrm{AgCl}$ electrodes suggest that the PPy electrodes are as effective as the widely used wet $\mathrm{Ag} / \mathrm{AgCl}$ electrodes for myoelectric control of prosthetic hands. The simultaneous measurements of source impedances and sEMG suggest that the source impedance imbalance is a main reason for the low $\mathrm{S} / \mathrm{N}$ ratio, and so the $\mathrm{S} / \mathrm{N}$ ratio may be improved by balancing the source impedances. We will continue to investigate the durability of the sEMG sensor using PPy electrodes and to improve it by optimizing the properties of the fabric sheet. Long-term home trial use together with prosthetic hands by amputees is under planning. PPy electrodes with different surface textures and geometric configurations will also be tested in the near future.

\section{AUTHOR CONTRIBUTIONS}

YJ, MT, BL, and HY conceived the study concept. YJ, MT, and HY developed the electrode with the polypyrrole-coated nonwoven fabric sheet. YJ and MT designed and executed the experiments. YJ, MT, BL, and $\mathrm{HY}$ interpreted the experimental data. YJ drafted the manuscript. MT, BL, and HY revised the manuscript.

\section{ACKNOWLEDGMENTS}

This research was partially supported by JSPS KAKENHI Grant Numbers JP16K01539, JP16K12951, and JP16K12503. It was also supported by the "Brain Machine Interface Development" and the "Adaptable and Seamless Technology Transfer Program through Target-driven R\&D” by the Japan Agency for Medical Research and Development (AMED). The authors gratefully acknowledge the support by Japan Viline for providing us with polypyrrole-coated nonwoven fabric sheet. Also, we appreciate the assistance of S. Sakoda in setting up the experimental system and S. Morishita in data analysis.

\section{REFERENCES}

Al-Timemy, A. H., Bugmann, G., Escudero, J., and Outram, N. (2013). Classification of finger movements for the dexterous hand prosthesis control with surface electromyography. IEEE J. Biomed. Health Inform. 17, 608-618. doi: 10.1109/JBHI.2013.22 49590

Amsüss, S., Goebel, P. M., Jiang, N., Graimann, B., Paredes, L., and Farina, D. (2014). Self-correcting pattern recognition system of surface EMG signals for upper limb prosthesis control. IEEE Trans. Biomed. Eng. 61, 1167-1176. doi: 10.1109/TBME.2013.2296274

Andreoli, E., Liao, K.-S., Haldar, A., Alley, N. J., and Curran, S. A. (2013). PPy:PSS as alternative to PEDOT:PSS in organic photovoltaics. Synthetic Metals 185-186, 71-78. doi: 10.1016/j.synthmet.2013.10.005 
Asghari Oskoei, M., and Hu, H. (2007). Myoelectric control systems-A survey. Biomed. Signal Process. Control 2, 275-294. doi: 10.1016/j.bspc.2007.07.009

Beck, T. W., Stock, M. S., and Defreitas, J. M. (2014). Shifts in EMG spectral power during fatiguing dynamic contractions. Muscle Nerve 50, 95-102. doi: $10.1002 /$ mus. 24098

Belter, J. T., Segi, J. L., Dollar, A. M., and Weir, R. F. (2013). Mechanical design and performance specifications of anthropomorphic prosthetic hands: a review. J. Rehabil. Res. Dev. 50, 599-618. doi: 10.1682/JRRD.2011.10.0188

Biddiss, E. A., and Chau, T. T. (2007). Upper limb prosthesis use and abandonment: a survey of the last 25 years. Prosthet. Orthot. Int. 31, 236-257. doi: 10.1080/0309364060099458

Chowdhury, R. H., Reaz, M. B. I., Ali, M. A. B. M., Bakar, A. A. A., Chellappan, K., and Chang, T. G. (2013). Surface electromyography signal processing and classification techniques. Sensors 13, 12431-12466. doi: 10.3390/s130912431

Cömert, A., and Hyttinen, J. (2014). Impedance spectroscopy of changes in skin-electrode impedance induced by motion. Biomed. Eng. Online 13, 1-19. doi: 10.1186/1475-925X-13-149

Cui, X., and Martin, D. C. (2003). Electrochemical deposition and characterization of poly(3,4-ethylenedioxythiophene) on neural microelectrode arrays. Sens. Actuators B 89, 92-102. doi: 10.1016/S0925-4005(02)00448-3

Degen, T., and Jackel, H. (2008). Continuous monitoring of electrode: skin impedance mismatch during bioelectric recordings. IEEE Trans. Biomed. Eng. 55, 1711-1715. doi: 10.1109/TBME.2008.919118

Dorgan, S. J., and Reilly, R. B. (1999). A model for human skin impedance during surface functional neuromuscular stimulation. IEEE Trans. Rehabil. Eng. 7, 341-348. doi: 10.1109/86.788470

Dosen, S., Cipriani, C., Kostic, M., Controzzi, M., Carrozza, M. C., and Popovic, D. B. (2010). Cognitive vision system for control of dexterous prosthetic hands: experimental evaluation. J. Neuroeng. Rehabil. 7:42. doi: 10.1186/1743-0003-7-42

Earley, E. J., Hargrove, L. J., and Kuiken, T. A. (2016). Dual window pattern recognition classifier for improved partial-hand prosthesis control. Front. Neurosci. 10:58. doi: 10.3389/fnins.2016.00058

Elschner, A., Kirchmeyer, S., Lovenich, W., Merker, U., and Reuter, K. (2010). PEDOT: Principles and Applications of an Intrinsically Conductive Polymer. Boca Raton, FL: CRC Press.

Fang, Y., Hettiarachchi, N., Zhou, D., and Liu, H. (2015). Multi-modal sensing techniques for interfacing hand prostheses: a review. IEEE Sens. J. 15, 6065-6076. doi: 10.1109/JSEN.2015.2450211

Hahne, J. M., Biessmann, F., Jiang, N., Rehbaum, H., Farina, D., Meinecke, F. C., et al. (2014). Linear and nonlinear regression techniques for simultaneous and proportional myoelectric control. IEEE Trans. Neural Syst. Rehabil. Eng. 22, 269-279. doi: 10.1109/TNSRE.2014.2305520

Hahne, J. M., Farina, D., Jiang, N., and Liebetanz, D. (2016). A novel percutaneous electrode implant for improving robustness in advanced myoelectric control. Front. Neurosci. 10:114. doi: 10.3389/fnins.2016.00114

Isik, M., Lonjaret, T., Sardon, H., Marcilla, R., Herve, T., Malliaras, G. G., et al. (2015). Cholinium-based ion gels as solid electrolytes for longterm cutaneous electrophysiology. J. Materials Chem. C 3, 8942-8948. doi: 10.1039/C5TC01888A

Jang, K. S., Lee, H., and Moon, B. (2004). Synthesis and characterization of water soluble polypyrrole doped with functional dopants. Synth. Met. 143, 289-294. doi: 10.1016/j.synthmet.2003.12.013

Jiang, N., Vujaklija, I., Rehbaum, H., Graimann, B., and Farina, D. (2014b). Is accurate mapping of EMG Signals on kinematics needed for precise online myoelectric control? IEEE Trans. Neural Syst. Rehabil. Eng. 22, 549-558. doi: 10.1109/TNSRE.2013.2287383

Jiang, Y., Sakoda, S., Hoshigawa, S., Ye, H., Yabuki, Y., Nakamura, T., et al. (2014a). "Development and evaluation of simplified EMG prosthetic hands," in Proceedings of the 2014 IEEE International Conference on Robotics and Biomimetics (Bali).

Jiang, Y., Sakoda, S., Togane, M., Morishita, S., Lu, B., and Yokoi, H. (2015). "A highly usable and customizable sEMG sensor for prosthetic limb control using polypyrrole-coated nonwoven fabric sheet," in Proceedings of the IEEE SENSORS 2015 (Busan).

Kato, R., Yokoi, H., Hernandez Arieta, A., Yu, W., and Arai, T. (2009). Mutual adaptation among man and machine by using f-MRI analysis. Rob. Auton. Syst. 57, 161-166. doi: 10.1016/j.robot.2008.07.005
Khodagholy, D., Doublet, T., Gurfinkel, M., Quilichini, P., Ismailova, E., Leleux, P., et al. (2011). Highly conformable conducting polymer electrodes for in vivo recordings. Adv. Mater. 23, H268-H272. doi: 10.1002/adma.201102378

Kim, M., Kim, T., Kim, D. S., and Chung, W. K. (2015). Curved microneedle arraybased sEMG electrode for robust long-term measurements and high selectivity. Sensors 15, 16265-16280. doi: 10.3390/s150716265

Lang, U., Naujoks, N., and Dual, J. (2009). Mechanical characterization of PEDOT:PSS thin films. Synth. Met. 159, 473-479. doi: 10.1016/j.synthmet.2008.11.005

Lee, C. Y., Lee, D. E., Joo, J., Kim, M. S., Lee, J. Y., Jeong, S. H., et al. (2001). Conductivity and EMI shielding efficiency of polypyrrole and metal compounds coated on (non) woven fabrics. Synthetic Metals (Basel) 119, 429-430. doi: 10.1016/S0379-6779(00)01280-7

Merrill, D. R., Lockhart, J., Troyk, P. R., Weir, R. F., and Hankin, D. L. (2011). Development of an implantable myoelectric sensor for advanced prosthesis control. Artif. Organs 35, 249-252. doi: 10.1111/j.1525-1594.2011.01219.x

Morishita, S., Sato, K., Watanabe, H., Nishimura, Y., Isa, T., Kato, R., et al. (2014). Brain-machine interface to control a prosthetic arm with monkey ECoGs during periodic movements. Front. Neurosci. 8:417. doi: 10.3389/fnins.2014.00417

Nagamine, K., Chihara, S., Kai, H., Kaji, H., and Nishizawa, M. (2016). Totally shape-conformable electrode/hydrogel composite for on-skin electrophysiological measurements. Sens. Actuators B 237, 49-53. doi: $10.1016 / j . s n b .2016 .06 .076$

Pan, L., Zhang, D., Jiang, N., Sheng, X., and Zhu, X. (2015). Improving robustness against electrode shift of high density EMG for myoelectric control through common spatial patterns. J. Neuroeng. Rehabil. 12:110. doi: 10.1186/s12984-015-0102-9

Pani, D., Dessi, A., Saenz-Cogollo, J. F., Barabino, G., Fraboni, B., and Bonfiglio, A. (2016). Fully textile, PEDOT:PSS based electrodes for wearable ECG monitoring systems. IEEE Trans. Biomed. Eng. 6, 540-549. doi: 10.1109/TBME.2015.2465936

Papaiordanidou, M., Takamatsu, S., Rezaei-Mazinani, S., Lonjaret, T., Martin, A., and Ismailova, E. (2016). Cutaneous recording and stimulation of muscles using organic electronic textiles. Adv. Healthc. Mater. 5, 2001-2006. doi: 10.1002/adhm.201600299

Popovic-Maneski, L., Kostic, M., Bijelic, G., Keller, T., Mitrovic, S., Konstantinovic, L., et al. (2013). Multi-pad electrode for effective grasping: design. IEEE Trans. Neural Syst Rehabil. Eng. 21, 648-654. doi: 10.1109/TNSRE.2013.22 39662

Richardson-Burns, S. M., Hendricks, J. L., Foster, B., Povlich, L. K., Kim, D. H., and Martin, D. C. (2007). Polymerization of the conducting polymer poly(3,4ethylenedioxythio-phene) (PEDOT) around living neural cells. Biomaterials 28 1539-1552. doi: 10.1016/j.biomaterials.2006.11.026

Roy, S. H., De Luca, G., Cheng, M. S., Johansson, A., Gilmore, L. D., and De Luca, C. J. (2007). Electro-mechanical stability of surface EMG sensors. Med. Biol. Eng. Comput. 45, 447-457. doi: 10.1007/s11517-007-0168-z

Searle, A., and Kirkup, L. (2000). A direct comparison of wet, dry and insulating bioelectric recording electrodes. Physiol. Meas. 21, 271-283. doi: 10.1088/0967-3334/21/2/307

Seki, T., Nakamura, T., Kato, R., Morishita, S., and Yokoi, H. (2013). "Development of five-finger multi-DoF myoelectric hands with a power allocation mechanism," in Proceedings of 2013 IEEE International Conference on Robotics and Automation (Karlsruhe).

Smith, L. H., Kuiken, T. A., and Hargrove, L. J. (2016). Evaluation of linear regression simultaneous myoelectric control using intramuscular emg. IEEE Trans. Biomed. Eng. 63, 737-746. doi: 10.1109/TBME.2015.2469741

Takamatsu, S., Lonjaret, T., Crisp, D., Badier, J. M., Malliaras, G. G., and Ismailova, E. (2015). Direct patterning of organic conductors on knitted textiles for long-term electrocardiography. Sci. Rep. 5:15003. doi: 10.1038/srep15003

Tsukada, S., Nakashima, H., and Torimitsu, K. (2012). Conductive Polymer Combined Silk Fiber Bundle for Bioelectrical Signal Recording. PLoS ONE, 7:e33689. doi: 10.1371/journal.pone.0033689

Vasluian, E., de Jong, I. G. M., Janssen, W. G. M., Poelma, M. J., van Wijk, I., Reinders-Messelink, H. A., et al. (2013). Opinions of youngsters with congenital below-elbow deficiency, and those of their parents and professionals concerning prosthetic use and rehabilitation treatment. PLOS ONE 8:e67101. doi: 10.1371/journal.pone.006710 
Xu, H., Konovalov, V. V., Contescu, C. I., Jaffe, S. M., and Madou, M. (2006). Water transport in a non-aqueous, polypyrrole electrochemical cell. Sens. Actuators $B$ 114, 248-253. doi: 10.1016/j.snb.2005. 04.039

Yamato, H., Ohwa, M., and Wernet, W. (1995). Stability of polypyrrole and poly(3,4-ethylenedioxythiophene) for biosensor application. J. Electroanalytical Chem. 397, 163-170. doi: 10.1016/0022-0728(95) 04156-8

Yokus, M. A., and Jur, J. S. (2016). Fabric-based wearable dry electrodes for body surface biopotential recording. IEEE Trans. Biomed. Eng. 63, 423-430. doi: 10.1109/TBME.2015.2462312

Young, A. J., Hargrove, L. J., and Kuiken, T. A. (2012). Improving myoelectric pattern recognition robustness to electrode shift by changing interelectrode distance and electrode configuration. IEEE Trans. Biomed. Eng. 59, 645-652. doi: 10.1109/TBME.2011.2177662

Young, A. J., Smith, L. H., Rouse, E. J., and Hargrove, L. J. (2013). Classification of simultaneous movements using surface emg pattern recognition.
IEEE Trans. Biomed. Eng. 60, 1250-1258. doi: 10.1109/TBME.2012.22 32293

Conflict of Interest Statement: The handling Editor declared a shared affiliation, though no other collaboration, with one of the author BL and states that the process nevertheless met the standards of a fair and objective review.

The other authors declare that the research was conducted in the absence of any commercial or financial relationships that could be construed as a potential conflict of interest.

Copyright (c) 2017 Jiang, Togane, Lu and Yokoi. This is an open-access article distributed under the terms of the Creative Commons Attribution License (CC BY). The use, distribution or reproduction in other forums is permitted, provided the original author(s) or licensor are credited and that the original publication in this journal is cited, in accordance with accepted academic practice. No use, distribution or reproduction is permitted which does not comply with these terms. 
OPEN ACCESS

Edited by:

Dingguo Zhang

Shanghai Jiao Tong University, China

Keum-Shik Hong,

University of Illinois at

Urbana-Champaign, USA

Jianjun Meng,

University of Minnesota, USA

*Correspondence:

Kalyana C. Veluvolu

veluvolu@ee.knu.ac.kr

Specialty section: This article was submitted to

Neural Technology, a section of the journal

Frontiers in Neuroscience

Received: 01 September 2016

Accepted: 16 January 2017

Published: 01 February 2017

Citation:

Wang Y and Veluvolu KC (2017) Evolutionary Algorithm Based Feature

Optimization for Multi-Channel EEG

Classification. Front. Neurosci. 11:28.

doi: 10.3389/fnins.2017.00028

\section{Evolutionary Algorithm Based Feature Optimization for Multi-Channel EEG Classification}

\author{
Yubo Wang ${ }^{1,2}$ and Kalyana C. Veluvolu ${ }^{2 *}$ \\ ${ }^{1}$ School of Life Science and Technology, Xidian University, Xi'an, China, ${ }^{2}$ School of Electronics Engineering, College of IT \\ Engineering, Kyungpook National University, Daegu, South Korea
}

The most $\mathrm{BCl}$ systems that rely on EEG signals employ Fourier based methods for time-frequency decomposition for feature extraction. The band-limited multiple Fourier linear combiner is well-suited for such band-limited signals due to its real-time applicability. Despite the improved performance of these techniques in two channel settings, its application in multiple-channel EEG is not straightforward and challenging. As more channels are available, a spatial filter will be required to eliminate the noise and preserve the required useful information. Moreover, multiple-channel EEG also adds the high dimensionality to the frequency feature space. Feature selection will be required to stabilize the performance of the classifier. In this paper, we develop a new method based on Evolutionary Algorithm (EA) to solve these two problems simultaneously. The real-valued EA encodes both the spatial filter estimates and the feature selection into its solution and optimizes it with respect to the classification error. Three Fourier based designs are tested in this paper. Our results show that the combination of Fourier based method with covariance matrix adaptation evolution strategy (CMA-ES) has the best overall performance.

Keywords: $\mathrm{BCl}$, evolutionary algorithm, Fourier linear combiner, feature optimization

\section{INTRODUCTION}

Brain-computer interface (BCI) is defined as an alternative communication pathway which translates the measured brain activity into control commands (Pfurtscheller et al., 2008). Among the existing brain activity measurement techniques, EEG has been extensively employed for $\mathrm{BCI}$ applications due to its non-invasiveness, ease of implementation, and cost-efficiency (Lotte et al., 2007). Recently, the dry-electrodes and wireless EEG systems have also been studied in various experimental settings which has further expanded the scope of EEG based BCI systems (Tangermann et al., 2012; Gao et al., 2014).

The motor imagery has been one of the successful methods for EEG based BCI systems (McFarland et al., 2010; Hill et al., 2014). The application of motor imagery task to BCI systems has enabled subjects to sufficiently control a moving cursor in 2D space or a quadcopter in 3D space (Wolpaw et al., 2004; LaFleur et al., 2013). As shown in Muller-Gerking et al. (1999) and Guger et al. (2003), a contra-lateral amplitude decrease in $\alpha$ band can be found in most subjects during motor imagery tasks. Therefore, the motor imagery based BCI systems require transforming the recorded EEG signal into frequency domain in quasi real-time. Then the extracted frequency domain features are fed to machine learning algorithms for classification (Lotte et al., 2007; Robinson et al., 2013). 
As frequency analysis of EEG is a major concern, many approaches such as the band pass filter bank, autoregressive (AR) model, Fourier transform, and Wavelet transform have been employed (Turnip and Hong, 2012; Wang et al., 2012b; Robinson et al., 2013; Hsu, 2014; Khan et al., 2014). However, all these methods have certain limitations. The frequency resolution of a band-pass filter is constrained by the order of the filter. The AR model coefficients only provide limited frequency information as the envelope of the spectrum reconstructed from AR coefficients is limited by the order of AR model (Shumway and Stoffer, 2011). The discrete wavelet transform is considered as a bandpass filter bank with orthogonal basis that has better frequency and temporal resolution (Wang et al., 2013b). However, the obtained wavelet coefficients are redundant in general. Further, post-processing is required to stabilize the performance of the classifier (Qin and He, 2005).

The EEG signal considered for BCI is generally bandlimited. To decompose such signal into frequency domain and to maintain the balance between temporal and frequency resolution, the band-limited multiple Fourier linear combiner (BMFLC) has been developed (Veluvolu et al., 2012). BMFLC models the signal by adopting a truncated Fourier series. Adaptive filter algorithm is then employed to obtain amplitude estimation of individual frequency components in a pre-fixed frequency band. For application to motor imagery, BMFLC is applied to provide time-frequency mapping of the $\mu$ rhythm. The estimated amplitude of each frequency is then fed to a classifier (Wang et al., 2012a, 2013b). It was found in Wang et al. (2012a) that the performance of the BMFLC based BCI systems is improved if a subject-specific narrow band can be identified within the range of $\mu$ rhythm. The identification criterion and existence of such band have been established in Veluvolu et al. (2012). The reduced dimensionality in the feature vector resulted in better performance of the classifier.

Earlier approaches based on BMFLC only employed EEG signals that were recorded from sensorimotor cortex (i.e., C3 and C4 from international 10/20 system, Jurcak et al., 2007; Wang et al., 2012a, 2013b). Since only two EEG channels were considered, it was straightforward to perform the subject-specific band identification on the averaged time-frequency mapping (Wang et al., 2013b). Although the two channel system is simple in implementation, there is very limited scope for performance improvement. Due to the volume conduction of skull and scalp, the signal generated by the cortex of interest may be distributed to a vicinity of the area on the scalp and picked up by surrounding electrodes with different amplitude (Blankertz et al., 2008). To further enhance the classification performance, more data from EEG channels is often required for more complex problems.

The increase in the number of EEG channels poses two challenges. First, as the number of channel increases, direct identification of the subject-dependent reactive band becomes infeasible and an automated identification process is required. Secondly, as the feature vector includes all frequency estimates from all the channels, the dimension increases drastically. High dimensionality in feature vector will effect the performance of the classifier (Muni et al., 2006).
The above mentioned two problems are related as they both require optimization of the features to reduce the overall dimensionality and preserve the class-related information. For BCI application, the common spatial filter (CSP) is usually employed to solve the above two problems by reducing the whole EEG montage to a few spatial filtered channels in which the task-related information is significant (Lotte, 2014; Meng et al., 2015). The CSP works to find a projection that maximizes signal variance in one class while minimizing the variance in another class (Lotte and Guan, 2011). It has been shown in Wang et al. (2013a) that the application of the Tikhonov regularized CSP (TRCSP) to pre-process the EEG signal, and followed by the application of BMFLC for feature extraction provides a better performance as compared to the common average referencing or variant of Laplacian based spatial filters. As BMFLC decomposes the scalar measurement of each EEG channel into a multi-dimensional frequency estimation, the spatial filter that is optimized with respect to the bandpass filtered EEG signal may not be optimal for all frequency components.

In general, the above mentioned problems can be considered as a feature optimization problem (Chen et al., 2009; Estévez et al., 2009). However, the popular greedy search-based feature selection algorithm are not ideal to deal with multi-EEG channels due to its computational complexity. In the worst case scenario, to identify a optimal subset of a $n$-dimension feature vector, it would require all possible combinations of the $n$-dimension vector and that results in repetition of the cost function evaluation by $\sum_{r=1}^{n} C_{r}^{n}$ times. With increase in $n$, it will be no longer feasible due to computational load. As an alternative, the EA-based feature optimization, which encodes a feature subset in its solution and then optimizes it with respect to the performance index of a classifier, could be employed (Muni et al., 2006; Aberg and Wessberg, 2007; Aler et al., 2012). The computation load of the EA-based algorithm is limited by the number of maximum generations or function evaluations. Further, it is less intensive in computation demand as compared to the greedy-search based algorithms. A genetic type EA-based feature optimization algorithm is popular for the binarized solution to find the optimal feature subset (Banerjee et al., 2007). Due to this reason, it is not suitable for optimizing real-valued function. With the recent development of real-valued EA, the classifier optimization and the feature selection can be performed in one setting (Aberg and Wessberg, 2007).

The application of EA also provides us with a new way of estimating the spatial filter. As the common spatial filter estimation is a maximization process, the cost function is generally limited to a convex form for efficient application of the existing optimization algorithms (Lotte and Guan, 2011). With the help of real-valued EA algorithms, direct optimization of the spatial filter with respect to the classification accuracy can be performed where the convexity may not be preserved in the cost function. In Aler et al. (2012), the spatial filter was estimated by using the covariance matrix adaptation evolution strategy (CMA-ES), a type of EA.

In this work, we employ two real-valued EAs for optimizing the features that are produced by BMFLC in a multi-channel EEG. Both spatial filter and feature selection are encoded in 
the solution vector and optimized simultaneously. Two realvalued EA's, namely global and local real-coded genetic algorithm (GLGA) and CMA-ES, are employed for optimization. The above two algorithms are selected due to their stable performance on various real-valued practical problems (García-Martínez et al., 2008; Hansen et al., 2009). The result of the proposed EA-based feature optimization (BMFLC-EA) is compared with the BMFLC with CSP, and BMFLC. Our results show that the BMFLC with EA outperforms the rest. The preliminary results of this work has been published in Wang et al. (2015). In the current version, we have modified the optimal number of spatial filters selection procedure, and provide a comprehensive comparison with the existing methods.

The remaining paper is organized as follows. Three configurations of BMFLC based classifiers are proposed in Section 2, where the necessary details of the algorithm are provided. The performance of the proposed method and comparison to the existing methods are presented in Section 3. Section 4 concludes this paper.

\section{METHODS}

In this section, three different configurations are proposed that are based on BMFLC for feature extraction. Various methods employed for this purpose are described in brief.

\subsection{Proposed BCI Architecture}

An overview of the EEG signal processing employed in this work is shown in Figure 1. The BCI system can be divided into a training phase and a testing phase. The feature optimization and classifier training is executed during the training phase. Once the optimal feature set and classifier has been obtained, the system can shift to the testing phase where feature optimization is no longer required. The reported classification accuracy is estimated during the testing phase of the BCI system. Before application to any feature extraction and optimization algorithm, the multiple channel EEG signals are first band-pass filtered with a Butterworth fifth order filter to the desired frequency band.
To analyze the motor imagery, the $\mu$ rhythm is employed in the current study.

Three configurations that are based on BMFLC are discussed in this work. The major differences between three BCI configurations lie in how the spatial filter is employed in combination with the BMFLC. The BMFLC is employed to obtain time-frequency mapping of the EEG signal in each configuration. The spatial filter is employed to reduce the number of electrodes to a smaller subset. It can substantially reduce the dimension in the feature vector.

The work flow of the three configurations is shown in Figure 2. Configuration 1 shown in Figure 2 is the simplest of the three configurations. As it is well-known that channels C3 and C4 in 10/20 systems have distinct patterns during motor imagery tasks, EEG signal from these two channels are selected. Thus, the spatial filter is formulated by a manual selection of channels in this configuration. The BMFLC is directly applied to the EEG data of C3 and C4.

Second configuration uses the Tikhonov regularized common spatial filter (TRCSP) to reduce the number of electrodes. Prior to the application of TRCSP, the EEG signal of each channel is band-pass filtered into the frequency band of interest, i.e., $\mu$ band. TRCSP then uses the band-pass filtered EEG to estimate spatial filters by maximizing the signal variance among classes. The number of spatial filters that are finally employed in the testing phase depends on the end application. The regularization parameter " $\alpha$ " in TRCSP is optimized based on the training data using the procedure developed in Lotte and Guan (2011). Later, the raw EEG signal in the testing set is multiplied by the selected spatial filters. The BMFLC is then employed to decompose the spatial filtered EEG signal to form the feature vector for the classifier.

Configuration 3 incorporates EA for estimating the spatial filter. In comparison with the earlier two configurations, the BMFLC is applied ahead of the spatial filter estimation. The dimension of the decomposed signal from BMFLC contains the full spectrum information with high dimensionality. EA is thus employed to find spatial filter in order to reduce the dimensionality of the decomposed signal. Compared to

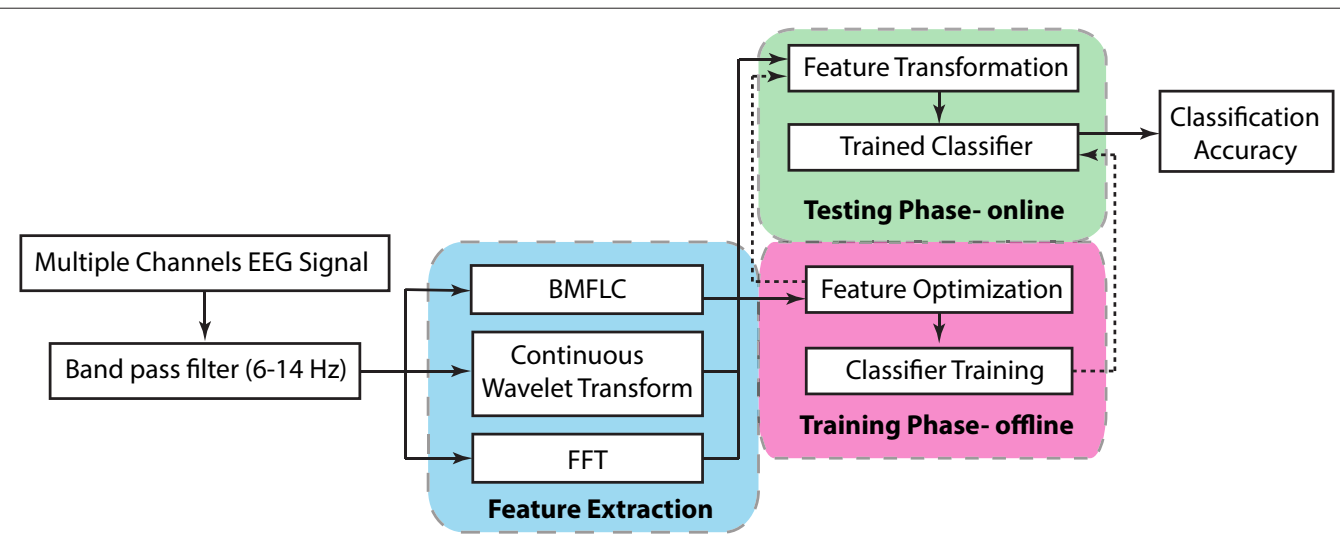

FIGURE 1 | Overview of the EEG signal processing chain. 


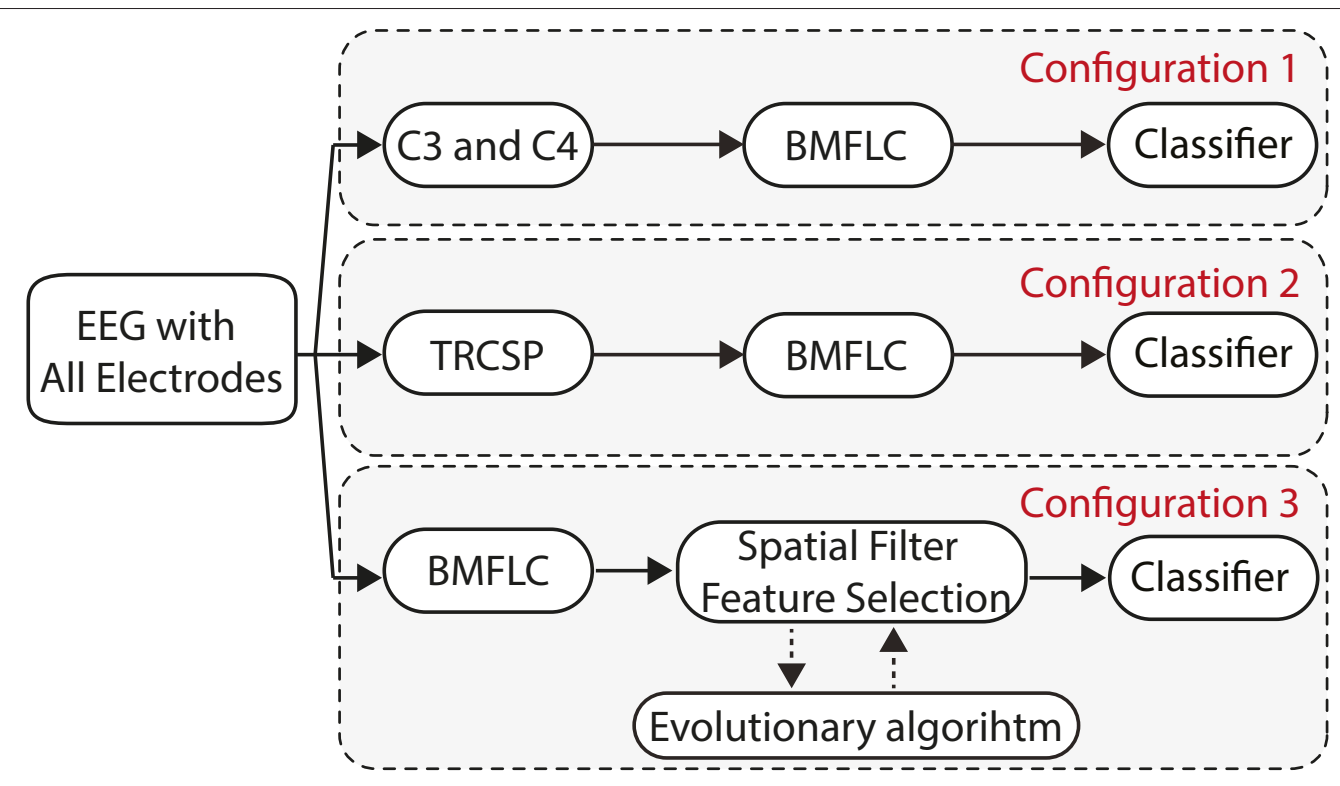

FIGURE 2 | Proposed configurations.

the optimization of the spatial filter in configuration 2, the optimization criterion in EA is the classification performance rather than the variance of the EEG signal in different classes. The importance of this difference will be more clear in the Results Section. Moreover, to further reduce the dimensionality of the feature vector, feature selection is also conducted by finding a subset of frequency components in the whole band that is provided by the BMFLC with EA.

\subsection{BMFLC-KF Based EEG Feature Extraction}

Common to all three configurations, the BMFLC is applied to decompose the EEG signal to obtain time-frequency mapping of the $\alpha$ band. The amplitude information of each frequency component at each time instant or averaged over a fixed moving window can be employed to form the feature vector for the classifier.

BMFLC divides a pre-defined frequency band $\left[\omega_{1}, \cdots, \omega_{n}\right]$ into $n$ equally distributed divisions with frequency spacing $\Delta_{f}$, and estimates the amplitude of each frequency component by using the Kalman filter as shown below (Wang et al., 2012b):

$$
\begin{aligned}
y_{k} & =\mathbf{x}_{k}^{T} \mathbf{w}_{k}+v_{k} \\
\mathbf{w}_{k+1} & =\mathbf{w}_{k}+\eta_{k}
\end{aligned}
$$

where $\mathbf{x}_{k}$ and $\mathbf{w}_{k}$ are defined as

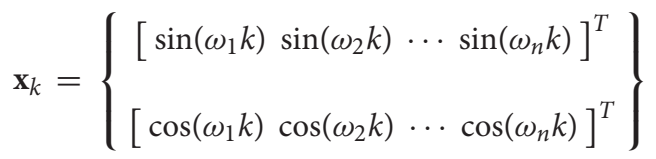

$$
\begin{aligned}
& \mathbf{w}_{k}=\left\{\begin{array}{c}
{\left[\begin{array}{cccc}
a_{1 k} & a_{2 k} & \cdots & a_{n k}
\end{array}\right]^{T}} \\
{\left[\begin{array}{cccc}
b_{1 k} & b_{2 k} & \cdots & b_{n k}
\end{array}\right]^{T}}
\end{array}\right\}
\end{aligned}
$$

Assume that $v_{k}$ and $\eta_{k}$ are independent Gaussian processes with 0 mean and covariances of $R$ and $\mathbf{Q}$, respectively. The Kalman filter is employed for the adaptation of weights estimate $\hat{\mathbf{w}}_{k}=\mathbf{E}\left[\mathbf{w}_{k} \mid \mathbf{y}_{k-1}\right]$. The detailed implementation of BMFLC-KF is provided in the Supplementary Material (S.2). The weight vectors of BMFLC represents the Fourier coefficients of the band-limited signal. The estimated weights are further combined as follows:

$$
\mathbf{W}_{k}=\left[\sqrt{a_{1 k}^{2}+b_{1 k}^{2}} \cdots \sqrt{a_{n k}^{2}+b_{n k}^{2}}\right]^{T}
$$

where $\mathbf{W}_{k}$ is the absolute weight vector of the frequency components at time instant $k$. The time-frequency mapping of a given signal is obtained by cascading the weights in $\mathbf{D}=$ $\left[\mathbf{W}_{1}, \ldots, \mathbf{W}_{n}\right]$.

\subsection{EA-Based Spatial Filter and Feature Selection Optimization}

As the usage and implementation of TRCSP in configuration 2 is straightforward, for the sake of brevity, the details are provided in Supplementary Material (S.1). In this subsection, we focus on developing EA-based spatial filter and feature selection optimization.

As EA has no limitations in the formation of solution vector, we need to encode the spatial filter and feature selection into the solution vector and then employ EA to optimize the solution vector with respect to the same cost function. Since the final objective of applying both spatial filter and feature selection is to improve the performance of the classifier, the cost function is selected to be the error rate of the classifier.

In this work, the covariance matrix adaptation evolution strategy (CMA-ES) and, global and local real-coded genetic algorithm (GLGA) are used due their superior performance in 
real-valued optimization (García-Martínez et al., 2008; Hansen et al., 2009). The algorithms for GLGA and CMA-ES are discussed in the following subsection. For more detailed information, please refer to S.3 and S.4 in Supplementary Material, respectively.

\subsubsection{Global and Local Real-Coded Genetic Algorithm (GLGA)}

Although we can encode a real-valued quantity into a binary string via some transformation and using traditional genetic algorithm (GA) (Hansen et al., 2009), the dimension of the solution becomes large as the number of required spatial filter grows. In this work, the GLGA, a real-valued version of GA, is employed (García-Martínez et al., 2008).

GLGA uses several operators to balance the exploration and exploitation. First, the solution vector in the current population is divided into male and female group by using female and male differentiation (FMD) operator. GLGA then uses parentcentric BLX- $\alpha$ (PBX- $\alpha)$ crossover operator to generate offspring. The $\mathrm{PBX}-\alpha$ takes one solution vector from each group, the generated offspring that lies in the vicinity of the female solution. The distance between the offspring and the female solution is governed by the selected male solution and a parameter $\alpha$. The parameter $\alpha$ determines the spread of offspring to its parents.

The uniform fertility selection (UFS) and the negative associative mating (NAS) are employed for the selection of female and male parents, respectively(García-Martínez et al., 2008). To apply UFS, a number of elite solutions in the current population are selected as a female parent. The number of times that a solution vector is used as female is tracked during the evolution. The less frequent solution is then selected as female parent. For selection of the male parent, the roulette wheel methods are applied to selected five candidate male parents. The Euclidean distance between the selected female parent and each of the possible male parent is calculated. The one with the highest distance is then selected as the male parent.

GLGA balances the global and local search by tuning the number of female and male parents. Let $N_{m}$ and $N_{f}$ denote the number of male and female parents, respectively. With a large $N_{f}$, GLGA focuses on exploring the solution space, whereas a large $N_{m}$ will restrict GLGA to concentrate on local area. GLGA is not operated by generation, rather it uses function evaluation as the criterion to shift from global to local search. GLGA-n\% indicates that $\mathrm{n} \%$ of function evaluations are used for global search and rest are used for local search. After certain number of function evaluations, GLGA assumes that the solutions have already entered into the vicinity of the global optimal, it thus reduces $N_{f}$ to fine tune the solution in that region. To maintain the population size unchanged during evolution, the "replace the worst strategy" is adopted. Each time when an offspring is generated, the fitness value of the offspring is compared to the solution in the current population. If it is better than any of the solution in the current population, the worst one is replaced.

\subsubsection{CMA-ES}

CMA-ES uses the second order statistics estimated from the solution space to find the optimal value of the target function. It is thus well-suited for optimizing the function with a complicated structure. It has been applied to both artificial functions and real practical problems and has shown to be performing better than the contemporary counterparts (Hansen et al., 2009; Elteto et al., 2012).

The $(\mu, \lambda)$ CMA-ES requires $\lambda$ number of solutions in each generation, and select the best $\mu$ number of solutions for updating the value in next step. The CMA-ES algorithm generates the solution $s_{k}^{(g)}$ by sampling from a Gaussian distribution with mean $m$, step-size $\delta$ and covariance estimation $\mathbf{C}$, where $g$ and $k$ are indexes for generation and solution, respectively. To find the solution for the next generation, CMA-ES estimates the mean, step-size, and covariance with the solution in the current generation considering the history path of the solution evolution. After each generation, the solutions are ranked based on their fitness value. The update of mean is carried out by a weighted summation of the $\mu$ best solutions in the current generation. The value of $\mathbf{C}$ is determined by the covariance of the estimated evolution path denoted by $p_{c}$ and the covariance estimated from the $\mu$ number of solutions in the current generation.

\subsubsection{Optimization of Spatial Filter and Feature Selection}

The key to the application of EA for optimizing the spatial filter and feature selection simultaneously is to develop a proper solution vector. The spatial filter is real-valued by default, unlike the genetic algorithm based feature solution. The binary valued feature selection is replaced with real-valued problem. It was shown in earlier studies that the subject-specific band has a maximum bandwidth of $3 \mathrm{~Hz}$ (Veluvolu et al., 2012). Therefore, the EA is employed to search for the starting frequency and the optimal bandwidth of the subject-specific band. Moreover, the dimensionality of the solution vector is significantly reduced by encoding the frequency selection in real-value compared to the binary encoding scheme. As the number of function evaluations required for a EA to converge depends on the dimension of the solution vector (Herrera et al., 1998), this approach should be able to reduce the computational complexity and further improve the solution quality when a fixed number of function evaluations is considered.

Thus, the solution vector for GLGA and CMA-ES is constructed as follows:

$$
s o l=[\overbrace{s f_{11}, \ldots, s f_{1 M}, \quad s f_{21}, \ldots, s f_{2 M}, \ldots,}^{\text {Spatial Filter }}, \overbrace{F S, \quad{ }_{B W}}^{\text {Frequency Selection }}]
$$

where $s f_{i j}$ denotes the $j$ th component in $i$ th spatial filter, FS is the starting frequency, and $B W$ is the bandwidth. $M$ is the total number of EEG channels. The number of resultant spatial filters depend on the application. For motor imagery BCI system, the number of classes is usually chosen as the number for spatial filters selection (Aler et al., 2012). As the EA is applied to the time-frequency mapping of BMFLC, FS has to lie in the range of $\left[f_{1}, f_{n}-B W\right]$, where $f_{1}$ and $f_{n}$ are the lower and upper cutoff frequency of BMFLC, respectively. As shown in Veluvolu et al. (2012), BW is constrained to have a maximum $3 \mathrm{~Hz}$ band. 
The classification error obtained from linear discriminant analysis (LDA) is employed as the optimization criterion for GLGA and CMA-ES. The frequency weights that are estimated from BMFLC-KF are used as the original feature set. To optimize the spatial filter and frequency selection, the frequency weights obtained from each channel is first combined to the following form:

$$
\mathbf{F}_{k}=\left[\begin{array}{ccc}
\mathbf{w}_{k, 1}^{f_{s}} & \ldots & \mathbf{w}_{k, M}^{f_{s}} \\
\vdots & \vdots & \vdots \\
\mathbf{w}_{k, 1}^{f e} & \ldots & \mathbf{w}_{k, M}^{f_{e}}
\end{array}\right]^{T}
$$

where $\mathbf{w}_{k, M}^{f_{s}}$ indicates the weights of frequency $f s$ at time instant $k$ in channel $M$. Then, the best solution vector in the current generation is separated into two parts, frequency selection and spatial filter. The optimal frequency band is extracted form the original feature space by setting $f s=F S$ and $f e=F S+B W$. The spatial filter component of the solution vector is re-organized as follows:

$$
\mathbf{S F}=\left[\begin{array}{lll}
s f_{11} & \ldots & s f_{1 M} \\
s f_{21} & \ldots & s f_{2 M}
\end{array}\right]^{T}
$$

To obtain the optimized feature set, the spatial filter $\mathbf{S F}^{T}$ is multiplied with the $\mathbf{F}_{k}$, where superscript $T$ denotes matrix transpose. The resultant feature vector as well as the corresponding label are then sent to the classifier. The error rate of the employed classifier is used to guide the EA to search for a better spatial filter and feature set. To avoid over-fitting, only the classification error obtained from the validation data is used. The solution vector obtained at the end of the evolution contains the optimized spatial filter and frequency selection.

\subsection{Dataset Description}

To test the performance of the proposed architecture, we choose two publically available BCI datasets. The first dataset (denoted as Dataset I) is from Brain Computer Interface Competition IV (Brunner et al., 2007). Dataset I contains EEG data of nine subjects. EEG was recorded from $22 \mathrm{Ag} / \mathrm{AgCl}$ electrodes sampled at $250 \mathrm{~Hz}$. All signals were recorded monopolarly with the left mastoid as reference and right mastoid as ground. Four classes of cue-based motor imagery tasks were carried out, namely the imagination of the movement of the left hand, right hand, both feet and tongue. Each subject data was recorded in two sessions on separate days. Each session consisted of six runs separated by short breaks. One run consisted of 48 trials. During the recording, the subjects sat on a comfortable armchair in front of a computer screen. At the beginning of each trial $(t=0 s)$, a fixation cross appeared on the black screen. Two seconds later, a cue in the form of an arrow pointing either to the left, right, down, or up displayed on the screen and lasted 1.25s. The subjects were asked to perform the motor imagery task until the fixation cross disappeared from the screen at $t=6 \mathrm{~s}$. For more details about data collection, see Brunner et al. (2007).

The second dataset (denoted as Dataset II) was taken from the BCI competition 2003, dataset 2a (McFarland et al., 1997).
The dataset contains motor imagery data from three subjects. All subjects were informed to modulate their sensorimotor rhythm to move a cursor from the left edge of the screen to one of the four designated locations appeared on the right edge of the screen. In this paper, we chose the top and bottom target out of totally four available targets. Totally, 64 channels EEG signal were recorded according to the international 10/20 system with a sampling frequency of $160 \mathrm{~Hz}$. Each subjects participated 10 sessions of the experiment. In this work, the top and bottom target in the training session (session 1-6) was employed.

\section{RESULTS}

\subsection{Experiment Settings}

As the EEG $\alpha$ band is of interest, the frequency range for BMFLC-KF is set to $f_{1}=6 \mathrm{~Hz}$ and $f_{n}=14 \mathrm{~Hz} . \Delta f$ is set to $0.5 \mathrm{~Hz}$ as it has been shown to offer better results in EEG signal decomposition (Wang et al., 2012b). For applying CMAES algorithm, the population size $\lambda$ is set to be five times the solution dimension. The number of spatial filters are selected to be equal to the number of classes in the dataset. The reason for this selection will be discussed in the later section.

The CMA-ES starts by setting the initial value to $s^{(0)}, \delta^{(0)}, \mathbf{C}^{(0)}$, and updating the solution and is similar to the implementation provided in Hansen and Kern (2004). The parameters provided in Hansen et al. (2009) are employed as they are shown to offer robust performance.

For GLGA, the parameters are chosen from García-Martínez et al. (2008). $\alpha$ is equal to 0.5 and $25 \%$ of function evaluations are employed for global search. For a fair comparison between CMAES and GLGA, the functional evaluation of GLGA is set to 10,000, as it approximately matches to the function evaluations used by CMA-ES. $N_{m}=N$ and $N_{f}=N / 2$ are used for global searching and $N_{m}=100$ and $N_{f}=5$ are employed for local searching, where $N$ is the population size. The algorithm terminates when the stopping criterion is met.

For performance analysis, the EEG dataset is divided into training set and testing set according to 10 -times cross validation scheme. Feature optimization including CSP and EA based methods are performed on the training set. The same training set is employed to train the final classifier. The classification accuracy which is obtained on the testing data is employed for performance comparison. It is important to note that, since the GLGA and CMA-ES relies on classification error as the cost function, a separate 10-times cross validation is also applied to the training set.

Another important factor that affects the performance is the number of spatial filter pairs employed for each algorithm/configuration. In this paper, the number of spatial filter pairs is selected for each algorithm separately to ensure optimal performance for each configuration. The rationale and procedure for this selection will be discussed in the later section.

For the sake of comparison, we consider other feature extraction and optimization algorithms that have been reported to have superior performance on BCI motor imagery tasks. The continuous wavelet transform (CWT) with was shown to have accurate time-frequency resolution in analyzing the EEG signal 
(Hsu, 2015). Thus, it has been included in our comparison analysis. We have also chosen Daubechies wavelets of order 4 (denoted as Db4) as suggested in Hsu (2015). The obtained wavelet features undergoes the same feature optimization procedure with CMA-ES (denoted as Db4-CMAES). Further, the filter bank common spatial pattern (FBCSP) is included as it was shown to have superior performance on the BCI competition dataset (Ang et al., 2012). In this work, we have implemented a BMFLC based FBCSP. In summary, the EEG signal is first decomposed by the BMFLC. Then, CSP filter was employed for each frequency estimate and the features were constructed as the logarithm power. The obtained features were then optimized by employing the mutual information criterion (Ang et al., 2012). Further, the algorithm developed in Aler et al. (2012) is also implemented (denoted as FFT-CMAES).

\subsection{Performance of GLGA and CMA-ES Based Feature Optimization}

The value of the cost function of CMA-ES for each generation is shown in Figure 3 for all subjects. The shaded area indicates the standard deviation of the classification error obtained from 10 -times cross validation scheme. For all the subjects, we observe that the classification error converges to a stable bound at the end of the evolution process. It can be noted that with CMAES, the error converges at a faster rate in the first 20 generations. Later on, the convergence rate is slowed up to 100 generations. After 100 generations, the classification error becomes stable. The simulations for 500 generations also yielded similar results.

Another observation from Figure 3 is that the standard deviation varies significantly across subjects. A small standard deviations can be identified in subject-1, -7 , and -9 . Whereas, large standard deviation can be observed in the rest of the subjects. The large standard deviation implies that CMA-ES converges to different values at cross validation runs. Further, it may be also due to the complexity of the cost function employed. However, this divergence in the evolution process does not affect the performance of the final classifier with CMA-ES as shown in Figure 7. This result indicates the superiority of CMA-ES in the optimization of a complex function as compared to GLGA.

Similarly, the cost function evolution of GLGA is shown in Figure 4. The vertical line in Figure 4 indicates the transition from global search to local search. The first observation is the slow error decay during the global search phase. After GLGA

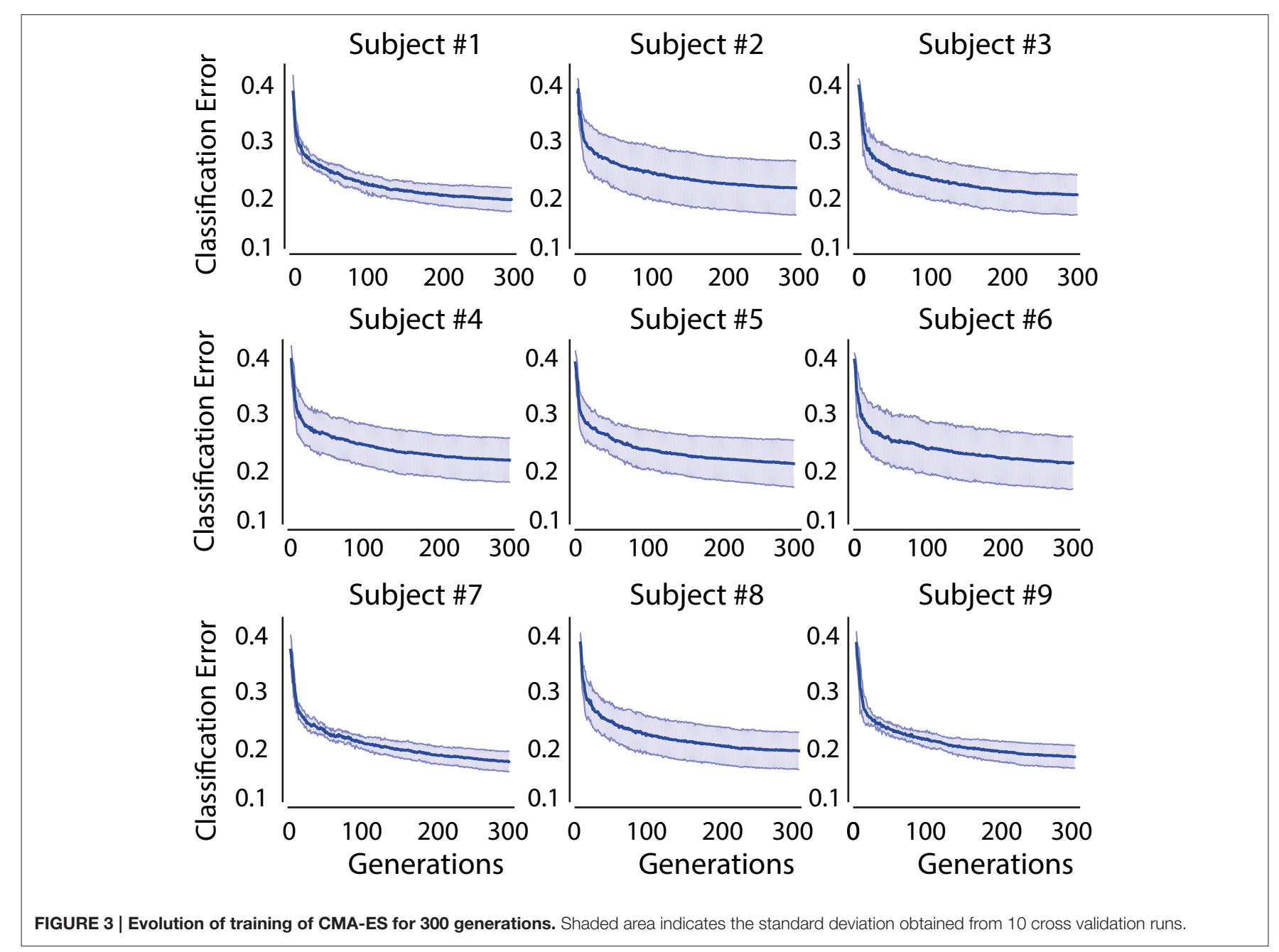



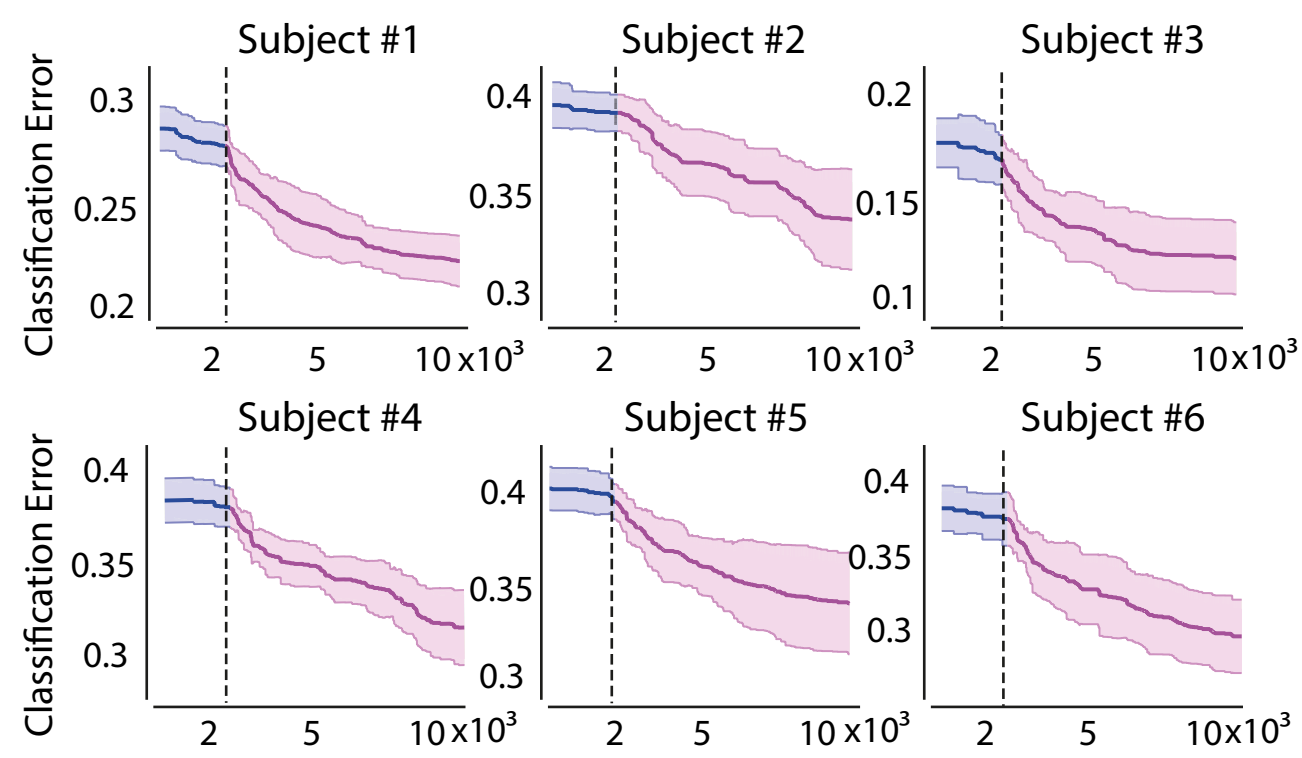

Subject \#6

\section{Subject \#7}

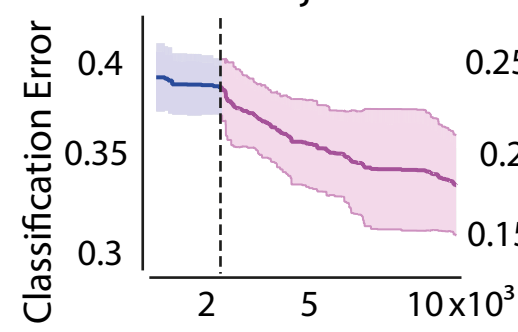

Subject \#8
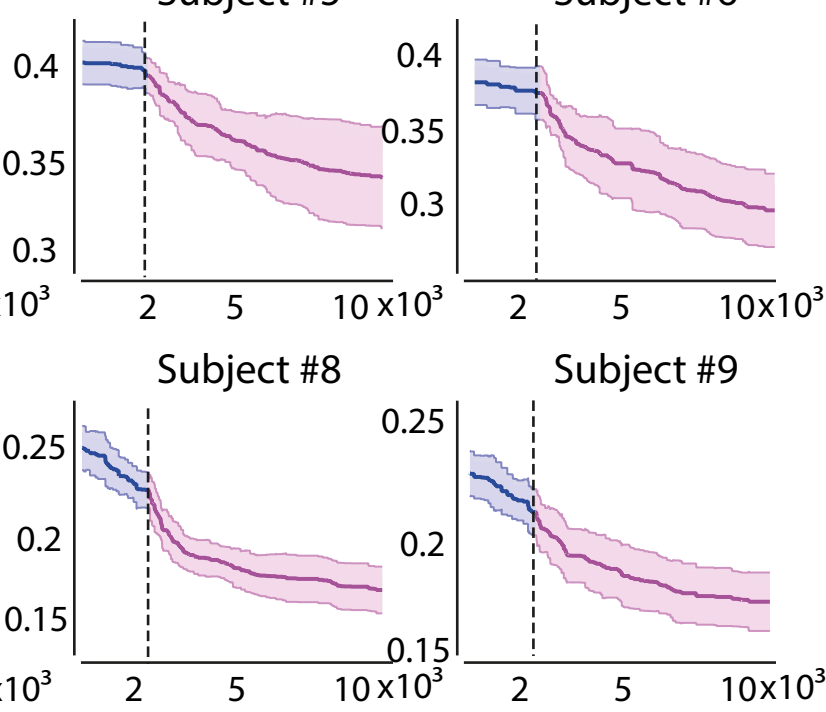

\section{Function Evaluation Function Evaluation Function Evaluation}

FIGURE 4 | Evolution of training of GLGA-25 for 10,000 function evaluations. Shaded area indicates the standard deviation obtained from 10 cross validation

runs. The vertical line indicates the transition from global searching to local searching.

moves to local search, initially, a fast error decay followed by a stable phase can be observed. The standard deviation is generally small during global search and the value increases when the algorithm approaches the end of the execution. The results suggest that GLGA tends to converge to different area after each global search during each cross validation run.

Comparing the error evolution of GLGA to CMA-ES, we first note that the GLGA has a higher standard deviation over all subjects, whereas the error evolution in CMA-ES varies with the subject with a smaller volatility. Moreover, CMA-ES converges to a small error bound at the end of the evolution process as compared to GLGA.

To further evaluate the performances of GLGA and CMA-ES, the overall error improvement of each algorithm is obtained for all subjects. The error improvement is calculated as the difference between the maximum error and the minimum error obtained during evolutionary process. It shows how well the employed EA explores the error surface. For comparison, the algorithm proposed in Aler et al. (2012) (named as FFT-CMAES) is also implemented and the results are shown in Figure 5. We observe CMA-ES based feature optimization outperforms the GLGA in terms of performance improvement. The BMFLC-CMAES shows superiority in performance compared to FFT-CMAES. BMFLCGA has the lowest improvement during training. The error improvement is stable for all the subjects with FFT-CMAES, as shown by small standard deviation.

\subsection{Performance of the Final Classifier for All Configurations}

To perform the comparison for all different configurations, the number of spatial filters required for each configuration needs to be identified. As the dataset employed in this work consists of 22 EEG recordings for motor imagery tasks, we can therefore obtain up to 11 pairs of spatial filters for each subject. In this work, we select the number of spatial filters for each configuration based on the generalization performance index. This index is used to quantify the performance for the selected number of spatial filters over all subjects. To calculate this index for a configuration, we first obtain the classification accuracy for all subjects and all spatial filter pairs in the training data set. Then for a particular subject, the classification accuracy for all spatial filter pairs is normalized to the range of [0 1$]$. The employed unity-based normalization ensures that the optimal spatial filter pair for each subject has the performance index of 1 , so that the difference in 


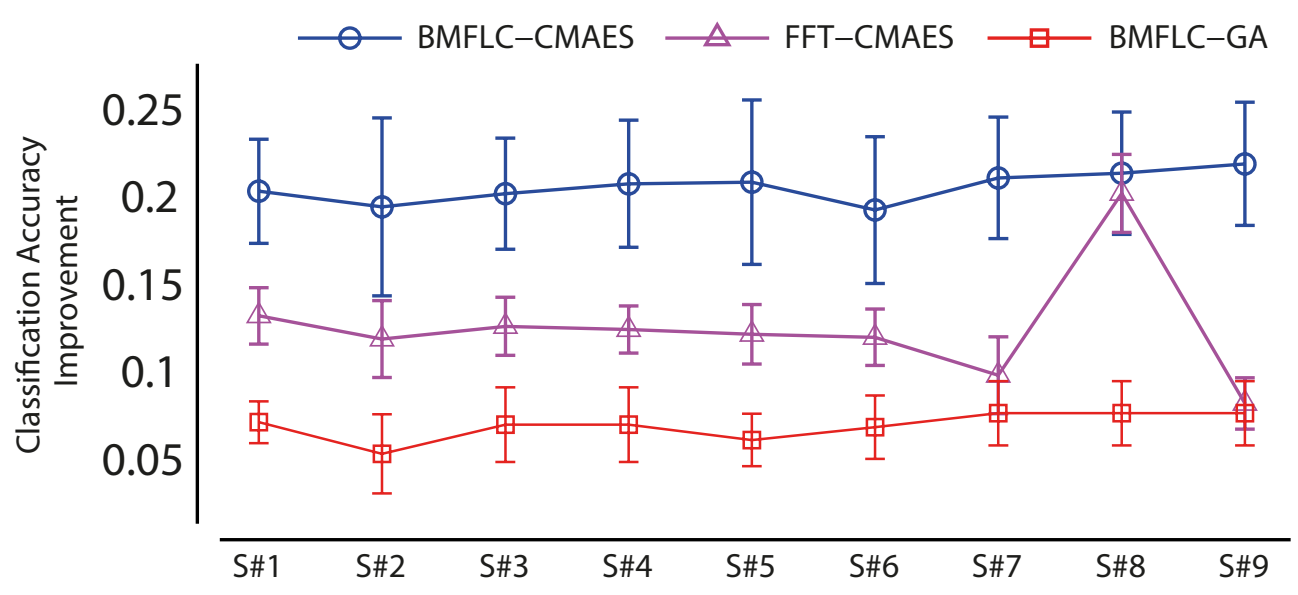

FIGURE 5 | Classification accuracy improvement BMFLC-CMAES, FFT-CMAES, and BMFLC-GA.

classification accuracy among subjects will not bias the spatial filter selection. Then, the generalization performance index for each spatial filter pair is the normalized classification accuracy averaged over all subjects. The results of generation performance index for all configurations is shown in Figure 6.

From Figure 6, we can observe that all configurations tends to have better generalization performance for fewer number of spatial filter pairs. The CSP and FFT-CMAES display maximum generalization performance when one pair of spatial filters is employed. BMFLC-CMAES peaks at two pair spatial filters, whereas BMFLC-GA requires three pairs of spatial filters. Thus, the obtained number of spatial filter pair is set to each configuration for comparison. After optimizing the features by employing CSP, CMA-ES, and GLGA with pre-selected parameters and number of spatial filter pairs, a final classifier is then built with the same training set and then finally evaluated on the testing set.

The performance of all subjects in Dataset I averaged over 10 -times cross validation is shown in Figure 7. Configuration 1 is denoted as BMFLC where no feature optimization has been employed. Configuration 2, where CSP is applied prior to BMFLC is denoted by CSP-BMFLC. The two variants of EA based feature optimization procedures are named as BMFLC-CMAES and BMFLC-GLGA, respectively.

In Figure 7, we observe that BMFLC-CMAES outperforms all other algorithms in seven out of nine subjects. Further, it has also the best performance when averaged over all subjects, and passes the Friedman test with $\alpha=0.05$. We also observe that the features obtained from BMFLC and later optimized by CMAES and GLGA display better performance compared to FFTCMAES on average. The BMFLC-FBCSP has better performance as compared to BMFLC and CSP-BMFLC. The performance varies across subject in CSP-BMFLC and is subject-dependent. It is also clear that feature optimization improves the overall performance.

The classification performance with Dataset II is shown in Table 1. We observe that the BMFLC-CMAES and Db4CMAES outperformed all other methods in mean accuracy. This

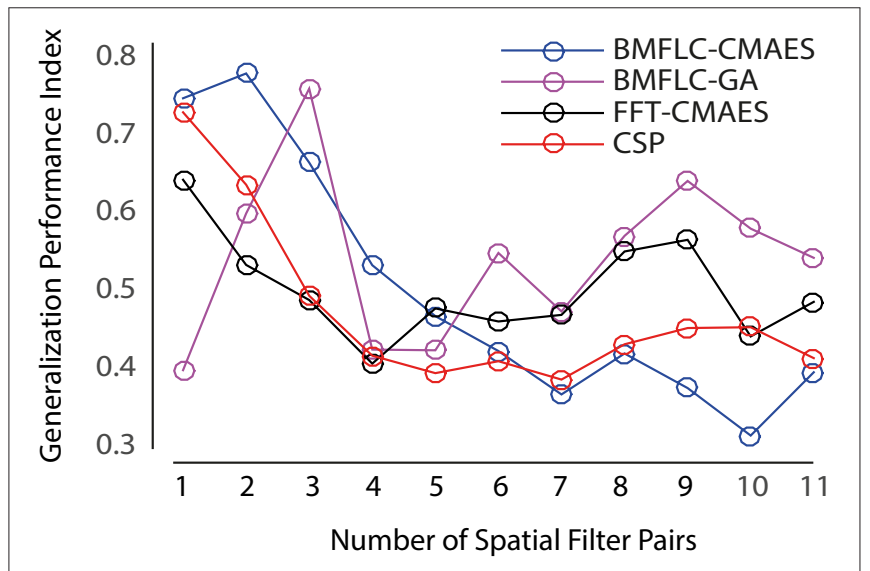

FIGURE 6 | Selection of optimal number of spatial filter pair.

observation clearly demonstrates the superiority of employing time-frequency decomposition for feature extraction. Further, we notice that the BMFLC-GA is inferior to the BMFLC-FBCSP method.

We also compared the number of occasions of one algorithm outperforming another algorithm by accumulating the results from the cross validation runs of all subjects in Dataset I. Since 10 cross validation were employed, a total of 90 ( 9 subject $\times 10$ cross validations runs) accuracy estimates per configuration were obtained. The results are shown in Figure 8. The total number and its corresponding percentage value by which one algorithm outperforms the other algorithm is provided in the middle of each block.

From Figure 8, it clearly demonstrates the necessity of feature optimization. The configurations that are combined with feature optimization have better classification accuracy when BMFLC based features are employed. The results in the first and second row when compared to the third row of Figure 8 further demonstrate the superiority of BMFLC and Db4 in extracting information from EEG as compared to FFT. 


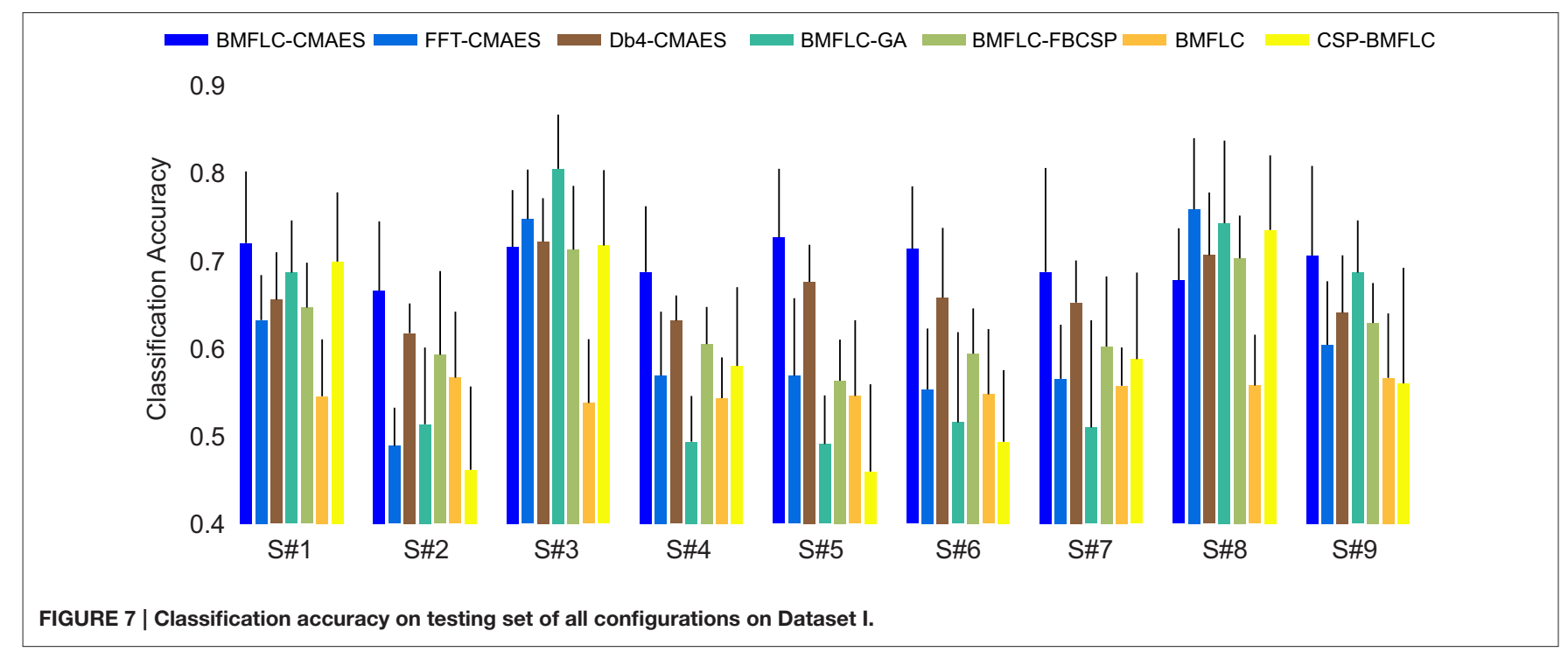

TABLE 1 | Classification accuracy on testing set of all configurations-Dataset II.

\begin{tabular}{|c|c|c|c|c|c|c|c|}
\hline \multirow[t]{2}{*}{ Subject No. } & \multicolumn{7}{|c|}{ Classification accuracy } \\
\hline & BMFLC-CMAES & FFT-CMAES & Db4-CMAES & BMFLC-GA & BMFLC-FBCSP & BMFLC & CSP-BMFLC \\
\hline Subject AA & $0.83 \pm 0.03$ & $0.73 \pm 0.08$ & $0.80 \pm 0.07$ & $0.75 \pm 0.12$ & $0.76 \pm 0.13$ & $0.58 \pm 0.15$ & $0.69 \pm 0.08$ \\
\hline Subject BB & $0.81 \pm 0.04$ & $0.78 \pm 0.08$ & $0.80 \pm 0.07$ & $0.67 \pm 0.10$ & $0.76 \pm 0.11$ & $0.68 \pm 0.13$ & $0.74 \pm 0.09$ \\
\hline Subject CC & $0.83 \pm 0.05$ & $0.75 \pm 0.07$ & $0.78 \pm 0.06$ & $0.75 \pm 0.07$ & $0.74 \pm 0.08$ & $0.68 \pm 0.11$ & $0.71 \pm 0.16$ \\
\hline Average & $0.83 \pm 0.04$ & $0.76 \pm 0.08$ & $0.80 \pm 0.08$ & $0.72 \pm 0.10$ & $0.76 \pm 0.10$ & $0.65 \pm 0.13$ & $0.72 \pm 0.11$ \\
\hline
\end{tabular}

Moreover, we also observe that all configurations that involve feature optimization outperform the traditional methods. The high standard deviation obtained with GLGA (Figure 4) is also reflected in the classification results as its overall performance is lower compared to BMFLC-CMAES.

To further consolidate our observed results, we have performed a repeated measure of ANOVA by collecting all accuracy estimates from all methods on all subjects. As the obtained accuracy estimates violated the sphericity assumption, Greenhouse-Geisser correction has been employed. Results show that the mean accuracy differences are statistically significant among all methods $\left[F_{(4.037,359.252)}=27.904, p<0.01\right]$. Post-hoc tests using the Bonferroni correction revealed that the proposed BMFLC-CMAES outperforms all comparison methods with $p<0.01$. All methods with feature optimization show superior performance as compared to BMFLC $(p<0.05)$. It highlights the necessity of employing feature optimization in BCI applications. Furthermore, the BMFLC-CMAES and Db4CMAES show statistically significant accuracy improvement over CSP-BMFLC and FFT-CMAES $(p<0.01)$, which indicates the merit of using time-frequency decomposition to expand the feature space.

\subsection{Runtime Complexity}

To show the applicability of the proposed methods to the realtime BCI applications, we conducted a runtime computational complexity analysis. The reported runtime estimates for each algorithm are obtained on a system with Intel(R) Core i5-6500 CPU and 8GB RAM. The time duration was measured using Matlab (Mathworks, USA) inbuilt function. Here, we reported the average time duration to finish one validation run. The obtained runtime estimates for all feature optimization methods are given in Table 2 .

The runtime requirement of the proposed feature optimization procedure consists of the feature extraction, optimization, and classification. The feature extraction method BMFLC has been shown to have superior computational complexity as compared to STFT and CWT (Wang et al., 2013b). In this work, we estimated that the time required for BMFLC to decompose a $2 \mathrm{~s}$ EEG epoch is $0.0349 \mathrm{~s} \pm 0.0037$. Also, the computational demand of LDA is negligible as only linear operation is involved. Therefore, the main contributor to the computational complexity of the proposed algorithm is the time taken by the employed optimization algorithm.

All EA based feature optimization algorithms require few minutes of run-time, as shown in Table 2, to attain optimized features. Compared to the CSP based methods, the EA based feature optimization has much higher computational demands. However, the feature optimization is usually conducted during the training phase of the BCI application. The feature optimization only needs to be finished in a reasonable time. We estimated that the BMFLC-CMAES requires $7 \mathrm{~min}$ to find the 


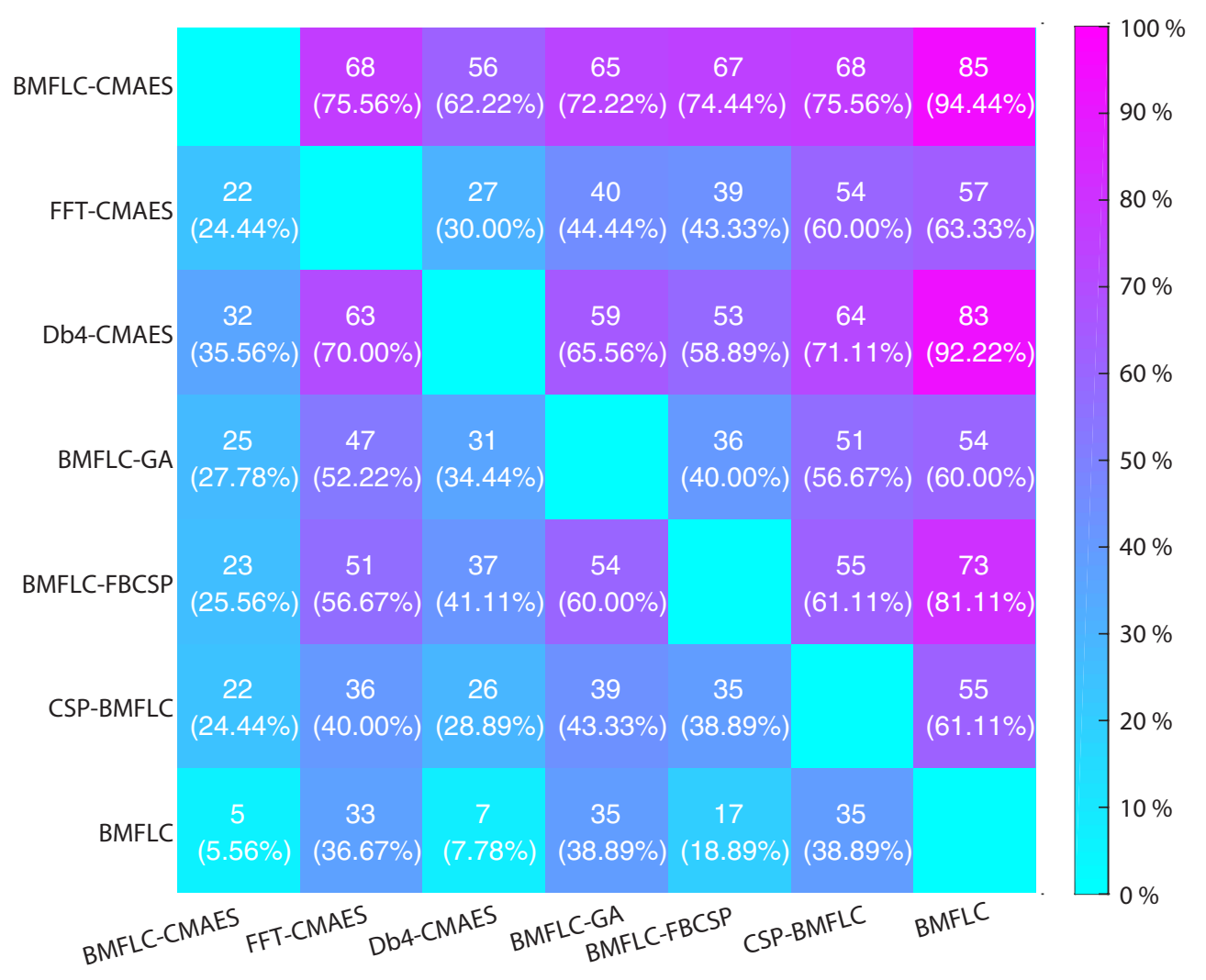

FIGURE 8 | Performance comparison for various configurations on Dateset I.

TABLE 2 | Runtime complexity of all configurations.

\begin{tabular}{|c|c|c|c|c|c|c|}
\hline Methods & BMFLC-CMAES & FFT-CMAES & Db4-CMAES & BMFLC-GA & BMFLC-FBCSP & CSP-BMFLC \\
\hline Time (s) & $471.84 \pm 19.33$ & $515.69 \pm 32.08$ & $494.05 \pm 47.13$ & $17151.27 \pm 839.07$ & $1.55 \pm 0.81$ & $0.01 \pm 0.01$ \\
\hline
\end{tabular}

optimized feature subspace. Once the optimized features have been identified during training, the system only needs to perform feature extraction and classification in real-time.

\section{DISCUSSION}

In this work, different BCI configurations which employ BMFLC based features for classification have been developed. As our results suggest, BMFLC-KF with manually selected channels has the lowest classification performance. However, without any optimization, the BMFLC feature offers the most stable performance. There are two possible reasons for this. First, the BMFLC-KF decomposes the EEG signal with high accuracy and this provides the classifier with a stable feature set. Secondly, the stability in the classification results may also indicate the lack of diversity and limited information available within the two channels. This result also highlights the necessity for employing the optimization for feature extraction.

It is evident that the performance of CSP-BMFLC is subject dependent. For three out of nine subjects in Dataset I, the
CSP-BMFLC has good testing accuracy. However, for the rest subjects, the CSP-BMFLC performance is similar to or even worse than applying BMFLC alone. As CSP algorithm relies on the training data to find the spatial filter, the unsatisfactory performance in test data may be due to over-fitting of the training data.

Comparing the results from the configurations which involve feature optimization, the BMFLC-CMAES outperforms Db4-CMAES, BMFLC-GA, FFT-CMAES, and BMFLC-FBCSP. In fact, BMFLC-CMAES, and Db4-CMAES have the best performance among all configurations considered in this work. Our results indicate that the feature optimization is critical in improving the testing performance for a given feature set.

We also observe that the performance of each EA involved configuration differs. BMFLC based system is degraded when GLGA is employed for feature optimization. As GLGA divides the evolution into two distinct phases, global, and local searches, the overall performance of GLGA depends on the quality of the global search phase. If the global search fails in finding the promising area, then the subsequent local search may 
not improve the results and this may introduce severe overfitting. However, if the global search successfully enters into the promising area, then the local search can fine-tune the solution and this results in good performance as shown in subject 3 and subject 8 in Figure 7.

Among all feature optimization methods that employ BMFLC, the BMFLC-CMAES has the best performance. Whereas, the BMFLC-GA provides inferior performance as compared to BMFLC-FBCSP. This result indicates that the careful selection of optimization algorithm is also critical to identify the suitable feature subset. Our results suggests that the CMAES is preferable for feature optimization.

BMFLC-CMAES, Db4-CMAES, and FFT-CMAES differ only in the feature extraction algorithm. The superior performance of BMFLC-CMAES and Db4-CMAES as compared to FFTCMAES highlights the ability of time-frequency decomposition in extracting the information from motion induced EEG signal as compared to the traditional FFT. Moreover, the BMFLCCMAES provides better performance as compared to Db4CMAES. However, the improvement failed to attain statistical significant level $(p<0.01)$. The current results are in line with our previous work that BMFLC has similar performance in terms of time-frequency decomposition of EEG signal (Wang et al., 2013b). However, as shown in Wang et al. (2013b), the BMFLC has less computational complexity as compared to CWT. Thus, it is more suitable for the real-time BCI applications.

The superiority in classification performance obtained from EA based feature optimization comes with a price. All EA based configurations require much higher computational resources compared to the rest of the configurations employed in this work. The computational complexity of the EA based feature optimization grows with the increasing number of EEG channels and the frequency band of interest. However, the frequency band for a BCI system is usually limited and the feature optimization is only required during training phase. We show that the required training time for BMFLC-CMAES is around $7 \mathrm{~min}$, which is tolerable in the current BCI applications. Thus, the computational burden posed by the EA has little impact to the real-time applicability of the proposed feature optimization algorithm.

Feature optimization is mainly intended to identify a suitable subset of features from the original feature space which can improve the classification accuracy and mitigate the requirement in computational demand. The most reliable method to solve the feature optimization problem is to enumerate all possible feature combinations from the the original feature space. However, such enumeration is only possible when the number of features in the original feature space is small. It has been shown in a fNIRS based BCI application that by selecting two out of six features, the testing accuracy is maximized (Naseer et al., 2016). However, the feature dimension in the current work is 17 per channel. With a 22 channels EEG recording, it results in a 374 dimensional feature vector. To enumerate on all combinations on such high feature space requires high computational requirements. The case is even worse when the number of available channels increases. Therefore, an evolution algorithm based approach was desirable to optimize the features in such high dimensional space.
Classification performance of the BCI applications can be improved with support vector machine (SVM) as the classifier (Schlögl et al., 2005; Naseer, 2014; Khan and Hong, 2015). The training of SVM is however computationally extensive when compared to a LDA classifier (Khan and Hong, 2015). Furthermore, to obtain better performance with SVM, its hyperparameters need to be fine-tuned (Vapnik, 1995; Suykens et al., 2002), which increases the computational cost for training a BCI system considerably. The main aim of the current paper is to show the impact of the feature exaction and feature optimization on the performance of BCI system. Therefore, for the illustration of classification performance with proposed optimal feature selection technique, we chose LDA as the classifier in this work. For completeness, we report that one can chose any advanced machine learning techniques as a classifier for the proposed feature extraction and optimization scheme.

\section{CONCLUSION}

In this work, the performance of BMFLC-KF with various combinations of FBCSP,CSP, CMA-ES, and GLGA for feature optimization is quantified with a two-class BCI application. Two publically available BCI competition datasets are employed to study the performance of the proposed feature extraction and feature optimization algorithm. The evolutionary algorithms (namely CMA-ES and GLGA) are employed to optimize the spatial filter and feature selection simultaneously. It is clear from our results that the performance of the final classifier can be improved by employing any of the above mentioned feature optimizing algorithms. Statistical analysis reveals that the proposed BMFLC-CMAES based feature optimization algorithm outperforms its counterparts. Moreover, the superior performance of time-frequency features obtained from BMFLC and CWT also highlights the necessity of employing time-frequency decomposition for feature extraction in BCI applications. The small execution time of CMA-ES indicates that it is more suitable for feature optimization as compared to GLGA.

\section{AUTHOR CONTRIBUTIONS}

This work was carried out by YW under the supervision of KV.

\section{FUNDING}

This research was supported by the Basic Science Research Program through the National Research Foundation of Korea (NRF) funded by the Ministry of Education, Science and Technology under the Grant NRF-2014R1A1A2A10056145 and in part by the BK21 Plus project funded by the Ministry of Education, Korea (21A20131600011).

\section{SUPPLEMENTARY MATERIAL}

The Supplementary Material for this article can be found online at: http://journal.frontiersin.org/article/10.3389/fnins. 2017.00028/full\#supplementary-material 


\section{REFERENCES}

Aberg, M. C., and Wessberg, J. (2007). Evolutionary optimization of classifiers and features for single-trial EEG discrimination. Biomed. Eng. Online 6:32. doi: 10.1186/1475-925X-6-32

Aler, R., Galván, I. M., and Valls, J. M. (2012). Applying evolution strategies to preprocessing EEG signals for brain-computer interfaces. Inform. Sci. 215, 53-66. doi: 10.1016/j.ins.2012.05.012

Ang, K. K., Chin, Z. Y., Wang, C., Guan, C., and Zhang, H. (2012). Filter bank common spatial pattern algorithm on BCI competition IV datasets $2 \mathrm{a}$ and $2 \mathrm{~b}$. Front. Neurosci. 6:39. doi: 10.3389/fnins.2012.00039

Banerjee, M., Mitra, S., and Banka, H. (2007). Evolutionary rough feature selection in gene expression data. IEEE Trans. Syst. Man Cybernet. C 37, 622-632. doi: 10.1109/TSMCC.2007.897498

Blankertz, B., Tomioka, R., Lemm, S., Kawanabe, M., and Müller, K.-R. (2008). Optimizing spatial filters for robust EEG single-trial analysis. IEEE Signal Process. Mag. 25, 41-56. doi: 10.1109/MSP.2008.44 08441

Brunner, C., Naeem, M., Leeb, R., Graimann, B., and Pfurtscheller, G. (2007). Spatial filtering and selection of optimized components in four class motor imagery data using independent components analysis. Pattern Recognit. Lett. 28, 957-964. doi: 10.1016/j.patrec.2007.01.002

Chen, X., Zhu, X., and Zhang, D. (2009). Use of the discriminant Fourier-derived cepstrum with feature-level post-processing for surface electromyographic signal classification. Physiol. Meas. 30, 1399-1413. doi: 10.1088/0967-3334/30/12/008

Elteto, T., Hansen, N., Germain-Renaud, C., and Bondon, P. (2012). Scalable structural break detection. Appl. Soft Comput. 12, 3408-3420. doi: 10.1016/j.asoc.2012.06.002

Estévez, P. A., Tesmer, M., Perez, C. A., and Zurada, J. M. (2009). Normalized mutual information feature selection. IEEE Trans. Neural Netw. 20, 189-201. doi: 10.1109/TNN.2008.2005601

Gao, S., Wang, Y., Gao, X., and Hong, B. (2014). Visual and auditory brain-computer interfaces. IEEE Trans. Biomed. Eng. 61, 1436-1447. doi: 10.1109/TBME.2014.2300164

García-Martínez, C., Lozano, M., Herrera, F., Molina, D., and Sánchez, A. M. (2008). Global and local real-coded genetic algorithms based on parent-centric crossover operators. Eur. J. Oper. Res. 185, 1088-1113. doi: 10.1016/j.ejor.2006.06.043

Guger, C., Edlinger, G., Harkam, W., Niedermayer, I., and Pfurtscheller, G. (2003). How many people are able to operate an EEG-based braincomputer interface (bci)? IEEE Trans. Neural Syst. Rehab. Eng. 11, 145-147. doi: 10.1109/TNSRE.2003.814481

Hansen, N., and Kern, S. (2004). "Evaluating the CMA evolution strategy on multimodal test functions," in Parallel Problem Solving from Nature \{PPSN VIII\}, Vol. 3242, LNCS, eds X. Yao, E. K. Burke, J. A. Lozano, J. Smith, J. J. Merelo-Guervós, J. A. Bullinaria, J. E. Rowe, P. Tiňo, A. Kabán, and H.-P. Schwefel (Birmingham: Springer), 282-291.

Hansen, N., Niederberger, A. S. P., Guzzella, L., and Koumoutsakos, P. (2009). A method for handling uncertainty in evolutionary optimization with an application to feedback control of combustion. IEEE Trans. Evol. Comput. 13, 180-197. doi: 10.1109/TEVC.2008.924423

Herrera, F., Lozano, M., and Verdegay, J. (1998). Tackling real-coded genetic algorithms: operators and tools for behavioral Analysis. Artifical Intell. Rev. 12, 265-319. doi: 10.1023/A:1006504901164

Hill, N. J., Ricci, E., Haider, S., McCane, L. M., Heckman, S., Wolpaw, J. R., et al. (2014). A practical, intuitive brain-computer interface for communicating 'yes' or 'no' by listening. J. Neural Eng. 11:035003. doi: 10.1088/1741-2560/11/3/035003

Hsu, W. Y. (2014). Improving classification accuracy of motor imagery EEG using genetic feature selection. Clin. EEG Neurosci. 45, 163-168. doi: 10.1177/1550059413491559

Hsu, W.-Y. (2015). Enhancing the performance of motor imagery EEG classification using phase features. Clin. EEG Neurosci. 48, 113-118. doi: $10.1177 / 1550059414555123$

Jurcak, V., Tsuzuki, D., and Dan, I. (2007). 10/20, 10/10, and 10/5 systems revisited: their validity as relative head-surface-based positioning systems. NeuroImage 34, 1600-1611. doi: 10.1016/j.neuroimage.2006.09.024
Khan, M. J., and Hong, K.-S. (2015). Passive BCI based on drowsiness detection: an fNIRS study. Biomed. Optics Express 6, 4063-4078. doi: 10.1364/BOE.6.004063

Khan, M. J., Hong, M. J., and Hong, K.-S. (2014). Decoding of four movement directions using hybrid NIRS-EEG brain-computer interface. Front. Hum. Neurosci. 8:244. doi: 10.3389/fnhum.2014.00244

LaFleur, K., Cassady, K., Doud, A., Shades, K., Rogin, E., and He, B. (2013). Quadcopter control in three-dimensional space using a noninvasive motor imagery-based brain-computer interface. J. Neural Eng. 10:046003. doi: 10.1088/1741-2560/10/4/046003

Lotte, F. (2014). "Chapter 7: A tutorial on EEG signal processing techniques for mental state recognition in brain-computer interfaces," in Guide to BrainComputer Music Interfacing, eds E. R. Miranda and J. Castet (New York, NY: Springer), 133-161.

Lotte, F., Congedo, M., Lecuyer, A., Lamarche, F., and Arnaldi, B. (2007). A Review of classification algorithm for EEG-based brain-computer interface. J. Neural Eng. 4, R1-R13. doi: 10.1088/1741-2560/4/2/R01

Lotte, F., and Guan, C. (2011). Regularizing common spatial patterns to improve BCI designs: unified theory and new algorithms. IEEE Trans. Biomed. Eng. 58, 355-362. doi: 10.1109/TBME.2010.2082539

McFarland, D. J., McCane, L. M., David, S. V., and Wolpaw, J. R. (1997). Spatial filter selection for EEG-based communication. Electroencephalogr. Clin. Neurophysiol. 103, 386-394. doi: 10.1016/S0013-4694(97)00022-2

McFarland, D. J., Sarnacki, W. A., and Wolpaw, J. R. (2010). Electroencephalographic (EEG) control of three-dimensional movement. J. Neural Eng. 7:36007. doi: 10.1088/1741-2560/7/3/036007

Meng, J., Yao, L., Sheng, X., Zhang, D., and Zhu, X. (2015). Simultaneously optimizing spatial spectral features based on mutual information for EEG classification. IEEE Trans. Biomed. Eng. 62, 227-240. doi: 10.1109/TBME.2014.2345458

Muller-Gerking, J., Pfurtscheller, G., and Flyvbjerg, H. (1999). Designing optimal spatial filters for single-trial EEG classification in a movement task. Clin. Neurophysiol. 110, 787-798. doi: 10.1016/S1388-2457(98) 00038-8

Muni, D. P., Pal, N. R., and Das, J. (2006). Genetic programming for simultaneous feature selection and classifier design. IEEE Trans. Syst. Man Cybernet. B 36, 106-117. doi: 10.1109/TSMCB.2005.854499

Naseer, N., Hong, M. J., and Hong, K.-S. (2014). Online binary decision decoding using functional near-infrared spectroscopy for the development of brain-computer interface. Exp. Brain Res. 232, 555-564. doi: 10.1007/s00221-013-3764-1

Naseer, N., Noori, F. M., Qureshi, N. K., and Hong, K.-S. (2016). Determining optimal feature-combination for LDA classification of functional near-infrared spectroscopy signals in brain-computer interface application. Front. Hum. Neurosci. 10:237. doi: 10.3389/fnhum.2016.00237

Pfurtscheller, G., Muller-Putz, G. R., Scherer, R., and Neuper, C. (2008). Rehabilitation with brain-computer interface systems. Computer 41, 1-8. doi: 10.1109/MC.2008.432

Qin, L., and He, B. (2005). A wavelet-based time-frequency analysis approach for classification of motor imagery for brain-computer interface applications. $J$. Neural Eng. 2, 65. doi: 10.1088/1741-2560/2/4/001

Robinson, N., Guan, C., Vinod, A. P., Ang, K. K., and Tee, K. P. (2013). Multiclass EEG classification of voluntary hand movement directions. J. Neural Eng. 10:056018. doi: 10.1088/1741-2560/10/5/056018

Schlögl, A., Lee, F., Bischof, H., and Pfurtscheller, G. (2005). Characterization of four-class motor imagery EEG data for the BCI-competition 2005. J. Neural Eng. 2, 14-22. doi: 10.1088/1741-2560/2/4/L02

Shumway, R. H., and Stoffer, D. S. (2011). Time Series Analysis and Its Applications. Springer Texts in Statistics. New York, NY: Springer.

Suykens, J. A. K., Gestel, T. V., Brabanter, J. D., Moor, B. D., and Vandewalle, J. (2002). Least Squares Support Vector Machines. Singapore: World Scientific.

Tangermann, M., Müller, K.-R., Aertsen, A., Birbaumer, N., Braun, C., Brunner, C., et al. (2012). Review of the BCI competition IV. Front. Neurosci. 6:55. doi: 10.3389/fnins.2012.00055

Turnip, A., and Hong, K.-S. (2012). Classifying mental activities from EEG-P300 signals using adaptive neural networks. Int. J. Innov. Comput. Inform. Control 8, 6429-6443.

Vapnik, V. N. (1995). The Nature of Statistical Learning Theory, Vol. 8. New York, NY: Springer. 
Veluvolu, K. C., Wang, Y., and Kavuri, S. S. (2012). Adaptive estimation of EEG-rhythms for optimal band identification in BCI. J. Neurosci. Methods 203, 163-172. doi: 10.1016/j.jneumeth.2011. 08.035

Wang, Y., Gonuguntla, V., Shafiq, G., and Veluvolu, K. C. (2013a). "Performance comparison of spatial filter with multiple BMFLCs for BCI applications," in Neural Information Processing, Vol. 8226, Series Lecture Notes in Computer Science, eds M. Lee, A. Hirose, Z.-G. Hou, and R. M. Kil (Daegu: Springer) 411-418.

Wang, Y., Mohanarangam, K., Mallipeddi, R., and Veluvolu, K. C. (2015). "Spatial filter and feature selection optimization based on EA for multi-channel EEG," in 37th Annual International Conference of the IEEE Engineering in Medicine and Biology Society (Milan), 2311-2314.

Wang, Y., Veluvolu, K. C., Cho, J.-H., and Defoort, M. (2012a), Adaptive estimation of EEG for subject-specific reactive band identification and improved ERD detection. Neurosci. Lett. 528, 137-142. doi: 10.1016/j.neulet.2012.09.001

Wang, Y., Veluvolu, K. C., and Lee, M. (2013b). Time-frequency analysis of band-limited EEG with BMFLC and Kalman filter for
BCI applications. J. NeuroEng. Rehab. 10:109. doi: 10.1186/1743-000310-109

Wang, Y., Wang, Y.-T., and Jung, T.-P. (2012b). Translation of EEG spatial filters from resting to motor imagery using independent component analysis. PLoS ONE 7:e37665. doi: 10.1371/journal.pone.0037665

Wolpaw, J. R., and McFarland, D. J. (2004). Control of a two-dimensional movement signal by a noninvasive brain-computer interface in humans. Proc. Natl. Acad. Sci. U.S.A. 101, 17849-17854. doi: 10.1073/pnas.0403504101v

Conflict of Interest Statement: The authors declare that the research was conducted in the absence of any commercial or financial relationships that could be construed as a potential conflict of interest.

Copyright (c) 2017 Wang and Veluvolu. This is an open-access article distributed under the terms of the Creative Commons Attribution License (CC BY). The use, distribution or reproduction in other forums is permitted, provided the original author(s) or licensor are credited and that the original publication in this journal is cited, in accordance with accepted academic practice. No use, distribution or reproduction is permitted which does not comply with these terms. 


\section{OPEN ACCESS}

Edited by:

Dingguo Zhang,

Shanghai Jiao Tong University, China

Reviewed by:

Vassiliy Tsytsarev,

University of Maryland, Baltimore,

USA

Victor Manuel Pulgar, Wake Forest School of Medicine, USA

GuangYe $\mathrm{Li}$,

Shanghai Jiao Tong University, China

*Correspondence:

Chaolin Ma

chaolinma@ncu.edu.cn

Jian Huang

huang_jan@mail.hust.edu.cn Jiping $\mathrm{He}$

jiping.he@asu.edu

Specialty section:

This article was submitted to

Neural Technology,

a section of the journal

Frontiers in Neuroscience

Received: 01 October 2016

Accepted: 20 January 2017

Published: 07 February 2017

Citation:

Ma X, Ma C, Huang J, Zhang P, Xu J and He J (2017) Decoding Lower Limb

Muscle Activity and Kinematics from

Cortical Neural Spike Trains during

Monkey Performing Stand and Squat

Movements. Front. Neurosci. 11:44.

doi: 10.3389/fnins.2017.00044

\section{Decoding Lower Limb Muscle} Activity and Kinematics from Cortical Neural Spike Trains during Monkey Performing Stand and Squat Movements

\author{
Xuan $\mathrm{Ma}^{1}$, Chaolin $\mathrm{Ma}^{2,3 *}$, Jian Huang ${ }^{1 *}$, Peng Zhang ${ }^{1}$, Jiang $\mathrm{Xu}^{4}$ and Jiping $\mathrm{He}{ }^{1,3,5,6 *}$ \\ ${ }^{1}$ Neural Interface and Rehabilitation Technology Research Center, School of Automation, Huazhong University of Science \\ and Technology, Wuhan, China, ${ }^{2}$ Center for Neuropsychiatric Disorders, Institute of Life Science, Nanchang University, \\ Nanchang, China, ${ }^{3}$ Center for Neural Interface Design, School of Biological and Health Systems Engineering, Arizona State \\ University, Tempe, AZ, USA, ${ }^{4}$ Department of Rehabilitation Medicine, Tongji Hospital, Tongji Medical College, Huazhong \\ University of Science and Technology, Wuhan, China, ${ }^{5}$ Collaborative Innovation Center for Brain Science, Huazhong \\ University of Science and Technology, Wuhan, China, ${ }^{6}$ Advanced Innovation Center for Intelligent Robots and Systems, \\ Beijing Institute of Technology, Beijing, China
}

Extensive literatures have shown approaches for decoding upper limb kinematics or muscle activity using multichannel cortical spike recordings toward brain machine interface (BMI) applications. However, similar topics regarding lower limb remain relatively scarce. We previously reported a system for training monkeys to perform visually guided stand and squat tasks. The current study, as a follow-up extension, investigates whether lower limb kinematics and muscle activity characterized by electromyography (EMG) signals during monkey performing stand/squat movements can be accurately decoded from neural spike trains in primary motor cortex (M1). Two monkeys were used in this study. Subdermal intramuscular EMG electrodes were implanted to 8 right leg/thigh muscles. With ample data collected from neurons from a large brain area, we performed a spike triggered average (SpTA) analysis and got a series of density contours which revealed the spatial distributions of different muscle-innervating neurons corresponding to each given muscle. Based on the guidance of these results, we identified the locations optimal for chronic electrode implantation and subsequently carried on chronic neural data recordings. A recursive Bayesian estimation framework was proposed for decoding EMG signals together with kinematics from M1 spike trains. Two specific algorithms were implemented: a standard Kalman filter and an unscented Kalman filter. For the latter one, an artificial neural network was incorporated to deal with the nonlinearity in neural tuning. High correlation coefficient and signal to noise ratio between the predicted and the actual data were achieved for both EMG signals and kinematics on both monkeys. Higher decoding accuracy and faster convergence rate could be achieved with the unscented Kalman filter. These results demonstrate that lower limb EMG signals and kinematics during monkey stand/squat can be accurately decoded from a group of M1 neurons with the proposed algorithms. Our findings provide new insights for extending current 
BMI design concepts and techniques on upper limbs to lower limb circumstances. Brain controlled exoskeleton, prostheses or neuromuscular electrical stimulators for lower limbs are expected to be developed, which enables the subject to manipulate complex biomechatronic devices with mind in more harmonized manner.

Keywords: lower limb motor control, primary motor cortex, cortical neuronal encoding, electromyography (EMG), Kalman filter, nonhuman primates

\section{INTRODUCTION}

Brain machine interface (BMI), which translates the activity of cortical neurons into specific motor output commands, has been assumed as a promising approach to help patients who have lost normal motor functions due to spinal cord injury or other neural impairments to restore fundamental skills in daily living (Serruya et al., 2002; Andersen et al., 2004; Schwartz, 2004; Lebedev and Nicolelis, 2006; Hatsopoulos and Donoghue, 2009). Extensive literatures have shown successful realization of the concept of BMI on both nonhuman primates (NHP) and human subjects (Wessberg et al., 2000; Taylor et al., 2002; Carmena et al., 2003; Hochberg et al., 2006; Kim et al., 2008; Velliste et al., 2008; Hwang and Andersen, 2009; Carpaneto et al., 2011; Hochberg et al., 2012; Wodlinger et al., 2014). Among the existing BMI studies, the majority is concerned with using motor cortical activity to decode the movements of upper limbs. Previous literatures have shown two-dimensional or three-dimensional hand/arm trajectories when monkey performing cursor tracking, joystick playing, target-pursuit or center-out tasks can be extracted from neural spike trains using linear regression (Moran and Schwartz, 1999; Gao et al., 2003; Hatsopoulos et al., 2004; Kemere et al., 2004; Paninski et al., 2004; Linderman et al., 2008), artificial neural networks (ANN) (Fang et al., 2010; Huang et al., 2011; Wang et al., 2014), or different variants of Bayesian filtering algorithms (Wu et al., 2002; Brockwell et al., 2004; Wu et al., 2006; Li et al., 2009; Wu et al., 2009; Wang and Principe, 2010; Kang et al., 2015; Menz et al., 2015; Hotson et al., 2016). Analogous approaches were also proposed for predicting arm electromyograph (EMG) signals from the discharge activity of an ensemble of motor neurons or multi-channel local field potentials (Morrow and Miller, 2003; Pohlmeyer et al., 2007; Nazarpour et al., 2012; Shin et al., 2012; Liao et al., 2015). In fact, among the existing population of neural trauma victims, a large portion suffer from the inability of standing and walking (Qin et al., 2010). With the expectation of helping them, researchers have begun to seek possible realizations of BMI systems for lower limb motor function restoration. Some studies have tried to unscramble the information correlating with user intentions (Kilicarslan et al., 2013) or lower limb kinematics (Presacco et al., 2011, 2012; He et al., 2014; Luu et al., 2016) or surface EMG signals (Paek et al., 2013) during treadmill walking or sitting/standing (Bulea et al., 2014) from noninvasive scalp electroencephalographic (EEG) signals. To gain more specific information about how cortical neurons modulate their activities to initiate and control lower limb movements, it is expected to directly collect neural activity from the cerebral cortical areas related to lower limbs using invasive electrodes. However, there are relatively scarce reports about these due to the lack of proper experimental paradigms or apparatus and the difficulties in locating the exact functional cortical areas related to lower limb movements compared to the cases in upper limb. Nicolelis et al. (Fitzsimmons et al., 2009) extracted kinematic parameters for monkey bipedal walking from cortical neuronal ensemble activity and proposed possible approaches to control artificial actuators that reproduce walking patterns.

The construction of BMI lies on the detection of cortical neuronal representations of limb movements. Generally speaking, the control and organization of movements are often considered different for upper limb and lower limb (Winters and Woo, 2012). For example, bipedal locomotion of human is dynamically controlled by the skillfully coordinated activations of the redundant lower limb muscles. Under normal conditions, the activations of these muscles are controlled by both specialized neural circuits in spinal cord known as central pattern generator (CPG) and command signals directly from the brain (Ogihara and Yamazaki, 2001; Vogelstein et al., 2006). Here we concentrate a fundamental lower limb voluntary movement: stand and squat. We previously reported a system for training monkeys to perform visually guided stand and squat tasks ( $\mathrm{Ma}$ and $\mathrm{He}$, 2010). Neuronal ensemble activity from the representation area of the lower limbs in the primary motor cortex (M1), subdermal intramuscular EMG signals in lower limb muscles, and motion trajectories were recorded synchronously with the task. In an acute data recording session, we have detected many M1 neurons tuned to certain task events (Ma et al., 2015).

In this study, we immediately followed our previous work to investigate issues related to neural decoding toward BMI applications. To what extent do neurons in M1 participate the initiation or maintenance of lower limb voluntary movements? Are the neurons showing correlation with certain task events we have observed sufficient for decoding both lower limb muscular activity and kinematics? Which computational models are better for describing the neuronal representation of such visual guided stand/squat movements? We were also curious about whether analogous methods which have been proven practical in upper limb scenario as mentioned above could achieve the same performance in lower limb cases. With the guidance provided by the data collected in acute recording sessions, we identified those locations where more task related neurons clustered, and subsequently carried out chronic electrode array implantation. A recursive Bayesian estimation framework was proposed for decoding EMG signals together with kinematics from cortical spike trains. Two specific algorithms were implemented and compared: a standard Kalman filter and an unscented Kalman filter. For the latter one, an artificial neural network was 
incorporated to deal with the nonlinearity in neural tuning. Our results indicate that lower limb EMG signals and kinematics during monkey stand/squat can be accurately decoded from the activity of M1 neurons.

\section{MATERIALS AND METHODS}

\subsection{Overview}

The data used in this study were collected from two male Rhesus monkeys (monkey 1 and monkey 2). During earlier experimental stage, acute neural recordings were performed for locating the exact brain areas corresponding to the stand/squat movements. After that, chronic electrode array implantation and data recordings were carried out subsequently. In both acute and chronic data recording sessions, subdermal intramuscular EMG signals and limb trajectories were synchronously recorded. Details about animal behavioral training, surgery and some preliminary results of the acute neural data recordings can be found in our previous papers (Ma and He, 2010; Ma et al., 2015). The animal behavioral training and the actual experimental protocols was designed and implemented at Arizona State University (ASU). The experiments were complied with NIH policy on Humane Care and Use of laboratory animals, and were approved by the institutional Animal Care and Use Committee of ASU.

\subsection{Behavioral Tasks}

The monkey performed stand/squat tasks following a series of visual cues in a virtual reality environment. Restrained in a special designed primate chair shown as Figure 1A, with the head and both arms fixed and both legs staying on the movable pedal, the monkey could stand upright by pushing down the movable pedal or squat down by contracting legs back. A marker for optical tracking was placed at the animal's right ankle, corresponding to a red ball in the virtual environment, which was defined as the ankle cursor.

The procedures of the behavioral task in a typical successful trial are shown in Figure 1B. The emerging of a green box at the bottom of the screen indicated the start of a trial (Center On). To proceed, the monkey must squat down and make the ankle cursor touch the green box (Center Hit). Once the box was touched, its color turned to red immediately. A short time later, a green ball (Target) emerged at the top of the screen (Target On). When perceiving this, the monkey should stand up and push down the pedal to move the ankle cursor toward the target until their positions were matched (Target Hit). The time point when the ankle cursor left the bottom box was defined as Center Release, at which the bottom box turned back to green. The monkey was trained to fully extend the legs to hit the target green ball and hold this standing posture up to about $400 \mathrm{~ms}$. After that, it was required to squat by retracting the legs and make the ankle cursor move toward the starting position (Target Release). When the ankle cursor touched the bottom box again (Center Hit Again), the phase of squatting was finished. About $100 \mathrm{~ms}$ later, a trial was completed, and the monkey will receive several drops of water as the reward. Each trial was preceded by an inter-trial interval (varied randomly from 5 to $10 \mathrm{~s}$ ) when the screen was illuminated with bright blank scene to prevent dark adaptation since the room was in low light condition.

\subsection{Data Collection and Preprocessing 2.3.1. Acute Neural Data Recording}

For acute neural data recordings, five independently controllable electrodes were transdurally insert into the target brain area (Figure 1C) in the left hemisphere with a multi-electrode microdrive (Thomas Recording, Germany). Extra-cellular neural signals were amplified, filtered and recorded with a 64 -channel Plexon MAP system (Plexon Inc., Dallas, TX) at $40 \mathrm{kHz} /$ channel sampling rate. Every 40 successful trials were grouped to form a recording dataset. When a dataset was finished, the locations or penetrating depth of the electrodes will be changed. The locations for electrode penetrating can be found in Figures 1D,E for each monkey. Putative neuron units were isolated through spike sorting based on the clustering of detected action potential waveforms in principal components (PC) feature spaces using the Offline Sorter (Plexon Inc., Dallas, TX) software. For each putative neuron, the coordinates corresponding to the electrode were recorded as its location. By calculating the spike counts in consecutive $30 \mathrm{~ms}$ nonoverlap bins, discrete spike times were transformed into a firing rate sequences for further analysis.

\subsubsection{EMG Data Collection}

Separate surgical procedures were performed to implant Teflon coated stainless steel wire electrodes into 8 muscles on each leg, as listed below: right and left soleus (RS and LS), right and left tibialis anterior (RTA and LTA), right and left semitendinosus (RST and LST), right and left recutus femoris (RRF and LRF), right and left extensor digitorum longus (REDL and LEDL), right and left flexor digitorum longus (RFDL and LFDL), right and left adductor magnus (RMG and LMG), right and left flexor hallucis longus (RFHL and LFHL). The EMG signals were recorded at a sampling rate of $1 \mathrm{kHz}$. Only signals from the right leg were used in further analysis. Raw EMG signals were full-wave rectified and filtered with a 4-pole $10 \mathrm{~Hz}$ low-pass Butterworth filter to get nonnegative envelops.

\subsubsection{Kinematic Data Collection}

The position of the ankle LED marker were captured by a VZ 2000 tracker (PTI, Canada) with a sampling interval of $15 \mathrm{~ms}$ and recorded synchronously with the neural data. The velocity of the ankle was approximated by calculating the difference between the position of the marker at current time and that at the previous time, while the acceleration from the velocity in the same way. Since the monkey was restrained on the primate chair when performing repetitive stand/squat behaviors, it is obvious that only the dimension corresponding to the axis perpendicular to the transverse plane ( $\mathrm{z}$ axis) changed significantly in different movement stages. In fact, motion data on the other two dimensions remained almost invariant during the whole movement. Therefore, we defined the kinematics vector as the instantaneous position, velocity and acceleration of the ankle marker at the $\mathrm{z}$ axis. The EMG signals and kinematics were also down-sampled to match the bin size of the neural firing rate sequences. 


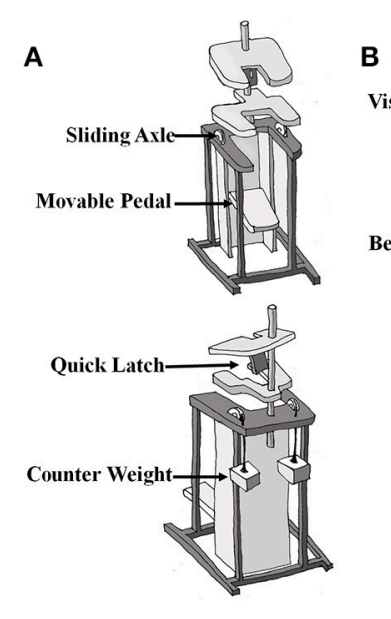

C

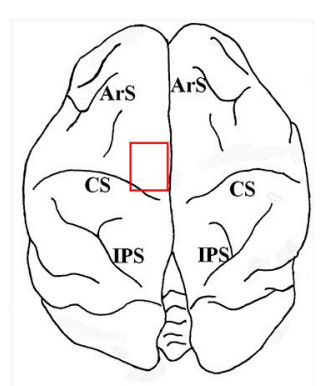

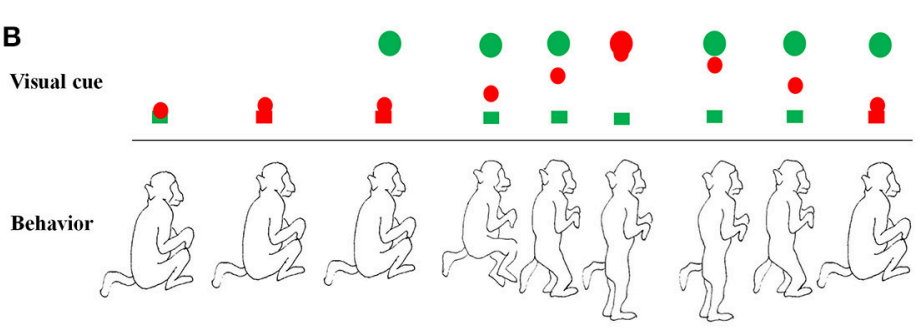

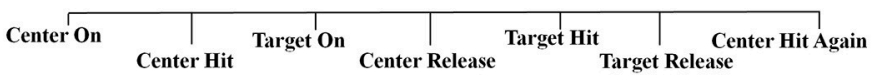

D

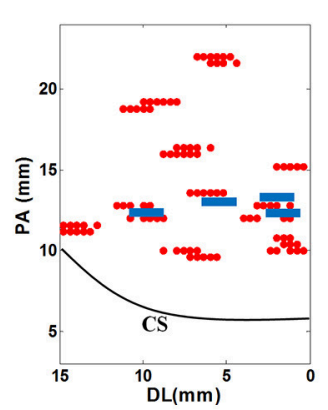

E

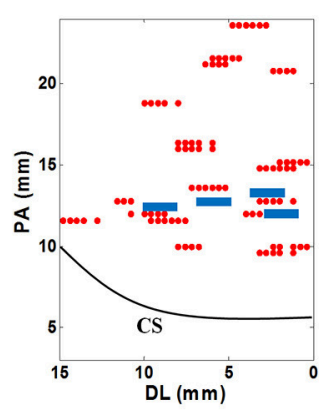

FIGURE 1 | The experimental setup. (A) The specially designed primate chair for the stand/squat tasks. (B) The monkey experimental protocol and the definition of behavior events. (C) The target brain area for electrode implantation. ArS, Arcuate Sulcus; CS, Central Sulcus; IPS, Intraparietal Sulcus. (D) Locations of acute electrode penetrations and chronic electrodes for monkey 1 . The red dots indicate the locations of acute electrode penetrations, and the 4 blue rectangles indicate the locations of the 4 chronically implanted microelectrode arrays. These locations are close to the midline $(0 \mathrm{~mm}$ of the horizontal axis). PA, posterior- anterior. DL, dorsallateral. (E) Locations of acute electrode penetrations and chronic electrodes for monkey 2. The meanings of markers in the plot are the same as those in (D).

\subsubsection{Identifying Neural Facilitation of Muscle Activation}

During the acute data recording session, we have explored a wide region in M1. To determine the optimal locations for chronic electrode implantation, it was necessary to identify those areas which clustered more neurons with high predictive power on EMG signals or motion trajectories. Here we calculated the spike triggered average (SpTA) of each neuron-muscle pair to examine if a given M1 neuron facilitated certain lower limb muscle activation.

Previous studies have demonstrated how the SpTA of EMG signals can be used to identify the synaptic connectivity between cortical neurons and the motor units of activated limb muscles (Fetz and Cheney, 1980; McKiernan et al., 1998; Griffin et al., 2008). By taking into account post-spike characteristics of the averaged EMG such as the onset latency, peak magnitude and duration, SpTA can be used as a predictor of the quality and strength of facilitative contribution that a detected cortical neuron may have in relation to a given muscle (Baker and Lemon, 1998; Aguayo et al., 2009).

For each neuron-muscle pair, the SpTA was calculated by averaging full-wave rectified EMG segments for all successful trials starting $20 \mathrm{~ms}$ prior to until $60 \mathrm{~ms}$ after a spike event. For each SpTA, a baseline mean and standard deviation (SD) were calculated using the $20-10 \mathrm{~ms}$ of the SpTA prior to the spike event. The onset latency is defined as the point that the SpTA crosses a threshold defined by the baseline mean +2 SD after the spiking events for the first time. Similarly, offset latency is defined as the point that the SpTA falls below a threshold (baseline mean $-2 \mathrm{SD})$. The peak magnitude is defined as the peak value of an SpTA after the spiking events minus the baseline mean. The peak width at half magnitude (PWHM) is defined as the width of the SpTA at a level that is half the measured peak. A neuron-muscle pair will be considered to have no dependency relationship and eliminated from statistics if the onset latency of the SpTA is less than $5 \mathrm{~ms}$ or greater than $15 \mathrm{~ms}$, or the PWHM of the SpTA is less than $0.75 \mathrm{~ms}$ or greater than $9 \mathrm{~ms}$.

After scanning enough datasets, a number of neurons which were considered to facilitate the activation of each given muscle were identified from the ensemble. The densities of different muscle-innervating neurons were calculated with regard to each muscle as the number of identified neurons divided by the total number of neurons recorded in an unit brain area. By plotting a series of density contours, the spatial distributions of 
these muscle-innervating neurons can be directly perceived and utilized to determine the locations for chronic implantation.

\subsubsection{Chronic Neural Data Recording}

About 1 month after the acute recording session, new surgeries were performed to implant 4 microelectrode arrays chronically into the selected brain areas for each monkey (Figures 1D,E). There were 16 polyimide insulated tungsten electrodes on each array as the recording channels, which were arranged with two rows. The diameter of each electrode was 50 microns. The spacing between electrodes in each row was 250 microns and the spacing between each row was 500 microns. In the following chronic neural data recording session, behavior tasks for monkey training and protocols for data recordings remained consistent.

\subsection{Neural Decoding Algorithms}

The purpose of the neural decoding algorithm is to use cortical spike trains to estimate the lower limb EMG signals together with the kinematics during the stand/squat tasks. We formulated the problem in a recursive Bayesian estimation framework (Bergman, 1999), where the activity of each muscle, characterized by corresponding EMG signals, or the motion of the limb, represented by the kinematics, were viewed as hidden states of a dynamic system, while cortical spiking data acquired by the implanted microelectrodes as constantly updated measurements to the system. In the following sections, we first introduce the general basics of the recursive Bayesian estimation theory we count on, and then describe the two specific algorithms for practical implementation in this study.

\subsubsection{Recursive Bayesian Estimation}

Here $\mathbf{x}_{k}$ denotes the system states at the $\mathrm{k}$-th time instant $t_{k}=k \Delta t$. For EMG decoding, $\mathbf{x}_{k}$ is specified as $\mathbf{x}_{k}=$ $\left[u_{1}(k), u_{2}(k), \cdots, u_{M}(k)\right]^{T}$, where $u_{m}(k), m=1, \cdots, M$ is the EMG envelop amplitude of the $m$ th muscle at time $t_{k}$, and $M$ is the total number of muscles. For kinematics decoding, $\mathbf{x}_{k}$ is specified as $\mathbf{x}_{k}=[z(k), v(k), a(k)]^{T}$, representing the $z$ position, $z$-velocity and $z$-acceleration of the ankle marker at $t_{k}$. Meanwhile, $\mathbf{y}_{k}$ denotes the measurement to the system at $t_{k}$, which is in fact a vector containing the spike counts of $C$ neurons in the $k$ th time bin. The estimation consists of two steps: prediction and update. The aim is to construct the a-posterior probability density function $p\left(\mathbf{x}_{k} \mid \mathbf{y}_{1: k}\right)$ for $\mathbf{x}_{k}$ conditioned on all available measurements up to $t_{k}\left(\mathbf{y}_{1: k}\right)$.

In the prediction step, the a-priori distribution of $\mathbf{x}_{k}$ is estimated from the states at previous time instants. In terms of an usually made assumption that $\mathbf{x}_{k}$ is generated by a Markov process, we have

$$
p\left(\mathbf{x}_{k} \mid \mathbf{y}_{1: k-1}\right)=\int p\left(\mathbf{x}_{k} \mid \mathbf{x}_{k-1}\right) p\left(\mathbf{x}_{k-1} \mid \mathbf{y}_{k-1}\right) d \mathbf{x}_{k-1}
$$

where $p\left(\mathbf{x}_{k} \mid \mathbf{x}_{k-1}\right)$ models how the system state evolves from current time to the next, and $p\left(\mathbf{x}_{k-1} \mid \mathbf{y}_{k-1}\right)$ corresponds to the a-posterior probability for the previous time instant.

In the update step, the a-posterior prediction is obtained by correcting the a-prior prediction with the information provided by the new measurement data $\mathbf{y}_{k}$ :

$$
p\left(\mathbf{x}_{k} \mid \mathbf{y}_{1: k}\right)=\frac{p\left(\mathbf{y}_{k} \mid \mathbf{x}_{k}\right) p\left(\mathbf{x}_{k} \mid \mathbf{y}_{1: k-1}\right)}{p\left(\mathbf{y}_{k} \mid \mathbf{y}_{1: k-1}\right)}=\alpha p\left(\mathbf{y}_{k} \mid \mathbf{x}_{k}\right) p\left(\mathbf{x}_{k} \mid \mathbf{y}_{1: k-1}\right)
$$

where $p\left(\mathbf{y}_{k} \mid \mathbf{x}_{k}\right)$ reflects the mapping between the system states and the measurements, called the likelihood term, and $p\left(\mathbf{y}_{k} \mid \mathbf{y}_{1: k-1}\right)$ is a normalization constant $\alpha$ that can usually be ignored.

Following Equations (1) and (2), $p\left(\mathbf{x}_{k} \mid \mathbf{y}_{1: k}\right)$ can be calculate recursively. In practical implementation, it requires to have closed form solutions to these general equations. Here we explored two specific algorithms, the Kalman filter (Grewal, 2011) and the unscented Kalman filter (UKF) (Julier et al., 1995) for estimating the EMG signals and kinematics recursively from the cortical signals, and details are described as follows.

\subsubsection{Kalman Filter}

For the implementation of the Kalman filter, the state transition and the measurement model were both assumed linear and with Gaussian noise. Supposing model parameters are time invariant, these models can be written as:

$$
\begin{aligned}
& \mathbf{x}_{k}=\mathbf{A} \mathbf{x}_{k-1}+\mathbf{w}_{k} \\
& \mathbf{y}_{k}=\mathbf{H} \mathbf{x}_{k}+\mathbf{q}_{k}
\end{aligned}
$$

where $\mathbf{A}$ is the state transition matrix, $\mathbf{H}$ is the measurement matrix. For EMG decoding, $\mathbf{A}, \mathbf{W} \in \mathbb{R}^{M \times M}, \mathbf{H} \in \mathbb{R}^{C \times M}$, and for kinematics decoding, $\mathbf{A}, \mathbf{W} \in \mathbb{R}^{3 \times 3}, \mathbf{H} \in \mathbb{R}^{C \times 3}$. The random variables $\mathbf{w}_{k} \sim \mathcal{N}(0, \mathbf{W})$ and $\mathbf{q}_{k} \sim \mathcal{N}(0, \mathbf{Q})$ denote the process and the measurement noise respectively. $\mathbf{A}$ and $\mathbf{H}$ are fitted from the training data via a least square linear regression approach, while $\mathbf{Q}$ and $\mathbf{W}$ are regression residuals. Let $\hat{\mathbf{x}}_{k}^{-}$and $\hat{\mathbf{x}}_{k}$ represent the a-prior estimation and the a-posterior estimation of the system state, the error covariance matrices can be defined as

$$
\mathbf{P}_{k}^{-}=E\left[\left(\mathbf{x}_{k}-\hat{\mathbf{x}}_{k}^{-}\right)\left(\mathbf{x}_{k}-\hat{\mathbf{x}}_{k}^{-}\right)^{T}\right]
$$

for the a-prior estimation, and

$$
\mathbf{P}_{k}=E\left[\left(\mathbf{x}_{k}-\hat{\mathbf{x}}_{k}\right)\left(\mathbf{x}_{k}-\hat{\mathbf{x}}_{k}\right)^{T}\right]
$$

for the a-posterior estimation.

The estimation started from a prediction step:

$$
\begin{aligned}
& \hat{\mathbf{x}}_{k}^{-}=\mathbf{A} \hat{\mathbf{x}}_{k-1} \\
& \mathbf{P}_{k}^{-}=\mathbf{A} \mathbf{P}_{k-1} \mathbf{A}^{T}+\mathbf{W}
\end{aligned}
$$

Subsequently, $\hat{\mathbf{x}}_{k}$ was obtained via an update step with the new measurement data at $\mathbf{t}_{k}$ :

$$
\begin{aligned}
& \hat{\mathbf{x}}_{k}=\hat{\mathbf{x}}_{k}^{-}+\mathbf{K}_{k}\left(\mathbf{y}_{k}-\mathbf{H} \hat{\mathbf{x}}_{k}^{-}\right) \\
& \mathbf{P}_{k}=\left(\mathbf{I}-\mathbf{K}_{k} \mathbf{H}\right) \mathbf{P}_{k}^{-}
\end{aligned}
$$

where $\mathbf{K}_{k}=\mathbf{P}_{k}^{-} \mathbf{H}^{T}\left(\mathbf{h} \mathbf{H} \mathbf{P}_{k}^{-} \mathbf{H}^{T}+\mathbf{Q}\right)^{-1}$ is known as the Kalman gain. 


\subsubsection{ANN Based Neural Tuning Model}

For the update procedure of recursive Bayesian estimation indicated by Equation (2), knowledge about the mapping from system states to the measurements is required. Here it means a more appropriate model describing the neuronal modulation of muscular activity and limb movements should be selected. Previous studies have indicated that different hierarchically organized components, including the motor cortex and several subcortical structures and also circuits in the spinal cord, are involved in the neural motor control process (Kandel et al., 1995; Harel et al., 2008). Therefore, much nonlinearity exists for the neuronal modulations to movements, which is difficult for modeling with classical parametric techniques. The linear model (4) is actually a straightforward approximation for the neuronal modulations. Although easy for implementation, it may not be consistent with the actual neural system. It is expected to have a model which can reflect the intrinsic nonlinearity of the neural control process.

Here we utilized a feed-forward single hidden layer ANN to construct the model for neuronal modulations to EMG and kinematics. With the ability of approximating any complex nonlinear mappings directly from the input samples, ANN has been widely applied in many fields (Suykens et al., 2012), and is assumed to be efficient for the modeling of the neuronal modulations. The proposed feed-forward network included an input layer, a hidden layer and an output layer. Each neuron in one layer had directed weighted connections to those in the subsequent layer. Sigmoid functions were applied as the activation functions for the hidden layer neurons. Parameters of the network, including the connection weights and activation thresholds, were determined through a learning process. The modeling errors were fed back through the network via a back-propagation mechanism, and a gradient descent method was adopted for parameters adjusting in every learning epoch (Graupe, 2013). The ANN based measurement model can be written as:

$$
\mathbf{y}_{k}=\operatorname{net}\left(\mathbf{x}_{k}\right)
$$

where net $(\cdot)$ represents the neural network. For EMG decoding, $n e t(\cdot): \mathbb{R}^{M} \rightarrow \mathbb{R}^{C}$ and for kinematics decoding, net $(\cdot): \mathbb{R}^{3} \rightarrow \mathbb{R}^{C}$.

\subsubsection{Unscented Kalman Filter}

As an improved algorithm to the Kalman filter, the unscented Kalman filter (UKF) incorporates nonlinear models in estimation, and uses a nonstochastic simulation to approximate the nonlinearity (Julier et al., 1995). The basic procedure for the UKF is the unscented transform, a deterministic sampling technique for approximating a given probability distribution with a series of weighted sample points (Sigma points), which has been proven superior to other ordinary linearization or approximation methods (Wan and Van Der Merwe, 2000).

In our implementation, the system states were still assumed to evolve according to a linear function as Equation (3) in the prediction step, while the mapping from the system states $\mathbf{x}_{k}$ to the measurements $\mathbf{y}_{k}$ was computed according to the ANN based model (7) in the update step.
Let $d$ denote the dimension of the state variable. A set of $2 d+1$ Sigma points were generated from $\hat{\mathbf{x}}_{k}^{-}$and $\mathbf{P}_{k}^{-}$through the unscented transform:

$$
\begin{aligned}
& \mathbf{X}_{k}^{-(0)}=\hat{\mathbf{x}}_{k}^{-} \\
& \mathbf{X}_{k}^{-(i)}=\hat{\mathbf{x}}_{k}^{-}+\sqrt{d+\lambda}\left[\sqrt{\mathbf{P}_{k}^{-}}\right]_{i} \\
& \mathbf{X}_{k}^{-(i+d)}=\hat{\mathbf{x}}_{k}^{-}-\sqrt{d+\lambda}\left[\sqrt{\mathbf{P}_{k}^{-}}\right]_{i}, i=1,2, \ldots, d
\end{aligned}
$$

where $[\cdot]_{i}$ denotes the $i$-th row of the matrix, $\lambda=\alpha^{2}(d+\kappa)-d$ is a scaling parameter and $\alpha$ and $\kappa$ determine the spread of the sigma points around $\hat{\mathbf{x}}_{k}^{-}$. The square root of the matrix $\mathbf{P}_{k}^{-}$ was obtained by calculating the Cholesky decomposition. These Sigma points were propagated through Equation (7) to get the transformed results:

$$
\hat{\mathbf{Y}}_{k}^{i}=\operatorname{net}\left(\mathbf{X}_{k}^{-(i)}\right), i=0,1, \ldots, d, \ldots, 2 d
$$

The predicted mean and covariance of the measurement, and the cross-covariance between the state and the measurement were calculated from these transformed Sigma points:

$$
\begin{aligned}
& \hat{\mathbf{Y}}_{k}=\sum_{i=0}^{2 d} w_{i} \hat{\mathbf{Y}}_{k}^{(i)} \\
& \mathbf{P}_{k}^{Y Y}=\sum_{i=0}^{2 d} w_{i}\left(\hat{\mathbf{Y}}_{k}^{(i)}-\hat{\mathbf{y}}_{k}\right)\left(\hat{\mathbf{Y}}_{k}^{(i)}-\hat{\mathbf{y}}_{k}\right)^{T}+\mathbf{Q} \\
& \mathbf{P}_{k}^{X Y}=\sum_{i=0}^{2 d} w_{i}\left(\mathbf{X}_{k}^{-(i)}-\hat{\mathbf{x}}_{k}^{-}\right)\left(\hat{\mathbf{Y}}_{k}^{(i)}-\hat{\mathbf{y}}_{k}\right)^{T}+\mathbf{Q}
\end{aligned}
$$

where $w_{i}$ are a series of constant weights:

$$
\begin{aligned}
& w_{0}=\frac{\lambda}{d+\lambda} \\
& w_{i}=\frac{1}{2(d+\lambda)}, i=1,2, \ldots, 2 d
\end{aligned}
$$

The a-posterior estimate $\hat{\mathbf{x}}_{k}$ and corresponding error covariance matrix $\mathbf{P}_{k}$ were finally obtained by:

$$
\begin{aligned}
& \hat{\mathbf{x}}_{k}=\hat{\mathbf{x}}_{k}^{-}+\mathbf{K}_{k}\left(\mathbf{y}_{k}-\hat{\mathbf{y}}_{k}\right) \\
& \mathbf{P}_{k}=\mathbf{P}_{k}^{-}-\mathbf{K}_{k} \mathbf{P}_{k}^{Y Y} \mathbf{K}_{k}^{T}
\end{aligned}
$$

where the Kalman gain was calculated as $\mathbf{K}_{k}=\mathbf{P}_{k}^{X Y}\left(\mathbf{P}_{k}^{Y Y}\right)^{-1}$. The initial value for the error covariance matrix $\mathbf{P}_{0}$ was set to the unit matrix. $\mathbf{P}_{k}$ will show a convergent feature as the calculation of Equations (8)-(11) at each time instant.

\subsubsection{Performance Evaluation}

Two metrics, Pearson's correlation coefficient (CC) and the signal to noise ratio (SNR) were used for the performance evaluation of the algorithms.

CC is calculated from the actual signal $x$ and the estimated value $\hat{x}$ for each channel by:

$$
C C=\frac{\sum_{k=1}^{K}\left(x_{k}-\bar{x}\right)\left(\hat{x}_{k}-\overline{\hat{x}}\right)}{\sqrt{\sum_{k=1}^{K}\left(x_{k}-\bar{x}\right)^{2}} \sqrt{\sum_{k=1}^{K}\left(\hat{x}_{k}-\overline{\hat{x}}\right)^{2}}}
$$


where $K$ is the length of the signal. An CC value close to 1 indicates better fit between the real and estimated data.

SNR is defined as the ratio between the variance of the actual data and the mean square error (MSE) of the estimates, and is calculated as:

$$
S N R=10 \log _{10} \frac{\sum_{k=1}^{K}\left(x_{k}-\bar{x}\right)^{2}}{\sum_{k=1}^{K}\left(\hat{x}_{k}-x_{k}\right)^{2}}
$$

for each channel. It is quantified in decibels $(\mathrm{dB})$ scale, where 0 $\mathrm{dB}$ means the signal and the noise are in equal proportion, and larger positive value means more useful signals are extracted. The SNR is sensitive to the errors introduced by amplitude scaling and offsets, and has also been adopted in Fitzsimmons et al. (2009).

\section{RESULTS}

\subsection{Synchronized Multiple Data Recordings}

Synchronized multiple data recorded during 2 successive trials from monkey 1 is shown in Figure 2, including the spike trains of 16 task-related neurons (Figure 2A), the raw EMG signals of 5 right lower limb muscles (Figure 2B), and the trajectories of the marker attaching on the monkey's right ankle (Figure 2C). The occurring time instants for certain behavior events are indicated by short bars at the bottom. Data within the inter-trial interval (about $5 \mathrm{~s}$ ) are omitted for displaying. Notably, the firing rates of most cortical neurons kept low when resting (quiet squatting, Center Hit to Target On). An ensemble of neurons began to discharge more frequently after target presenting (Target On), while their firing rates have been on a significant increase during such pre-movement epoch (Target On to Center Release). Later, amplitude oscillations were observed for some EMG channels around the initiation of the standing (Center Release), indicating the activation of corresponding muscles. For the subsequent epochs involving standing up (Center Release to Target Hit), standing still (Target Hit to Target Release), and squatting down (Target Release to Center Hit Again), some obvious event-related neural discharging patterns can be revealed via a series of perievent histogram analyses, which have been reported in our previous study (Ma et al., 2015). Compared to the previous work, here we may go a further step to explore the neuronmuscle associations more quantitatively for the neural decoding problems based on these observational or statistical results.

\subsection{Spatial Distributions of Different Muscle-Innervating Neurons}

In the acute neural recording session, we explored the M1 area in the left hemisphere by changing the locations and penetrating depths of independent movable electrodes at a distance of 1 $\mathrm{mm}$. The exact range of the region was in the coordinate system determined by the stereotaxic apparatus: $8.00-22.00 \mathrm{~mm}$ from anterior to posterior (PA), $0.00-16.00 \mathrm{~mm}$ from dorsal to lateral (DL), and $0.00-5.00 \mathrm{~mm}$ in the depth. A total number of 96 datasets were collected, and 1598 task-related neurons were isolated (909 from Monkey 1, 689 from Monkey 2).
Based on the SpTA analyses mentioned above, the neurons which may facilitate the activation of each given muscle were identified from the ensemble. Figure 3 shows the density contours of the identified neurons with regard to 8 muscles in the horizontal (Figure 3A) and the coronal (Figure 3B) view using the data from monkey 1 . The central sulcus (CS), as a prominent landmark, is also indicated designedly in the plots. All density values are normalized to $[0,1]$ and smoothed by a median filter. The hotter color indicates the higher density. These plots are to some extent "maps" for finding the neurons which may contribute to improving the accuracy of the EMG decoding. In the horizontal view, several concentration centers of muscleinnervating neurons for the 8 muscles can be clearly identified (Figure 3A). In the coronal view, we noticed that the depth between $0.5 \sim 2.5 \mathrm{~mm}$ was where muscle-innervating neurons aggregated most densely (Figure 3B).

It is notable that some density contours are quite analogous, like those for the RRF-innervating neurons and the RTAinnervating neurons. But clear divergence exists for some other groups, like the RRF and the REDL. Here we evaluated the similarities of the spatial distributions of different muscleinnervating neurons via a distance measure based cluster method. To be specific, the density contours for different types of muscle-innervating neurons can be viewed essentially a series of matrices. The Euclidean distances between every two matrices were calculated for a cluster analysis. The results of the cluster are shown in Figure 4, for the horizontal (Figure 4A) and the coronal view (Figure 4B) respectively, using the data from Monkey 1. Several groups of muscles could be identified from the cluster results: RRF and RMG, RFHL and RFDL, RTA and REDL, either in the horizontal or the coronal view. Dramatically, the results using the data from Monkey 2 are almost the same. We seek possible illustrations for these results with some knowledge in anatomy. In fact, RMG and RRF are both thigh muscles. RFHL and RFDL are leg muscles, and are both innervated by the Tibial Nerve. RTA and REDL are also leg muscles, but are innervated by the Deep Fibular Nerve (Moore et al., 2011). These results suggest that, for muscles sharing larger similarities in anatomy, the corresponding M1 neurons have closer spatial distributions.

\subsection{EMG Decoding}

The chronic neural recording session lasted 2 weeks and 13 datasets were collected for each monkey. In each dataset, $40 \pm$ 5 successful stand/squat trials were recorded, and an average of 34 task-related neurons can be identified from all detected units. A 4-fold cross-validation was employed for model parameter estimation, in which 3 -folds were used for training and 1-fold for testing.

Figure 5 shows typical plots of the predicted EMG envelops(blue dashed line, by the standard Kalman filter; red dash dot line, by the UKF) from a test subset in comparison with the actual EMG signals(dark gray solid line) during 4 successive trials conducted by Monkey 1 . The amplitude of all signals are normalized to the interval $[0,1]$. The exact time of 5 important behavior events are indicated in each subplot (light gray dashed line). It is perceived from the plots that both 


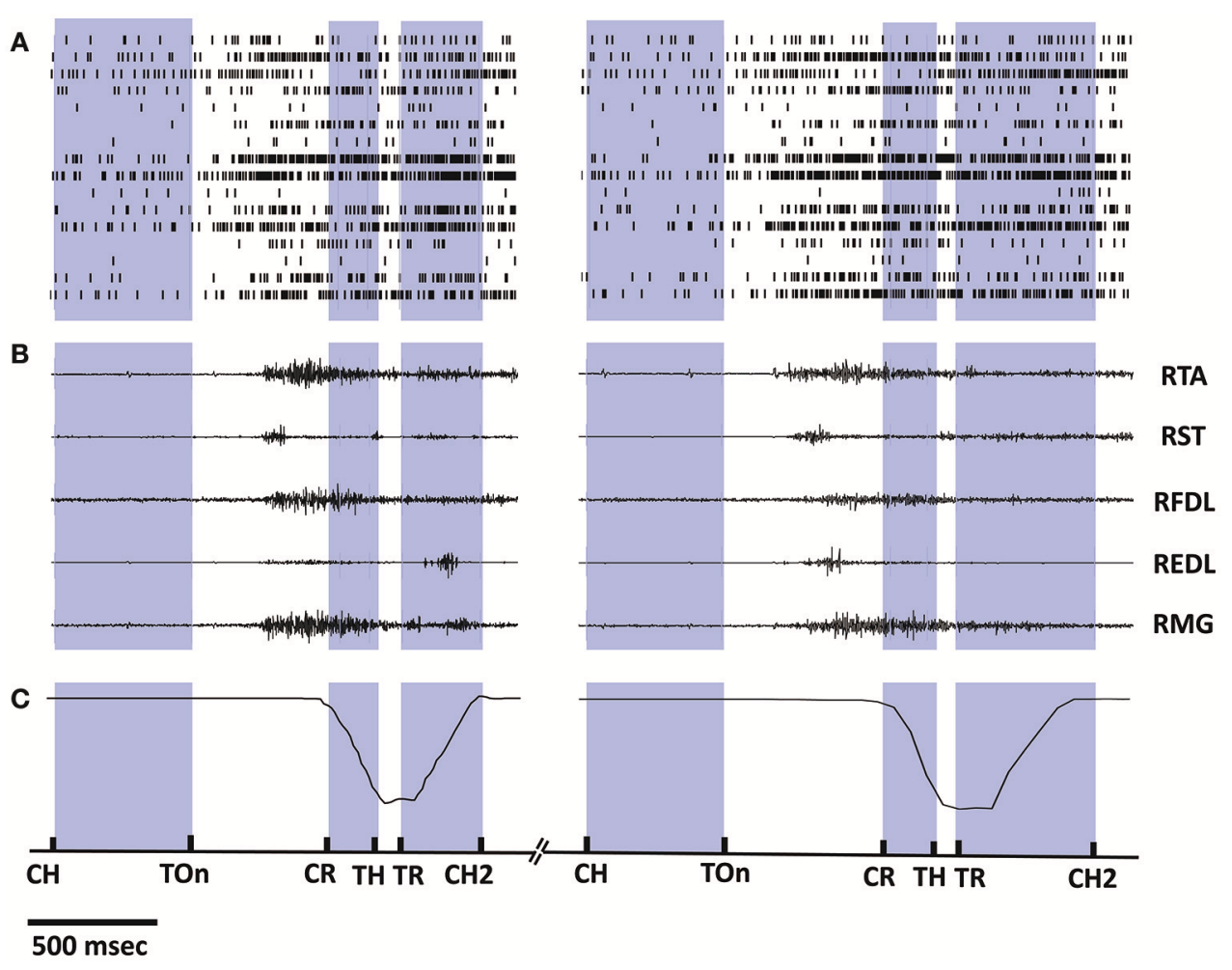

FIGURE 2 | Synchronized multiple data recorded during 2 successive trials from monkey 1. (A) Spike trains of 16 cortical neurons. Short bars indicates the actual spiking time and each row corresponds to a neuron. (B) EMG signals of 5 lower limb muscles. (C) Position trajectories of the marker on the right ankle of the monkey. The exact time instances of important behavior events are indicated on the bottom time line by short bars: Center Hit (CH), Target On (TOn), Center Release $(\mathrm{CR})$, Target Hit (TH), Target Release (TR), Center Hit Again (CH2), and the exact meaning of these events can be found in Section 2.2. Data within the inter-trial interval $(2-5 \mathrm{~s})$ are omitted for displaying.

of the proposed decoding methods were capable to accurately predict the overall trends of the EMG signals during the standing and squatting task, as well as some significant waveform features such as the quiescence before Target On, the gradual amplitude increase during the pre-movement epoch (Target On to Center Release), and the transient changes during the movement stages. Compared to the standard Kalman filter, the UKF method could produce more approximate EMG envelop values. These results clearly imply that not only the timings for muscle activation/relaxation, but also the strength of lower limb muscular activity can be extracted from the cortical ensemble signals.

Figure 6 shows the CC and SNR between the predicted and real EMG signals in each channel for the 2 monkeys, demonstrating the mean and the standard error of the mean (SEM). It is suggested that the UKF based decoding method can achieve higher CC and SNR values. For Monkey 1, the mean CC values for 7 channels of EMG signals (RFDL, RRF, RST, RTA, RMG, RS, REDL) were greater than 0.75 , and the mean SNR values for 6 channels (RFDL, RRF, RST, RTA, RMG, RS) exceeded 3.5, when using the UKF as the decoding algorithm. In contrast, when using the standard Kalman filter to predict the EMG signals, there were only 4 channels (RFDL, RRF, RTA, and RMG) of which the mean CC values exceeded 0.75 , and only 3 channels (RRF, RTA, and RMG) of which the mean SNR value was greater than 3.5. Similar results can be perceived for Monkey 2. Afterwards, we performed a right tail $t$-test with 416 observations (13 datasets, 4 -folds, 8 channels of EMG signals) and significance level $\alpha=0.05$ for each monkey to test whether the UKF based method improved the EMG decoding performance significantly. The $t$-test verified that the predicted signals with the UKF had higher CC and SNR values compared to those with the standard Kalman filter for all EMG channels $(p<$ 0.001). In fact, the UKF improved the CC for $13.6 \%$ and the SNR for $17.5 \%$.

\subsection{Kinematics Decoding}

The same procedures for model training and testing as in EMG decoding were adopted for the kinematics decoding. Figure 7 presents the predicted trajectories for the position, velocity and acceleration of the ankle marker in comparison with the real data (dark gray solid line). It is shown that both methods can provide reliable tracking for the trajectories of the position when the legs of the monkey were moving either downward or upward. For the velocity, the predictions can approximate the overall trend but discrepancies were found within some time intervals. However, both methods cannot achieve satisfactory predictions for the acceleration. Table 1 


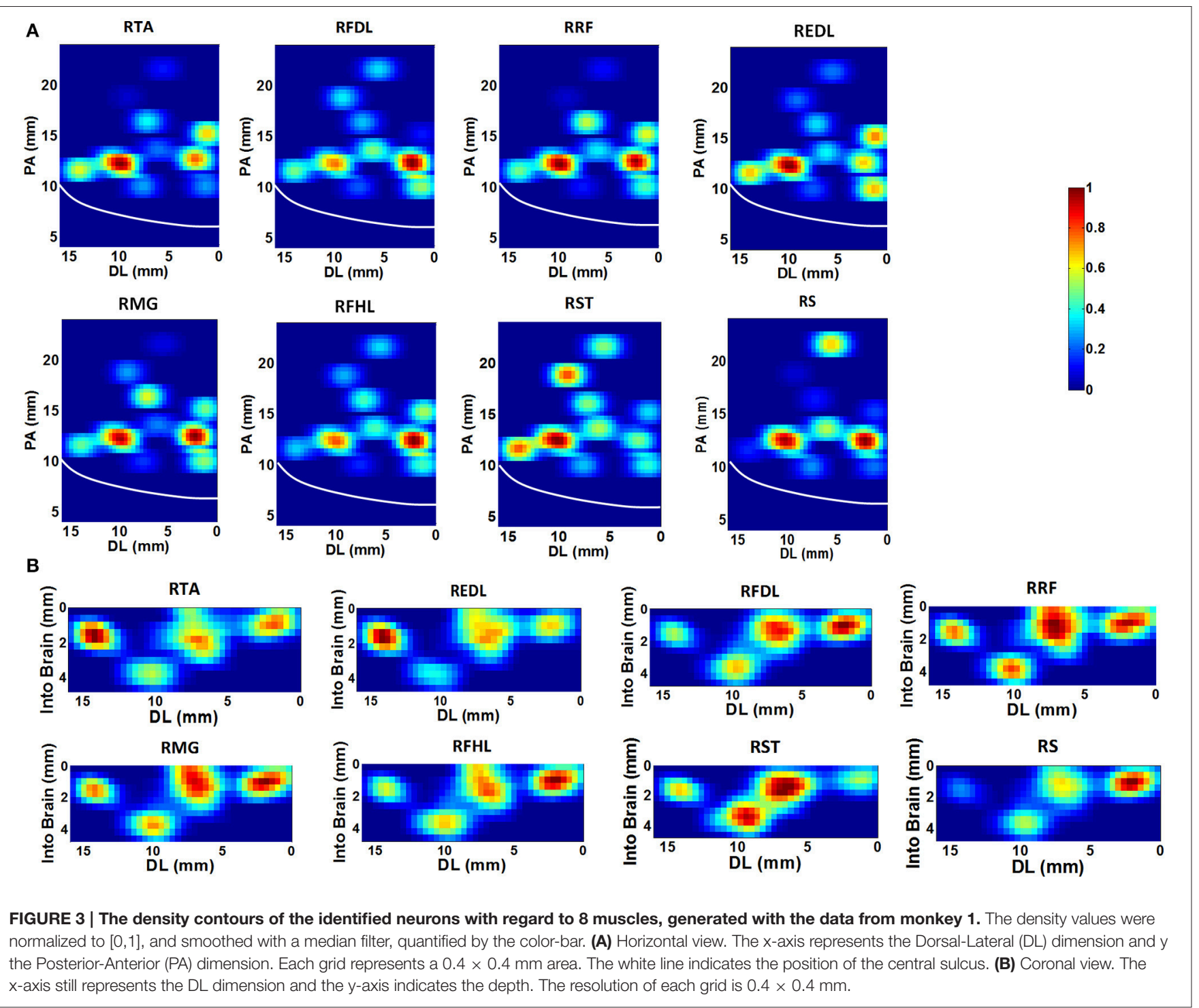

A

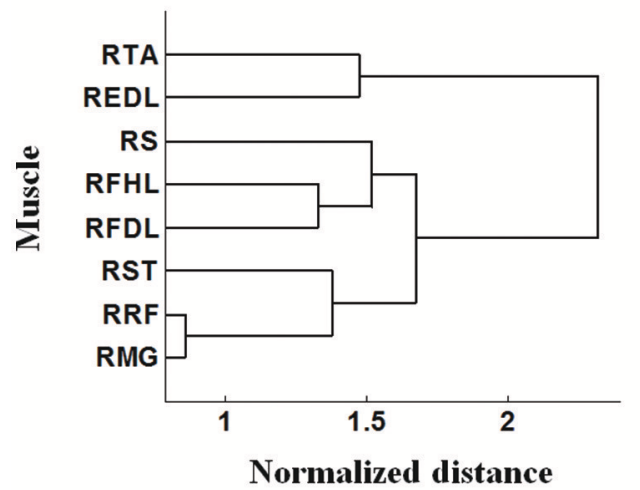

B

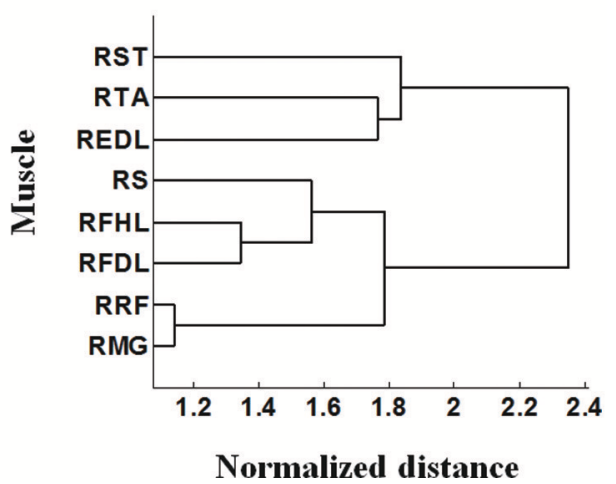

FIGURE 4 | Cluster analysis based on the pattern of density contours of different muscle-innervating neurons corresponding to each given muscle. (A) Clustering results on the horizontal view (DL-PA). (B) Clustering results on the coronal view (DL-depth). 

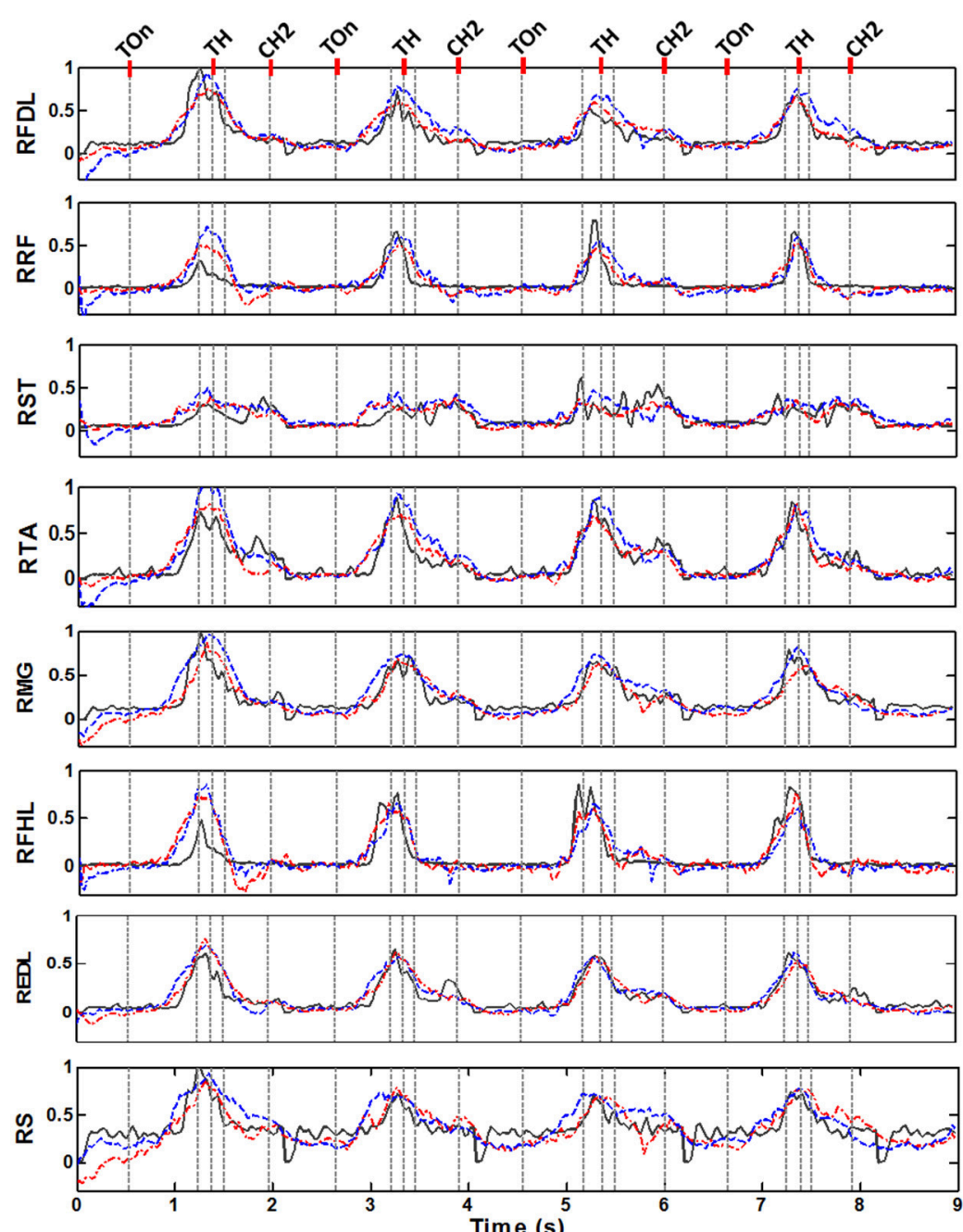

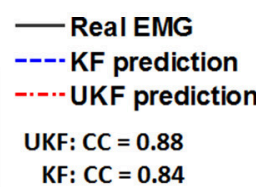

UKF: $\mathrm{CC}=0.81$

KF: $\mathrm{CC}=\mathbf{0 . 7 2}$

\section{UKF: $C C=0.78$ \\ $\mathrm{KF}: \mathrm{CC}=0.71$}

UKF: $C C=0.87$

$\mathrm{KF}: \mathrm{CC}=\mathbf{0 . 8 1}$

\section{UKF: $\mathrm{CC}=\mathbf{0 . 8 8}$}

KF: $\mathrm{CC}=\mathbf{0 . 8 3}$

UKF: $\mathrm{CC}=\mathbf{0 . 7 5}$

$\mathrm{KF}: \mathrm{CC}=\mathbf{0 . 7 1}$

UKF: $C C=0.85$

$\mathrm{KF}: \mathrm{CC}=\mathbf{0 . 8 2}$

UKF: $\mathrm{CC}=\mathbf{0 . 7 7}$

KF: $\mathrm{CC}=\mathbf{0 . 7 2}$

FIGURE 5 | Normalized actual EMG envelop waveforms (dark gray solid) and the reconstructed results by the standard Kalman filter (KF, blue dashed) and the UKF (red dash dot) in four successive stand/squat trials. The data are from one of the chronic datasets from Monkey 1 . The subplot in each row corresponds to a muscle, where the name is labeled on the left. The occurring time of 5 important behavior events are indicated by a series of light gray dashed lines in each subplot, where the names of 3 events could be found on the top line of the figure: Target On (TOn), Target Hit (TH), and Center Hit Again (CH2). The event occurring right before Target Hit is Center Release, and the event right after Target Hit is Target Release. The exact meaning of the behavior events can be found in Section 2.2. The CC between the actual and predicted signals are listed on the right of each subplot for the two algorithms.

summarizes the CC and SNR between the predicted and real trajectories (mean \pm SEM). For tracking the position, high mean CC and SNR values were achieved. A right tail $t$-test with 52 observations (13 datasets, 4-folds) and significance level $\alpha=$ 0.05 for each monkey verified the superiority of the UKF based method $(p<0.001)$. For approximating the velocity, the CC and SNR values were relatively low, but higher than 0.5. For decoding the acceleration, both algorithms encountered not good enough performance, with the mean of the $\mathrm{CC}<0.5$, and SNR $<1$.

\subsection{Stability Analysis of the Neural Decoding Algorithms}

We examined the stability of the neural decoding algorithms by investigating the convergence of the a-posteriori estimate error covariance matrix $\mathbf{P}_{k}$ and the Kalman gain $\mathbf{K}_{k}$. The matrix $\mathbf{P}_{k}$ defines the estimation error at each time step, while $\mathbf{K}_{k}$ represents the relative importance of the measurement residual with respect to the a-prior estimation $\hat{\mathbf{x}}_{k}^{-}$. For reliable estimations, it is expected that the errors converge to considerable small values as the recursive iteration increases. As $\mathbf{P}_{k}$ gets small, it means there is no requirement to alter the estimation too much at each time instant, therefore, $\mathbf{K}_{k}$ will also get small. So the numerical stability of $\mathbf{P}_{k}$ and $\mathbf{K}_{k}$ are appropriate indexes for evaluating the stability of the neural decoding algorithms. Figure 8 plots the $L^{2}$ norm of $\mathbf{P}_{k}$ and $\mathbf{K}_{k}$ across iterations. It is shown that the $L^{2}$ norm of both $\mathbf{P}_{k}$ and $\mathbf{K}_{k}$ converge to a small value after about 20-30 iterations. It is also suggested that the UKF can achieve faster convergence rate for $\mathbf{P}_{k}$ and $\mathbf{K}_{k}$. 
A

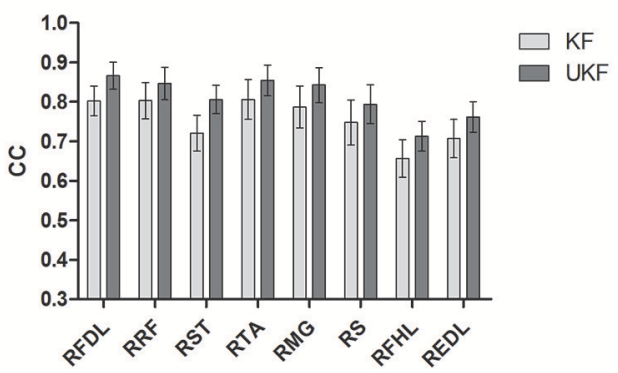

C

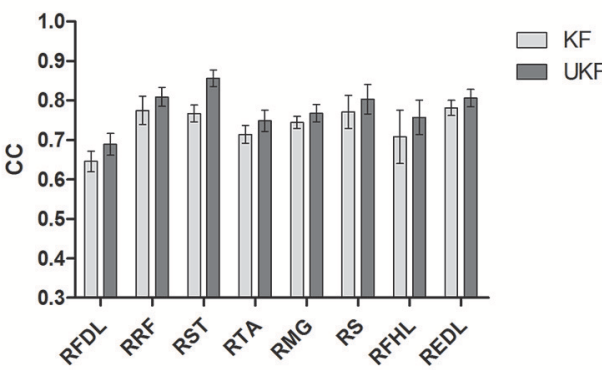

B

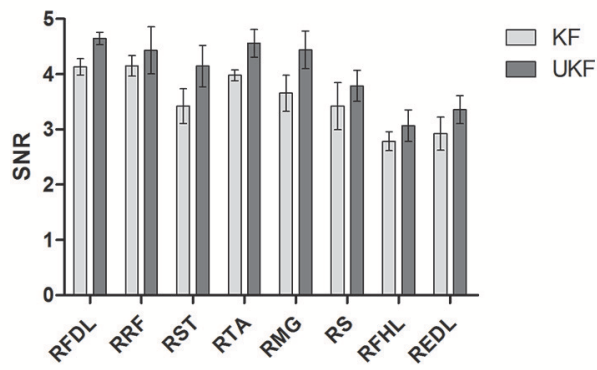

D

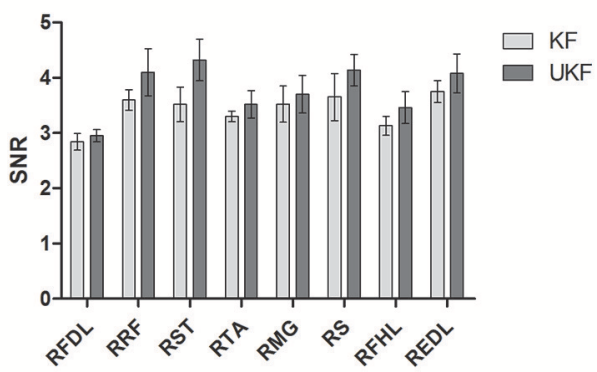

FIGURE 6 | The CC and SNR for the EMG decoding. The mean and the standard error of the mean (SEM) are shown. The SEM is indicated by the error bar. (A,B) are for Monkey 1. (C,D) are for Monkey 2.
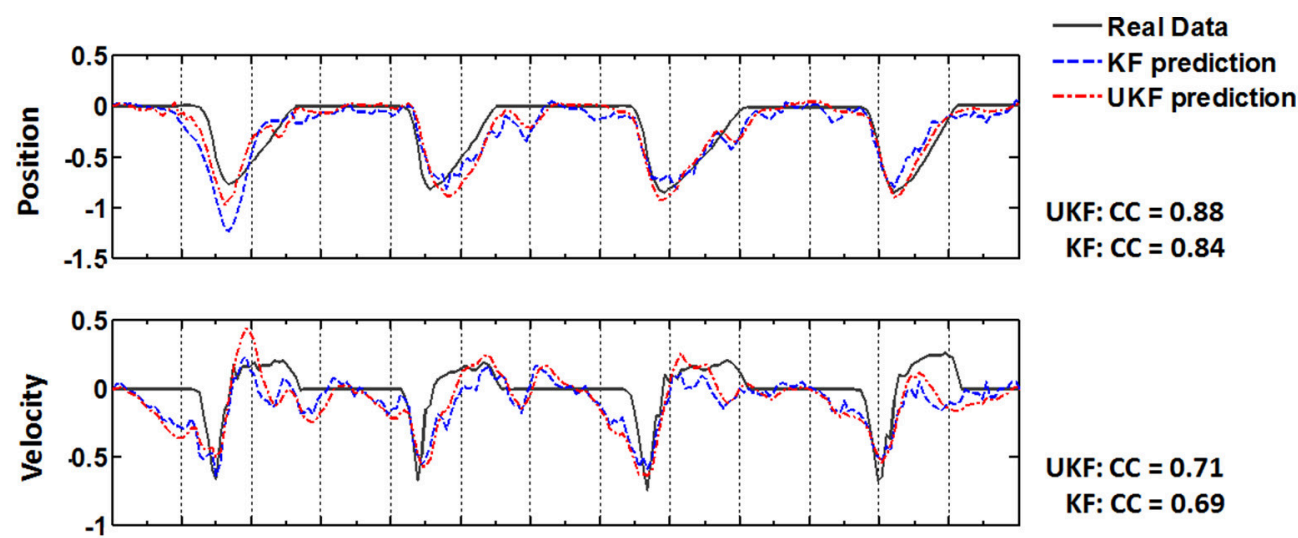

UKF: $\mathrm{CC}=\mathbf{0 . 7 1}$

$\mathrm{KF}: \mathrm{CC}=0.69$

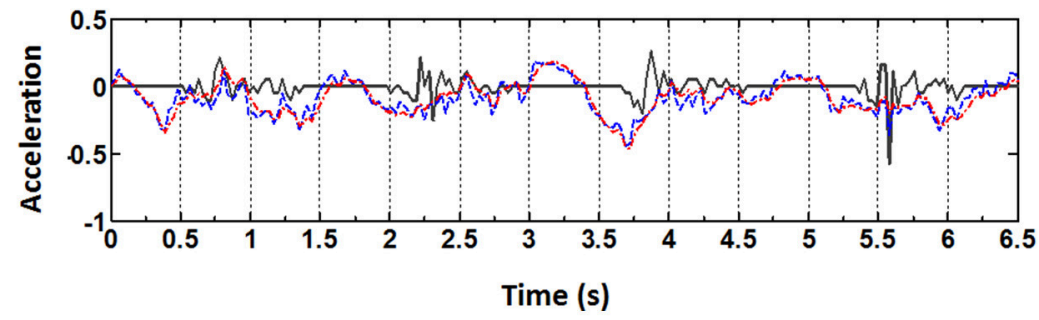

UKF: $\mathrm{CC}=0.33$

$\mathrm{KF}: \mathrm{CC}=\mathbf{0 . 3 8}$

FIGURE 7 | Real position, velocity and acceleration (dark gray solid) and the reconstructed results by the standard Kalman filter (blue dashed, KF) and the UKF (red dash dot) in four successive trials. The CC values between the actual and reconstructed trajectories are listed on the right for each subplot. 
TABLE 1 | The CC and SNR values between the predicted and actual data for kinematics decoding (mean \pm SEM).

\begin{tabular}{|c|c|c|c|c|c|}
\hline & & \multicolumn{2}{|c|}{ Monkey 1} & \multicolumn{2}{|c|}{ Monkey 2} \\
\hline & & CC & $\operatorname{SNR}(\mathrm{dB})$ & CC & SNR(dB) \\
\hline Prediction using & Position & $0.85 \pm 0.05$ & $5.53 \pm 0.44$ & $0.83 \pm 0.05$ & $5.05 \pm 0.52$ \\
\hline the standard & Velocity & $0.61 \pm 0.06$ & $1.58 \pm 0.75$ & $0.64 \pm 0.05$ & $1.67 \pm 0.84$ \\
\hline Kalman filter & Acceleration & $0.37 \pm 0.05$ & $0.55 \pm 0.34$ & $0.33 \pm 0.03$ & $0.45 \pm 0.33$ \\
\hline Prediction using & Position & $0.90 \pm 0.05$ & $6.12 \pm 0.83$ & $0.88 \pm 0.05$ & $5.93 \pm 0.74$ \\
\hline \multirow[t]{2}{*}{ the UKF } & Velocity & $0.66 \pm 0.08$ & $1.89 \pm 0.95$ & $0.68 \pm 0.08$ & $1.52 \pm 1.17$ \\
\hline & Acceleration & $0.36 \pm 0.10$ & $0.53 \pm 0.58$ & $0.34 \pm 0.08$ & $0.47 \pm 0.27$ \\
\hline
\end{tabular}
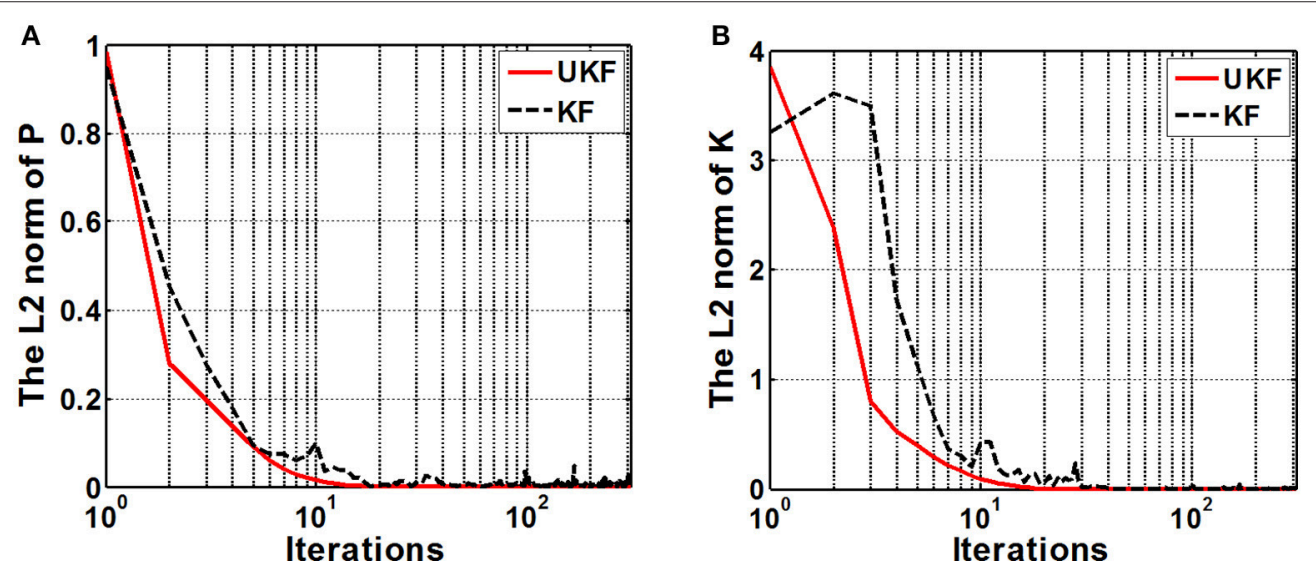

FIGURE 8 | The $L^{2}$ norm of the a-posteriori estimate error covariance matrix $\mathbf{P}_{\boldsymbol{k}}(\mathbf{A})$ and the Kalman gain matrix $\mathbf{K}_{k}(\mathbf{B})$. The $x$-axis indicates the iterations $(k)$ and is expressed in logarithm form. Red solid line and black dashed line represent the UKF and the standard Kalman filter situation respectively.

\section{DISCUSSION}

In this study, we demonstrate feasible approaches for decoding lower limb muscular activity and kinematics from cortical neural signals during monkey performing a series of visual cued stand/squat tasks in a virtual environment. This study provides important insight regarding the cortical innervation of voluntary lower limb movements, while previous work have paid ample attentions to the upper limbs. The results clearly demonstrate that lower limb EMG signals and kinematics can be predicted from the firing rate sequences of multiple cortical neurons, which suggests the possibility for more comprehensive lower limb BMI systems.

The work in Bulea et al. (2014) has demonstrated sitting and standing intention can be discriminated from scalp EEG signals. However, more details about the timing and strength of multiple muscle activity and kinematics are still difficult to be extracted from the general noninvasive signals. There are not too many literatures concerning lower limb motor control using invasive methods due to the lack of systematic experimental protocols like Center-Out in upper limb circumstance and the uncertainty about brain areas for electrode implantation. Similar to the work in Fitzsimmons et al. (2009), we performed invasive neural recordings, but we concentrated voluntary stand/squat instead of the cyclic bipedal walking. By exploring a wide region in M1 with movable electrodes, we have identified the spatial distributions of different muscle-innervating neurons corresponding to each given muscle, which can provide important guidance for implanting chronic electrodes. The recursive Bayesian estimation based methods proposed here are proved effective for implementation, and could produce accurate EMG and kinematic estimation.

In Pohlmeyer et al. (2007), the prediction of EMG signals of several arm and hand muscles in button pressing or grasp movements captured most EMG features, and got $R^{2}$ values between $0.60 \sim 0.70$. In $\mathrm{Li}$ et al. (2009), the moving trajectories of the hand of the monkey can be reconstructed off-line with SNR between 2 and $5 \mathrm{~dB}$, or CC between 0.6 and 0.9. In our study, the neural decoding accuracies of the 8 lower limb muscles and the ankle marker position are comparable to these studies (Figure 6, Table 1). Such results suggest that current upper limb BMI design concepts can be extended to lower limb occasions.

\subsection{Implications for the Cortical Neural Encodings of Voluntary Lower Limb Movements}

Previous upper limb BMI studies found neurons in M1 (Moran and Schwartz, 1999; Paninski et al., 2004; Wahnoun et al., 2006; Truccolo et al., 2008), ventral premotor cortex (Prabhu et al., 
2009; Carpaneto et al., 2011), dorsal premotor cortex (Hao et al., 2013) encode various task related parameters, like reaching direction, joint orientation, or hand gestures, and have developed different neural decoding algorithms based on such observations (Hatsopoulos et al., 2004; Hao et al., 2013, 2014). As indicated in Winters and Woo (2012), motor control process of lower limbs is often considered different from that of upper limbs. We wonder either abstract representation of movements or explicit encodings of muscular activity and limb states can be extracted from the neural responses in M1 lower limb area, which determines the feasibility for constructing lower limb BMI systems.

Our findings suggest M1 neural ensemble may provide specific encodings to the execution of such visual guided standing/squatting task. When the visual cue was launched at Target On, the animal commenced to plan its movement in order to get success (Figure 1B). At this moment, the firing rates of a large portion of neurons have begun to increase. After the movement was initiated (at Center Hit), a good control about the position of the legs must be maintained since the monkey was required to match the ankle marker to the figures on the screen in right sequence, while most neurons were observed keeping relatively high firing rate. We think this fact reflects the sustained activity of task-related neurons from planning to executing in voluntary movement. In contrast, most recorded M1 neurons showed high variant firing patterns and exhibited weak modulation to the movement in a treadmill bipedal walking task (Huo et al., 2010). The execution of walking can be attributed to sort of cyclic movements, during which low level centers, like the CPG, take over more control details. Thus, the synchronization of M1 neural activity were absent. However, also in Huo et al. (2010), almost all recorded neurons became increasingly synchronized in firing patterns to each step, especially before and during stance phase, when the monkey walked on the treadmill after a spinal cord hemi-section surgery. These observations imply that M1 neurons are intensively involved into the control of walking when spinal cord is impaired. For the visual guided standing/squatting tasks, accurate responses to certain cues must be produced in time, of which the complexity is far more than the cyclic walking, and beyond the spinal cord circuits can handle. To sum up, M1 neurons participate the specific control of lower limb movements more in those circumstances during which low level centers are damaged or unable to produce accurate responses for purposeful tasks. Compared to another bipedal walking study (Fitzsimmons et al., 2009), the SNR for the predictions of EMG signals on the right leg musculature was $1.55 \pm 0.39$, which was lower than ours (Figure 6). This fact indicates the neural activity patterns during the visual cued standing/squatting is sufficient for achieving better decoding performance than the cyclic walking.

\subsection{Functional Division of M1 Neurons Corresponding to Muscles}

To determine the best locations for chronic electrode implantation, we investigated the contribution of a number of M1 neurons to facilitate given muscles. In fact, the elaborate organization of M1 neurons in lower limb control process can be revealed by plotting the density contours of neurons corresponding to each given muscle (Figure 3). The detected neurons appear sort of functional division corresponding to muscles or muscle groups. On one hand, there are significant discrepancies among the spatial distributions of different muscle-innervating neurons. On the other hand, analogous or overlapping spatial distributions of neurons can be identified for muscles innervated by the same nerve (Figure 4). The spatial range of the lower limb representative area in M1 has been repeatedly confirmed in the last decades using imaging techniques such as functional magnetic resonance imaging (fMRI), but the exact muscle-neuron associations and the specific locations of different muscle-innervating neurons are still not clear. Compared to these methods, the simultaneous multiple data recordings in this study achieved better time and spatial resolutions. Since the actual number of neurons is huge, we cannot yet declare we have constructed an exact map between M1 neurons and lower limb muscles. The most direct benefit is that we are more clear about which locations are optimal for the chronic electrode implantation. Meanwhile, these findings are helpful for understanding the organizations of the motor control pathway for lower limbs.

\subsection{Nonlinear Method Achieves Better Decoding Performance}

We try to seek better computational models to relate the multidimensional neural firing rate sequence to the EMG signals or kinematics. It is noticeable that higher decoding accuracy was achieved when using the UKF with an ANN based neural tuning model to estimate the EMG signals and the trajectories of the ankle position. Considering the intrinsic nonlinearity in the neural system as described before, a linear model as 4 may not fit the real neural data very well, though it is easy for implementation. The superiority of incorporating nonlinear models for neural decoding has been revealed by some previous studies. In Li et al. (2009), a quadratic neural tuning model relating position, velocity with the binned neural firing rates can considerably improve the decoding accuracy of the $2 \mathrm{D}$ hand moving trajectory. In Pohlmeyer et al. (2007), the $R^{2}$ between the real and predicted upper limb EMG signals was 0.69 when using linear estimation method, and increased to 0.75 when using nonlinear estimation method. The results in these reports are consistent with ours as mentioned above. The ANN reflected the nonlinearities between the system states to be estimated and the observed multi-dimensional neural firing rate sequences, and improved the decoding accuracy significantly when it was incorporated as the measurement model in the recursive estimation process.

\subsection{EMG Decoding vs. Kinematics Decoding}

We notice that the decoding accuracies for the EMG signals and kinematics are different. As shown in Figure 6, the mean CC values for the 8 channels of decoded EMG signals are all above 0.6 , and the mean SNR values are all above $2 \mathrm{~dB}$. For the trajectories of the ankle marker position, the prediction is accurate (mean $\mathrm{SNR}>5$ and mean $C C>0.75$ ). For velocity, 
the prediction captures the overall trend (SNR around 2 and $\mathrm{CC}$ around 0.6). However, the estimation of the acceleration is not satisfactory. In fact, the same situation have been noticed in previous studies. In Wu et al. (2002), the reconstruction for hand position and velocity are satisfactory while for acceleration is poor. They thought the acceleration was a second order difference of position, thus the measurements of it tended to be very noisy in real data. They also indicated the acceleration has a weak effect on the model relative to position and velocity.

After Target On, the monkey was in a plan stage, but actual movement has not been initiated until Center Release. The actual position, velocity and acceleration during the plan stage retained still (Figure 7, real data). Although the overall prediction accuracy for the ankle position was very high, the estimated values often failed to capture such quiescence (Figure 7, upper panel, predicted data). However, many neurons have begun to fire intensively and the amplitudes of the EMG signals have shown an analogous activated profile at the same time (Figure 7). In contrast to the ankle position, the estimated EMG values in this stage captured the dramatic increase of the real signals. Generally speaking, EMG signals are stochastic and noisy, and have greater bandwidth, which suggests more difficulties for the neural decoding algorithms. So the mean CC and SNR for the estimated ankle position trajectories are higher than those for the estimated EMG signals. But compared to the kinematics, EMG signals are closer to the cortical neural activity. When initiating and maintaining a movement, M1 neurons modulate their firing patterns to facilitate the contractile force in the skeletal muscles via a hierarchic downstream pathway. The planned movement are produced by the synergic activity of multiple muscles, and is more indirect with the cortical neurons. So the estimated EMG signals from the cortical activity can capture the trend of the real signals well even in the pre-movement epoch.

\subsection{Potential Applications for FES and Mind Controlled Prostheses}

The BMI technology constructs a bridge between the subject's mind and the external actuators. It has been assumed as the most promising technique for neuro-prostheses control and neurorehabilitation implementation. Here we expect the findings in our explorations on monkey stand/squat behaviors could be helpful for transforming BMI design concepts to potential clinical applications.

One of the possible applications is using the predicted EMG signals as the inputs of the functional electric stimulation (FES) to activate specific lower limb muscles of the paralyzed patients. FES is an effective method for preventing the muscle mass loss and promoting the functional recovery after nervous system lesion. In Moritz et al. (2008), a monkey can directly control stimulation of muscles using the activity of neurons in M1, thereby restoring goal-directed movements to a transiently paralyzed arm. Here we have demonstrated the timing and strength of the activity of multiple lower limb muscles can be predicted from cortical neural spike trains. With the predicted data, the timing and intensity of the FES for target muscles can be tuned according to the subject's mind to conduct more purposeful stimulation. Such approaches are expected to achieve similar results as in Moritz et al. (2008) for restoring various voluntary lower limb movements, like standing and squatting.

The predicted lower limb kinematics could be incorporated as the control source to drive artificial legs, exoskeleton or walking assistant robots designed for lower limb paralyzed patients or amyotrophic lateral sclerosis (ALS) patients. The actuators can be controlled to track the estimations of position and velocity extracted from cortical neural signals. Compared to traditional methods using accelerometer or other sensors (Reza et al., 2014), brain control enables the patients to manipulate complex biomechatronic devices in a more harmonized manner.

Meanwhile, the proposed algorithms are easy for implementation in micro-chip or real time embedded system. The Kalman filters does not require long windows to collect data as the multiple linear regression method and provides an explicit generative model, which is propitious to real time implementations for capturing rapid motions. The realization of the ANN and the unscented transform have also been proven feasible on current programmable micro controllers.

\section{CONCLUSIONS}

In this study, we focus on neural signals collected during monkey performing visual guided stand/squat tasks. We have demonstrated lower limb EMG signals and ankle moving kinematics during stand/squat could be accurately decoded from a group of M1 neurons with the proposed algorithms. Our findings provide new insights for extending current BMI design concepts on upper limbs to lower limb circumstances. Brain controlled exoskeleton, prostheses or neuromuscular electrical stimulators for lower limbs are expected to be developed for the future extension of this work.

\section{AUTHOR CONTRIBUTIONS}

Conceived the project and designed the technical detail: $\mathrm{JH}$ (Jiping He). Performed the experiments: CM, JX. Analyzed the data: XM, CM, PZ, JH (Jian Huang). Drafted the manuscript: XM, CM. Revised the manuscript: XM, CM, JH (Jian Huang), JH (Jiping He).

\section{FUNDING}

This work is supported by the National Natural Science Foundation of China (31460263, 61233015), 973 Project of China (2013CB329506), and Jiangxi Natural Science Foundation (12004630).

\section{ACKNOWLEDGMENTS}

Authors wish to express their appreciation for the technical helps provided by Dr. Hang Zhang, Dr. Xinying Cai, Mr. Tedd Brandon and other members of the Neural Control Laboratory in Arizona State University. 


\section{REFERENCES}

Aguayo, J. J., Zhang, H., and He, J. (2009). "Time series analysis of neural facilitation of muscle activation recorded using chronically implanted microwire arrays," in 2009 International Conference on Complex Medical Engineering (Tempe, AZ), 1-5.

Andersen, R. A., Burdick, J. W., Musallam, S., Pesaran, B., and Cham, J. G. (2004). Cognitive neural prosthetics. Trends Cogn. Sci. 8, 486-493. doi: 10.1016/j.tics.2004.09.009

Baker, S. N., and Lemon, R. N. (1998). Computer simulation of post-spike facilitation in spike-triggered averages of rectified emg. J. Neurophysi. 80, 1391-1406.

Bergman, N. (1999). Recursive Bayesian Estimation. Doctoral dissertation, Department of Electrical Engineering, Linköping University, Linköping Studies in Science and Technology, 579.

Brockwell, A. E., Rojas, A. L., and Kass, R. E. (2004). Recursive bayesian decoding of motor cortical signals by particle filtering. J. Neurophysiol. 91, 1899-1907. doi: $10.1152 /$ in. 00438.2003

Bulea, T. C., Prasad, S., Kilicarslan, A., and Contreras-Vidal, J. L. (2014). Sitting and standing intention can be decoded from scalp eeg recorded prior to movement execution. Front. Neurosci. 8:376. doi: 10.3389/fnins.2014.00376

Carmena, J. M., Lebedev, M. A., Crist, R. E., O’Doherty, J. E., Santucci, D. M., Dimitrov, D. F., et al. (2003). Learning to control a brainmachine interface for reaching and grasping by primates. PLoS Biol. 1:e42. doi: 10.1371/journal.pbio.0000042

Carpaneto, J., Umiltà, M. A., Fogassi, L., Murata, A., Gallese, V., Micera, S., et al. (2011). Decoding the activity of grasping neurons recorded from the ventral premotor area F5 of the macaque monkey. Neuroscience 188, 80-94. doi: 10.1016/j.neuroscience.2011.04.062

Fang, H., Wang, Y., and He, J. (2010). Spiking neural networks for cortical neuronal spike train decoding. Neural Comput. 22, 1060-1085. doi: $10.1162 /$ neco.2009.10-08-885

Fetz, E. E., and Cheney, P. D. (1980). Postspike facilitation of forelimb muscle activity by primate corticomotoneuronal cells. J. Neurophysiol. 44, 751-772.

Fitzsimmons, N. A., Lebedev, M. A., Peikon, I. D., and Nicolelis, M. A. (2009). Extracting kinematic parameters for monkey bipedal walking from cortical neuronal ensemble activity. Front. Integr. Neurosci. 3:3. doi: 10.3389/neuro.07. 003.2009

Gao, Y., Black, M. J., Bienenstock, E., Wu, W., and Donoghue, J. P. (2003). “A quantitative comparison of linear and non-linear models of motor cortical activity for the encoding and decoding of arm motions," in International IEEE Embs Conference on Neural Engineering, 2003. Conference Proceedings (Capri), 189-192.

Graupe, D. (2013). Principles of Artificial Neural Networks, Vol. 7. Singapore: World Scientific.

Grewal, M. S. (2011). Kalman Filtering. Berlin: Springer.

Griffin, D. M., Hudson, H. M., Belhaj-Saif, A., McKiernan, B. J., and Cheney, P. D. (2008). Do corticomotoneuronal cells predict target muscle emg activity? J. Neurophysiol. 99, 1169-1186. doi: 10.1152/jn.00906.2007

Hao, Y., Zhang, Q., Zhang, S., Zhao, T., Wang, Y., Chen, W., et al. (2013). Decoding grasp movement from monkey premotor cortex for real-time prosthetic hand control. Chinese Sci. Bullet. 58, 2512-2520. doi: 10.1007/s11434-013-5840-0

Hao, Y., Zhang, Q., Controzzi, M., Cipriani, C., Li, Y., Li, J., et al. (2014). Distinct neural patterns enable grasp types decoding in monkey dorsal premotor cortex. J. Neural Eng. 11:066011. doi: 10.1088/1741-2560/11/6/066011

Harel, R., Asher, I., Cohen, O., Israel, Z., Shalit, U., Yanai, Y., et al. (2008). Computation in spinal circuitry: lessons from behaving primates. Behav. Brain Res. 194, 119-128. doi: 10.1016/j.bbr.2008.07.013

Hatsopoulos, N. G., and Donoghue, J. P. (2009). The science of neural interface systems. Annu. Rev. Neurosci. 32:249. doi: 10.1146/annurev.neuro.051508. 135241

Hatsopoulos, N. G., Joshi, J., and O'Leary, J. G. (2004). Decoding continuous and discrete motor behaviors using motor and premotor cortical ensembles. J. Neurophysiol. 92, 1165-1174. doi: 10.1152/jn.01245.2003

He, Y., Nathan, K., Venkatakrishnan, A., Rovekamp, R., Beck, C., Ozdemir, R., et al. (2014). "An integrated neuro-robotic interface for stroke rehabilitation using the NASA X1 powered lower limb exoskeleton," in International Conference of the IEEE Engineering in Medicine and Biology Society (Chicago, IL), 3985-3988.
Hochberg, L. R., Bacher, D., Jarosiewicz, B., Masse, N. Y., Simeral, J. D., Vogel, J., et al. (2012). Reach and grasp by people with tetraplegia using a neurally controlled robotic arm. Nature 485, 372-375. doi: 10.1038/nature11076

Hochberg, L. R., Serruya, M. D., Friehs, G. M., Mukand, J. A., Saleh, M., Caplan, A. H., et al. (2006). Neuronal ensemble control of prosthetic devices by a human with tetraplegia. Nature 442, 164-171. doi: 10.1038/nature04970

Hotson, G., Smith, R. J., Rouse, A. G., and Schieber, M. H. (2016). High precision neural decoding of complex movement trajectories using recursive bayesian estimation with dynamic movement primitives. IEEE Robot. Autom. Lett. 1, 676-683. doi: 10.1109//ra.2016.2516590

Huang, J., Wang, Y., Zhu, Q., and He, J. (2011). Modelling of monkey's motor cortical signals by an extended adaptive liquid state machine: an integrated procedure from model, identification, experiments, data fitting, to validation. Int. J. Syst. Control Commun. 3, 287-301. doi: 10.1504/IJSCC.2011.042435

Huo, Y., Ma, C., Zhang, H., Li, P., and He, J. (2010). "Synchronization of motorcortical neurons after spinal cord injury," in 2010 4th International Conference on Bioinformatics and Biomedical Engineering (Chengdu: IEEE), $1-4$.

Hwang, E. J., and Andersen, R. A. (2009). Brain control of movement execution onset using local field potentials in posterior parietal cortex. J. Neurosci. 29, 14363-14370. doi: 10.1523/JNEUROSCI.2081-09.2009

Julier, S. J., Uhlmann, J. K., and Durrant-Whyte, H. F. (1995). "A new approach for filtering nonlinear systems," in Proceedings of the 1995 American Control Conference, Vol. 3 (Seattle, WA: IEEE), 1628-1632.

Kandel, E. R., Schwartz, J. H., and Jessel, T. M. (1995). Essentials of Neural Science and Behavior. New York, NY: McGraw-Hill.

Kang, X., Sarma, S. V., Santaniello, S., Schieber, M., and Thakor, N. V. (2015). Task-independent cognitive state transition detection from cortical neurons during 3D reach-to-grasp movements. IEEE Trans. Neural Syst. Rehabil. Eng. 23, 676-682. doi: 10.1109/TNSRE.2015.2396495

Kemere, C., Shenoy, K. V., and Meng, T. H. (2004). Model-based neural decoding of reaching movements: a maximum likelihood approach. IEEE Trans. Biomed. Eng. 51, 925-932. doi: 10.1109/TBME.2004.826675

Kilicarslan, A., Prasad, S., Grossman, R. G., and Contreras-Vidal, J. L. (2013). "High accuracy decoding of user intentions using eeg to control a lower-body exoskeleton," in International Conference of the IEEE Engineering in Medicine and Biology Society (Osaka), 5606-5609.

Kim, S. P., Simeral, J. D., Hochberg, L. R., Donoghue, J. P., and Black, M. J. (2008). Neural control of computer cursor velocity by decoding motor cortical spiking activity in humans with tetraplegia. J. Neural Eng. 5, 455. doi: $10.1088 / 1741-2560 / 5 / 4 / 010$

Lebedev, M. A., and Nicolelis, M. A. (2006). Brain-machine interfaces: past, present and future. Trends Neurosci. 29, 536-546. doi: 10.1016/j.tins.2006. 07.004

Li, Z., O’Doherty, J. E., Hanson, T. L., Lebedev, M. A., Henriquez, C. S., and Nicolelis, M. A. L. (2009). Unscented kalman filter for brain-machine interfaces. PLOS ONE 4:e6243. doi: 10.1371/journal.pone.0006243

Liao, Y., She, X., Wang, Y., Zhang, S., Zhang, Q., Zheng, X., et al. (2015). Monte carlo point process estimation of electromyographic envelopes from motor cortical spikes for brain-machine interfaces. J. Neural Eng. 12:066014. doi: 10.1088/1741-2560/12/6/066014

Linderman, M., Santhanam, G., Kemere, C., Gilja, V., O'Driscoll, S., Yu, B., et al. (2008). Signal processing challenges for neural prostheses. IEEE Signal Process. Mag. 25, 18-28. doi: 10.1109/MSP.2008.4408439

Luu, T. P., He, Y., Brown, S., Nakagame, S., and Contreras-Vidal, J. L. (2016). Gait adaptation to visual kinematic perturbations using a real-time closed-loop brain-computer interface to a virtual reality avatar. J. Neural Eng. 13:036006. doi: 10.1088/1741-2560/13/3/036006

Ma, C., and He, J. (2010). A method for investigating cortical control of stand and squat in conscious behavioral monkeys. J. Neurosci. Methods 192, 1-6. doi: 10.1016/j.jneumeth.2010.06.027

Ma, C., Ma, X., Zhang, H., Xu, J., and He, J. (2015). Neuronal representation of stand and squat in the primary motor cortex of monkeys. Behav. Brain Funct. 11:1. doi: 10.1186/s12993-015-0061-0

McKiernan, B. J., Marcario, J. K., Karrer, J., and Cheney, P. D. (1998) Corticomotoneuronal postspike effects in the shoulder, elbow, wrist, digit, and intrinsic hand muscles during a reach and prehension task. J. Neurophysiol. 80 1961-1980. 
Menz, V. K., Schaffelhofer, S., and Scherberger, H. (2015). Representation of continuous hand and arm movements in macaque areas $\mathrm{ml}$, f5, and aip: a comparative decoding study. J. Neural Eng. 12, 5203-5211. doi: 10.1088/1741-2560/12/5/056016

Moore, K. L., Agur, A. M., and Dalley, A. F. (2011). Essential Clinical Anatomy. Philadelphia, PA: Lippincott Williams \& Wilkins.

Moran, D. W., and Schwartz, A. B. (1999). Motor cortical representation of speed and direction during reaching. J. Neurophysiol. 82, 2676-2692.

Moritz, C. T., Perlmutter, S. I., and Fetz, E. E. (2008). Direct control of paralysed muscles by cortical neurons. Nature 456, 639-642. doi: 10.1038/nature07418

Morrow, M. M., and Miller, L. E. (2003). Prediction of muscle activity by populations of sequentially recorded primary motor cortex neurons. J. Neurophysiol. 89. 2279-2288. doi: 10.1152/jn.00632.2002

Nazarpour, K., Ethier, C., Paninski, L., Rebesco, J. M., Miall, R. C., and Miller, L. E. (2012). Emg prediction from motor cortical recordings via a nonnegative point-process filter. IEEE Trans. Biomed. Eng. 59, 1829-1838. doi: 10.1109/TBME.2011.2159115

Ogihara, N., and Yamazaki, N. (2001). Generation of human bipedal locomotion by a bio-mimetic neuro-musculo-skeletal model. Biol. Cybernet. 84, 1-11. doi: 10.1007/PL00007977

Paek, A. Y., Brown, J. D., Gillespie, R. B., O’Malley, M. K., Shewokis, P. A., and Contreras-Vidal, J. L. (2013). "Reconstructing surface emg from scalp eeg during myoelectric control of a closed looped prosthetic device," in Conference Proceedings of Annual International Conference of the IEEE Engineering in Medicine and Biology Society (Osaka), 5602-5605.

Paninski, L., Shoham, S., Fellows, M. R., Hatsopoulos, N. G. and Donoghue, J. P. (2004). Superlinear population encoding of dynamic hand trajectory in primary motor cortex. J. Neurosci. 24, 8551-8561. doi: 10.1523/JNEUROSCI.0919-04.2004

Pohlmeyer, E. A., Solla, S. A., Perreault, E. J., and Miller, L. E. (2007). Prediction of upper limb muscle activity from motor cortical discharge during reaching. $J$. Neural Eng. 4, 369-379. doi: 10.1088/1741-2560/4/4/003

Prabhu, G., Shimazu, H., Cerri, G., Brochier, T., Spinks, R. L., Maier, M. A., et al. (2009). Modulation of primary motor cortex outputs from ventral premotor cortex during visually guided grasp in the macaque monkey. J. Physiol. 587, 1057-1069. doi: 10.1113/jphysiol.2008.165571

Presacco, A., Forrester, L. W., and Contreras-Vidal, J. L. (2012). Decoding intra-limb and inter-limb kinematics during treadmill walking from scalp electroencephalographic (eeg) signals. IEEE Trans. Neural Syst. Rehabil. Eng. 20, 212-219. doi: 10.1109/TNSRE.2012.2188304

Presacco, A., Goodman, R., Forrester, L., and Contreras-Vidal, J. L. (2011). Neural decoding of treadmill walking from noninvasive electroencephalographic signals. J. Neurophysiol. 106, 1875-87. doi: 10.1152/jn.00104.2011

Qin, W., Bauman, W. A., and Cardozo, C. (2010). Bone and muscle loss after spinal cord injury: organ interactions. Ann. N.Y. Acad. Sci. 1211, 66-84. doi: 10.1111/j.1749-6632.2010.05806.x

Taslim Reza, S. M. T, Ahmad, N., Choudhury, I. A., and Ghazilla, R. A. R. (2014). A fuzzy controller for lower limb exoskeletons during sit-to-stand and stand-to-sit movement using wearable sensors. Sensors 14, 4342-4363. doi: $10.3390 / s 140304342$

Schwartz, A. B. (2004). Cortical neural prosthetics. Annu. Rev. Neurosci. 27, 487-507. doi: 10.1146/annurev.neuro.27.070203.144233

Serruya, M. D., Hatsopoulos, N. G., Paninski, L., Fellows, M. R., and Donoghue, J. P. (2002). Brain-machine interface: instant neural control of a movement signal. Nature 416, 141-142. doi: 10.1038/416141a

Shin, D., Watanabe, H., Kambara, H., Nambu, A., Isa, T., Nishimura, Y., et al. (2012). Prediction of muscle activities from electrocorticograms in primary motor cortex of primates. PLoS ONE 7:e47992. doi: 10.1371/journal.pone.0047992

Suykens, J. A., Vandewalle, J. P., and de Moor, B. L. (2012). Artificial Neural Networks for Modelling and Control of Non-linear Systems. New York, NY: Springer Science \& Business Media.
Taylor, D. M., Tillery, S. I., and Schwartz, A. B. (2002). Direct cortical control of 3D neuroprosthetic devices. Science 296, 1829-1832. doi: 10.1126/science.1070291

Truccolo, W., Friehs, G. M., Donoghue, J. P., and Hochberg, L. R. (2008). Primary motor cortex tuning to intended movement kinematics in humans with tetraplegia. J. Neurosci. 28, 1163-1178. doi: 10.1523/JNEUROSCI.441507.2008

Velliste, M., Perel, S., Spalding, M. C., Whitford, A. S., and Schwartz, A. B. (2008). Cortical control of a prosthetic arm for self-feeding. Nature 453, 1098-1101. doi: 10.1038/nature06996

Vogelstein, R. J., Tenore, F., Etienne-Cummings, R., Lewis, M. A., and Cohen, A. H. (2006). Dynamic control of the central pattern generator for locomotion. Biol. Cybernet. 95, 555-566. doi: 10.1007/s00422-006-0119-z

Wahnoun, R., He, J., and Tillery, S. I. (2006). Selection and parameterization of cortical neurons for neuroprosthetic control. J. Neural Eng. 3, 162. doi: 10.1088/1741-2560/3/2/010

Wan, E. A., and Van Der Merwe, R. (2000). "The unscented kalman filter for nonlinear estimation," in IEEE 2000 Adaptive Systems for Signal Processing, Communications, and Control Symposium (Lake Louise, AB: IEEE), 153-158.

Wang, Y., and Principe, J. C. (2010). Instantaneous estimation of motor cortical neural encoding for online brain-machine interfaces. J. Neural Eng. 7, 2899. doi: 10.1088/1741-2560/7/5/056010

Wang, Y., Wang, F., Xu, K., Zhang, S., and Zheng, X. (2014). Neural control of a tracking task via attention-gated reinforcement learning for brain-machine interfaces. EEE Trans. Neural Syst. Rehabil. Eng. 7:e47992. doi: $10.1109 /$ tnsre.2014.2341275

Wessberg, J., Stambaugh, C. R., Kralik, J. D., Beck, P. D., Laubach, M., Chapin, J. K., et al. (2000). Real-time prediction of hand trajectory by ensembles of cortical neurons in primates. Nature 408, 361-365. doi: 10.1038/35042582

Winters, J. M. and Woo, S. L. (2012). Multiple Muscle Systems: Biomechanics and Movement Organization. New York, NY: Springer Science \& Business Media.

Wodlinger, B., Downey, J. E., Tyler-Kabara, E. C., Schwartz, A. B., Boninger, M. L., and Collinger, J. L. (2014). Ten-dimensional anthropomorphic arm control in a human brain- machine interface: difficulties, solutions, and limitations. J. Neural Eng. 12:016011. doi: 10.1088/1741-2560/12/1/016011

Wu, W., Black, M. J., Gao, Y., Bienenstock, E., Serruya, M., and Donoghue, J. P. (2002). "Inferring hand motion from multi-cell recordings in motor cortex using a kalman filter," in SAB 02-Workshop on Motor Control in Humans and Robots: On the Interplay of Real Brains and Artificial Devices (Washington, DC), 66-73.

Wu, W., Gao, Y., Bienenstock, E., Donoghue, J. P., and Black, M. J. (2006). Bayesian population decoding of motor cortical activity using a kalman filter. Neural Comput. 18, 80-118. doi: 10.1162/089976606774841585

Wu, W., Kulkarni, J. E., Hatsopoulos, N. G., and Paninski, L. (2009). Neural decoding of hand motion using a linear state-space model with hidden states. IEEE Trans. Neural Syst. Rehabil. Eng. 17, 370-378. doi: 10.1109/TNSRE.2009.2023307

Conflict of Interest Statement: The authors declare that the research was conducted in the absence of any commercial or financial relationships that could be construed as a potential conflict of interest.

The reviewer GL and handling Editor declared their shared affiliation, and the handling Editor states that the process nevertheless met the standards of a fair and objective review.

Copyright $\odot 2017 \mathrm{Ma}, \mathrm{Ma}$, Huang, Zhang, Xu and He. This is an open-access article distributed under the terms of the Creative Commons Attribution License (CC BY). The use, distribution or reproduction in other forums is permitted, provided the original author(s) or licensor are credited and that the original publication in this journal is cited, in accordance with accepted academic practice. No use, distribution or reproduction is permitted which does not comply with these terms. 
OPEN ACCESS

Edited by:

Wenwei Yu,

Chiba University, Japan

Reviewed by:

Stefano Vassanelli,

University of Padua, Italy

Erika Kristine Ross,

Mayo Clinic, USA

*Correspondence:

Xiaohong Sui

suixhong@sjtu.edu.cn

${ }^{\dagger}$ These authors have contributed equally to this work.

Specialty section: This article was submitted to

Neural Technology,

a section of the journal

Frontiers in Neuroscience

Received: 15 December 2016 Accepted: 18 April 2017

Published: 16 May 2017

Citation:

Zhu K, Li L, Wei X and Sui X (2017) A $3 D$ Computational Model of

Transcutaneous Electrical Nerve Stimulation for Estimating A Tactile Nerve Fiber Excitability.

Front. Neurosci. 11:250. doi: 10.3389/fnins.2017.00250

\section{A 3D Computational Model of Transcutaneous Electrical Nerve Stimulation for Estimating A $\beta$ Tactile Nerve Fiber Excitability}

\author{
Kaihua Zhu ${ }^{\dagger}$, Liming $\mathrm{Li}^{\dagger}$, Xuyong Wei and Xiaohong Sui * \\ School of Biomedical Engineering, Shanghai Jiao Tong University, Shanghai, China
}

Tactile sensory feedback plays an important role in our daily life. Transcutaneous electrical nerve stimulation (TENS) is widely accepted to produce artificial tactile sensation. To explore the underlying mechanism of tactile sensation under TENS, this paper presented a novel 3D TENS computational model including an active $A \beta$ tactile nerve fiber (TNF) model and a forearm finite element model with the fine-layered skin structure. The conduction velocity vs. fiber diameter and strength-duration relationships in this combined TENS model matched well with experimental data. Based on this validated TENS model, threshold current variation were further investigated under different stimulating electrode sizes with varied fiber diameters. The computational results showed that the threshold current intensity increased with electrode size, and larger nerve fibers were recruited at lower current intensities. These results were comparable to our psychophysical experimental data from six healthy subjects. This novel 3D TENS model would further guide the floorplan of the surface electrodes, and the stimulating paradigms for tactile sensory feedback.

Keywords: 3D computational modeling, tactile sensory feedback, transcutaneous electrical nerve stimulation, $A \beta$ tactile nerve fiber, double-cable model, psychophysical experiments

\section{INTRODUCTION}

Tactile sense is important for us to gather information about the world, and tactile sensory feedback plays a great role in our daily life. Both tactile sensation and motor function are important for reali zing dexterous manipulation. In recent years, incorporation of artificial tactile sensory feedback has obtained great interest worldwide to help achieve the fine closed-loop control for the prosthetic hand (Kim et al., 2014; Tan et al., 2014; Tee et al., 2015).

For stroke patients with severe loss of hand sensation, their grasp function could be greatly improved by incorporation of the fingers' artificial tactile sensation based on mechanical stimulation of other normal skin area such as shoulder (Kita et al., 2013). By combining both tactile and visual feedback, the virtual reality-based training technology made a significant role in rehabilitation for patients with stroke or Parkinson disease (Saposnik et al., 2010; Liao et al., 2014). For the amputees, accomplishing tactile sensory feedback of prosthetics will improve their acceptance for the artificial limb (Antfolk et al., 2013).

Normally, our ability to feel the richness of our tactile environment relies on the cutaneous mechanoreceptors. These receptors detect and convert mechanical stimuli to corresponding electrical signals in the cutaneous nerve fibers. These signals can be delivered to the central nervous 
system, where they are further processed and interpreted as our tactile sensation (Gardner, 2010; Woo et al., 2015). Usually, thin-myelinated $\mathrm{A} \delta$ - or unmyelinated $\mathrm{C}$ - fibers mediate thermal and noxious stimuli which can produce pain sensation. The myelinated $\mathrm{A} \beta$ sensory afferents mostly innervate cutaneous mechanoreceptors, and are thought to produce an innocuous tactile sensation (Matsumoto et al., 2008). On the other hand, it was reported that some of $\mathrm{A} \beta$ fibers could innervate with the nociceptors (Djouhri and Lawson, 2004), and A $\beta$ fiber perception could be transmitted to spinal neurons, which originally received only $\mathrm{A} \delta$ - and C-fiber-mediated pain transmission, in animals with nerve injury (Matsumoto et al., 2008). Besides, $A \beta$ fibers can activate a significant proportion of superficial dorsal horn GABAergic neurons for maintaining separation of touch and pain modalities (Daniele and MacDermott, 2009), and the electrical stimulation of the lowthreshold $A \beta$ afferent fibers may treat chronic neuropathic pain (Sdrulla et al., 2015). Then the A $\beta$ fibers play an important role in tactile and pain sensation.

There are various kinds of ways to realize artificial tactile feedback. The mechanical tactile feedback can be obtained by using a skin pusher to convey tactile information. The typical example is the pneumatic system which was composed of pressure pads and a tube exerting pressure to the residual limb from the prosthetic fingers (Childress, 1980). The tactile feedback can also be achieved by electrical stimulation. By implanting microelectrode arrays in the somatosensory cortex, the electrodes can record the action potentials related to tactile sensation (Schwartz, 2004). Direct stimulation of the peripheral nerves is another way to elicit tactile feedback (Dhillon and Horch, 2005; Raspopovic et al., 2014). With a flat interface nerve electrode, the common nerve trunk can be selectively recruited (Tyler and Durand, 2002). Recently, the transcutaneous electrical nerve stimulation (TENS) was applied to accomplish tactile sensation for the amputee (Chai et al., 2015) and healthy subjects (Forst et al., 2015). Due to the non-invasiveness, the TENS could be promising in wide clinical applications to elicit tactile feedback. By attaching a surface electrode to the target skin area, various sensory afferents including $\mathrm{A} \beta, \mathrm{A} \delta$, or $\mathrm{C}$ fibers can be directly recruited without the activation of mechanoreceptors to produce artificial tactile or nociceptive sensation (Frahm et al., 2013). The excitatory state of sensory afferents such as $A \beta$ nerve fibers can be modulated by different kinds of stimulating waveform parameters (Mahmud and Vassanelli, 2016).

To describe how the TENS evokes tactile neural activities and leads to psychophysical responses, the computational modeling is a feasible and efficient way, and a solid tactile nerve fiber model is the key to investigate the fiber excitation patterns. Although part of the $A \beta$ nerve fibers contribute to pain sensation, they mostly play a significant role in feeling the pressure/light touch (Gardner, 2010). Most of the previous computational work about TENS focused on nociceptive A $\delta$ - or C-type fibers (Yang et al., 2015), or motor nerve fibers (Kuhn et al., 2010; Goffredo et al., 2014). Although some papers established TENS model considering tactile sensory nerve fiber (Kajimoto et al., 2004; Mørch et al., 2011), the fiber model is passive without explicit ion channel distribution. The active tactile sensory nerve fiber model is more comparable to neurophysiological properties and important for TENS modeling. McNeal et al. established the first active multi-compartment cable model in 1976 to study the response patterns of action potentials. The model assumed the myelin sheath as a perfect insulator, and contained a few voltage-gated sodium and potassium channels at the nodes of Ranvier (McNeal, 1976). In 1995, Schwartz et al. recorded the action potentials and membrane currents in single human myelinated nerve fibers by means of the current and voltageclamp techniques. They applied the obtained experimental data to the mathematical model derived from the FrankenhaeuserHuxley equations (Schwarz et al., 1995). In 1999, Wesselink et al. implemented a human myelinated sensory nerve fiber model for direct spinal cord electrical stimulation (Wesselink et al., 1999). McIntyre et al. (2002) built a McIntyre-Richard-Grill (MRG) neuron model for mammalian motor nerve fibers on the foundation of Hodgkin-Huxley $(\mathrm{H}-\mathrm{H})$ model. In recent years, some researches adopted the MRG model as the sensory nerve fiber model in their computational modeling work (Bourbeau et al., 2011; Åström et al., 2015). However, the MRG model was derived from mammalian motor nerve fibers, and motor and sensory fibers were different in recovery cycle, action potential shape, and the properties of excitability (Howells et al., 2012). Consequently, a solid active tactile $\mathrm{A} \beta$ sensory nerve fiber is necessary for TENS modeling.

In our present study, we developed a novel three-dimensional (3D) computational model of TENS on the forearm to investigate the human subcutaneous $A \beta$ tactile nerve fiber (TNF) excitation. The 3D model establishment was achieved by combining finite element model of the whole forearm, and the active TNF counterpart. The solid active tactile sensory nerve fiber model was optimized with integral ionic and morphological parameters based on the $\mathrm{H}-\mathrm{H}$ model. In order to clearly figure out the excitability of TNFs under the TENS, the skin model was delaminated into multiple layers in terms of physiological structures. In addition, the effects of electrode size on the tactile sensation threshold were further compared between computational and psychophysical experimental results on healthy subjects. This novel 3D TENS model would lay a solid basis for optimization of surface electrodes in the transcutaneous electrical nerve stimulation.

\section{METHODS}

\section{The 3D Finite Element Model}

A 3D finite element model (FEM) of the forearm was created using COMSOL software (COMSOL Multiphysics 4.2a, Sweden). The FEM model was established to calculate the electrical potential distribution in the subcutaneous area during electrical stimulation. The stimulating waveform was biphasic chargebalanced cathodic-first current pulse. The FEM models were computed in the AC/DC module with a stationary electric current. According to the charge relaxation theory, the charge relaxation time $\tau$ is described as follows:

$$
\tau=\frac{\varepsilon}{\sigma}
$$


where $\varepsilon$ and $\sigma$ are respectively the permittivity and conductivity of the tissues. In neural tissue, the values of these parameters are $\varepsilon=10-7 \mathrm{~F} / \mathrm{m}$ and $\sigma=0.1 \mathrm{~S} / \mathrm{m}$ (Gabriel et al., 1996a). As a result, the $\tau=10-6 \mathrm{~s}$, which is much smaller than the external time scale such as the stimulation pulse duration on the order of hundreds of microseconds. When applying current-regulated TENS, the influence of capacitance in the intermediate tissues can be neglected (Kuhn et al., 2009a).

Mimicking the circumstance of psychophysical experiments on the forearm, a $150 \mathrm{~mm}$-long cylindrical geometric structure with stimulating and return electrodes was modeled as shown in Figure 1. The computational model consisted of seven concentric layers: stratum corneum, epidermis, dermis, fat, muscle, cortical bone, and bone marrow. The detailed electrical properties and thicknesses were listed in Table 1.

In this model, the epidermis, dermis, and muscle layers are anisotropic, while the other tissues are regarded as isotropic. The electric scalar potential $\left(\mathrm{V}_{\mathrm{FE}}\right)$ in the FEM was described by Laplace's equation.

$$
-\nabla \cdot\left(\sigma \nabla V_{F E}\right)=0
$$

The $\sigma$ is conductivity. By free tetrahedral meshing, there are more than 10 million elements in total with extremely tiny meshes due to the thin layers of epidermis and dermis. The computation was carried out using a computer workstation with an Intel Xeon CPU E5 and 64 GB of RAM.

\section{Subcutaneous A $\beta$ Tactile Nerve Fiber Model Morphological Parameters}

In our model, we developed $\mathrm{A} \beta$ nerve fibers with distribution density of approximate $2 / \mathrm{mm}^{2}$ under the skin (Lacour et al., 1991; Lesniak et al., 2014). As shown in Figure 1B, A $\beta$ nerve

TABLE 1 | Morphological and electric parameters in the FEM.

\begin{tabular}{|c|c|c|c|c|}
\hline & \multicolumn{3}{|c|}{ Conductivity(S/m) } & \multirow[t]{2}{*}{ Thickness (mm) } \\
\hline & Min & Typical & Max & \\
\hline Stratum corneum & $e-5^{[a, b]}$ & $2 e-5^{[a, b]}$ & $e-4^{[a, b]}$ & $0.0290^{[e, f]}$ \\
\hline Epidermis(axial) & & $0.9500^{[b, c]}$ & & $0.0600^{[e, f]}$ \\
\hline Epidermis(radial) & & $0.1500^{[b, c]}$ & & \\
\hline Dermis(axial) & $2^{\star}$ & $2.5700^{[b, c]}$ & $3.8^{*}$ & $1.4110^{[b, c]}$ \\
\hline Dermis(radial) & $1^{\star}$ & $1.6200^{[b, c]}$ & $2.8^{*}$ & \\
\hline Fat & $0.0017^{[\mathrm{d}]}$ & $0.01^{[\mathrm{d}]}$ & $0.1000^{[\mathrm{d}]}$ & $2.5000^{[\mathrm{d}]}$ \\
\hline Muscle(axial) & $0.2000^{[\mathrm{d}]}$ & $0.3333^{[\mathrm{d}]}$ & $0.5000^{[\mathrm{d}]}$ & $13.5000^{[\mathrm{d}]}$ \\
\hline Muscle(radial) & $0.0667^{[\mathrm{d}]}$ & $0.1111^{[\mathrm{d}]}$ & $0.1667^{[\mathrm{d}]}$ & \\
\hline Cortical bone & $0.0167^{[\mathrm{d}]}$ & $0.0200^{[d]}$ & $0.0250^{[\mathrm{d}]}$ & $6.0000^{[\mathrm{d}]}$ \\
\hline Bone marrow & $0.0667^{[\mathrm{d}]}$ & $0.08^{[\mathrm{d}]}$ & $0.1000^{[\mathrm{d}]}$ & $6.5000^{[\mathrm{d}]}$ \\
\hline Gel & $0.0001^{[\mathrm{d}]}$ & $0.0200^{[d]}$ & $1.0000^{[\mathrm{d}]}$ & $1.0000^{[\mathrm{d}]}$ \\
\hline Electrode(Pt) & & $8.9 \mathrm{e} 6$ & & 0.0100 \\
\hline
\end{tabular}

[a] Yamamoto and Yamamoto (1976); [b] Gabriel et al. (1996b); [c] Tavernier et al. (1993); ${ }^{[0]}$ Kuhn et al. (2009b); ${ }^{[e]}$ Neerken et al. (2004); ${ }^{[f]}$ Sandby-Moller et al. (2003) and the *notation denotes the values are adjusted for the sensitivity analysis. Through the thickness of the skin varied with many conditions, but the generally the total thickness is thought to be $1.5 \mathrm{~mm}$ (Kuhn et al., 2009a). Consequently the thickness of the dermis is determined by the stratum corneum and epidermis thicknesses and the whole skin thickness.
A

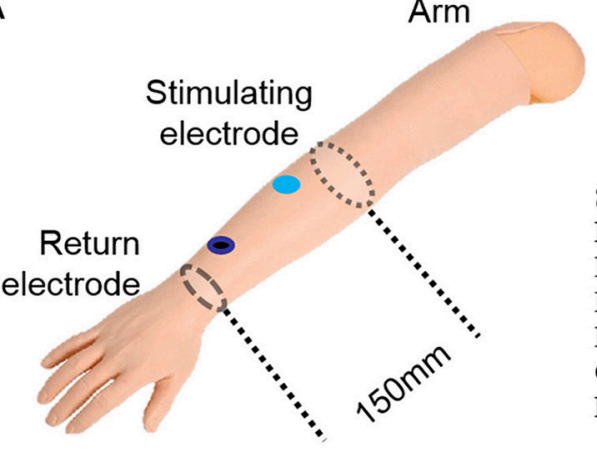

B

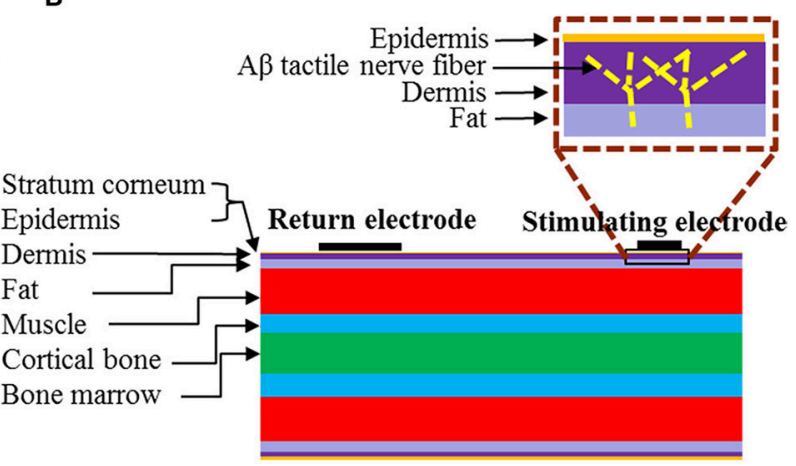

D

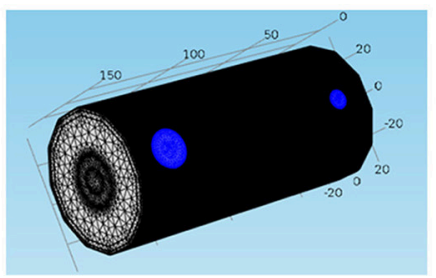

E

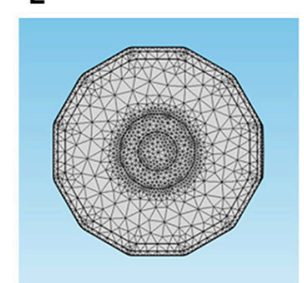

FIGURE 1 | Geometric structure of the 3D FEM for TENS. (A) The schematic diagram of TENS on a subject's forearm. The distance between stimulating electrode and return electrode is $120 \mathrm{~mm}$, and the return electrode diameter fixed to be $18 \mathrm{~mm}$ in diameter. The axial length of our total 3D FEM is $150 \mathrm{~mm}$. (B) The layered structure of the forearm. In the box the bright dashed line illustrated the location of an A $\beta$ tactile nerve fiber branching in the dermis layer. (C) Trimetric view of the 3D model. (D,E) Trimetric and front views of the model in extreme tiny tetrahedron mesh. The blue circle area denotes the two electrodes. 
fibers protruded from the muscle layer, and then aroused vertically to the skin which terminated in the dermis. This fiber length was $7 \mathrm{~mm}$ long, and was perpendicular to the skin except for the distal branching endings.

The TNFs were developed including parallel double cable models with nodes of Ranvier, paranodal, juxtaparanodal, and internodal sections, and the myelin sheath, etc. based on previous studies (McIntyre et al., 2002; Li et al., 2013). These fiber models have similar structures with the same kinds of ionic channels but with different spatial distribution of ion channels as shown in Figure 2 .

The internodal section consists of two paranodal myelin attachment segments, juxtaparanodal segments and six internodal segments such that relationship remains valid for the compartments in all models. The Node-to-node length represents the distance between two adjacent Ranvier node centers, as shown in Equation (3).

$$
\begin{aligned}
\text { Node }- \text { to }- \text { node length }= & \text { nodal length } \\
& +2 \times \text { paranodal length } \\
& +2 \times \text { juxtaparanodal length } \\
& +6 \times \text { internodal length }
\end{aligned}
$$

The models were built in NEURON v7.3 environment, and solved using backward Euler implicit integration with a time step of $0.001 \mathrm{~ms}$.
It was reported that the axonal diameter of $A \beta$ fibers was about $10 \mu \mathrm{m}$ (McGlone and Reilly, 2010), while such big diameter was always measured on the ulnar or median nerve trunk. It was found that some mechanoreceptor-innervated nerve fibers such as Ruffini corpuscle afferent axons measured 2-4 $\mu \mathrm{m}$ in diameter (Halata et al., 1985). And, the $3 \mu \mathrm{m}$ was most common diameter in the superficial cutaneous layer (Provitera et al., 2007). So the diameter of cutaneous $A \beta$ nerve fiber is much compatible to the sural nerve fiber with $3.11 \mu \mathrm{m}$ (Van Veen et al., 1995). The number of myelin lamellae and axon diameter was not constant, but decreased toward the distal end. Caruso $\mathrm{G}$ et al. found that fiber diameters in the skin were at least 3 times thinner than the respective proximal segments in the nerve trunk (Caruso et al., 1992). Besides, Provitera denoted that the subcutaneous fiber diameter should be about 2-8 $\mu \mathrm{m}$ (Provitera et al., 2007). As a result, under TENS of the forearm, the tactile $\mathrm{A} \beta$ afferents were selected as small myelinated nerve fibers in our present work. However, no detailed morphological parameters were reported adaptable to small $A \beta$ fibers. The morphological parameters for the fibers were listed in Table 2. Some parameters varied with diameters, and the others were fixed according to the experimental data. So we used numeric interpolation and extrapolation to estimate the morphological parameters of $A \beta$ tactile fibers with different diameters as shown in Figure 3. Then, the varied morphological properties can be determined including node-to-node length (X1), the number of myelin lamella (X2),

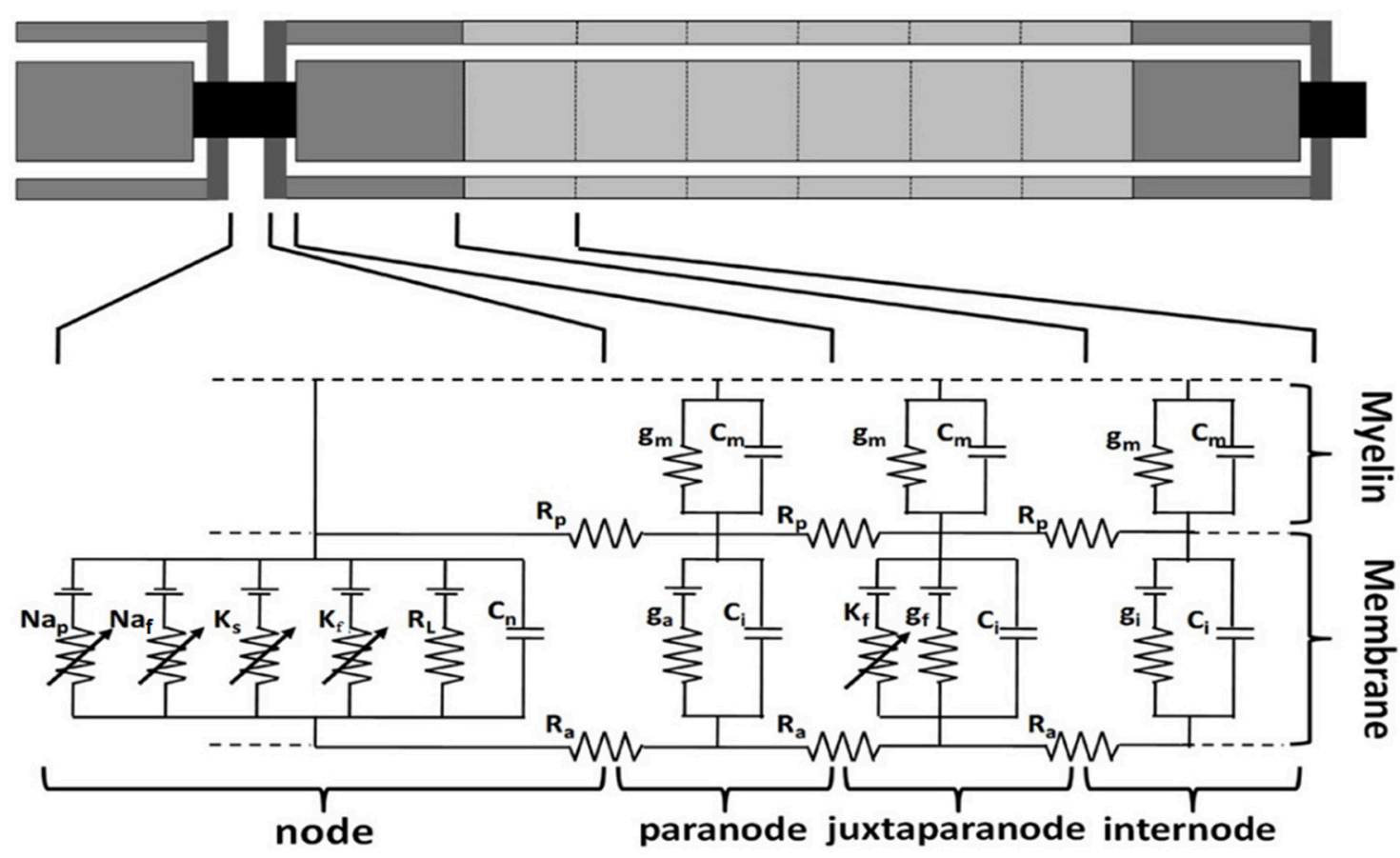

FIGURE 2 | Multi-compartment double cable model of a human sensory fiber. The nodal membrane dynamics included fast sodium (Naf), persistent sodium $(\mathrm{Nap})$, slow potassium $\left(\mathrm{K}_{\mathrm{S}}\right)$, fast potassium $\left(\mathrm{K}_{f}\right)$, and linear leakage conductance $\left(\mathrm{R}_{\mathrm{L}}\right)$ in parallel with the nodal capacitance $\left(\mathrm{C}_{n}\right)$. The juxtaparanode segments have fast potassium $\left(K_{f}\right)$ ion channel and linear leakage conductance $\left(g_{f}\right)$ in parallel with the internodal capacitance $\left(C_{j}\right)$. $g_{m}$ and $C_{m}$ are the conductance and capacitance separately in myelin sheath. $R_{p}$ is periaxonal resistivity, $R_{a}$ is axoplasmic resistivity. $g_{a}$ and $g_{i}$ are the conductance in paranode and internode segments separately (modified from Li et al., 2013). 
TABLE 2 | Geometric parameters for A $\beta$ TNF.

\begin{tabular}{lc}
\hline Variables & Value \\
\hline Node-to-node length $^{\mathrm{a}}$ & $\mathrm{X}_{1} \mu \mathrm{m}$ \\
The number of Myelin lamella $^{\mathrm{a}}$ & $\mathrm{X}_{2}$ \\
Node length & $2 \mu \mathrm{m}$ \\
Node diameter $^{\mathrm{a}}$ & $\mathrm{X}_{3} \mu \mathrm{m}$ \\
Paranode length & $3 \mu \mathrm{m}$ \\
Paranode diameter $^{\mathrm{a}}$ & $\mathrm{X}_{3} \mu \mathrm{m}$ \\
Paranode psw $^{\mathrm{b}}$ & $0.002 \mu \mathrm{m}$ \\
Juxtaparanode length $^{\mathrm{a}}$ & $\mathrm{X}_{4} \mu \mathrm{m}$ \\
Juxtaparanode diameter $^{\mathrm{a}}$ & $\mathrm{X}_{5} \mu \mathrm{m}$ \\
Juxtaparanode psw $^{\mathrm{b}}$ & $0.004 \mu \mathrm{m}$ \\
Internode length $^{\mathrm{c}}$ & $\left(\mathrm{X}_{1}-2 \mathrm{X}_{4}-7\right) / 6 \mu \mathrm{m}$ \\
Internode diameter $^{\mathrm{a}}$ & $\mathrm{X}_{5} \mu \mathrm{m}$ \\
Internode periaxonal space width & $0.004 \mu \mathrm{m}$
\end{tabular}

These fixed values are taken from previous studies. (Mclntyre et al., 2002, 2004; Sotiropoulos and Steinmetz, 2007).

a Values of these parameters are all dependent variables of fiber diameters (Figure 3).

${ }^{b}$ psw: periaxonal space width

${ }^{c}$ Derived from Equation (3).

node diameter (X3), juxtaparanode diameter (X4), internode length (X5).

\section{The lon Channel and Membrane Dynamics in the A $\beta$ TNF Fiber Model}

To simulate the behavior of the axon under electrical stimulation, the model we implemented contains both linear and nonlinear membrane dynamics. There are many kinds of voltage-gated ion channels in the axon, and the ion channels located in the axonal membrane carry out action potential initiation and conduction by governing the amplitude and shape of the unitary spike and pattern of repetitive firing (Debanne et al., 2011). We developed a kind of human peripheral TNF model based on the double cable model structure (McIntyre et al., 2002; Li et al., 2013). The node Ranviers consisted of slow $\mathrm{K}^{+}$, fast $\mathrm{K}^{+}$, fast $\mathrm{Na}^{+}$, and persistent $\mathrm{Na}^{+}$conductance. The juxtaparanodal consisted of fast $\mathrm{K}^{+}$conductance in parallel with the membrane and leakage conductance. The dynamics of the nodal and internodal ion channels (Equations in Appendix) were based on the experimental data of sensory nerve fibers (Howells et al., 2012). The maximum density of fast $\mathrm{K}^{+}$in the sensory nerve fiber (Howells et al., 2012) is far more than that in the motor fiber (McIntyre et al., 2002), so it was incorporated to our TNF model. Then the novel $A \beta$ tactile sensory nerve fiber model was achieved. All of the parameter values were derived from the reported experimental data, and dynamic equations form for sodium ionic channel was based on ionic conductance. The electrical parameters of the A $\beta$ TNF model are shown in Table 3.

\section{The Combined 3D TENS Modeling}

This combined 3D TENS model is accomplished by integration of the forearm FEM model implemented in COMSOL and TNF model developed in NEURON. Stimulating currents were applied to the surface electrode on the forearm FEM model in COMSOL, and then the subcutaneous spatially interpolated

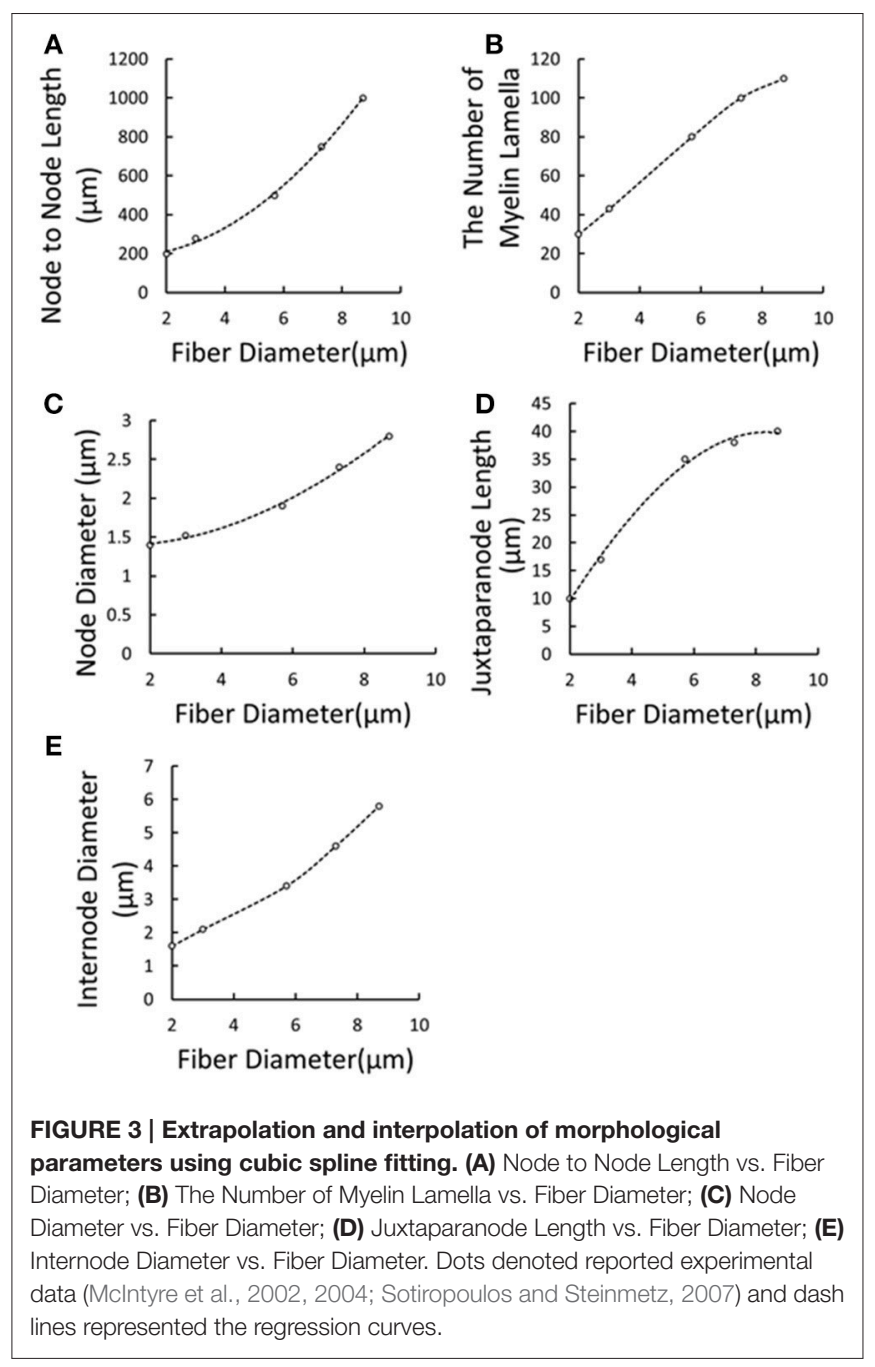

electric potentials $V_{\mathrm{FE}}$ in COMSOL were exported to NEURON as the corresponding extracellular voltages $\mathrm{Ve}\left(\mathrm{Ve}=\mathrm{V}_{\mathrm{FE}}\right)$ of the TNFs. Thus, the excitability of the TNFs under TENS can be quantitatively determined, and the excitation threshold was defined as the minimum current intensity to recruit one fiber.

\section{Model Validation}

By adopting typical FEM model parameters in Table 1 and TNF properties in Tables 2, 3, the 3D TENS model was validated in terms of conduction velocity of the TNFs and the strength-duration relationship of the surface electrical stimulation. The conduction velocities were computed in NEURON corresponding to different fiber diameters ranging from 2 to $8 \mu \mathrm{m}$. The curves of conduction velocity vs. fiber diameters were compared with previously reported sensory fiber modeling results (Wesselink et al., 1999) and experimental data (Van Veen et al., 1995). In addition, with the stimulating frequency of $50 \mathrm{~Hz}$ and pulse duration of $200 \mu \mathrm{s}$, the strengthduration relationship in our 3D TENS model were achieved for a given $\Phi-9 \mathrm{~mm}$ electrode and an $\Phi-3 \mu \mathrm{m} \mathrm{A} \beta$ nerve fiber with typical electrical properties. The stimulating pulse durations were selected increasing from $10 \mu \mathrm{s}$ to $1 \mathrm{~ms}$, and the corresponding 
TABLE 3 | Electrical parameters for the cable model.

\begin{tabular}{|c|c|}
\hline Model electrical parameters & Accepted values \\
\hline Nodal capacitance $\left(c^{n}\right)$ & $2.8 \mu \mathrm{F} / \mathrm{cm}^{2}[\mathrm{a}]$ \\
\hline Internodal capacitance $\left(c^{i}\right)$ & $2.8 \mu \mathrm{F} / \mathrm{cm}^{2}[\mathrm{a}]$ \\
\hline Myelin capacitance $\left(c^{m}\right)$ & $0.1 \mu \mathrm{F} / \mathrm{cm}^{2}[\mathrm{~b}]$ \\
\hline Axoplasmic resistivity $\left(\mathrm{R}^{\mathrm{a}}\right)$ & $33 \Omega \mathrm{cm}^{[\mathrm{a}]}$ \\
\hline Periaxonal resistivity $\left(\mathrm{R}^{\mathrm{p}}\right)$ & $33 \Omega \mathrm{cm}^{[\mathrm{a}]}$ \\
\hline Myelin conductance $\left(g^{m}\right)$ & $0.001 \mathrm{~S} / \mathrm{cm}^{2}[\mathrm{~b}]$ \\
\hline Myelin attachment conductance $\left(\mathrm{g}^{\mathrm{m}}\right)$ & $0.001 \mathrm{~S} / \mathrm{cm}^{2}[\mathrm{~b}]$ \\
\hline Paranodal conductance $\left(\mathrm{g}^{\mathrm{a}}\right)$ & $0.0001 \mathrm{~S} / \mathrm{cm}^{2}[\mathrm{~b}]$ \\
\hline Internodal conductance $\left(\mathrm{g}^{\mathrm{i}}\right)$ & $0.0001 \mathrm{~S} / \mathrm{cm}^{2}[\mathrm{~b}]$ \\
\hline Max transient $\mathrm{Na}^{+}$conductance $\left(\mathrm{Na}^{\mathrm{f}}\right)$ & $3^{[b]}$ \\
\hline Persistent $\mathrm{Na}^{+}$conductance $\left(\mathrm{Na}^{\mathrm{P}}\right)$ & $0.0321^{[\mathrm{d}]}$ \\
\hline Max slow $\mathrm{K}^{+}$conductance $\left(\mathrm{K}^{\mathrm{S}}\right)$ & $0.06 \mathrm{~S} / \mathrm{cm}^{2}[\mathrm{a}]$ \\
\hline Max fast $\mathrm{K}^{+}$conductance $\left(\mathrm{K}^{f}\right)$ & $0.03 \mathrm{~S} / \mathrm{cm}^{2}[\mathrm{a}]$ \\
\hline Max juxtaparanodal fast $\mathrm{K}^{+}$conductance $\left(\mathrm{K}^{\dagger}\right)$ & $0.3 \mathrm{~S} / \mathrm{cm}^{2}[\mathrm{~d}]$ \\
\hline Nodal leakage conductance $\left(R^{L}\right)$ & $0.06 \mathrm{~S} / \mathrm{cm}^{2}[\mathrm{a}]$ \\
\hline $\mathrm{Na}^{+}$Nernst potential (Ena) & $43.7 \mathrm{mV}[\mathrm{a}]$ \\
\hline $\mathrm{K}^{+}$Nernst potential $\left(\mathrm{E}^{\mathrm{k}}\right)$ & $-84 m V^{[c]}$ \\
\hline Leakage reversal potential $\left(\mathrm{E}^{\mathrm{L}}\right)$ & $-84 \mathrm{mV}[\mathrm{c}]$ \\
\hline Rest potential (V'rest) & $-80 m V^{[d]}$ \\
\hline
\end{tabular}

[a] Wesselink et al. (1999); [b] Mclntyre et al. (2002); [c] Schwarz et al. (1995); [d] Howells et al. (2012).

threshold current intensities were obtained. Strength-duration curves were further compared among our computational, psychophysical experimental and reported modeling results.

\section{Forearm Model Parameter Sensitivity Analyses}

The tactile sensation threshold varied between different individuals under TENS. To potentially explore what caused the threshold variance between individuals, we made several sensitivity analyses on the tissue properties. The thickness and conductivity in fat, muscle and dermis layers were changed to explore what tissue properties would contribute most to the TENS difference. As shown in Table 1, the fat conductivity changed between 0.0017 and $0.1 \mathrm{~S} / \mathrm{m}$, the muscle conductivity (axial) between 0.2 and $0.5 \mathrm{~S} / \mathrm{m}$. The muscle conductivity (radial) between 0.0667 and $0.1667 \mathrm{~S} / \mathrm{m}$, the dermis conductivity (axial) between 2 and $3.8 \mathrm{~S} / \mathrm{m}$, and the dermis conductivity (radial) between 1 and $2.8 \mathrm{~S} / \mathrm{m}$. The corresponding thickness variation ranges were as follows: $1.5-4 \mathrm{~mm}$ for Fat layer, $1.2-1.7 \mathrm{~mm}$ for Dermis layer, and 8-18 mm for Muscle layer. Many combinations can be obtained with the ranges from different parameters. In order to get the threshold current relationship with different conductivities or thicknesses, the specific parameter changes within a variation range, and the remaining geometric and electrical properties were kept constant as the typical values in Table 1.

\section{Threshold Currents under the 3D TENS Modeling}

Based on this validated 3D TENS model, the effects of electrode size and fiber diameters on the threshold current intensity were investigated. Fiber diameters were selected from 2 to $8 \mu \mathrm{m}$ with a step of $1 \mu \mathrm{m}$, the surface electrode size from 5 to $12 \mathrm{~mm}$, pulse duration from $10 \mu$ s to $1 \mathrm{~ms}$. With the $\Phi-9 \mathrm{~mm}$ surface electrode and $\Phi-3 \mu \mathrm{m}$ fiber, the spatial distribution of threshold current intensity below the surface electrode was obtained, and the fiber excitation characteristics were further observed under different stimulating currents.

\section{Psychophysical Experiments}

The psychophysical experiments were carried out for five consecutive days on six healthy subjects (aged 22-30 years, 3 males, 3 females) without clinical or neuro-physiological disorders. All subjects were provided the informed written consent prior to commencement of the experiment. All experiments were conducted in accordance with the Declaration of Helsinki and approved by the Ethics Committee of Human and Animal Experiments of School of Biomedical Engineering at Shanghai Jiao Tong University (No. 2016012). In our experiments, we used Master-9 Pulse Stimulator with two isolators (Iso-Flex, A.M.P.I. Company, Israel) to generate biphasic charge-balanced cathodic-first stimulating current pulses. The gold stimulating electrode was placed on the volar part of forearm $2 \mathrm{~cm}$ from the proximal wrist crease and the return electrode approximately $12 \mathrm{~cm}$ apart from the stimulating electrode on the same side. The same-size thinlayer conductive gel was adhered to the stimulating electrode during the TENS. An $\Phi-25 \mathrm{~mm}$ non-woven surface electrode (Shanghai Kongren Medical, Inc.) was used as the return electrode. The subjects sit in a chair with a comfortable position, and the small current intensities were adopted to electrically stimulate the forearm skin through the electrode to produce the pressure sensation. The experiments started only when the room temperature was stable at $27^{\circ} \mathrm{C}$. During all the experiments, the subjects were given 5 -min relaxation every 20 min to assure the comfort during the whole psychophysical experiments.

The tactile perception thresholds were defined as the least current pulse intensity or amplitude that a subject could detect, and was determined by using a staircase paradigm. The pulse amplitude was first increased with a step of $0.1 \mathrm{~mA}$, and then a step of $0.01 \mathrm{~mA}$ to estimate the potential perception threshold current. Immediately after each stimulation, the subjects were required whether or not the stimulus was perceived. Tens of stimulating trials were conducted near the potential threshold currents, and the exact perception threshold was determined in terms of 50 percent level of the trails. In the psychophysical experiments, the effects of diameters of the gold stimulating electrode (5-12 mm) and the pulse duration (50-400 $\mu \mathrm{s})$ on the tactile perception threshold were also investigated.

The effects of individual subject difference on the perception threshold were also statistically evaluated in the psychophysical experiments. The independent variable was "individual subject," and the dependent variable was "perception threshold." A oneway analysis of variance (ANOVA) was performed and the F-test was used to find out the significance of the independent variable. The significance level was chosen as 0.05 . 


\section{RESULTS}

\section{Model Validation}

The typical propagating characteristics of action potentials in an $\Phi-3 \mu \mathrm{m} \mathrm{A} \beta$ nerve fiber were shown in Figure 4 under biphasic charge-balanced cathodic-first current pulse stimulation. The pulse duration was $200 \mu \mathrm{s}$, and the stimulating intensity was $2.84 \mathrm{~mA}$ which is 1.2 times the stimulating threshold. The action potential was excited at node $S$, and then propagated along both directions of the fiber. The other nodes were first hyperpolarized, and then depolarized when the propagating action potential arrived. The conduction velocity of this typical TNF was $8.27 \mathrm{~m} / \mathrm{s}$ in terms of Figure 4. The conduction velocities corresponding to varied fiber diameter were also shown in Figure 5. With the fiber diameter increasing from 2 to $8 \mu \mathrm{m}$, the corresponding conduction velocity increased from 5.68 to $33.14 \mathrm{~m} / \mathrm{s}$, which matched well with experimental data (Wesselink et al., 1999), and previously published modeling results in terms of smalldiameter tactile sensory nerve fibers (Van Veen et al., 1995). The conduction velocity is dependent on a host of factors such as myelin sheath thickness and internodal length (Waxman, 1980). These morphological parameters were interpolated from various existing models as shown in Figure 3. Other parameters such as axoplasmic resistivity which have various alternatives were also optimized to match the experimental results. The ultimate optimal parameters as for the morphological parameters and electrical properties were denoted in Tables 2,3 , respectively.

With standard 3D TENS model parameters selected, the strength-duration relationship was illustrated in Figure 6, where the $\Phi-3 \mu \mathrm{m} \mathrm{A} \beta$ nerve fibers and $\Phi-9 \mathrm{~mm}$ stimulating electrodes were adopted. As illustrated in Figure 6, multiples of the rheobase current was selected to describe the stimulus threshold. With pulse duration enhancing, the threshold decreased sharply within short pulse durations and maintained stable at durations larger

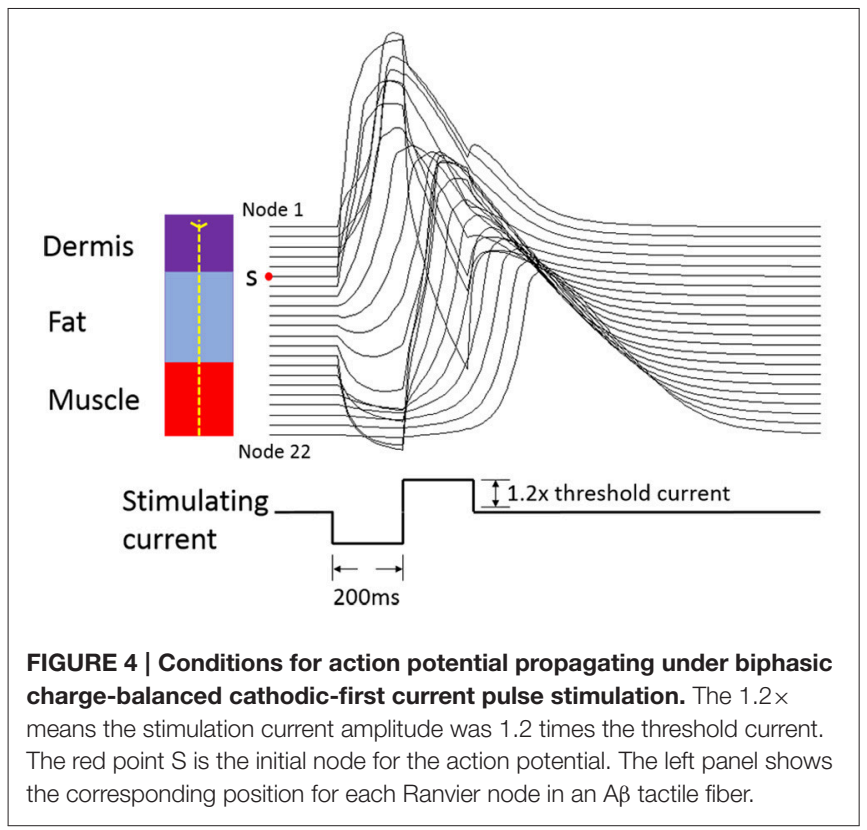

than $500 \mu \mathrm{s}$. The threshold currents for tactile perception were obtained based on psychophysical experiments from 6 healthy volunteers. Figure $\mathbf{6}$ showed that the strength-duration tendency was closely consistent with our psychophysical experimental results.

\section{Forearm Model Parameter Sensitivity Analyses}

Figure 7 showed the typical subcutaneous distribution of current density and electrical field strength during the TENS. The current density reached the maximum in the dermis layer, especially near the electrode edge. On the contrary, the strongest electrical field occurred in the fat layer due to the lower conductivity. The
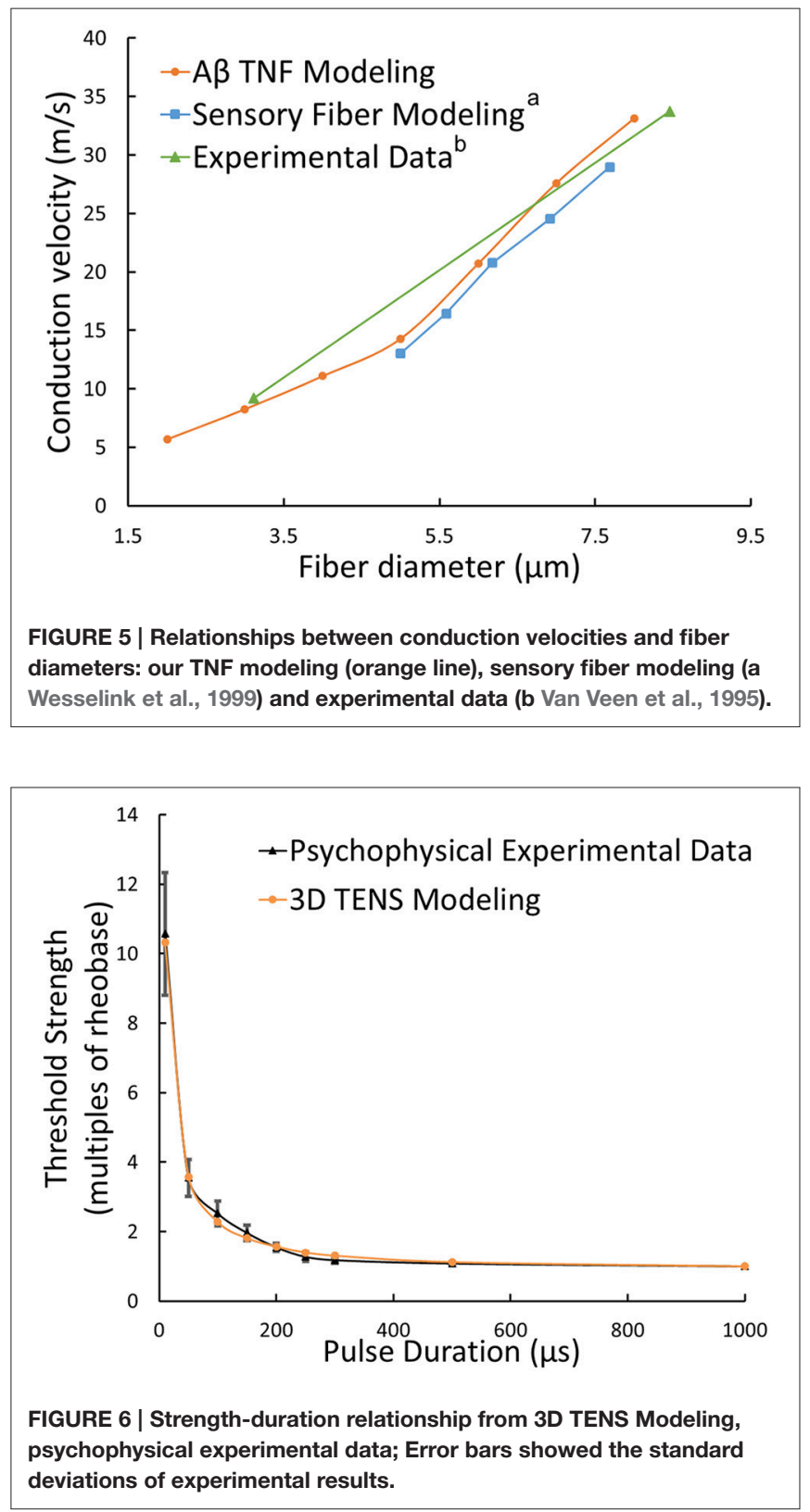
properties in dermis and fat layers may play an important role in the tactile sensation threshold. Figure 8A showed that the threshold current rose with the increasing electrical conductivity of fat and dermis layers while descending with the increasing electrical conductivity of the muscle layer. Within the given variation range shown in Table 1, the threshold current was most sensitive to the electrical conductivity of fat tissue. Figure $\mathbf{8 B}$ showed that the threshold current rose with the increasing thickness of fat and dermis layers, while descended a bit when increasing the muscle thickness during the given range. The thickness of fat and dermis layers showed obvious effect on the threshold current.

\section{Threshold Currents under the TENS}

The threshold currents were further investigated by means of both the 3D TENS computation and psychophysical experimental work. The threshold currents in terms of 6 subjects were shown in Figure 9. The one-way ANOVA results indicated that the different subject showed a significant variance on the tactile perception threshold $\left[F_{(5,24)}=24.565, p<\right.$ 0.0001]. The tactile sensation threshold were between 0.5 and $1.5 \mathrm{~mA}$ in our psychophysical experiments, and the corresponding strength-duration relationship was illustrated in Figure 6, which showed similar tendency to that of our modeling results.

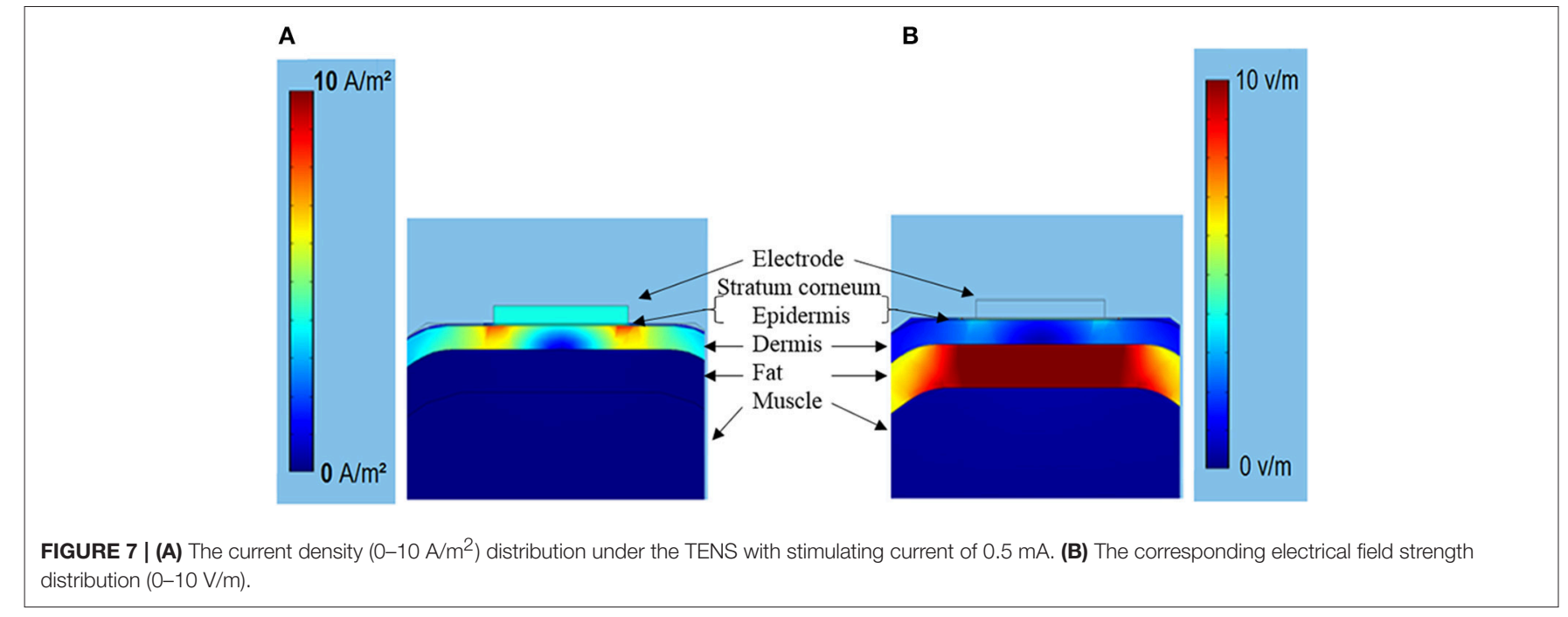

B
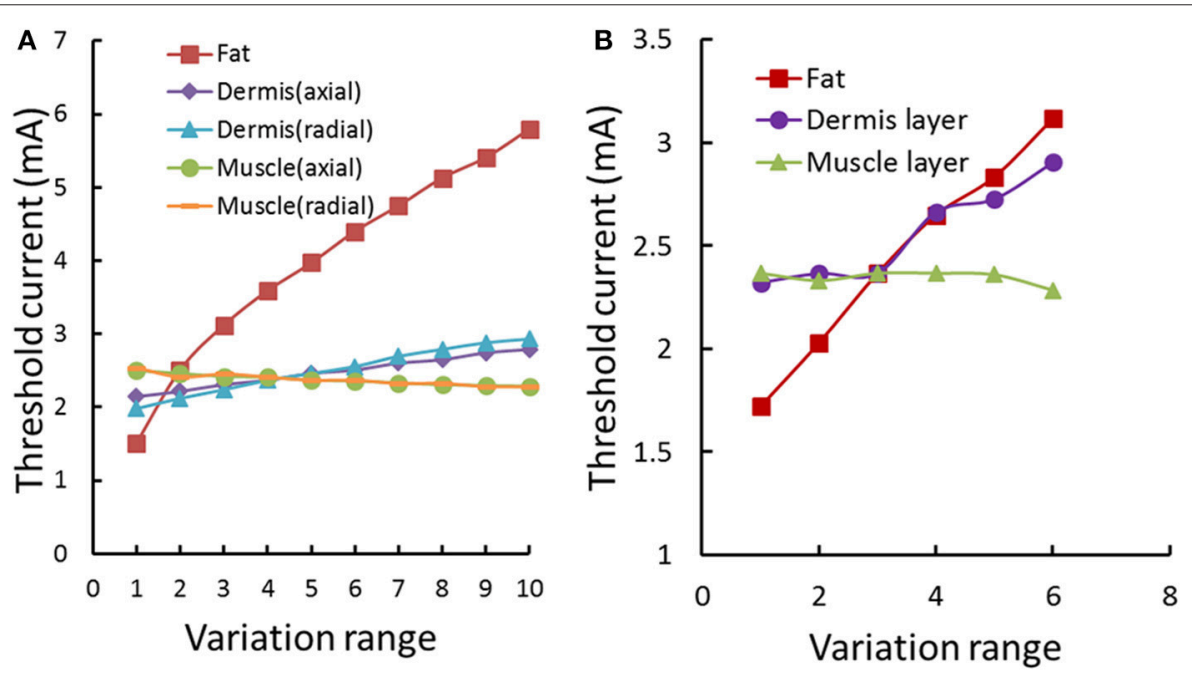

FIGURE 8 | Threshold current variation under the forearm model sensitivity analysis. (A) The threshold current variation with electrical conductivity changing of human arm tissues. The different tissue conductivities varied in a given range as shown in Table 1. The Fat conductivity of $0.0017-0.1 \mathrm{~S} / \mathrm{m}$; The muscle conductivity (axial) of $0.2-0.5 \mathrm{~S} / \mathrm{m}$; The muscle conductivity (radial) of $0.0667-0.1667 \mathrm{~S} / \mathrm{m}$; The dermis conductivity (axial) of 2-3.8 S/m; The dermis conductivity (radial) of $1-2.8 \mathrm{~S} / \mathrm{m}$. The range of conductivity was segmented into 10 evenly spaced parts. (B) The threshold current variation with thickness of human arm tissues. The fat thickness is between 1.5 and $4 \mathrm{~mm}$; The muscle thickness is between 8 and $18 \mathrm{~mm}$; The dermis thickness is between 1.2 and $1.7 \mathrm{~mm}$. The range of the thickness in each tissue was segmented into 6 evenly spaced parts. 


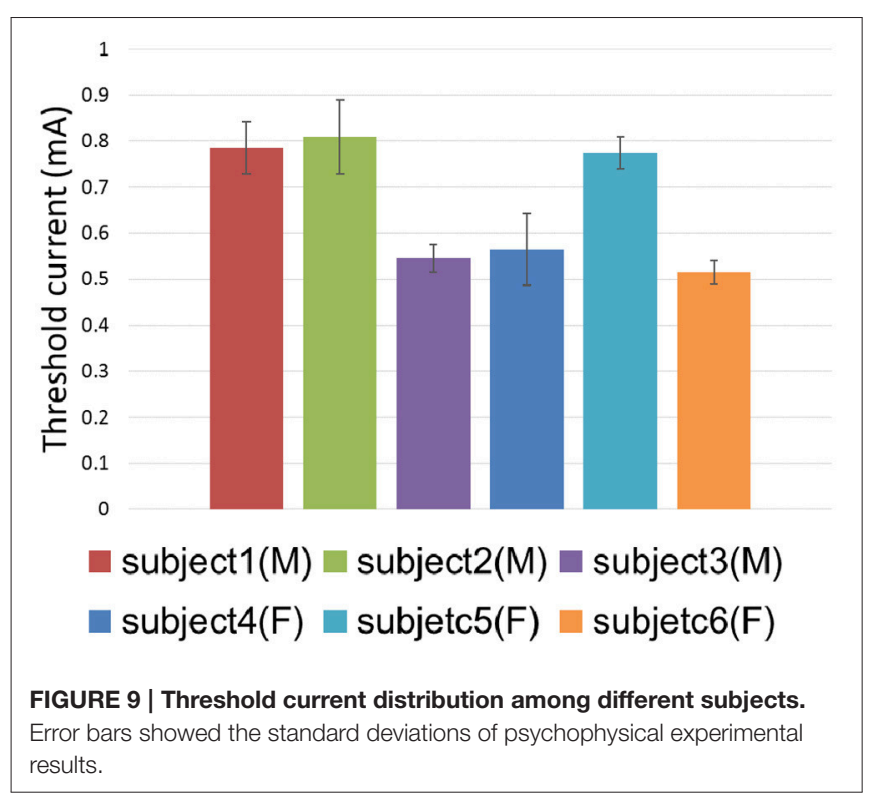

Figure 10A showed the spatial distribution of threshold current in terms of $\Phi-3 \mu \mathrm{m}$ axons under the $\Phi-9 \mathrm{~mm}$ stimulating electrode. It was clear that the least excitation threshold occurred axially below the stimulating electrode corresponding to one fiber excitation. The threshold increased apparently with a further distance from the electrode center. The threshold current below the edge of the electrode almost two times than that of the electrode center. Figure 10B showed that when stimulating current increased a little more than the threshold, the fiber excitation area extended greatly. When applying current intensity 1.4 times the threshold, the excitation area was close to size of the $\Phi-9 \mathrm{~mm}$ stimulating electrode.

Figure 10C illustrated the nerve excitation threshold during 3D TENS computation for different axon diameters and pulse durations. Larger nerve fibers were recruited at lower current amplitudes. The threshold current intensity decreased with fiber diameter from 2 to $8 \mu \mathrm{m}$, and varied sharply with small pulse duration stimulation. For instance, with pulse duration of $50 \mu \mathrm{s}$ for $\Phi-3 \mu \mathrm{m}$ fibers, the threshold was $1 / 3$ compared with that at $10 \mu$ s duration. While, with tenfold variation of pulse duration from 100 to $1,000 \mu \mathrm{s}$, the threshold current at $1,000 \mu \mathrm{s}$ only decreased to be about $1 / 2$ at $100 \mu \mathrm{s}$. Besides, at short durations, the threshold difference among different-size fibers was more obvious than that of long durations. For example, the threshold currents at $10 \mu \mathrm{s}$ for $\Phi-2 \mu \mathrm{m}$ fibers were $22 \mathrm{~mA}$ bigger than that of the $\Phi-8 \mu \mathrm{m}$ fibers, and the difference decreased to be $3.3 \mathrm{~mA}$ at $200 \mu$ s correspondingly.

In addition, under typical pulse duration of $200 \mu \mathrm{s}$ and frequency of $50 \mathrm{~Hz}$, the effects of different fiber diameters and stimulating electrode sizes on the threshold current were further compared between 3D TENS computational results and the psychological experimental data in Figure 10D. Computational results showed that the threshold current amplitude increased gradually with electrode size enlarging from 5 to $12 \mathrm{~mm}$. With fiber diameter increasing from 3 to $8 \mu \mathrm{m}$, the threshold current decreased. The black square line depicted the psychophysical experimental results averaged from 6 healthy volunteers with similar changing tendency. This line fitted closely to the changing tendency of the $\Phi-7 \mu \mathrm{m}$ fiber in the computational results.

\section{DISCUSSION}

In this study we developed a novel general 3D TENS computational model which could guide theoretical estimation of the recruitment of tactile afferent fibers. Although sensory nerve model had been studied for many years (Schwarz et al., 1995; Wesselink et al., 1999; Smit et al., 2009; Howells et al., 2012), the specific active model of tactile sensory fibers had not been studied in detail during the TENS. Our tactile nerve fiber model adopted the widely-accepted double-cable-layer model structure. The ionic types and kinetics parameters in this TNF model were derived from the recent study results (Howells et al., 2012), which were different from the previous parameters associated with the motor fiber model (McIntyre et al., 2002). The slow potassium channels in the internode were removed due to the low density distribution (Howells et al., 2012). The other electrical parameters were optimized by mathematical fitting based on the previous separate physiological experiments. This novel fiber model was validated by comparing the velocity vs. diameter and strength vs. duration relationships between our modeling and experimental results. As for the finite element modeling work, the $3 \mathrm{D}$ forearm was modeled, and the skin structure was finely layered into stratum, epidermis and dermis layers. This fine structure would be more practicable in comparison with the non-layered skin structure in some previous work (Kajimoto et al., 2004; Kuhn et al., 2009a). Based on the combined 3D TENS model consisting of finite element model of forearm and tactile nerve fiber model, the threshold current variation under TENS in terms of different electrode size and fiber diameters were investigated. The TENS computational results showed high consistency with psychophysical experimental results among 6 healthy volunteers.

The 3D TENS model were validated based on the action potential propagation, conduction velocity vs. fiber diameter relationship, and the strength-duration relationship. The action potential was excited in the Ranvier node $S$, and then spread along both directions of the axon. The node $S$ is the closest Ranvier node to the dermis-fat junction where the electrical field intensity was enhanced due to the fat layers. The action potentials showed similar propagating characteristics under cathodic current stimulation with the reported computational result (Rattay, 1999). The conduction velocity matched well with reported experimental (Van Veen et al., 1995) and other sensory nerve fiber modeling work (Wesselink et al., 1999).

In strength-duration relationship, the chronaxie was not adopted in our model validation for the reason that many factors can affect the accuracy of the chronaxie measurement (Geddes, 2004), and then we used multiples of rheobase as our comparison standard between modeling and psychophysical experimental work in Figure 6. It is more meaningful to have the specific model parameters for each subject which provided similar trend 


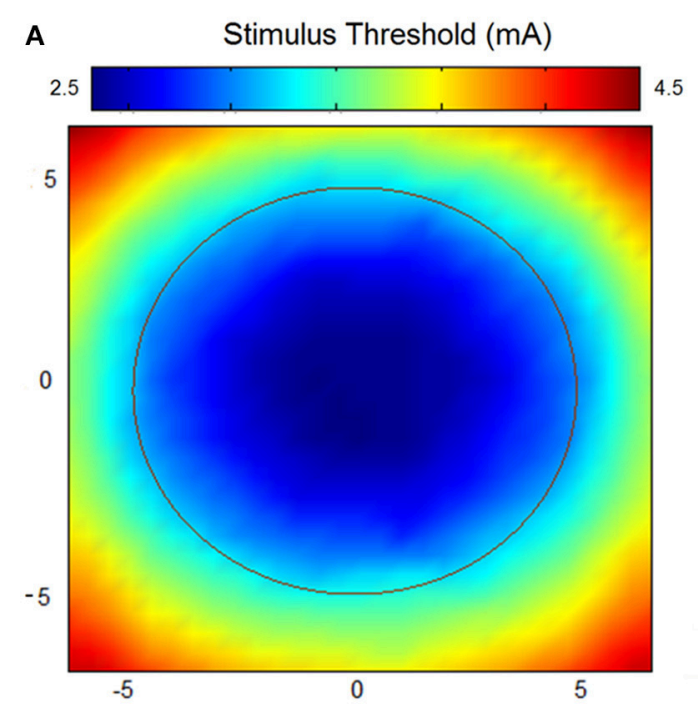

B

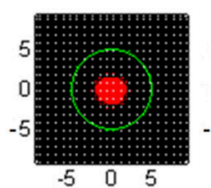

$1.05 x$
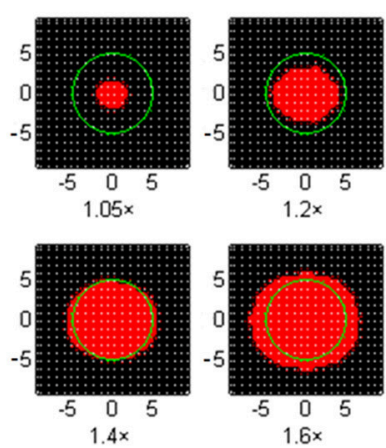

$1.2 \times$

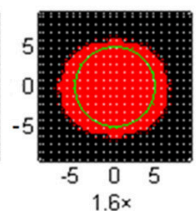

C
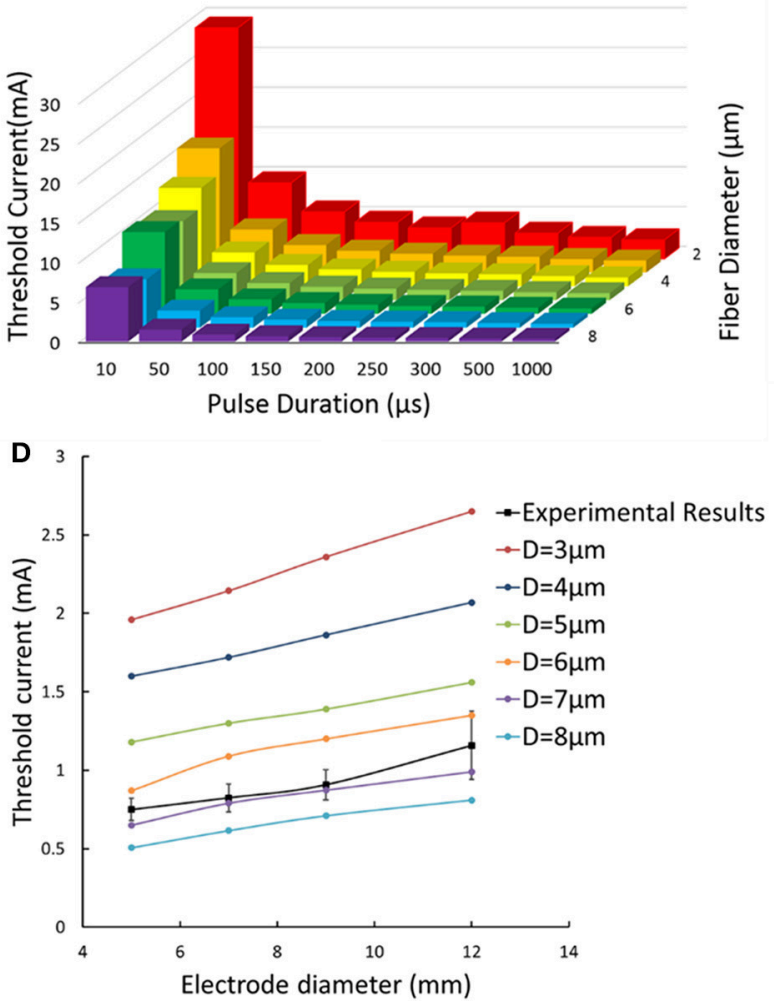

FIGURE 10 | A $\beta$ tactile nerve fiber excitability under different electrical stimulation. (A) The nerve excitation threshold distribution under the TENS ( $\Phi-9$ mm electrode). The color map corresponds to the threshold distribution. The middle circle corresponds to the 9 mm stimulating electrode. (B) The nerve excitation area (red) varies with different stimulating current. The $1.05 \times$ means the stimulating current was 1.05 times the least threshold current in the skin. The same as $1.2 \times, 1.4 \times$, and $1.6 \times$. (C) The fiber excitation thresholds under different pulse duration with different-diameter fibers. (D) The threshold current for $3-8 \mu \mathrm{m}$ diameter axons under different size of electrode stimulation. The dashed line corresponds to the experimental threshold current density. Error bars show the standard deviations of experimental results.

between the threshold current vs. duration. While, Figure 6 does not show the relationship between the threshold current and duration, and the Y-axis represents the threshold strength with the unit of multiples of rheobase, which was equal to the threshold current divided by the respective rheobase current. From the sensitivity analyses results, it is clear that conductivity and thickness of the fat layer play a key role in the variation of threshold current during TENS. So the specific fat properties for each subject should be incorporated into the model parameters to have similar trends between the threshold current and duration among different subject. This work will be carried out in the future work.

Threshold current intensities showed significant difference between individuals in our psychophysical experiments, which was consistent with previous studies (Larkin et al., 1986; Ara et al., 2012). The sensitivity analyses of the electrical properties in the forearm tissue clearly showed that both the thickness and electric conductivity in the fat layer greatly affected the variation of threshold current intensities. It could be inferred that fat would play an important role in the tactile perception threshold in the psychophysical experiments.

As shown in Figure 10D, the relationship of threshold currents vs. electrode sizes in the psychophysical experiments was most comparable to the $\Phi-7 \mu \mathrm{m} \mathrm{A} \beta$ fibers in the computational modeling results. It was indicated that $A \beta$ nerve fibers with diameters of no less than $7 \mu \mathrm{m}$ would be more associated with the comfortable tactile perception for the subjects. Nevertheless, there still exists modeling limitation. Although the strengthduration trend matches well in Figure 6, the threshold strength only represented multiples of rheobase, and the threshold current for a $3 \mu \mathrm{m}$ diameter fiber is far more than the experimental values in Figure 10D. As a result, in our forthcoming work, a coefficient might be included to the computation of the threshold current, and to achieve similar threshold currentduration trends between computational and experimental results. 
By establishing this novel 3D TENS model, we would directly observe the natural tactile sensation activities under the electrical stimulation. Some other issues can also be solved based on this model such as the relationship of nerve fibers activation with the two point discrimination under electrical stimulation (Solomonow et al., 1977) and the influence of nerve fiber regeneration or degeneration on the electrical stimulation (Simpson et al., 2013; Hebert et al., 2014).

\section{CONCLUSION}

In this paper, we introduced a novel $A \beta$ TNF cable model used for TENS. The model is validated with previous studies and psychological experiments. At the same time, by combining 3D FEM and TNF modeling methods, we developed a novel $3 \mathrm{D}$ TENS model to explore the A $\beta$ TNF excitatory threshold currents under TENS. This novel 3D TENS model was validated by comparing computational and experimental results in terms of conduction velocity vs. fiber diameter and strength-duration relationships. Then we investigated the effects of electrode size and fiber diameters on the threshold current intensities. The computational results illustrated that the larger the nerve fibers, the lower the threshold current intensities. These computational results corresponded to our psychophysical experimental data on six healthy subjects. This presented novel 3D TENS model would further guide the generation of artificial tactile sensation based on the surface electrodes.

\section{ETHICS STATEMENT}

This study was carried out in accordance with the recommendations of the Ethics Committee of Human and Animal Experiments of the Med-X Research Institute at Shanghai Jiao Tong University with written informed consent from all subjects. All subjects gave written informed consent

\section{REFERENCES}

Antfolk, C., D’Alonzo, M., Rosén, B., Lundborg, G., Sebelius, F., and Cipriani, C. (2013). Sensory feedback in upper limb prosthetics. Expert Rev. Med. Devices 10, 45-54. doi: 10.1586/erd.12.68

Ara, J., Hwang, S. H., Song, T., and Khang, G. (2012). Electrically-elicited tactile sensation for different modulation types, polarities and waveforms of stimulation pulse trains. Int. J. Precision Eng. Manuf. 13, 1911-1916. doi: 10.1007/s12541-012-0251-8

Åström, M., Diczfalusy, E., Martens, H., and Wårdell, K. (2015). Relationship between neural activation and electric field distribution during deep brain stimulation. IEEE Trans. Biomed. Eng. 62, 664-672. doi: 10.1109/TBME.2014.2363494

Bourbeau, D. J., Hokanson, J. A., Rubin, J. E., and Weber, D. J. (2011). A computational model for estimating recruitment of primary afferent fibers by intraneural stimulation in the dorsal root ganglia. J. Neural Eng. 8:056009. doi: 10.1088/1741-2560/8/5/056009

Caruso, G., Massini, R., Crisci, C., Nilsson, J., Catalano, A., Santoro, L., et al. (1992). The relationship between electrophysiological findings, upper limb growth and histological features of median and ulnar nerves in man. Brain 115, 1925-1945. doi: 10.1093/brain/115.6.1925

Chai, G., Sui, X., Li, S., He, L., and Lan, N. (2015). Characterization of evoked tactile sensation in forearm amputees with transcutaneous electrical in accordance with the Declaration of Helsinki. The protocol was approved by the Ethics Committee of Human and Animal Experiments of the School of Biomedical Engineering at Shanghai Jiao Tong University.

\section{AUTHOR CONTRIBUTIONS}

XS contributed to the design of the overall experiment and simulation approaches; KZ conducted the simulation and psychophysical experiments. $\mathrm{KZ}$ wrote the first draft and XS also contributed to the whole manuscript revision. LL contributed to fiber model programming, and the manuscript revision. XW contributed to fiber model programming. All authors were active in the editing and revising processes of the manuscript. All authors read and approved the final manuscript.

\section{FUNDING}

This research is supported by the National Natural Science Foundation of China (81671801, 61671300, 61472247), and the SJTU SMC-Morning Star Excellent Young Scholar-B (14X100010047).

\section{ACKNOWLEDGMENTS}

The authors would like to thank Dr. Menghui Li and Si Li for the modeling advice, Mengnan Li and Ying Chen for help in the psychophysical experiments.

\section{SUPPLEMENTARY MATERIAL}

The Supplementary Material for this article can be found online at: http://journal.frontiersin.org/article/10.3389/fnins. 2017.00250/full\#supplementary-material

nerve stimulation. J. Neural Eng. 12:066002. doi: 10.1088/1741-2560/12/6/ 066002

Childress, D. S. (1980). Closed-loop control in prosthetic systems: historical perspective. Ann. Biomed. Eng. 8, 293-303. doi: 10.1007/BF02363433

Daniele, C. A., and MacDermott, A. B. (2009). Low-threshold primary afferent drive onto GABAergic interneurons in the superficial dorsal horn of the mouse. J. Neurosci. 29, 686-695. doi: 10.1523/JNEUROSCI.5120-08.2009

Debanne, D., Campanac, E., Bialowas, A., Carlier, E., and Alcaraz, G. (2011). Axon physiology. Physiol. Rev. 91, 555-602. doi: 10.1152/physrev.00048.2009

Dhillon, G. S., and Horch, K. W. (2005). Direct neural sensory feedback and control of a prosthetic arm. IEEE Trans. Neural Syst. Rehabil. Eng. 13, 468-472. doi: 10.1109/TNSRE.2005.856072

Djouhri, L., and Lawson, S. N. (2004). A $\beta$-fiber nociceptive primary afferent neurons: a review of incidence and properties in relation to other afferent A-fiber neurons in mammals. Brain Res. Rev. 46, 131-145. doi: 10.1016/j.brainresrev.2004.07.015

Forst, J. C., Blok, D. C., Slopsema, J. P., Boss, J. M., Heyboer, L. A., and Tobias, C. M. (2015). Surface electrical stimulation to evoke referred sensation. J. Rehabil. Res. Dev. 52:397. doi: 10.1682/JRRD.2014.05.0128

Frahm, K. S., Mørch, C. D., Grill, W. M., and Andersen, O. K. (2013). Experimental and model-based analysis of differences in perception of cutaneous electrical stimulation across the sole of the foot. Med. Biol. Eng. Comput. 51, 999-1009. doi: 10.1007/s11517-013-1079-9 
Gabriel, S., Lau, R. W., and Gabriel, C. (1996a). The dielectric properties of biological tissues: II. Measurements in the frequency range $10 \mathrm{~Hz}$ to $20 \mathrm{GHz}$. Phys. Med. Biol. 41:2251. doi: 10.1088/0031-9155/41/11/002

Gabriel, S., Lau, R. W., and Gabriel, C. (1996b). The dielectric properties of biological tissues: III. Parametric models for the dielectric spectrum of tissues. Phys. Med. Biol. 41:2271. doi: 10.1088/0031-9155/41/11/003

Gardner, E. P. (2010). "Touch," in Encyclopedia of Life Sciences, ed D. J. Perkel (Chichester: John Wiley \& Sons, Ltd.), 1-12.

Geddes, L. A. (2004). Accuracy limitations of chronaxie values. IEEE Trans. Biomed. Eng. 51, 176-181. doi: 10.1109/TBME.2003.820340

Goffredo, M., Schmid, M., Conforto, S., Bilotti, F., Palma, C., Vegni, L., et al. (2014). A two-step model to optimise transcutaneous electrical stimulation of the human upper arm. COMPEL Int. J. Comput. Math. Electrical Electron. Eng. 33, 1329-1345. doi: 10.1108/COMPEL-04-2013-0118

Halata, Z., Rettig, T., and Schulze, W. (1985). The ultrastructure of sensory nerve endings in the human knee joint capsule. Anat. Embryol. 172, 265-275. doi: 10.1007/BF00318974

Hebert, J. S., Olson, J. L., Morhart, M. J., Dawson, M. R., Marasco, P. D., Kuiken, T. A., et al. (2014). Novel targeted sensory reinnervation technique to restore functional hand sensation after transhumeral amputation. IEEE Trans. Neural Syst. Rehabil. Eng. 22, 765-773. doi: 10.1109/TNSRE.2013.2294907

Howells, J., Trevillion, L., Bostock, H., and Burke, D. (2012). The voltage dependence of Ih in human myelinated axons. J. Physiol. 590, 1625-1640. doi: 10.1113/jphysiol.2011.225573

Kajimoto, H., Kawakami, N., Maeda, T., and Tachi, S. (2004). "Electro-tactile display with tactile primary color approach," in International Conference on Intelligent Robots and Systems (IROS) (Sendai).

Kim, J., Lee, M., Shim, H. J., Ghaffari, R., Cho, H. R., Son, D., et al. (2014). Stretchable silicon nanoribbon electronics for skin prosthesis. Nat. Commun. 5:5747. doi: $10.1038 /$ ncomms6747

Kita, K., Otaka, Y., Takeda, K., Sakata, S., Ushiba, J., Kondo, K., et al. (2013). A pilot study of sensory feedback by transcutaneous electrical nerve stimulation to improve manipulation deficit caused by severe sensory loss after stroke. J. Neuroeng. Rehabil. 10:55. doi: 10.1186/1743-0003-10-55

Kuhn, A., Keller, T., Lawrence, M., and Morari, M. (2009a). A model for transcutaneous current stimulation: simulations and experiments. Med. Biol. Eng. Comput. 47, 279-289. doi: 10.1007/s11517-008-0422-z

Kuhn, A., Keller, T., Lawrence, M., and Morari, M. (2010). The influence of electrode size on selectivity and comfort in transcutaneous electrical stimulation of the forearm. IEEE Trans. Neural Syst. Rehabil. Eng. 18, 255-262. doi: 10.1109/TNSRE.2009.2039807

Kuhn, A., Keller, T., Micera, S., and Morari, M. (2009b). Array electrode design for transcutaneous electrical stimulation: a simulation study. Med. Eng. Phys. 31, 945-951. doi: 10.1016/j.medengphy.2009.05.006

Lacour, J. P., Dubois, D., Pisani, A., and Ortonne, J. P. (1991). Anatomical mapping of Merkel cells in normal human adult epidermis. Br. J. Dermatol. 125, 535-542. doi: 10.1111/j.1365-2133.1991.tb14790.x

Larkin, W. D., Reilly, J. P., and Kittler, L. B. (1986). Individual differences in sensitivity to transient electrocutaneous stimulation. IEEE Trans. Biomed. Eng. 33, 495-504. doi: 10.1109/TBME.1986.325736

Lesniak, D. R., Marshall, K. L., Wellnitz, S. A., Jenkins, B. A., Baba, Y., Rasband, M. N., et al. (2014). Computation identifies structural features that govern neuronal firing properties in slowly adapting touch receptors. Elife 3:e01488. doi: 10.7554/eLife.01488

Li, M. H., Yan, Y., Wang, Q. X., Zhao, H. H., Chai, X. Y., Sui, X. H., et al. (2013). A simulation of current focusing and steering with penetrating optic nerve electrodes. J. Neural Eng. 10:066007. doi: 10.1088/1741-2560/10/6/066007

Liao, Y.-Y., Yang, Y.-R., Cheng, S.-J., Wu, Y.-R., Fuh, J.-L., and Wang, R.-Y. (2014). Virtual reality-based training to improve obstacle-crossing performance and dynamic balance in patients with Parkinson's disease. Neurorehabil. Neural Repair 29, 658-667. doi: 10.1177/1545968314562111

Mahmud, M., and Vassanelli, S. (2016). Differential modulation of excitatory and inhibitory neurons during periodic stimulation. Front. Neurosci. 10:62. doi: $10.3389 /$ fnins.2016.00062

Matsumoto, M., Xie, W., Ma, L., and Ueda, H. (2008). Pharmacological switch in A $\beta$-fiber stimulation-induced spinal transmission in mice with partial sciatic nerve injury. Mol. Pain 4:25. doi: 10.1186/1744-8069-4-25
McGlone, F., and Reilly, D. (2010). The cutaneous sensory system. Neurosci. Biobehav. Rev. 34, 148-159. doi: 10.1016/j.neubiorev.2009.08.004

McIntyre, C. C., Grill, W. M., Sherman, D. L., and Thakor, N. V. (2004). Cellular effects of deep brain stimulation: model-based analysis of activation and inhibition. J. Neurophysiol. 91, 1457-1469. doi: 10.1152/jn.00989.2003

McIntyre, C. C., Richardson, A. G., and Grill, W. M. (2002). Modeling the excitability of mammalian nerve fibers: influence of afterpotentials on the recovery cycle. J. Neurophysiol. 87, 995-1006. doi: 10.1152/jn.00353.2001

McNeal, D. R. (1976). Analysis of a model for excitation of myelinated nerve. IEEE Trans. Biomed. Eng. 23, 329-337. doi: 10.1109/TBME.1976.324593

Mørch, C. D., Hennings, K., and Andersen, O. K. (2011). Estimating nerve excitation thresholds to cutaneous electrical stimulation by finite element modeling combined with a stochastic branching nerve fiber model. Med. Biol. Eng. Comput. 49, 385-395. doi: 10.1007/s11517-010-0725-8

Neerken, S., Lucassen, G. W., Bisschop, M. A., Lenderink, E., and Nuijs, T. A. (2004). Characterization of age-related effects in human skin: a comparative study that applies confocal laser scanning microscopy and optical coherence tomography. J. Biomed. Opt. 9, 274-281. doi: 10.1117/1.1645795

Provitera, V., Nolano, M., Pagano, A., Caporaso, G., Stancanelli, A., and Santoro, L. (2007). Myelinated nerve endings in human skin. Muscle Nerve 35, 767-775. doi: $10.1002 /$ mus.20771

Raspopovic, S., Capogrosso, M., Petrini, F. M., Bonizzato, M., Rigosa, J., Di Pino, G., et al. (2014). Restoring natural sensory feedback in real-time bidirectional hand prostheses. Science Transl. Med. 6:222ra19. doi: 10.1126/scitranslmed.3006820

Rattay, F. (1999). The basic mechanism for the electrical stimulation of the nervous system. Neuroscience 89, 335-346. doi: 10.1016/S0306-4522(98)00330-3

Sandby-Moller, J., Poulsen, T., and Wulf, H. C. (2003). Epidermal thickness at different body sites: relationship to age, gender, pigmentation, blood content, skin type and smoking habits. Acta Derm. Venereol. 83, 410-413. doi: 10.1080/00015550310015419

Saposnik, G., Teasell, R., Mamdani, M., Hall, J., McIlroy, W., and Cheung, D. (2010). Effectiveness of virtual reality using Wii gaming technology in stroke rehabilitation a pilot randomized clinical trial and proof of principle. Stroke 41, 1477-1484. doi: 10.1161/STROKEAHA.110.584979

Schwartz, A. B. (2004). Cortical neural prosthetics. Annu. Rev. Neurosci. 27, 487-507. doi: 10.1146/annurev.neuro.27.070203.144233

Schwarz, J. R., Reid, G., and Bostock, H. (1995). Action potentials and membrane currents in the human node of Ranvier. Pflugers Arch. 430, 283-292. doi: 10.1007/BF00374660

Sdrulla, A. D., Xu, Q., He, S.-Q., Tiwari, V., Yang, F., Zhang, C., et al. (2015). Electrical stimulation of low-threshold afferent fibers induces a prolonged synaptic depression in lamina II dorsal horn neurons to high-threshold afferent inputs in mice. Pain 156, 1008-1017. doi: 10.1097/01.j.pain.0000460353.15460.a3

Simpson, A. H., Gillingwater, T. H., Anderson, H., Cottrell, D., Sherman, D. L., Ribchester, R. R., et al. (2013). Effect of limb lengthening on internodal length and conduction velocity of peripheral nerve. J. Neurosci. 33, 4536-4539. doi: 10.1523/JNEUROSCI.4176-12.2013

Smit, J. E., Hanekom, T., and Hanekom, J. J. (2009). Modelled temperaturedependent excitability behaviour of a generalised human peripheral sensory nerve fiber. Biol. Cybern. 101, 115-130. doi: 10.1007/s00422-009-0324-7

Solomonow, M., Lyman, J., and Freedy, A. (1977). Electrotactile two-point discrimination as a function of frequency, body site, laterality, and stimulation codes. Ann. Biomed. Eng. 5, 47-60. doi: 10.1007/BF02409338

Sotiropoulos, S. N., and Steinmetz, P. N. (2007). Assessing the direct effects of deep brain stimulation using embedded axon models. J. Neural Eng. 4:107. doi: 10.1088/1741-2560/4/2/011

Tan, D. W., Schiefer, M. A., Keith, M. W., Anderson, J. R., Tyler, J., and Tyler, D. J. (2014). A neural interface provides long-term stable natural touch perception. Sci. Transl. Med. 6:257ral38. doi: 10.1126/scitranslmed.3008669

Tavernier, A., Dierickx, M., and Hinsenkamp, M. (1993). Tensors of dielectric permittivity and conductivity of in vitro human dermis and epidermis. Bioelectrochem. Bioenerg. 30, 65-72. doi: 10.1016/0302-4598(93)80063-Z

Tee, B. C. K., Chortos, A., Berndt, A., Nguyen, A. K., Tom, A., McGuire, A., et al. (2015). A skin-inspired organic digital mechanoreceptor. Science 350, 313-316. doi: $10.1126 /$ science.aaa9306 
Tyler, D. J., and Durand, D. M. (2002). Functionally selective peripheral nerve stimulation with a flat interface nerve electrode. IEEE Trans. Neural Syst. Rehabil. Eng. 10, 294-303. doi: 10.1109/TNSRE.2002.806840

Van Veen, B. K., Schellens, R. L. L. A., Stegeman, D. F., Schoonhoven, R., and Gabreëls-Festen, A. A. W. M. (1995). Conduction velocity distributions compared to fiber size distributions in normal human sural nerve. Muscle Nerve 18, 1121-1127. doi: 10.1002/mus. 880181008

Waxman, S. G. (1980). Determinants of conduction velocity in myelinated nerve fibers. Muscle Nerve 3, 141-150. doi: 10.1002/mus.880030207

Wesselink, W. A., Holsheimer, J., and Boom, H. B. (1999). A model of the electrical behaviour of myelinated sensory nerve fibres based on human data. Med. Biol. Eng. Comput. 37, 228-235. doi: 10.1007/BF02513291

Woo, S. H., Lumpkin, E. A., and Patapoutian, A. (2015). Merkel cells and neurons keep in touch. Trends Cell Biol. 25, 74-81. doi: 10.1016/j.tcb.2014.10.003

Yamamoto, T., and Yamamoto, Y. (1976). Electrical properties of the epidermal stratum corneum. Med. Biol. Eng. 14, 151-158. doi: 10.1007/BF02478741
Yang, H., Meijer, H. G., Doll, R. J., Buitenweg, J. R., and van Gils, S. A. (2015). Computational modeling of Adelta-fiber-mediated nociceptive detection of electrocutaneous stimulation. Biol. Cybern. 109, 479-491. doi: $10.1007 /$ s00422-015-0656-4

Conflict of Interest Statement: The authors declare that the research was conducted in the absence of any commercial or financial relationships that could be construed as a potential conflict of interest.

Copyright (c) $2017 \mathrm{Zhu}, \mathrm{Li}$, Wei and Sui. This is an open-access article distributed under the terms of the Creative Commons Attribution License (CC BY). The use, distribution or reproduction in other forums is permitted, provided the original author(s) or licensor are credited and that the original publication in this journal is cited, in accordance with accepted academic practice. No use, distribution or reproduction is permitted which does not comply with these terms. 


\section{OPEN ACCESS}

Edited by:

Dingguo Zhang,

Shanghai Jiao Tong University, China

Reviewed by:

Domen Novak,

University of Wyoming, USA

Nicolas Garcia-Aracil,

Universidad Miguel Hernández de

Elche, Spain

*Correspondence:

Carlos Rodriguez-Guerrero

carodrig@vub.ac.be

Specialty section

This article was submitted to

Neural Technology,

a section of the journal

Frontiers in Neuroscience

Received: 30 December 2016

Accepted: 12 April 2017

Published: 01 May 2017

Citation:

Rodriguez-Guerrero C, Knaepen K,

Fraile-Marinero JC, Perez-Turiel J, Gonzalez-de-Garibay $V$ and Lefeber $D$ (2017) Improving Challenge/Skill Ratio

in a Multimodal Interface by

Simultaneously Adapting Game

Difficulty and Haptic Assistance

through Psychophysiological and Performance Feedback.

Front. Neurosci. 11:242.

doi: 10.3389/fnins.2017.00242
Improving Challenge/Skill Ratio in a Multimodal Interface by Simultaneously Adapting Game Difficulty and Haptic Assistance through Psychophysiological and Performance Feedback

\author{
Carlos Rodriguez-Guerrero ${ }^{1 *}$, Kristel Knaepen ${ }^{2,3}$, Juan C. Fraile-Marinero ${ }^{4}$, \\ Javier Perez-Turiel ${ }^{4}$, Valentin Gonzalez-de-Garibay ${ }^{5}$ and Dirk Lefeber ${ }^{1}$ \\ ${ }^{1}$ Robotics and Multibody Mechanics, Flanders Make, Vrije Universiteit Brussel, Brussels, Belgium, ${ }^{2}$ Institute for Movement \\ and Neurosciences, German Sport University Cologne, Cologne, Germany, ${ }^{3}$ Human Physiology Research Group, Vrije \\ Universiteit Brussel, Brussels, Belgium, ${ }^{4}$ Biomedical Engineering, Fundacion CARTIF, Centro Tecnologico de Boecillo, \\ Valladolid, Spain, ${ }^{5}$ Department of Statistics and Operative Research, Universidad de Valladolid, Valladolid, Spain
}

In order to harmonize robotic devices with human beings, the robots should be able to perceive important psychosomatic impact triggered by emotional states such as frustration or boredom. This paper presents a new type of biocooperative control architecture, which acts toward improving the challenge/skill relation perceived by the user when interacting with a robotic multimodal interface in a cooperative scenario. In the first part of the paper, open-loop experiments revealed which physiological signals were optimal for inclusion in the feedback loop. These were heart rate, skin conductance level, and skin conductance response frequency. In the second part of the paper, the proposed controller, consisting of a biocooperative architecture with two degrees of freedom, simultaneously modulating game difficulty and haptic assistance through performance and psychophysiological feedback, is presented. With this setup, the perceived challenge can be modulated by means of the game difficulty and the perceived skill by means of the haptic assistance. A new metric (Flowlndex) is proposed to numerically quantify and visualize the challenge/skill relation. The results are contrasted with comparable previously published work and show that the new method afforded a higher Flowlndex (i.e., a superior challenge/skill relation) and an improved balance between augmented performance and user satisfaction (higher level of valence, i.e., a more enjoyable and satisfactory experience).

Keywords: psychophysiology, human-robot interaction, biocooperative, rehabilitation robotics, haptics, multimodal interfaces, biomechatronics 


\section{INTRODUCTION}

\section{Human-Robot Interaction}

In recent years, human-robot interaction (HRI) research has mostly focused on a master-slave relationship. However, as a natural step forward, robotics designers have found new and exciting frontiers to expand the existing research toward human-centered fields. Robots are now physically interacting with humans, especially in the medical domain (Colombo et al., 2001; Hochberg et al., 2012; Bonatti et al., 2014). This whole new field of robotics is evolving toward closing the gap between mechatronic systems and humans. In particular, robots have proved to be an excellent research tool in areas of neuroscience such as human motor control (Reinkensmeyer et al., 2004; Huang and Krakauer, 2009) and neurorehabilitation (Daly and Wolpaw, 2008; Hidler and Sainburg, 2011). Besides robots, other emerging technologies, such as virtual reality (Lewis and Rosie, 2012) and haptics (Okamura, 2009), are nowadays in the spotlight of neuroscientists. These new tools allow hacking and augmenting the human visual, auditory, or vibrotactile systems in a controlled way, thus opening up new routes for groundbreaking research.

\section{Patient-Cooperative Control}

In neurorehabilitation, there are many examples of mechatronic systems designed for human-robot cooperative tasks. The main idea behind these systems is to assist the subject in completing a given task (e.g., reaching or grasping), typically by using a haptic controlled robotic device to deliver the forces needed for the correct completion of the objective (Liu et al., 2006; Nef et al., 2009; Song et al., 2013; Squeri et al., 2014; Pignolo et al., 2016). The intended output of these therapeutic robots is to promote engagement, human effort, and intention of movement and, therefore, to accelerate motor learning and induce neural plasticity (Reinkensmeyer et al., 2004; Edgerton et al., 2008; Huang and Krakauer, 2009; Pennycott et al., 2012). The control strategies of interactive robotic devices are typically designed based on the paradigm "assistance-as-needed," where human and robot cooperate to successfully complete a task, minimizing the intervention from the robotic device and maximizing that of the human. This patient-cooperative control is mostly based on performance measures such as kinematics, kinetics, muscle activity, etc.

Yet, in spite of human-robot cooperative tasks being "performed" accurately and successfully through these patientcooperative control paradigms, patient outcome and recovery did not improve significantly with the introduction of "patient-incharge" robotic rehabilitation devices. Issues such as emotional and cognitive stress, excessive physical work demand, discomfort, pain, boringness, and lack of motivation are rarely taken into account, yet play a key role in human motor control performance (Guadagnoli and Lee, 2004). For example, emotions such as anxiety, frustration, or stress can have a large impact on motor performance, speed, and variability (Coombes et al., 2006). As early as 1908, Yerkes and Dodson (1908) showed that human performance changes in relation to the level of arousal depending on the difficulty of the task: for a difficult task, high levels of arousal can be counterproductive, but if the task lies within the subject's capabilities, high levels of arousal can be beneficial instead (Yerkes and Dodson, 1908; Diamond et al., 2007).

According to Mihály Csíkszentmihályi (1992), cognitive states (e.g., focused, bored, motivated) can also change in relation to the challenge and skill level perceived by a given subject. These cognitive states can either increase or decrease performance accordingly. For instance, a state of boredom will tend to decrease performance, while a focused cognitive state will lead to better results by promoting mental engagement (Guadagnoli and Lee, 2004; Holden, 2005).

\section{Assessment of Emotion}

Emotions are accompanied by a set of somatic responses associated with autonomic nervous system (ANS) activity. For a specific emotional state, there exists a probable set of somatic and ANS outputs, although it remains unclear which kind of emotion is associated with which exact autonomic signature (Kreibig, 2010). Nevertheless, psychophysiology has been used to indirectly measure ANS-related responses to external stimuli that affect a person's mood and engagement in a variety of interactive scenarios (Kreibig, 2010). Autonomic responses of emotion are present in parameters such as heart rate (HR), skin temperature (SKT), galvanic skin response (GSR), and respiration rate (RR). A complete overview of these parameters has been summarized in a tag cloud based on 134 publications and explained in detail in Kreibig (2010).

\section{Biocooperative Control}

In an attempt to harmonize existing mechatronic systems with human beings, physiological computing has appeared as an enabling technology to give machines exteroceptive capabilities to measure and record physiological data on the emotional state of the user, allowing proactive and implicit adaptations of HRI in real time (Fairclough, 2009, 2011). This concept includes the human in the control loop and gives birth to a whole new set of possible biomechatronic devices capable of "biocooperative adaptation" (Pope et al., 1995; Prinzel et al., 2000).

In 2008-2009, the first ideas of using ANS-related signals as feedback information to close a biocooperative control loop took shape (Bonarini et al., 2008; Mihelj et al., 2009; Novak et al., 2009; Riener et al., 2009; Rodriguez Guerrero et al., 2009). In 2010, Novak et al. (2010a,b) attempted to determine which psychophysiological responses provide the most reliable information about the subject's psychological state during an upper-limb virtual reality (VR) task with the HapticMaster robot in healthy subjects (Novak et al., 2010a) and stroke patients (Novak et al., 2010b; Goljar et al., 2011). Koenig et al. (2010, 2011b) took this research a step further and determined the most suitable psychophysiological parameters to estimate the psychological state of subjects and neurological patients during walking in a driven gait orthosis Lokomat while completing a cognitively demanding VR task (Koenig et al., 2010, 2011e). Also in 2010, Rodriguez Guerrero et al. (2010) presented a step forward toward a working prototype of a biocooperative closed loop that included HR feedback to modulate a set of assistive forces in a robotic-aided neurorehabilitation scenario. Soon after, Novak et al. (2011a,b), Koenig et al. (2011a), Badesa 
et al. (2012, 2014), and Morales et al. (2014) implemented a more complex closed-loop system that included feedback parameters related to HR, RR, GSR, and SKT (Novak et al., 2011a,b; Koenig et al., 2011b; Badesa et al., 2012, 2014; Morales et al., 2014). These studies aimed to change the difficulty level of the VR task depending on the emotional and cognitive load experienced by the subjects (Koenig et al., 2011b; Novak et al., 2011a,b; Badesa et al., 2012, 2014; Morales et al., 2014). In a previous work, Rodriguez Guerrero et al. (2013) developed a closed biocooperative loop with an auto-tuning fuzzy logic classifier to modulate the haptic assistance given to subjects using HR and GSR feedback. Notice that in contrast with the work by Koenig and Novak (Koenig et al., 2011b; Novak et al., 2011a,b), the goal was not to adapt the game difficulty and thus the perceived challenge level, but to adapt the level of assistance given, thereby attempting to affect the perceived skill level instead. Although the conclusions of Rodriguez Guerrero et al. have suggested that psychophysiological feedback alone can help improve the user experience, independent of whether there exists contextual information about the performance or not, they also suggested that the biocooperative system could be further improved by blending the haptic modulation together with adaptations in game difficulty (Rodriguez Guerrero et al., 2013).

\section{Article Contribution}

The Yerkes and Dodson model (Yerkes and Dodson, 1908; Diamond et al., 2007) suggests that arousal levels have a strong impact on performance. It also suggests that high levels of arousal can be counterproductive, especially when the presented task becomes overwhelming to the subject. In contrast, if the task lies within the subject's capabilities, high levels of arousal can be beneficial instead. The contribution of this work expands upon this logic and improves the biocooperative architecture presented by Rodriguez Guerrero et al. (2013) by augmenting the system with contextual performance information that, when blended with the individual's psychophysiological feedback, provides a superior and more individualized continuous adaptation of the system to keep the task difficulty within its user's capabilities, improving subject performance and more importantly the overall user experience. Moreover, as there is still controversy on which psychophysiological parameters are eligible for use in biocooperative enhanced systems, the present study will also take into consideration, as was previously done by Novak et al. (2010a) and Knaepen et al. (2015), which psychophysiological parameters are the most suited for use in a closed biocooperative loop. Finally, a new metric (i.e., FlowIndex) is proposed to numerically quantify and visualize the challenge/skill relation.

\section{MATERIALS AND METHODS}

\section{Subjects}

Eleven subjects participated in this study involving an openloop and a closed-loop experiment. The subjects had no clinical records of neural or motor deficiencies. Six subjects [five males and one female, mean age 30.5 years $(S D=3.83)$ ] participated in the open-loop experiment and 11 subjects [eight males and three females, mean age 35 years $(S D=7.34)$ ] participated in the closed-loop experiment. All experimental procedures were performed according to the standards set by the Declaration of Helsinki for medical research involving human subjects. Upon arrival in the lab, subjects signed a written informed consent form. This research was approved by the medical ethics committee of Fundación CARTIF.

\section{Instrumentation \\ Virtual Reality Game}

The virtual scenario was based on a popular "catch the falling droplet" reaching game (see Rodriguez Guerrero et al., 2013 for more details), which has also been used by other robotic rehabilitation platforms such as Armeo (Merians et al., 2009; Schwickert et al., 2011; Wittmann et al., 2016). The game interface and its mechanics are very simple and demand basically no explanation or extra human intervention. Droplets were spawned one at a time at a given velocity, $v_{\text {droplet }}$, defined in pixels/sec. Only one droplet was rendered at a time, to avoid pre-emptive maneuvers from the subject and maintain focus on the actual target. The subject was expected to catch the falling droplet by moving the PHYSIOBOT end effector, which was represented by a cup in the VR environment. Movements of the robot were projected back to the screen, thereby acting as a visual feedback. The initial positions of the droplets were predefined and generated in advance as an array of 100 positions. This array size is sufficiently large to prevent subjects from memorizing the positions, therefore avoiding habituation issues. The difficulty level of the task could be changed by altering either the distance between droplets or the falling speed. However, by keeping the same initial positions, the total amount of power needed for the task to be successfully completed in a certain amount of time was homogeneous among sessions with a defined velocity (i.e., experiments in the open loop). Therefore, the difficulty modulation was reduced to make changes in the velocity of the falling droplets only. The faster the droplets fall, the greater the amount of power needed to catch them in time, due to the permanent velocity-dependent force field rendered by the haptic device.

\section{Haptic Assistance}

The PHYSIOBOT is capable of rendering a wide range of $3 \mathrm{D}$ forces and primitives such as haptic walls, dampers, springs, textures, or a combination thereof (Rodriguez Guerrero et al., 2009). In this setup, a viscoelastic force tunnel was rendered so that it created resistive forces in the sagittal and vertical axes. On the task axis (i.e., transverse axis), a constant-viscosity force field $(150 \mathrm{Ns} / \mathrm{m})$ was rendered, such that velocity-dependent resistive forces made the physical task of moving the virtual cup a demanding exercise without the appropriate haptic assistance. The robot could also help the subject by applying assistive forces in the task direction. Forces could be modulated, placed, or removed online at any time.

Optimally, haptic assistance should be computed as the force needed for the robot to take the subject to the target with minimum intervention, and simultaneously promote engagement and self-initiated movements. Therefore, the 
assistive forces were designed along the task axis, as a critically damped second-order system. A critically damped response allows the system to marginally get to the target in time with the minimum amount of assistance needed to complete the task (see Rodriguez Guerrero et al., 2013 for further details of this calculation). Besides the actuation strategy in terms of magnitude, the timing of the assistive forces needed to be determined. Most of the existing physical human-robot cooperative systems, such as those in rehabilitation robotics, render assistive forces to correct erroneous movements with the help of virtual springs. The problem with this method is that it creates the feeling of being helped rather than the feeling of being more capable/in control of moving the virtual object. The haptic assistance in PHYSIOBOT enhances, rather than corrects, the subject's movements by only giving assistance when the subject has initiated the movement in the target direction. The goal is to "hide" the sensation of being helped and to promote the feeling of being more skillful and capable, a very important feature achieved in this design.

\section{Biocooperative Control}

In a biocooperative control loop, the human plays a key central role. Figure 1A shows the human as a plant in a classical control scheme. The human block is where the final action lies, and also where the primary feedback is taken from. Figure 1B shows a greatly simplified human control system that only involves some specific portions of the nervous system directly affected by the multimodal interface: the audiovisual stimuli coming from the virtual environment and the interaction forces coming from the physical human-robot interaction with the PHYSIOBOT.

The biocooperative high-level controller used was a TakagiSugeno-type fuzzy inference system (FIS) synthesizing several fuzzy rules (see Appendix A in Supplementary material for a complete list of the rules used). Fuzzy logic has already been used in psychophysiological and emotion modeling (Mandryk and Atkins, 2007; Badesa et al., 2012, 2014; Rodriguez Guerrero et al., 2013; Lledo et al., 2015). This system combines data fusion, classification, and control in one simple and wellknown framework. The system has four inputs and two outputs (Figure 2).

The results from the open-loop experiments suggested (Section Open-Loop Results: Open-Loop Results) that the most useful signals to use as inputs for the controller were HR and the tonic (i.e., skin conductance level, SCL) and phasic (i.e., skin conductance response frequency, SCR frequency) components of the GSR signal. Performance was the fourth input of the highlevel controller. The outputs were the haptic assistance and the changes in game difficulty (i.e., changes in the speed at which the droplets fell). The haptic assistance output was a value between 0 and 1, where 0 represents no assistance and 1 represents full critical assistance. This allows the system to act accordingly when either of the two undesired situations (i.e., under- or overchallenge) occurs for the subject. For instance, if the overall state of the subject indicates that he/she is underchallenged, the assistance will cease, and the difficulty of the task will be augmented accordingly. If, on the other hand, the overall state of the subject indicates that he/she is overchallenged, the assistance will be computed and delivered according to his/her ANS activity, and the game difficulty may be reduced as well.

An intrinsic problem in biocooperative controller design is the inter-/intrasubject variability. In Badesa et al. (2012), fuzzy logic was used but no adaptation algorithm was implemented to deal with this effect. This is important as any consumed substance such as $\beta$-blockers, antidepressants, coffee, or sugar may drastically impact baseline ANS readings. In order to deal with the problem of the inter-/intrasubject variability, the FIS was tuned to adapt to the immediate state of each subject. Every FIS has two basic tunable sets of parameters, the rules base, and the membership functions (MFs) for each I/O. The MFs were automatically calibrated after an open-loop "calibration task" designed primarily for that purpose. In the 3-min non-hapticassisted VR calibration task, the difficulty level was adapted such that every time the subject caught a droplet, the system augmented the falling speed by 2 pixels/s; on the contrary, if the subject missed a droplet, the system reduced the speed by the same amount. The idea behind the calibration task was that the subject's score would steadily increase over time until the challenge level started to approach his/her skill level. After reaching this tipping point, the subject typically started to get drastically aroused as their skill limit was constantly being challenged by the difficulty of the game. The data obtained at the end of the calibration task can be used to tune the MF of the HR, SCL, and SCR frequency. We refer the reader to Rodriguez Guerrero et al. (2013) for more details regarding MF auto-tuning. Besides these two parameters, the rules base and the MFs, a performance parameter (i.e., based on the subject's performance during the VR game) was used in the high-level controller of the biocooperative feedback loop.

The algorithm shown in Figure 3 was used to compute the "performance" input to the fuzzy controller based on the trend of the global score. The idea behind this design was that, no matter how well subjects were performing globally (i.e., their total game score), small sustained changes in performance would always influence their emotions. A "trend" or winning/losing streak will often boost or deteriorate our confidence (i.e., dominance value). Therefore, if two droplets were caught $/ \mathrm{missed}$ in a row, the performance sign changed accordingly in order to take preemptive actions in the controller logic to avoid frustration.

The fuzzy controller worked at a sample rate of $100 \mathrm{~Hz}$, although both of its outputs (i.e., haptic assistance and game difficulty) were updated every epoch ( $\mathrm{n}$; the period between two consecutive droplets). Thus, the output of an epoch (n) was the result of the average of the calculations made in the epoch $(n-1)$. As such, the calculations and effects of the inputs that manage the signals were synchronized with the performance input, which was updated every epoch (n). This averaging contributes to filtering the outputs, especially the haptic assistance, so that the subject does not feel as though it is changing continuously, thereby producing a more natural, less noisy feeling of the haptic assistance and avoiding a constantly varying droplet speed.

\section{Experimental Design}

Upon arrival in the lab, the purpose and procedure of the experiment were explained and the subjects signed the written 
A

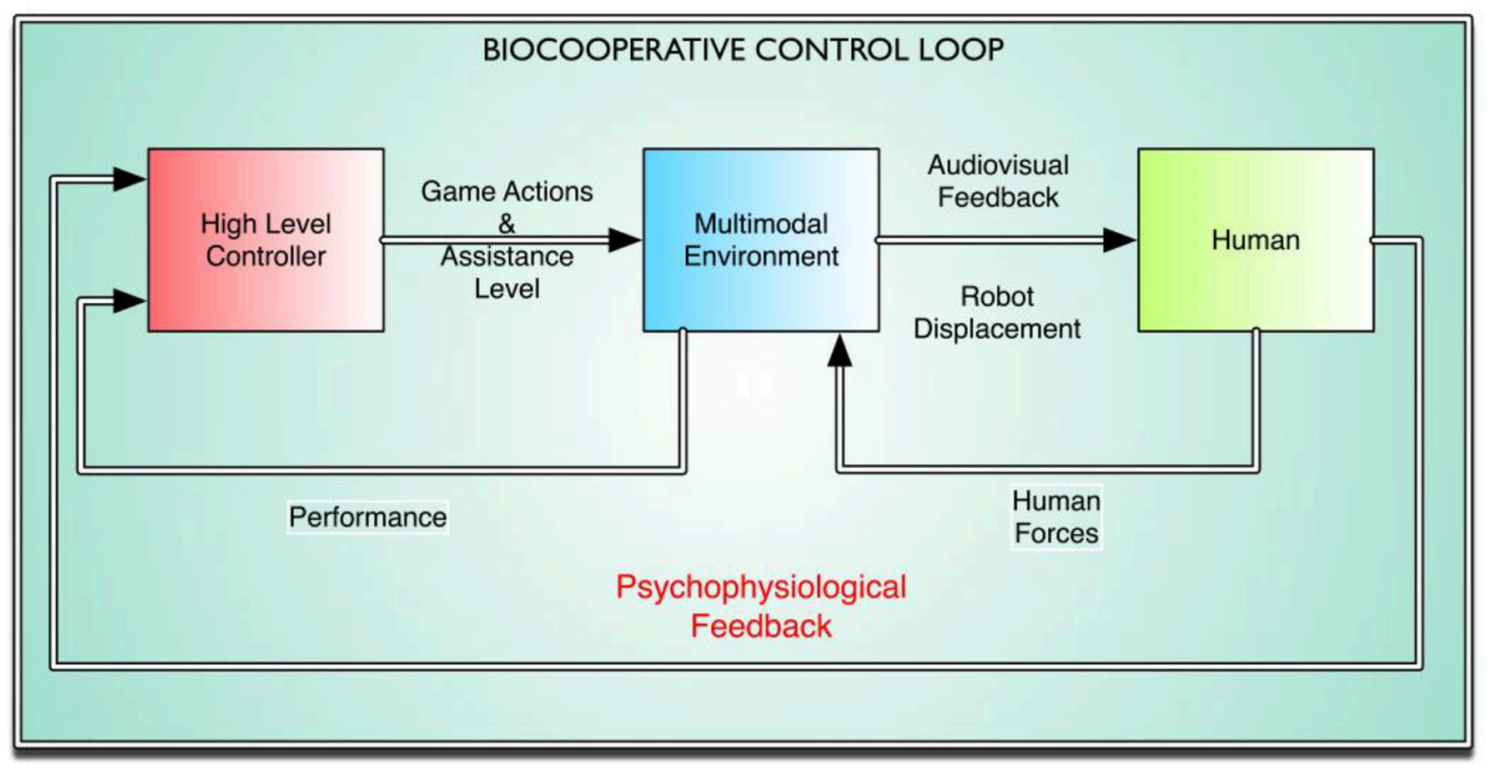

B

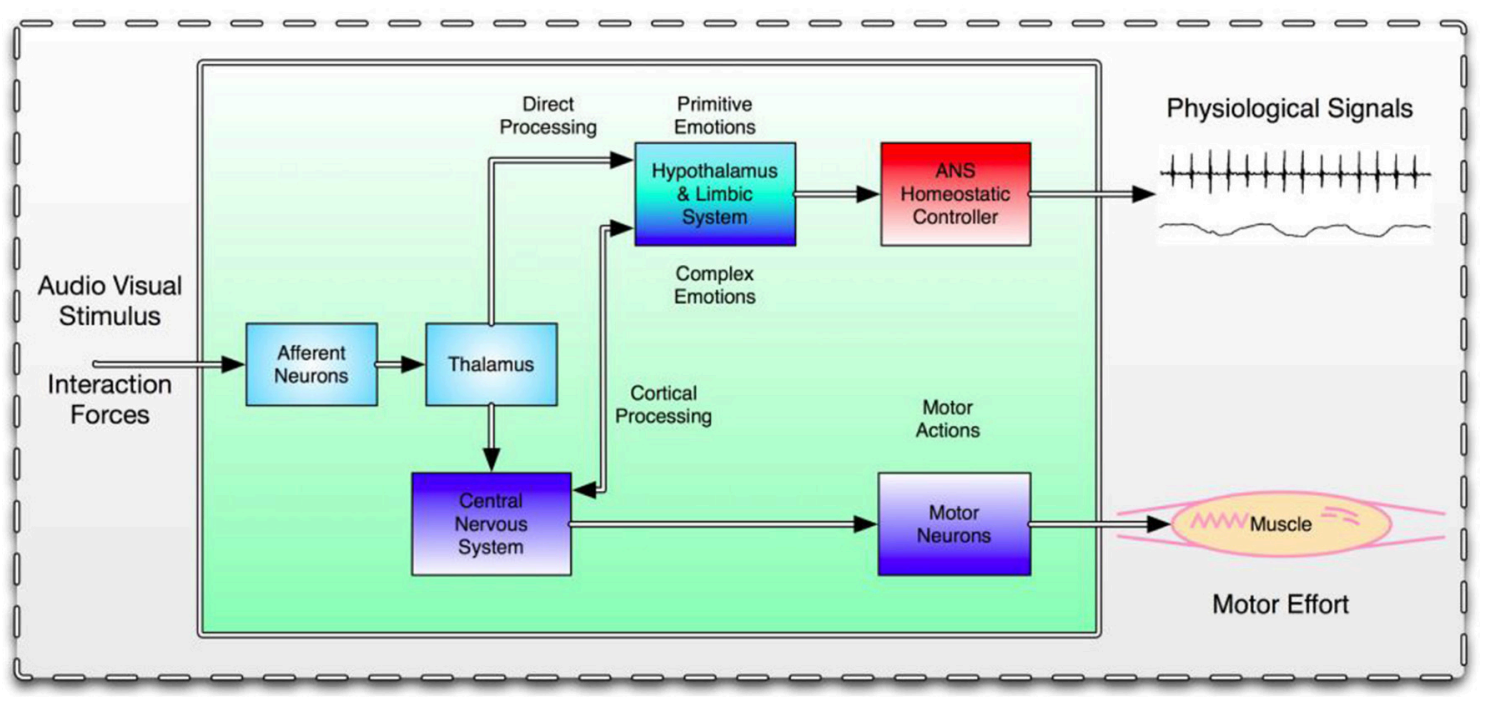

FIGURE 1 | (A) Macro view of the biocooperative control loop. Three different interacting blocks can be distinguished. From left to right, the red block represents the high-level controller, which makes decisions based on the overall state of the system, including the subject, and sends actions regarding the difficulty of the game and the level of haptic assistance to the multimodal environment represented by the central blue block. The yellow block represents the human, whose responses to the audiovisual and haptic feedback generate ANS responses and interaction forces, which are, in turn, used to close the biocooperative loop. (B) Effects of the multimodal interface (i.e., PHYSIOBOT and VR) on the human control system. External stimuli are captured by the afferent neurons in the peripheral nervous system (PNS) and distributed over different cortical and subcortical levels to the CNS: the thalamus, the limbic system, and the cortex. All external sensory input is received by the thalamus, which sends information simultaneously to the cortex for higher-level processing, and directly to the limbic system (LeDoux, 1995). Part of the information goes to the hypothalamus, which secretes neurohormones that either stimulate or inhibit the secretion of the pituitary hormones that modulate the behavior of the ANS. Simultaneously, the external stimuli make their way to the sensorimotor cortex, which modulates ongoing movements and motor effort, and the prefrontal cortex, which controls the subject's psychophysiological state and turns it into a conscious state and thus a mood or feeling. ANS signals are then fed to the high-level controller and the interaction forces are fed to the multimodal environment as "human in the loop" feedback signals.

informed consent form and were equipped with the Biopac MP150 data acquisition system for measuring ANS responses (i.e., ECG, GSR, and SKT). Next, subjects participated in two different experiments involving a VR task of catching falling droplets by manipulating the PHYSIOBOT end effector (see
Rodriguez Guerrero et al., 2013 for a picture of the experimental setup). During each session, electrophysiological data, game performance, and physical performance were measured, and immediately following each session, the emotional status of the subject was registered. 


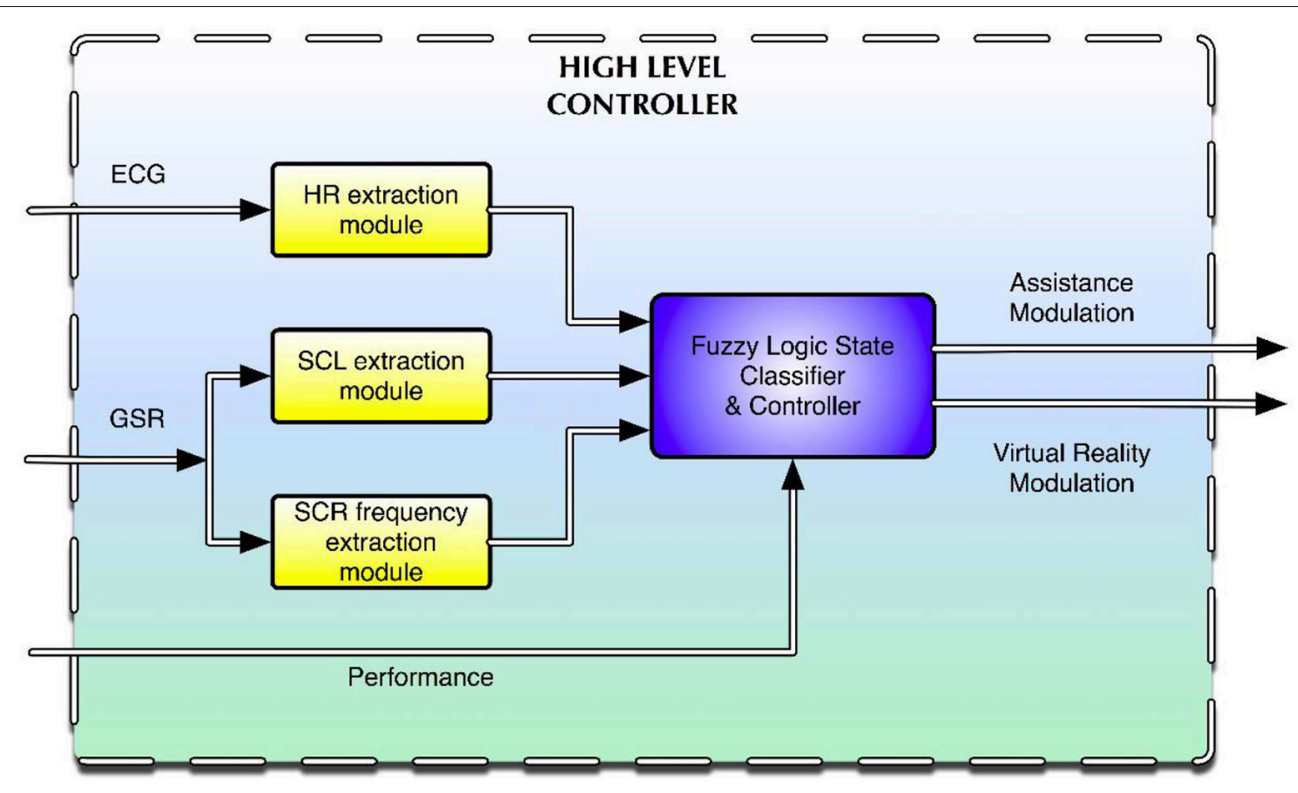

FIGURE 2 | High-level controller driving the interaction between the subject and the multimodal environment.

\section{Open-Loop Experiment}

The first experiment was an open-loop experiment $(n=6)$ consisting of a warm-up of 3 min followed by four game sessions of $5 \mathrm{~min}$ each. The 3-min warmup time (i.e., no physical or mental exercises were performed) was used to stabilize the sensor readings to a baseline. The four experimental sessions contained four different levels of difficulty ranging from very easy to very hard, starting with difficulty level 3 , then difficulty 4 , followed by difficulty 2, and finally difficulty level 6 (Table 1). All difficulty levels were chosen in advance, prior to the experiment.

\section{Closed-Loop Experiment}

The second experiment was a closed-loop experiment $(n=11)$ consisting of a control task of 5 min (i.e., the calibration task, hereafter referred to as CT), followed by a 5 -min resting period and a 5-min closed-loop trial (hereafter referred to as haptic and difficulty modulation (HDM) controller). The obtained data were compared to the results obtained in Rodriguez Guerrero et al. (2013), where a haptic-assisted-only controller was used (hereafter referred to as $\mathrm{HAO}$ ).

\section{Data Acquisition and Analysis VR Score}

The score on the VR task was calculated based on the game score, which added one point for each droplet caught and subtracted one point if a droplet was missed. The score mean value with standard deviation (SD) is presented for each open- and closed-loop experiment in the results.

\section{Physical Performance}

Physical performance was computed by measuring the interaction force with the PHYSIOBOT haptic device by means of a force sensor mounted between the end effector and the human. The mean absolute force (MAF) expressed in newtons (N) with SD was used as an indicator of physical performance and was computed by extracting the mean of the absolute value of all of the forces over the task axis during a session, sampled at a rate of $1 \mathrm{kHz}$.

\section{Electrophysiological Assessment}

For the physiological signal recording, the Biopac MP150 data acquisition system ${ }^{1}$ with three amplifiers, ECG100c ${ }^{2}$, GSR $100 c^{3}$, and SKT100c ${ }^{4}$, was used. All recordings were collected online during the game time through UDP/IP at a frequency of 100 Hz. All physiological measurements were recorded through noninvasive, ambulatory sensors, using simple, and fast Velcro attachment electrodes for GSR and SKT and sticky disposable electrodes for the ECG. The mean values for the 5-min experimental sessions (i.e., open- and closed-loop experiments) were calculated. From the ECG, the intervals between two heartbeats (NN intervals) were extracted in order to calculate the mean HR value with SD. Two components were extracted from the GSR signal: SCL and SCR frequency. The mean SCL with SD, which is the baseline level of skin conductance, was calculated for each game difficulty level. The SCR frequency represents increases in skin conductance followed by a return to the tonic level. The mean SCR frequency with SD as well as mean SKT with $\mathrm{SD}$ are presented in the results.

\footnotetext{
${ }^{1}$ http://www.biopac.com/data-acquisition-system-mp150-system-glp-win.

${ }^{2}$ http://www.biopac.com/ecg-electrocardiogram-amplifier.

${ }^{3}$ http://www.biopac.com/gsr-eda-galvanic-skin-response-amplifier-

electrodermalactivity.

${ }^{4} \mathrm{http}: / /$ www.biopac.com/skin-temperature-amplifier.
} 


\section{Emotion Assessment}

To subjectively assess emotion, the self-assessment manikin (SAM) test was used (Posner et al., 2005, 2009) (see Appendix A in Supplementary Material). We implemented a computer

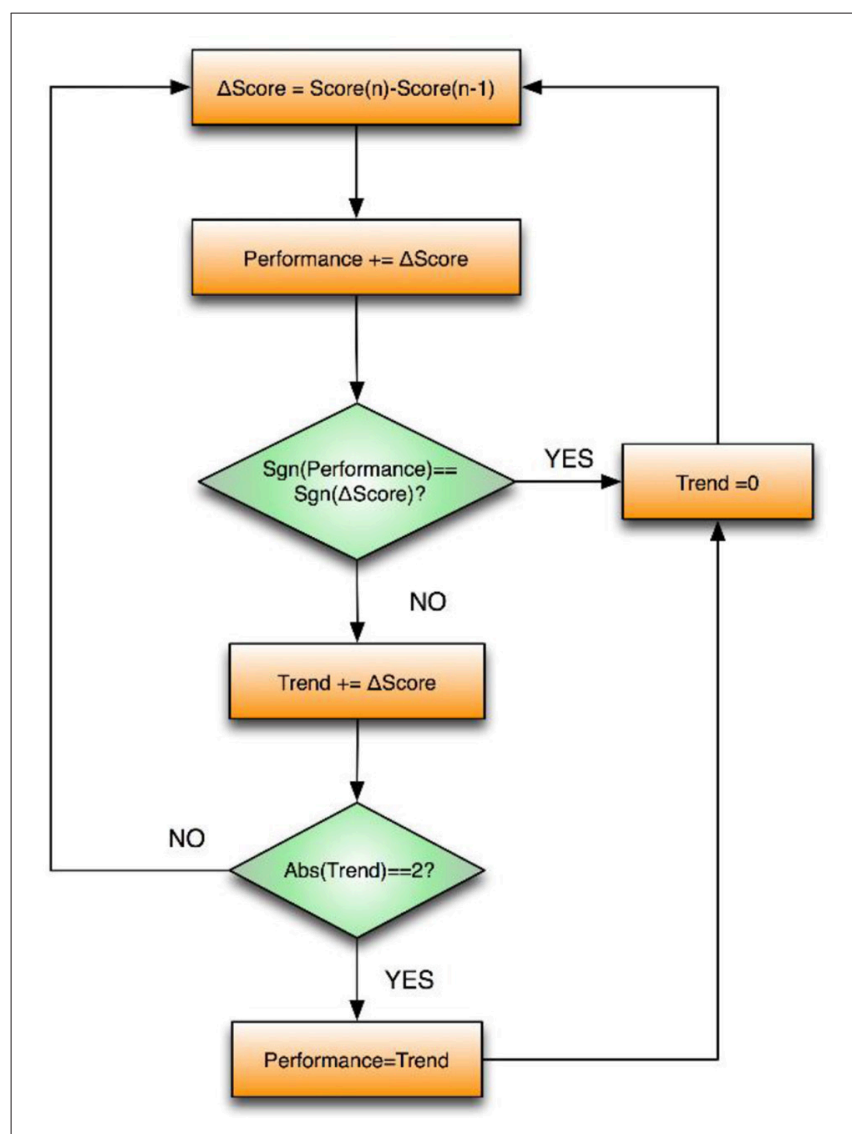

FIGURE 3 | Schematic overview of the logic for calculating the performance trend in the VR game.

TABLE 1 | The four levels of task difficulty in the open-loop experiment $(n=6)$.

\begin{tabular}{llc}
\hline $\begin{array}{l}\text { Difficulty } \\
\text { level }\end{array}$ & Description & $\begin{array}{c}\text { Presentation } \\
\text { order }\end{array}$ \\
\hline 2 & $\begin{array}{l}\text { Very easy: This task is meant as a baseline period. It } \\
\text { maintains the subject's focus and helps to reduce } \\
\text { psychophysiological responses elicited by random } \\
\text { mental thoughts or memories. }\end{array}$ & 3rd \\
\hline 3 & $\begin{array}{l}\text { Normal: This task makes a good transition between } \\
\text { the baseline period and the following trials. }\end{array}$ & 1st \\
\hline 4 & $\begin{array}{l}\text { Challenging: This is a more demanding task than } \\
\text { level 3, but in general, it fits better with the average }\end{array}$ & 2nd \\
subject preferences. & & \\
\hline 6 & $\begin{array}{l}\text { Very hard: Although this level can be quite hard, it is } \\
\text { not designed to be frustrating but demanding } \\
\text { instead. It is meant to force the subject into an } \\
\text { aroused state. }\end{array}$
\end{tabular}

program that presented the SAM test at the end of each session in an automated way, such that the subjects were able to select their choice without any external human intervention that might have otherwise biased their answers. The three emotional dimensions used were arousal, valence (i.e., pleasure), and dominance (i.e., sense of control of the situation). Each dimension was rated on a nine-point scale (i.e., 1-9). For each dimension, the mean value with SD is presented.

\section{Vector Representation, Flowlndex, and FlowDir}

In this article, a new and simple set of tools is proposed for analyzing, visualizing, and comparing the impact of a biocooperative controller based on arousal and dominance scores: the vector representation, FlowIndex, and FlowDir. These vectors are tools to graphically and numerically evaluate the results of a given control strategy designed to act on the challenge/skill perception of a particular task.

The vector representation (Figure 4) is a visual way to present the challenge/skill ratio and compare the [dominance, arousal] results (i.e., from the SAM questionnaire or as a result of the output of an automatic classifier) of one or more tasks (i.e., vector A) relative to the maximal/optimal challenge/skill ratio (i.e., reference vector $\mathbf{F}$ ). This metric is a mathematical representation inspired by a theory used in psychology termed "Flow statem," where an optimal experience motivates people to further learn or stay committed to a task (Csikszentmihalyi and LeFevre, 1989; Engeser and Rheinberg, 2008). The challenge level relates to the arousal domain and can be modulated by means of the game difficulty level, and the skill level is related to the dominance domain and can be modulated by means of the haptic assistance; see Equation (Hochberg et al., 2012). Therefore, the equivalence

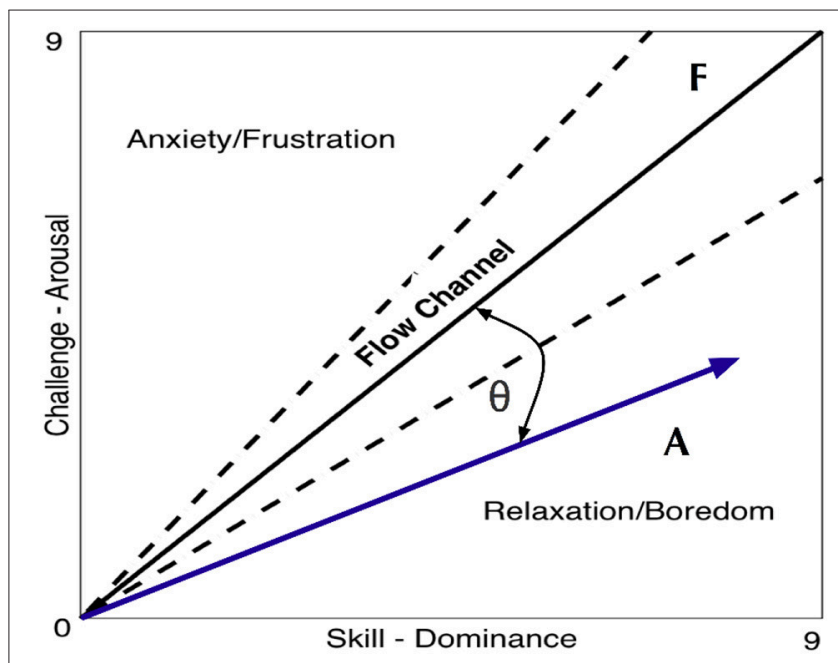

FIGURE 4 | Vector $F$ is the reference with components [dominance, arousal $]=[9,9]$ and vector $A$ is used as an example of a given output obtained within a session with coordinates [8,5]. The vector

representation affords a quick glance into the performance of a given session. In this example, the results point toward the relaxation/boredom zone of the figure and therefore it is unlikely that the experience was enjoyable or engaging but rather boring instead. 
for their ratios can be defined as:

$$
\frac{\text { Challenge }}{\text { Skill }} \cong \frac{\text { Arousal }}{\text { Dominance }}
$$

Since this relation has two components, it can be expressed and plotted as a vector (Figure 4).

FlowIndex uses the obtained pair $\mathbf{A}$ [dominance, arousal] represented in vector form, and quantifies how alike vector $\mathbf{A}$ (blue) and the ideal vector $\mathbf{F}$ (solid black) are (Figure 4). This form of vector similarity first uses the normalized scalar projection (NSP) (Equation 2) of $\mathbf{A}$ projected onto $\mathbf{F}$. The result of this calculation is a scalar bounded between 0 and 1 that gives information on how $\mathbf{A}$ projects onto $\mathbf{F}$. Then, the angular similarity (AS) (Equation 3) is used to calculate the similarity between the two vector angles and gives again a value bounded between 0 and 1 (i.e., where 0 is completely orthogonal and 1 is perfectly parallel). By multiplying the two obtained results (Equation 4), the AS penalizes vectors that are diverging from $\mathbf{F}$ (i.e., the angle between them is $>0$ ). Notice that both NSP and AS are bounded between 0 and 1 and, therefore, the FlowIndex results will always be bounded in the same way. The FlowIndex is then a scalar value that represents how alike $\mathbf{A}$ and $\mathbf{F}$ are in terms of both angle and magnitude.

The higher the FlowIndex value is, the closer we are to being within the flow channel (i.e., the dotted lines delimiting the ideal range of challenge/skill ratio in Figure 4) and, therefore, the more pleasant and exciting the experience will be.

$$
\begin{aligned}
\text { NSP } & =\frac{A . \hat{F}}{\|F\|} \\
A S=\cos \theta & =\left(\frac{A . F}{\|A\|\|F\|}\right) \\
\text { FlowIndex } & =N S P * A S
\end{aligned}
$$

Since FlowIndex is always positive and bounded between 0 and 1 , this metric alone cannot reveal if the resulting vector is pointing toward the frustration or relaxation zone. Nevertheless, the direction of the FlowIndex can be calculated. This computation is straightforward and can be performed as follows:

$$
\text { FlowDir }= \begin{cases}\text { Frustration } & \operatorname{sign}\left(A_{y}-A_{x}\right)=1 \\ \text { Flow } & \operatorname{sign}\left(A_{y}-A_{x}\right)=0 \\ \text { Relaxation } & \operatorname{sign}\left(A_{y}-A_{x}\right)=-1\end{cases}
$$

Equation (5) gives a value of 0 whenever vectors $\mathbf{A}$ and $\mathbf{F}$ are parallel (i.e., flow), 1 whenever vector $\mathbf{A}$ is pointing toward the frustration zone, and -1 whenever it is pointing toward the relaxation zone. For this given example, the FlowIndex of $\mathbf{A}=$ $[8,5]$ is 0.65 with FlowDir = Relaxation.

\section{Statistical Analysis}

Statistical analysis was performed using the Statistical Package for Social Sciences 24.0 for Windows statistical software (SPSS Inc., Chicago, IL, USA). Data are presented as mean with SD. Statistical significance was accepted at $p<0.05$. As $n=6$, nonparametric statistics were applied and effect sizes were calculated.
Eight variables were extracted from all recorded data: valence, dominance, and arousal were extracted from the SAM; MAF from the haptic device; and SKT, HR, SCL, and SCR frequency were derived from physiological recordings. To assess differences between the four difficulty levels in the open-loop experiment $(n=6)$, a Friedman's analysis of variance (i.e., ANOVA) was used. As an estimate of effect size, Kendall's $W$ coefficient was calculated, where a value of $W=0$ indicates no relationship and a value of $W=1$ indicates a perfect relationship (Tomczak and Tomczak, 2014). The following null hypothesis was tested, for which $\mu_{i}$ is the mean of the observed variable for each difficulty level (i.e., subindex $i$ corresponds to difficulty levels 2,3 , 4, and 6): no significant differences between the four difficulty levels, $\mathrm{H}_{0}: \mu_{2}$ $=\mu_{3}=\mu_{4}=\mu_{6}$.

Furthermore, Spearman's rank correlation coefficient was used to measure the strength of the association between the subjective data from the SAM questionnaire and objective data such as HR, SCL, SCR, SKT, and MAF.

\section{RESULTS}

\section{Open-Loop Experiment}

Figure 5 shows the mean values for all of the eight measured variables $(n=6)$ in the order of difficulty i.e., $2,3,4$, and 6 (note that the difficulty levels were presented to the subjects in a different order; see Table 1). Table 2 shows the outcome of all eight variables for the tested hypothesis $\left(\mathrm{H}_{0}\right)$ with the corresponding effect size. Significant differences $(p<0.05)$ and large effect sizes between all four difficulty levels were found for arousal, dominance, MAF, SCR frequency, HR, and SCL (Figure 5 and Table 2).

The results of the Spearman's rank correlation analysis between the subjective variables of arousal, dominance, and valence, on the one hand, and objective psychophysiological responses (i.e., HR, SCL, SCR, SKT) and physical effort (i.e., $\mathrm{MAF}$ ), on the other hand, can be found in Table 3.

\section{Closed-Loop Experiment}

Table 4 shows the mean values with SD for the total game score, MAF, HR, SCL, SCR frequency, arousal, dominance, and valence during the 5-min calibration task and the 5-min closed-loop task. The percentage change between the results of the closedloop experiment and the baseline, i.e., the calibration task, are presented in Figure 6.

\section{DISCUSSION}

\section{Open-Loop Results}

For a psychophysiological signal to be useful as feedback information for control purposes, it has to change considerably and coherently in relation to the task difficulty. From the results shown in Table 2, we can infer that arousal, dominance, MAF, SCR frequency, HR, and SCL changed significantly across the four difficulty levels (i.e., $p<0.05$ ). These results are coherent with previous studies on psychophysiological changes during mixed physical/psychological HRI tasks (Rodriguez Guerrero et al., 2010; Novak et al., 2010a,b, 2011a,b; Koenig et al., 2011a,c; 

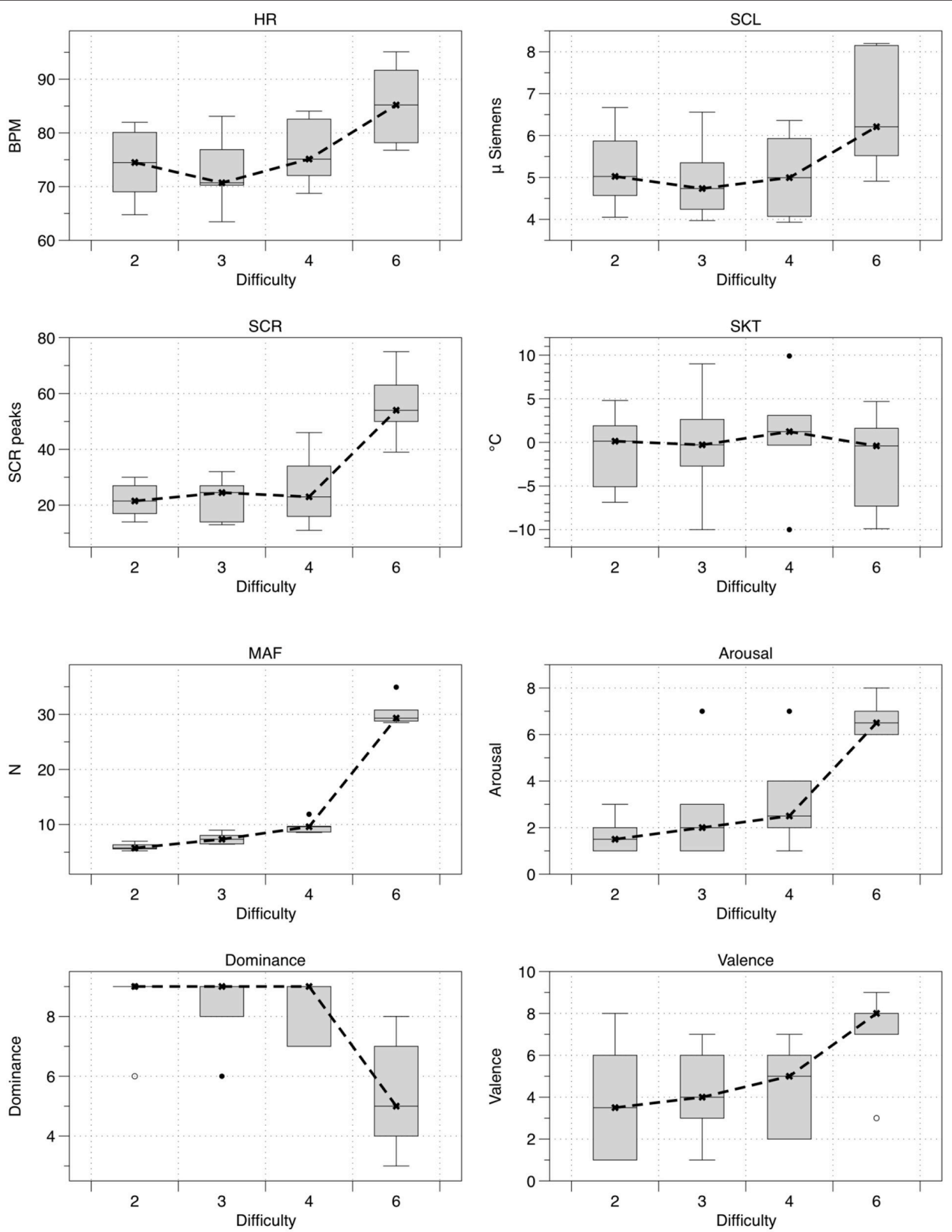

FIGURE 5 | Boxplots with mean values of HR, SCL, SCR frequency, SKT, MAF, arousal, dominance, and valence for the four levels of game difficulty (n =6). The shaded boxes represent the range in which 50\% of the data fell (i.e., interquartile range). The "l" shaped whiskers represent all of the data that fell within 3 SD of the mean (i.e., the black horizontal bars). The open (o) and closed (•) circles above the whiskers represent outliers (i.e., individual scores very different from the overall scores in the shaded box). Difficulty is presented in ascending order, although the presentation order in the experiments was 3, 4, 2, 6 (see Table 1). 
TABLE 2 | Significant differences and effect sizes between game difficulty levels for the eight measured variables.

\begin{tabular}{lcc}
\hline & \multicolumn{1}{c}{$\mathbf{H}_{\mathbf{0}}$} & Effect size $\boldsymbol{W}$ \\
\hline Arousal & $p=0.001^{*}$ & $W=0.883$ \\
Dominance & $p=0.002^{\star}$ & $W=0.818$ \\
Valence & $p=0.125$ & $W=0.319$ \\
MAF & $p<0.001^{*}$ & $W=1.00$ \\
SCR frequency & $p=0.006^{\star}$ & $W=0.698$ \\
HR & $p=0.002^{\star}$ & $W=0.833$ \\
SKT & $p=0.642$ & $W=0.093$ \\
SCL & $p=0.008^{\star}$ & $W=0.656$
\end{tabular}

*Significant difference, $p<0.05$.

TABLE 3 | Correlations between subjective and objective measurements.

\begin{tabular}{llllll}
\hline & HR & SCL & SCR & SKT & MAF \\
\hline Arousal & $p=0.221$ & $p=0.003^{\star}$ & $p=0.011^{*}$ & $p=0.110$ & $p<0.001^{\star}$ \\
& $r=0.259$ & $r=0.572$ & $r=0.512$ & $r=-0.334$ & $r=0.708$ \\
\hline Dominance & $p=0.807$ & $p=0.001^{*}$ & $p<0.001^{*}$ & $p=0.414$ & $p=0.004^{*}$ \\
& $r=-0.053$ & $r=-0.620$ & $r=-0.681$ & $r=0.175$ & $r=-0.560$ \\
\hline Valence & $p=0.525$ & $p=0.044^{*}$ & $p=0.061$ & $p=0.706$ & $p=0.044^{\star}$ \\
& $r=-0.136$ & $r=0.415$ & $r=0.388$ & $r=-0.081$ & $r=0.415$
\end{tabular}

${ }^{*}$ Significant correlation, $p<0.05$.

Badesa et al., 2012, 2014; Morales et al., 2014) and show that, in this experiment, HR, SCL, and SCR were the most sensitive physiological parameters to discriminate between the difficulty levels of the VR task. For HR, the observations made went further and showed that this variable exhibited a considerable inertia when preceded by a higher level of physical activity, as can be seen in Figure 5 (i.e., delayed effect of task presentation order, see Table 1; more specifically, a continued effect of level 4 on level 2 can be seen). This delay in HR decrease during recovery from a higher level of physical effort is a well-known effect (Shetler et al., 2001; Vijayalakshmi et al., 2014). In healthy subjects, a decrease of 15-20 beats per minute in the first minute of recovery has been shown to be typical (Shetler et al., 2001). This slower vagal reactivation may have to be taken into account in future research, as such inertial effects may become important when the subject is exposed to a wider range of physical loads over an extended period of time. In such cases, adding an appropriate corrective factor to the HR might be beneficial. The SCL and SCR behaved similarly to HR and increased significantly with increasing level of game difficulty. This is comparable to what has been found in other studies (Novak et al., 2010a; Badesa et al., 2012, 2014; Morales et al., 2014; Knaepen et al., 2015). The SKT was the only physiological variable that did not differ significantly between the four difficulty levels. Although decreases in SKT have been put forward as a marker to detect changes in mental workload (Ohsuga et al., 2001; Koenig et al., 2011e), other studies have found no significant differences for SKT with changes in mental workload (Novak et al., 2010a;
Badesa et al., 2012; Knaepen et al., 2015) or found that variations depended on the test subject (Morales et al., 2014). Novak et al. (2010a) proposed that a certain threshold of mental workload should probably be exceeded before the SKT starts to decrease significantly (Novak et al., 2010a). The hardest level (i.e., level 6 , see Table 1) in this study was designed to be demanding but not frustrating, and as such this threshold might not have been exceeded, which could explain why the SKT did not change significantly. Moreover, the slow response of the SKT, as also shown by Novak et al. (2010a), makes it difficult to use it in a closed-loop biocooperative scenario. Therefore, we have discarded SKT as a possible feedback signal for the proposed closed-loop controller. As a result, HR, SCR, and SCL proved to be the most useful indicators of psychophysiological activity in a mixed physical/psychological load scenario.

To date, it remains unclear which kinds of emotion are associated with which kinds of objective autonomic signature (Kreibig, 2010). Therefore, objectively measuring emotion through physiological signals remains challenging. In our study, a subjective SAM test was used in order to afford a numerical measurement of perceived emotion. However, it is difficult to integrate a subjective measure of emotion into the control loop of assistive robotic devices. Therefore, we also performed correlations between SAM scores and the other psychophysiological and physical parameters. Results from the Spearman correlation analysis (Table 3) showed that, while HR did not correlate with any of the subjective emotional scores, arousal was positively correlated $(r>0, p<0.05)$ with SCL and SCR. Dominance, on the other hand, was negatively correlated with SCL and SCR $(r<0, p<0.05)$, while valence was only positively correlated with SCL $(r>0, p<$ $0.05)$. These few significant correlations show that it remains difficult to achieve mapping between physiological signals and the emotional state of a subject based on a few physiological measures. The significant correlations between MAF, on the one hand, and arousal, valence, and dominance, on the other hand, further confirm that additional contextual information and probably more physiological parameters are necessary to accurately predict the emotional states of subjects during HRI. This corresponds to what Kreibig (2010) pointed out: as emotions consist of an integrated variety of physiological responses, it is important to select a sufficient number of response measures to allow for the response pattern and its variations to be accurately identified (Kreibig, 2010). Further research will have to carefully consider which additional physiological parameters should be included in the biocooperative controller in order to correctly identify and influence emotional states of the subjects.

\section{Biocooperative Closed-Loop Control}

Results from the SAM questionnaires and FlowIndex suggest that the user satisfaction and challenge/skill relation were effectively improved in this work, where haptic and difficulty modulation (HDM) was implemented, compared to the calibration task (CT) and the haptic-assisted-only (HAO) results presented in Rodriguez Guerrero et al. (2013). We invite the reader to take a look at Supplementary Figure 1 (i.e., the SAM scale) in 
TABLE 4 | Mean values with SD for the eight measured variables during the 5-min calibration and 5-min closed-loop tasks for the HDM and HAO controller.

\begin{tabular}{|c|c|c|c|c|c|}
\hline Variable & Unit & CT_HDM & HDM & CT_HAO & HAO \\
\hline Total game score & Points & $29.72 \pm 2.68$ & $56.72 \pm 9.43$ & $30.09 \pm 2.11$ & $62.63 \pm 18.53$ \\
\hline MAF & $\mathrm{N}$ & $34.45 \pm 5.92$ & $25.27 \pm 5.45$ & $34.27 \pm 5.85$ & $27.82 \pm 5.48$ \\
\hline $\mathrm{HR}$ & BPM & $88.99 \pm 5.24$ & $80.55 \pm 5.01$ & $87.75 \pm 5.91$ & $86.57 \pm 5.73$ \\
\hline SCL & $\mu S$ & $6.56 \pm 1.11$ & $5.96 \pm 0.81$ & $6.4 \pm 1.17$ & $6.52 \pm 1.32$ \\
\hline SCR frequency & Counts per task & $67.27 \pm 8.24$ & $49 \pm 9.51$ & $67.27 \pm 9.88$ & $58 \pm 10.16$ \\
\hline Arousal & $\mathrm{N} / \mathrm{A}$ & $8.45 \pm 0.52$ & $6.72 \pm 0.9$ & $8.36 \pm 0.67$ & $7.45 \pm 0.68$ \\
\hline Dominance & $\mathrm{N} / \mathrm{A}$ & $3.72 \pm 1.1$ & $8.09 \pm 0.7$ & $3.90 \pm 1.70$ & $7 \pm 0.77$ \\
\hline Valence & $\mathrm{N} / \mathrm{A}$ & $4.09 \pm 1.37$ & $8.18 \pm 0.75$ & $4.54 \pm 1.63$ & $6.27 \pm 1.34$ \\
\hline
\end{tabular}

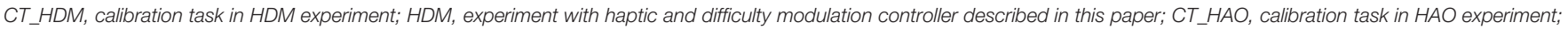
HAO, haptic-assistance-only experiment from 2013 (Rodriguez Guerrero et al., 2013); MAF, mean absolute force; HR, heart rate; SCL, skin conductance level.

Appendix A, to provide visual support during the upcoming discussion.

\section{User Satisfaction}

The dominance value obtained for HDM (8.09) was superior compared to those obtained for CT (3.72) and for the HAO controller (Hidler and Sainburg, 2011; Table 4). An improved dominance values translates into an improved "perceived skill." Besides dominance, the valence scores showed the most interesting and desired improvements. The HDM controller scored 8.18 on the valence scale, while values of 4.09 and 6.27 were obtained for the CT and the HAO, respectively. The differences in standard deviation between HAO and HDM for both dominance and valence can be neglected (i.e., 0.07 and 0.59 , respectively). The combination of high dominance (i.e., feeling under control of the situation) and high valence (i.e., pleasure) while still being challenged is likely to influence motivation and, thus, to promote future practice and total duration of training, which, in turn, might influence the success of motor learning (Shetler et al., 2001; Guadagnoli and Lee, 2004; Vijayalakshmi et al., 2014).

\section{User Performance}

The results for the total game score obtained with HDM (56.72) were greatly improved compared with the scores obtained for the CT (29.72). However, when compared to the total game score for HAO (62.63), the results ended up being lower by a mean of six points per session. Score variability was expected, as not only the skill but also the assistance given to each subject was different. The assistance given depended entirely on the subject's individual performance and ANS activity, which, of course, is highly dependent on how each subject reacts to pleasant/unpleasant situations. As the spawn frequency of the droplets was also being controlled by the game difficulty, the maximum possible number of points varied with each subject's skill. The more skillful a user was, the more challenging the session would likely be and the more droplets would be rendered. Nevertheless, as the HAO difficulty was not controlled, HDM might be better suited for longer training sessions, as it can provide larger/faster adaptation steps and preemptive maneuvers when fatigue starts to appear, by using the extra information from the performance input.

\section{Vector Representation, Flowlndex, and FlowDir}

Within the framework of this article, a new set of tools was proposed based on arousal and dominance scores, to indirectly evaluate the impact of a biocooperative controller: the vector representation, FlowIndex, and FlowDir. These tools allow the graphical and numerical evaluation of the results of a given control strategy designed to improve the perception of a given task. An optimal flow level, or optimal challenge/skill ratio, results in the high commitment of a person to a given task (Engeser and Rheinberg, 2008) and also stimulates motor learning (Guadagnoli and Lee, 2004). Previous studies have shown that, for optimal motor learning, the motor task should challenge, arouse, and excite the subject, while not being too stressful or boring (Maclean and Pound, 2000; Guadagnoli and Lee, 2004; Koenig et al., 2011d; Rodriguez Guerrero et al., 2013). The arousal results for HDM (6.72) decreased compared to those for the CT (8.45) and HAO (7.45). Since HDM is able to control the game difficulty (i.e., challenge), arousal can also be greatly influenced by its actions. The decrease in arousal obtained by the HDM controller seems to have played a favorable role for this controller, keeping the FlowDir in the relaxation zone (Figure 7). This is in contrast with the FlowDir for HAO, which was located in the frustration zone (Figure 7). HDM also performed better on the FlowIndex (i.e., 0.82 vs. 0.80 obtained for the HAO controller; see Table 5), meaning that it was closer to the optimal challenge/skill ratio. Although this difference in FlowIndex between HAO and HDM is small, HDM also afforded considerably higher values for valence (Figure 6). It seems that when subjects are close to the flow zone, they perform better under the relaxation zone rather than under the frustration zone (Figure 7). While the evidence for the previous statements is yet to be fully proved, the assumptions are supported by the Yerkes and Dodson model (Yerkes and Dodson, 1908; Diamond et al., 2007), as high levels of arousal (such as those triggered by frustration) can be counterproductive, and the fact that this controller setup led to a more enjoyable experience. Not only did the biocooperative controller presented in this work perform better, it also added greater flexibility to tune the system with 


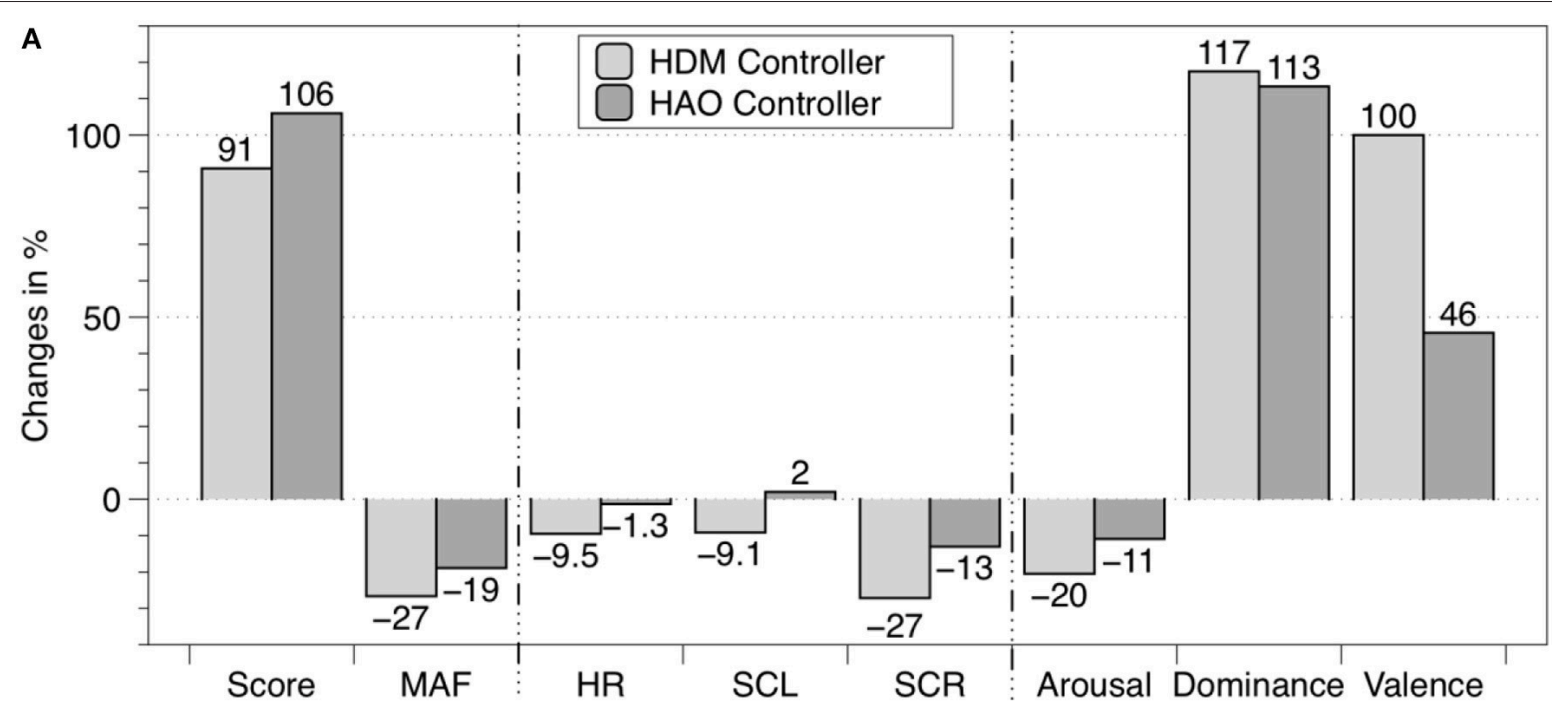

B

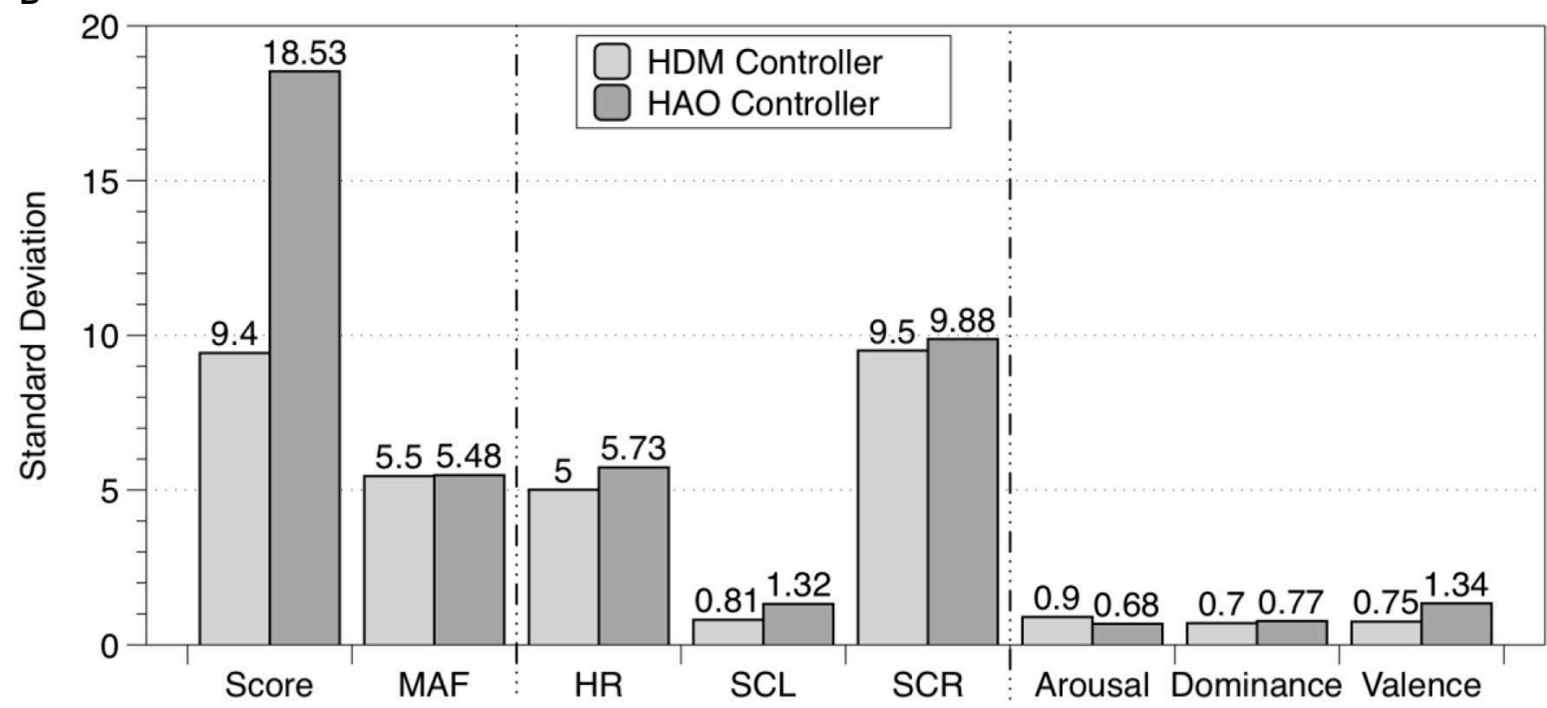

FIGURE 6 | (A) Percentage change between baseline (i.e., calibration task) and the closed-loop experiments for the eight measured variables. (B) The corresponding standard deviations. Light gray bars represent the data obtained for the HDM controller described in this article, and dark gray bars represent data from the HAO controller published in Rodriguez Guerrero et al. (2013). In both cases the sample sizes were $n=11$.

an extra degree of freedom by controlling the difficulty of the game. This allows modulation of the challenge/skill relation, with challenge being controlled by the game difficulty and skill by the haptic assistance. This could certainly be exploited in order to fine-tune the system rules and, thus, to maximize the FlowIndex and obtain a FlowDir closer to the optimal flow zone.

\section{Study Limitations}

The sample size used here, especially for the open-loop setup ( $n$ $=6$ ), was small. Therefore, caution should be taken in attempting to generalize the statistical results. The significant differences and large effect sizes indicate an interesting effect that should be confirmed in future studies with a larger sample size and thus a higher statistical power. Although, the difficulty settings for the open-loop trials were satisfactory for the subjects who tested them prior to the experiments, it seems that we could have added another higher level of game difficulty. In this study, we visualized more the effects of the low difficulty range (i.e., game difficulty levels 2, 3, and 4). Only one level of higher game difficulty was present (level 6). The addition of another session with an even higher level of difficulty would have allowed us to more thoroughly explore the effects of the biocooperative controller, as with the difficulty levels used the subjects never really became frustrated and their dominance levels therefore started to decay only at level 6. It would have been useful to explore these effects over longer periods of time to better understand the signal dynamics and compensate for phenomena such as signal inertia. 


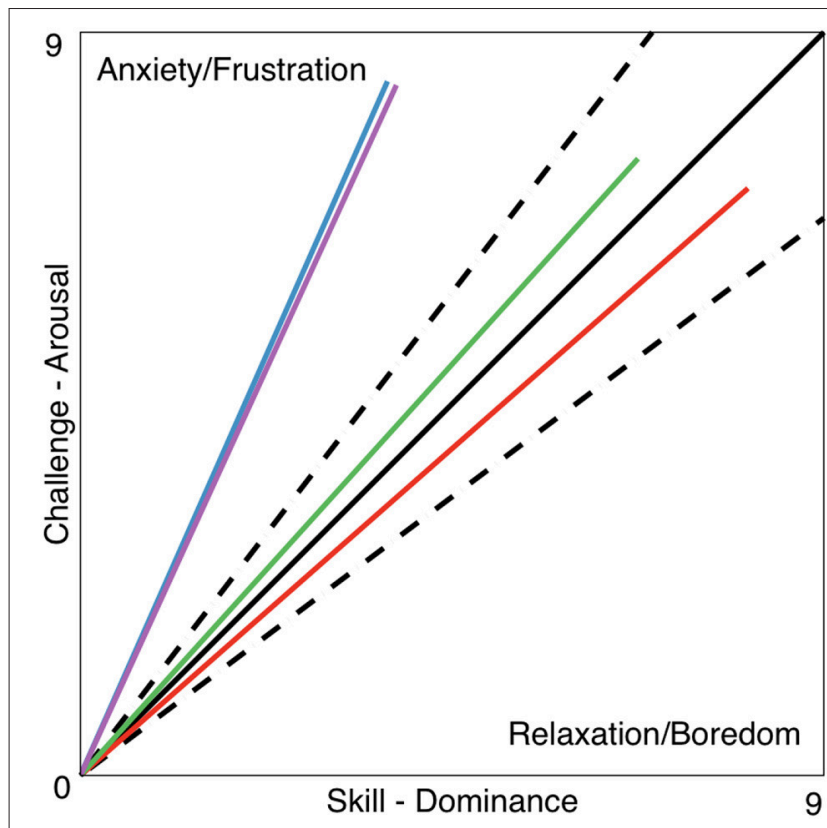

FIGURE 7 | Vector representation of the challenge/skill relation for the CT_HDM (solid blue line) and HDM (solid red line) tasks. The data for CT_HAO (solid purple) and HAO (solid green) tasks are also shown. The dotted lines delimit the flow channel, i.e., the range reflecting an ideal challenge/skill ratio, and the solid black line represents the maximal challenge/skill ratio $\mathbf{F}=[9,9]$. The presented data is taken from Table $\mathbf{5}$.

It would also have been useful to investigate and compare the $\mathrm{HAO}$ controller to the HDM controller over longer sessions. It is probable that the improved HDM adaptation could have clearer benefits (i.e., higher scores for FlowIndex and valence) over longer sessions, where fatigue, boredom, or frustration would be more frequent.

\section{CONCLUSION}

User satisfaction and augmented human performance are the most important features that any human-machine interface can achieve. The architecture and control strategies presented in this work effectively delivered a well-balanced tradeoff between these key design points. For a human-cooperative controlled interface such as the one presented in this work, augmenting human performance could be as easy as delivering the needed assistance to complete the task. However, this lacks the engagement component that drives motivation and, therefore, it always coincides with the tradeoff of deteriorating user satisfaction. Consequently, a biocooperative interface should ideally deliver individually tailored adaptation based on the user's psychophysiological state and (if available) contextual performance information. Augmenting the system with contextual performance information together with the individual's psychophysiological feedback provides a superior and more individualized adaptation of the system to maintain the task difficulty within its user's capabilities, improving subject performance and more importantly the overall user experience.
TABLE 5 | Flowlndex and FlowDir for the 5-min calibration and 5-min closed-loop tasks for the HDM and HAO controllers.

\begin{tabular}{lllll}
\hline & CT_HDM & HDM & CT_HAO & HAO \\
\hline Flowlndex & 0.63 & 0.82 & 0.64 & 0.8 \\
FlowDir & Frustration & Relaxation & Frustration & Frustration
\end{tabular}

CT_HDM, calibration task in HDM experiment; HDM, experiment with haptic and difficulty modulation controller described in this paper; CT_HAO, calibration task in HAO experiment; HAO, haptic-assistance-only experiment from 2013 (Novak et al., 2011b).

By modulating both haptic assistance and game difficulty, designers have greater potential to control dominance and arousal levels. This has a direct impact on the capabilities to control the challenge/skill relation of a given task.

Psychophysiological activity under physically demanding scenarios can be measured by using ANS-related signals such as those used in this work. However, the direct mapping against subjective emotional scales like arousal, valence, and dominance seems to remain a challenge that could soon be possible to achieve with modern deep-learning algorithms. Nevertheless, by using psychophysiological feedback in conjunction with additional contextual information (i.e., performance), an overall estimation of the emotional state of a subject can still be made and exploited for control purposes with good results.

Although the given inputs for FlowIndex and FlowDir are based on the SAM scale, future work needs to be carried out to achieve mapping between arousal and dominance, on the one hand, and psychophysiological signals, on the other hand, so that the calculations can be performed online in real time. This could allow using the index for control or optimization purposes, taking actions based on minimizing the error between the expected output (i.e., flow vector) and the given desired input and, thus, maintaining an optimal flow level.

\section{ETHICS STATEMENT}

This study was carried out in accordance with the recommendations of "the medical ethics committee of Fundación CARTIF" with written informed consent from all subjects. All subjects gave written informed consent in accordance with the Declaration of Helsinki. The protocol was approved by "the medical ethics committee of Fundación CARTIF."

\section{AUTHOR CONTRIBUTIONS}

CR, DL, JF, and JP conceived and designed the experiments. CR developed the technology for the methods to build the experimental setup, robotics and VR methods, metrics, algorithms, and data processing. KK contributed to the design and layout of the article, tables, additions to the bibliography, and extensive revisions. DL, JF, and JP helped to obtain the funding for the project that financed this research. VG helped with the formulation of the null hypothesis and data processing for the statistics. 


\section{FUNDING}

This research was funded by the Spanish Ministry of Sciences and Innovation (DPI2009-10658) and a research grant (SAN/126/2009) from the Castilla y Leon Council of Health.

\section{ACKNOWLEDGMENTS}

The authors would like to acknowledge DL from the Vrije Universiteit Brussel for the economical and motivational

\section{REFERENCES}

Badesa, F. J., Morales, R., Garcia-Aracil, N., Sabater, J. M., Casals, A., and Zollo, L. (2014). Auto-adaptive robot-aided therapy using machine learning techniques. Comput. Methods Progr. Biomed. 116, 123-130. doi: 10.1016/j.cmpb.2013.09.011

Badesa, F. J., Morales, R., Garcia-Aracil, N., Sabater, J. M., Perez-Vidal, C., and Fernandez, E. (2012). Multimodal Interfaces to Improve Therapeutic Outcomes in Robot-Assisted Rehabilitation. IEEE Trans. Sys, Man Cyber. Part C Appli. Rev. 42, 1152-1158. doi: 10.1109/TSMCC.2012.2201938

Bonarini, A., Mainardi, L., Matteucci, M., Tognetti, S., and Colombo, R. (eds.). (2008). "Stress recognition in a robotic rehabilitation task," in Proceedings of "Robotic Helpers: User Interaction, Interfaces and Companions in Assistive and Therapy Robotics," A Workshop at ACM/IEEE HRI (Amsterdam).

Bonatti, J., Vetrovec, G., Riga, C., Wazni, O., and Stadler, P. (2014). Robotic technology in cardiovascular medicine. Nat. Rev. Cardiol. 11, 266-275. doi: $10.1038 /$ nrcardio. 2014.23

Colombo, G., Wirz, M., and Dietz, V. (2001). Driven gait orthosis for improvement of locomotor training in paraplegic patients. Spinal Cord. 39, 252-255. doi: $10.1038 /$ sj.sc.3101154

Coombes, S. A., Cauraugh, J. H., and Janelle, C. M. (2006). Emotion and movement: activation of defensive circuitry alters the magnitude of a sustained muscle contraction. Neurosci. Lett. 396, 192-196. doi: 10.1016/j.neulet.2005.11.048

Csikszentmihalyi, M., and LeFevre, J. (1989). Optimal experience in work and leisure. J. Pers. Soc. Psychol. 56, 815-822. doi: 10.1037/0022-3514.56.5.815

Daly, J. J., and Wolpaw, J. R. (2008). Brain-computer interfaces in neurological rehabilitation. Lancet Neurol. 7, 1032-1043. doi: 10.1016/S1474-4422(08) 70223-0

Diamond, D. M., Campbell, A. M., Park, C. R., Halonen, J., and Zoladz, P. R. (2007). The temporal dynamics model of emotional memory processing: a synthesis on the neurobiological basis of stress-induced amnesia, flashbulb and traumatic memories, and the Yerkes-Dodson law. Neural Plast. 2007:60803. doi: $10.1155 / 2007 / 60803$

Edgerton, V. R., Courtine, G., Gerasimenko, Y. P., Lavrov, I., Ichiyama, R. M., Fong, A. J., et al. (2008). Training locomotor networks. Brain Res. Rev. 57, 241-254. doi: 10.1016/j.brainresrev.2007.09.002

Engeser, S., and Rheinberg, F. (2008). Flow, performance and moderators of challenge-skill balance. Motiv Emot. 32, 158-172. doi: 10.1007/s11031-008-9102-4

Fairclough, S. H. (2009). Fundamentals of physiological computing. Inter. Comp. 21, 133-145. doi: 10.1016/j.intcom.2008.10.011

Fairclough, S. H. (2011). "Physiological computing: interfacing with the human nervous system," in Sensing Emotions in Context, ed M. Ouwerkerk (Dordrecht: Springer), 1-20.

Goljar, N., Javh, M., Poje, J., Ocepek, J., Novak, D., Ziherl, J., et al. (2011). "Psychophysiological responses to robot training in different recovery phases after stroke," in IEEE International Conferenece on Rehabilitation Robot (Zurich).

Guadagnoli, M. A., and Lee, T. D. (2004). Challenge point: a framework for conceptualizing the effects of various practice conditions in motor learning. J. Mot. Behav. 36, 212-224. doi: 10.3200/JMBR.36.2.212-224 support. Last but not least, we would also like to acknowledge all of the people who voluntarily participated in this study.

\section{SUPPLEMENTARY MATERIAL}

The Supplementary Material for this article can be found online at: http://journal.frontiersin.org/article/10.3389/fnins. 2017.00242/full\#supplementary-material

Hidler, J., and Sainburg, R. (2011). Role of Robotics in Neurorehabilitation. Top Spinal Cord Inj. Rehabil. Summer 17, 42-49. doi: 10.1310/sci1701-42

Hochberg, L. R., Bacher, D., Jarosiewicz, B., Masse, N. Y., Simeral, J. D., Vogel, J., et al. (2012). Reach and grasp by people with tetraplegia using a neurally controlled robotic arm. Nature 485, 372-375. doi: 10.1038/nature 11076

Holden, M. K. (2005). Virtual environments for motor rehabilitation: review. Cyberpsychol. Behav. 8, 187-211. doi: 10.1089/cpb.2005.8.187

Huang, V. S., and Krakauer, J. W. (2009). Robotic neurorehabilitation: a computational motor learning perspective. J. Neuroeng. Rehabil. 6:5. doi: 10.1186/1743-0003-6-5

Knaepen, K., Marusic, U., Crea, S., Rodriguez Guerrero, C. D., Vitiello, N., Pattyn, N., et al. (2015). Psychophysiological response to cognitive workload during symmetrical, asymmetrical and dual-task walking. Hum. Mov. Sci. 40C, 248-263. doi: 10.1016/j.humov.2015.01.001

Koenig, A., Caruso, A., Bolliger, M., Somaini, L., Omlin, X., Morari, M., et al. (2011a). "Model-based heart rate control during robot-assisted gait training," in IEEE International Conference on Robotics and Automation (Shanghai) 4151-4156.

Koenig, A., Novak, D., Omlin, X., Pulfer, M., Perreault, E., Zimmerli, L., et al. (2011b). Real-time closed-loop control of cognitive load in neurological patients during robot-assisted gait training. IEEE Trans. Neural Syst. Rehabil. Eng. 19, 453-464. doi: 10.1109/TNSRE.2011.2160460

Koenig, A., Omlin, X., Bergmann, J., Zimmerli, L., Bolliger, M., Muller, F., et al. (2011c). Controlling patient participation during robot-assisted gait training. J. Neuroeng. Rehabil. 8:14. doi: 10.1186/1743-0003-8-14

Koenig, A., Omlin, X., Novak, D., and Riener, R. (2011d). A review on biocooperative control in gait rehabilitation. IEEE Int. Conf. Rehabil. Robot. 2011:5975454. doi: 10.1109/icorr.2011.5975454

Koenig, A., Omlin, X., Zimmerli, L., Sapa, M., Krewer, C., Bolliger, M., et al. (2011e). Psychological state estimation from physiological recordings during robot-assisted gait rehabilitation. J Rehabil. Res. Dev. 48, 367-385. doi: 10.1682/JRRD.2010.03.0044

Koenig, A., Pulfer, M., Omlin, X., Perreault, E., Zimmerli, L., and Riener, R. (2010). "Automatic estimation of cognitive load during robot-assisted gait training," in AUTOMED - Automatisierungstechnische Verfahren für die Medizin (Zürich).

Kreibig, S. D. (2010). Autonomic nervous system activity in emotion: a review. Biol. Psychol. 84, 394-421. doi: 10.1016/j.biopsycho.2010.03.010

LeDoux, J. E. (1995). Emotion: clues from the brain. Annu. Rev. Psychol. 46, 209-235. doi: 10.1146/annurev.ps.46.020195.001233

Lewis, G. N., and Rosie, J. A. (2012). Virtual reality games for movement rehabilitation in neurological conditions: how do we meet the needs and expectations of the users? Disabil. Rehabil. 34, 1880-1886. doi: 10.3109/09638288.2012.670036

Liu, J., Cramer, S. C., and Reinkensmeyer, D. J. (2006). Learning to perform a new movement with robotic assistance: comparison of haptic guidance and visual demonstration. J. Neuroeng. Rehabil. 3:20. doi: 10.1186/1743-0 003-3-20

Lledo, L. D., Badesa, F. J., Almonacid, M., Cano-Izquierdo, J. M., Sabater-Navarro, J. M., Fernandez, E., et al. (2015). Supervised and dynamic neuro-fuzzy systems to classify physiological responses in robot-assisted neurorehabilitation. PLoS ONE 10:e0127777. doi: 10.1371/journal.pone.0127777 
Maclean, N., and Pound, P. (2000). A critical review of the concept of patient motivation in the literature on physical rehabilitation. Soc. Sci. Med. 50, 495-506. doi: 10.1016/S0277-9536(99)00334-2

Mandryk, R. L., and Atkins, M. S. (2007). A fuzzy physiological approach for continuously modeling emotion during interaction with play technologies. Int. J. Hum. Comp. Stud. 65, 329-347. doi: 10.1016/j.ijhcs.2006.11.011

Merians, A. S., Tunik, E., and Adamovich, S. V. (2009). Virtual reality to maximize function for hand and arm rehabilitation: exploration of neural mechanisms. Stud. Health Technol. Inform. 145, 109-125. doi: 10.3233/978-1-60750-01 8-6-109

Mihály Csíkszentmihályi, I. S. (1992). Optimal Experience: Psychological Studies of Flow in Consciousness. Cambridge: Cambridge University Press.

Mihelj, M., Novak, D., and Munih, M. (2009). "Emotion-aware system for upper extremity rehabilitation," in IEEE Virtual Rehabilitation International Conference (Haifa), 160-165.

Morales, R., Badesa, F. J., Garcia-Aracil, N., Perez-Vidal, C., Sabater, J. M., Papaleo, E., et al. (2014). Patient-tailored assistance: a new concept of assistive rbotic device that adapts to inidivdual users. IEEE Robot. Automat. Magazine 21, 123-133. doi: 10.1109/MRA.2014.2304051

Nef, T., Guidali, M., and Riener, R. (2009). ARMin III-arm therapy exoskeleton with an ergonomic shoulder actuation. Appl. Bion. Biomechan. 6, 127-142. doi: 10.1155/2009/962956

Novak, D., Mihelj, M., and Munih, M. (2009). "Using psychophysiological measurements in physically demanding virtual environments," in 12th IFIP Conference on Human-Computer Interaction - INTERACT (Uppsala), 490-493.

Novak, D., Mihelj, M., and Munih, M. (2010a). Psychophysiological responses to different levels of cognitive and physical workload in haptic interaction. Robotica 29, 367-374. doi: 10.1017/S0263574710000184

Novak, D., Mihelj, M., Ziherl, J., Olensek, A., and Munih, M. (2011a). Psychophysiological Measurements in a Biocooperative Feedback Loop for Upper Extremity Rehabilitation. IEEE Transactions on Neural Systems and Rehabilitation Engineering. 19, 400-410. doi: 10.1109/TNSRE.2011. 2160357

Novak, D., Mihelj, M., Ziherl, J., Olensek, A., and Munih, M. (2011b). "Task difficulty adjustment in biocooperative rehabilitation using psychophysiological responses," in IEEE International Conference on Rehabilitation Robotics (Zurich).

Novak, D., Ziherl, J., Olensek, A., Milavec, M., Podobnik, J., Mihelj, M., et al. (2010b). Psychophysiological responses to robotic rehabilitation tasks in stroke. IEEE Trans. Neural Syst. Rehabil. Eng. 18, 351-361. doi: 10.1109/TNSRE.2010.2047656

Ohsuga, M., Shimono, F., and Genno, H. (2001). Assessment of phasic work stress using autonomic indices. Int. J. Psychophysiol. 40, 211-220. doi: 10.1016/S0167-8760(00)00189-6

Okamura, A. M. (2009). Haptic feedback in robot-assisted minimally invasive surgery. Curr. Opin. Urol. 19, 102-107. doi: 10.1097/MOU.0b013e3283 $1 \mathrm{a} 478 \mathrm{c}$

Pennycott, A., Wyss, D., Vallery, H., Klamroth-Marganska, V., and Riener, R. (2012). Towards more effective robotic gait training for stroke rehabilitation: a review. J. Neuroeng. Rehabil. 9, 65. doi: 10.1186/1743-0003-9-65

Pignolo, L., Lucca, L. F., Basta, G., Serra, S., Pugliese, M. E., Sannita, W. G., et al. (2016). A new treatment in the rehabilitation of the paretic upper limb after stroke: the ARAMIS prototype and treatment protocol. Ann. Ist Super Sanita. 52, 301-308. doi: 10.4415/ANN_16_02_25

Pope, A. T., Bogart, E. H., and Bartolome, D. S. (1995). Biocybernetic system evaluates indices of operator engagement in automated task. Biol. Psychol. 40, 187-195. doi: 10.1016/0301-0511(95)05116-3

Posner, J., Russell, J. A., Gerber, A., Gorman, D., Colibazzi, T., Yu, S., et al. (2009). The neurophysiological bases of emotion: an fMRI study of the affective circumplex using emotion-denoting words. Hum. Brain Mapp. 30, 883-895. doi: 10.1002/hbm.20553

Posner, J., Russell, J. A., and Peterson, B. S. (2005). The circumplex model of affect: an integrative approach to affective neuroscience, cognitive development, and psychopathology. Dev. Psychopathol. Summer17, 715-734. doi: $10.1017 / \mathrm{s} 0954579405050340$

Prinzel, L. J., Freeman, F. G., Scerbo, M. W., Mikulka, P. J., and Pope, A. T. (2000). A closed-loop system for examining psychophysiological measures for adaptive task allocation. Int. J. Aviat. Psychol. 10, 393-410. doi: 10.1207/S15327108IJAP1004_6

Reinkensmeyer, D. J., Emken, J. L., and Cramer, S. C. (2004). Robotics, motor learning, and neurologic recovery. Annu. Rev. Biomed. Eng. 6, 497-525. doi: 10.1146/annurev.bioeng.6.040803.140223

Riener, R., Koenig, A., Bolliger, M., Wieser, M., duschau-Wicke, A., and Vallery, H. (eds.). (2009). "Bio-cooperative robotics: controlling mechanical, physiological and mental patient states," in IEEE 11th International Conference on Rehabilitation Robotics; Kyoto International Conference Center (Kyoto).

Rodriguez Guerrero, C. D., Fraile Marinero, J. C., Perez Turiel, J., and Rivera Farina, P. (2010). "Bio-cooperative robotic platform for motor function recovery of the upper limb after stroke," in Conference Proceeding IEEE Engineering in Medicine and Biology Society (Buenos Aires), 4472-4475.

Rodriguez Guerrero, C. D., Marinero, J. C. F., and Turiel, J. P. (2009). "Robot adaptive behavior to suit patient needs and enable more intensive rehabilitation tasks," in IEEE International Conference on Mechatronics ICM (Malaga), 1-6.

Rodriguez Guerrero, C., Fraile Marinero, J. C., Perez Turiel, J., and Munoz, V. (2013). Using "human state aware" robots to enhance physical human-robot interaction in a cooperative scenario. Comp. Methods Progr. Biomed. 112, 250-259. doi: 10.1016/j.cmpb.2013.02.003

Schwickert, L., Klenk, J., Stahler, A., Becker, C., and Lindemann, U. (2011). Robotic-assisted rehabilitation of proximal humerus fractures in virtual environments: a pilot study. Z. Gerontol. Geriatr. 44, 387-392. doi: 10.1007/s00391-011-0258-2

Shetler, K., Marcus, R., Froelicher, V. F., Vora, S., Kalisetti, D., Prakash, M., et al. (2001). Heart rate recovery: validation and methodologic issues. J. Am. Coll. Cardiol. 38, 1980-1987. doi: 10.1016/S0735-1097(01)01652-7

Song, R., Tong, K. Y., Hu, X., and Zhou, W. (2013). Myoelectrically controlled wrist robot for stroke rehabilitation. J Neuroeng. Rehabil. 10:52. doi: 10.1186/1743-0003-10-52

Squeri, V., Masia, L., Giannoni, P., Sandini, G., and Morasso, P. (2014). Wrist rehabilitation in chronic stroke patients by means of adaptive, progressive robot-aided therapy. IEEE Trans. Neural Syst Rehabil. Eng. 22, 312-325. doi: 10.1109/TNSRE.2013.2250521

Tomczak, M., and Tomczak, E. (2014). The need to report effect size estimates revisited. An overview of some recommended measures of effect size. Trends Sport Sci. 1, 19-25.

Vijayalakshmi, I., Subha, K. C., and Niruba, R. (2014). Comparison of cardiac vagal activity between pre and postmenopausal women using heart rate recovery. Int. J. Clin. Trials. 1, 105-109. doi: 10.5455/2349-3259.ijct20141104

Wittmann, F., Held, J. P., Lambercy, O., Starkey, M. L., Curt, A., Hover, R., et al. (2016). Self-directed arm therapy at home after stroke with a sensor-based virtual reality training system. J. Neuroeng. Rehabil. 13, 75. doi: 10.1186/s12984-016-0182-1

Yerkes, R. M., and Dodson, J. D. (1908). The relation of strength of stimulus to rapidity of habit-formation. J. Comp. Neurol. Psychol. 18, 459-482. doi: $10.1002 / \mathrm{cne} .920180503$

Conflict of Interest Statement: The authors declare that the research was conducted in the absence of any commercial or financial relationships that could be construed as a potential conflict of interest.

Copyright (c) 2017 Rodriguez-Guerrero, Knaepen, Fraile-Marinero, Perez-Turiel, Gonzalez-de-Garibay and Lefeber. This is an open-access article distributed under the terms of the Creative Commons Attribution License (CC BY). The use, distribution or reproduction in other forums is permitted, provided the original author(s) or licensor are credited and that the original publication in this journal is cited, in accordance with accepted academic practice. No use, distribution or reproduction is permitted which does not comply with these terms. 


\title{
Physiological Signal-Based Method for Measurement of Pain Intensity
}

\author{
Yaqi Chu ${ }^{1}$, Xingang Zhao ${ }^{1 *}$, Jianda Han ${ }^{1}$ and Yang $\mathrm{Su}^{2}$ \\ ${ }^{1}$ State Key Laboratory of Robotics, Shenyang Institute of Automation, Chinese Academy of Sciences, Shenyang, China, \\ ${ }^{2}$ Shengjing Hospital of China Medical University, Shenyang, China
}

OPEN ACCESS

Edited by:

Dingguo Zhang,

Shanghai Jiao Tong University, China

Reviewed by:

Jun Xie,

Xi'an Jiaotong University, China

Long Cheng,

Institute of Automation (CAS), China

*Correspondence: Xingang Zhao

zhaoxingang@sia.cn

Specialty section:

This article was submitted to

Neural Technology,

a section of the journal

Frontiers in Neuroscience

Received: 18 February 2017

Accepted: 01 May 2017

Published: 26 May 2017

Citation:

Chu Y, Zhao X, Han J and Su Y (2017) Physiological Signal-Based Method for Measurement of Pain Intensity.

Front. Neurosci. 11:279.

doi: 10.3389/fnins.2017.00279
The standard method for prediction of the absence and presence of pain has long been self-report. However, for patients with major cognitive or communicative impairments, it would be better if clinicians could quantify pain without having to rely on the patient's self-description. Here, we present a newly pain intensity measurement method based on multiple physiological signals, including blood volume pulse (BVP), electrocardiogram (ECG), and skin conductance level (SCL), all of which are induced by external electrical stimulation. The proposed pain prediction system consists of signal acquisition and preprocessing, feature extraction, feature selection and feature reduction, and three types of pattern classifiers. Feature extraction phase is devised to extract pain-related characteristics from short-segment signals. A hybrid procedure of genetic algorithm-based feature selection and principal component analysis-based feature reduction was established to obtain high-quality features combination with significant discriminatory information. Three types of classification algorithms-linear discriminant analysis, $k$-nearest neighbor algorithm, and support vector machine-are adopted during various scenarios, including multi-signal scenario, multi-subject and between-subject scenario, and multi-day scenario. The classifiers gave correct classification ratios much higher than chance probability, with the overall average accuracy of $75 \%$ above for four pain intensity. Our experimental results demonstrate that the proposed method can provide an objective and quantitative evaluation of pain intensity. The method might be used to develop a wearable device that is suitable for daily use in clinical settings.

Keywords: feature extraction, feature selection and reduction, pain intensity quantification, physiological signals, pattern classification

\section{INTRODUCTION}

From an evolutionary angle of vision, pain is considered as an unpleasant emotional and sensory experience that may be associated with a real or potential tissue damage (Hudspith et al., 2006; Loeser and Treede, 2008). Nowadays, pain is one of the most significant clinical symptoms that can be utilized to detect the acuteness and degree of a patient's injury. Moreover, pain is considered as a warning to danger, and often indicates the site of a lesion; its intensity serves as an indicator of patient well-being. A precise prediction of pain intensity could provide valuable insights in situations in which it can be utilized effectively to ultimately determine the position of pain and accordingly to formulate a reasonable therapeutic schedule. Therefore, pain prediction could enhance the quality of daily life for patients in the health-related field of rehabilitation, in-home healthcare and medical emergency services.

As a subjective first-person experience (Loeser and Treede, 2008), pain does not only reflect perceptual status but would also be substantially affected by psycho-physiological conditions (e.g., 
fluctuations in attention or alertness) and even psychosocial contexts (e.g., the age or gender of individuals) (Aslaksen et al., 2007). In clinical practice, self-description is the gold standard approach for the determination of the absence, presence, and intensity of pain (Cruccu et al., 2010; Haanpää et al., 2011), such as Numeric Pain Rating Scales (NPRS), Verbal Rating Scales (VRS), and Visual Analog Scales (VAS) (Frampton and Hughes-Webb, 2011). These self-reported scales are especially well applied and validated in cancer patients (Caraceni et al., 2002). In addition, the McGill Pain Questionnaire (MPQ) and Brief Pain Inventory are also used to assess the wider pain perception in multidimensional scales (Frampton and HughesWebb, 2011). While self-descripted pain provides important clinical reference indicators and proves to be a valid method for the adequate therapy of patients suffered from pain in most situations (Brown et al., 2011), it would fail to be applied in certain vulnerable populations. Individuals with communicative impairments or disturbance of consciousness, including older adults with dementia and intensive care unit patients in vegetative state, coma, and minimally conscious state (Herr et al., 2006; Schnakers and Zasler, 2007), may not be able to provide effective and credible self-reports of pain ( $\mathrm{Li}$ et al., 2008). For those populations, lack or any inaccuracy indicators used to evaluate pain may lead to suboptimal or inappropriate treatment of pain, which may bring about various additional clinical issues, such as the deterioration of chronic pain and psychological distress or depression (Roulin and Ramelet, 2012). Furthermore, the self-reported pain is very subjective and unable to be obtained in real-time.

Recent developments in objective pain assessment have concentrated mainly on recognition and prediction from human behaviors, such as vocalizations (Puntillo et al., 2004), body motions (Young et al., 2006), and facial expressions (Lucey et al., 2011; Kaltwang et al., 2012; Irani et al., 2015). While behavioral methods exist, they also may be inapplicable in individuals with paralysis or other motor disorders affecting behaviors. By observing the face of an individual, a huge number of features related with affective state can be extracted, including pain state. However, facial expression-based pain recognition need track the special facial regions of the users, which can be very cumbersome and tedious in the clinical application. Meanwhile, researchers' effort has been shifted to target toward a physiology-driven pain prediction that does not rely on individual's facial or volitional behaviors (Shankar et al., 2009; Treister et al., 2012). Those studies have focused on diverse bio-physiological signals, such as heart rate variability (De Jonckheere et al., 2010, 2012; Faye et al., 2010; Logier et al., 2010), skin conductance or electrodermal activity (Harrison et al., 2006; Treister et al., 2012), electromyography (Oliveira et al., 2012), electroencephalography (Nir et al., 2010; Huang et al., 2013), and functional magnetic resonance imaging (fMRI) (Marquand et al., 2010; Brown et al., 2011). Recently, pain assessment method implemented by multimodality signals has been confirmed to be highly effective, some even outperforming single-signal mode markedly (Werner et al., 2014; Kächele et al., 2015). However, the most physiology-driven pain researches just provide statistically significant correlations between several bio-physiologic signals and the presence of pain.
They are qualitative assessments for discriminating the presence or absence of pain. Few measurements can predict the intensity of pain as a level indicator. Hence, despite many remarkable researches, there is no acceptable pain assessment method based on physiological signals of human.

With the increasing availability of wearable smart devices, many researchers focus on developing a non-invasive integrated system for health monitoring. An appropriate interpretation of the signals recorded by wearable devices equipped with sensors can help monitor, evaluate and eventually solve health problems related pain. In this article, the focused goal is the quantitative measurement of pain intensity from multi-physiological signals obtained by wearable sensors in the customer market. At present, only few studies have been carried out the procedure of automatic recognition of pain intensity from physiological signals. Olugbade et al. applied electromyography (EMG) and body motions in combination with Support Vector Machines (SVM) and Random Forests (RF) as classifiers to recognize three pain intensity (Olugbade et al., 2015). Kachele et al. used EMG, skin conductance level (SCL) and electrocardiogram (ECG) incorporated with unsupervised and semi-supervised learning to establish a personalized system of continuous pain intensity recognition (Kachele et al., 2016). However, these assessment procedures are quite complicated and timeconsuming, especially feature extraction. Moreover, the latter focuses on a personalization scenario where the recognition process may not be widely used in the general population.

In this paper, we proposed a convenient and objective method of pain intensity recognition based on multiple physiological signals. The technique uses a hybrid of genetic algorithm (GA) with principal component analysis (PCA) to obtain optimized feature combination related with pain states. And three kinds of classification methods are compared to establish more appropriate recognition models. The presented system that complements the self-reported pain can thus be adapted to general persons.

The remainder of this paper is structured as follows. In Section Dataset and Experimental Protocol, we provide a short presentation of physiological signals used in pain recognition and experiment of pain induced by electrical stimulation. Section Feature Processing elaborates a thorough feature processing phase, including feature extraction, feature selection, and feature reduction. In Section Pattern Classification, we present the three classifiers for pain intensity recognition system. Thorough experimental results and discussion are described in Section Experimental Results and Discussion. Section Conclusions and Outlook provides our findings and ideas for future study in this field.

\section{DATASET AND EXPERIMENTAL PROTOCOL}

\section{Subjects}

Six subjects (4 males and 2 females, numbered 1-6) aged 22-25 years (standard deviation $[S D]=3.0$ ) were recruited in this study. All participants were healthy, without any history of medical 
illness, neurological or psychopathological disorders, and none had a history of chronic pain. All the experimental protocols of this study were approved by the ethics committee of China Medical University. Moreover, all subjects were fully informed about the procedures, risk, and benefits of the study, and written informed consent was obtained from all subjects before the study.

The algorithm design and performance validation in this pain intensity measurement were implemented for the databases constructed from physiological signals of each subject. The signals were obtained with the procedure described in Section Physiological Signals and Preprocessing and Pain Induction Protocol. Moreover, we continuously collected 1-week data from the same participant, thus generating a 7-day dataset. For the database of each subject, we randomly chosen $75 \%$ of the samples as the training set, and the rest as the testing set. For the 7day database, data from six randomly chosen days were used as the training set, and the rest were used as the testing set (leave-one-out method).

\section{Physiological Signals and Preprocessing}

Acquisition of high-quality physiological data is of utmost importance for the pain intensity recognition system. The selection of available physiological signals that are used as input is the first consideration in the pain detection system. It is expected that the physiological signals reflect the effect of pain on the activity level of the nervous system. However, unlike selfreporting or facial recognition of pain, where the truth class labels of pain intensity for a given sample are self-evident, a highconfidence physiological signal in an underlying pain intensity is not easy to be acquired. Furthermore, it is difficult to determine whether the variability of the physiological signals is specific to pain or whether they represent a general response to factors such as thought, cognition, emotion and environment.

Since we were decided to exploit a practical quantitative and convenient system, there was a limitation on the selection of available signals. Though facial electromyograms and electroencephalograms would be expected to be useful, the attachment of electrodes to the face and scalp is complicated and not suitable for practical application. In our study, the selected physiological signals were blood volume pulse (BVP), electrocardiogram (ECG), and skin conductance level (SCL). These signals reflect the activity level of the autonomic nervous system, which is connected with the secretory activity of cardiac muscles and internal organs. As a wearable device, we use the Infiniti 3000A platform developed by Thought Technology Ltd., Quebec, Canada. The Infiniti 3000A apparatus is a compact sensing platform with 10 isolated channels for recording signals. It is small enough to attach to a portable computer and integrates to commercial sensors via custom cabling. The sampling frequency was fixed at $256 \mathrm{~Hz}$ for all the three channels.

The BVP signal is derived from a photoplethysmographic (PPG) sensor that monitors blood volume in capillaries and arteries by emitting an infrared light through the tissues. Vasomotor activity, which controls blood vessel diameter, is regulated by the sympathetic nervous system (Babchenko et al., 2001). Hence, changes in BVP amplitude reflect instantaneous sympathetic activation. Most PPG sensors can be placed anywhere on the body, with the finger as the most common location for recording a BVP signal. In this study, A BVPFlex/Pro sensor was placed against the palmar surface of the middle finger of the right hand with an elastic strap or a short strip of adhesive tape to acquire BVP signals (Figure 1A). In the preprocessing phase, a 4-order Butterworth bandpass filter with gain 3 , cutoff frequencies $[30,200] \mathrm{Hz}$ was applied to eliminate the bursts in the BVP signal.

The ECG, an electro-physiological signal that is associated with the electrical activity of the sinuatrial node, reflects the cardiovascular activity. Additionally, ECG responses to external stimuli (such as pain stimuli and stress) can produce large variability in a given subject's physiological signal (Sriram et al., 2009). Therefore, we can employ ECG signal to extract universal information about pain state or intensity. The ECG signal was measured from both surface of upper arms with the twoelectrode method based on lead I in our experiment (Figure 1A). For the preprocessing of the ECG trace collected by using noninvasive surface electrodes, a typical high-pass and movingaverage filter (Milanesi et al., 2008; Sidek et al., 2014) were used to remove a low frequency baseline drift due to electrode contact noise, respiratory effects, and motion artifacts. Otherwise, we employed an adaptive linear interpolation algorithm to perform baseline correction before feature extraction.

The SCL is another signal that can easily be acquired from the body surface. It reveals variance in the electrical properties of the skin due to the secretory activity of the perspiratory glands. As it is directly regulated by the sympathetic nervous system, it is a good indicator of psychological or physiological arousal level due to external stimuli. Changes in the SCL signal induced by a painful stimulus were validated to be used as a tool to monitor nociceptive stimulation and pain (Harrison et al., 2006; Storm, 2008). The SCL signal was traced from two Ag/ AgCl electrodes attached to the tip of the index and ring fingers on the palm-side of the right hand in this study (Figure 1A). In the preprocessing phase, a moving average smoothing was carried out to subtract artifacts. Furthermore, the raw SCL data was down-sampled by a factor of $1 / 2$ to reduce the computational complexity in feature extraction phase.

\section{Pain Induction Protocol}

As in many pain researches, there are variety ways that can be used to induce pain, such as thermal or cold pain stimulation (Appelhans and Luecken, 2008; Kachele et al., 2016), mechanical pain elicitation (Matsunaga et al., 2005; Shankar et al., 2009), and electrical shock (Oliveira et al., 2012; Zhang et al., 2016). In our study, pain was induced by an electrical stimulator (MotionStim8; Medel GmbH, Hamburg, Germany), which can generate a current square wave with a certain pulse width. The amplitude and frequency of the current are adjustable. The value of the pulse amplitude can be used as an objective index of pain intensity. In the experiment, the frequency of MotionStim8 was set to $2 \mathrm{~Hz}$. In order to avoid interference of the stimulator with the sensors, especially the ECG sensor, the stimulation electrodes were placed on the tibialis anterior muscle of the right leg, as far away as possible from the sensors (Figure 1A). 


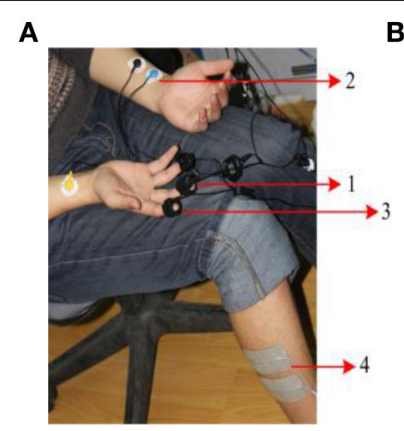

B

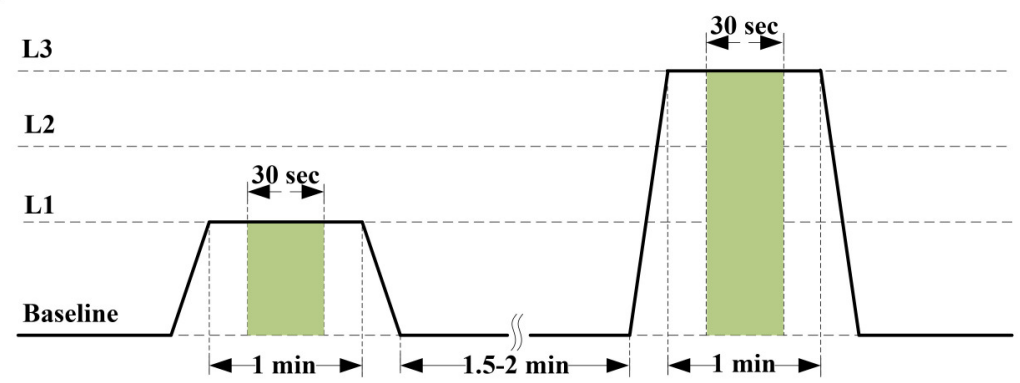

FIGURE 1 | (A) Pain recording scenario. Positioning of sensors and electrical stimulation electrodes: (1) BVP-Flex/Pro sensor, (2) EKG-Flex/Pro sensor, (3) SC-Flex/Pro sensor, and (4) Electrical stimulation electrodes. (B) Pain induction protocol. The stimulus levels are represented by current intensity L1 to L3. The features are extracted from the green window of length $30 \mathrm{~s}$.

Before stimulation phase, the current intensity was calibrated according to the subjects' self-reports. An electrical stimulus with $20 \mathrm{~mA}$ induced the sensation of pain was as pain starts (threshold). An electrical stimulus with $40 \mathrm{~mA}$ induced intense pain was as barely bearable pain (tolerance). Then, we divided the range between threshold and tolerance into 3 equally spaced intervals, 20-mA stimulus (stim20, L1), 30-mA stimulus (stim30, L2), and 40-mA stimulus (stim40, L3) with additional prestimulus (baseline, L0). During the stimulation experiment, each of the different current intensity was proceed for $1 \mathrm{~min}$ followed by a recovery period of $1.5-2 \mathrm{~min}$ (Figure 1B). To eliminate stimulation adaptability and time correlations, the sequence of stimuli and duration of the recovery period were randomly designed. Pain elicitation was executed 30 sessions for each of the 3 calibrated intensities (L1-L3) in the same day. With the addition of baseline phase, a total number of 120 stimulation trials were obtained for each subject. Each session took approximately $15 \mathrm{~min}$. To minimize motion artifacts, the participants were requested to be as relaxed as possible during the stimulation phase. Moreover, the recording scenario that led to the multi-day dataset was continuously carried out 7 days for one person in the same condition. Figure 2 shows physiological traces from a participant in the four pain states.

\section{FEATURE PROCESSING}

The overflow of the proposed pain intensity estimation system can be seen in Figure 3. The pipeline consists of raw signal input, independent preprocessing according to Section Physiological Signals and Preprocessing, feature processing, and finally pain intensity recognition. In this section, the proposed feature processing phase is introduced, including feature extraction, Section Feature Selection and Feature Reduction.

\section{Feature Extraction}

From the Figure 2, we can see that the amplitude of physiological signals obviously varied according to the level of electrical stimulus. Based on this observation, we gathered a variety of basic statistical features to evaluate the response to the pain-inducing stimuli, such as maximum, minimum, median values of data.
Furthermore, these features can easily be calculated in an online way, which makes them more suitable for real-time recognition system of pain intensity.

The statistical features can be calculated for each of the signal channels as follows. Let the three preprocessed signalsthe digitized BVP, ECG and SCL waveforms from any one of the four pain states segments-be designated by $X^{i}, i=1,2,3$ respectively. Let $X_{n}^{i}$ represent the value of the $n^{\text {th }}$ sample of the $i^{\text {th }}$ signal, where $n=1,2, \ldots, N$ with $N$ in range of 3,840 to 7,680 . Let $\tilde{X}_{n}^{i}$ refer to the normalized signal (zero mean, unit variance), formulated as:

$$
\tilde{X}_{n}^{i}=\frac{X_{n}^{i}-\mu^{i}}{\sigma^{i}} \quad i=1,2,3
$$

where $\mu^{i}$ and $\sigma^{i}$ are the means and standard deviations of $X^{i}$ as presented below. Let $f_{j}^{i}$ represent the $j^{\text {th }}$ features of the $i^{\text {th }}$ signal.

- The means of the signals: $f_{1}^{i}$ :

$$
\mu^{i}=\frac{1}{N} \sum_{n=1}^{N} X_{n}^{i} \quad i=1,2,3
$$

- The standard deviations of the signals: $f_{2}^{i}$ :

$$
\sigma^{i}=\left(\frac{1}{N-1} \sum_{n=1}^{N}\left(X_{n}^{i}-\mu^{i}\right)^{2}\right)^{1 / 2} i=1,2,3
$$

- The means of the absolute values of the first differences of the signals: $f_{3}^{i}$ :

$$
\theta_{1}^{i}=\frac{1}{N-1} \sum_{n=1}^{N-1}\left|X_{n+1}^{i}-X_{n}^{i}\right| \quad i=1,2,3
$$

- The means of the absolute values of the first differences of the normalized signals: $f_{4}^{i}$ :

$$
\tilde{\theta}_{1}^{i}=\frac{1}{N-1} \sum_{n=1}^{N-1}\left|\tilde{X}_{n+1}^{i}-\tilde{X}_{n}^{i}\right|=\frac{\theta_{1}^{i}}{\sigma^{i}} \quad i=1,2,3
$$



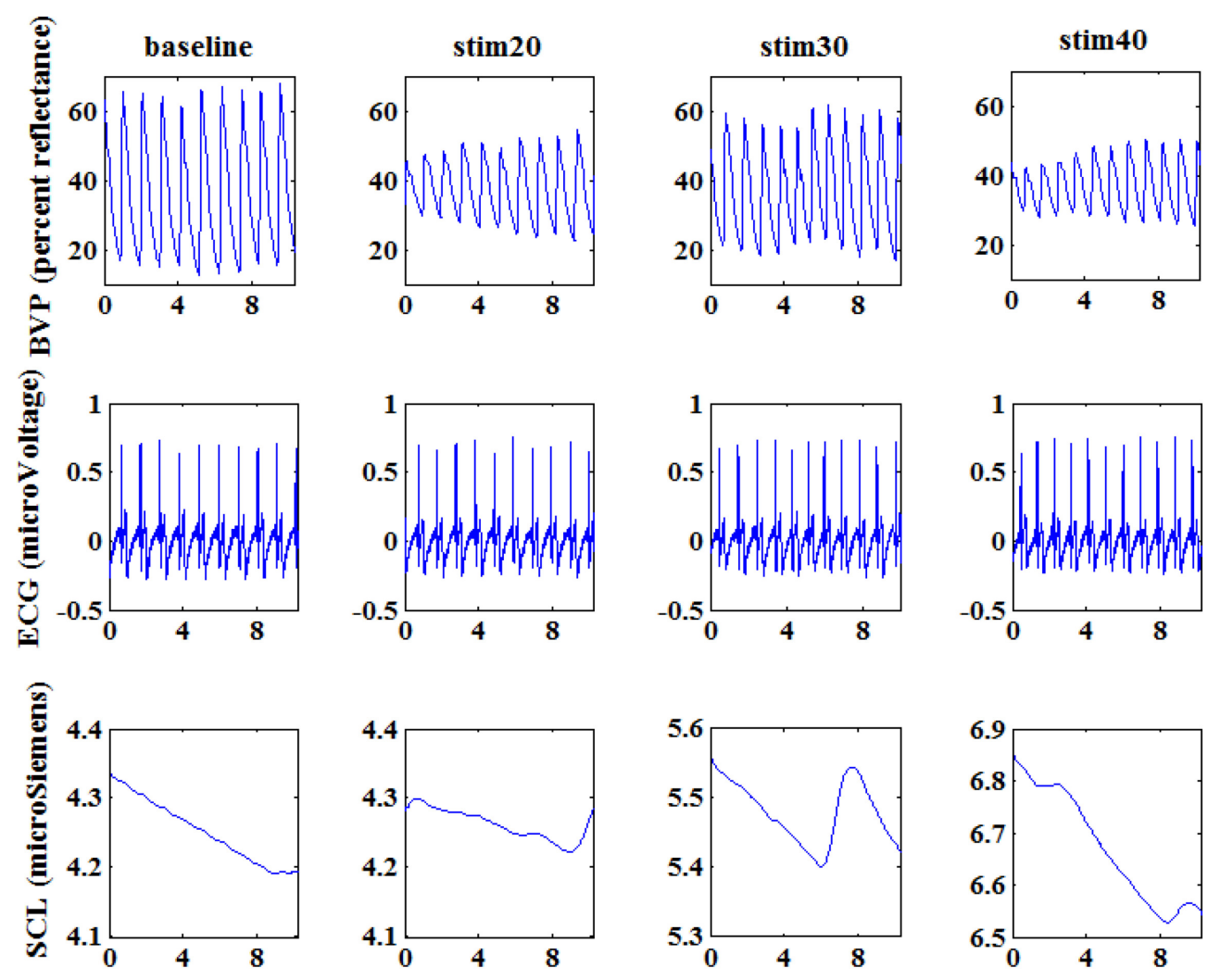

Time in seconds

FIGURE 2 | Physiological signals of a subject at baseline, stim20, stim30, and stim40. From top to bottom: blood volume pressure (BVP; percent reflectance), electrocardiogram (ECG; microVoltage), and skin conductance (SCL; microSiemens). Each graph shows 10 s of response. The segments shown here are visibly different for the four states.

- The means of the absolute values of the second differences of the signals: $f_{5}^{i}$ :

$$
\theta_{2}^{i}=\frac{1}{N-2} \sum_{n=1}^{N-2}\left|X_{n+2}^{i}-X_{n}^{i}\right| \quad i=1,2,3
$$

- The means of the absolute values of the second differences of the normalized signals: $f_{6}^{i}$ :

$$
\tilde{\theta}_{2}^{i}=\frac{1}{N-2} \sum_{n=1}^{N-2}\left|\tilde{X}_{n+2}^{i}-\tilde{X}_{n}^{i}\right|=\frac{\theta_{2}^{i}}{\sigma^{i}} \quad i=1,2,3
$$

- The minimum: $f_{7}^{i}$ :

$$
\operatorname{Min}=\operatorname{minimum}\left(X_{n}^{i}\right) \quad i=1,2,3
$$

- The maximum: $f_{8}^{i}$ :

$$
\operatorname{Max}=\operatorname{maximum}\left(X_{n}^{i}\right) \quad i=1,2,3
$$

- The minimum ratio: $f_{9}^{i}$ :

$$
\operatorname{minRatio}=\frac{\text { Min }}{N}
$$

Here, $N$ represents the length of the signal.

- The maximum ratio: $f_{10}^{i}$ :

$$
\text { maxRatio }=\frac{\text { Max }}{N}
$$

Here, $N$ represents the length of the signal.

- The range of the signal: $f_{11}^{i}$ :

$$
\text { range }=\text { Max }- \text { Min }
$$

Besides, we also calculated the median value of the signal as $f_{12}^{i}$. Hence, each channel was characterized by 12 features. Considering the time lag of pain reaction, we selected $30 \mathrm{~s}$ signal for feature analysis in the 1-min data. For obtaining the subtle changes of the signals in a trial, we segmented the $30 \mathrm{~s}$ signal by a sliding window with $3 \mathrm{~s}$ sliding step (the window length was $3 \mathrm{~s}$ ) to obtain the feature windows. Hence, a sample dataset of $40 \times 36$ can be obtained for each experiment session consisted of 4 trials. For single subject with 30 sessions, the size of sample dataset was 1,200 with 36 feature dimensions.

The BVP, ECG, and SCL are dependent on each participant's initial physiological level. Even when these signals are measured from the same person, they are likely day-dependent due to 


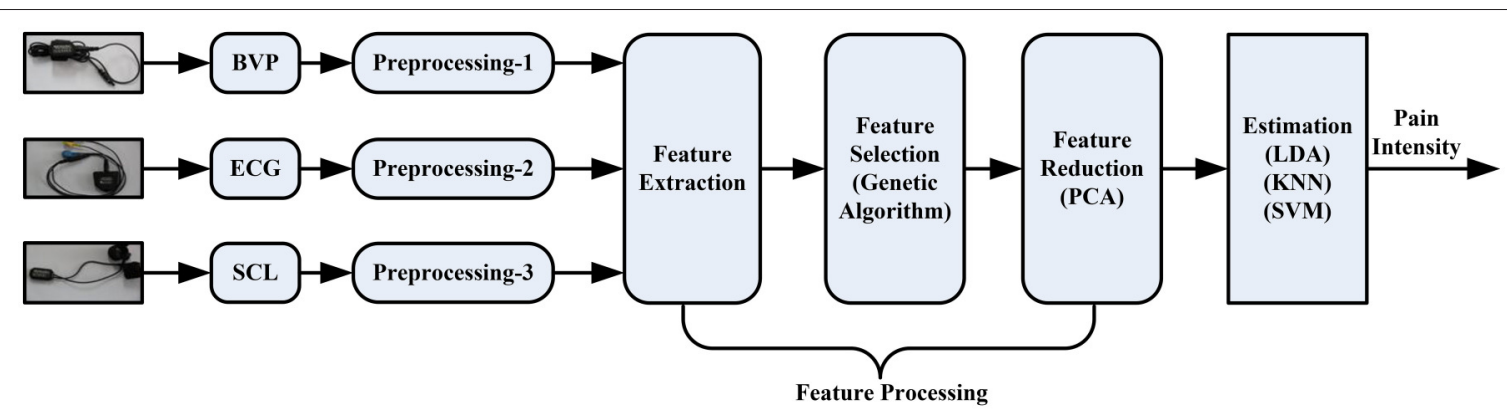

FIGURE 3 | Processing pipeline of the pain intensity recognition system.

variations in mental state affected by emotion, variations in physiology caused by sleep or diet, or variations in the sensor's connectivity with skin (Sun et al., 2010). To eliminate the intrasubject and day-to-day variation factor, a normalization stage for each feature was applied in feature vector set $F$. Refer to (13), the first was to subtract the minimum value from each feature. Then, the features are divided by the range to make all the values lie between 0 and 1 .

$$
F(m)_{n o r m}=\frac{F(m)-V_{\text {min }}(m)}{V_{\max }(m)-V_{\min }(m)}
$$

where, $V_{\min }(m)=\min \left\{F_{n}(m)\right\}, V_{\max }(m)=\max \left\{F_{n}(m)\right\}$, $\forall n \in|F|$.

\section{Feature Selection and Reduction}

Note that not all of these features are independent. Some features are a linear combination of other features (e.g., $\tilde{\theta}_{1}^{i}$ and $\tilde{\theta}_{2}^{i}$ ) or a non-linear property. To obtain high-quality features with significant discriminatory information, a hybrid combination of genetic algorithm (GA) and principal component analysis (PCA) was proposed. This combination was motivated by the roles of the two algorithms: GA selects from a feature set to remove useless and redundant features, while PCA linearly transforms a feature set. Since feature selection is non-linear, the hybrid of these two ways could provide a more powerful separation than either alone.

The GA is a biologically inspired stochastic algorithm based on some optimization criterion that mimics natural evolution. The GA is naturally applicable to feature selection since the problem has an exponential search space (Oh et al., 2004). Furthermore, the distinct specialty of this algorithm is that it always maintains a set of solutions (called chromosomes or individuals) in a population. To simulate the biological evolution, the GA selects fitter chromosomes at each generation by some genetic operations, such as crossover and mutation. The traditional procedures of steady-state GA are described below.

1. Initialize population $P$;

2. Repeat \{

3. select two parent chromosomes $p_{1}$ and $p_{2}$ from $P$;

4. $\quad$ offspring $=\operatorname{crossover}\left(p_{1}, p_{2}\right)$;

5. mutation (offspring);

6. $\quad$ replace $(P$, offspring);
7. fitness (offspring);

8. \} until (stopping condition);

For the feature selection problem, a finite bit string with $d$ binary digits is usually used to represent chromosomes. A binary digit represents a feature. A value of 1 or 0 in the chromosomes means that the corresponding feature is selected or removed, respectively. For example, a chromosome 10100010 means that the first, third and seventh features are selected. Since we wanted to improve recognition rate, the classification accuracy from the classifiers was directly used as the fitness function to evaluate a chromosome (Lin et al., 2015). Based on the initial fitness evaluation, the chromosome for the next generation was selected by a selection mechanism that ensures fitter chromosomes have a higher probability to survive. In our design, a traditional roulette wheel selection strategy was applied. We also used the standard two-point crossover operator to obtain the offspring and Gaussian mutation operations at probability on each of the offspring produced from crossover. The crossover rate and mutation rate were set to 0.8 and 0.01 , respectively. Besides, the size of population and the maximum generation in GA were set to 100 and 30 , respectively. These parameters are tunable to be suitable for a specific dataset to improve performance.

The principal component analysis (PCA) is a well-known statistics method of multi-variable analysis and used orthogonal transformation to convert a set of observations of possibly correlated variables into a set of values of linearly uncorrelated variables (Karamizadeh et al., 2013), also known as principal components. Moreover, PCA is a tool to reduce dimensionality by searching a linear projection matrix of the dataset to a feature space with lower dimensions while retaining most of the information. This linear projection matrix is a meaningful basis to filter out the noise and reveal hidden information in the original feature space. The key advantages of the PCA are the decreased requirements for memory and capacity, it low noise sensitivity, and increased efficiency in a lower dimension. By solving the covariance matrix, a series of eigenvalues with the corresponding eigenvectors are computed and rank-ordered descendingly by quantifying how "principal" each variance is. The $k$ largest eigenvalues are selected and the corresponding eigenvectors ( $k$ principal components) are used to construct the linear projection matrix. Then, a feature space with $N$ dimensions is transformed to a space of $k$ dimensions by this reduction 
matrix. For a detailed description of the PCA algorithm about mathematical derivation, the reader is referred to Bro and Smilde (2014).

A hybrid GA with PCA method is proposed, evaluated and implemented for comparison. As mentioned above, the GA algorithm provides a subset of features selected from raw features. Instead of feeding the PCA algorithm with all possible features, we used the selected subset of features as the input to the PCA. Note that the PCA algorithm is applied here for transforming features into a linearly uncorrelated space.

\section{PATTERN CLASSIFICATION}

The features undergo processing were feeding to classifiers to identify a subject' pain intensity. We used three types of learning methods to train and investigate the performance of classifiers, including linear discriminant analysis (LDA), $k$-nearest neighbor (KNN) algorithm, and support vector machine (SVM). We evaluated classification performance for single-signal datasets and multi-signal datasets, as well as for multi-subject datasets and multi-day datasets.

The LDA is a commonly used statistical method that uses a linear discriminant function to classify a new data points. In the calculation process of the algorithm, a hyperplane is constructed by finding a linear combination of features to separate or characterize two classes of objects or events. The optimal linear combination or projection in a classical LDA is constructed by minimizing the within-class distance and maximizing the between-class distance simultaneously, thereby achieving maximum class separation (Yan et al., 2014). Since a LDA algorithm is a linear binary classifier, it is not suitable in multi-class situations. In our pain study, the classes are three pain intensity levels and the baseline state. Thus, we need a multi-task method to inference pain states. A one-vs.-rest strategy was used to address multi-class problems. For each class $l$, an independent LDA classifier was trained to separate data of class $l$ and the rest class. In this paper, four trained LDA classifiers were applied in the test set. For a test sample, the recognition result was determined by selecting the class with the highest output value. Moreover, the classification accuracy of LDA was used as the fitness in the GA.

The KNN is a simple multi-class technique that operates by finding the $k$ objects in the training set which are closest to the test sample by some metrics, such as measures based on distance (Weinberger et al., 2005). In order to classify an instance of the test set into a class, KNN calculates the distance between each instance of the training set and this test sample. Then, a prediction is determined as the most common class among this set of nearest neighbors, with each neighbor's vote being assigned a weight inversely proportional to that neighbor's distance from the test sample. The performance of a KNN classifier is primarily determined by the choice of $k$ as well as the distance metric applied (Xiao and Chaovalitwongse, 2016). By effectively using prior knowledge such as distribution of the features, Euclidean distances were applied as the distance metric in our KNN classifier. In addition, the configurable parameter $k$ of the $k$-nearest neighbor algorithm was set to 3 by multiple testing.

The SVM is a supervised learning algorithm by finding a highdimensional discriminant hyperplane to separate observations into two class with a maximum margin, generally a soft margin (Vapnik, 2005). A regularization parameter was applied in the soft margin to penalize misclassification. By using the "kernel trick" to map the testing data points into a higher dimensional space, a support vector machine can construct an optimized separation hyperplane and make the mapped data easier to be separated. For pain recognition, randomization was executed using a computer-based list randomizer to construct the training data set for each subject. The preferential kernel function was the Gaussian or Radial Basis Function (RBF) kernel in this study. The SVM model was trained by cross-validation method with a grid search to find the optimal parameters of regularization and kernel. In this study, the SVM is optimal with a regularization of 5 and a Radial Basis Function kernel with width of 2.58.

In both of the KNN and SVM methods applied in pain recognition, the processed features were uniformed to zero mean and unit variance. Accordingly, the effect of feature variations in distance-based inference algorithms was reduced.

\section{EXPERIMENTAL RESULTS AND DISCUSSION}

\section{Initial Results -Single-Signal Analysis}

Previous studies have performed qualitative analyses of pain based on a single physiological signal. For example, Harrison et al. revealed SCL as a promising indicator to predict the presence or absence of pain in hospitalized infants during painful and non-painful medical procedures (Harrison et al., 2006). However, in this study, we applied pattern recognition techniques to quantify pain intensity based on a set of physiological signals. Since there was not yet a priori knowledge of pain recognition, we originally established a quantitative model for pain intensity from individual's single physiological signal. For each signal, we extracted 12 statistical features to construct three types of feature set, including BVP-features, ECG-features, and SCLfeatures. The classification accuracy of the LDA algorithm was evaluated by using these three types of feature set. Figure 4 presents the recognition results gained with LDA algorithm for each physiological signal in each of the six study subjects.

From the Figure 4, the experimental results provide evidence to support our hypothesis that a single signal can be used to quantify pain intensity in some degree, with the average classification accuracy of $50 \%$ above. Furthermore, since the BVP and SCL signal were highly affected by the intensity of pain stimuli in our experiment, the classification accuracies were even higher than that of ECG signal alone. Especially, the average classification accuracy of SCL signal was $68.39 \%$ $( \pm 2.32 \%)$, and that of ECG signal was $53.01 \%( \pm 3.40 \%)$. Unlike ECG, it can be observed that even the original SCL signal already presents an obvious variance with the pain stimulation in Figure 2. As the experimental results suggest, the extracted SCL features are a good indicator to identify the pain intensity. 


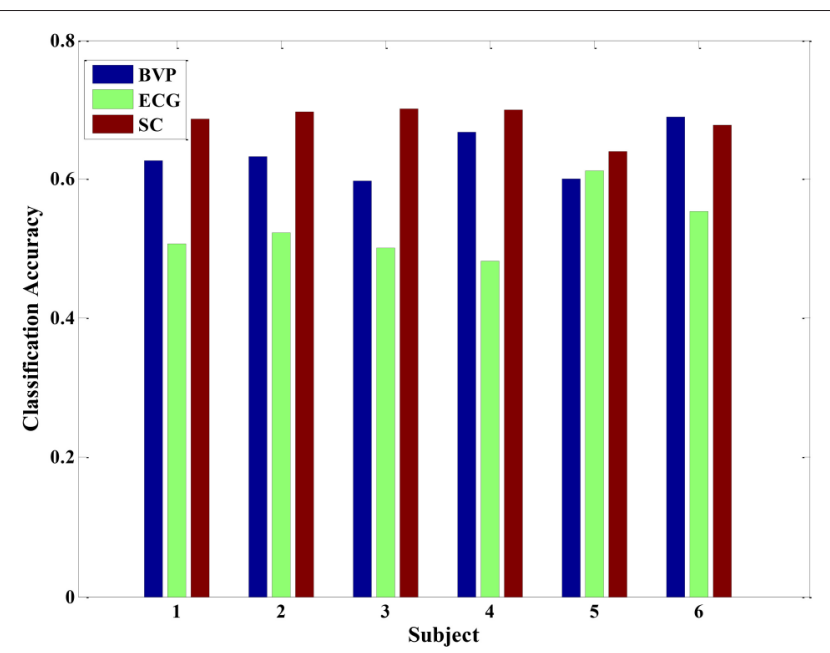

FIGURE 4 | The classification accuracy of the LDA algorithm for each physiological signal in each of the six subjects.

This is particularly suitable when it comes to integrate the proposed methods into wearable device. Since the SCL sensors are less intrusive and more convenient attached than BVP or ECG sensors, it can be easily embedded into wearable devices. Although the performance of LDA model for a single signal is considerable, the correct classification rate is relatively low. For improving the classification performance and obtaining better models, we considered applying multiple physiological signals with all-feature combination to quantify pain intensity. The idea was inspired to take full advantages of pain information contained in various signals.

\section{Results of the Multi-Signal Model}

Since we used multiple signals (BVP, ECG, and SCL), the total number of extracted features per sample was 36 (12 features per signal). We also selected the LDA algorithm as the classifier. The recognition accuracies of running 10 trials LDA are shown in Figure 5. The average accuracy of the LDA model was $42.49 \%( \pm 14.31 \%)$ for subject $1,53.78 \%( \pm 20.84 \%)$ for subject $2,65.12 \%( \pm 16.57 \%)$ for subject $3,51.07 \%( \pm 20.52 \%)$ for subject $4,54.30 \%( \pm 13.62 \%)$ for subject 5 , and $55.52 \%( \pm 11.43 \%)$ for subject 6 , respectively. The maximum classification accuracy of 10 trials LDA algorithm in the condition of multiple signals was approximately $90 \%$ (such as subjects 2 and 3). However, the variation of classification accuracy was large, ranging from $19.64 \%$ (subject 1 ) to $87.61 \%$ (subject 3 ). This finding indicated that the robustness of the multi-signal model established by LDA was insufficient. This may be because some extracted features are not independent and may be correlated. Furthermore, some features do not contribute or contribute little to the performance of the classification. A poor feature combination may greatly degrade the performance of the classifier. Hence, feature selection was needed to select a subset of good features.

In this study, we applied the genetic algorithm technique to seek and identify the potential informative features combinations, which contribute most to the performance of the classification. From other researches, it is obvious that the best accuracy of the LDA by using cross-validation method is considered as the indicator of the fitness function. Table 1 provide the experimental results of GA selection. The GA was run 15 times; each row lists the results from the corresponding run. In Table 1, the column "Best generation" indicates the generation number in which the optimal set of features was selected. And the average number of selected features was roughly 18 . The performance of LDA was significantly improved with average accuracy of $72 \%$ above. In detail, the feature $f_{2}^{i}, f_{3}^{i}, f_{4}^{i}$, $f_{5}^{i}, f_{6}^{i}$, and $f_{7}^{i}$ computed from the three physiological signals were almost repeatedly selected by each running of the GA, yielding 18 features: $\mu^{i}, \sigma^{i}, \theta_{1}^{i}, \tilde{\theta}_{1}^{i}, \theta_{2}^{i}, \tilde{\theta}_{2}^{i}, i \in(B V P, E C G, S C L)$. It means that these features are the more robust feature combination than those that are rarely selected in the context of this pain experiment.

Although the trend of the feature combination proves their efficacy in distinguishing pain intensity, the 18 features selected by GA are not the best informative features for classification. This is because the genetic algorithm cannot easily eliminate the linear features. To solve this problem, we applied the feature reduction technique. Figure 6 shows samples of 18-dimension feature space projected onto the first three features from subject 1. The selected feature vectors from the same class aggregated a cluster with a large amount of variation, whereas the feature vectors from the different classes overlapped dramatically. Then, a PCA method was applied to reduce the redundant linear features. After data transformation, the feature vector samples within same class were clustered more compactly than did samples before transformation, and the feature vector samples from different classes are farther away from one another (see in Figure 7).

After the feature processing stage by using the hybrid GA with PCA, the samples were fed to the LDA classifier to investigate the performance. In addition, we also tested the KNN and SVM algorithms for comparison. The corresponding recognition accuracy is presented in Figure 8. Using the optimized feature set, the average classification accuracy of three classifiers reached $80 \%$ or higher. Particularly, the performance of LDA algorithm was better than the other two algorithms, with $98 \%$ average accuracy. Those results prove that the proposed feature processing method is effective. And, the LDA classifier is more suitable in physiology-based pain intensity recognition for a single-subject scenario.

\section{Results of the Multi-Subject Model}

In addition to discussing the recognition model of a single subject, we also analyzed samples constructed from multiple subjects. A sample set of multiple subjects was generated based on the six participants. Similarly, GA-based feature selection and PCA-based dimension reduction technique were carried out on the sample set comprising multiple subjects. The LDA, KNN, and SVM algorithms were implemented to train the corresponding models. Figure 9 shows the pain intensity recognition accuracy of running 10 times for the multi-subject model. The average categorize accuracy was $84.28,83.94$, and $96.47 \%$ for LDA, 

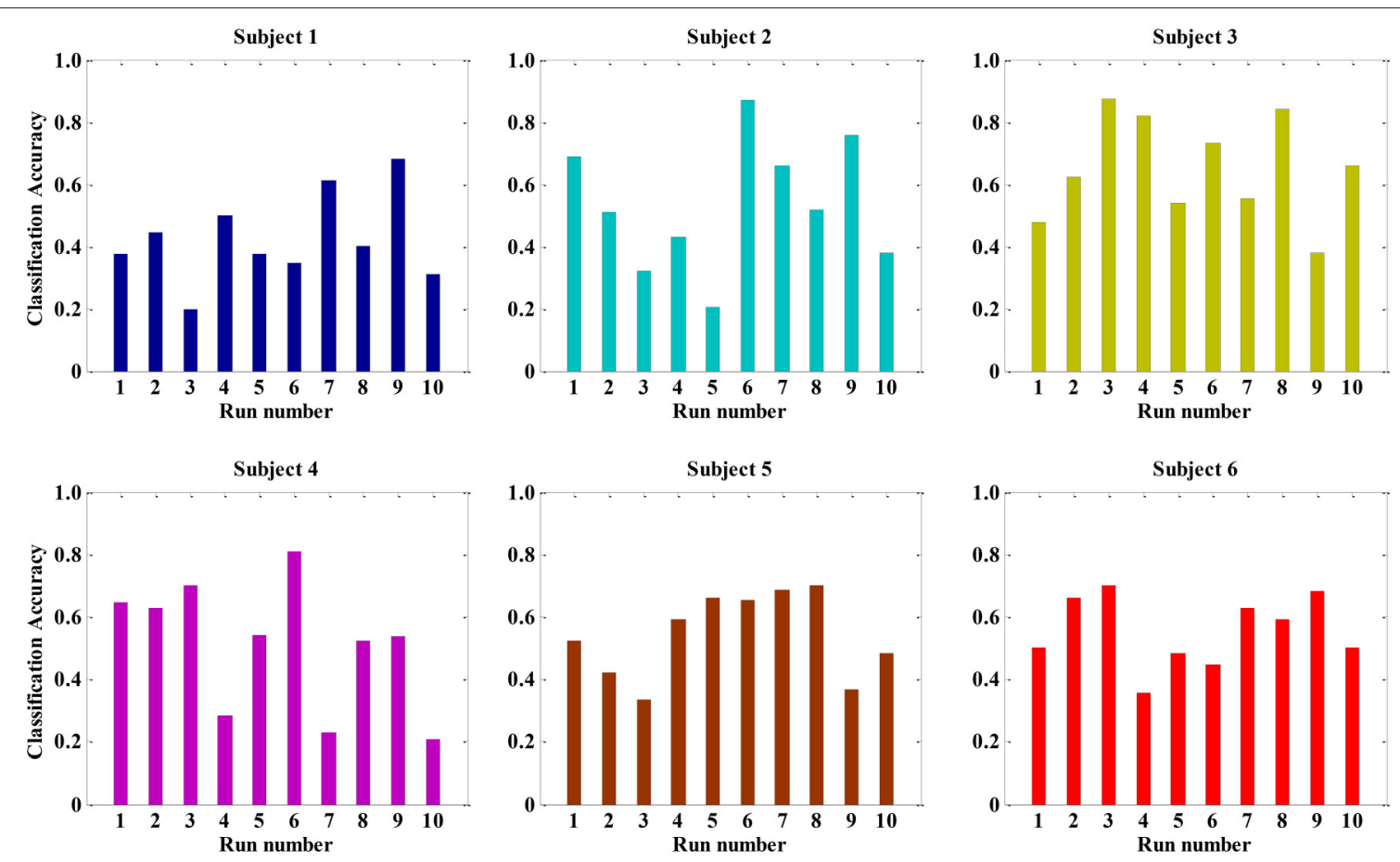

FIGURE 5 | The classification accuracy of running ten times LDA for all 36 features in each subject.

TABLE 1 | Experimental results of 15 runs of the GA.

\begin{tabular}{|c|c|c|c|c|c|c|}
\hline \multirow[t]{2}{*}{ Runs } & \multirow[t]{2}{*}{ Best generation } & \multirow[t]{2}{*}{ Number of features } & \multicolumn{3}{|c|}{ Features selected } & \multirow[t]{2}{*}{ Best accuracy (\%) } \\
\hline & & & BVP & ECG & SCL & \\
\hline 1 & 20 & 16 & 100110100100 & 111011000010 & 011110000100 & 77.80 \\
\hline 2 & 15 & 17 & 111011000010 & 001111000001 & 111011000001 & 84.20 \\
\hline 3 & 10 & 17 & 011111000000 & 100110001000 & 111111100001 & 86.89 \\
\hline 4 & 25 & 19 & 110111100100 & 011011000100 & 111011001100 & 79.95 \\
\hline 5 & 17 & 20 & 111011000101 & 111011000001 & 111111001000 & 82.38 \\
\hline 6 & 13 & 20 & 101101101000 & 011111001001 & 111011010010 & 75.38 \\
\hline 7 & 19 & 18 & 110111000100 & 111011001100 & 101111000000 & 88.89 \\
\hline 8 & 22 & 18 & 111011010010 & 010100100100 & 011111001001 & 84.28 \\
\hline 9 & 18 & 19 & 011111001001 & 111100000100 & 111011100010 & 85.25 \\
\hline 10 & 27 & 20 & 101111101101 & 010100110010 & 011011010010 & 72.30 \\
\hline 11 & 19 & 17 & 111011100000 & 001111100001 & 111011000000 & 82.10 \\
\hline 12 & 15 & 18 & 110111000100 & 100110001100 & 111100001110 & 75.35 \\
\hline 13 & 22 & 17 & 111001000100 & 010111110000 & 011111100000 & 80.82 \\
\hline 14 & 15 & 20 & 101110010001 & 111011110000 & 101111110000 & 79.08 \\
\hline 15 & 25 & 20 & 011110001110 & 010111000110 & 110111100100 & 84.27 \\
\hline
\end{tabular}

KNN and SVM, respectively. Overall, these three algorithms effectively identified pain intensity. The relevant multi-subject models established by LDA, KNN and SVM are feasible and appropriate. Besides, the multi-subjects model gained from SVM algorithm was better than the other two models. Every time we used the training SVM model to test the rest samples, the predicted accuracy was $92 \%$ or higher. Therefore, the trained
SVM model can be used as a general model to quantify pain intensity for a multiple-subject scenario.

Meanwhile, between-subjects' experimental validation was conducted by using the LDA, KNN, and SVM in a leave-onesubject-out cross-validation method, which is standard when dealing with multiple subjects. Here, the recognition modes were trained with dataset from randomly selected six subjects 


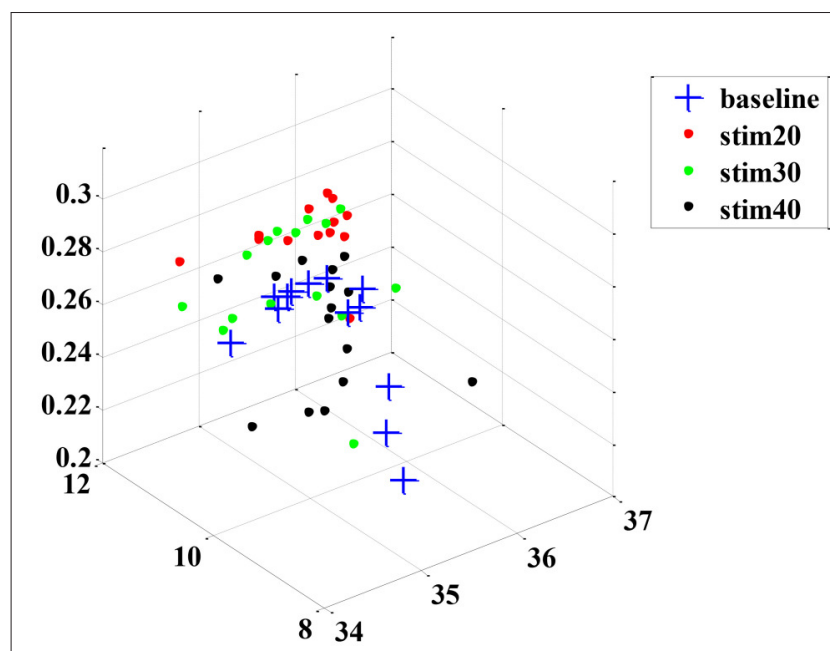

FIGURE 6 | An example sample set from subject 1 before dimension reduction projected onto the first three features.

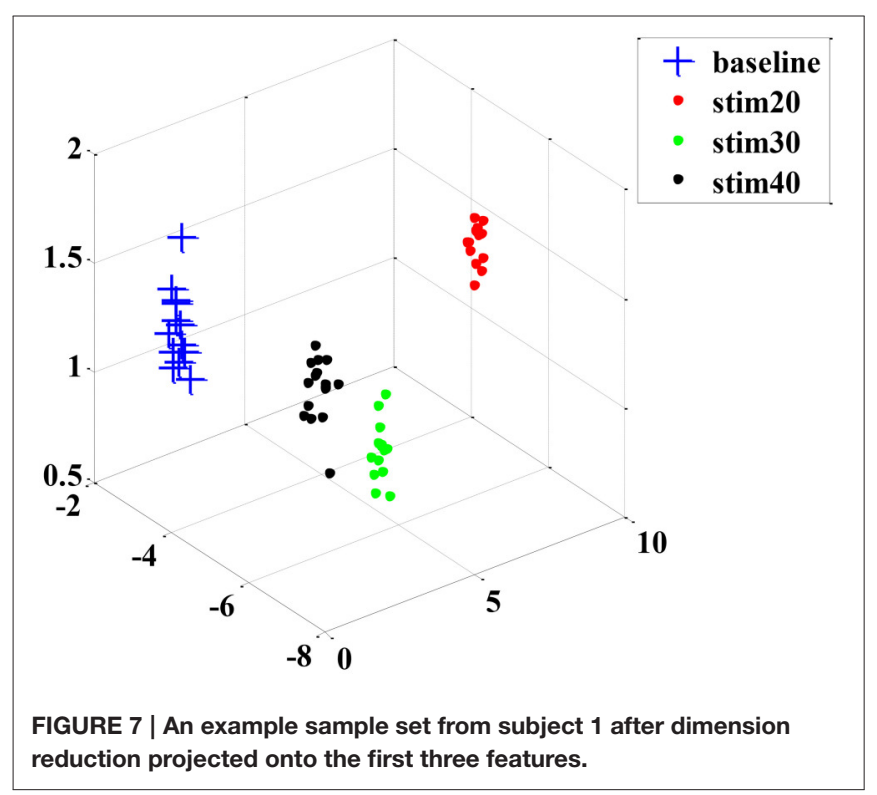

to predict the pain intensity of the remaining subject. It was repeated until all of the subjects had been part of the testing dataset. For a given between-subject scenario, we repeated 20 times and calculated the average accuracy for each pain intensity, respectively (see Figure 10). In detail, the average recognition accuracy of pain intensity with baseline, stim20, stim30, stim 40 was $78.01,90.10,82.35,82.90 \%$, respectively. This is a more realistic condition in which the distribution of the training dataset and the testing dataset are dissimilar due to inter-individual variability. Moreover, the SVM algorithm was performed better than the KNN and LDA, with average accuracy of $91.18,76.14$, and $82.83 \%$, respectively. These results demonstrate that the SVM classifier outperforms the other two classifiers for a between-subject scenario. Furthermore, the

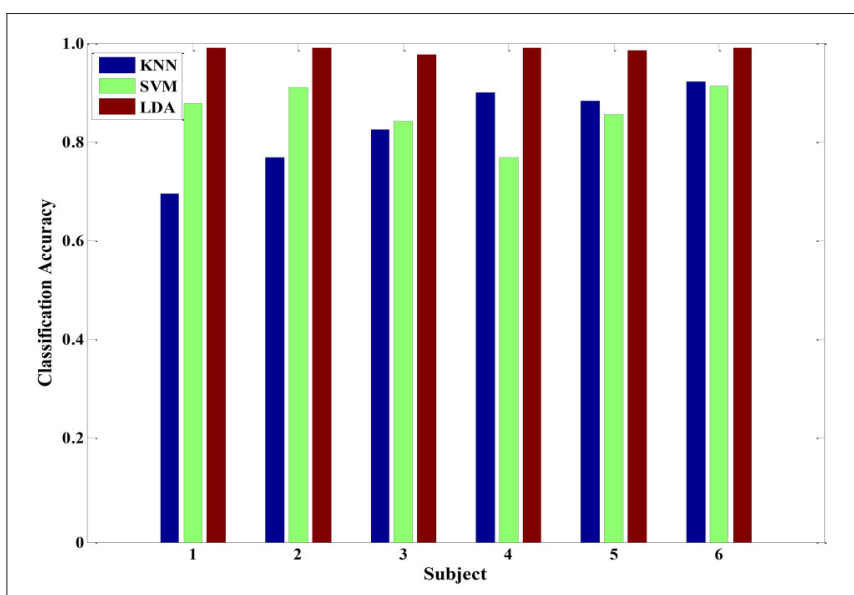

FIGURE 8 | The classification accuracy of three classifiers (KNN, SVM and LDA) after feature processing in each of the six subjects.

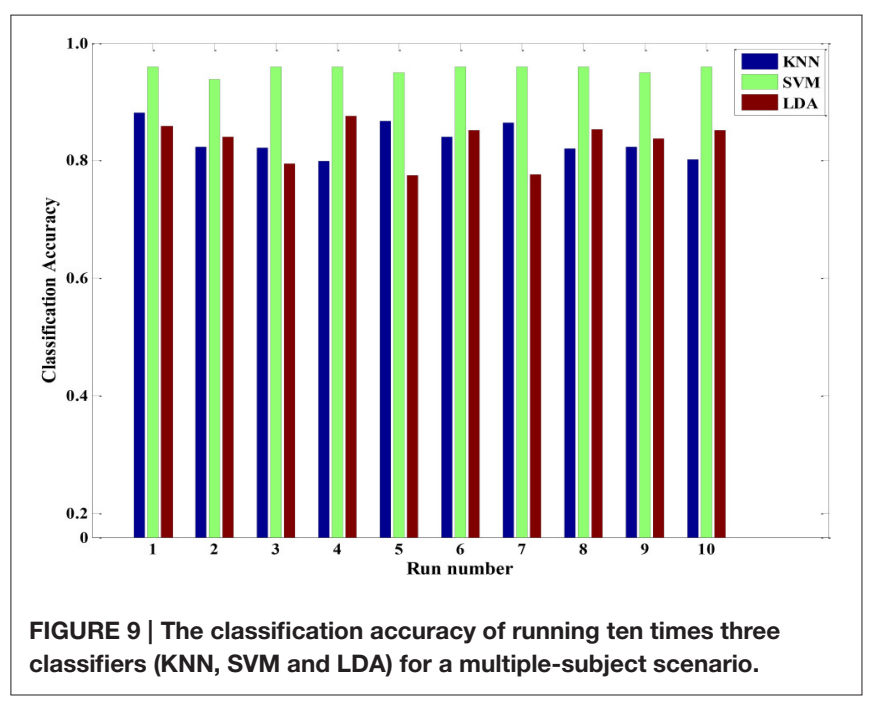

proposed system in the context of pain prediction would mitigate the inter-personal variance while preserving precise scalability.

\section{Results of the Multi-Day Model}

Furthermore, we also conducted experimental evaluation by using the three types of classifiers in a multi-day scenario. For one person, we continuously carried out the pain induction experiment of 7 days and collected a seven-day dataset. A leaveone-day-out cross-validation method was applied to obtain the pain intensity of one person. The results of multi-day model are given in Figure 11. The average recognition rate was 71.02, 81.39, and $76.93 \%$ for KNN, SVM, and LDA, respectively. It implied that the trained SVM model seemed to be more suitable as a special model to quantify pain intensity of multiple days for one subject. In addition, the overall accuracy was roughly reduced from $87 \%$ of 1 -day scenario to $70 \%$ of multi-day scenario. This may be mainly because that the signals measured from the same person are likely day-dependent. The quality of signals 


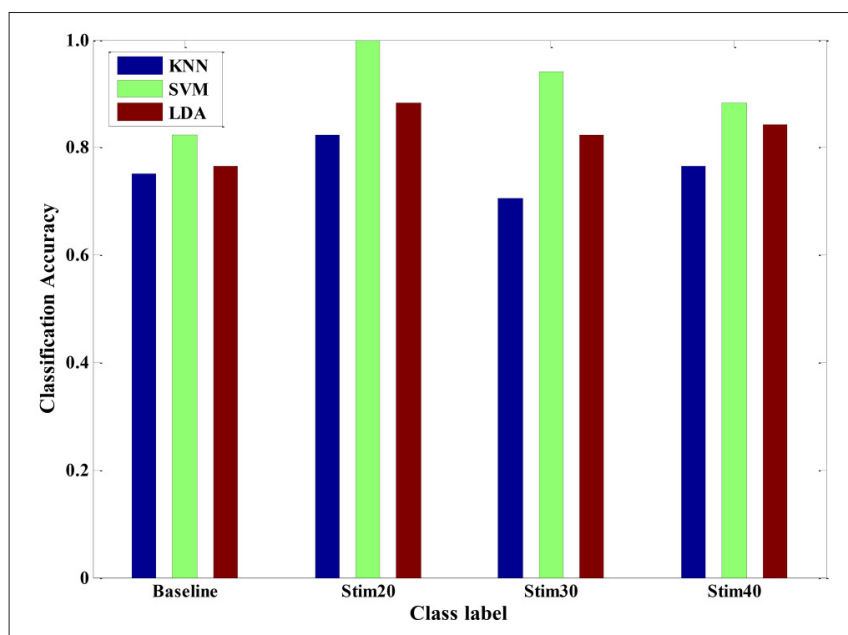

FIGURE 10 | Pain intensity recognition of three classifiers (KNN, SVM and LDA) for a between-subject scenario.

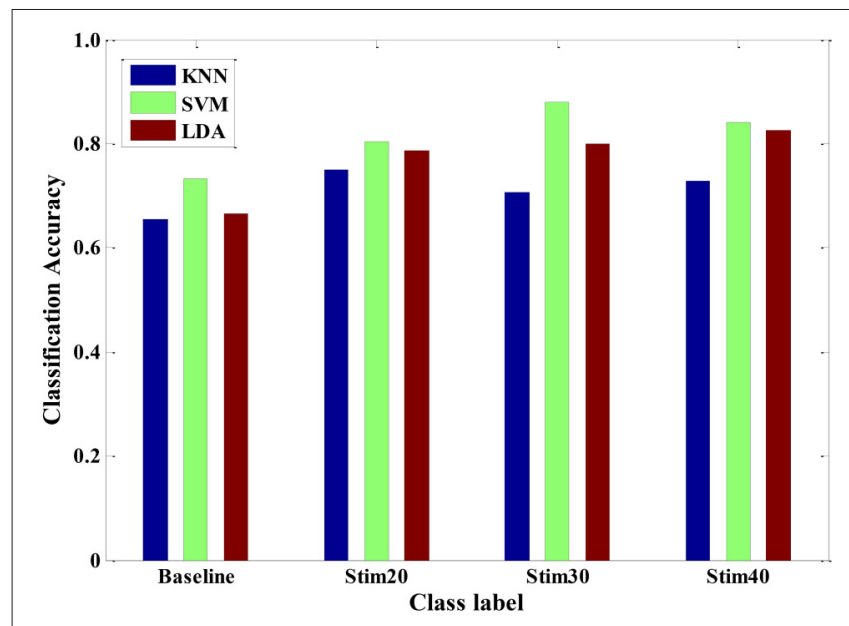

FIGURE 11 | Pain intensity recognition of three classifiers (KNN, SVM and LDA) for a multi-day scenario.

is affected by the intro-individual variance in mental state and environmental factor. However, our proposed pain intensity system is relatively fitted for practical applications of continuous and long-term pain monitoring.

\section{CONCLUSIONS AND OUTLOOK}

In this paper, we proposed a physiological signal-based method for convenient quantification of pain intensity induced by the external electrical stimulation. The proposed measurement provides a valid, physiology-based substitution of self-reported pain. The newly developed pain intensity measurement system consists of signal acquisition and preprocessing, feature extraction, GA-based feature selection and PCA-based feature reduction, and three types of pattern classifiers, including LDA, $\mathrm{KNN}$, and SVM. Using the hybrid feature processing of GA with PCA, the training samples can be optimized to obtain a higher recognition accuracy while simultaneously reducing computational complexity as unrelated features are removed, such as maximum, minimum, median, et al.

In various experimental validations, our method was tested and the performance of three classifiers were evaluated, including single-signal analysis, multi-signal model, multi-subject scenario, and multi-day scenario. For a LDA model trained by a single signal, the SCL signal seems to provide a better result than that of the BVP or ECG signal. Moreover, after a series of feature process, the performance of LDA classifier was significantly improved for multi-signal model. And, the LDA classifier is more suitable in physiology-based pain intensity recognition for a single-subject scenario compared to the KNN and SVM. For the samples constructed from multiple subjects, the KNN, LDA, and SVM could categorize pain intensity with 83.94, 84.28, and $96.47 \%$ accuracy, respectively. We also compared the performance of the three classifiers for a between-subject scenario. It seems that the performance of SVM algorithm was slightly better. Therefore, the trained SVM model can be used as a general model to quantify pain intensity for other unseen population. Furthermore, the three classifiers could also reach high accuracy for a multi-day scenario. These findings suggest that the proposed pain intensity measurement method can be suitable for practical applications of continuous pain monitoring. From Figures 10, 11, we can see that the recognition accuracy of different pain intensities was different. Especially, the recognition rate at baseline was relatively low. This could be caused by the large amount of variation of physiological signals for various subjects and various days at baseline. There are numerous causes that may influence the physiological baseline signals, such as cognition, physical movement, and even physical or psychological status. These factors may also affect the model selection and even deteriorate the recognition ratio. In summary, our system was able to predict well above chance level between three levels of pain intensity with respect to previous researches (Olugbade et al., 2015; Kachele et al., 2016).

For our future work, an online recognition experiment of pain intensity based on multi-physiological signals will be conducted. Then, a system of continuously pain monitor will be developed. Eventually, we expect that the sensors of our proposed system would be executed as a wearable device that is suitable for daily use in clinical settings. In the case of online recognition, the input signal cannot be preprocessed and standardized in advance. A large amount of signal from previous offline experiments in various population must be collected before using to deal with the online prediction of unseen samples. Besides, the latency of the online system must be considered. The final prediction system is to provide a trade-off between lower latency and higher accuracy. Since the dataset generated in the current study was obtained by artificial electrical stimulation to induce different intensities of pain, this procedure is not appropriate for an online prediction system. Hence, more ways should be investigated in order to predict and quantify pain intensity perceived by a person. We furthermore plan to research various methods of feature selection to access to a comparison result. 


\section{AUTHOR CONTRIBUTIONS}

$\mathrm{YC}, \mathrm{XZ}, \mathrm{JH}$, and YS conceived the conception and design of this research. YC executed the experiments, including acquisition and analysis of data for the work. YC, XZ, JH, and YS interpreted the experimental data. YC drafted the manuscript. XZ, JH, and YS revised the manuscript.

\section{FUNDING}

This research was partially supported by the National Nature Science Foundation of China (Grants nos. 61273355, 61573340),

\section{REFERENCES}

Appelhans, B. M., and Luecken, L. J. (2008). Heart rate variability and pain: associations of two interrelated homeostatic processes. Biol. Psychol. 77, 174-182. doi: 10.1016/j.biopsycho.2007.10.004

Aslaksen, P. M., Myrbakk, I. N., Høifødt, R. S., and Flaten, M. A. (2007). The effect of experimenter gender on autonomic and subjective responses to pain stimuli. Pain 129, 260-268. doi: 10.1016/j.pain.2006.10.011

Babchenko, A., Davidson, E., Ginosar, Y., Kurz, V., Faib, I., Adler, D., et al. (2001). Photoplethysmographic measurement of changes in total and pulsatile tissue blood volume, following sympathetic blockade. J. Physiol. Meas. 22, 389-396. doi: $10.1088 / 0967-3334 / 22 / 2 / 310$

Bro, R., and Smilde, A. K. (2014). Principal component analysis. Anal. Methods 6, 2812-2831. doi: 10.1039/c3ay41907j

Brown, J. E., Chatterjee, N., Younger, J., and Mackey, S. (2011). Towards a physiology-based measure of pain: patterns of human brain activity distinguish painful from non-painful thermal stimulation. PLoS ONE 6:e24124. doi: 10.1371/journal.pone.0024124

Caraceni, A., Cherny, N., Fainsinger, R., Kaasa, S., Poulain, P., Radbruch, L., et al. (2002). Pain measurement tools and methods in clinical research in palliative care: recommendations of an Expert Working Group of the European Association of Palliative Care. J. Pain Symptom. Manag. 23, 239-255. doi: 10.1016/S0885-3924(01)00409-2

Cruccu, G., Sommer, C., Anand, P., Attal, N., Baron, R., Garcia-Larrea, L., et al. (2010). EFNS guidelines on neuropathic pain assessment: revised 2009. Eur. J. Neurol. 17, 1010-1018. doi: 10.1111/j.1468-1331.2010.02969.x

De Jonckheere, J., Logier, R., Jounwaz, R., Vidal, R., and Jeanne, M. (2010). “From pain to stress evaluation using heart rate variability analysis: development of an evaluation platform," in Proceedings of th 2010 IEEE International Conference on Engineering in Medicine and Biology Society (Buenos Aires).

De Jonckheere, J., Rommel, D., Nandrino, J., Jeanne, M., and Logier, R. (2012). "Heart rate variability analysis as an index of emotion regulation processes: interest of the Analgesia Nociception Index (ANI)," in Proceedings of the 2012 IEEE International Conference on Engineering in Medicine and Biology Society (San Diego).

Faye, P. M., De Jonckheere, J., Logier, R., Kuissi, E., Jeanne, M., Rakza, T., et al. (2010). Newborn infant pain assessment using heart rate variability analysis. Clin. J. Pain 26, 777-782. doi: 10.1097/AJP.0b013e3181ed1058

Frampton, C. L., and Hughes-Webb, P. (2011). The measurement of pain. J. Clin. Oncol. 23, 381-386. doi: 10.1016/j.clon.2011.04.008

Haanpää, M., Attal, H., Backonja, M., Baron, R., Bennett, M., Bouhassira, D., et al. (2011). NeuPSIG guidelines on neuropathic pain assessment. Pain 152, 14-27. doi: 10.1016/j.pain.2010.07.031

Harrison, D., Boyce, S., Loughnan, P., Dargaville, P., Storm, H., and Johnston, L. (2006). Skin conductance as a measure of pain and stress in hospitalised infants. Earl. Hum. Dev. 82, 603-608. doi: 10.1016/j.earlhumdev.2005.12.008

Herr, K., Bjoro, K., and Decker, S. (2006). Tools for assessment of pain in nonverbal older adults with dementia: a state-of-the-science review. J. Pain Sym. Man. 31, 170-192. doi: 10.1016/j.jpainsymman.2005.07.001

Huang, G., Xiao, O., Hung, Y. S., Iannetti, G. D., Zhang, Z. G., and Hu, L. (2013). A novel approach to predict subjective pain perception and in part by the National High Technology Research and Development Program of China (863 Program) under Grant 2015AA042301 and Youth Innovation Promotion Association CAS.

\section{ACKNOWLEDGMENTS}

The authors gratefully acknowledge the support by State Key Laboratory of Robotics and China Medical University for providing us with the acquisition devices. Also, we appreciate the assistance of Lingguo Kong in setting up the experimental system and Lipeng An in data analysis. from single-trial laser-evoked potentials. Neuroimage 81, 283-293. doi: 10.1016/j.neuroimage.2013.05.017

Hudspith, M. J., Siddall, P. J., and Munglani, R. (2006). Physiology of pain. Found. Anesthesia 2, 267-285. doi: 10.1016/B978-0-323-03707-5.50029-2

Irani, R., Nasrollahi, K., and Moeslund, T. B. (2015). "Pain recognition using spatiotemporal oriented energy of facial muscles," in Proceedings of the 2015 IEEE Confernece on Computer Vision and Pattern Recognition (CVPR) Workshops (Boston, MA).

Kachele, M., Thiam, P., Amirian, M., Schwenker, F., and Palm, G. (2016). Methods for person-centered continuous pain intensity assessment from bio-physiological channels. IEEE J. Sel. Topics Signal Process. 10, 854-864. doi: 10.1109/JSTSP.2016.2535962

Kächele, M., Werner, P., Al-Hamadi, A., Palm, G., Walter, S., and Schwenker, F. (2015). Bio-visual fusion for person-independent recognition of pain intensity. Mult. Classif. Syst. 9132, 220-230. doi: 10.1007/978-3-319-20248-8_19

Kaltwang, S., Rudovic, O., and Pantic, M. (2012). Continuous pain intensity estimation from facial expressions. In Int. Sym. Vis. Comput. 7432, 368-377. doi: 10.1007/978-3-642-33191-6_36

Karamizadeh, S., Abdullah, S. M., Manaf, A. A., Zamani, M., and Hooman, A. (2013). An overview of principal component analysis. J. Signal Inform. Process. 4, 173-175. doi: 10.4236/jsip.2013.43B031

Li, D., Puntillo, K., and Miaskowski, C. (2008). A review of objective pain measurement for use with critical care adult patients unable to self-report. Pain 9, 2-10. doi: 10.1016/j.jpain.2007.08.009

Lin, C. T., Prasad, M., and Saxena, A. (2015). An improved polynomial neural network classifier using real-coded genetic algorithm. IEEE Trans. Syst. Man, Cybern. Syst. 45, 1389-1401. doi: 10.1109/TSMC.2015.2406855

Loeser, J. D., and Treede, R. D. (2008). The Kyoto protocol of IASP Basic Pain Terminology. Pain 137, 473-477. doi: 10.1016/j.pain.2008.04.025

Logier, R., Jeanne, M., Dassonneville, A., Delecroix, M., and Tavernier, B. (2010). "PhysioDoloris: a monitoring device for analgesia/nociception balance evaluation using heart rate variability analysis," in Proceedings of the 2010 IEEE International Conference on Engineering in Medicine and Biology Society (Buenos Aires).

Lucey, P., Cohn, J. F., Matthews, I., Lucey, S., Sridharan, S., Howlett, J., et al. (2011). Automatically detecting pain in video through facial action units. IEEE Trans. Syst. Man Cybern. B Cybern. 3, 664-674. doi: 10.1109/TSMCB.2010.20 82525

Marquand, A., Howard, M., Brammer, M., Chu, C., Coen, S., and MourãoMiranda, J. (2010). Quantitative prediction of subjective pain intensity from whole-brain fMRI data using Gaussian processes. Neuroimage 49, 2178-2189. doi: 10.1016/j.neuroimage.2009.10.072

Matsunaga, N., Kuroki, A., and Kawaji, S. (2005). "Superficial pain model using ANNs and its application to robot control," in Proceedings of the 2005 IEEE International Conference on Advanced Intelligent Mechatronics (Monterey).

Milanesi, M., Martini, M., Vanello, N., Positano, V., Santarelli, M. F., and Landini, L. (2008). Independent component analysis applied to the removal of motion artifacts from electrocardiographic signals. Med. Biol. Eng. Comput. 46, 251-261. doi: 10.1007/s11517-007-0293-8

Nir, R. R., Sinai, A., Raz, E., Sprecher, E., and Yarnitsky, D. (2010). Pain assessment by continuous EEG: association between subjective perception of tonic pain 
and peak frequency of alpha oscillations during stimulation and at rest. Brain Res. 1344, 77-86. doi: 10.1016/j.brainres.2010.05.004

Oh, I. S., Lee, J. S., and Moon, B. R. (2004). Hybrid genetic algorithms for feature selection. IEEE Trans. Pattern Anal. Mach. Intell. 26, 1424-1437. doi: 10.1109/TPAMI.2004.105

Oliveira, M. I., Machado, A. R. P., Chagas, V. G. S., Granado, T. C., Pereira, A. A., and Andrade, A. O. (2012). "On the use of evoked potentials for quantification of pain," in Proceedings of the 2012 IEEE International Conference on Engineering in Medicine and Biology Society (San Diego, CA).

Olugbade, T. A., Bianchi-Berthouze, N., Marquardt, N., and Williams, A. C. (2015). "Pain level recognition using kinematics and muscle activity for physical rehabilitation in chronic pain," in Proceedings of the 2015 IEEE International Conference on Affective Computing and Intelligent Interaction (ACII) (Xian).

Puntillo, K. A., Morris, A. B., Thompson, C. L., Stanik-Hutt, J., White, C. A., and Wild, L. R. (2004). Pain behaviors observed during six common procedures: results from Thunder Project II. Crit. Care Med. 32, 421-427. doi: 10.1097/01.CCM.0000108875.35298.D2

Roulin, M. J., and Ramelet, A. S. (2012). Pain indicators in brain-injured critical care adults: an integrative review. Aust. Crit. Care 25, 110-118. doi: 10.1016/j.aucc.2011.10.002

Schnakers, C., and Zasler, N. D. (2007). Pain assessment and management in disorders of consciousness. Curr. Opin. Neurol. 20, 620-626. doi: 10.1097/WCO.0b013e3282f169d9

Shankar, K., Subbiah, B. V., and Jackson, D. (2009). "An empirical approach for objective pain measurement using dermal and cardiac parameters," in Proceedings of the 2009 IEEE International Conference on Biomedical Engineering (Singapore).

Sidek, K. A., Khalil, I., and Jelinek, H. F. (2014). ECG biometric with abnormal cardiac conditions in remote monitoring system. IEEE Trans. Syst. Man Cybern. Syst. 44, 1498-1509. doi: 10.1109/TSMC.2014.2336842

Sriram, J. C., Shin, M., Choudhury, T., and Kotz, D. (2009). “Activity-aware ECGbased patient authentication for remote health monitoring," in Proceedings of the 2009 International Conference on Multimodal Interfaces (Cambridge).

Storm, H. (2008). Changes in skin conductance as a tool to monitor nociceptive stimulation and pain. Curr. Opin. Anesthesiol. 21, 796-804. doi: 10.1097/ACO.0b013e3283183fe4

Sun, F. T., Kuo, C., Cheng, H. T., Buthpitiya, S., Collins, P., and Griss, M. (2010). “Activity-aware mental stress detection using physiological sensors," in
International Conference on Mobile Computing, Application, and Services (Santa Clara).

Treister, R., Kliger, M., Zuckerman, G., Aryeh, I. G., and Eisenberg, E. (2012). Differentiating between heat pain intensities: the combined effect of multiple autonomic parameters. Pain 153, 1807-1814. doi: 10.1016/j.pain.2012.04.008

Vapnik, V. (2005). Universal learning technology: support vector machines. NEC J. Adv. Technol. 2, 137-144.

Weinberger, K. Q., Blitzer, J., and Saul, L. K. (2005). "Distance metric learning for large margin nearest neighbor classification," in Proceedings of the 18th International Conference on Neural Information Processing System (British Columbia).

Werner, P., Al-Hanadi, A., Niese, R., Walter, S., Gruss, S., and Traue, H. C. (2014). "Automatic pain recognition from video and biomedical signals," in Proceedings of the 2014 IEEE International Conference on Pattern Recognition (Stockholm: ICPR).

Xiao, C., and Chaovalitwongse, W. A. (2016). Optimization models for feature selection of decomposed nearest neighbor. IEEE Trans. Syst. Man Cybern. Syst. 46, 177-184. doi: 10.1109/TSMC.2015.2429637

Yan, Y., Ricci, E., Subramanian, R., Liu, G., and Sebe, N. (2014). Multitask linear discriminant analysis for view invariant action recognition. IEEE Trans. Image Process. 23, 5599-5611. doi: 10.1109/TIP.2014.2365699

Young, J., Siffleet, J., Nikoletti, S., and Shaw, T. (2006). Use of a Behavioural Pain Scale to assess pain in ventilated, unconscious and/or sedated patients. Intensive Crit. Care Nurs. 22, 32-39. doi: 10.1016/j.iccn.2005.04.004

Zhang, D., Xu, F., Xu, H., Shull, P. B., and Zhu, X. (2016). Quantifying different tactile sensations evoked by cutaneous electrical stimulation using electroencephalography features. Int. J. Neur. Syst. 26:1650006. doi: $10.1142 /$ S0129065716500064

Conflict of Interest Statement: The authors declare that the research was conducted in the absence of any commercial or financial relationships that could be construed as a potential conflict of interest.

Copyright (C) 2017 Chu, Zhao, Han and Su. This is an open-access article distributed under the terms of the Creative Commons Attribution License (CC BY). The use, distribution or reproduction in other forums is permitted, provided the original author(s) or licensor are credited and that the original publication in this journal is cited, in accordance with accepted academic practice. No use, distribution or reproduction is permitted which does not comply with these terms. 


\section{OPEN ACCESS}

Edited by:

Dingguo Zhang,

Shanghai Jiao Tong University, China

Reviewed by:

Ranu Jung,

Florida International University, USA

Rong Song,

Sun Yat-sen University, China

*Correspondence:

Simona Crea

simona.crea@santannapisa.it

${ }^{\dagger}$ These authors have contributed equally to this work

Specialty section:

This article was submitted to

Neural Technology,

a section of the journal

Frontiers in Neuroscience

Received: 24 September 2016

Accepted: 21 April 2017

Published: 12 May 2017

Citation:

Crea S, Cempini M, Mazzoleni S,

Carrozza MC, Posteraro F and

Vitiello N (2017) Phase-Il Clinical

Validation of a Powered Exoskeleton

for the Treatment of Elbow Spasticity.

Front. Neurosci. 11:261.

doi: 10.3389/fnins.2017.00261

\section{Phase-II Clinical Validation of a Powered Exoskeleton for the Treatment of Elbow Spasticity}

\author{
Simona Crea ${ }^{1 *}$, Marco Cempini $^{1,2,3}$, Stefano Mazzoleni ${ }^{1,4}$, Maria Chiara Carrozza ${ }^{1}$, \\ Federico Posteraro ${ }^{4,5+}$ and Nicola Vitiello ${ }^{1,6 \dagger}$ \\ ${ }^{1}$ The BioRobotics Institute, Scuola Superiore Sant'Anna, Pisa, Italy, ${ }^{2}$ Shirley Ryan AbilityLab, Chicago, IL, USA, ${ }^{3}$ Feinberg \\ School of Medicine, Northwestern University, Chicago, IL, USA, ${ }^{4}$ Rehabilitation Bioengineering Laboratory, Volterra, Italy, \\ ${ }^{5}$ Rehabilitation Department, Versilia Hospital, Azienda USL Toscana Nord Ovest, Viareggio, Italy, ${ }^{6}$ Fondazione Don Carlo \\ Gnocchi, Firenze, Italy
}

Introduction: Spasticity is a typical motor disorder in patients affected by stroke. Typically post-stroke rehabilitation consists of repetition of mobilization exercises on impaired limbs, aimed to reduce muscle hypertonia and mitigate spastic reflexes. It is currently strongly debated if the treatment's effectiveness improves with the timeliness of its adoption; in particular, starting intensive rehabilitation as close as possible to the stroke event may counteract the growth and postpone the onset of spasticity. In this paper we present a phase-II clinical validation of a robotic exoskeleton in treating subacute post-stroke patients.

Methods: Seventeen post-stroke patients participated in 10 daily rehabilitation sessions using the NEUROExos Elbow Module exoskeleton, each one lasting 45 min: the exercises consisted of isokinetic passive mobilization of the elbow, with torque threshold to detect excessive user's resistance to the movement. We investigated the safety by reporting possible adverse events, such as mechanical, electrical or software failures of the device or injuries or pain experienced by the patient. As regards the efficacy, the Modified Ashworth Scale, was identified as primary outcome measure and the NEEM metrics describing elbow joint resistance to passive extension (i.e., maximum extension torque and zero-torque angle) as secondary outcomes.

Results: During the entire duration of the treatments no failures or adverse events for the patients were reported. No statistically significant differences were found in the Modified Ashworth Scale scores, between pre-treatment and post-treatment and between post-treatment and follow-up sessions, indicating the absence of spasticity increase throughout (14 days) and after (3-4 months follow-up) the treatment. Exoskeleton metrics confirmed the absence of significant difference in between pre- and post-treatment data, whereas intra-session data highlighted significant differences in the secondary outcomes, toward a decrease of the subject's joint resistance.

Conclusions: The results show that our robotic exoskeleton can be safely used for prolonged sessions in post-stroke and suggest that intensive early rehabilitation 
treatment may prevent the occurrence of spasticity at a later stage. Moreover, the NEEM metrics were found to be reliable compared to the Modified Ashworth Scale and sensitive to revealing intra-session changes of elbow resistance to passive extension, in agreement with clinical evidences.

Keywords: robotics, rehabilitation, stroke, upper limb, exoskeleton, spasticity

\section{INTRODUCTION}

Stroke has been recognized as one of the major causes of long-term movement disabilities in the elderly, and in the last years its contingency is continuously increasing (Feigin et al., 2003). A huge number of stroke-related disabilities dramatically influence the lives of stroke survivors, of which spasticity is a significant component (O'Brien et al., 1996). Clinically, spasticity is defined as the occurrence of increased involuntary resistance to passive movements. However, this phenomenon reflects a wider spectrum of clinical problems, including abnormal limb posture and hyperactive cutaneous and tendon reflexes. After stroke, the initial usual occurrence of flaccidity and hypotonia is followed by spasticity in about $20 \%$ of patients, after a time interval that is highly variable and may occur in the short-, medium-, or longterm post-stroke period depending on a large number of factors (Ward, 2012). Clinical studies have emphasized that preventing spasticity and treating emerging spasticity in a timely manner is essential to improve the neurologic and articular pattern of the impaired limb (Ottenbacher and Jannell, 1993).

Typical rehabilitation therapy for stroke survivors helps them relearn skills that are lost due to brain damage; in particular, patients are prompted to engage in passive or active rangeof-motion exercises to strengthen their impaired limbs, as an integral part of daily management (Langhorne et al., 2011). Indeed, while they tend to avoid using paretic limbs, it has been widely demonstrated that their repetitive mobilization (either passive or active) can reduce muscle hypertonia, thus lowering joint spasticity and encouraging brain plasticity (Lum P. S. et al., 2002; Colombo et al., 2005; Masiero et al., 2007). Among the different rehabilitation exercises (e.g., passive, active-assisted, active as needed, active-resisted), the passive movement of the patient's joints has been demonstrated to maintain the range of motion $(R O M)$ at the joints and flexibility in the muscles and connective tissue, as well as reduce muscle hypertonia and resistance to passive movement (Schmit et al., 2000; Lum P. et al., 2002; Nuyens et al., 2002). One of the key components of effective post-stroke rehabilitation is also the early mobilization of the paretic limb (Langhorne and Pollock, 2002).

When treating muscle contractures, the mobilization of contracted articulations involves close physical contact between the therapist and the patient: the therapist needs to regulate the strength and speed of the motion of the patient's articulations, in order to correct any compensatory strategies and comply with sudden spasticity. In this scenario, using robotic platforms that are capable of automating the repetitive motions usually performed by the physical therapist (Kwakkel et al., 2007) and of complying with sudden spasticity may facilitate high-intensity rehabilitation.
Many devices have been specifically developed for the neurorehabilitation of stroke patients, allowing patients to perform specific movements of the limbs. Extensive reviews of robotic devices for upper-limb neuro-rehabilitation have been carried out (Pizzi et al., 2005; Turchetti et al., 2014): many upperlimb robotic devices have been applied in stroke rehabilitation and their effectiveness has been evaluated at the start and end of treatment by means of widely used clinical assessment scales (Volpe et al., 2000; Masiero et al., 2007; Mazzoleni et al., 2013a,b) and sometimes even by measurements performed using robotic systems (Colombo et al., 2005; Mazzoleni et al., 2011).

Within the above framework, at The BioRobotics Institute of Scuola Superiore Sant'Anna we recently developed the NEUROExos, a powered elbow exoskeleton, designed to perform both passive and active elbow joint mobilization in post-stroke patients (Vitiello et al., 2013). A modified version of the device, the NEUROExos Elbow Module (NEEM) holds certification as a class-II medical device (compliant with EU regulations for medical devices, i.e., IEC EN 60601-1:2007 and EN ISO 14971:2012) and is approved for in-clinic use (Vitiello et al., 2016).

In this paper, a group of 17 post-stroke patients in their sub-acute phase were treated with the NEEM system on a daily basis, in addition to traditional physical therapy. The outcome was assessed in terms of system safety and efficacy. Safety was investigated by reporting possible adverse events, such as mechanical, electrical or software failures of the device or injuries or pain experienced by the patient. To test the treatment efficacy, the Modified Ashworth Scale (MAS, Ashworth, 1964; Bohannon and Smith, 1987; Pandyan et al., 1999) was identified as primary outcome measure and the NEEM metrics describing elbow joint resistance to passive extension (i.e., maximum extension torque and zero-torque angle) as secondary outcomes. We expected that intense therapy performed in the sub-acute phase following the stroke would be effective in preventing elbow spasticity from occurring at a later stage (i.e., 3-4 months after the stroke). Similar to other phase-II studies in the field of poststroke rehabilitation (van Heugten et al., 1998), the present study included only one group of patients. A non-controlled group of study was essential to understand whether the proposed therapy could provide positive results, and was intended to be preparatory for a phase-III randomized control trial, where the efficacy of the proposed therapy will be compared with other conventional therapies. As a second objective, similarly to other studies with robot-assisted therapy (Colombo et al., 2005), the results from the robot metrics were validated against the outcomes from the MAS and clinical evidence from the state-of-the-art. 


\section{METHODS}

\section{The NEEM System}

While a detailed description of the mechatronic modules of the NEEM platform, along with its performance, is given in Vitiello et al. (2016), for the sake of clarity we will summarize its main features below. The NEEM is a powered elbow exoskeleton designed to ensure maximum comfort and safety to the patient (Figure 1). It embodies many key design solutions for enhanced ergonomics. Firstly, a compact and light-weight mechanical structure, with double-shelled links and a wide physical humanrobot interaction area, optimizes interaction comfort. Secondly, a four-degree-of-freedom passive mechanism relieves the elbow articulation from loads other than the actuated flexion/extension torque, and at the same time ensures appropriate alignment between human and robot joint axes. Thirdly, a series elastic actuation (SEA) unit (Giovacchini et al., 2014) allows compliant human-robot interaction as well as reliable measurement of the powered torque. The actuation unit is housed remotely in a box on the exoskeleton stand, in order to minimize the weight of the moving part. The actuation unit employs a $400 \mathrm{~W}$ DC brushless motor (EC motor EC60, Maxon Motor ${ }^{\circledR}$, Sachseln, Switzerland), a Harmonic Drive reduction stage (transmission ratio 1:80), a torque limiter acting as safety clutch on the gear output, which disengages the actuation unit from the transmission means in case of torque values higher than a pre-defined threshold (set to $35 \mathrm{Nm}$ in this study), and a grooved pulley. Torque is transmitted from this pulley to the exoskeleton actuated joint through a tendon-sheath system: two antagonist tendons, routed by flexible Bowden hoses, transmit rotation from the actuation unit pulley to the exoskeleton joint. A torsion spring is deployed between the actuated pulley and the exoskeleton. This element provides series elasticity, ensuring compliant interaction and accurate force transduction. Two 32-bit absolute encoders (RESOLUTE $^{\text {TM }}$, RESA30USA052B ring plus RA32BAA052B30F readhead, Renishaw ${ }^{\circledR}$, Wotton-under-Edge, UK) sensorize the spring, one for each extremity. The encoder more proximal to the human joint (i.e., the joint position encoder) provides the elbow joint angular value, namely the measured joint angle, while the difference between the two encoder readings gives torsion spring deformation and, indirectly, the value of the transmitted torque. The wearable and moving part of the system (the powered orthosis) hangs from an adjustable spherical gimbal, which is mounted on an extensible horizontal cantilever bar supported by the main vertical stand. It allows manual adjustments to best fit the orthosis to the patient's body. Finally, the entire system was assembled onto a wheeled platform, in order to allow easy transportation within the clinical setting.

The NEEM control system is based on a two-layered hierarchical architecture. The NEEM low-level control implements two basic working modalities: (a) position control and (b) torque control. Under the position control mode, the robot joint is displaced along pre-defined angular trajectories (i.e., the desired angle) thanks to the action of a closed-loop proportional-integrative (PI) regulator acting on the error between the desired and measured joint angle. The output of the PI regulator is a desired current which is supplied to the DC motor by means of a commercial servo amplifier (EPOS2 70/10, Maxon Motor ${ }^{\circledR}$, Sachseln, Switzerland). Under the torque control mode, a PI closed-loop compensator is used to control torsional spring deformation and so apply a desired torque to the user joint. Under this control mode, when the reference torque is null, the exoskeleton joint becomes "transparent" and the user can freely move.

The high-level control layer has the form of a finite-state machine, which implements a warm-up routine and a set of pre-defined rehabilitation exercises (i.e., passive, active-assisted, active-resisted, similar to those described in Prange et al., 2006). Once the system is powered on, the finite-state machine enters the so-called "Initialization" state: the exoskeleton holds the current angular position, under the position control mode action. At this point, the physical therapist (namely the experimenter) can switch the machine to the so-called "Wear" state: the system smoothly switches to the torque control mode with null torque as a desired reference, i.e., the exoskeleton joint is free to move. This way the therapist can help the patient wear the exoskeleton. Once
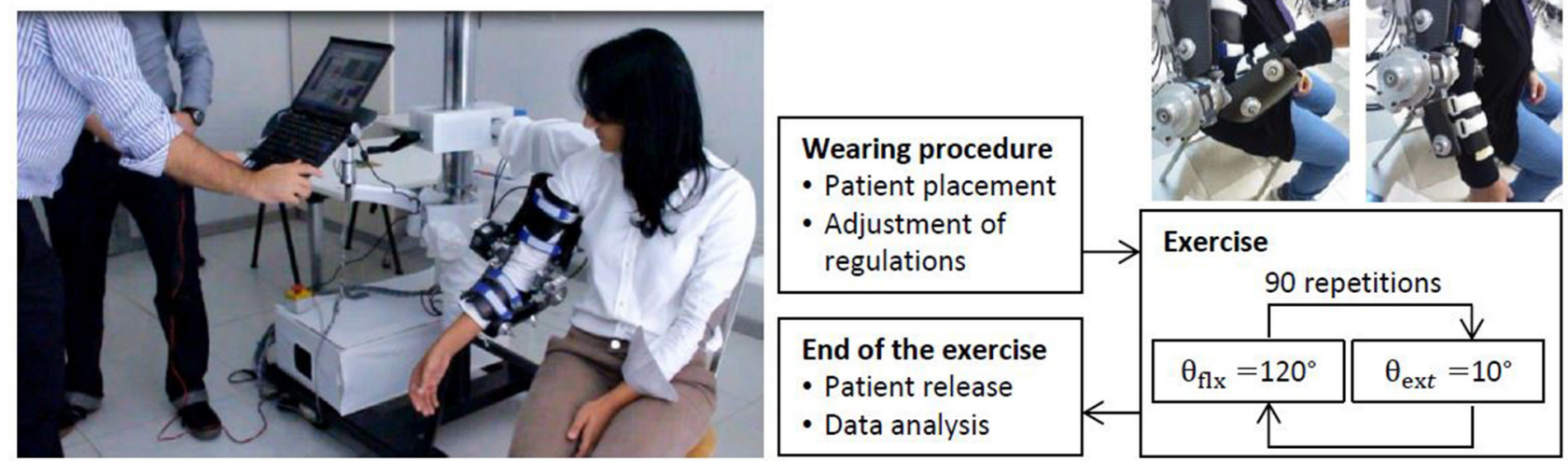

FIGURE 1 | Overview of the NEEM system in the clinical scenario. 
the exoskeleton is fastened to the patient's limb, the therapist can select and start any of the desired rehabilitation exercises (Figure 1).

\section{Rehabilitation Exercise}

The rehabilitation exercise that was selected for this work was the so-called isokinetic passive mobilization with torque threshold. The exoskeleton was programmed to displace - under the position control mode- the elbow joint of the patient along a predetermined flexion/extension trajectory (henceforth named "cycle"). In particular, the exoskeleton moved the elbow joint from an initial flexed configuration (i.e., $\theta_{f l x}=120^{\circ}, \theta$ being the patient's elbow angle measured by the exoskeleto) to a full-extended configuration (i.e., $\theta_{\text {ext }}=10^{\circ}$ ) at a constant velocity. For safety purposes, threshold values for the maximum allowed torque, in flexion, $\tau_{f l x}^{t h}$, and extension, $\tau_{\text {ext }}^{\text {th }}$, applied by the exoskeleton were set for each subject: when the transmitted torque exceeded one of these thresholds, as in the case of any abnormal/spastic muscle activity, the system switched to the torque-control mode with the desired torque set to zero. This way, the robot could smoothly comply with the user's spontaneous movement and prevent spastic muscle strength from increasing, with the consequent worsening of stretch reflexes and hypertonia. Each flexion-extension cycle can be divided into four phases (Figure 2), namely: (i) "elbow extension," the exoskeleton moved at a speed of $20^{\circ} / \mathrm{s}$ from $\theta_{f l x}$ to $\theta_{\text {ext }}$; (ii) "rest," the exoskeleton held the fully extended position for $2 \mathrm{~s}$; (iii) "elbow flexion," the exoskeleton moved at the same speed from $\theta_{\text {ext }}$ to $\theta_{f l x}$; and (iv) "rest," the exoskeleton kept the fully flexed position for $2 \mathrm{~s}$. Exoskeleton speed was kept at $20^{\circ} / \mathrm{s}$ to reduce the probability to evoke stretch reflexes during execution of the movement (Levin et al., 2000). Speed, as well as the values of $\theta_{\text {ext }}$ and $\theta_{f l x}$ were constant across the patients. The threshold values for the applied torque were set as follows. For the flexion (positive) torque, the threshold was equal for all patients and set to $\tau_{f l x}^{\text {th }}=7 \mathrm{~N} \cdot \mathrm{m}$. For the extension (negative) torque, the threshold changed among subjects in the range $\tau_{\text {ext }}^{\text {th }}=-5 \pm 1$ $\mathrm{N} \cdot \mathrm{m}$.

\section{Experimental Protocol}

The experimental protocol was approved by the local ethical committee (Azienda Ospedaliera Universitaria of Pisa, reference number 3919, year 2014) and carried out in accordance with the principles stated by the Declaration of Helsinki. The trial was also registered on ClinicalTrials.gov (Identifier is NCT02934646). All participants signed a written informed consent before starting the experiment. A physical therapist assessed the participants' elbow ROM, Chedoke-McMaster Stroke Assessment Measure (CMSA) (Gowland et al., 1993), and MAS before the beginning of the treatment. After the first assessment was completed, the subjects were seated next to the exoskeleton, on either an armless chair or their own wheelchair, as depicted in Figure 1. When using the wheelchair, the right armrest was removed for the entire duration of the exercise. A physical therapist helped the participant place his/her forearm on the lower link of the exoskeleton, and his/her upper arm on the upper link. The exoskeleton vertical and horizontal position and rotation on the main stand were adjusted to best adapt the patient's arm to the powered orthosis: the upper and lower back shells of the exoskeleton were adjusted so that they adhered completely to the limbs and so that the height of the patient's right shoulder was symmetrical to the left side. After adjusting the exoskeleton, the device was fastened to the user's arm by means of two belts. Subjects participated in 10 rehabilitation sessions, on consecutive working days, for $45 \mathrm{~min}$, i.e., 90 flexion/extension cycles per session. When the subjects showed any discomfort in maintaining the seated position for 45 min, the duration of the treatment was set to $15 \mathrm{~min}$. Subjects were allowed to skip up to two consecutive sessions. Subjects who skipped three consecutive sessions were excluded from the protocol. It is worth noting that after the first session, the subjectspecific settings of the exoskeleton were not changed for the entire duration of treatment. After completing the last session, the subjects were evaluated once more by a physical therapist who quantified the post-treatment elbow ROM values and MAS scores. After 3 months from the stroke, the subjects were asked to participate in a follow-up screening to assess MAS and ROM.

The first assessment of the subject's $R O M$ was used to set the limits on $\theta_{\text {ext }}$ and $\theta_{f l x}$ and the torque threshold $\tau_{\text {ext }}^{\text {th }}$ so that the applied torque allowed to fully extend the patient's elbow without causing pain.

\section{Participants}

Subjects enrolled in the study ranged in the 15th to the 35th day after stroke, at the beginning of the therapy. Plegia on the right upper limbs due to stroke was considered an inclusion criteria, while subjects with orthopedic pathologies at the elbow, e.g., articular blocks, were excluded from the study. Seventeen poststroke subjects completed the protocol (aged $71 \pm 14$ years); 12 of them suffered from ischemic stroke, and the rest from hemorrhagic stroke. On average, subjects started the robotassisted treatment in their subacute phase, i.e., the 25 th day after the stroke ( \pm 5 days). Two additional subjects initially started the protocol but were then excluded since they skipped more than two sessions. Two out of 17 subjects performed treatment $15 \mathrm{~min}$ a day instead of 45 min because they could not maintain a sitting position for prolonged sessions. Out of the 17 participants, only 13 were able to attend the follow-up assessment.

All subjects fit into the maximum $R O M$ of the NEEM, i.e., $\theta_{f l x}=120^{\circ}$ and $\theta_{\text {ext }}=10^{\circ}$ and could perform the movement at the pre-defined constant speed of $20^{\circ}$ s.

\section{NEEM Data Collection and Analysis}

We collected the following variables from the exoskeleton sensing apparatus: the applied torque $\tau$, and the elbow desired $\theta_{\text {des }}$ and measured $\theta$ angle. All variables were sampled and stored at 100 Hz. After completion of each session, data were downloaded from the NEEM control unit and stored for offline data analysis. We extracted the following metrics from the variables of each flexion-extension cycle, as indicators of the resistance to the elbow extension (Figure 2):

- Maximum Extension Torque (MET): the torque exerted by the NEEM at the fully extended position; 

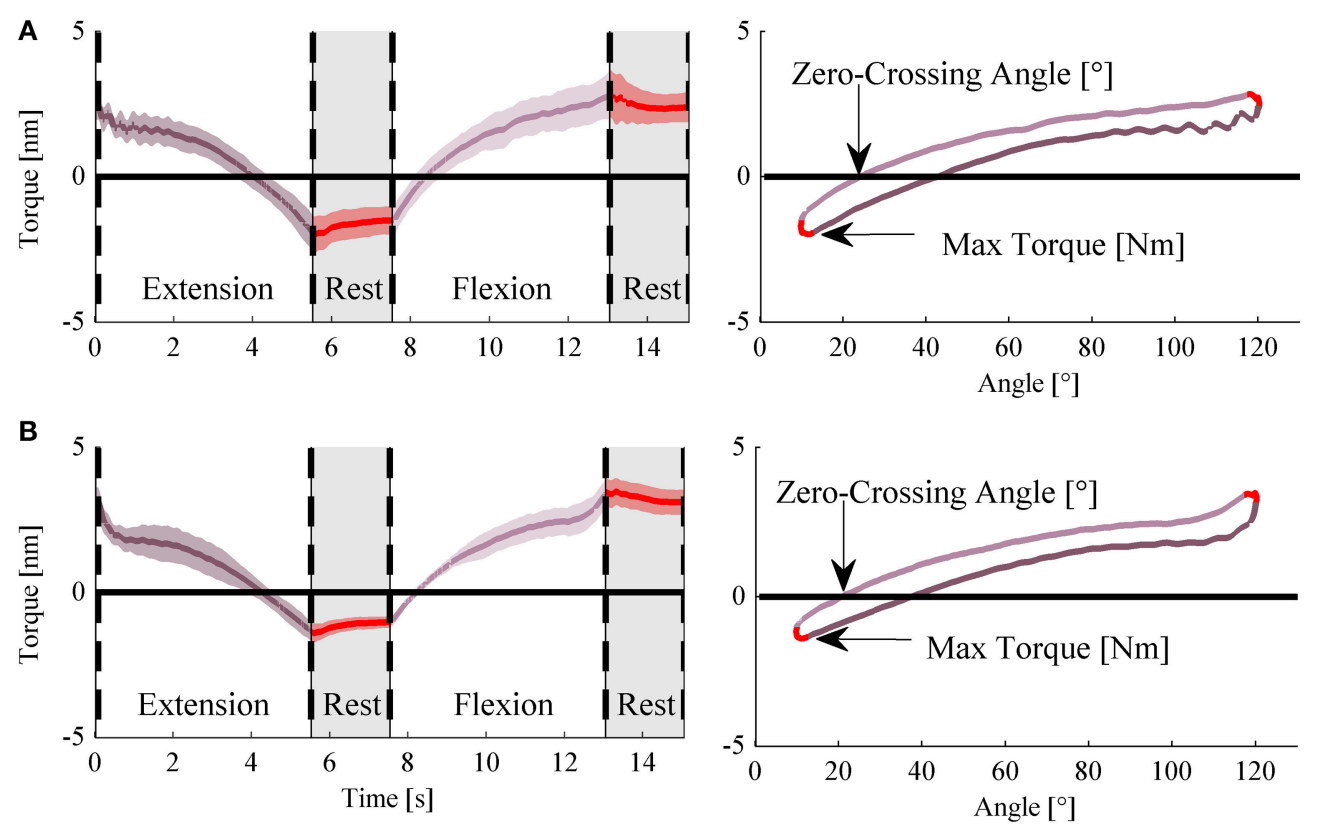

FIGURE 2 | Figure 1 NEEM measurement and indices for a representative subject. (A) Average applied torque during a flexion/extension cycle, during the first treatment session (Day \#1). (B) Average applied torque and measured angle during a flexion/extension exercise, during the last treatment session (Day \#10).

- Zero-Torque Angle (ZTA): the angle at which the applied torque was zero during the elbow extension phase of the cycle.

Notably, the corresponding flexion parameters were not considered in this study as all the participants had spasticity on the elbow flexors, thus the resistance to elbow extension is more informative about the status of the spasticity.

\section{Statistical Analysis}

The overall effect of the robot-mediated treatment was quantified using two approaches.

(1) MAS. Friedman repeated measures analysis of variance on ranks was performed to detect the overall effects of the treatment on the MAS scores, in pre-treatment, posttreatment and follow-up data. Post-hoc pairwise multiple comparison of the MAS scores was performed using the Tukey test (i.e., pre-treatment with post-treatment data, post-treatment with follow-up data and pre-treatment with follow-up data).

(2) NEEM metrics. The metrics extracted from the initial and final rehabilitative sessions were compared as explained hereafter. For each session, MET and ZTA were considered on the last 20 flexion/extension cycles (corresponding to the last $10 \mathrm{~min}$ of the trial). Data from the first and last 2 days of the treatment were grouped and median values extracted for each subject, to respectively compute representative values of the two initial sessions (i.e., $M E T_{\text {Init Session, }}$ $Z T A_{\text {Init Session }}$ ) and two final sessions (i.e., $M E T_{\text {Final Session }}$ and $\left.Z T A_{\text {Final Session }}\right)$. Across-subject statistical analysis was performed separately for MET and ZTA, using a Wilcoxon signed-rank test.
In addition, we analyzed the NEEM metrics to assess the intrasession effect of the treatment, by comparing the initial and final cycles of each session. For each session, median values of the first and last 5 flexion/extension cycles were extracted. The median values of all the indices extracted from the initial and final cycles of each session (from Days 1 to 10 ) were computed. $M E T$ and ZTA data from all participants were thus grouped in four vectors, namely $M E T_{\text {Init Cycle }}, M E T_{\text {Final Cycle }}, Z T A_{\text {Init Cycle }}$,

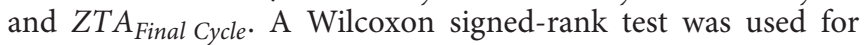
comparison of initial and final cycles values.

Statistical significance was set at $p<0.05$. SigmaStat software (version 3.5) (Systat Software, San Jose, CA, USA) was used for statistical analysis.

\section{RESULTS}

\section{Safety}

All participants easily wore the exoskeleton without reporting any discomfort. Subjects had different weights, arm sizes and upper-arm circumferences, so different inner-shell sizes were used to provide a comfortable wearability of the exoskeleton. Moreover, subjects had different levels of upper-limb motor impairments (CMSA scores ranging from 1 to 6 ). In addition, some of them could walk easily and sit on a normal armless chair while most of them needed to sit on their own wheelchair. All participants could easily hold a correct sitting posture next to the exoskeleton to effectively complete the rehabilitation sessions.

The device was tested for a total of more than $120 \mathrm{~h}$. During the entire duration of the treatment no mechanical or electrical failures or reliability-related issues were reported; the clinical 
validation was completed without any damages to the device (or any of its components) or any undesired effects on the patients.

\section{Efficacy of the Treatment \\ Overall Assessment Based on MAS Scores}

Table 1 reports the MAS scores and the ROM values for all the participants assessed in the pre-treatment, post-treatment and follow-up sessions. The CMSA scores are reported only for the pre-treatment evaluation. Friedman test revealed no changes in the MAS scores in pre-treatment, post-treatment and follow-up session $\left[\chi^{2}(2,17)=0.5, p=0.779\right]^{1}$, thus post-hoc pairwise comparison was not performed.

At the beginning of the treatment all patients showed a MAS score 0 or 1 and a full elbow ROM as they were recruited during the subacute phase when spasticity is not usually arisen.

Fifteen out of 17 participants presented the same value of $M A S$ score and ROM in pre- and post-treatment. A decrease of the MAS score from 1 to 0 was observed in one subject (\#16), and in another (\#6) an increase from 1 to $1+$. Ten out of 13 subjects who participated in the follow-up, showed the same MAS score in the follow-up and the post-treatment sessions; two subjects (\#7 and \#15) showed a decrease from 1 to 0 , whereas an increase from 0 to 1 was observed in one subject (\#16). In the follow-up session, one participant revealed moderate spasticity $(M A S=1+)$.

\section{Overall Assessment Based on NEEM Metrics}

Figure 3 reports the results of the two indices extracted from the NEEM measurements, MET and ZTA. Data are presented both for each subject and as aggregated form. Comparison between the initial and final treatment sessions did not show any statistical difference in MET ( $p=0.09)$ and ZTA $(p=0.28)$, in accordance with the MAS results. Table S1 (in Supplementary Materials) reports the numerical data.

Subject \#6, who presented an increased MAS score between pre- and post-treatment, also showed an increased MET $(+27 \%$, absolute value) and an increased ZTA ( $+28 \%)$; subject \#16, who showed a decreased MAS score in the post-treatment, presented a decreased $M E T$ ( $-27 \%$, absolute value) and a decreased ZTA (-25\%). Figure 4 shows $M E T$ and ZTA values from subject \#6

${ }^{1}$ The software is able to consider missing data in the computation of statistical tests and subject \#16 over all the treatment sessions. The regression line is calculated using the least-square technique and confirms the trend over the whole treatment.

\section{Intra-Session Treatment Assessment Based on NEEM Metrics}

Figure 5 shows the comparison between MET and ZTA values referred to the first and last cycles of the rehabilitative sessions. Data are presented both for each participant and as aggregated form. Comparison between the initial and final values revealed a statistical difference in $\operatorname{MET}(p=0.020)$ and ZTA $(p=0.003)$.

In 10 subjects, when comparing the initial and final cycles of the treatment sessions, both MET and ZTA values substantially decreased (at least $-10 \%$ from their initial values on both parameters). One subject (\#7) showed increased MET and ZTA values (over $+10 \%$ on both parameters), whereas the others showed variations in the range of $\pm 10 \%$ of the initial values (\#3, \#4, \#5, \#8, \#13, \#14).

\section{DISCUSSION}

\section{Overall Usability and Safety}

The use of robotic devices for rehabilitation can provide numerous advantages in terms of intensity and repeatability of the treatment for the patient and decrease of physical effort for the physiotherapists (Turchetti et al., 2014). Moreover, robot-assisted rehabilitation treatments may provide better optimization of healthcare resources (e.g., therapists can supervise more than one patient at a time, without reducing the effort dedicated to each patient). In addition robotic devices are able to provide quantitative evaluation of motor recovery by means of specific parameters that can characterize the rate of improvement of the patients (Colombo et al., 2005). However, for the treatment of upper-limb spasticity, studies using the MITManus (Krebs et al., 2004), Bi-Manu Track (Hesse et al., 2003), and REHAROB (Fazekas et al., 2007) robots proved that passive repetitive exercises could improve spasticity in chronic stroke patients, but few of them correlated the treatments with the rising of spasticity at a later stage in sub-acute patients. Moreover, just recently it has been demonstrated that from a functional perspective, there is significant effect in favor of robot-assisted therapy with respect to traditional therapies (Staubli et al., 2009;

TABLE 1 | MAS scores and ROM values for each subject, assessed by physical therapists before and after the rehabilitation therapy with NEUROExos.

\begin{tabular}{|c|c|c|c|c|c|c|c|c|c|c|c|c|c|c|c|c|c|}
\hline Subject [\#] & 1 & 2 & 3 & 4 & 5 & 6 & 7 & 8 & 9 & 10 & 11 & 12 & 13 & 14 & 15 & 16 & 17 \\
\hline CMSA & 6 & 5 & 5 & 6 & 6 & 2 & 6 & 6 & 6 & 6 & 6 & 6 & 6 & 1 & 2 & 2 & 1 \\
\hline Initial MAS & 0 & 1 & 0 & 0 & 1 & 1 & 1 & 0 & 0 & 0 & 0 & 0 & 0 & 0 & 1 & 1 & 0 \\
\hline Initial ROM & 150 & 150 & 150 & 115 & 150 & 150 & 150 & 150 & 150 & 150 & 150 & 150 & 150 & 150 & 150 & 150 & 150 \\
\hline Final MAS & 0 & 1 & 0 & 0 & 1 & $1+$ & 1 & 0 & 0 & 0 & 0 & 0 & 0 & 0 & 1 & 0 & 0 \\
\hline Final ROM & 150 & 150 & 150 & 115 & 150 & 150 & 150 & 150 & 150 & 150 & 150 & 150 & 150 & 150 & 150 & 150 & 150 \\
\hline Follow-up MAS & 0 & 1 & 0 & 0 & 1 & $1+$ & 0 & 0 & 0 & 0 & 0 & NA & NA & NA & 0 & 1 & NA \\
\hline Follow-up ROM & 150 & 150 & 150 & 115 & 150 & 150 & 150 & 150 & 150 & 150 & 150 & NA & NA & NA & 150 & 150 & NA \\
\hline
\end{tabular}

NA, Not Available. 

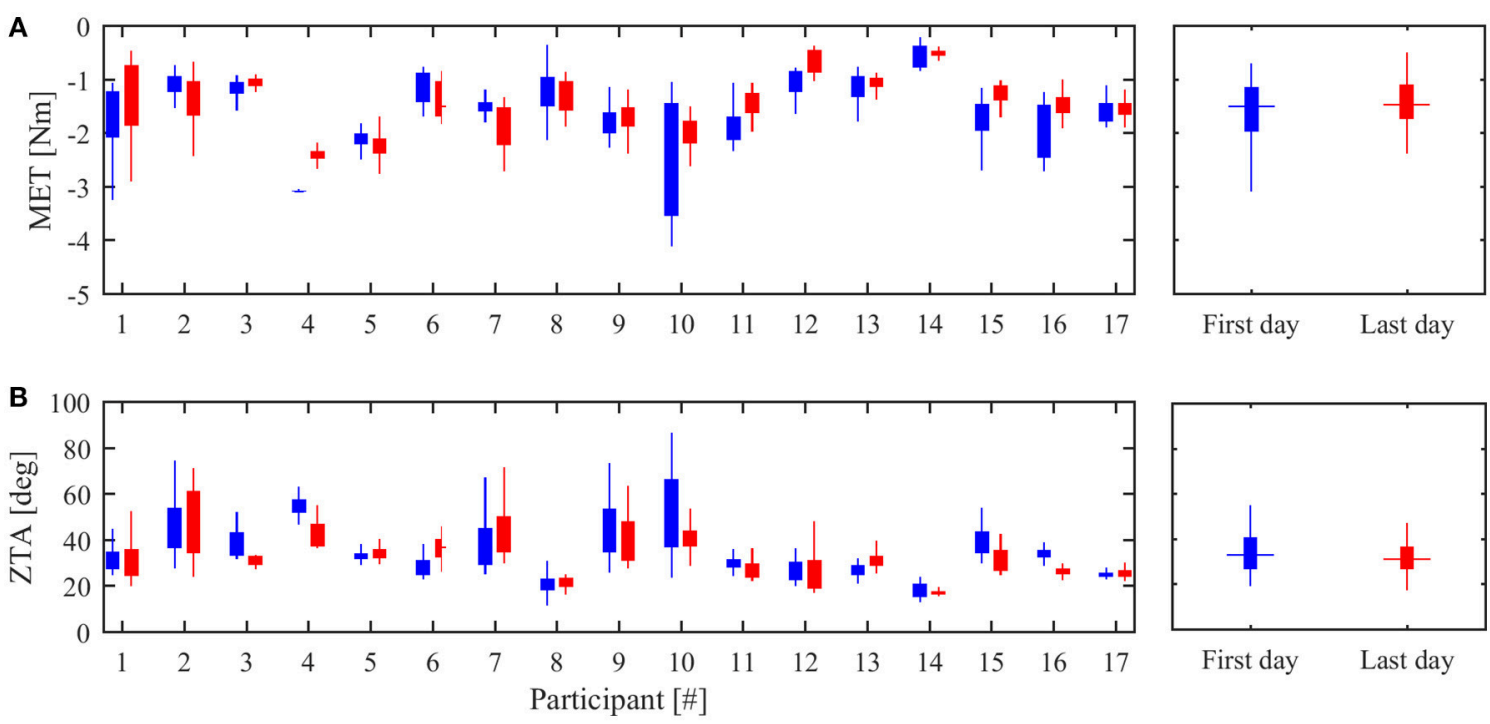

FIGURE 3 | Overall therapy effectiveness. (A) Maximum extension torque (MET) in the first (blue) and last (red) day of treatment. (B) Zero-Torque Angle (ZTA) in the first (blue) and last (red) day of treatment. The left panels show the median values and $90 \%$ confidence interval for each subject. The right panel reports the inter-subject median value and confidence interval.
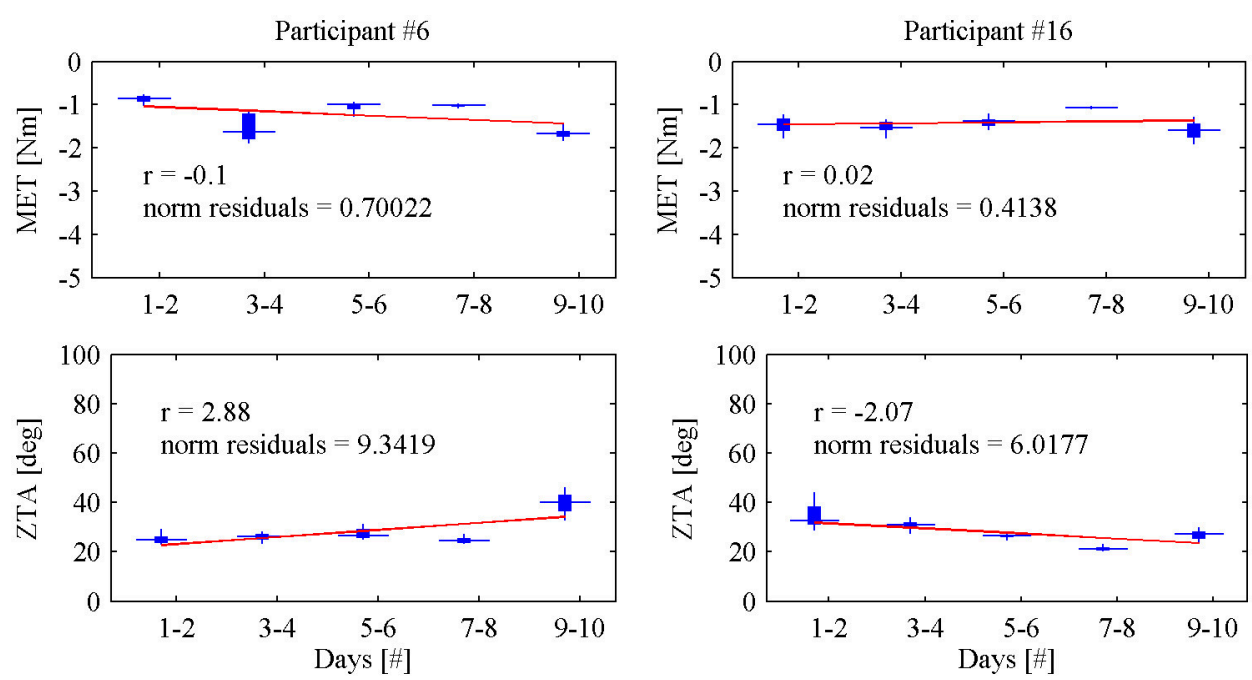

FIGURE 4 | Maximum extension torque (MET) and Zero-Torque Angle (ZAT) for Participant \#6 and Participant \#16 over all the treatment sessions.

Mehrholz et al., 2012), whereas previous review studies could not prove it (Prange et al., 2006; Kwakkel et al., 2007).

Subjects having different levels of motor impairments were able to complete the rehabilitation sessions characterized by the same flexion and extension angle and speed values.

This study shows the safety of the device for treating patients with different body sizes and motor impairments, and for prolonged sessions.

Moreover, it contributes to the investigation of efficacy-related aspects in robotic post-stroke rehabilitation.

\section{Efficacy of the Therapy: Early Mobilization of the Paretic Limb Could Contribute to Reduced Risk of Developing Spasticity}

Although a growing number of studies evaluated the prevalence of spasticity among the post-stroke population, the huge variability in time of onset of spasticity still impedes the determination of a universal prevalence rate (Ward, 2012).

One of the most recent studies on this topic reported that within 2 weeks after stroke, $24.5 \%$ of patients develop increased muscle tone (i.e., $M A S \geq 1$ ), while after 16 weeks spasticity increases only slightly, up to $26.7 \%$. Results highlight that in 

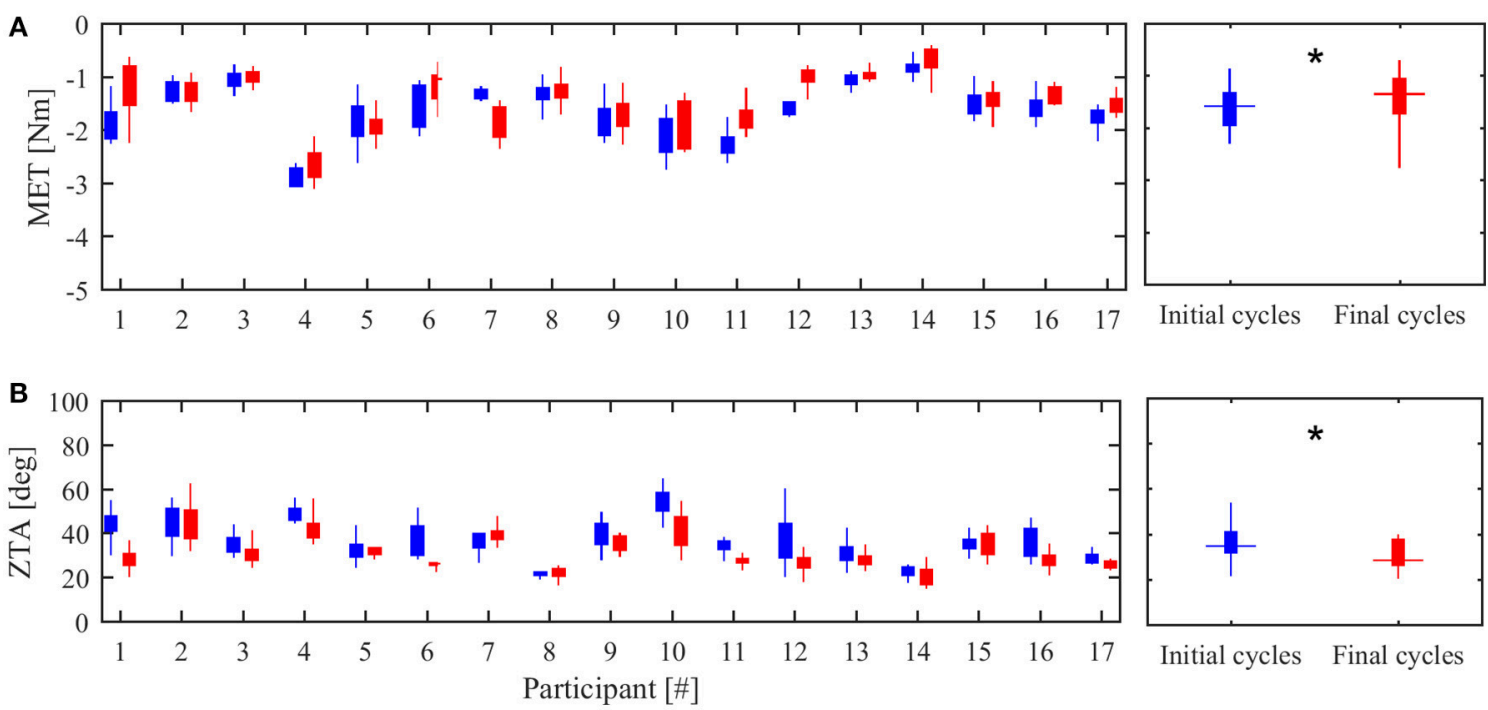

FIGURE 5 | Intra-day therapy effectiveness. (A) Maximum extension torque (MET) in the initial (blue) and final (red) cycles of treatment. (B) Zero-Torque Angle $(Z T A)$ in the initial (blue) and final (red) cycles of treatment. The left panels show the median values and $90 \%$ confidence interval for each subject. The right panel reports the inter-subject median value and confidence interval. *indicates statistical significant difference $(p<0.05)$.

$98 \%$ of the patients who developed spasticity within the first 2 weeks, this is still present after 16 weeks (Wissel et al., 2010). Another study reports a similar finding: patients who develop spasticity after 3 months from stroke (i.e., 19\% of patients), in general continue to report spasticity one and a half years later (Sommerfeld et al., 2004). At the same time, some patients stopped reporting spasticity, even though this outcome is rather rare. In line with the above mentioned studies (Sommerfeld et al., 2004; Wissel et al., 2010), in our population all the patients, but one, reporting spasticity in the pre-evaluation session (i.e., MAS $\geq 1$ ), reported the same after treatment and in the follow-up.

Recently, Kong and colleagues (Kong et al., 2012) reported that 3 months after stroke, $33 \%$ of patients present spasticity, $65 \%$ of which moderate to severe spasticity (i.e., MAS $\geq$ $1+)$. Strikingly, our results show that among the 13 subjects who reported spasticity in the follow-up $(M A S>0)$, only one participant showed moderate spasticity (with $M A S=1+$ ), and none reported severe spasticity (i.e., $M A S>1+$ ). The possible reasons to explain this positive outcome can be two-fold.

On the one hand the spasticity treatment we provided through the NEEM in the early post-stroke phase, prior to the progression of moderate or severe spasticity (Paolucci et al., 2000; Leathley et al., 2004), could effectively prevent the rising of spasticity in the later stage. This hypothesis is grounded on results reported by several studies. In particular, O'Dwyer and colleagues examined 24 hemiparetic patients recruited within 13 months of stroke and demonstrated how preventing or treating contractures in the early rehabilitation phase, is paramount to reduce the occurrence of spasticity (O'Dwyer et al., 1996). The intense rehabilitation treatment provided in this study by means of the NEEM can doubtfully be replicated by physical therapists, due to the huge amount of time and effort, and such intense early mobilization could have contributed to preventing the rising of severe spasticity in the following months.

On the other hand, it might be possible that prevalence rates calculated on our relatively-small group of patients, despite being similar to many similar studies on robot-assisted robotic treatment, can be slightly different from the statistics reported by the studies carried out on larger populations. It is worth noting that in this study, the absence of a control group prevents us from drawing conclusions about the relative efficacy of robot-assisted therapy with respect to other conventional physical therapies or spontaneous improvements: in the future, a randomized control trial will be necessary to evaluate the effects of different rehabilitation approaches to treating emerging spasticity.

\section{NEEM Metrics Could Reliably Assess the Outcome of the Rehabilitation}

NEEM metrics has been validated against two benchmarks, i.e., the MAS scores and evidence from the state-of-the-art.

First of all, NEEM metrics was used to assess the overall treatment effects and outcome compared to the MAS scores. In agreement with results obtained from the MAS scores, both $M E T$ and ZTA values showed no statistical differences between preand post-treatment values. The qualitative comparison between device measurements and traditional clinical scales revealed that the robot measurements could detect changes in patients who reported a change in the MAS score (see subject \#6 and \#16). At the same time, the device revealed to be more sensitive in detecting small changes in joint resistance to passive extension since some participants who did not reported any MAS changes from pre- to post-treatment, showed visible differences in MET and ZTA. 
Second, we validated intra-session results from NEEM metrics with evidence from the state of the art. It has been widely demonstrated that within a rehabilitation session with passive mobilization of the joint, its resistance to extension decreases (Nuyens et al., 2002). Indeed, muscle stretching and joint mobilization is a common intervention for treatment of spastic hypertonia or muscle contractures, which is based on the clinical experience that resistance decreases during repeated passive movements. Thus, we expected this result in almost all the participants and NEEM indices confirmed a statistical reduction of the elbow resistance, highlighting at the same time the reliability of the measurements extracted from the variable collected by the device.

The benchmark with the MAS scores, that is a golden standard for the assessment of joint spasticity, and evidences from the state of the art proved that the robot measurements could reliably describe the overall outcome of a rehabilitation treatment and suggests the possible use of the device not only for providing the treatment, but also as a tool to assess the outcome of a therapeutic treatment in an objective manner.

\section{CONCLUSIONS}

The results of this study proved the usability and safety of the NEEM in rehabilitation; moreover, the results suggested that intense early rehabilitation could contribute to preventing elbow spasticity from occurring at a later stage (3-4 months after stroke); however the small sample size does not allow deriving straightforward conclusions applicable to larger populations.

The metrics extracted from the robot measurements were consistent with the MAS scale suggesting that the device could be potentially used as a tool to assess the outcome of rehabilitation. Notably, other features can be extracted from the NEEM measures (e.g., positive/negative delivered energy or power, active $\mathrm{ROM}$, zero-torque angle and maximum torque in the

\section{REFERENCES}

Ashworth, B. (1964). Preliminary trial of carisoprodal in multiple sclerosis. Practitioner 192, 540-542.

Bohannon, R. W., and Smith, M. B. (1987). Interrater reliability of a modified Ashworth scale of muscle spasticity. Phys. Ther. 67, 206-207. doi: $10.1093 / \mathrm{ptj} / 67.2 .206$

Colombo, R., Pisano, F., Micera, S., Mazzone, A., Delconte, C., Carrozza, M. C., et al. (2005). Robotic techniques for upper limb evaluation and rehabilitation of stroke patients. Neural Syst. Rehabil. Eng. IEEE Trans. 13, 311-324. doi: 10.1109/TNSRE.2005.848352

Fazekas, G., Horvath, M., Troznai, T., and Toth, A. (2007). Robot-mediated upper limb physiotherapy for patients with spastic hemiparesis: a preliminary study. J. Rehabil. Med. 39, 580-582. doi: 10.2340/16501977-0087

Feigin, V. L., Lawes, C. M., Bennett, D. A., and Anderson, C. S. (2003). Stroke epidemiology: a review of population-based studies of incidence, prevalence, and case-fatality in the late 20th century. Lancet Neurol. 2, 43-53. doi: 10.1016/S1474-4422(03)00266-7

Giovacchini, F., Cempini, M., Vitiello, N., and Carrozza, M. C. (2014). Torsional Transmission Element with Elastic Response. PCT patent application no. PCT/IB2014/062735, Application date: June 30. flexion phase) and it can be easily synchronized with commercial electromiographic devices and allow measuring muscle activity.

The positive findings of this work invite further investigations in order to test the effectiveness of the proposed approach in a large-scale clinical study, where different therapy modalities, such as active-assisted or active-resisted exercises, can be proved to be able to prevent the occurrence of spasticity.

\section{AUTHOR CONTRIBUTIONS}

Study concept and design: SC, MC, NV, MCC, FP. Acquisition of data: SC. Statistical analysis and interpretation of data: SC, MC, NV, FP, SM, MCC. Drafting of manuscript: SC, MC, NV, FP, SM, MCC. Study supervision: NV, FP.

\section{FUNDING}

This study was partly supported by: the European Commission under the H2020 AIDE project (GA 645322) and by Regione Toscana under the RONDA project (Bando PAR FAS 20072013).

\section{ACKNOWLEDGMENTS}

The authors would like to thank Francesco Giovacchini, Matteo Moisè, and Mario Cortese for their support in the development of the NEEM, and Stefano Romiti for his help with the platform installation and data acquisition.

\section{SUPPLEMENTARY MATERIAL}

The Supplementary Material for this article can be found online at: http://journal.frontiersin.org/article/10.3389/fnins. 2017.00261/full\#supplementary-material

Table S1 | Absolute value of MET and ZTA values for each subject. Medians and $50 \%$ confidence intervals are reported.
Gowland, C., Stratford, P., Ward, M., Moreland, J., Torresin, W., Van Hullenaar, S., et al. (1993). Measuring physical impairment and disability with the ChedokeMcMaster Stroke Assessment. Stroke 24, 58-63. doi: 10.1161/01.STR.24.1.58

Hesse, S., Schulte-Tigges, G., Konrad, M., Bardeleben, A., and Werner, C. (2003). Robot-assisted arm trainer for the passive and active practice of bilateral forearm and wrist movements in hemiparetic subjects. Arch. Phys. Med. Rehabil. 84, 915-920. doi: 10.1016/S0003-9993(02)04954-7

Kong, K. H., Lee, J., and Chua, K. S. (2012). Occurrence and temporal evolution of upper limb spasticity in stroke patients admitted to a rehabilitation unit. Arch. Phys. Med. Rehabil. 93, 143-148. doi: 10.1016/j.apmr.2011. 06.027

Krebs, H. I., Ferraro, M., Buerger, S. P., Newbery, M. J., Makiyama, A., Sandmann, M., et al. (2004). Rehabilitation robotics: pilot trial of a spatial extension for MIT-Manus. J. Neuroeng. Rehabil. 1:5. doi: 10.1186/1743-0003-1-5

Kwakkel, G., Kollen, B. J., and Krebs, H. I. (2007). Effects of robot-assisted therapy on upper limb recovery after stroke: a systematic review. Neurorehabil. Neural Repair. 22, 111-121. doi: 10.1177/1545968307305457

Langhorne, P., Bernhardt, J., and Kwakkel, G. (2011). Stroke rehabilitation. Lancet 377, 1693-1702. doi: 10.1016/S0140-6736(11)60325-5

Langhorne, P., and Pollock, A. (2002). What are the components of effective stroke unit care? Age Ageing 31, 365-371. doi: 10.1093/ageing/31.5.365 
Leathley, M. J., Gregson, J. M., Moore, A. P., Smith, T. L., Sharma, A. K., and Watkins, C. L. (2004). Predicting spasticity after stroke in those surviving to 12 months. Clin. Rehabil. 18, 438-443. doi: 10.1191/0269215504cr727oa

Levin, M. F., Selles, R. W., Verheul, M. H., and Meijer, O. G. (2000). Deficits in the coordination of agonist and antagonist muscles in stroke patients: implications for normal motor control. Brain Res. 853, 352-369. doi: 10.1016/S0006-8993(99)02298-2

Lum, P., Reinkensmeyer, D., Mahoney, R., Rymer, W. Z., and Burgar, C. (2002). Robotic devices for movement therapy after stroke: current status and challenges to clinical acceptance. Top. Stroke Rehabil. 8, 40-53. doi: 10.1310/9KFM-KF81-P9A4-5WW0

Lum, P. S., Burgar, C. G., Shor, P. C., Majmundar, M., and Van der Loos, M. (2002). Robot-assisted movement training compared with conventional therapy techniques for the rehabilitation of upper-limb motor function after stroke. Arch. Phys. Med. Rehabil. 83, 952-959. doi: 10.1053/apmr.2001.33101

Masiero, S., Celia, A., Rosati, G., and Armani, M. (2007). Robotic-assisted rehabilitation of the upper limb after acute stroke. Arch. Phys. Med. Rehabil. 88, 142-149. doi: 10.1016/j.apmr.2006.10.032

Mazzoleni, S., Posteraro, F., Forte, F., Micera, S., Dario, P., and Carrozza, M. C. (2011). Biomechanical assessment of reaching movements in post-stroke patients during a robot-aided rehabilitation. Appl. Bionics Biomech. 8, 39-54. doi: 10.1155/2011/298926

Mazzoleni, S., Sale, P., Franceschini, M., Bigazzi, S., Carrozza, M. C., Dario, P., et al. (2013a). Effects of proximal and distal robot-assisted upper limb rehabilitation on chronic stroke recovery. NeuroRehabilitation 33, 33-39. doi: 10.3233/NRE-130925

Mazzoleni, S., Sale, P., Tiboni, M., Franceschini, M., Carrozza, M. C., and Posteraro, F. (2013b). Upper limb robot-assisted therapy in chronic and subacute stroke patients: a kinematic analysis. Am. J. Phys. Med. Rehabil. 92(10 Suppl. 1), e26-e37. doi: 10.1097/PHM.0b013e3182ale852

Mehrholz, J., Hädrich, A., Platz, T., Kugler, J., and Pohl, M. (2012). Electromechanical and robot-assisted arm training for improving generic activities of daily living, arm function, and arm muscle strength after stroke. Cochrane Database Syst. Rev. 13:CD006876. doi: 10.1002/14651858.cd006876.pub3

Nuyens, G. E., De Weerdt, W. J., Spaepen, A. J., Kiekens, C., and Feys, H. M. (2002). Reduction of spastic hypertonia during repeated passive knee movements in stroke patients. Arch. Phys. Med. Rehabil. 83, 930-935. doi: 10.1053/apmr.2002.33233

O'Brien, C. F., Seeberger, L. C., and Smith, D. B. (1996). Spasticity after stroke. Drugs Aging 9, 332-340. doi: 10.2165/00002512-199609050-00004

O'Dwyer, N. J., Ada, L., and Neilson, P. D. (1996). Spasticity and muscle contracture following stroke. Brain 119, 1737-1749. doi: 10.1093/brain/ 119.5.1737

Ottenbacher, K. J., and Jannell, S. (1993). The results of clinical trials in stroke rehabilitation research. Arch. Neurol. 50, 37-44. doi: 10.1001/archneur.1993. 00540010033014

Pandyan, A. D., Johnson, G. R., Price, C. I., Curless, R. H., Barnes, M. P., and Rodgers, H. (1999). A review of the properties and limitations of the Ashworth and modified Ashworth scales as measures of spasticity. Clin. Rehabil. 13, 373-383. doi: 10.1191/026921599677595404

Paolucci, S., Antonucci, G., Grasso, M. G., Morelli, D., Troisi, E., Coiro, P., et al. (2000). Early versus delayed inpatient stroke rehabilitation: a matched comparison conducted in italy. Arch. Phys. Med. Rehabil. 81, 695-700. doi: 10.1016/S0003-9993(00)90095-9

Pizzi, A., Carlucci, G., Falsini, C., Verdesca, S., and Grippo, A. (2005). Evaluation of upper-limb spasticity after stroke: a clinical and neurophysiologic study. Arch. Phys. Med. Rehabil. 86, 410-415. doi: 10.1016/j.apmr.2004 10.022

Prange, G. B., Jannink, M. J., Groothuis-Oudshoorn, C. G., Hermens, H. J., and IJzerman, M. J. (2006). Systematic review of the effect of robot-aided therapy on recovery of the hemiparetic arm after stroke. J. Rehab. Res. Dev. 43, 171-184. doi: 10.1682/JRRD.2005.04.0076

Schmit, B. D., and Dewald, J. P., and Rymer, W. Z. (2000). Stretch reflex adaptation in elbow flexors during repeated passive movements in unilateral brain-injured patients. Arch. Phys. Med. Rehabil. 81, 269-278. doi: 10.1016/S0003-9993(00)90070-4

Sommerfeld, D. K., Eek, E. U., Svensson, A. K., Holmqvist, L. W., and von Arbin, M. H. (2004). Spasticity after stroke: its occurrence andassociation with motor impairments and activity limitations. Stroke 35, 134-139. doi: 10.1161/01.STR.0000105386.05173.5E

Staubli, P., Nef, T., Klamroth-Marganska, V., and Riener, R. (2009). Effects of intensive arm training with the rehabilitation robot ARMin II in chronic stroke patients: four single-cases. J. Neuroeng. Rehabil. 6:46. doi: 10.1186/1743-0003-6-46

Turchetti, G., Vitiello, N., Trieste, L., Romiti, S., Geisler, E., and Micera, S. (2014). Why effectiveness of robot-mediated neuro-rehabilitation does not necessarily influence its adoption? IEEE Rev. Biomed. Eng. 7, 143-153. doi: 10.1109/RBME.2014.2300234

van Heugten, C. M., Dekker, J., Deelman, B. G., Van Dijk, A. J., and StehmannSaris, J. C. (1998). Outcome of strategy training in stroke patients with apraxia: a phase ii study. Clin. Rehabil. 12, 294-303. doi: 10.1191/026921598674468328

Vitiello, N., Cempini, M., Crea, S., Giovacchini, F., Cortese, M., Moisè, M., et al. (2016). Functional design of a powered elbow orthosis towards its clinical employment. Mechatronics IEEE Trans. 21, 1880-1891. doi: 10.1109/TMECH.2016.2558646

Vitiello, N., Lenzi, T., Roccella, S., De Rossi, S. M. M., Cattin, E., Giovacchini, F., et al. (2013). Neuroexos: a powered elbow exoskeleton for physical rehabilitation. Robot. IEEE Trans. 29, 220-235. doi: 10.1109/TRO.2012.2211492

Volpe, B. T., Krebs, H. I., Hogan, N., Edelstein, L., Diels, C., and Aisen, M. (2000). A novel approach to stroke rehabilitation robot-aided sensorimotor stimulation. Neurology 54, 1938-1944. doi: 10.1212/WNL.54.10.1938

Ward, A. B. (2012). A literature review of the pathophysiology and onset of post-stroke spasticity. Eur. J. Neurol. 19, 21-27. doi: $10.1111 / \mathrm{j} .1468-1331.2011 .03448 . \mathrm{x}$

Wissel, J., Schelosky, L. D., Scott, J., Christe, W., Faiss, J. H., and Mueller, J. (2010). Early development of spasticity following stroke: a prospective, observational trial. J. Neurol. 257, 1067-1072. doi: 10.1007/s00415-010-5463-1

Conflict of Interest Statement: The authors declare that the research was conducted in the absence of any commercial or financial relationships that could be construed as a potential conflict of interest.

SC, MCC, and NV have interests in the commercial exploitation of the patent application protecting the design of the NEEM torsional spring.

Copyright (C) 2017 Crea, Cempini, Mazzoleni, Carrozza, Posteraro and Vitiello. This is an open-access article distributed under the terms of the Creative Commons Attribution License (CC BY). The use, distribution or reproduction in other forums is permitted, provided the original author(s) or licensor are credited and that the original publication in this journal is cited, in accordance with accepted academic practice. No use, distribution or reproduction is permitted which does not comply with these terms. 


\section{OPEN ACCESS}

Edited by:

Dingguo Zhang,

Shanghai Jiao Tong University, China

Reviewed by:

Jason Scott Metcalfe,

US Army Research Laboratory Human Research and Engineering Directorate,

United States

Victor Manuel Pulgar

Wake Forest School of Medicine,

United States

${ }^{*}$ Correspondence:

Jacopo Buzz

jacopo.buzzi@polimi.it

Specialty section

This article was submitted to

Neural Technology,

a section of the journal

Frontiers in Neuroscience

Received: 10 January 2017 Accepted: 11 September 2017

Published: 26 September 2017

Citation:

Buzzi J, Ferrigno G, Jansma JM and De Momi E (2017) On the Value of Estimating Human Arm Stiffness during Virtual Teleoperation with

Robotic Manipulators.

Front. Neurosci. 11:528

doi: 10.3389/fnins.2017.00528

\section{On the Value of Estimating Human Arm Stiffness during Virtual Teleoperation with Robotic Manipulators}

\author{
Jacopo Buzzi ${ }^{1 *}$, Giancarlo Ferrigno ${ }^{1}$, Joost M. Jansma ${ }^{2}$ and Elena De Momi ${ }^{1}$ \\ ${ }^{1}$ Department of Electronics, Information and Bioengineering, Politecnico of Milan, Milan, Italy, ${ }^{2}$ Mechanical Engineering \\ Department, Delft University of Technology, Delft, Netherlands
}

Teleoperated robotic systems are widely spreading in multiple different fields, from hazardous environments exploration to surgery. In teleoperation, users directly manipulate a master device to achieve task execution at the slave robot side; this interaction is fundamental to guarantee both system stability and task execution performance. In this work, we propose a non-disruptive method to study the arm endpoint stiffness. We evaluate how users exploit the kinetic redundancy of the arm to achieve stability and precision during the execution of different tasks with different master devices. Four users were asked to perform two planar trajectories following virtual tasks using both a serial and a parallel link master device. Users' arm kinematics and muscular activation were acquired and combined with a user-specific musculoskeletal model to estimate the joint stiffness. Using the arm kinematic Jacobian, the arm end-point stiffness was derived. The proposed non-disruptive method is capable of estimating the arm endpoint stiffness during the execution of virtual teleoperated tasks. The obtained results are in accordance with the existing literature in human motor control and show, throughout the tested trajectory, a modulation of the arm endpoint stiffness that is affected by task characteristics and hand speed and acceleration.

Keywords: human-robot interaction, surgical teleoperation, arm end-point impedance, musculoskeletal model, master devices comparison

\section{INTRODUCTION}

Teleoperated robotic systems are widely used in several long-short range application fields, from plant decommissioning (Cragg and Hu, 2003), environment exploration (Mitsou et al., 2006), hazardous material handling and surgery (Johnson and Somu, 2016). In order to control the remotely operated system, the user interacts with a master interface, a robot whose precise and accurate manipulation allows task execution at the slave side. The design of master interfaces is a fundamental aspect in teleoperation, indeed several studies focused on the master devices physical and control characteristics to achieve control transparency while assuring system stability (Fischer et al., 1990; Conti et al., 2014; Enayati et al., 2016). In teleoperation, stability of humanrobot interaction (Colgate, 1994) is guaranteed by closing the control loop with the user sensory and motor systems and, in this context, both the robot and the user are considered as passive elements (Lee and Spong, 2006). Therefore, although the design of master devices architecture 
relies on the human user to achieve stability, the high variability that characterizes human control strategies are usually neglected. In fact, in human motor control, task stability is achieved by regulating the dynamic proprieties of the limbs through muscular activation. The arm impedance and its components (viscosity, inertia and stiffness) can be modified to adapt to different tasks and desired interactions by tuning muscle contractions and varying joint angular position,. As the most predominant component, the arm stiffness also directly depends on the joint angular velocity, reflex modulation and the presence of expected perturbations (Flash and Mussa-Ivaldi, 1990). Previous studies (Burdet et al., 2001) demonstrated the existence of control strategies employed to achieve stability through the regulation of the arm endpoint stiffness in terms of maximal value and orientation. Other studies showed the importance of stiffness regulation during complex tasks execution and motor learning (Osu et al., 2002; Gribble et al., 2003; Buzzi et al., 2017).

Up to now, the dynamic proprieties of the human arm have not been fully considered in the control and optimization design of the master interfaces (Hadavand et al., 2014; Qiu et al., 2014). Through robust and continuous estimation of arm stiffness, it would be possible to implement a master controller able to adapt and regulate the physical interaction between the robot and the human (Tsumugiwa et al., 2002; Ajoudani et al., 2012) in order to achieve better performance, higher resistance to external perturbations, and possibly reducing muscular fatigue (Wiker et al., 1989).

In order to estimate the human arm stiffness, several methods and devices have been proposed in literature: Flash et al. (Flash and Mussa-Ivaldi, 1990) as well as Gomi et al. (Gomi and Kawato, 1997) used planar robotized handles fitted with force sensors to record the interaction forces between the subjects hand and the robot when known displacements were applied. Force and displacement variations were used to compute hand stiffness in multiple directions. While these methods produce a measurement of the stiffness during postural maintenance, they cannot be applied to the study of stiffness during movement and task execution without interfering with arm kinematics. To overcome this limitation, microscopic displacements combined with a time frequency analysis were used to estimate the mechanical proprieties of the arm during a single reaching movement (Piovesan et al., 2013). Following an electromyography (EMG) based approach, recent works (Darainy et al., 2004; Shin et al., 2009; Ajoudani et al., 2015), estimated the arm endpoint stiffness using simplified planar musculoskeletal models and recorded surface muscle activations from couples of shoulder and arm antagonist muscles. Muscular models were used to estimate the force direction and arm of muscular units, while force intensities were obtained through calibration from the recorded EMG signals and the maximal voluntary contractions. Although the simplified musculoskeletal models showed the ability to estimate the arm endpoint stiffness during task execution and without applying perturbations to the user kinematics, stiffness computation was limited to specific planes and directions thus neglecting the effects of other couples of muscles, such as the wrist flexor/ extensors.
In this work, we present a non-disruptive method for the computation of the arm endpoint stiffness based on a user specific 7 degrees of freedom (DoF) musculoskeletal model of the upper limb (Delp et al., 2007). The dynamic characteristics of the model as well as the activation dynamics of muscle units are used in conjunction with joint kinematics and EMG signals to obtain a continuous estimation of the arm stiffness (Pizzolato et al., 2015).

The aim of this work is to evaluate how different master devices and tasks influence the regulation of arm endpoint stiffness and its relation with task performance, hand speed and acceleration. We developed two planar tasks consisting in the position and orientation control of a virtual tool. The two task variations were intended to trigger the use of different biomechanical DoFs to demonstrate the influence of such constraints on the estimation of end-effector stiffness. We compared a parallel link (PL) and a serial link (SL) master device, to test how the differences between the two manipulators would elicit different levels of arm end-point stiffness during virtual teleoperation. During task performance, arm kinematics and EMG signals were acquired using an optical and a magnetic tracking systems).

Our primary hypothesis is that end-effector stiffness would be modulated according to both the mechanical features of the master device and the task characteristics. In particular, we expect the users to generate higher overall end-point stiffness when teleoperating with the serial link master device, characterized by lower structural stiffness and apparent mass. We also expect the users to increase arm stiffness while performing the second task; due to the increased complexity of simultaneously controlling position and orientation of the virtual tool. Our second hypothesis is that end-point stiffness would be modulated accordingly to curvature variations throughout the trajectory. More specifically, we believe that high curvature can be associated with higher complexity in executing the task, leading to higher values of arm end point stiffness. As third and final hypothesis, we expect a correlation between the hand speed and acceleration and arm end-point stiffness so that the users would generate the maximal levels of stiffness during slow and non accelerated movements.

\section{MATERIALS AND METHODS}

In order to analyze the stiffness regulation during virtual teleoperated tasks, we created a simple teleoperation scenario in which the users interacted with master devices to control virtual tools used to perform specific tasks.

\subsection{Tasks Design}

Simple repeatable and cyclical planar virtual tasks were developed to challenge the users with different levels of complexity without requiring any surgical expertise.

\section{Half Cloverleaf (HC)}

Figure 1, 1 shows the first virtual trajectory which represents half the cloverleaf motion as presented by Levit-Binnun et al. (2006). A virtual stylus shaped tool was manipulated in the simulated environment to follow the trajectory, starting from 


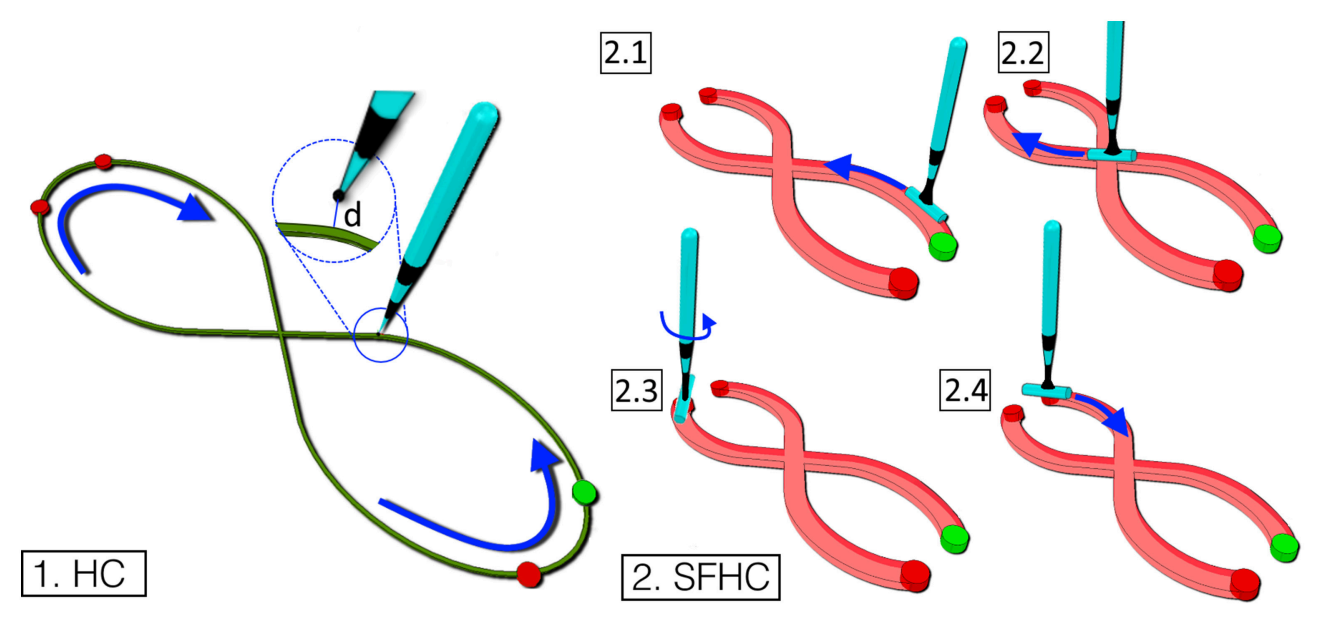

FIGURE 1 | The figure represents the two trajectories designed. Task 1. A line following task shaped as an half cloverleaf (HC). The aim to to finely follow the trajectory starting from the green dot and moving anti-clockwise. Task 2. In the task, shaped as a thicker half cloverleaf (SFHC), the users have to orient the tool's cylindrical end-effector along the trajectory (2.1-2.4).

the initial position (green dot in Figure 1, 1) and moving first counter-clockwise, then straight crossing the intersection in the middle, finally in a clock-wise direction to return to the starting position. In order to make the subject perform the task approximately in the $\mathrm{x}-\mathrm{y}$ plane of a three-dimensional Cartesian reference frame, a visual cue was provided in the form of the path color which turned green when the tool-tip of the stylus was in the said plane $(-1 \mathrm{~mm}<z<1 \mathrm{~mm})$, red otherwise.

2. Shape Fitted Half Cloverleaf (SFHC)

The second task was designed to include wrist rotation as the subjects were asked to navigate a virtual cylindrical shaped tool tip along the path, as shown in Figure 1, 2. The trajectory that the users were requested to follow was identical to the one of the previous task, with the added complexity of following the trajectory silhouette using the tool end-effector. Starting from the green dot (Figure 1,2), the users were asked to slide the tool end-effector over the shape fitted trajectory (see Figure 1, 2.1) . In order to follow the first portion of the trajectory, the users needed to flex the wrist until the central intersection was reached (see Figure 1,2.2) and then to extend it to reach the top left. At the trajectory upper end (red dot in Figure 1, 2.2), the users had to flex the wrist and abduct the shoulder to reposition the tool end-effector on the shape fitted trajectory (see Figure 1, 2.3). Similarly, the users had to reach the lower open end (see Figure 1, 2.4) to complete the task. As in the previous task, the visual feedback consisted in the change of the path color to green when the tool tip lied in the desired plane.

\subsection{Experimental Setup}

\subsubsection{Master Devices}

The subjects were controlling the virtual tools position and orientation using two different master devices: a 7 DoFs parallel links haptic interface (PL) and a 6 DoFs serial links haptic interface (SL).

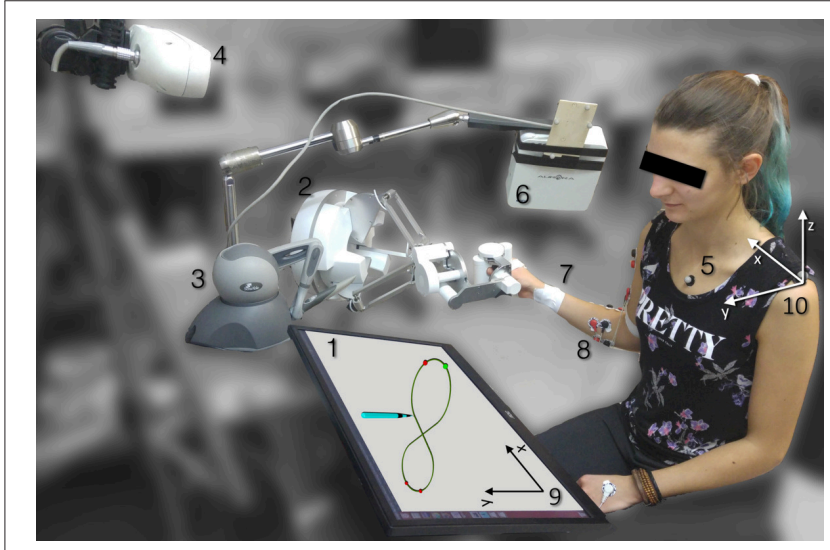

FIGURE 2 | Experimental setup: the user performs the task looking at a monitor laid flat on a table (1) while teleoperating using either a parallel link haptic device PL (2) or a serial link haptic device SL (3) while the thorax and arm kinematic are acquired with an optical camera (4) and reflective markers (5) and with electromagnetic tracker (6) and markers (7). The EMG activation is also acquired with bipolar electrodes (8). The virtual reference frame (9) and the shoulder reference frame (10) are grossly aligned in the setup phase.

A Force Dimension Sigma7 (Force Dimension, Nyon, Switzerland) was used as PL (see Figure 2, 2). The master device, gravity compensated, is characterized by 6 DoFs plus a grip control, has a resolution of $0.0015 \mathrm{~mm}$ and $0.013^{\circ}$ and an elliptical dome workspace with radiuses of approximately $190 \times$ $130 \mathrm{~mm}$. Thanks to its design, the translational and rotational degrees of freedom are completely decoupled, and the grasping unit has an apparent mass of $259 \mathrm{~g}$ (Tobergte and Helmer, 2013). The parallel-link structure also contributes to produce a system stiffness of approximately $14 \mathrm{~N} / \mathrm{mm}$.

A Phantom Omni (3D Systems, South Carolina, USA) was used as SL (see Figure 2, 3). The device is characterized by a $0.055 \mathrm{~mm}$ resolution, a $160 \times 120 \times 70 \mathrm{~mm}$ workspace and its 
controlled through a stylus end-effector. Even though the device is not gravity compensated, which means that the tele-operator had to sustain the stylus and part of the links weight when manipulating it, it is characterized by an apparent mass at the tip of $45 \mathrm{~g}$. Due to its design, the system stiffness components are not constant in the three axes, ranging from $1.02 \mathrm{~N} / \mathrm{mm}$ to 2.31 $\mathrm{N} / \mathrm{mm}$.

Such differences in the master devices dynamic proprieties, both in terms of apparent mass of the end-effector and system stiffness, contribute to create a higher sense of stability while teleoperating with PL.

For both the SL and PL master devices custom impedance controllers were developed using the proprietary API. In both master devices, the users hand movements were downscaled with a factor of 2 . The described tasks were designed to fit within the workspaces of both devices in order to avoid the necessity of using the devices clutching option. If used, the clutching would allow to decouple the virtual tool position from the master device end-effector position, allowing to reconfigure the arm when hitting the workspace limits. This option was excluded from the experiments since it would have caused significant kinematic variability during task execution.

\subsubsection{Acquisition Framework}

The users thorax and arm position and configuration were acquired using two localization devices calibrated to the same reference frame with a hand eye calibration approach (Horaud and Dornaika, 1995). The thorax position was acquired using an optical localization system (see Figure 2, 4) [Vicra, Northern Digital, Ontario, Canada, $20 \mathrm{~Hz}$ sampling rate, $0.25 \mathrm{~mm}$ position Root Mean Squared Error (RMSE)] using three passive retroreflective markers attached to the right and left acromions and next to the jugular notch.

The arm configuration was measured using an electromagnetic localization system (see Figure 2, 6) (Aurora, Northern Digital, Ontario, Canada, $30 \mathrm{~Hz}$ sampling rate, $0.48 \mathrm{~mm}$ and $0.3^{\circ}$ position and orientation RMSE, dome shaped field with a radius of approximately $500 \mathrm{~mm}$ ) and three 6-DoF $1.8 \times 9 \mathrm{~mm}$ electromagnetic sensors (see Figure 2, 7) that were used to generate 6 corresponding virtual markers calibrated on the users recognizable bony landmark on elbow, wrist and hand.

EMG signals were recorded through a TMSi Porti device (Twente Medical Systems International, Oldenzaal, Nederland, 32 channel acquisition system, 2,048 Hz sampling rate) using 10 bi-polar electrodes (see Figure 2, 8). Three couples of electrodes were used to acquire the electromyographic signals from the anterior, lateral and posterior deltoid fiber bands (Figure 3, 12-3). Two couples of electrodes were used to acquire the long and lateral triceps brachii heads (Figure 3, 4-5) and a single couple was used to acquire the biceps muscle (Figure 3, 6). Four electrode couples were used on the forearm to measure the activation of the brachioradialis, flexor carpi ulnaris and radialis and extensor digitorum (Figure 3, 7-8-9-10). The monopolar electrode used as reference was attached to the users' left hand. The muscular maximal voluntary contraction (MVC) was recorded right before the experiments: each subject was asked to perform a set of different isometric contractions against

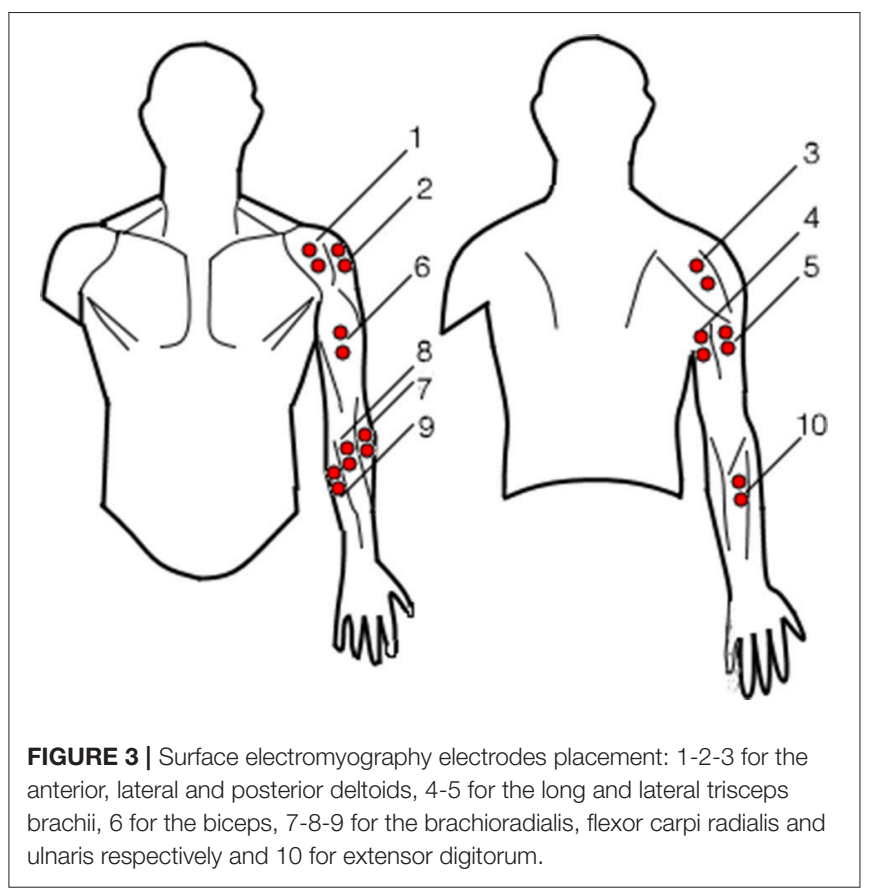

static resistance with different joint configurations (Lehman and McGill, 1999). The different movements were designed to elicit the activation of the muscles responsible for the same kinematic function: for instance, the biceps' MVC was recorded by asking the users to push the hand palm against a tabletop bottom while flexing the elbow; similarly, the forearm flexors' MVC were acquired during isometric wrist flexions against static objects. The contractions lasted for about $5 \mathrm{~s}$ and were followed by a second trial after $30 \mathrm{~s}$, to avoid muscular fatigue. The mean of the two maximal absolute values registered during the two repeated movements was extracted from the conditioned signals for each muscle. The MVC signals were processed with the same procedure as the EMG signals recorded during the experiments.

Retroreflective and electromagnetic markers movements and EMG signals were acquired, recorded and synchronized using custom developed software based on the Robotic Operating System (ROS, http://www.ros.org/).

\subsubsection{Experimental Protocol}

We recruited 4 healthy subjects ( 2 female and 2 male, mean age $23 \pm 1.5)$ who provided informed written consent, in accordance with the recommendations of Politecnico di Milano Ethical committee Board. All subjects gave written informed consent in accordance with the Declaration of Helsinki.

The subjects were sitting in a comfortable chair without arm rests, in front of a $2 \mathrm{D}$ monitor screen where the virtual tasks were displayed (Figure 2,1). The chair position was adjusted in order to allow an easy interfacing between the subjects and the tasks. The monitor was laid as flat as possible on the table to be approximately parallel to the plane in which the tasks were performed, allowing for the most intuitive control, in which the users hand movement directly corresponded to the virtual tool movement. 
TABLE 1 | Experiments list and description.

\begin{tabular}{lllc}
\hline Experiment acronym & Master device & Task & Number of trials \\
\hline PL1 & $\mathrm{PL}$ & HC & 10 \\
PL2 & $\mathrm{PL}$ & $\mathrm{SFHC}$ & 10 \\
SL1 & $\mathrm{SL}$ & $\mathrm{HC}$ & 10 \\
$\mathrm{SL2}$ & $\mathrm{SL}$ & $\mathrm{SFHC}$ & 10 \\
\hline
\end{tabular}

Each user was asked to perform ten trials of every task while trying to maintain the hand movement on a constant plane, exploiting visual color feedback as explained in section 2.1. Subjects were instructed to perform HC and SFHC tasks finding a personal trade-off between precision and execution speed. Table 1 describes the four experiments performed: for each subject the experiment order was randomized so that users performed the 10 repetitions of a randomly selected condition before moving on the next randomly selected one.

\subsection{Stiffness Computation}

The kinematic and dynamic characteristics of the user arm movements, as well as their muscular activations were obtained and elaborated to compute the arm end-point stiffness during task execution.

\subsubsection{Kinematic Analysis}

The arm and thorax spatial configuration were reconstructed using the experimental markers data and the musculoskeletal model implemented in OpenSim (Delp et al., 2007). The model, whose capabilities in predicting the arm dynamics were previously assessed (Saul et al., 2015), is derived from Holzbaur's model (Holzbaur et al., 2005) and composed by seven DoFs activated by 32 muscle compartments. The model was scaled to fit the anthropometric characteristics of each subject and its virtual markers were displaced to the average real markers position acquired during a static pose.

The markers position data were filtered by applying an IIR second order Butterworth filter twice, reversing the time in the second filtering in order to cancel the non linear phase shift. Filter cutoff frequency was set to $4 \mathrm{~Hz}(-6 \mathrm{~dB})$ and the filtered data were used as input for the inverse kinematics. The inverse kinematic algorithm solves the minimization problem in Equation (1) for each set of marker coordinates.

$$
\min _{\mathbf{q}}\left[\sum_{k \in N=\text { number of markers }} w_{k}\left\|\mathbf{x}_{k}^{\exp }-x_{k}(\mathbf{q})\right\|^{2}\right]
$$

Where $\mathbf{q}$ is the vector of joint angles, $\mathbf{x}_{k}^{\exp }$ is the experimental $3 \mathrm{D}$ position of the $k^{\text {th }}$ marker, acquired using the optical or the magnetic tracking device, $\mathbf{x}_{k}(\mathbf{q})$ is the position of the corresponding virtual marker on the model that depends on the vector of joint angles $\mathbf{q}$, and $w_{k}$ is the $k^{\text {th }}$ marker weight thorough which is possible to account for the uncertainty related to each marker position detection. For each set of marker coordinates, the algorithm finds the best joint vector $\mathbf{q}$ that minimizes the error between the experimental marker position vector $\mathbf{x}^{\text {exp }}$ and the virtual markers position vector $x(\mathbf{q})$, which is a function of the fixed model geometry and joint vector, $\mathbf{q}$.
In order to define the correct weighting vector $\mathbf{w}=$ $\left[w_{1}, \ldots, w_{k}, \ldots, w_{N}\right]$ with $N=$ number of markers, we analyzed the markers positions during a static recording. The ratio between the standard deviations from optical markers and electromagnetic markers is comparable with the ratio between the RMSE characteristics of each device. We therefore weighted the thorax optical markers with a coefficient of 1 , while the arm, forearm and hand electromagnetic markers with a coefficient of 0.52 (obtained from the ratio between the nominal RMSE for the optical tracker, $0.25 \mathrm{~mm}$ and the electromagnetic tracking device RMSE, $0.48 \mathrm{~mm}$ ).

\subsubsection{Dynamic Analysis}

The obtained joint angles were filtered with an IIR second order Butterworth filter (cutoff frequency $4 \mathrm{~Hz}$ ) twice, compensating the nonlinear phase shift. The filtered data were used as input for the inverse dynamic reconstruction, along with the model dynamic characteristics (masses and inertial proprieties of each model segment). Equation (2) shows the dynamic equation when no external forces are applied to the arm; $\boldsymbol{\tau}$ is the unknown (7 $\times 1$ ) vector of joint torques, $\mathbf{M}$ is the system mass matrix, $\mathbf{C}$ is the vector of Coriolis and centrifugal forces, $\mathbf{G}$ is the vector of gravitational forces, while $\boldsymbol{q}, \dot{\boldsymbol{q}}, \ddot{\boldsymbol{q}}$ are the vectors of joint position, velocities and accelerations, respectively.

$$
\boldsymbol{\tau}=M(\mathbf{q}) \ddot{\mathbf{q}}-C(\mathbf{q}, \dot{\mathbf{q}})-G(\mathbf{q})
$$

\subsubsection{EMG-Informed Torque Estimation}

A refined estimation of the joint torques was obtained using the Calibrated EMG-informed Neuro-Musculoskeletal (CEINMS) modeling toolbox in which the musculotendon unit (MTU) force arms obtained with the inverse kinematics are used with the joint torques estimated by the inverse dynamics and the recorded EMG signals.

The tool firstly calibrates the experimental muscle excitations derived from the surface EMG acquisition with the muscle activation patters, obtained from the inverse dynamic algorithm (Lloyd and Besier, 2003; Sartori et al., 2012). The resultant calibrated model is then used to predict MTU forces, joint moments, and muscle activations solving a set of differential equations that relates the muscle excitation with the electrical activity recorded (Shao et al., 2009). To be used as an input to the EMG-informed inverse dynamic algorithm, the EMG data was previously high pass filtered at $30 \mathrm{~Hz}$, full wave rectified and filtered with a zero-lag second order Butterworth filter (6 $\mathrm{Hz}$ cutoff frequency) (Lloyd and Besier, 2003). The experimental muscle excitations were normalized using each users maximal voluntary contractions (MVC).

\subsubsection{Stiffness Computation}

For each model DoF, the joint stiffness $(7 \times 7)$ matrix $\left(\mathbf{K}_{\mathbf{j}}\right)$ was computed using Equation (3), where $\boldsymbol{\tau}(i)$ is the joint torque vector that is associated with the $\mathbf{q}(i)$ arm configuration at the $i^{\text {th }}$ time instant (McIntyre et al., 1996).

$$
\mathbf{K}_{j}(i)=\frac{d \boldsymbol{\tau}(i)}{d \mathbf{q}(i)}
$$


In order to obtain the Cartesian representation of the arm stiffness, the arm end-point Cartesian stiffness $\left(\mathbf{K}_{\mathbf{e}}\right)$ has been obtained using the arm kinematic Jacobian $\mathbf{J}(7 \times 6)$, as follows:

$$
\mathbf{K}_{\mathbf{e}}(i)=\mathbf{J}^{T^{-1}}(i) \mathbf{K}_{\mathbf{j}}(i) \mathbf{J}^{-1}(i)
$$

Through the Singular Value Decomposition (SVD), the left singular vectors and non-zero singular values of $\mathbf{K}_{\mathbf{e}}$ were obtained to draw the end-point stiffness ellipsoid orientation and dimensions that are used as a way of visually represent the stiffness (McIntyre et al., 1996). The maximal singular value of $\mathbf{K}_{\mathbf{e}}$ is the maximal stiffness value $\left(K_{\max }(i)\right)$ in the $i^{\text {th }}$ time instant.

\subsection{Metrics and Statistical Analysis}

The following performance-related parameters were evaluated:

- $2 \mathrm{D}$ distance from the trajectory at the $i t h$ time frame, $d_{i}$ : the distance is computed from the tool tip to the closest point on the trajectory center on the task plane (see Figure 1); it is calculated as shown in Equation (5).

$$
d(i)=\sqrt[2]{\left(x_{\text {target }}(i)-x_{\text {tool }}(i)\right)^{2}+\left(y_{\text {target }}(i)-y_{\text {tool }}(i)\right)^{2}}
$$

Where $(x, y)_{\text {target }}$ are the tool tip's $2 \mathrm{D}$ coordinates and $(x, y)_{\text {tool }}$ are the $2 \mathrm{D}$ coordinates of the closest point on the trajectory. The $\mathrm{z}$-component of the displacement from the trajectory was excluded from the distance metric computation due to the significant differences that the color visual feedback could introduce with respect to the other two dimensions.

- Maximal stiffness $\boldsymbol{K}_{\text {max }}$ : the end-point stiffness ellipsoid main axis was computed in 29 (for HC) and 23 (for SFHC) equally spaced points along the task trajectory (for SFHC, the points corresponding to the open ends were excluded from the analysis). For each task trial and for each point selected, the three closest virtual tool positions were searched and the corresponding maximal stiffness $\left(\boldsymbol{K}_{\max }(j), \boldsymbol{K}_{\max }(j+1)\right.$ and $\left.\boldsymbol{K}_{\max }(j-1)\right)$ were averaged to obtain the mean maximal stiffness estimation as in Equation (6)

$$
\overline{\boldsymbol{K}}_{\max }=\frac{1}{3}\left(\boldsymbol{K}_{\max }(j-1)+\boldsymbol{K}_{\max }(j)+\boldsymbol{K}_{\max }(j+1)\right)
$$

With $j=1 . . . M-1$ number of points along the trajectory.

- Curvature $C$ : In both tasks, the trajectory was created to fit the same Bernulli's Lemniscate function. The generic function of a Bernulli Lemniscate curve in a planar $x-y$ plane with the main axis oriented along the $\mathrm{x}$ axis is

$$
\left(x^{2}+y^{2}\right)^{2}=2 a^{2}\left(x^{2}-y^{2}\right)
$$

Where $a$ is the parameter that defines the position of the curve foci. The task trajectory curvature $(C)$ can be computed in polar coordinates using:

$$
C(t)=\frac{3 * \sqrt{2} * \cos (t)}{\sqrt{3-\cos (2 t)}}
$$

with $t$ that spans from 0 to $2 \pi$. For each $3 \mathrm{D}$ point of the trajectory selected for the stiffness analysis, the corresponding curvature was computed.
- Hand speed and acceleration: the position and orientation of the hand in the shoulder reference frame (see Figure 2, 10) was computed. The speed was obtained performing numerical differentiation on the $x-y-z$ coordinates, smoothing the signal with a second order Butterworth filter with cutoff frequency of $4 \mathrm{~Hz}$ twice forward and backward. Acceleration was computed and filtered from hand speed following the same procedure previously described.

For all the metrics, the statistical distribution normality was tested for each user in the four experiments PL1, PL2, SL1, SL2 (see Table 1) using a one-sample Kolmogorov-Smirnov test with $1 \%$ significance level. The arm stiffness data resulted to be non-normal, therefore, using the natural logarithm function (as in Wilson and Worcester, 1945), the data was normalized: the same Kolmogorov-Smirnov test was performed for each user, repetition and experiment, identifying as normal the $97 \%$ of the distributions. The distance metric, as well as the hand speed and acceleration had a normal distribution (Kolmogorov-Smirnov $\alpha=0.01)$.

\subsubsection{Analysis Performed and Hypothesis Tested}

All the inferential statistic analysis that will be presented were conducted with the Statistics and Machine Learning Toolbox for Matlab 2016b (Mathworks, Natick, Massachusetts, US).

1. Maximal stiffness through the trajectory - The stiffness maximal values $K_{\max }$ were first of all studied as a function of the task type and controller. The distributions of the normalized stiffness data were compared between users and repetition, since no significant differences were found, the users data were grouped together for each experiment

Hypothesis 1 \& 2: To analyze whether the task and master device types, as well as level of trajectory curvature affected the maximal values of stiffness, the data from the 29 (HC) and 23 (SFHC) points on the trajectory for each experiment were grouped. The mean values of maximal stiffness for each region of curvature and repetition were extracted and a threeway ANOVA was performed on the log-normalized data (task, master device type and curvature region as fixed factors, user number as random-effect factor). To further test the effects of the different factors on the end-point stiffness, the same ANOVA model was also applied to the stiffness variability in the various regions and repetitions. To test the presence of possible correlations between stiffness and curvature the Pearson rank test ( $\alpha=0.05)$ was adopted.

2. Stiffness against hand speed and acceleration - Using two separate two-ways ANOVAs (task and master device type as fixed factors, user number as random-effect factor) the hand speed and acceleration distributions in the four experiments were analyzed. Hypothesis 3: we tested the hypothesis that a relation between hand speed and acceleration with respect to maximal stiffness could exist. For each of the ten trajectory repetitions, 4 levels of hand speed and acceleration were obtained in the four experiments and for the 4 users. Two separated three-way ANOVAs were performed on the corresponding values of stiffness (task, master device type and 
level of speed/acceleration as fixed factors and user number as random effect factor).

3. Distance metric - The difference between different trials of the same experiment for each user was tested using a one-way ANOVA test $(\alpha=0.05)$. Since no statistical difference emerged, the mean values of distance throughout each repetition and user were extracted. The four experiments' performance distributions were then modeled with a twoway ANOVA statistical model, where task and master device type were considered as fixed factors, while user number was considered as random effect factor.

\section{RESULTS}

\subsection{Maximal Stiffness through the Trajectory}

Figure 4 shows the results obtained when the mean stiffness values through the trajectory among users and trials for each experiment are grouped together. The stiffness values below a threshold of $0.1 \mathrm{~N} / \mathrm{m}$ were removed from the analysis: the threshold was obtained from previous arm end-point stiffness estimations (Gomi and Kawato, 1997; Tsumugiwa et al., 2002) and the percentage of values discarded was less than $1 \%$ of the total dataset. The results of the three-way ANOVA analysis showed no significant interaction between the factors, and a statistical difference between tasks (HC and SFHC) $\left[F_{(1,624)}=\right.$

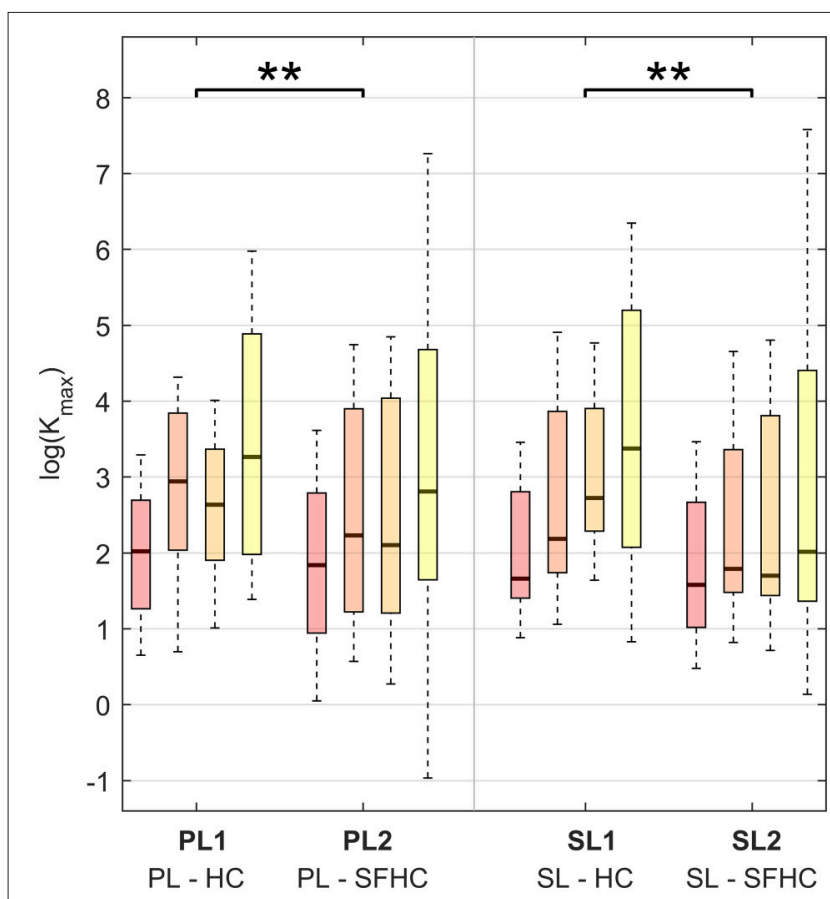

FIGURE 4 | Maximal stiffness $\left(K_{\max }\right.$ ) distribution in the four experiments for the four increasing levels of curvature. The results are presented using boxplot indicating median, first and third quartile, minimal and maximal values. Horizontal lines over the boxes indicate statistical difference while the number of stars indicate different levels of significance $\left({ }^{\star \star} p<0.01\right)$.
10.06, $p<0.005$ ] while no significant difference was found between the master devices $\left[F_{(1,624)}=0.14, p=0.70\right]$. Overall, users elicited significantly higher arm stiffness when performing the HC task with respect to SFHC. Figure 5 shows the endpoint stiffness variability in the four experiments. The three-way ANOVA test showed no significant interactions among factors and statistical difference between both tasks $\left[F_{(1,624)}=7.75, p<\right.$ $0.01]$ and master devices $\left[F_{(1,624)}=7.64, p<0.01\right]$. Interestingly while the stiffness variance increases in the SFHC task when teleoperating with PL, when teleoperating with the SL master device, instead, users decreased the arm stiffness variance when performing SFHC with respect to HC. Overall, users explored the highest range of end-point stiffness in $\mathrm{HC}$ when teleoperating with SL and showed the lowest variance with the same master device in the SFHC task.

To further analyze stiffness modulation through the 29 (for $\mathrm{HC}$ ) and 23 (for SFHC) points along the trajectory while teleoperating with the PL and SL master devices, Figure 6 shows the median values obtained for each experiment. The results are presented as a tri-dimensional graph where each point mean $K_{\max }$ value is represented as a colored column (see Figure 6). No evident trends can be found in stiffness maximal values modulation through the trajectory points.

Regarding the end-point stiffness mean values relation with curvature, the three-way ANOVA showed significant difference $\left[F_{(3,624)}=28.19, p<0.0001\right]$ between the four different levels of curvature. The box-plots in Figure 7, 3 show the maximal

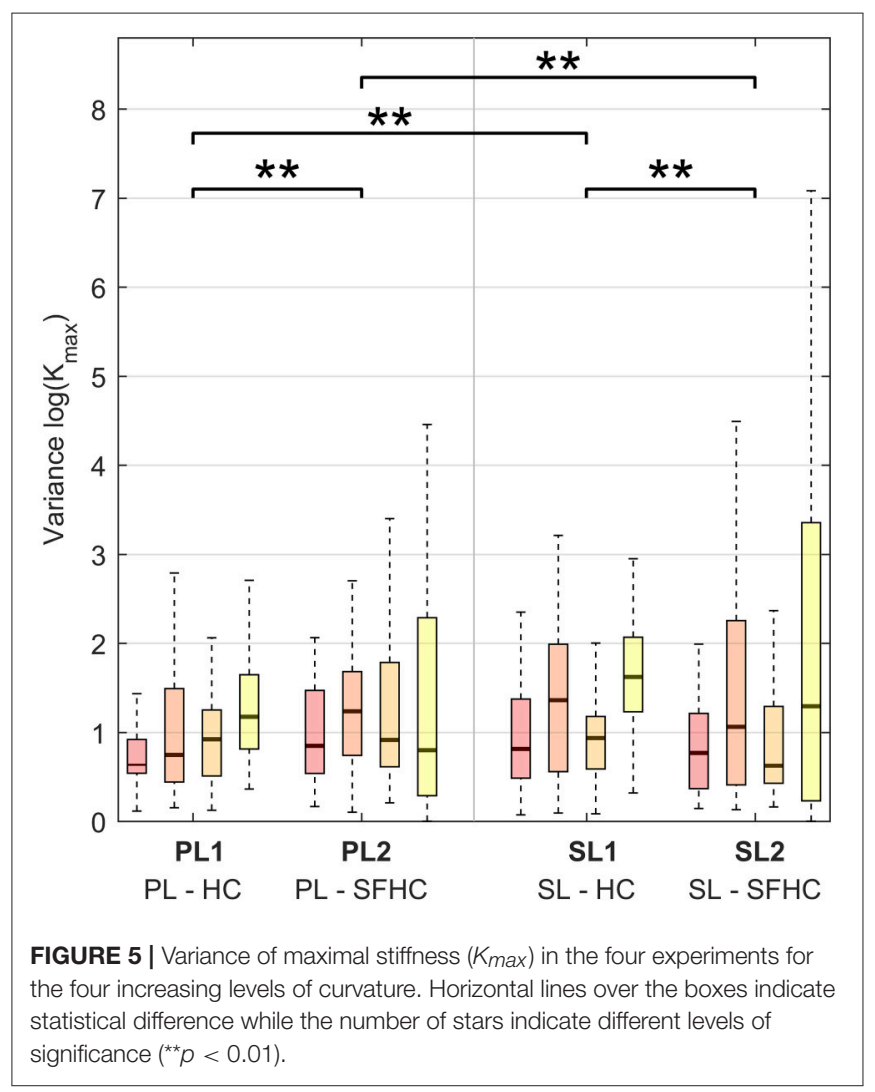




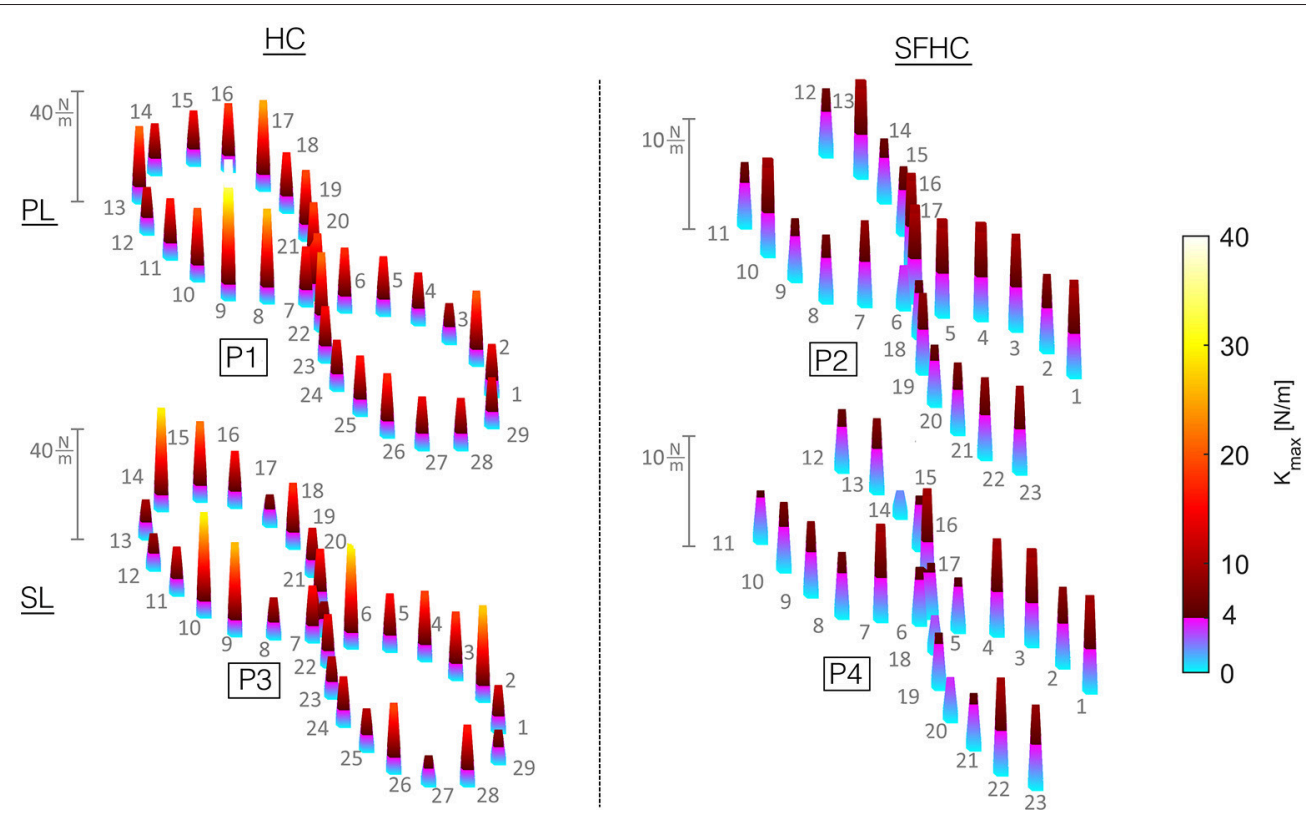

FIGURE 6 | Maximal stiffness $K_{\max }$ from all the users and trials in each point along the trajectory is presented: the height and color of each column indicates the corresponding point median. To account for the difference in terms of median values from experiments PL1 and SL1 compared with PL2 and SL2, a custom color-map was designed. $K_{\max }$ values from 0 to $4 \mathrm{~N} / \mathrm{m}$ are plotted with colors from light blue to purple, while $K_{\max }$ values from 4 to $40 \mathrm{~N} / \mathrm{m}$ range from a dark red to bright yellow.

stiffness distribution as function of four levels of trajectory curvature for PL and for SL. Although characterized by high interquartile distances, the similar behavior can be observed for PL and SL and in the two tasks: the smallest $K_{\max }$ mean values are registered where the normalized curvature is $<0.25$ while the maximal values appear in the range between 0.75 and 1. Interestingly, while for the hybrid parallel-serial link master device (PL) the end-point stiffness behavior in $\mathrm{HC}$ and $\mathrm{SFCH}$ shows the same trend, with an apparent plateau in the middle curvature zones, different behaviors can be seen with SL. In this case, HC shows a clear increase in the mean value of maximal stiffness with increasing levels of curvature, while in SFHC users were eliciting very small variations of arm end-point stiffness. Due to the high variance in the data, Pearson correlation analysis showed non-significant $(p>0.05)$ correlations for all the experiments. Furthermore, regarding the stiffness variability in the four regions of curvature, the three-way ANOVA test showed no significant differences $\left[F_{(3,624)}=1.54\right.$, $p=0.20]$.

\subsection{Stiffness against Hand Speed and Acceleration}

Figure 8 shows the speed and acceleration distribution in the four experiments; the two separated two-ways ANOVAs showed significant differences in tasks and master devices in both speed $\left[F_{(1,156)}=998 p<0.0001\right.$ and $F_{(1,156)}=36 p<0.0001$, respectively] and acceleration $\left[F_{(1,156)}=1,300 p<0.0001\right.$, $F_{(1,624)}=180 p<0.0001$, respectively]. The two separate threeway ANOVAs showed no significant interaction among task, master device type and level of speed or acceleration; a main significant effect was found for the task type $\left[F_{(1,624)}=10.23\right.$, $p<0.005$ when the data was grouped in levels of speed and $F_{(1,624)}=10.23, p<0.005$ for levels of acceleration] while no significant effects were found for the master device type and the levels of speed or acceleration. Figure 9 shows the endpoint stiffness distribution with respect to increasing levels of speed and acceleration for the four experiments. To emphasize the differences in the stiffness distribution, the non-normalized stiffness values are presented. The Pearson correlation tests between the log-normalized data and the hand speed and acceleration in the four experiments showed low ( $|p|$ ranging from 0.1 to 0.3$)$ non significant $(\rho>0.1)$ negative correlations.

\subsection{Distance Metric}

The mean distance value for each repetition was obtained for each of the 10 repetitions and users and the obtained data sets were grouped in the four experiments. The results of the twoway ANOVA with subjects' number as random-effect parameter showed a difference between HC and SFHC $\left[F_{(1,156)}=548\right.$ $p<0.0001]$ and no difference between PL and SL $\left[F_{(1,156)}=\right.$ $2.43 p=0.1214]$. The users were capable of achieving the best performances, testified by lower distances, with the parallel link master device (PL) and in the first task (HC). Similarly as seen with the maximal stiffness values (see Figure 4), the interquartile distances for SFHC are significantly higher than in HC.

\section{DISCUSSION}

We evaluated the arm end-point stiffness modulation adopted by novice teleoperators in performing two tasks with two 

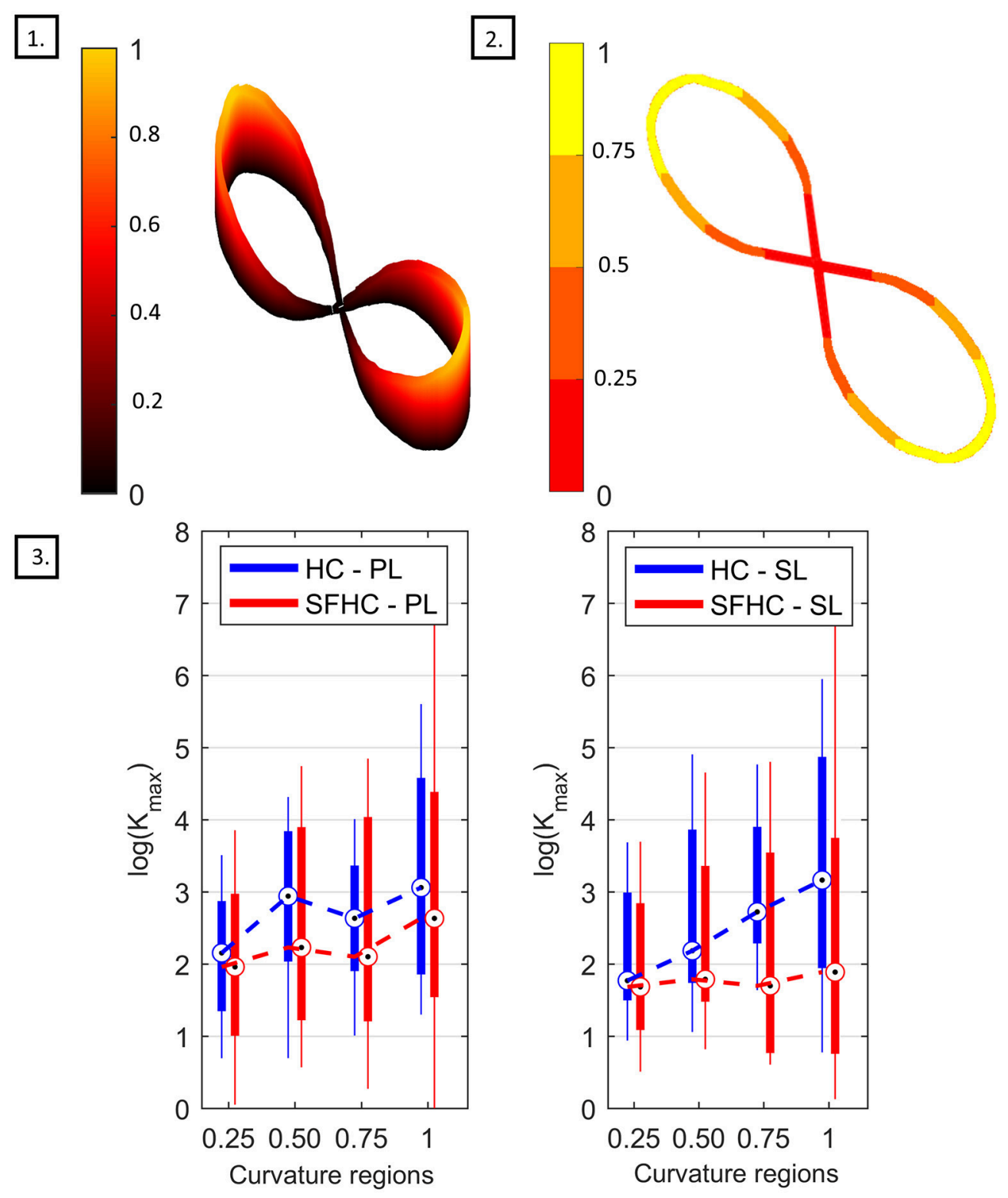

FIGURE 7 | Stiffness with respect to curvature analysis. (1) Normalized curvature through the trajectory. (2) Definition of the four regions of normalized curvature. (3) Box-plot comparing the maximal stiffness value $K_{\max }$ for the PL master device, on the left, and for the SL master device on the right. The boxes represent the first and third quartile, while the whiskers represent the minimal and maximal values.

different master devices as a reflection of the control strategies adopted by the central nervous system to increase the hand resistance to internal and external noise. Using kinematic and muscular parameters, we estimated the stiffness and its relation with the trajectory characteristics and the hand speed and acceleration.

The difference in maximal stiffness values between the different task types (as presented in Figure 4) proves that the users were adapting their stiffness modulation strategies to the different task characteristics. We were expecting higher values of end-point stiffness during the execution of the SFHC task, due to the increased complexity added by the requests to orient the tool; instead, the stiffness is significantly higher in the HC tasks. A possible explanation to this result may be that the users were discouraged to increase the arm stiffness in order to comfortably activate the wrist joint. In fact, to increase the overall arm stiffness, it would be necessary to increase the level of co-contraction also for the wrist flexor-extensors, potentially impairing the free rotation of the wrist. Therefore, this significant difference in stiffness values between $\mathrm{HC}$ and SFHC may be explained by the different levels of wrist flexionextension activations that were registered in the two tasks. As an example, Figure 10 shows the wrist flexion-extension patterns found during the execution of $\mathrm{HC}$ and SFHC for a single user 


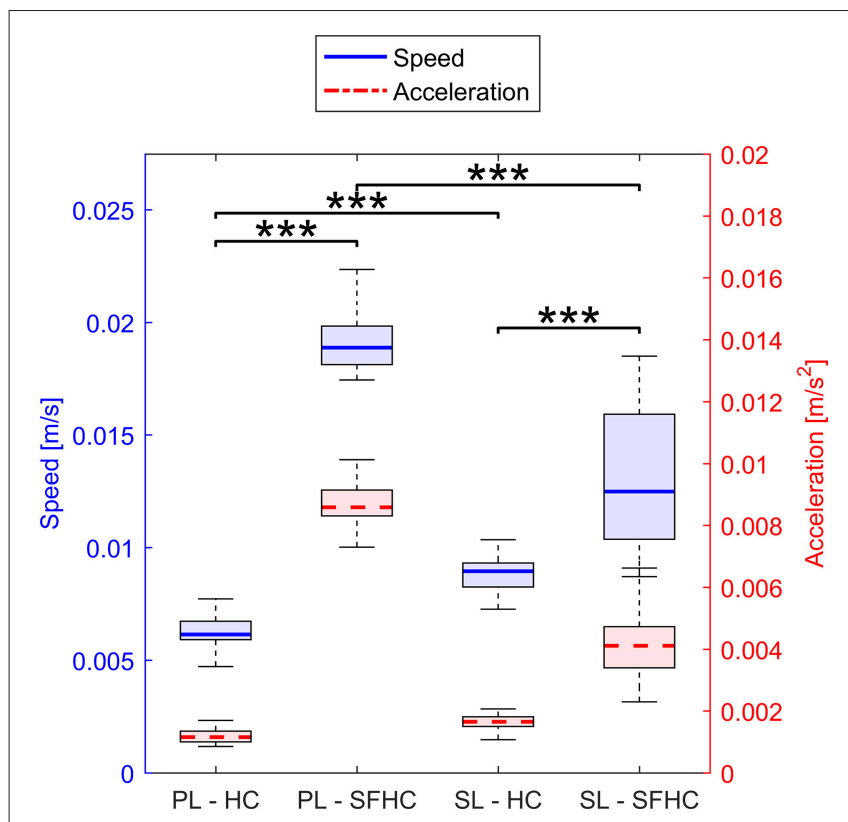

FIGURE 8 | Distribution of speed (in blue, solid line) and acceleration (in red, dashed line) in the four experiments. Horizontal lines over the boxes indicate statistical difference while the number of stars indicate different levels of significance $\left({ }^{\star \star \star} p<0.0001\right)$.

with the PL master device. The same significant difference can be seen in all the acquired users.

The fact that users were eliciting similar values of stiffness when teleoperating with the two master devices seems to suggest that users can compensate for the significant differences in the master devices mechanical proprieties assuring the same overall dynamic performances.

Users showed the highest end-point stiffness variability when teleoperating with the serial link robot during the execution of the HC task, possibly hinting that for that specific task, the SL master device's characteristics offered a less stable and reliable interaction with respect to PL. Interestingly, while for the PL master device the request to orient the tool along the trajectory introduced higher variability in the elicited stiffness, the opposite can be seen for SL. This discrepancy could be caused by the significant differences in the master device structural construction: in PL the hand rotations are completely decoupled from translations while in SL rotations are less hand-centered. This finding would therefore endorse the hypothesis that different kind of tasks may require different master device mechanical characteristics.

The statistical difference in arm stiffness mean values between different regions of curvature could suggest a relation between trajectory curvature and stiffness maximal values: in fact, the users were generally generating the lowest stiffness in the lowcurvature portion of the trajectory. Under the hypothesis that straight trajectories are easier to follow than highly curved ones, it is possible that the users were relying on higher stiffness to increase their performance in the most difficult parts of the trajectory. On the other hand, this modulation seems particularly affected by the task and master device characteristics. By looking

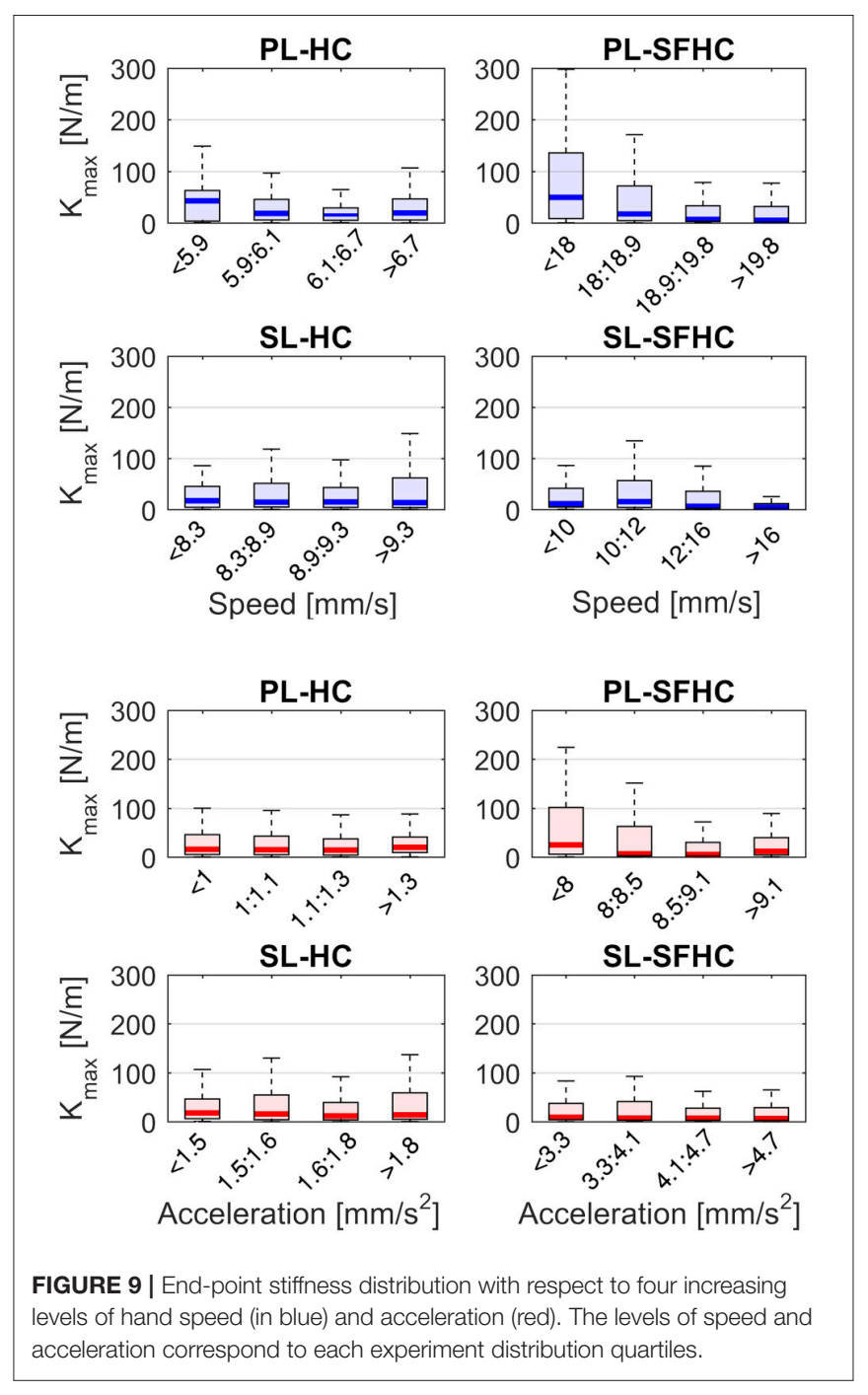

at the stiffness distributions in Figure 7, it seems that while the PL master device offers a rather consistent interface for both tasks, allowing more or less the same modulation in $\mathrm{HC}$ and SFHC, the SL master device showed different behaviors in the two tasks. Specifically, while an increase in the mean stiffness at high curvature can be seen for the HC task, arm end-point stiffness was almost constant for SFHC. This discrepancy may suggest that, during the SFHC task performance, when teleoperating with the SL master device, users weren't able or didn't felt the necessity to adopt the same kinetic strategy that they used otherwise.

The disparity in the speed and acceleration distributions between the two tasks could lie in the differences in the task graphical representation: in order to make the SFHC task more clear, the trajectory thickness is increased with respect to the $\mathrm{HC}$. In the same way, the tool-tip changes from a small point (in $\mathrm{HC}$ ) to a cylinder (in SFHC). These differences could have decreased the users perception of the distance from the target trajectory thus reducing the feedback on their actual performance. Consequently the users performed the task with higher speed. This explanation seems to be endorsed by the statistically higher mean distances 


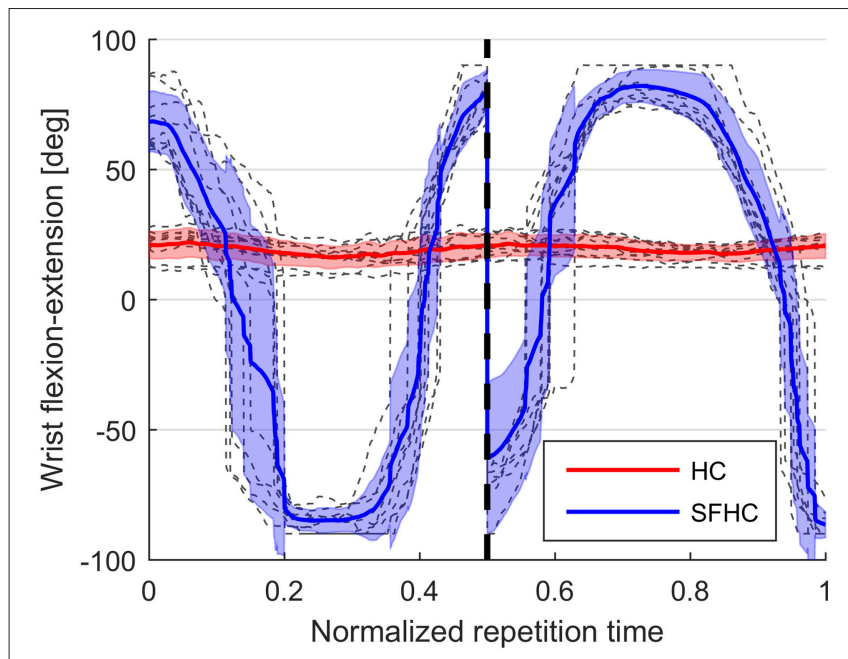

FIGURE 10 | Wrist flexion-extension patterns during the execution of $\mathrm{HC}$ and SFHC for a single user: in gray the single normalized task repetitions, red and blue lines represent the mean joint angle signal for $\mathrm{HC}$ and SFHC, while red and blue areas represent the standard deviation interval.

recorded in SFHC with both master devices. The differences in mean speed and acceleration between the master devices are affected by the task request: during HC users where slower using PL while significantly faster using SL. On the contrary, the maximal speed for SFHC was obtained with PL, showing how different tasks may require different types of master devices in order to be efficiently executed. No statistical difference was found between the arm stiffness at different levels of speed and acceleration, but the higher end-point stiffness values and variability that were seen for some experiments, suggest that, in those experiments, high stiffness is more likely to occur at lower speed and accelerations. On the other hand, it appears that, even though joint speed and acceleration are fundamental components in the joint stiffness computation, their relation with arm end-point stiffness can't be modeled with simple correlations.

A limitation of this work can be found, first of all, in the small number of subjects; the number of trials for each task may not have been enough to account for the high variability that characterizes human motor control. Another limitation lies in the described differences between the two tasks; the SFHC task, in fact, differs from HC not only for the necessity or re-orienting the tool end-effector, but also in terms of trajectory thickness and tool-tip dimension.

In conclusion, the results obtained suggest that the users tend to modulate the arm endpoint stiffness with respect to different

\section{REFERENCES}

Ajoudani, A., Fang, C., Tsagarakis, N. G., and Bicchi, A. (2015). “A reducedcomplexity description of arm endpoint stiffness with applications to teleimpedance control," in Intelligent Robots and Systems (IROS), 2015 IEEE/RSJ International Conference on (Hamburg: IEEE), 1017-1023. tasks and interfaces and that this modulation is influenced by both the trajectory characteristics and the users' hand kinematics. The users were coping with the difference in task design and master device by adapting their arm stiffness modulation both in terms of central tendencies and variability. Although affected by some limitations, these findings prove that the arm dynamic proprieties are highly variable and that there could be significant benefits from the estimation of the kinetic proprieties of the users' arm during teleoperation. For instance, knowing how the users modulate their stiffness would allow to develop master devices able to match and to enhance this modulation, potentially reducing the energetic cost of the teleoperators while maintaining high precision. The results obtained could also be used to improve human-robot interactions during cooperative tasks, as in Beretta et al. (2015a,b).

\subsection{Future Developments}

An interesting possible future development would be the inclusion in the research of a higher number of subjects as well as expert teleoperators, in order to study and compare the stiffness modulation strategies that they may have developed with expertise. To further understand which are the characteristics and parameters that play a fundamental role in the humanrobot interaction, it would also be advisable to study how stiffness relates to other dynamic parameters such as damping and inertia whose relation to human-robot system stability have been recently investigated (Dyck et al., 2013). These studies proved that the human arm behavior can change from passive to active based on the task performed and on the magnitude of the force perturbation applied.

\section{AUTHOR CONTRIBUTIONS}

JB developed the acquisition framework, system synchronization software, performed the experiments, analyzed data and wrote the paper; with ED, he conceived the methods and analysis. JJ helped in designing and performing the experiments, also writing the corresponding portion of the manuscript. GF and ED supervised, reviewed and approved the work.

\section{ACKNOWLEDGMENTS}

This project has received funding from the European Unions Horizon 2020 research and innovation programme under grant agreement No. H2020-ICT-2016-732515. The authors would like to thank Alexandra Stefanescova for the precious help with the Scaling and Inverse Kinematics. 
Beretta, E., Nessi, F., Ferrigno, G., and De Momi, E. (2015b). "Force feedback enhancement for soft tissue interaction tasks in cooperative robotic surgery," in Intelligent Robots and Systems (IROS), 2015 IEEE/RSJ International Conference on (Hamburg: IEEE), 209-215.

Burdet, E., Osu, R., Franklin, D. W., Milner, T. E., and Kawato, M. (2001). The central nervous system stabilizes unstable dynamics by learning optimal impedance. Nature 414, 446-449. doi: 10.1038/35106566

Buzzi, J., Gatti, C., Ferrigno, G., and De Momi, E. (2017). Analysis of joint and hand impedance during teleoperation and free-hand task execution. IEEE Robot. Autom. Lett. 2, 1733-1739. doi: 10.1109/LRA.2017.2678546

Colgate, J. (1994). Coupled stability of multiport systems theory and experiments. J. Dyn. Syst. Meas. Control 116, 419-428. doi: 10.1115/1.2899237

Conti, F., Park, J., and Khatib, O. (2014). "Interface design and control strategies for a robot assisted ultrasonic examination system," in Experimental Robotics, eds O. Khatib, V. Kumar, and G. Sukhatme (Berlin; Heidelberg: Springer), 97-113.

Cragg, L., and Hu, H. (2003). "Application of mobile agents to robust teleoperation of internet robots in nuclear decommissioning," in Industrial Technology, 2003 IEEE International Conference on, Vol. 2 (Maribor: IEEE), 1214-1219.

Darainy, M., Malfait, N., Gribble, P. L., Towhidkhah, F., and Ostry, D. J. (2004). Learning to control arm stiffness under static conditions. J. Neurophysiol. 92, 3344-3350. doi: 10.1152/jn.00596.2004

Delp, S. L., Anderson, F. C., Arnold, A. S., Loan, P., Habib, A., John, C. T., et al. (2007). Opensim: open-source software to create and analyze dynamic simulations of movement. IEEE Trans. Biomed. Eng. 54, 1940-1950. doi: 10.1109/TBME.2007.901024

Dyck, M., Jazayeri, A., and Tavakoli, M. (2013). "Is the human operator in a teleoperation system passive?," in World Haptics Conference (WHC), 2013 (Daejeon: IEEE), 683-688.

Enayati, N., De Momi, E., and Ferrigno, G. (2016). Haptics in robotassisted surgery: challenges and benefits. IEEE Rev. Biomed. Eng. 9, 49-65. doi: 10.1109/RBME.2016.2538080

Fischer, P., Daniel, R., and Siva, K. (1990). "Specification and design of input devices for teleoperation," in Robotics and Automation, 1990. Proceedings., 1990 IEEE International Conference on (Cincinnati, OH: IEEE), 540-545.

Flash, T., and Mussa-Ivaldi, F. (1990). Human arm stiffness characteristics during the maintenance of posture. Exp. Brain Res. 82, 315-326. doi: 10.1007/BF00231251

Gomi, H., and Kawato, M. (1997). Human arm stiffness and equilibriumpoint trajectory during multi-joint movement. Biol. Cybern. 76, 163-171. doi: $10.1007 /$ s004220050329

Gribble, P. L., Mullin, L. I., Cothros, N., and Mattar, A. (2003). Role of cocontraction in arm movement accuracy. J. Neurophysiol. 89, 2396-2405. doi: $10.1152 /$ jn. 01020.2002

Hadavand, M., Mirbagheri, A., Behzadipour, S., and Farahmand, F. (2014). A novel remote center of motion mechanism for the force-reflective master robot of haptic tele-surgery systems. Int. J. Med. Robot. Comput. Assisted Surg. 10, 129-139. doi: 10.1002/rcs.1515

Holzbaur, K. R., Murray, W. M., and Delp, S. L. (2005). A model of the upper extremity for simulating musculoskeletal surgery and analyzing neuromuscular control. Ann. Biomed. Eng. 33, 829-840. doi: 10.1007/s10439-005-3320-7

Horaud, R., and Dornaika, F. (1995). Hand-eye calibration. Int. J. Robot. Res. 14, 195-210. doi: 10.1177/027836499501400301

Johnson, B., and Somu, G. (2016). Robotic telesurgery: benefits beyond barriers. BMH Med. J. 3, 51-54.

Lee, D., and Spong, M. W. (2006). Passive bilateral teleoperation with constant time delay. IEEE Trans. Robot. 22, 269-281. doi: 10.1109/TRO.2005.862037

Lehman, G. J., and McGill, S. M. (1999). The importance of normalization in the interpretation of surface electromyography: a proof of principle. J. Manipulat. Physiol. Therapeut. 22, 444-446. doi: 10.1016/S0161-4754(99)70032-1

Levit-Binnun, N., Schechtman, E., and Flash, T. (2006). On the similarities between the perception and production of elliptical trajectories. Exp. Brain Res. 172, 533-555. doi: 10.1007/s00221-006-0355-4
Lloyd, D. G., and Besier, T. F. (2003). An emg-driven musculoskeletal model to estimate muscle forces and knee joint moments in vivo. J. Biomech. 36, 765-776. doi: 10.1016/S0021-9290(03)00010-1

McIntyre, J., Mussa-Ivaldi, F., and Bizzi, E. (1996). The control of stable postures in the multijoint arm. Exp. Brain Res. 110, 248-264. doi: 10.1007/BF00228556

Mitsou, N. C., Velanas, S. V., and Tzafestas, C. S. (2006). "Visuo-haptic interface for teleoperation of mobile robot exploration tasks," in ROMAN 2006The 15th IEEE International Symposium on Robot and Human Interactive Communication (Hatfield, UK: IEEE), 157-163.

Osu, R., Franklin, D. W., Kato, H., Gomi, H., Domen, K., Yoshioka, T., et al. (2002). Short-and long-term changes in joint co-contraction associated with motor learning as revealed from surface EMG. J. Neurophysiol. 88, 991-1004.

Piovesan, D., Pierobon, A., DiZio, P., and Lackner, J. R. (2013). Experimental measure of arm stiffness during single reaching movements with a timefrequency analysis. J. Neurophysiol. 110, 2484-2496. doi: 10.1152/jn.01013.2012

Pizzolato, C., Lloyd, D. G., Sartori, M., Ceseracciu, E., Besier, T. F., Fregly, B. J., et al. (2015). Ceinms: a toolbox to investigate the influence of different neural control solutions on the prediction of muscle excitation and joint moments during dynamic motor tasks. J. Biomech. 48, 3929-3936. doi: 10.1016/j.jbiomech.2015.09.021

Qiu, T., Hamel, W. R., and Lee, D. (2014). "Design and control of a low cost 6 dof master controller," in 2014 IEEE International Conference on Robotics and Automation (ICRA) (Hong Kong: IEEE), 5313-5318.

Sartori, M., Reggiani, M., Farina, D., and Lloyd, D. G. (2012). EMG-driven forward-dynamic estimation of muscle force and joint moment about multiple degrees of freedom in the human lower extremity. PLOS ONE 7:e52618. doi: 10.1371/journal.pone.0052618

Saul, K. R., Hu, X., Goehler, C. M., Vidt, M. E., Daly, M., Velisar, A., et al. (2015). Benchmarking of dynamic simulation predictions in two software platforms using an upper limb musculoskeletal model. Comput. Methods Biomech. Biomed. Eng. 18, 1445-1458. doi: 10.1080/10255842.2014.916698

Shao, Q., Bassett, D. N., Manal, K., and Buchanan, T. S. (2009). An EMG-driven model to estimate muscle forces and joint moments in stroke patients. Comput. Biol. Med. 39, 1083-1088. doi: 10.1016/j.compbiomed.2009.09.002

Shin, D., Kim, J., and Koike, Y. (2009). A myokinetic arm model for estimating joint torque and stiffness from emg signals during maintained posture. $J$. Neurophysiol. 101, 387-401. doi: 10.1152/jn.00584.2007

Tobergte, A., and Helmer, P. (2013). "A disturbance observer for the sigma. 7 haptic device," in Intelligent Robots and Systems (IROS), 2013 IEEE/RSJ International Conference on (Tokyo: IEEE), 4964-4969. doi: 10.1109/IROS.2013.6697073

Tsumugiwa, T., Yokogawa, R., and Hara, K. (2002). "Variable impedance control based on estimation of human arm stiffness for human-robot cooperative calligraphic task," in Robotics and Automation, 2002. Proceedings. ICRA'02. IEEE International Conference on, Vol. 1 (Washington, DC: IEEE), 644-650.

Wiker, S. F., Hershkowitz, E., and Zik, J. (1989). "Teleoperator comfort and psychometric stability: criteria for limiting master-controller forces of operation and feedback during telemanipulation," in Proceeding of the NASA Conference on Space Telerobotics 1, Vol. 1 (Pasadena, CA), 99-107.

Wilson, E. B., and Worcester, J. (1945). The normal logarithmic transform. Rev. Econ. Stat. 27, 17-22. doi: 10.2307/1927163

Conflict of Interest Statement: The authors declare that the research was conducted in the absence of any commercial or financial relationships that could be construed as a potential conflict of interest.

Copyright $\odot 2017$ Buzzi, Ferrigno, Jansma and De Momi. This is an open-access article distributed under the terms of the Creative Commons Attribution License (CC $B Y)$. The use, distribution or reproduction in other forums is permitted, provided the original author(s) or licensor are credited and that the original publication in this journal is cited, in accordance with accepted academic practice. No use, distribution or reproduction is permitted which does not comply with these terms. 


\title{
Generation of a Movement Scheme for Positive Training
}

\author{
Lin Liu ${ }^{1}$, Le Xie ${ }^{1,2 *}$, Yun-Yong Shi ${ }^{2}$ and Bing-Chen $\mathrm{An}^{3}$ \\ ${ }^{1}$ Institute of Forming Technology and Equipment, Shanghai Jiao Tong University, Shanghai, China, ${ }^{2}$ School of Biomedical \\ Engineering, Shanghai Jiao Tong University, Shanghai, China, ${ }^{3}$ HuaDong Hospital Affiliated with Fudan University, Shanghai, \\ China
}

Rehabilitation robots have been demonstrated to be an efficient tool in the field of rehabilitation training. Meanwhile, there are varieties of tasks designed for motion training. These tasks need to be transmitted to motion data for rehabilitation robots. In this paper, we designed a drinking task and captured the motion data as the ground truth, through sensors of an exoskeleton device named Neo-Arm. To verify the effectiveness of Neo-Arm, we used a Vicon system to capture the same motion task without Neo-Arm for comparison. Eight subjects participated in the experiment. The motion data of the drinking task, including the range of motion (ROM) and the velocity of each joint, are obtained. The result shows that the Neo-Arm can achieve the suitable precision and be fit for other kinds of upper limb motion tasks.

\section{OPEN ACCESS}

Edited by:

Wenwei Yu,

Chiba University, Japan

Reviewed by:

Giuseppe D'Avenio,

Istituto Superiore di Sanità, Italy

Waldemar Karwowski,

University of Central Florida, USA

${ }^{*}$ Correspondence:

LeXie

lexie@sjtu.edu.cn

Specialty section:

This article was submitted to

Neural Technology,

a section of the journal

Frontiers in Neuroscience

Received: 28 September 2016

Accepted: 14 February 2017

Published: 01 March 2017

Citation:

Liu L, Xie L, Shi Y-Y and An B-C

(2017) Generation of a Movement

Scheme for Positive Training.

Front. Neurosci. 11:96.

doi: 10.3389/fnins.2017.00096
Keywords: rehabilitation, training, upper limb, robot, trajectory

\section{INTRODUCTION}

A stroke, also called a cerebral vascular accident (CVA), is one kind of acute disease in the blood vessels in the brain characterized by varying degrees of neurological deficit, which leads to series of sequelae and symptoms. Among all the symptoms, hemiplegia is the most common. However, it is quite difficult for hemiplegic patients to regain their motion function of the upper limb, which leads to a difficult life with a constant reliance on family assistance and medical care. Hence, a variety of rehabilitation robots have been developed to help hemiplegic patients receive better rehabilitation training.

Early studies on rehabilitation robots are mainly based on the design using end-effecter mechanisms. For example, Stanford university developed an upper limb rehabilitation robot called MIME (Lum et al., 2004, 2006), based on the PuMA550 robot. Meanwhile, MIT-MANUS (Krebs et al., 2004), GENTLE/S (Coote et al., 2008), and iPAM (Holt et al., 2007) were developed. Although, these robots can lead the arm to move in space with multiple degree of freedom (multi-DOF) movements, they are not capable of controlling each joint of the upper limb during each period of motion. To control the upper limb from each joint, a variety of rehabilitation robots were designed as exoskeleton mechanisms, such as T-WREX (Housman et al., 2007), Intelliarm (Park et al., 2008), MEDARM (Ball et al., 2007), RUPERT (He et al., 2005), Dampace (Stienen et al., 2009), SUEFUL-7 (Gopura et al., 2009), and Armin I, II, and III (Nef and Riener, 2005; Mihelj et al., 2007; Nef et al., 2009). Nevertheless, they are not suitable for capturing motion information from active movement. Because these robots are heavy for subjects, motors, and corresponding drive mechanisms add more weight to the robots. In this case, subjects have to use more strength and change how they move to offset the weight and the inertia of the mechanism. 
To capture movement, optical measurement equipment has been widely used, which can record the trajectory of the marked point in the skin of the subject, such as Kinect (Chang et al., 2013; Uttarwar and Mishra, 2015) and Vicon (Chung et al., 2011; Henmi et al., 2014).

However, there is relative motion between the skin and inner bones during the upper limb movement, especially for internal/external rotation, which can lead to angular errors. Meanwhile, upper limb movement is complicated with multiDOFs in 3-D space, which can lead to a blocking problem when optical equipment is used to capture movement trajectories.

The objective in this research is to capture healthy people's trajectory in a motion task using an upper limb exoskeleton device. The tasks was designed as a series of movements for drinking. Eight healthy subjects participated in the experiment. They were instructed to wear Neo-Arm to perform the drinking task and to perform the same task using the Vicon system for comparison. During the task, angular information was recorded by both Neo-Arm and the Vicon system. Finally, the 3D trajectory of the upper limb movement was obtained.

\section{MATERIALS AND METHODS \\ Device Description \\ Neo-Arm}

A 7-DOF rehabilitation device for active training was developed in our earlier research (Yu et al., 2015), called the Neo-Arm device, as shown in Figure 1. The device is equipped with an angular displacement transducer at each DOF, which can capture the position information of real-time movement. NeoArm can be used to capture the 3D trajectory of the rehabilitation movement in positive training.

At each joint of Neo-Arm, an angular displacement sensor is equipped through the axis of the rotation, by which the angular information can be captured and transformed from electrical signals to angular data. Through the training system, data are saved as a file for the subject. The coordinate system of NeoArm is shown in Figure 2. Through the coordinate system and the data captured by sensors, we can calculate the $3 \mathrm{D}$ trajectory of the endpoint of the upper limb.

The Denavit-Hartenberg (D-H) matrix is adopted as Equation (1):

$$
{ }_{6}^{0} T={ }_{1}^{0} T_{2}^{1} T_{3}^{2} T_{4}^{3} T_{5}^{4} T_{6}^{5} T=\left[\begin{array}{cccc}
n_{x} & o_{x} & a_{x} & p_{x} \\
n_{y} & o_{y} & a_{y} & p_{y} \\
n_{z} & o_{z} & a_{z} & p_{z} \\
0 & 0 & 0 & 1
\end{array}\right]
$$

where, $\left\{p_{x} p_{y} p_{z}\right\}^{T}$ is the location of the endpoint of the robot.

Table 1 is also needed as the demand of $\mathrm{D}-\mathrm{H}$ matrix to calculate the endpoint position of the robot in $3 \mathrm{D}$ space.

In Table 1, the values of $d_{i}$ and $a_{i-1}$ are the length parameters of Neo-Arm. The length parameters for upper limb and NeoArm are shown in Table 2.

In Table 1, the values of $\theta i$ are the angle parameters of the DOF of the human upper limb. The values of $\mathrm{J}_{\mathrm{i}}$ can be captured by an

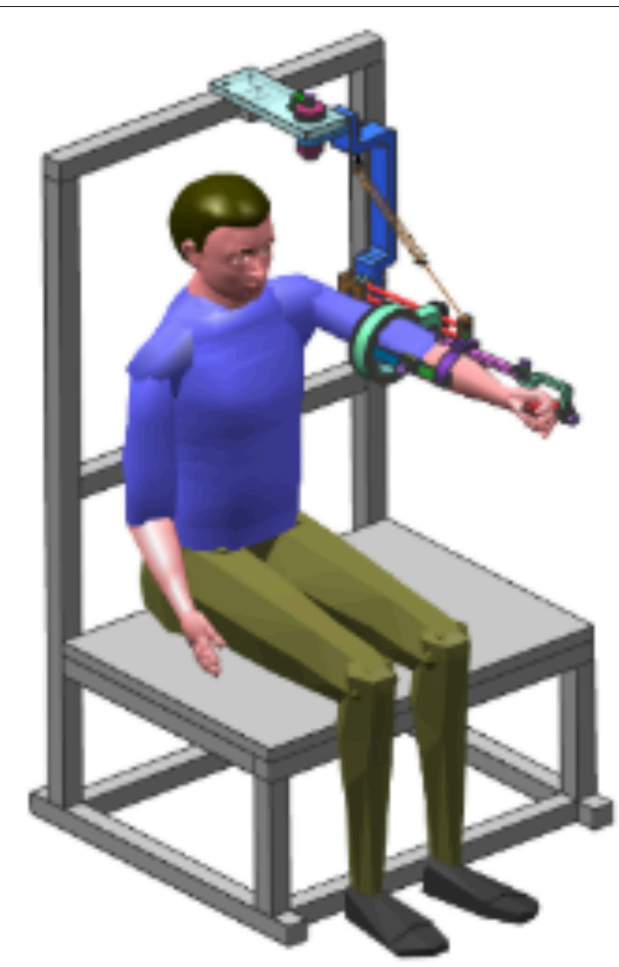

FIGURE 1 | Neo-Arm device.

angular displacement sensor. The angle parameters for the upper limb and Neo-Arm are shown in Table 3.

\section{Vicon System}

The optional motion capture system used in the experiment is a Vicon system (Oxford Metrics Ltd., Oxford, UK), in Ruijin Hospital, Shanghai Institute of Orthopedics and Traumatology, Shanghai, China, see Figure 3A. It is composed of 10 cameras and an MX13 motion capture system. The body of the subject is marked with 39 markers (14 $\mathrm{mm}$ in diameter), which are used to record trajectories in a sampling frequency of $100 \mathrm{~Hz}$. The motion data are processed in Vicon polygon software, see Figure 3B.

\section{Subjects}

Eight male subjects were selected to participate in the experiment. This study was carried out in accordance with the recommendations of Ethics Committee at Shanghai Ninth People's Hospital affiliated to Shanghai Jiao Tong University School of Medicine (No. 2013011). All subjects provided written informed consent prior to participation. Subjects are similar in age and sitting height, as well as their upper limb dimensions, as described in Table 4.

Meanwhile, the dimensions of the Neo-Arm device can be adjusted to accommodate the distinctive dimensions of the upper limb for different subjects, by which means each joint of the whole mechanism can be correlated with the corresponding joint of the subject's limb joint. The dimension information can be 


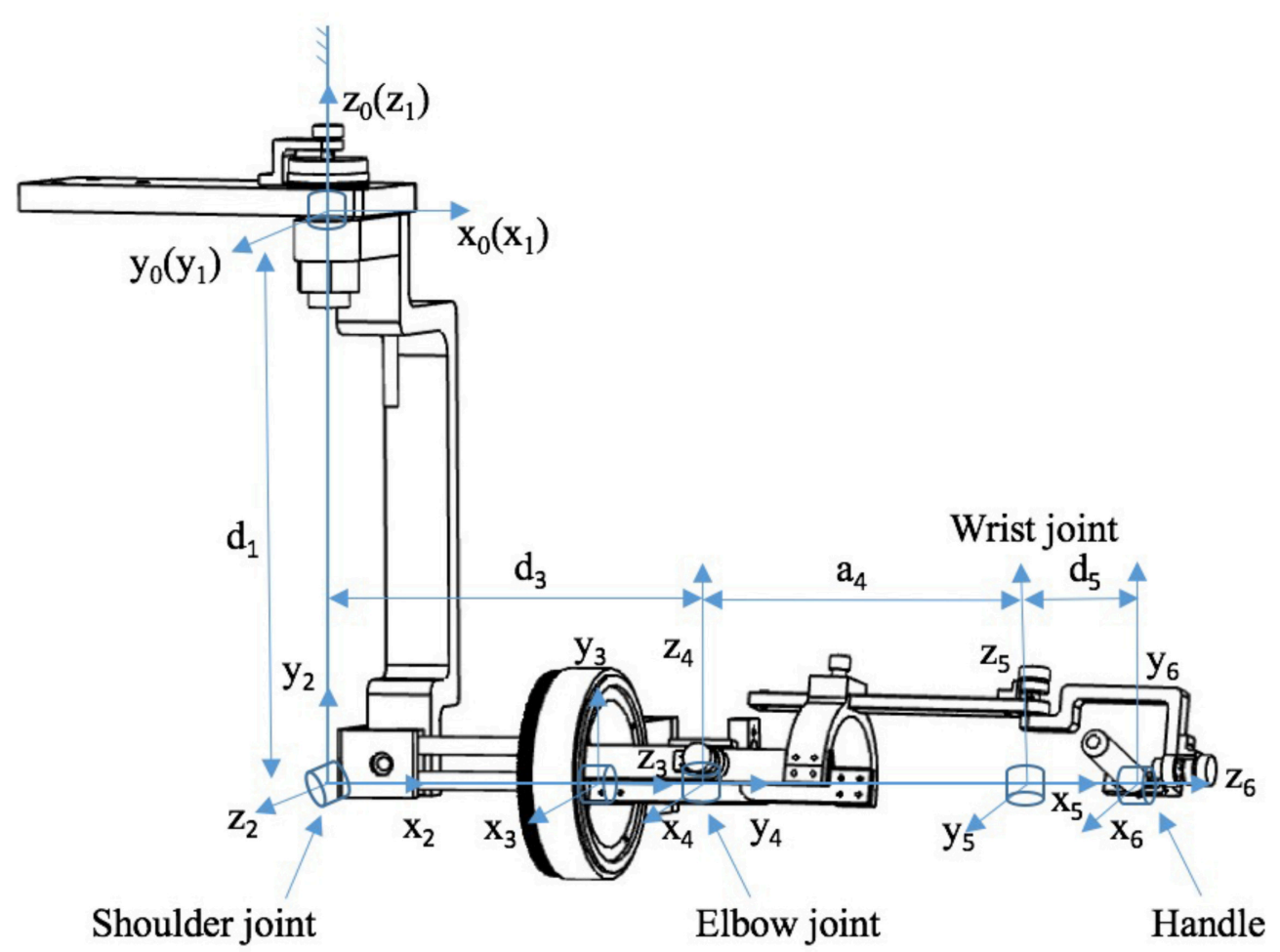

FIGURE 2 | Coordinate system of the Neo-Arm robot.

TABLE 1 | D-H table.

\begin{tabular}{ccccc}
\hline $\boldsymbol{I}$ & $\boldsymbol{\theta}_{\boldsymbol{i}}$ & $\mathbf{a}_{\boldsymbol{i}-\mathbf{1}}$ & $\mathbf{d}_{\boldsymbol{i}}$ & $\boldsymbol{\alpha}_{\boldsymbol{i}-\mathbf{1}}$ \\
\hline 1 & $\theta_{1}$ & 0 & $\mathrm{~d}_{1}$ & 0 \\
2 & $\theta_{2}$ & 0 & 0 & $\pi / 2$ \\
3 & $\theta_{3}$ & 0 & $\mathrm{~d}_{3}$ & $\pi / 2$ \\
4 & $\theta_{4}$ & 0 & 0 & $\pi / 2$ \\
5 & $\theta_{5}$ & $\mathrm{a}_{4}$ & 0 & $\pi / 2$ \\
6 & $\theta_{6}$ & 0 & $\mathrm{~d}_{5}$ & $\pi / 2$ \\
\hline
\end{tabular}

TABLE 2 | Length parameters of the upper limb and Neo-Arm.

\begin{tabular}{lll}
\hline Definition & Upper limb & Neo-Arm \\
\hline Distance between the base point and shoulder joint & $L 1$ & $\mathrm{~d}_{1}$ \\
Length of the upper arm & $\llcorner 2$ & $\mathrm{d}_{3}$ \\
Length of the forearm & $L 3$ & $\mathrm{a}_{4}$ \\
Distance between the elbow joint and handle & $\llcorner 4$ & $\mathrm{d}_{5}$ \\
\hline
\end{tabular}

saved as a record. The basic parameters of the device are shown in Table 5.

\section{Task Design Requirement}

The activities of daily living (ADL) include various self-care activities people perform in everyday lives, such as bathing, feeding, drinking, and dressing. Since the assessment of ADL has
TABLE 3 | Angle parameters of the upper limb and Neo-Arm.

\begin{tabular}{llll}
\hline $\begin{array}{l}\text { Joints of } \\
\text { upper limb }\end{array}$ & DOF of upper limb & $\begin{array}{l}\text { Angle of } \\
\text { upper limb }\end{array}$ & $\begin{array}{l}\text { Angle of } \\
\text { Neo-Arm }\end{array}$ \\
\hline Shoulder & Flexion/extension & $\theta_{1}$ & $\mathrm{~J} 1$ \\
& Abduction/adduction & $\theta_{2}$ & $\mathrm{~J} 2$ \\
& Internal rotation/external rotation & $\theta_{3}$ & $\mathrm{~J} 3$ \\
Elbow & Flexion/extension & $\theta_{4}$ & $\mathrm{~J} 4$ \\
Forearm & Pronation/supination & $\theta_{5}$ & $\mathrm{~J} 5$ \\
Wrist & Flexion/extension & $\theta_{6}$ & $\mathrm{~J} 6$ \\
& Ulnar deviation/radial deviation & $\theta_{6}$ & $\mathrm{~J}^{\prime}$ \\
\hline
\end{tabular}

played an important role in rehabilitation training, it is significant that in this research subjects should follow the mode of motion in ADL. Given that drinking is a basic movement performed repeatedly in everyday life, drinking was proposed and discussed as a task in this paper.

To simulate drinking in daily life, the task was designed based on three principles. First, each time subjects should perform a different movement. Second, the movement that subjects perform should be a motion combination including picking up a cup, fetching water, and drinking. Third, the ROM of the device should be within the ROM demanded in the task. Different movement trajectories combined with motion combinations can avoid the simple advance and return movement in rehabilitation training. In this way, subjects are led to finish the task by actively thinking. 
A

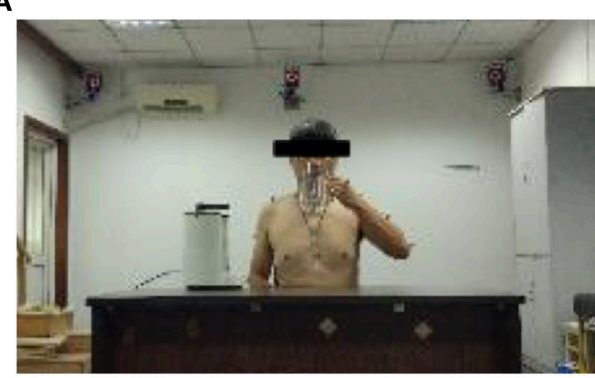

Vicon motion capture system

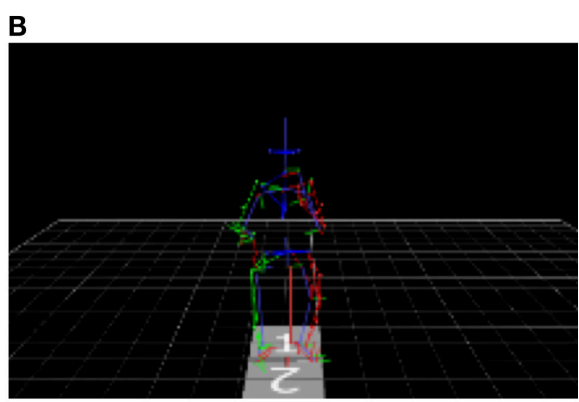

Vicon polygon software

FIGURE 3 | The motion captured in the Vicon system. (A) Vicon motion capture system. (B) Vicon polygon software.

TABLE 4 | Subjects.

\begin{tabular}{|c|c|c|c|c|c|c|c|}
\hline Subjects & Sex & Age & Weight (kg) & Height (cm) & L2 (mm) & L3 (mm) & $\mathrm{L} 4$ (mm) \\
\hline 1 & $M$ & 32 & 75 & 175 & 358 & 272 & 73 \\
\hline 2 & $\mathrm{M}$ & 27 & 68 & 173 & 359 & 270 & 72 \\
\hline 3 & $\mathrm{M}$ & 25 & 71 & 171 & 357 & 268 & 72 \\
\hline 4 & $\mathrm{M}$ & 25 & 70 & 173 & 356 & 268 & 75 \\
\hline 5 & $\mathrm{M}$ & 30 & 80 & 175 & 359 & 271 & 72 \\
\hline 6 & $M$ & 26 & 79 & 182 & 355 & 279 & 71 \\
\hline 7 & $\mathrm{~F}$ & 26 & 71 & 172 & 356 & 276 & 70 \\
\hline 8 & $\mathrm{~F}$ & 28 & 66 & 170 & 355 & 272 & 72 \\
\hline
\end{tabular}

TABLE 5 | Neo-Arm device parameters.

\begin{tabular}{lccc}
\hline L1 & L2 & L3 & L4 \\
\hline $435 \mathrm{~mm}$ & $360 \mathrm{~mm}$ & $270 \mathrm{~mm}$ & $70 \mathrm{~mm}$ \\
\hline
\end{tabular}

\section{Equipment Layout}

According to the first principle, three cup points are set up for $\mathrm{P} 2$, which leads to different movement trajectories during A2 and A3 actions. Three cup points were set up, as shown in Figure 4, including the left point (Point $\mathrm{L}$ ), right point (Point $\mathrm{R}$ ), and bottom point (Point $\mathrm{B}$ ). The locations of the chair, the desk, the bottle, and J1 DOF are also fixed.

In the experiment, movement parameters for different cup points were recorded. At first, subjects were instructed to perform the task for one cup point without any practice. After practicing, they were instructed to perform it at the same point again. This point was chosen as the left point. Then, subjects proceeded to complete the task with random cup points assigned by the researcher.

\section{Task Description}

According to the second and the third principles, a series of movements for drinking was proposed in the experiment. The subject had to use two hands coordinating with each other. NeoArm I was worn on the left upper limb to perform a series of movements, including five actions and five hovering postures,

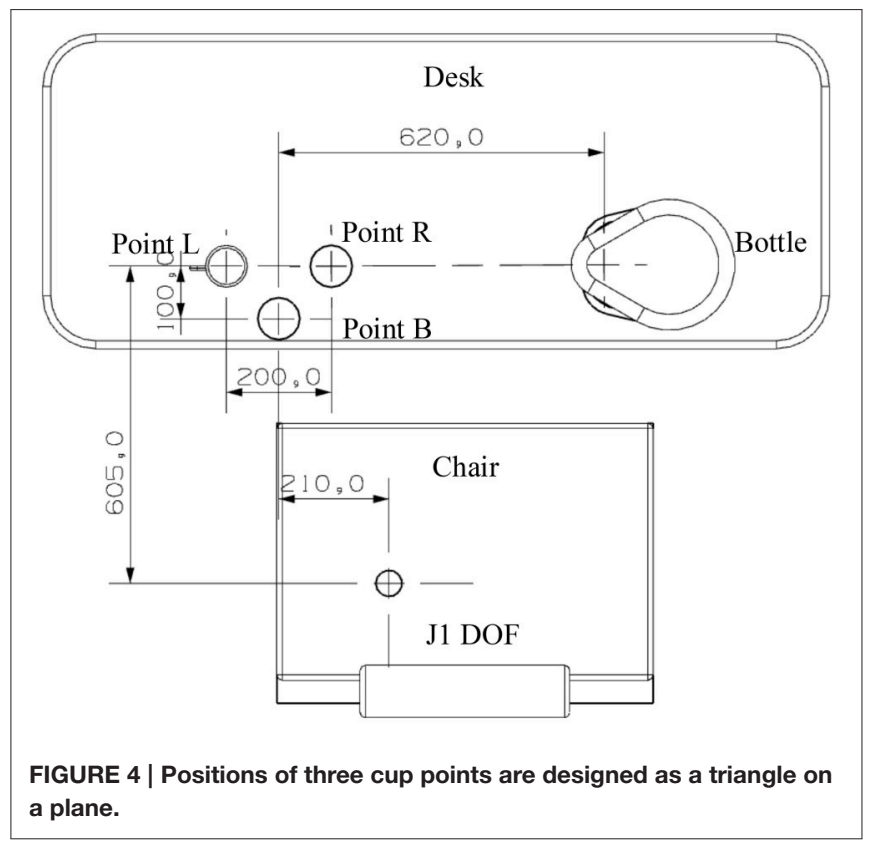

as shown in Figure 5: zero position posture (P0), ready action (A1), ready posture (P1), fetching cup action (A2), holding cup posture (P2), getting water action (A3), getting water posture (P3), drinking action (A4), drinking posture (P4), homing action (A5), and homing posture (P5). 
The first posture (P0) is zero position posture, as shown in Figure 5A, which is a fixed initial posture when the subjects put on the device in the task. P1 is the position when subjects get ready to fetch the cup, which is shown in Figure 5B. A1 is the action in which the upper limb is moved from P0 to $\mathrm{P} 1 . \mathrm{A} 2$ is the action in which subjects move the upper limb to fetch the cup. Although, it is essential that the subject completes the action of picking up the cup in real-world situations, hand function training is not the focus of this paper. Therefore, the catching action is simplified as moving the handle of the device and reaching the cup point. The posture where the upper limb hovers at the cup point is P2, as shown in Figure 5C. Then, the subject should move the left upper limb from the cup point to the bottle through A3 action. At P3, although the right upper limb is not tested in this research, the subject has to use his/her right hand to press the button to coordinate with the motion of the left upper limb, shown as in Figure 5D. After that, the subject should move the handle from the bottle to the side of his/her mouth and perform the drinking movement through the A4 action. Meanwhile, the right side of the upper limb must help to hold the cup. Finally, the upper limb should be moved to the ready posture.

\section{RESULTS}

\section{ROM}

According to the different periods of the whole movement, the movement in the task was divided into five actions and six hovering postures. Since the reaching cup motion, the getting water motion, and the drinking motion play important roles in
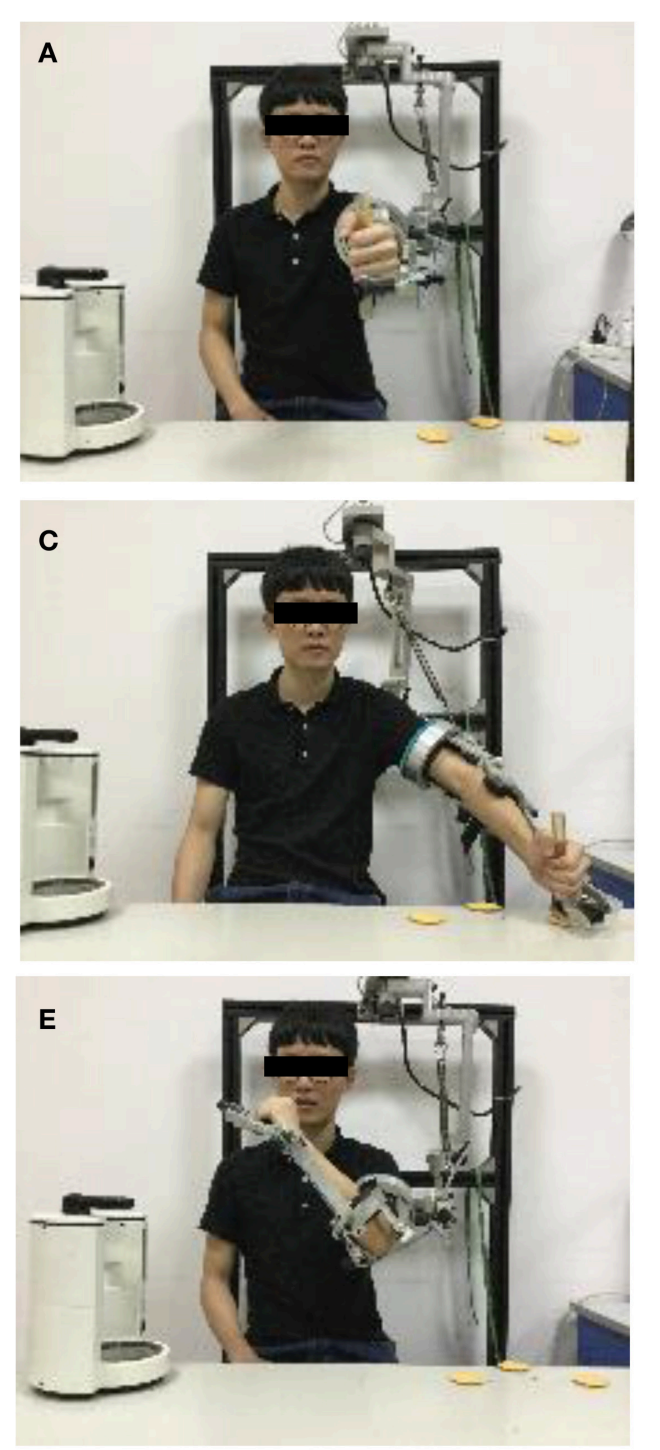
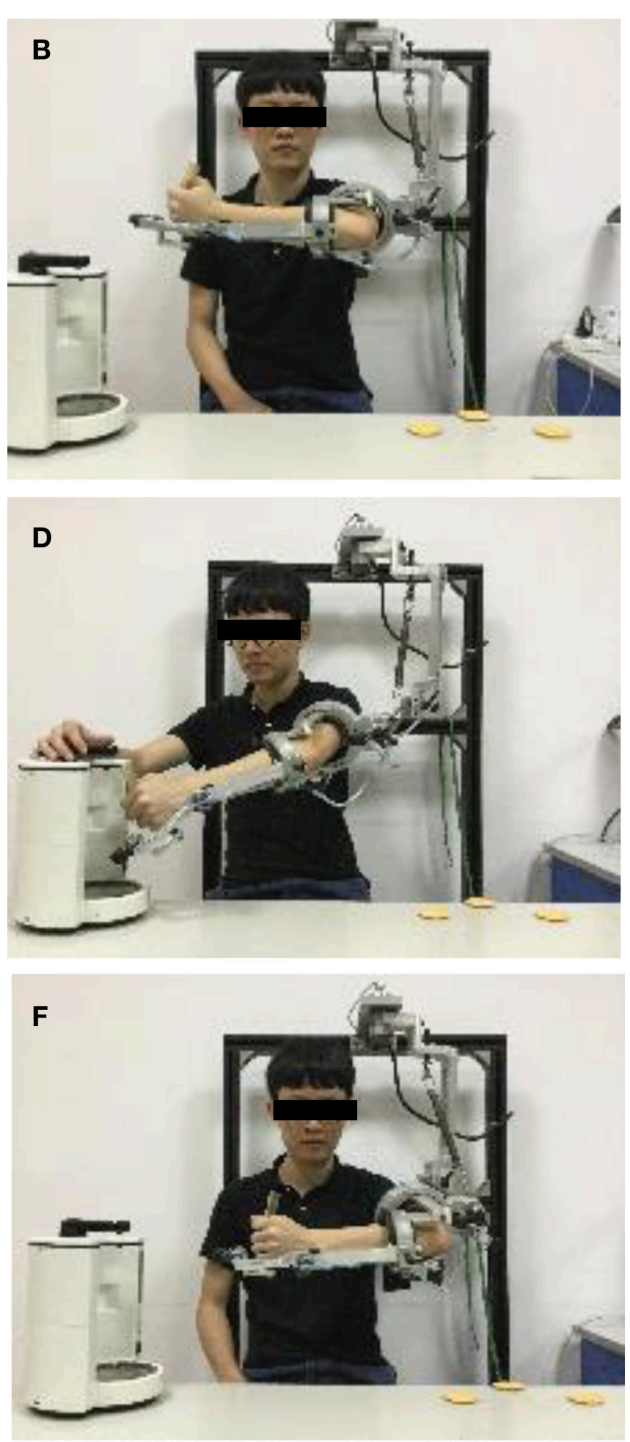

FIGURE 5 | Movement design. (A) P0 posture. (B) P1 posture. (C) P2 posture. (D) P3 posture. (E) P4 posture. (F) P5 posture. A1 action is the motion from P0 to P1; A2 action is the motion from P1 to P2; A3 action is the motion from P2 to P3; A4 action is the motion from P4; A5 action is the motion from P4 to P5. 
the task, the angle of each DOF from all subjects at the P2, P3, P4, and P5 postures are listed in Figures 6-10.

The data for P2 at the L point are shown in Figure 6. The mean angles of J1 from Neo-arm and Vicon are $-66.28 \pm 1.42$ and $-63.26 \pm 1.95 \mathrm{~mm}(p=0.038)$; the mean angles of $\mathrm{J} 2$ are $32.96 \pm 0.49,28.50 \pm 1.31 \mathrm{~mm}(p=0.046)$; the mean angles of $\mathrm{J} 3$ are $-25.14 \pm 0.27,-28.48 \pm 1.28 \mathrm{~mm}(p=0.031)$; the mean angles of $\mathrm{J} 4$ are $47.84 \pm 2.74,52.65 \pm 2.36 \mathrm{~mm}(p=0.042)$; the mean angles of $\mathrm{J} 5$ are $6.21 \pm 1.03,10.56 \pm 3.48 \mathrm{~mm}(p=0.11)$; the mean angles of $\mathrm{J} 6$ are $27.78 \pm 2.4,22.65 \pm 4.65 \mathrm{~mm}(p=0.12)$.

The data for P2 at B point are shown in Figure 7. The mean angles of J1 from Neo-arm and Vicon are $-84.86 \pm 4.8,-80.26$ $\pm 5.31 \mathrm{~mm}(p=0.062)$, the mean angles of $\mathrm{J} 2$ are $57.16 \pm 6.1$, $54.19 \pm 7.49 \mathrm{~mm}(p=0.053)$, the mean angles of J3 are -14.55 $\pm 1.46,-12.33 \pm 1.66 \mathrm{~mm}(p=0.037)$, the mean angles of $\mathrm{J} 4$ are $83.89 \pm 1.39,79.67 \pm 1.88 \mathrm{~mm}(p=0.046)$, the mean angles of J5 are $21.06 \pm 3.1,18.35 \pm 5.46 \mathrm{~mm}(p=0.032)$, the mean angles of $\mathrm{J} 6$ are $22.04 \pm 3.16,18.61 \pm 7.62 \mathrm{~mm}(p=0.067)$.

The data for $\mathrm{P} 2$ at the $\mathrm{R}$ point are shown in Figure 8. The mean angles of $\mathrm{J} 1$ from Neo-arm and Vicon are $-50.86 \pm 4.52$, $-45.64 \pm 3.88 \mathrm{~mm}(p=0.055)$, the mean angles of $\mathrm{J} 2$ are 27.26
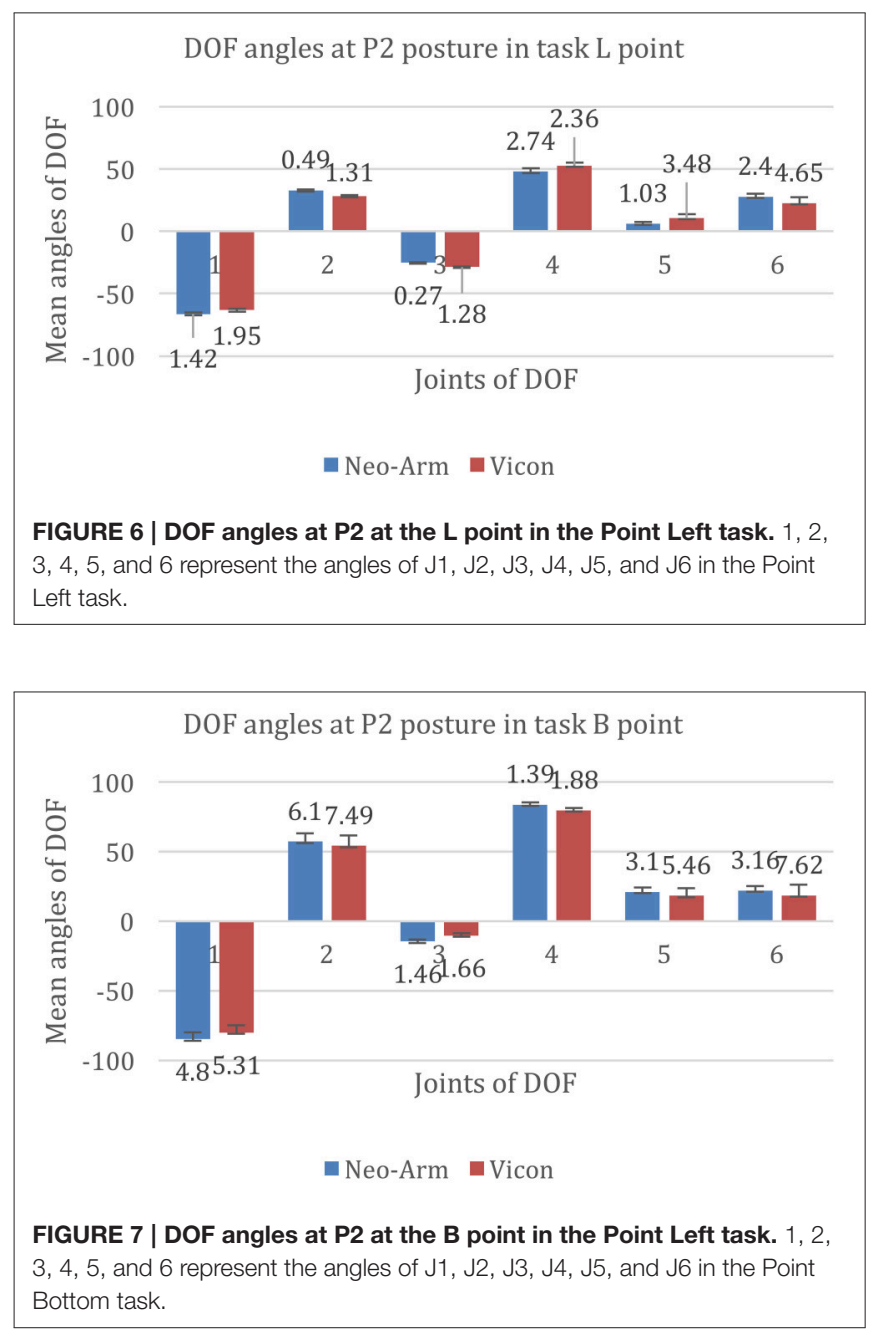
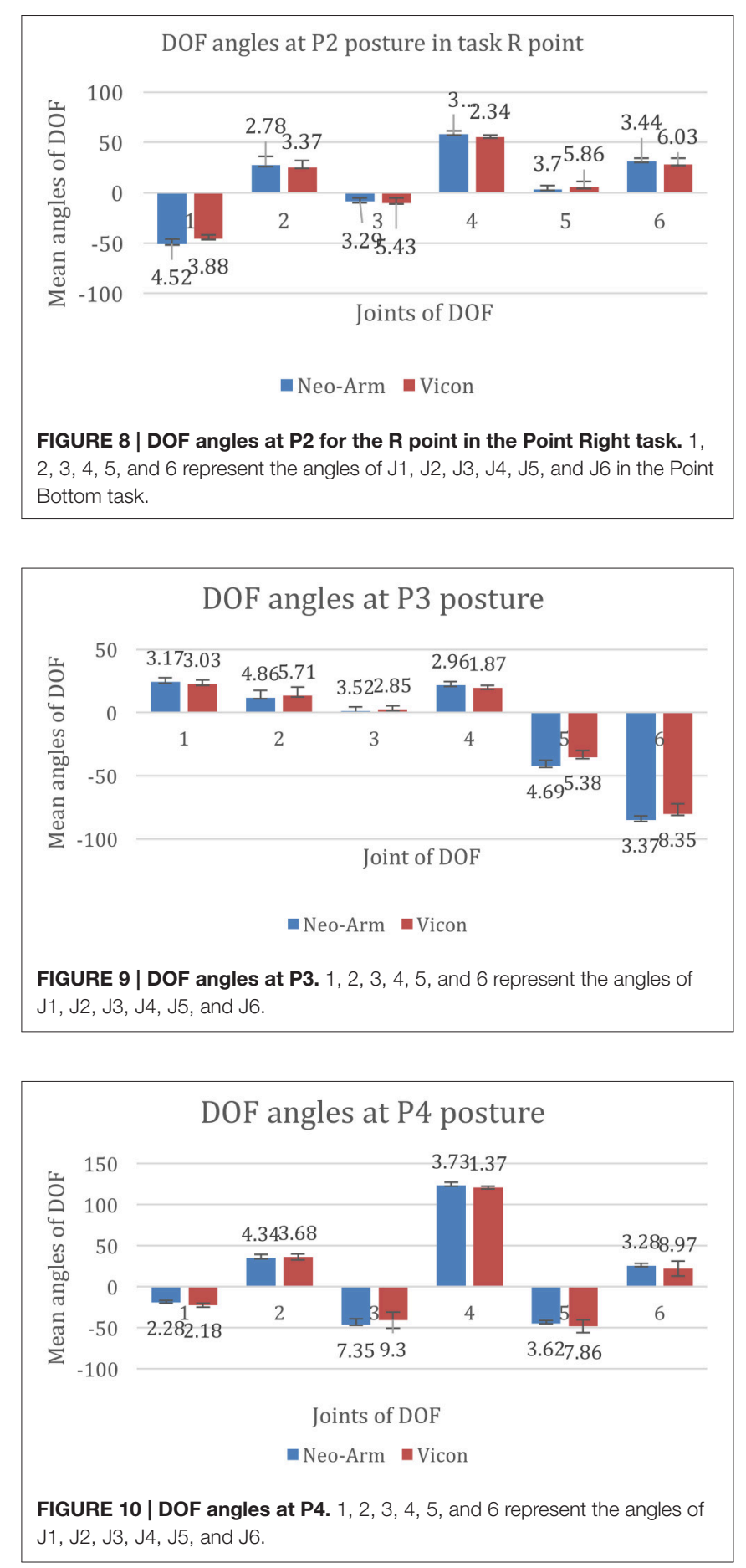

$\pm 2.78,25.37 \pm 3.37 \mathrm{~mm}(p=0.036)$, the mean angles of $\mathrm{J} 3$ are $-8.86 \pm 3.29,-10.50 \pm 5.43 \mathrm{~mm}(p=0.044)$, the mean angles of $\mathrm{J} 4$ are $58.30 \pm 3.11,55.16 \pm 2.34 \mathrm{~mm}(p=0.036)$, the mean angles of $\mathrm{J} 5$ are $3.37 \pm 3.70,5.35 \pm 5.86 \mathrm{~mm}(p=0.067)$, the mean angles of J6 are 28.16 $\pm 6.03,31.00 \pm 3.44 \mathrm{~mm}(p=0.072)$.

The data for P3 posture are shown in Figure 9. The mean angles of J1 from Neo-arm and Vicon are 24.36 $\pm 3.17,22.67 \pm$ $3.03 \mathrm{~mm}(p=0.028)$, the mean angles of $\mathrm{J} 2$ are $11.91 \pm 4.86$, 
$13.38 \pm 6.71 \mathrm{~mm}(p=0.043)$, the mean angles of $\mathrm{J} 3$ are $1.07 \pm$ $3.52,2.55 \pm 2.88 \mathrm{~mm}(p=0.035)$, the mean angles of $\mathrm{J} 4$ are 21.63 $\pm 2.96,19.57 \pm 1.97 \mathrm{~mm}(p=0.038)$, the mean angles of J5 are $-42.28 \pm 4.69,-35.38 \pm 5.68 \mathrm{~mm}(p=0.24)$, the mean angles of $\mathrm{J} 6$ are $-85.20 \pm 3.37,-80.36 \pm 8.35 \mathrm{~mm}(p=0.14)$.

The data for P3 are shown in Figure 10. The mean angles of $\mathrm{J} 1$ from Neo-arm and Vicon are $-19.20 \pm 2.28,-22.13 \pm 2.18$ $\mathrm{mm}(p=0.041)$, the mean angles of $\mathrm{J} 2$ are $34.84 \pm 4.34,-22.13$ $\pm 2.18 \mathrm{~mm}(p=0.032)$, the mean angles of $\mathrm{J} 3$ are $-46.32 \pm 7.35$, $-40.35 \pm 9.3 \mathrm{~mm}(p=0.058)$, the mean angles of $\mathrm{J} 4$ are 123.28 $\pm 3.73,120.89 \pm 1.37 \mathrm{~mm}(p=0.044)$, the mean angles of J5 are $-44.39 \pm 3.62,-48.32 \pm 7.86 \mathrm{~mm}(p=0.13)$, the mean angles of $\mathrm{J} 6$ are $25.56 \pm 3.28,22.13 \pm 8.97 \mathrm{~mm}(p=0.17)$.

Considering the differences among subjects, the average and the median angles of each DOF are listed in Table 6.

\section{Velocity}

In the experiment, during each action, the average angular velocities are listed in Table 7. During the A2 and A3 periods, since different cup points lead to different actions, they are listed with v_L, v_R, and v_B, which represent the velocity of different actions for $\mathrm{A} 2$ and $\mathrm{A} 3$.

\section{Measured ROM}

Figure 11 shows the motion of J1 from eight subjects in the Point Left task. J1 represents the shoulder abduction/adduction. To compare all curves, the suspending period has been adjusted within $2 \mathrm{~s}$. Besides, the other five DOFs were solved with the same manner as the J1 DOF.

\section{Movement Scheme}

Since the average angular and the average velocity have been obtained, the movement scheme is shown in Figure 12. It is shown that the angle of each joint, which is displayed as a distinctive color, varies with time. Meanwhile, since the velocity of the endpoint cannot reflect the whole movement comprehensively, the velocity scheme is proposed, as represented by the gradient of each line.

\section{D trajectory}

According to the movement scheme, the $3 \mathrm{D}$ trajectory is shown in Figures 13, 14. Considering that the movement of the wrist would reflect the whole movement trajectory with interference, the intersection of the forearm pronation/supination axis and wrist flexion/extension axis was chosen as the endpoint. The $y-z$ plane represents the horizontal plane. The zero point represents the ending point of the upper limb when it is at the zero posture position, the cup point represents the position where the cup is to be placed, the bottle point represents the position where the water bottle is to be placed. The segment of line 1 represents the movement of reaching for the cup, the segment of line 2 represent the movement of moving the cup to the water bottle, and the segment of line 3 represents the movement of drinking.

\section{DISCUSSION}

\section{Comparison between Neo-Arm and Vicon}

When the hand reached the P2 posture for the cup and P3 for the bottle, the marks on the wrist and the hand were blocked by the cup and the bottle. In this situation, the marks in the Vicon software are lost in these zones, see Figure 15A. To generate a continuous trajectory, the Vicon polygon software simulated the motions of the lost mark based on the trajectory captured previously and afterward. Meanwhile, when one mark is blocked, the Vicon polygon system can still calculate the results from the motion data of adjacent marks. For example, in the two simulated trajectories, J6 DOF varied in two ways, see Figure 15B. According to the simulation, both ways are reasonable. However, during this process, the simulated values of the angles varied for different trajectories, which resulted in a larger standard deviation and $p>0.05$ for three DOF of the wrist in the Vicon system than in Neo-Arm.

\section{Motion Coordination and ROM for the Point Left Task}

According to Figure 12, there are similar patterns in three points tasks. Especially in the shoulder joints and the elbow, four DOFs change with time in a similar pattern.

TABLE 6 | Median and average angles of each DOF in three points of the task.

\begin{tabular}{|c|c|c|c|c|c|c|c|}
\hline & & J1 & J2 & J3 & J4 & J5 & J6 \\
\hline \multirow[t]{5}{*}{ P2 } & Median (L) & -66.75 & 32.34 & -25.04 & 44.97 & 4.3 & 28.9 \\
\hline & Avg (L) & -66.57 & 32.11 & -25.42 & 45.26 & 4.49 & 28.87 \\
\hline & Median (R) & -50.02 & 28.61 & -6.82 & 59.7 & 1.71 & 28.37 \\
\hline & Avg (R) & -50.15 & 28.84 & -6.69 & 60.08 & 2.12 & 28.62 \\
\hline & Median (B) & -86.63 & 56.55 & -15.32 & 83.84 & 20.24 & 20.92 \\
\hline \multirow[t]{2}{*}{ P3 } & Median & 23.82 & 11.74 & -0.02 & 22.12 & -42.16 & -85.38 \\
\hline & Avg & 24.22 & 11.85 & 0.15 & 21.7 & -42.2 & -85.12 \\
\hline \multirow[t]{2}{*}{ P4 } & Median & -19 & 35.06 & -46.26 & 123.36 & -44.02 & 25.71 \\
\hline & Avg & -19.2 & 34.84 & -46.32 & 123.28 & -44.39 & 25.56 \\
\hline
\end{tabular}


TABLE 7 | Velocity of each joint during each motion.

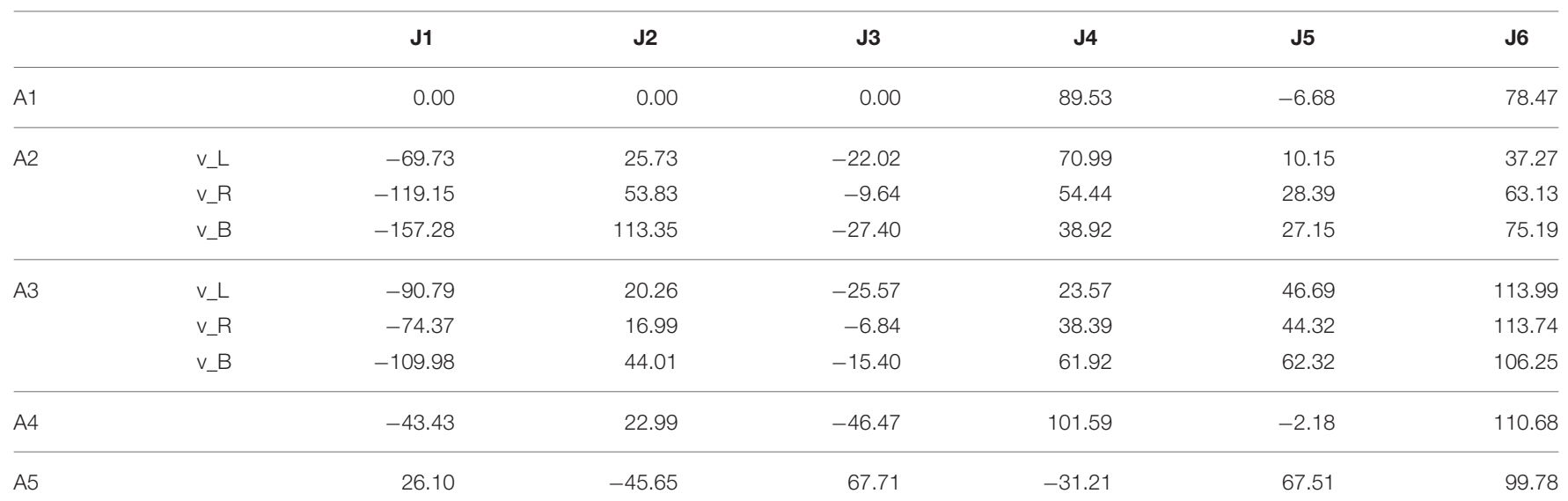

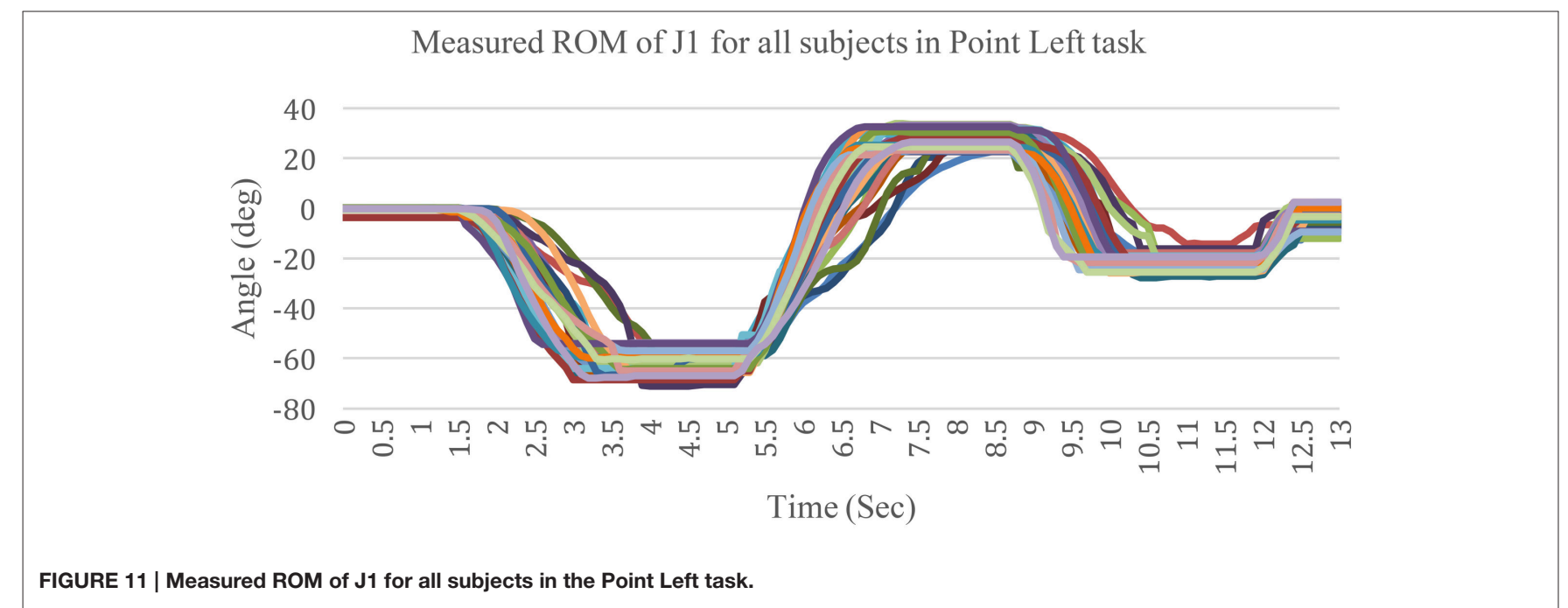

During the A2 period, in the first half, J1 rotated from the zero position posture to the left side, with a maximum of $-66.57^{\circ}$ on average, while $\mathrm{J} 3$ started to increase from $0^{\circ}$ at a low velocity. In the second half of A2, the angle displacement of J3 increased at a high velocity, while J4 rotated from the maximum to the minimum. The changes in this period mean that when subjects moved their left arm to reach the cup, which was placed on the left side, they were inclined to perform shoulder abduction first, accompanied by slight shoulder external rotation synchronously. After that, subjects tried to press their elbow by the way of shoulder extension, while the elbow extended at a maximum angle to open their arm. However, the motion of J5 exhibited two patterns. Subjects tended to pronate the forearm to $4.3^{\circ}$ on average. By this way, the wrist is adjusted to perform radial deviation, so the subjects could move J5 in the tangential direction of the space trajectory.

During A3, the angular variation of J2 and J3 led the arm move in advance. The two joints started to rotated back to $\sim 0^{\circ}$. During the second half of this process, J1 started to increase to a maximum angle of $23.82^{\circ}$ on average, while $\mathrm{J} 4$ rotated to $22.12^{\circ}$ on average. J5 moved to an angle of -42.16 on average. To perform this action, subjects first lifted their elbow using shoulder flexion and internal rotation. Before they finished the lifting, the shoulder began to adduct, accompanied by elbow flexion. All these variations stopped simultaneously when the hand reached the water bottle position. The forearm and the wrist remained still in this process.

During A4, J1 decreased at a high velocity while J4 increased at a high velocity. In this process, J3, J5, and J6 increased slightly. In this process, the elbow flexed while the shoulder abducted. By this movement, subjects moved the hand to the mouth position. At the same time, the forearm pronated to maintain a drinking gesture.

\section{Comparison between Two Patterns of variations of velocity}

During A2 and A3, two major modes of angular velocity curves were presented for J1, referred to as the M-curve and N-curve, as shown in Figure 16. The major difference between these two types of curves is that there are two wave crests in the M-curve, 


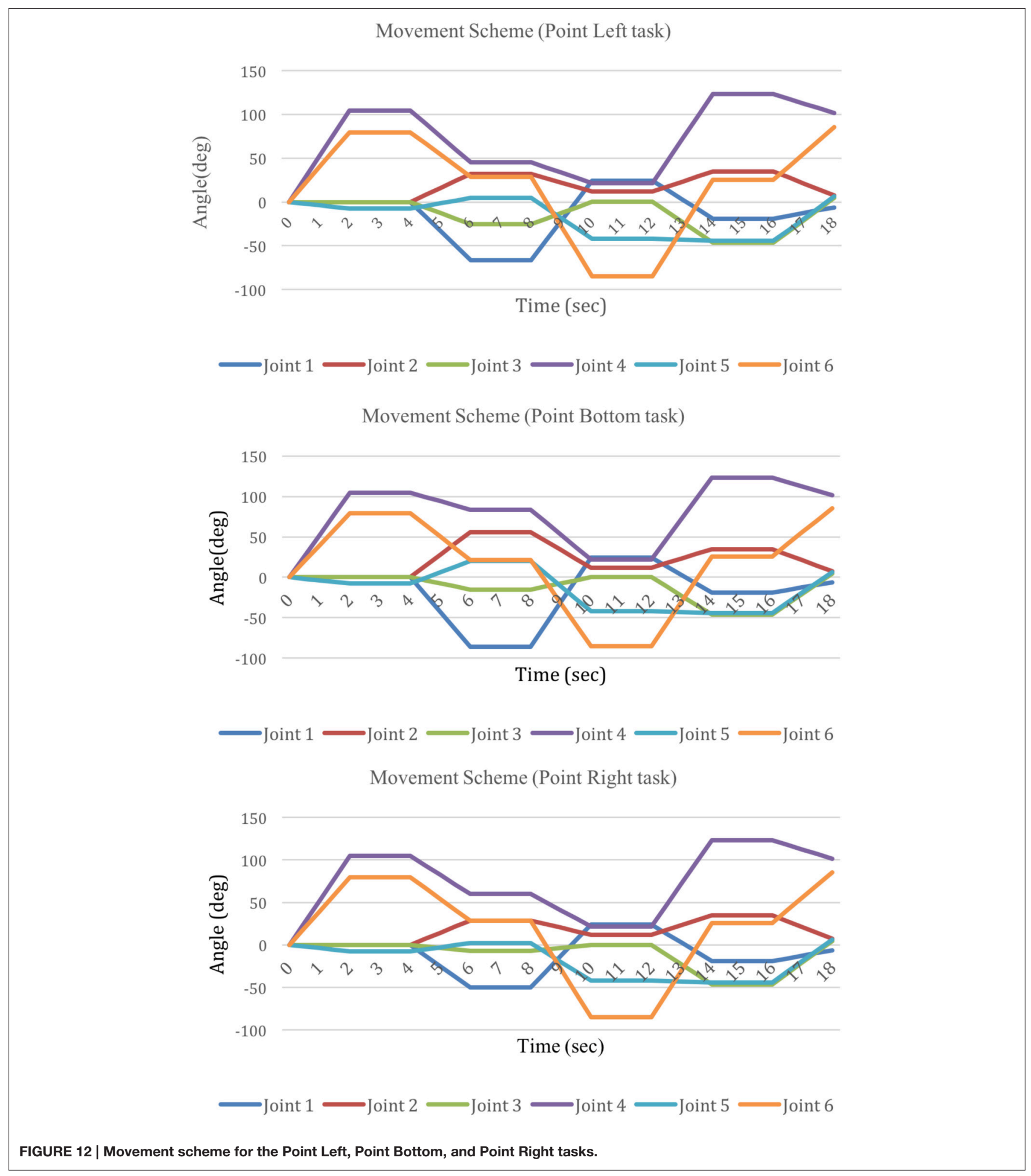

between which there is a wave trough. Meanwhile there is only one wave crest in the $\mathrm{N}$-curve.

In the experiment, this difference involves three kinds of conditions. The first is whether the subjects have practiced with the device and the task. When subjects performed the fixed cup point task without any practice, the angular velocity varied in the M-curve mode. Meanwhile, the angular velocity varied in the $\mathrm{N}$-curve mode after subjects experienced the practice. The 

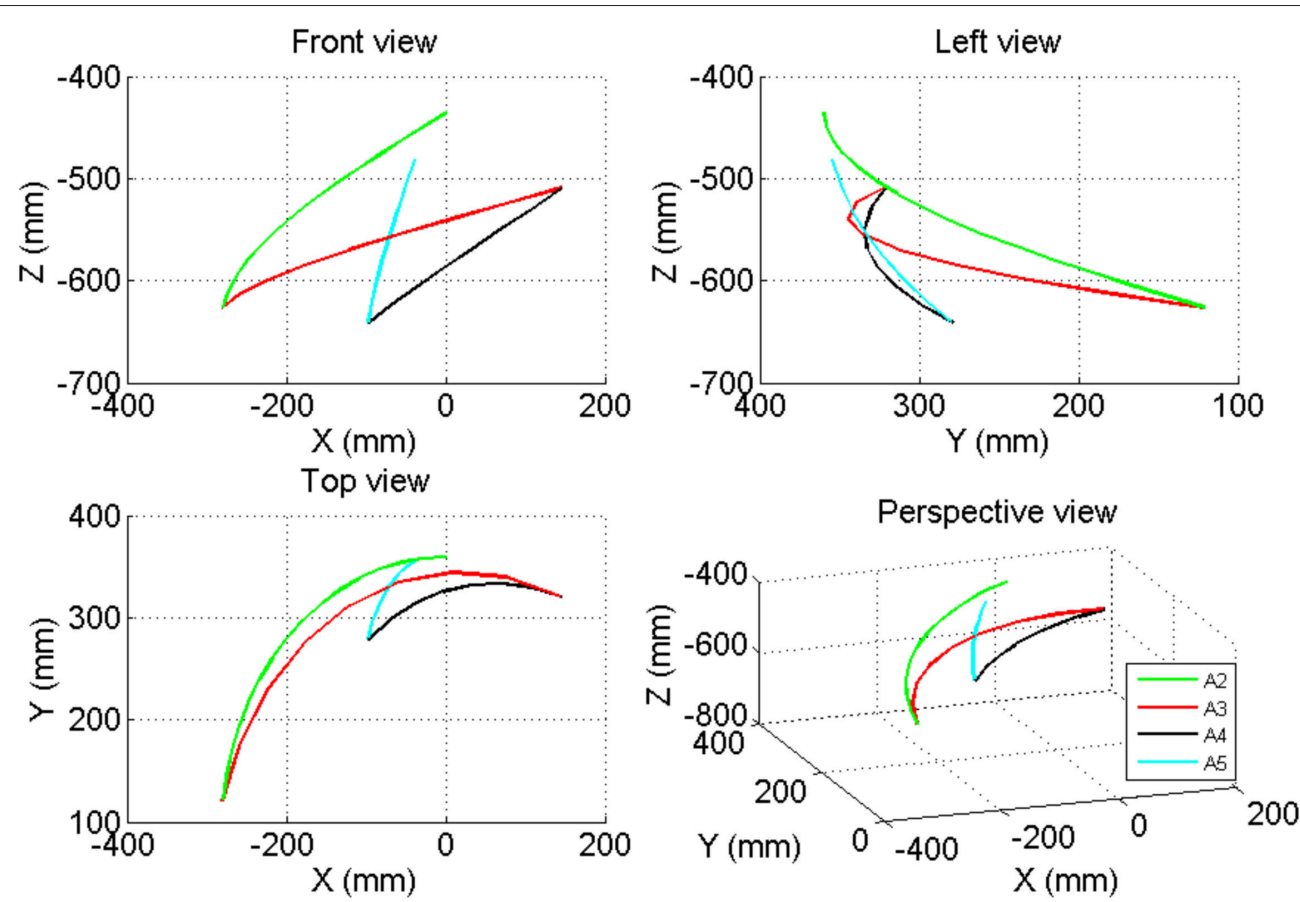

FIGURE 13 | The 3-D trajectory of the movement of the elbow joint in the Point Left task.
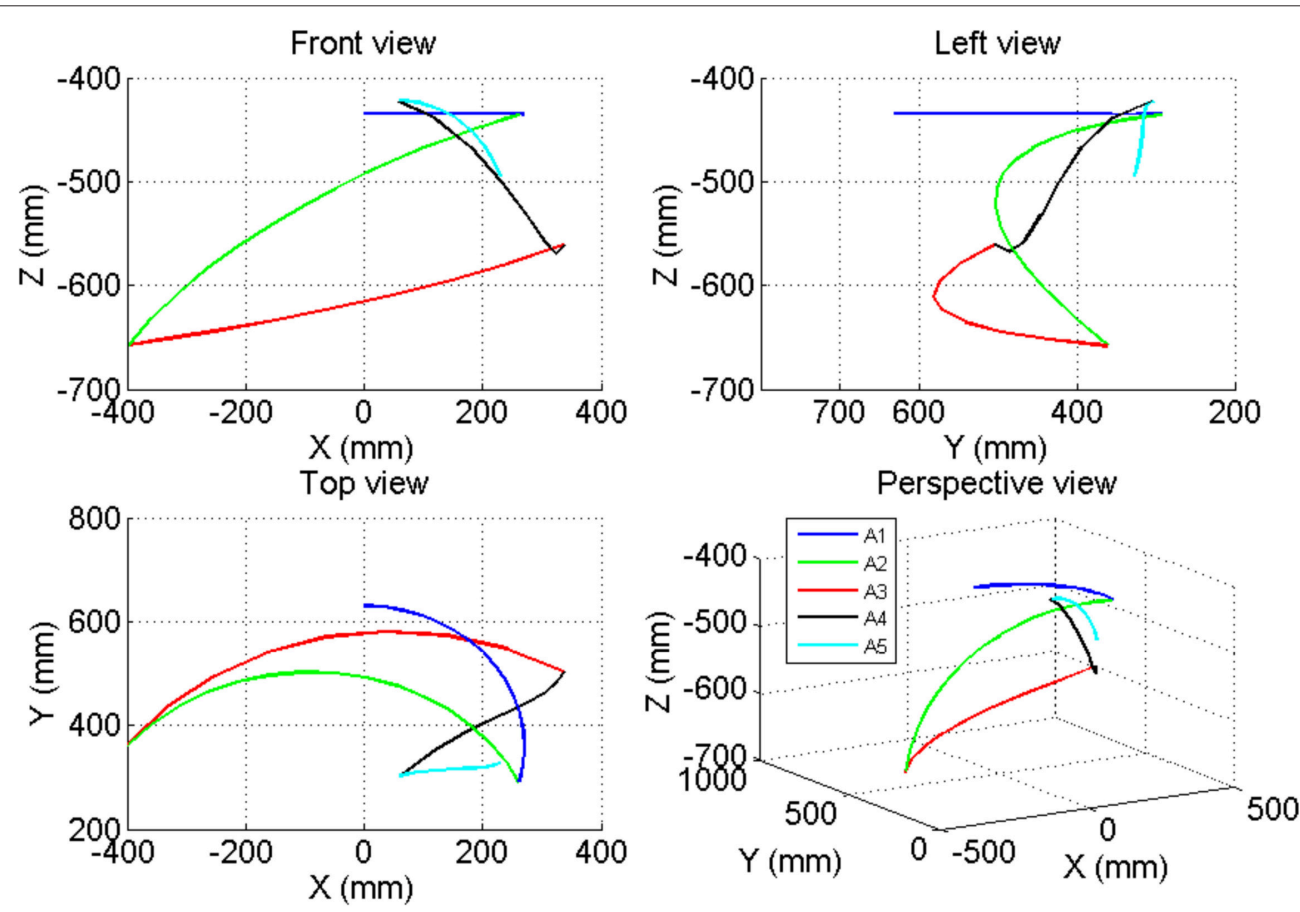

FIGURE 14 | The 3-D trajectory of the movement of the wrist joint in the Point Left task.

reason for this phenomenon is that the practice made subjects more skillful in the task. The more skilled the subjects became, the more smoothly they moved.
Second, even after practice, when the testing mode was changed from a fixed cup point to random cup points, the angular velocity curve appeared to be in the M-curve mode. This is 

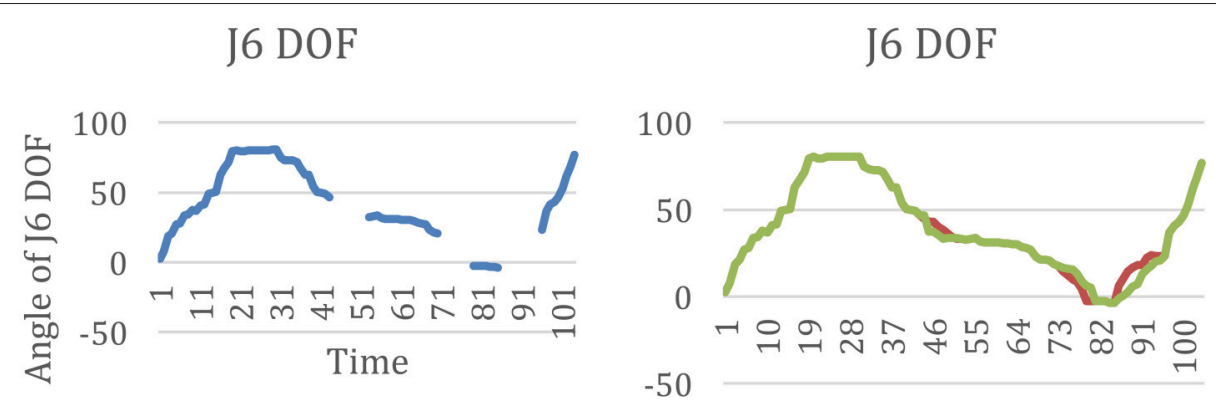

FIGURE 15 | (A) The lost points of J6 DOF in the movement before simulation from Vicon, (B) the two results of the simulation motion data of J6 from Vicon.
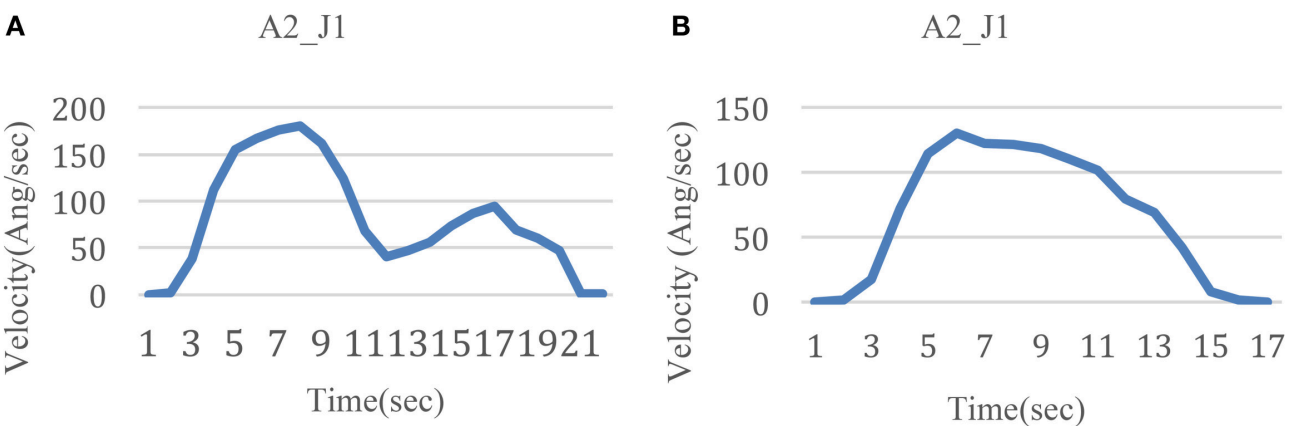

FIGURE 16 | Two modes of velocity variation. (A) M-curve for J1 during A2 (B) N-curve for J1 during A2.

because compared with the fixed cup point task, mechanical repetition was avoided. And the more familiar they became with the task, the less their conscious thought process was involved. However, when performing the random cup point task, subjects had to focus on the task goal and made corresponding movement adjustments. As such, the random cup point task motivated their conscious thought process more than the fixed cup point.

Third, this difference occurred obviously in J1 and J4 during A2 and A3. This is because horizontal movements played a main role in the two actions. The range of abduction and adduction of J1 for the shoulder was large. Other movements of joints were also accompanied during the same period, which lead to a reduction in the movement smoothness.

\section{Comparison between the Three Points}

The functional movement in daily life is not limited in fixed posture and motion. In order to provide a task with different range of motion (ROM), we proposed three cup points in this research. Through the three points, the subject need to move their upper limb in different ROM. At each point, the posture of the upper limb is distinctive. In the task, according to the different points, it is shown that the angle of each DOF is distinctive at each posture, see Figures 6-10.

Meanwhile, when the task is set with one cup point, the subject can be familiar with the task soon, and form a mechanical movement, without the active consciousness. But in the task of random points mode, the velocity of DOF varied in a M-curve type, see Figure 16. It is considered that this velocity variation mode is caused by the active consciousness from the subject. So, the three points task is one solution for the upper limb active training. In this case, the three points movement scheme should be obtained from the research, see Figure 12.

\section{Movement Scheme}

For Point Left task, the time length of the period of hovering is set at two seconds. During this time interval, all the joints should be kept still. This period can be prolonged as required. But it is still necessary that each interval last for at least $2 \mathrm{~s}$. This time period is set for the subject to adjust his/her movement. Since A1 is for reparation and $\mathrm{A} 5$ is for backing, which includes simple rotation for DOF, it is detailed discussed on A2, A3, and A4 as below.

In the scheme, A2 lasts for $2 \mathrm{~s}$. The objective is to open the upper limb to reach a cup placed on the left side. This action is designed in two parts. For 4-6s, the shoulder extends slightly at a low velocity. The shoulder abduction is accomplished within the first $1 \mathrm{~s}$, and the forearm pronation is accomplished within the first $0.5 \mathrm{~s}$. This forearm pronation is needed to adjust the wrist at an angle by which the fist can move along the tangential direction of the space trajectory. In this way, the forearm can move more easily and fluently. The elbow merely extends slightly, while the forearm follows the shoulder extension. For 6-8s, the shoulder abduction stops at the maximum angle. The elbow is pressed down as a result of shoulder external rotation and extension, which is accompanied by wrist radial deviation. At the same time, the elbow extends with vast angular displacement at a high velocity. It is obvious that during the A2 period, shoulder external 
rotation and elbow extension occur near the end of shoulder abduction.

A3 lasts for $2 \mathrm{~s}$. First, for 8-10 s, the elbow moves up with the assistance of the gravity compensation mechanism by means of shoulder flexion and internal rotation. Meanwhile, the elbow has slight flexion. Then, for 9-10 s, the shoulder accomplishes the adduction swiftly while the elbow finishes the remaining part of the flexion. A4 lasts for 12-14 s. During this period, the shoulder abducts greatly and the elbow flexes to move the hand to the mouth position to maintain a drinking gesture. The forearm pronates at an angle to perform the gesture of maintaining the cup.

This movement scheme can be stored as a training task on a computer and applied for upper limb rehabilitation training with exoskeleton robots.

\section{CONCLUSION}

In the research, subjects were instructed to move the left upper limb from the center at the front of the chest to the left side, and then from the left side to the right side, and then back to the body. During these movements, subjects were inclined to adjust the upper arm to lead the distal joints when the task was to move the upper limb from a proximal to a distal location. Meanwhile, subjects were inclined to adjust the distal joints when the task was to move the upper limb from a distal to a proximal location.

Motion data were obtained using two systems, Neo-Arm and Vicon. The motion of each DOF of the upper arm was directly

\section{REFERENCES}

Ball, S. J., Brown, I. E., and Scott, S. H. (2007). "MEDARM: a rehabilitation robot with 5DOF at the shoulder complex," in IEEE/ASME International Conference on Advanced Intelligent Mechatronics (Zurich).

Chang, Y. J., Han, W. Y., and Tsai, Y. C. (2013). A Kinect-based upper limb rehabilitation system to assist people with cerebral palsy. Res. Dev. Disabil. 34, 3654-3659. doi: 10.1016/j.ridd.2013.08.021

Chung, W. M., Yeung, S., Pak, C. H., and Lee, R. (2011). Repeatability of vicon motion analysis system for upper limb kinematic measurement during fencing lunge action. Hong Kong Physiother. J. 29, 96. doi: 10.1016/j.hkpj.2011.08.011

Coote, S., Murphy, B., Harwin, W., and Stokes, E. (2008). The effect of the GENTLE/s robot-mediated therapy system on arm function after stroke. Clin. Rehabil. 22, 395-405. doi: 10.1177/0269215507085060

Gopura, R. A. R. C., Kiguchi, K., and Li, Y. (2009). "SUEFUL-7: A 7DOF upperlimb exoskeleton robot with muscle-model-oriented EMG-based control," in IEEE/RSJ International Conference on Intelligent Robots and Systems (St. Louis, MO).

He, J., Koeneman, E., Schultz, R., Herring, D., Wanberg, J., Huang, H., et al. (2005). "RUPERT: a device for robotic upper extremity repetitive therapy," in 27th Annual International Conference of the Engineering in Medicine and Biology Society (Shanghai).

Henmi, S., Yonenobu, K., Masatomi, T., and Oda, K. (2014). A biomechanical study of activities of daily living using neck and upper limbs with an optical three-dimensional motion analysis system. Mod. Rheumatol. 16, 289-293. doi: 10.3109/s10165-006-0499-x

Holt, R., Makower, S., Jackson, A., Culmer, P., Levesley, M., Richardson, R., et al. (2007). "User involvement in developing rehabilitation robotic devices: an essential requirement," in IEEE 10th International Conference on Rehabilitation Robotics (Noordwijk). captured by Neo-Arm, while with Vicon, it was calculated by the trajectories of reflective marks. The results indicate that the NeoArm can achieve the suitable precision and be fit for other kinds of upper limb motion tasks. The motion data of three points in task are captured and stored as a scheme in the task library on a computer.

\section{AUTHOR CONTRIBUTIONS}

LL: main author who have did this research and wrote the manuscript. LX: the doctoral supervisor of the LL and YS, who proposed the research and direct us in the experiment. YS: assist in the experiment and part of work in data processing. BA: proposed the opinions from the medicine, and proposed the control experiment.

\section{ACKNOWLEDGMENTS}

The work described in this paper was supported by The project of National Natural Science Foundation of China (No. 61672341), The Major Program of National Natural Science Foundation of China (No. 61190124, 61190120), National High Technology Research and Development Program of China (No. 2015AA043203), The project of the Science and Technology Commission of Shanghai Municipality (14441900800, 14DZ1941100, 14DZ1941103, 15DZ1942103), National Key Technology Support Program (No. 2009BAI71B06).

Housman, S. J., Le, V., Rahman, T., Sanchez, R., and Reinkensmeyer, D. J. (2007). "Arm-training with T-WREX after chronic stroke: preliminary results of a randomized controlled trial," in IEEE 10th International Conference on Rehabilitation Robotics (Noordwijk).

Krebs, H. I., Ferraro, M., Buerger, S. P., Newbery, M. J., Makiyama, A., Sandmann, M., et al. (2004). Rehabilitation robotics: pilot trial of a spatial extension for MIT-Manus. J. Neuroeng. Rehabil. 1:5. doi: 10.1186/1743-0 003-1-5

Lum, P. S., Burgar, C. G., Loos, M. V. D., Shor, P. C., Majmundar, M., and Yap, R. (2006). MIME robotic device for upper-limb neurorehabilitation in subacute stroke subjects: a follow-up study. J. Rehabil. Res. Dev. 43:631. doi: 10.1682/JRRD.2005.02.0044

Lum, P. S., Burgar, C. G., and Shor, P. C. (2004). Evidence for improved muscle activation patterns after retraining of reaching movements with the MIME robotic system in subjects with post-stroke hemiparesis. IEEE Trans. Neural Syst. Rehabil. Eng. 12, 186-194. doi: 10.1109/TNSRE.2004.8 27225

Mihelj, M., Nef, T., and Riener, R. (2007). “ARMin II - 7 DoF rehabilitation robot: mechanics and kinematics," in IEEE International Conference on Robotics and Automation (Roma).

Nef, T., Guidali, M., and Riener, R. (2009). ARMin III - arm therapy exoskeleton with an ergonomic shoulder actuation. Appl. Bionics Biomech. 6, 127-142. doi: $10.1155 / 2009 / 962956$

Nef, T., and Riener, R. (2005). "ARMin - design of a novel arm rehabilitation robot," in 9th International Conference on Rehabilitation Robotics (Chicago).

Park, H.-S., Ren, Y., and Zhang, L.-Q. (2008). "IntelliArm: an exoskeleton for diagnosis and treatment of patients with neurological impairments," in 2nd IEEE RAS \& EMBS International Conference on Biomedical Robotics and Biomechatronics (Scottsdale, AZ). 
Stienen, A. H. A., Hekman, E. E. G., Prange, G. B., Jannink, M. J. A., Aalsma, A. M. M., van der Helm, F. C. T., et al. (2009). Dampace: design of an exoskeleton for force-coordination training in upper-extremity rehabilitation. J. Med. Devices 3:031003. doi: $10.1115 / 1.3191727$

Uttarwar, P., and Mishra, D. (2015). "Development of a kinect-based physical rehabilitation system," in Third International Conference on Image Information Processing (Shimla).

Yu, H., Xie, L., Lv, C., Shao, W., Wang, Y., Wang, J., et al. (2015). A system for upper limb rehabilitation and motor function evaluation. J. Mech. Med. Biol. 15:1550010. doi: 10.1142/S0219519415500104
Conflict of Interest Statement: The authors declare that the research was conducted in the absence of any commercial or financial relationships that could be construed as a potential conflict of interest.

Copyright (c) $2017 \mathrm{Liu}, \mathrm{Xie}$, Shi and An. This is an open-access article distributed under the terms of the Creative Commons Attribution License (CC BY). The use, distribution or reproduction in other forums is permitted, provided the original author(s) or licensor are credited and that the original publication in this journal is cited, in accordance with accepted academic practice. No use, distribution or reproduction is permitted which does not comply with these terms. 


\section{OPEN ACCESS}

Edited by:

Wenwei Yu,

Chiba University, Japan

Reviewed by:

Giancarlo Ferrigno,

Polytechnic University of Milan, Italy

Waldemar Karwowski,

University of Central Florida, USA

*Correspondence:

Christopher Jarrett

cjar160@aucklanduni.ac.nz

Specialty section: This article was submitted to

Neural Technology,

a section of the journal

Frontiers in Neuroscience

Received: 22 November 2016

Accepted: 15 February 2017

Published: 14 March 2017

Citation:

Jarrett C and McDaid A (2017) Virtual Normalization of Physical Impairment:

A Pilot Study to Evaluate Motor

Learning in Presence of Physical Impairment. Front. Neurosci. 11:101.

doi: 10.3389/fnins.2017.00101

\section{Virtual Normalization of Physical Impairment: A Pilot Study to Evaluate Motor Learning in Presence of Physical Impairment}

\author{
Christopher Jarrett * and Andrew McDaid \\ Department of Mechanical Engineering, Faculty of Engineering, University of Auckland, Auckland, New Zealand
}

Motor learning is a critical component of the rehabilitation process; however, it can be difficult to separate the fundamental causes of a learning deficit when physical impairment is a confounding factor. In this paper, a new technique is proposed to augment the residual ability of physically impaired patients with a robotic rehabilitation exoskeleton, such that motor learning can be studied independently of physical impairment. The proposed technique augments the velocity of an on-screen cursor relative to the restricted physical motion. Radial Basis Functions (RBFs) are used to both model velocity and derive a function to scale velocity as a function of workspace position. Two variations of the algorithm are presented for comparison. In a cross-over pilot study, healthy participants were recruited and subjected to a simulated impairment to constrain their motion, imposed by the cable-driven wrist exoskeleton. Participants then completed a sinusoidal tracking task, in which the algorithms were statistically shown to augment the cursor velocity in the constrained state such that it matched position-dependent velocities recorded in the healthy state. A kinematic task was then designed as a motor-learning case study where the algorithms were statistically shown to allow participants to achieve the same performance when their motion was constrained as when unconstrained. The results of the pilot study provide motivation for further research into the use of this technique, thus providing a tool with which motor-learning can be studied in neurologically impaired populations. This could be used to give physiotherapists greater insight into underlying causes of motor learning deficits, consequently facilitating and enhancing subject-specific therapy regimes.

Keywords: virtual normalization, biomechatronics, robotic assessment, motor learning, rehabilitation robotics

\section{INTRODUCTION}

A major neuroscience and clinical question is how best to invoke motor learning, a critical component of the rehabilitation process (Krakauer, 2006). It is difficult to completely understand why neurologically impaired patients cannot learn to perform certain tasks, as there are many underlying factors that contribute to motor learning deficits. Often the presence of physical impairment, such as issues with strength or range of motion (ROM), can confound results. This can make it unclear whether the inability to perform aspects of a task is physical, a neurological motor learning deficit or a combination of both. 
This is illustrated in multiple domains. One example is the administration of botulinum toxin type A (BoNT-A), commonly used to reduce spasticity in children with cerebral palsy (Boyd and Graham, 1999; Wissel et al., 1999; Wallen et al., 2004; FattalValevski et al., 2008, 2010; Molenaers et al., 2009, 2010; Tedroff et al., 2009). BoNT-A addresses issues due to neural spasticity and in cases where patients exhibit motion impairment after injections, it can be unclear whether this is a result of dosage, issues with the structural properties of muscle, or a fundamental incapacity for neural plasticity and motor learning. Similarly confounding issues have been reported in other studies, alluding to the uncertainty of drawing conclusions when motor learning appears poor in children with CP (van Abswoude et al., 2015). Self-exploration and problem solving are critical to the motor learning process, but these can be impacted by motor difficulties, preventing optimal learning (Valvano and Rapport, 2006).

The emergence of bio-mechatronics systems, specifically rehabilitation robotics, has provided researchers with a new suite of tools for interacting with humans and hence the ability to investigate the fundamental mechanisms of rehabilitation and motor learning (Boudreau et al., 2010; Turner et al., 2013). Robots have served as a useful research platform, igniting debate about the most efficient way to stimulate motor learning. Some authors promote the well-developed "assist-as-needed" (AAN) paradigm (Jezernik et al., 2004) while others suggest that erroraugmentation (EA) is more effective (Patton et al., 2006). The most recent evidence suggests that the answer depends on a range of factors, from frequency of feedback to initial skill level (Wulf et al., 2010; Burtner et al., 2014; Marchal-Crespo et al., 2015; Fujii et al., 2016). In other words, the potential for motor learning is largely subject-dependent. Moreover, recent reviews indicate that a gap exists between current technology and true clinical needs (Cordella et al., 2016).

In order to optimize rehabilitation a therapist must better comprehend the fundamental subject-specific causes of motion deficit (Lipp and Tomassini, 2015). Recent studies have investigated the separation of neural components of torque such as spasticity, from non-neural components such as muscular stiffness (Bar-On et al., 2014, 2015; Lin et al., 2016). While this provides a valuable analysis of motion, and enables the effectiveness of various treatments to be analyzed, in these studies the cognitive ability to motor learn was not considered as a component of observed motion. Given the importance of motor learning in rehabilitation and to provide further insight into the causes of motion issues, it would be useful to evaluate motor learning independently of confounding physical impairment.

Melendez-Calderon et al. (2015) conducted a study which demonstrated motor learning could be achieved without physical motion. This built on studies focused on adaptation to environments after virtual training with visual feedback (Sarlegna et al., 2010; Melendez-Calderon et al., 2011; Rotella et al., 2014). In the case of physical impairment force production might be limited, which could affect both virtual exploration and conclusions regarding motor learning. Participants' motion could also be scaled in a purely kinematic sense and with no regard to dynamic models, as was previously used to investigate transfer of knowledge between scaling conditions (Ojakangas and Ebner,
1991; Smiley-Oyen et al., 2003; Paz et al., 2005). To the best of our knowledge no conclusive method has been developed to specifically study motor learning independently of physical impairment.

In this study, we ask whether it is possible to use a robotic exoskeleton as a tool to evaluate the motor learning capacity of an impaired individual by normalizing their physical abilities in a virtual environment and investigating their ability to learn a task in this environment. The robotic exoskeleton rehabilitation device is used to simulate a physical impairment on healthy participants and visually augment the residual motion on a display; thus "normalizing" the "impairment". The "impairment" is manifested as a set of constraints on motion. A case study is presented, where we hypothesize that the virtual normalization of participants' abilities can be used to evaluate the cognitive ability to motor learn irrespective of physical impairment. The exoskeleton will therefore be used as a tool to diagnose sources of motion deficit and evaluate the motor learning capacity of impaired individuals, serving as a valuable aid for clinicians to create optimized, subject-specific therapy routines. This also has potential to lead to enhanced and harmonized human-robotic interaction for people with impairment. Moreover, it provides a platform for further research into motor learning in the presence of impairment.

\section{MATERIALS AND METHODS}

\section{System Architecture and Model of Learning}

The architecture of the entire human-exoskeleton system proposed in this paper is described in Figure 1, with the human represented by the blocks encompassed by the dashed lines.

A movement is planned, resulting a motor command which is translated into a desired Cartesian coordinate, $\underline{x}_{d}$ which is converted via inverse kinematics to a desired joint angle(s), $\underline{\theta}_{d}$. The Neuromuscular System block encompasses the neuromuscular dynamics of the human, the output of which is a wrist torque, $\underline{\tau}_{w}$. This human torque along with the external torque exerted on the wrist by the robotic exoskeleton, $\underline{\tau}_{e x}$, determine the wrist kinematics. The human and robot torques are fed back internally to inform the human's motor planning. The exoskeleton torque is comprised of the resistive torque due to the constraints imposed by the simulated impairment, while the Neuromuscular System block encompasses any real impairment, which then affects the generated wrist torque.

As the wrist is coupled to the exoskeleton, the angle of the exoskeleton is equal to the angle of the wrist, $\underline{\theta}_{w}$. This kinematic output, $\underline{\theta}_{w}$, is converted into Cartesian coordinates, $x_{w}$, then passed through the normalization (which is unity in the healthy state) to be presented visually on screen as feedback (in the form of the cursor) to the participant, $\underline{x}_{c}$. The behavior of the dynamic target, $\underline{x}_{t}$, is related to the normalized wrist kinematics by a simulated model, in this case a viscous damper. The target and cursor locations are viewed by the human to inform motor planning for the subsequent motion. This has been intentionally modeled as a black-box because, while it is understood the relationship between wrist movement and corresponding target movement can be learnt, literature 


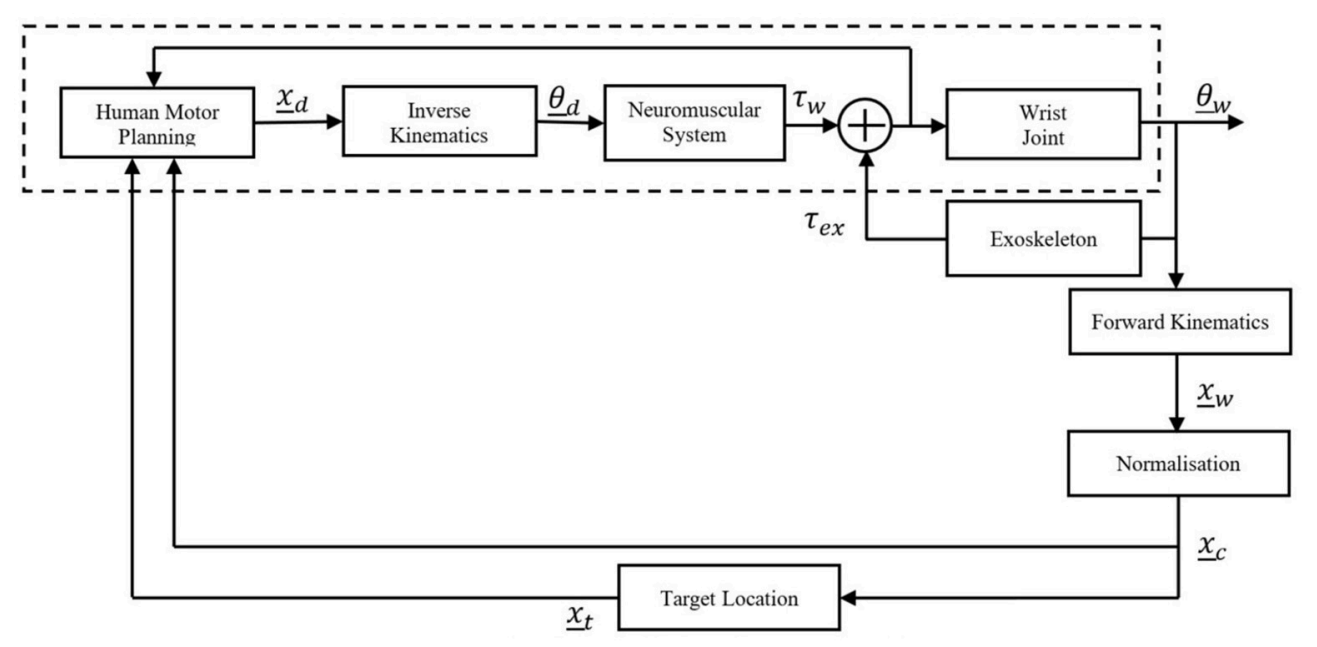

FIGURE 1 | Human-robot system architecture.

documents multiple mechanisms by which learning occurs (Haith and Krakauer, 2013) and this is not of direct relevance to this work.

\section{Participants}

A convenience sample of 10 participants, 8 male and 2 female, with no cognitive or physical impairments were recruited for the study. The study was approved under the guidelines of the University of Auckland Human Participants Ethics Committee (UAHPEC), reference number \#013729 and written and informed consent was obtained from all participants in accordance with these guidelines. Study size was considered sufficient, given the similarity of the results in the healthy population and exploratory nature of the study, where the aim was to technically and experimentally prove the concept of virtual normalization. All participants were coincidentally dominant in the right hand.

\section{Exoskeleton Device}

The 3 degree-of-freedom (DOF) AW-TEX robotic wrist exoskeleton was used in this study (McDaid, 2015). Flexion/extension (FE) and radial/ulnar deviation (RU) are actively controlled. The third DOF is passive, designed to accommodate forearms of differing lengths. The exoskeleton is shown in Figure 2.

Series Elastic Actuators (SEAs) are used at the active joints and are driven by remotely located motors with Bowden cables. An inner controller based on sliding mode control regulates the relative displacement of the SEA components, thus controlling the torque in an impedance-based architecture (Jarrett and Mc Daid, 2017).

\section{Implementation of Simulated Impairment}

The present study is performed with healthy participants with a simulated CP-like impairment so that we can use each person as their own control in a cross-over study. CP describes a group of disorders with a variety of phenotypic manifestations and studies have varied in their attempts to describe the "average" kinematics of people with cerebral palsy (Barroso et al., 2011; Klingels et al., 2012). In this study, the impairment is modeled with two components: (i) a simulated contraction, implemented by a linear torque spring restricting motion in the extension direction from $5^{\circ}$; (ii) a velocity-dependent torque, simulating restricted torque production and applied throughout the workspace. The simulated impairment is manifested as a set of "constraints," and is mathematically described in Equations (1) and (2).

$$
\begin{gathered}
\tau_{f e}=\left\{\begin{array}{c}
-\operatorname{sgn}\left(\dot{\theta}_{f e}\right)\left[K_{p}\left(\theta_{f e}-5\right)+K_{d} \dot{\theta}_{f e}+K_{v} \dot{\theta}_{f e}\right], \theta_{f e}>5^{o} \\
-s g n(1)
\end{array}\right. \\
\left.\tau_{r u}=-\operatorname{sgn}\left(\dot{\theta}_{f e}\right)\left[K_{v} \dot{\theta}_{f e}\right], \theta_{f e}<5_{v} \dot{\theta}_{r u}\right], \forall \theta_{r u}
\end{gathered}
$$

where $\tau_{f e}$ and $\tau_{r u}$ are the torques applied to the wrist in the flexion/extension and radial/ulnar deviation axes, $\theta_{f e}$ and $\theta_{r u}$ are the flexion/extension and radial/ulnar deviation angles of the wrist, $\dot{\theta}_{f e}$ and $\dot{\theta}_{r u}$ are the angular velocities of the wrist in the flexion/extension and radial/ulnar deviation axes, $K_{p}$ and $K_{d}$ are the stiffness and damping of the wall that restricts the ROM; set to $2 \mathrm{Nmdeg}^{-1}$ and $0.1 \mathrm{Nmsdeg}^{-1}$ respectively, $K_{v}$ is the gain applied to implement the velocity-based resistance; set to $0.02 \mathrm{Nmsdeg}^{-1}$ and $s g n$ is the sign function. Operation of the exoskeleton in this mode is hereafter referred to as the "constrained" state.

\section{Impairment Assessment}

A calibration routine was developed to evaluate the taskindependent physical ability of patients in the healthy (exoskeleton in backdriven mode) and constrained states. This extracted three subject-specific measurements:

(1) Self-selected neutral position. These coordinates were used as the participants' origins for the remainder of the experiments.

(2) ROM, obtained by asking participants to move their wrist as far as possible in each direction. 


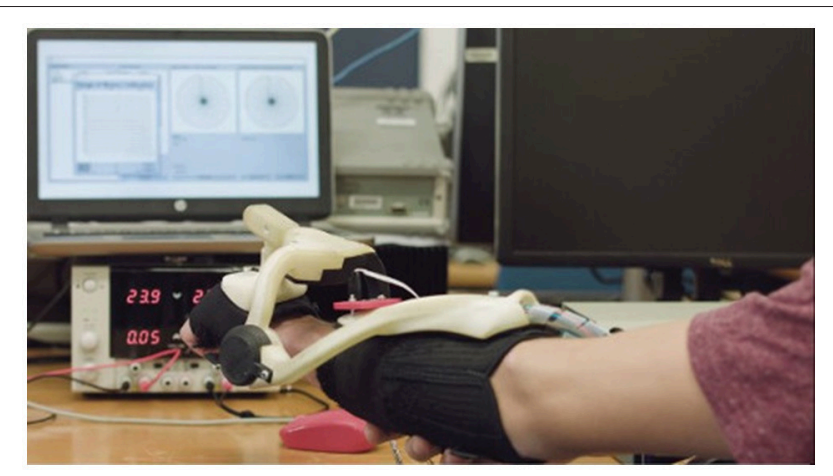

FIGURE 2 | AW-TEX Wrist Exoskeleton.

(3) Position-dependent velocity, this was recorded by asking participants to perform eight blocks of five linear movements, alternating between the flexion/extension and radial/ulnar deviation axes, (a total of four blocks per axis). Figure 3 illustrates the visual representation of the workspace shown to participants, displayed as nodes placed at $10^{\circ}$ intervals in the workspace measured by the preceding test. They were the locations at which average velocities were measured. One minute rest periods were enforced after four blocks of movement to eliminate effects of fatigue. The routine was completed twice: first in the healthy state, then with the constraints applied and separated by a 2 min break.

\section{Kinematic Normalization}

To normalize the ability of the participants in their constrained states, two steps were required. The first is to shift the position of the cursor such that the constrained ROM centers on $(0$, 0 ). The second step is to apply a position-dependent scaling factor to the velocity of the cursor such that it matches the position-dependent velocity recorded in the healthy state. This was achieved with the aid of Gaussian Radial Basis Functions (GRBFs). GRBFs are advantageous due to their ability to describe any continuous function; and have been used in other robotic rehabilitation research to model patient torque as a function of workspace position (Wolbrecht et al., 2008; Oboe and Pilastro, 2014; Pehlivan et al., 2015). The nodes at which velocities were recorded during the calibration phase served as the locations of the RBFs.

The first step was to compress and shift the node coordinates in the healthy workspace into the constrained workspace, illustrated by the green plot in Figure 4. The compressed healthy $\mathrm{RBF}$ was then evaluated at the nodes describing the constrained workspace. This allows the calculation of the ratio of healthy to constrained velocity at each of the constrained velocity nodes, as described by Equation (3).

$$
S F_{n}=\frac{v_{h n}}{v_{i n}}
$$

where $S F_{n}$ is the scaling factor at the $n^{\text {th }}$ constrained node, $v_{h n}$ is the estimated healthy velocity at the $n^{\text {th }}$ constrained node

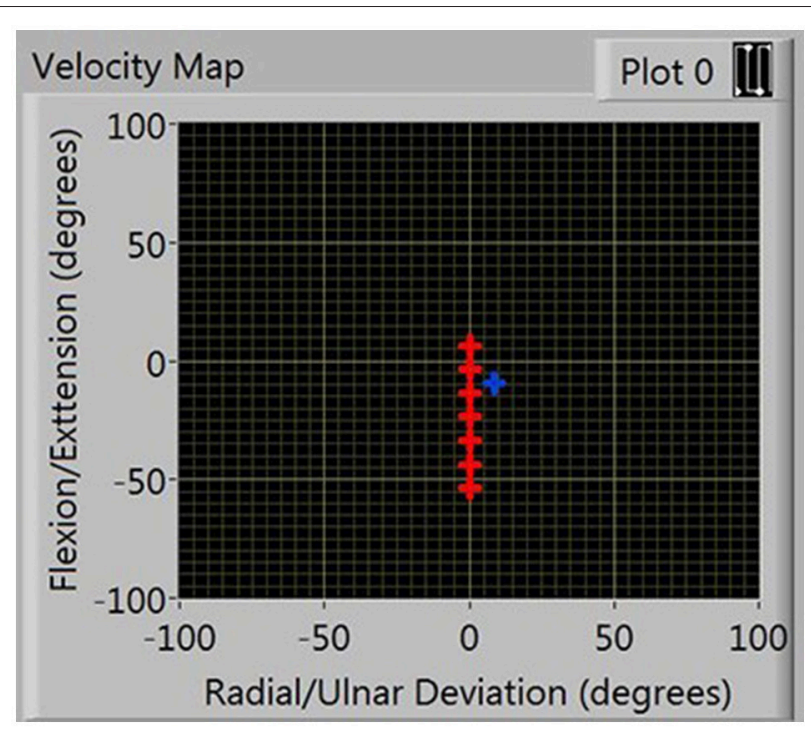

FIGURE 3 | Presentation of nodes in FE workspace during velocity calibration.

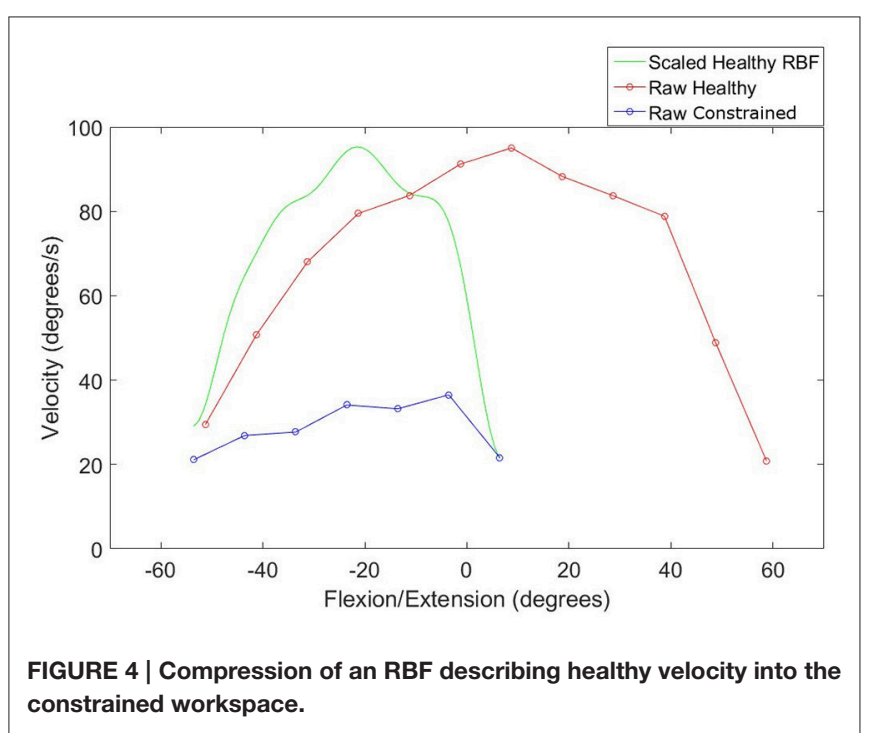

and $v_{i n}$ is the constrained velocity at the $n^{\text {th }}$ constrained node. This creates a discrete set of scaling factors which are used to create a second RBF that normalizes velocity in the constrained workspace. The RBF allows the algorithm to capture velocity characteristics that vary with wrist position.

Equation (4) describes the RBF that was used to derive the scaling factor:

$$
S F_{j}\left(\theta_{j}\right)=Y a+w
$$

where $S F_{j}\left(\theta_{j}\right)$ is the scaling factor as a function of motion at joint $j, \theta_{j}, Y$ is the regressor matrix, composed of Gaussian basis functions as illustrated in Equation (5), $a$ is a set of weightings applied to each basis function and $w$ is a linear bias term applied 
to the output layer, calculated by multiplying an additional weighting coefficient by the current position.

$$
Y=\left[\begin{array}{cc}
g_{1} & 0 \\
0 & g_{n}
\end{array}\right]
$$

where $n$ is equivalent to the number of nodes that were obtained in the ROM test and $g_{n}$ refers to the Gaussian radial basis function, defined in Equation (6).

$$
g_{n}=e^{-\frac{d_{n}^{2}}{2 \sigma^{2}}}
$$

where $e$ is the exponential function, $d_{n}$ is the distance of the cursor from the nth node and $\sigma$ is the width of each basis function; defined as the average distance between nodes.

Equation (4) contains a linear bias term, the purpose of which is to reproduce global behavior of the RBF function (Li and Verma, 2015). In previous work in rehabilitation robotics involving RBFs, the linear bias term was omitted (Wolbrecht et al., 2008; Oboe and Pilastro, 2014; Pehlivan et al., 2015). In this research, we set the value of $w$ to 0 in the RU axis, to mirror the previous studies and investigate if the linear bias term can significantly improve the normalization algorithm.

An example of the resulting scaling factor is illustrated in Figure 5 for both axes.

\section{Validation of Normalization Algorithms}

To validate the normalization, participants were subjected to the constraints with their cursor velocity normalized by the subjectspecific scaling factor RBF. They were asked to try to track the moving target, which was a recording of their movement during the healthy calibration. The velocity of the cursor was recorded for comparison with the velocity of the healthy movement. Four movement blocks were performed, in the same structure as the calibration routine.

\section{Case Study: Motor Learning Dynamic Cursor Model}

To evaluate the use of the algorithm in conducting kinematic motor learning experiments a task where the target has a massdamper relationship with the cursor is implemented. The aim was to prove that when their constrained velocity was normalized, participants could perform the task to the same standard with the same amount of learning as they did in the healthy state. Eight participants, six males, and two females, continued onto this phase of the study.

\section{Study Design}

The participants were randomly separated into two groups, to conduct a cross-over study. Each group was tested for performance in the task across two sessions; each conducted in one of the healthy and constrained states. Each session consisted of a familiarization phase, followed by three blocks of movement in an acquisition phase, details below. Group A conducted the first session in the healthy state, followed by the constrained state 1 week later and vice versa for Group B. The design is illustrated in Figure 6. In the constrained state, participants' residual motion was augmented to allow them to reach the same velocity in task space as they could under "healthy" states. The 1 week break between experimental states served as a washout period, similar to comparable motor learning crossover studies (Abdollahi et al., 2014).

\section{Task Description}

Participants were seated in front of a computer screen, upon which their wrist position was represented by a blue cursor and a target was illustrated in green, Figure 7. During task execution, the yellow origin was always visible. The goal of the task was to reach the target, with "success" occurring when participants had been within a $2^{\circ}$ radius for $750 \mathrm{~ms}$. Only one target appeared at a time. Targets were initially placed at a distance equal to $20 \%$ of the smallest ROM measurement across both axes. One participant's targets were placed at $25 \%$ of this distance, due to a small $\mathrm{ROM}\left(\approx 10^{\circ}\right.$ in the radial direction $)$ which would have placed the initial location of the targets within the $2^{\circ}$ threshold for target achievement. To make the task non-trivial and require some level of adaptation, the targets were programmed to be virtually connected to the participants' movement as a function of wrist velocity, according to Equation (5):

$$
\ddot{x}_{t}=\frac{c_{t}\left(\dot{x}_{c}-\dot{x}_{t}\right)}{m_{t}}
$$

where $\ddot{x}_{t}$ is the acceleration of the virtual target, $m_{t}$ is the virtual mass of the target, $c_{t}$ is the damping coefficient, $\dot{x}_{c}$ is the velocity of the cursor and $\dot{x}_{t}$ is the velocity of the target. The movement of the target is thus related to the kinematics of the participants' wrists and the difficulty can be varied. Based on pilot trials the mass and damping were set to $5 \mathrm{~kg}$ and $18 \mathrm{kgms}^{-1}$ respectively, to ensure the task was non-trivial and requires some learning and adaptation. No external torque field was applied (except the constraints) and consequently, the task requires pure kinematic adaptation.

Participants were instructed to: "Try and catch each target by moving toward it. The target will disappear if you have been successful and turn red if you fail to achieve it." They were further informed that a successful reach required that they were over the target for approximately $1 \mathrm{~s}$ and they had $10 \mathrm{~s}$ to reach each target. Targets were considered failed if their position exceeded the outer boundary (illustrated as the red circle in Figure 7) or if the participant had not achieved it within $10 \mathrm{~s}$ of it appearing.

\section{Experimental Procedure Familiarization phase}

Prior to conducting the task, participants underwent a familiarization phase, consisting of reaching 16 randomly ordered, stationary targets in both the positive and negative directions for each axis. Targets were placed at the same distance as for the main task.

\section{Acquisition phase}

Following familiarization, participants performed three blocks of the main task, separated by a 1-3 min break. Both targets were 
A

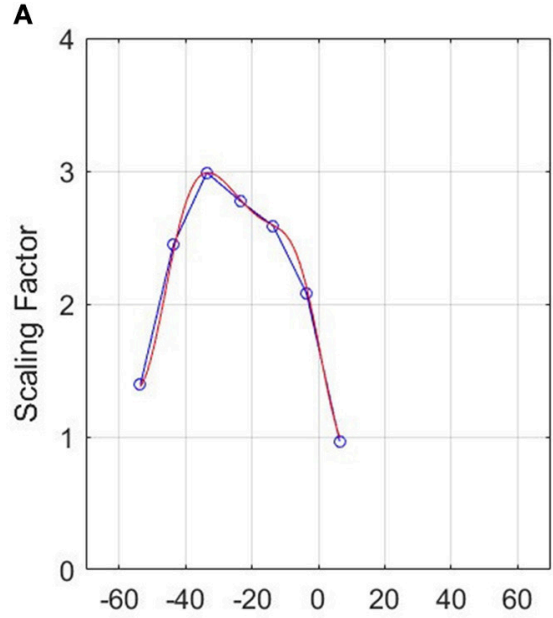

Flexion/Extension (degrees)
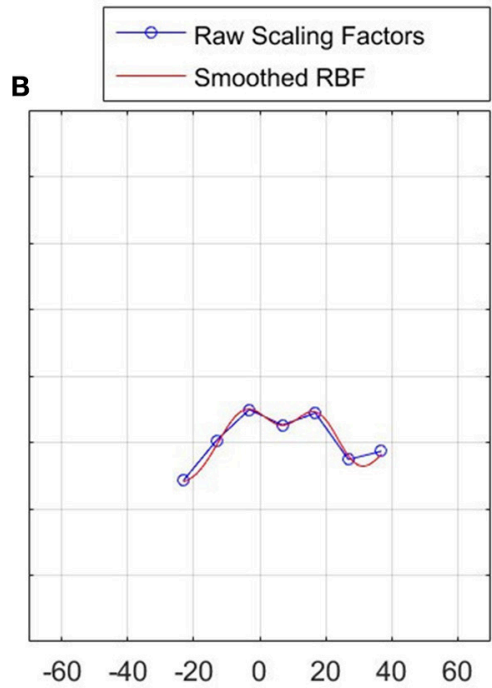

Radial/UInar Deviation (degrees)

FIGURE 5 | Scaling factor function to normalize velocity in the constrained workspace. Left: FE (A), Right: RU (B).

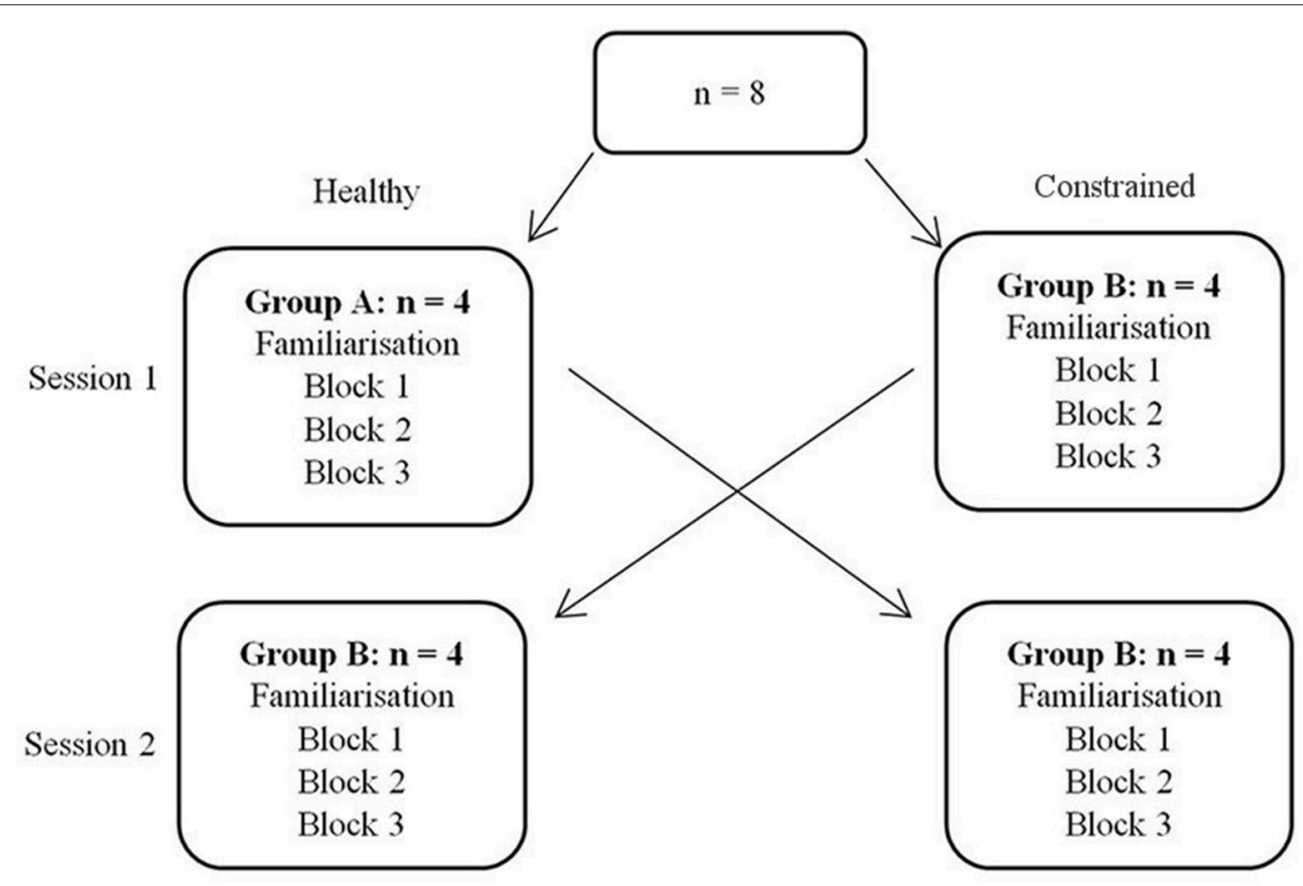

FIGURE 6 | Design of cross-over study.

visited 15 times each, in a randomized order, after which the RU target was made to reappear as a stationary target, serving as a catch trial to test for after-effects. Ideally, there would have been a subsequent catch trial in the FE axis as well; however, this would have been predictable, affected by a prior catch trial in the RU axis and generally confounded results (Focke et al., 2013; Stockinger et al., 2014).

\section{RESULTS}

\section{Validation of Normalization Algorithm from} Calibration

A representative response from the validation of the normalization algorithms is presented in Figure 8. Three metrics from the healthy and constrained velocity profiles are 


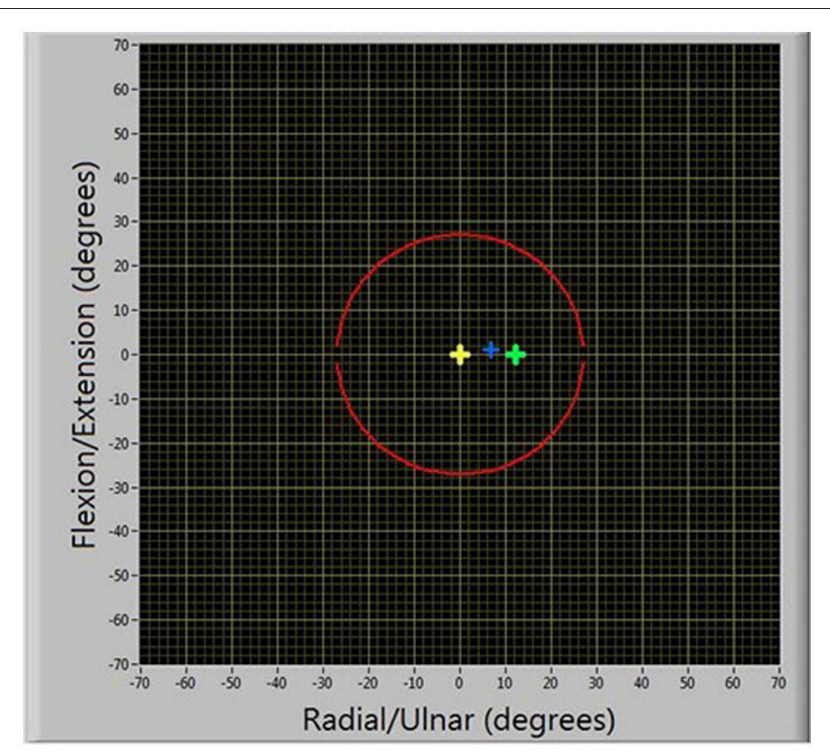

FIGURE 7 | Illustration of motor learning task.

extracted and presented in Figure 9. In Tables 1, 2, the median values for all participants' metrics across the four trials from the validation are presented, in addition to the RMS error normalized as a percentage of the healthy velocity. All statistical analyses used an $\alpha$ level of 0.05 .

The dominant frequency was measured by a Fast Fourier Transform (FFT). When the dominant frequency data was tested with a Wilcoxon signed rank test, there was no evidence of a difference between the healthy and constrained velocity profiles for either the $\mathrm{RU}(p=0.50)$ or $\mathrm{FE}(p=0.75)$ axes. Also reported are the power components measured at the dominant frequencies, a measure of the amplitude of the signal at that frequency. A Wilcoxon signed rank test on this data revealed no evidence of a statistical difference in the FE axis $(p=$ $0.19)$ and strong evidence of a difference in the RU axis ( $p=$ $0.0098)$.

The absolute peak velocities of the constrained and healthytarget states were extracted and the median of these was used as an indicator of the peak velocity in each state. A Wilcoxon signed rank test revealed no evidence of a difference in the peak velocities for the FE axis $(p=0.32)$ but strong evidence of a difference in the RU axis velocities $(p=0.027)$, with the medians reported in Tables 1, 2. This suggests the specific normalization algorithm may have had an effect. The median RMS errors are also reported as a percentage of the maximum healthytarget velocity and were 21.0 and $22.1 \%$ for the RU and FE axes respectively.

\section{Motor Learning Task}

Participant 6's adaptation to achieving the RU Target is shown in Figure 10, comparing performance between the first RU Target in Block 1 (a), the last RU target in Block 3 (b) and the catch trial (c). In (a), the participant has failed to achieve the target before it

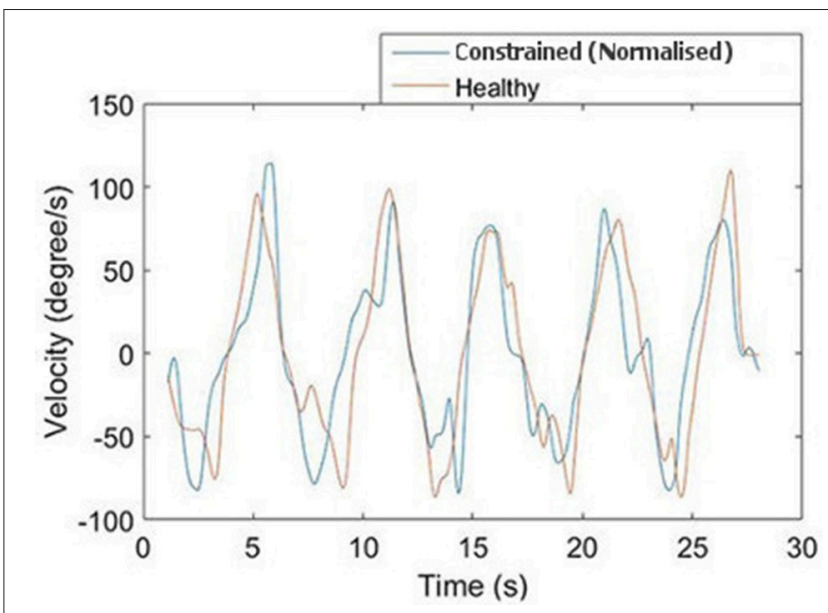

FIGURE 8 | Comparison of normalized and healthy velocities for the FE axis.

reached the boundary. In (b), the target was successfully achieved, and (c) illustrates a clear after-effect as the participant overshot before settling on the stationary target.

Two metrics were used to measure task performance; (i) number of successful targets achieved; (ii) sum-of-squared-error (SSE) measured from the time of target appearance to success (to reflect aspects of both settling time and overshoot); in addition to magnitude of after-effects observed on the catch trial (to quantify the amount of learning).

Qualitatively observing participants indicated that they used one of two methods to complete the task. Either they would immediately move toward the target, or initially move away from the target, giving themselves more "run-up" to reach the target. Therefore, for a given participant, the after-effect is either the maximum overshoot of the target (when participants immediately moved toward the target) or the maximum initial error (when participants chose to move away from the target initially). For each participant, the metric was calculated as the median value across the three blocks.

Figure 11 illustrates one participant's task metrics across the three blocks of trials for the RU Target in the constrained state. An increase in the number of targets achieved is accompanied by a decrease in SSE, representing an improvement in performance.

Both inter-session and inter-state analyses are presented. Inter-session analysis is performed using a Wilcoxon signed rank test, where statistical differences between sessions would imply that 1 week was insufficient time to washout learning. These results are summarized in Figure 12 and Table 3. There was no evidence of a difference between Sessions 1 and 2 for either target (FE, RU) for the number of targets achieved $(p=0.89, p=0.94)$ or the SSE $(p=0.15, p=0.20)$. There was also no evidence of a difference for the magnitude of after-effect on the catch trial $(p=$ 0.74 ). The results conclude that 1 week was indeed sufficient to washout learning.

Healthy and constrained states are also compared and the results summarized in Figure 13 and Table 4. Wilcoxon signed rank tests were performed and for the SSE, there was no evidence 

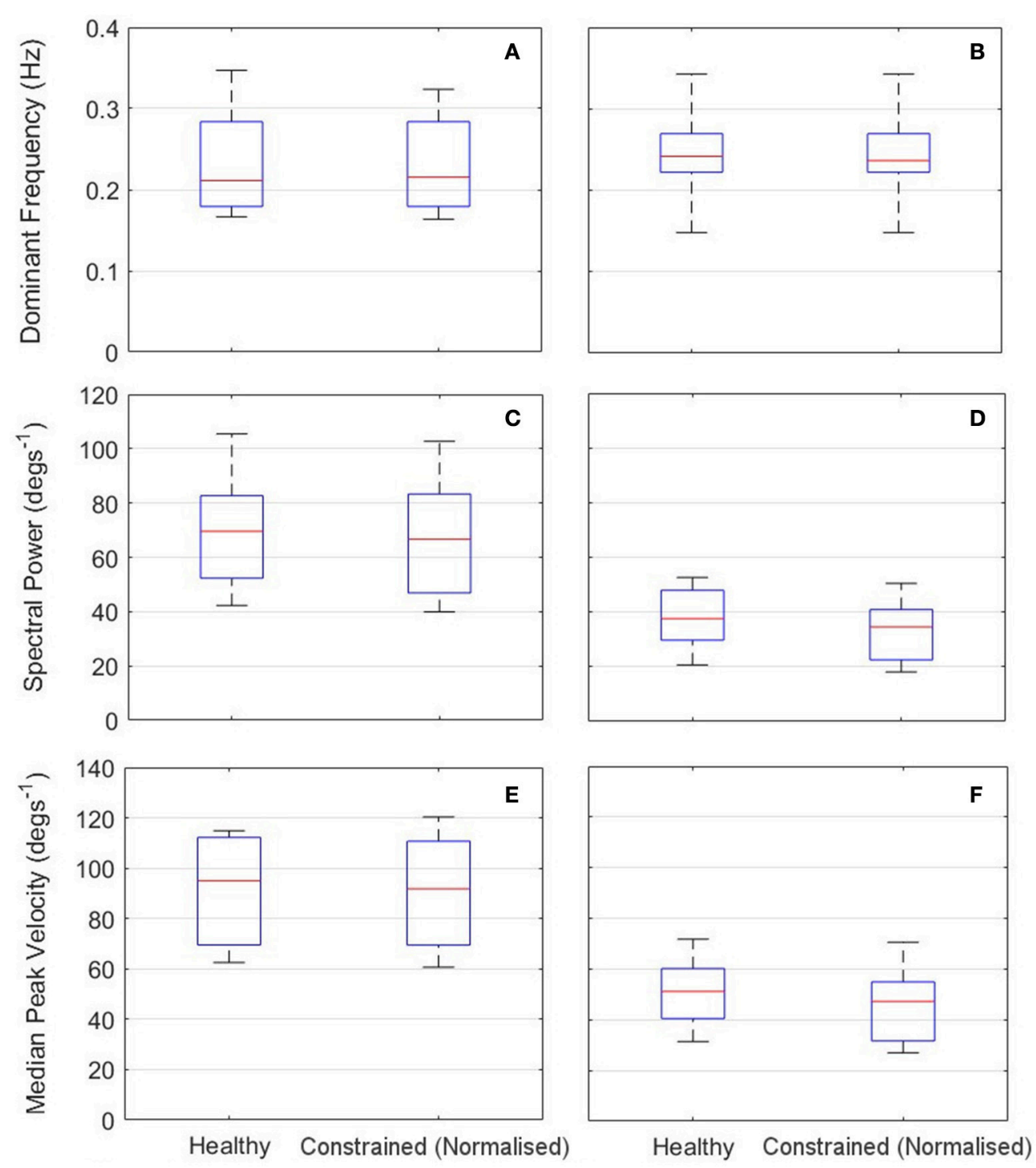

FIGURE 9 | Results of calibration validation for all participants $(\boldsymbol{n}=\mathbf{I 0})$. Left: RU axis (A,C,E); Right: FE axis (B,D,E). Top: Dominant Frequency (A,B); Middle: Spectral Power Component at Dominant Frequency (C,D). Bottom: Median Peak Velocity (E,F).

TABLE 1 | Summary statistics for algorithm validation in RU axis.

\begin{tabular}{|c|c|c|c|c|c|c|c|}
\hline & \multicolumn{2}{|c|}{ Dominant Frequency $(\mathrm{Hz})$} & \multicolumn{2}{|c|}{ Power Component (degs $^{-1}$ ) } & \multicolumn{2}{|c|}{ Peak Velocity (degs ${ }^{-1}$ ) } & Normalized RMS Error (\%) \\
\hline Median $(n=10)$ & 0.24 & 0.24 & 37.6 & 34.5 & 51.2 & 47.3 & 21.0 \\
\hline
\end{tabular}

of a difference for either the FE or RU target $(p=0.55, p=$ $0.15)$. For the number of targets achieved, there was no evidence of a difference for the RU target $(p=0.16)$ but there was evidence to suggest a difference in the metric for the FE target $(p=0.016)$. For the magnitude of the after-effect on the catch trial, there was no evidence of a difference between the states $(p=0.74)$.

\section{DISCUSSION}

\section{Validation of Normalization Algorithm in Calibration}

Overall, the results from the calibration phase show that the algorithms were able to adequately normalize the velocity of the participants' cursor, despite their motion being constrained. For 
TABLE 2 | Summary statistics for algorithm validation in FE axis.

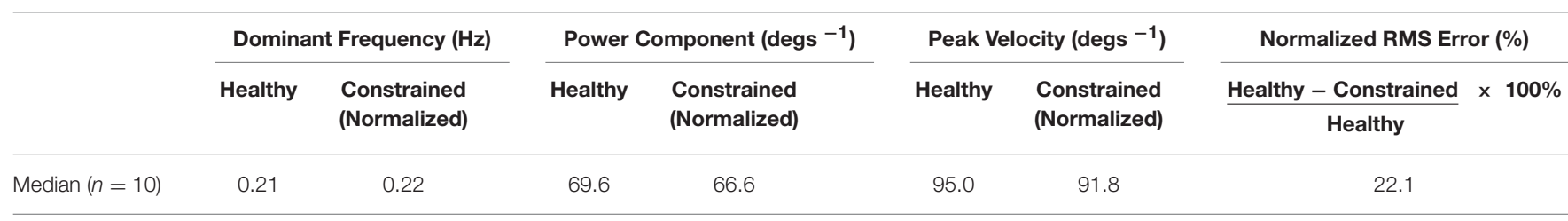

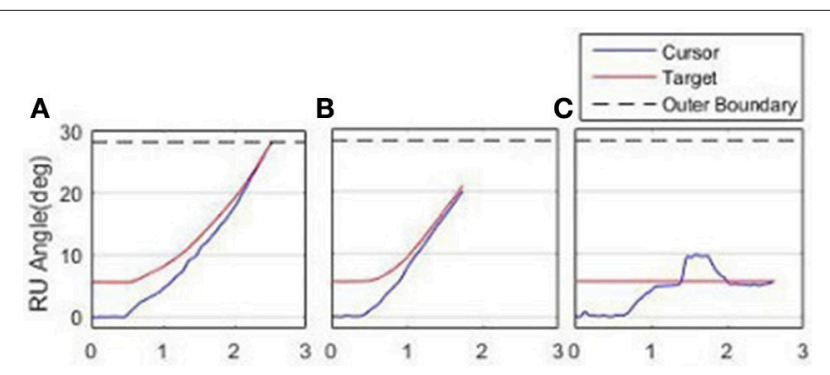

FIGURE 10 | Adaptation to achieving the RU Target. (A) First attempt, Block I. (B) Final attempt, Block 3. (C) Catch Trial.

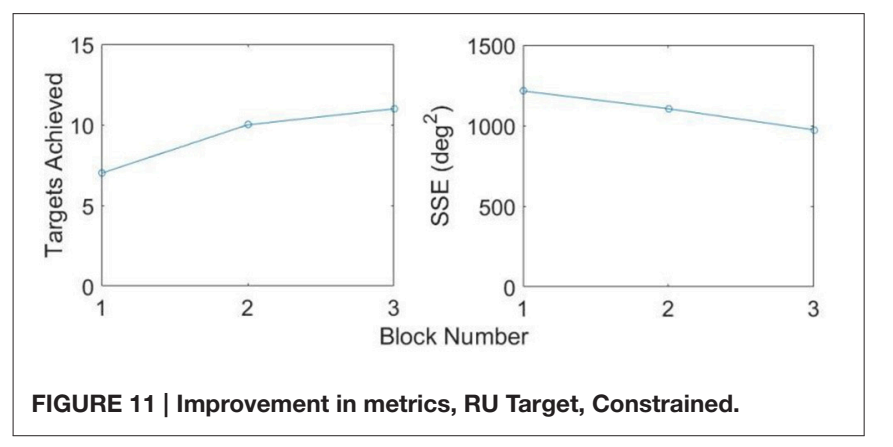

the majority of participants, the dominant frequency of motion of the normalized cursor matched that of the healthy-target motion. Where there is a difference, it is on the scale of 0.05 $\mathrm{Hz}$ and can be considered negligible. This is reinforced by a Wilcoxon signed rank test, suggesting no evidence of a difference in dominant frequency in either the RU or FE axes. When the power components of the dominant frequencies are considered, there is strong evidence of a difference for the RU axis, ( $p=$ 0.0098 ) and no evidence to suggest that the power components are different for the FE axis $(p=0.19)$.

The discrepancy in power components for the RU axis is not surprising, given the additional cognitive load associated with target tracking in comparison to free motion; participants constantly adjust to try and stay on the target and thus perfect tracking cannot be expected. This is reflected in the high frequency components of the graph shown in Figure 8. Moreover, the median values were similar for the constrained and healthy velocities in the RU axis $\left(37.6\right.$ degs $^{-1}$ and $34.5 \mathrm{degs}^{-1}$ ), indicating that despite being unable to conclude they are statistically similar, the values are reasonably close.
Some further insight can be gained by examining the peak velocities attained in the healthy and normalized states. A Wilcoxon signed rank test on these values revealed no statistically significant differences for the FE axis, but strong evidence of a difference for the RU axis. However, the median values of healthy and constrained velocity in the RU axis are $51.2 \mathrm{degs}^{-1}$ and 47.3 degs $^{-1}$ respectively, which is a relatively small error of 7.62\%. Furthermore, the boxplots in Figure 9 visually appear very similar. This suggests that the constrained velocity peaks in the RU axis were comparably close to the healthy velocity peaks.

As with the power content of the dominant frequency, some differences between peak velocities are to be expected, due to the difficulties associated with target tracking. While these metrics provide some insight into the performance of the algorithm, they are also strongly linked to individuals' cognitive abilities in target tracking. Considering the frequency components of motion, as opposed to peak velocities, offers an analysis more closely focused on the non-cognitive aspect of the motion.

The normalized RMS error was quite large, albeit similar in both the RU and FE axes, at 21.0 and $22.1 \%$ respectively. Less weight should be attached to the RMS values, however, since they are not representative indicators of the algorithms' performance due to phase lag and high-frequency motion components induced by the cognitive load of target tracking. The healthy-target was intended as a guide; thus we are not interested in matching its time-dependent position exactly. The values of the RMS error should therefore be regarded as a measure of comparing the two algorithms to each other, rather than explicitly evaluating their performance individually. From the RMS metric, it is obvious that the two algorithms have performed comparably.

Overall, we can conclude that the velocity in the constrained state was restored reasonably closely to the original healthy-target velocity by both algorithms. This justifies further exploration of this method as a tool to normalize patient physical ability in tasks requiring motor learning.

\section{Motor Learning Task}

In general, the weight of evidence from this experiment suggests that both algorithms were able to normalize constrained velocities such that learning of the task was not hindered. In terms of task metrics, the only case in which there was evidence of a statistical difference was the number of times the FE target was achieved $(p=0.016)$. Further information can be gleaned by examining the median number of times the FE target was achieved in the healthy and constrained states: 12.5 and 10 respectively; implying that it was easier to reach in the healthy state. 

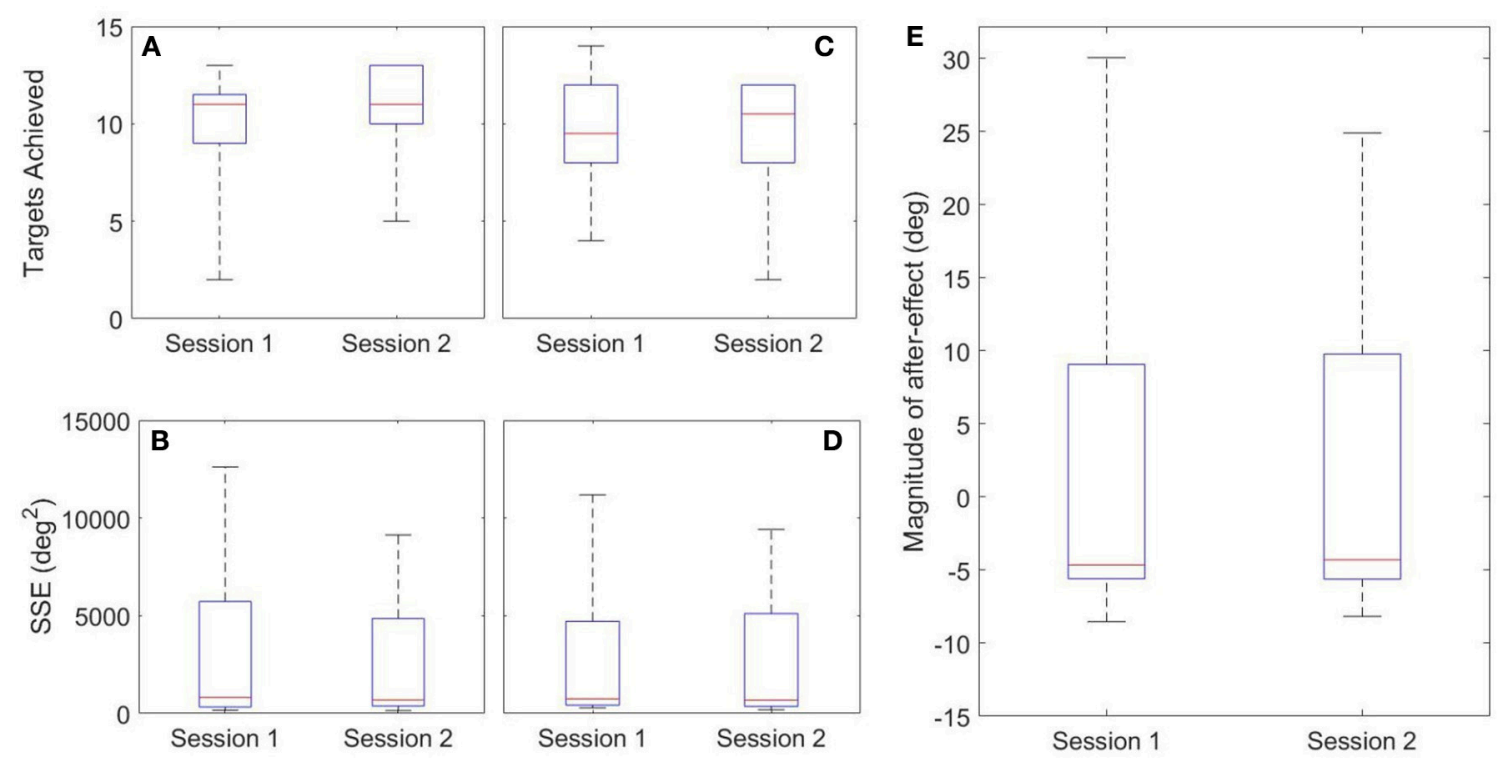

FIGURE 12 | Inter-session Results. Left: FE Target (A,B); Middle: RU Target (C,D); Right: After-Effects (E).

TABLE 3 | Inter-session summary statistics.

\begin{tabular}{|c|c|c|c|c|c|c|c|c|c|c|}
\hline \multirow{3}{*}{$\begin{array}{l}\text { Metric } \\
\text { Session }\end{array}$} & \multicolumn{4}{|c|}{ FE target } & \multicolumn{4}{|c|}{ RU target } & \multirow{2}{*}{\multicolumn{2}{|c|}{$\begin{array}{c}\text { After effect magnitude } \\
\text { Distance (deg) }\end{array}$}} \\
\hline & \multicolumn{2}{|c|}{ Targets achieved } & \multicolumn{2}{|c|}{ SSE } & \multicolumn{2}{|c|}{ Targets achieved } & \multicolumn{2}{|c|}{ SSE } & & \\
\hline & 1 & 2 & 1 & 2 & 1 & 2 & 1 & 2 & 1 & 2 \\
\hline Median $(n=8)$ & 11 & 11 & 746.6 & 694.4 & 9.5 & 10.5 & 813.9 & 697.9 & -4.65 & -4.31 \\
\hline
\end{tabular}

One reason may be due to the differing algorithms used. The normalization algorithm in the FE axis was supplemented with a linear bias. Simultaneously, most participants qualitatively noted that motion with the exoskeleton felt less restrained in the FE axis. This led to greater sensitivity in the FE axis, which could have led to less consistent performance, potentially explaining the non-significant result for the FE target. Additional human biomechanical and/or exoskeleton dynamics may have contributed to differences in each axis.

One of the primary aims of this research was to create a tool with which motor learning can be studied; thus we must attempt to quantify the amount of learning that took place. The fact that task performance was similar when the algorithm was applied is promising. However, this alone does not reflect the amount of learning that occurred. Haith and Krakauer (2013) distinguish between model-based learning, requiring an internal model, and model-free learning, operating on a trial-and-error basis with no internal model formed. From a technical viewpoint, it does not matter whether the learning that occurred was model-based or model free, since both fall under the accepted definition of "motor learning," and thus it suffices to show that the normalization allows equivalent learning across states.

Catch trials are often included in motor learning studies to test for after-effects (Abdollahi et al., 2014; Melendez-Calderon et al., 2015); and are commonly cited as evidence that an internal model was formed, since they are predictive errors (Shadmehr and Brashers-Krug, 1997; Krakauer, 2006; Haith and Krakauer, 2013). In the present study's catch trials, participants displayed substantial after-effects and no evidence of a difference was found between the healthy and constrained states for the magnitude of after-effects $(p=0.74)$. This provides evidence that the learning was the same despite the physical limitations imposed by the constraints. A limitation of the study is that the catch trial was only conducted in the RU axis and thus conclusions cannot be drawn with regards to the FE axis. This was done so as to avoid subsequent catch trials affecting each other (Focke et al., 2013; Stockinger et al., 2014). A further limitation of the catch trial analysis in this study is that two participants used different strategies to reach the target, initially pulling away from it. Therefore, the absolute values of their metrics are larger than the rest of the participants' data. While this affects the spread of the data presented, the statistical analyses remain valid, as after-effects were consistent for a given participant and paired Wilcoxon tests were used.

Haith and Krakauer (2013) also cite adaptation time as an indicator of the mechanism of motor learning. In the present study, participants required minimal adaptation time, suggesting model-based learning occurred; however, adaptation time is also 

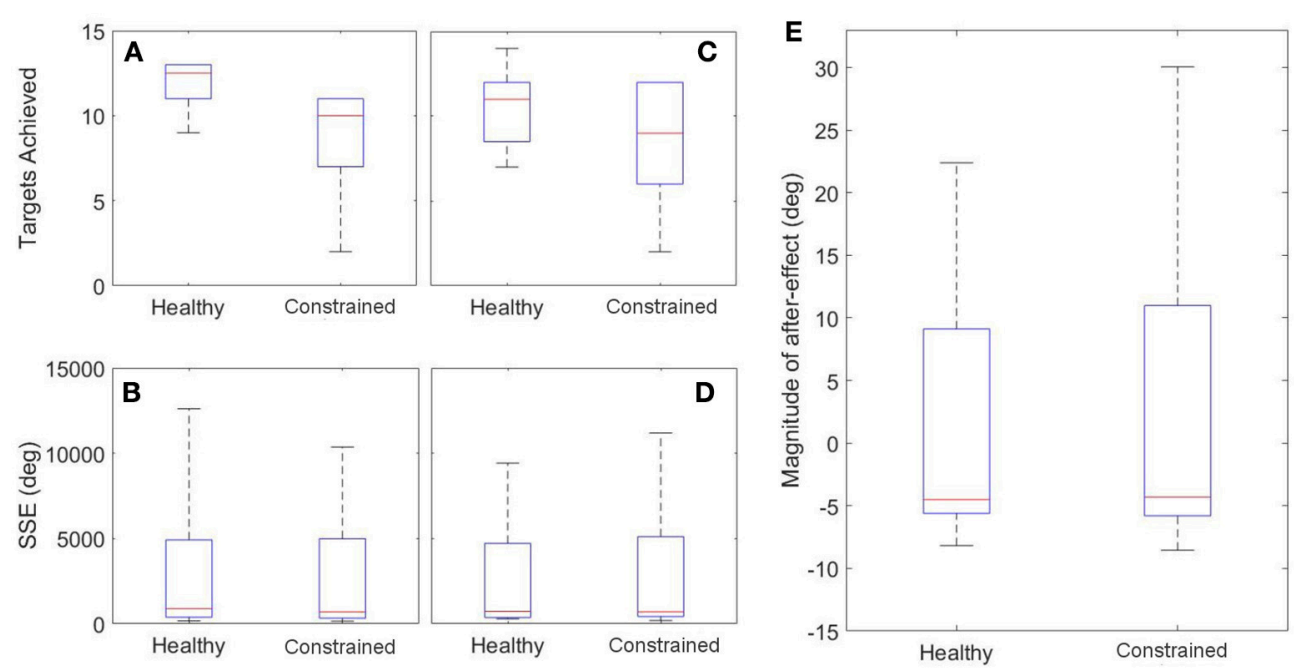

FIGURE 13 | Inter-state Results. Left: FE Target (A,B); Middle: RU Target (C,D); Right: After-Effects (E).

TABLE 4 | Inter-state summary statistics (Healthy - Constrained).

\begin{tabular}{|c|c|c|c|c|c|c|c|c|c|c|}
\hline \multirow{3}{*}{$\begin{array}{l}\text { Metric } \\
\text { State }\end{array}$} & \multicolumn{4}{|c|}{ FE target } & \multicolumn{4}{|c|}{ RU target } & \multirow{2}{*}{\multicolumn{2}{|c|}{$\frac{\text { After effect magnitude }}{\text { Distance }(\mathrm{deg})}$}} \\
\hline & \multicolumn{2}{|c|}{ Targets achieved } & \multicolumn{2}{|c|}{ SSE } & \multicolumn{2}{|c|}{ Targets achieved } & \multicolumn{2}{|c|}{ SSE } & & \\
\hline & Healthy & Constrained & Healthy & Constrained & Healthy & Constrained & Healthy & Constrained & Healthy & Constrained \\
\hline Median $(n=8)$ & 12.5 & 10 & 734.6 & 706.4 & 11 & 9 & 879.9 & 697.9 & -4.50 & -4.31 \\
\hline
\end{tabular}

influenced by task difficulty. Nevertheless, the presence of aftereffects provides quantifiable evidence that learning did occur, regardless of the mechanism by which it transpired.

One limitation of the study is the simulation of physical impairment; this adds dynamics that participants must compensate for. The familiarization phases in both the impairment assessment and in the motor learning case study were designed to ensure that participants had "learnt" the constraints as much as possible before the tasks were conducted. However, in previous studies, it was found that learning of dynamic and kinematic transformations occur independently of, and in parallel to, each other (Krakauer et al., 1999). Consequently, in this study it may be that "learning" of the constraints did not interfere with learning the kinematics required to complete the task. The lack of a systematic difference in metrics between the healthy and constrained states strengthens this conclusion. However, there may be some interference in learning the transformation between physical and normalized motion with the learning of the task kinematics.

The majority of metrics used to evaluate motor learning and task performance displayed no significant differences between the states. Thus the weight of evidence concludes that the normalization allowed participants to learn and complete the task to the same standard in the constrained state as in the healthy state. This significant because it lends justification to the potential use of the normalization algorithms to conduct studies on patients with physical impairment. Physical impairment has often been cited as a confounding factor in motor learning studies, as researchers are unable to determine whether poor results are due to an inability to motor learn or because of physical barriers (Valvano and Rapport, 2006; Burtner et al., 2014; van Abswoude et al., 2015). Using normalization, therapists may be able to evaluate the cognitive ability of a patient independently of their physical impairment, alleviating the compounding factors surrounding motor learning.

It is acknowledged that the task presented here is quite specific and that it may not be considered as useful for restoring function. However, we argue that it is the concept that is important, rather than the specific task, and the concept is easily generalized to more complex tasks. Furthermore, the purpose of the normalization is not to provide restoration of physical function, but as a tool with which therapists can fairly compare individuals' (with and without impairment) inherent ability to motor learn and provide subject-specific, optimized therapy programs informed by this knowledge. This study is intended as an exploratory investigation of the concept of the normalization and future work will examine the use of this technique in patients with cerebral palsy, which will lead to further understanding of motor learning in impaired participants.

\section{Comparison of Algorithms}

The final matter to address is the comparison of each algorithm. Both algorithms were able to individually restore velocity capabilities to a decent standard. When the validation of the calibration is considered, it was the algorithm used in the FE axis that provided better results. However, it is important to consider 
that the only metrics where it significantly outperformed the RU algorithm were the measurement of peak velocities and the power component of the dominant frequency, which are affected by cognitive difficulties and are not considered the optimal measures of performance. Furthermore, for both algorithms, the summary statistics for all metrics were comparably close between the healthy and constrained velocity profiles.

The algorithm used in the RU axis overall provided better performance in the motor learning task, where no evidence of a difference between healthy and constrained states was found for any metric. This contrasts with the FE axis, where significantly more targets were achieved in the healthy state.

It is therefore difficult to say conclusively which algorithm was superior. The linear bias added to the algorithm in the FE axis gave no clear advantage over the standard algorithm in this application. In contrast, the algorithm in the RU axis performed sufficiently, despite the lack of a supplementing bias factor, and matches the structure of RBF used in the research that originally inspired our use of RBFs (Wolbrecht et al., 2008; Oboe and Pilastro, 2014; Pehlivan et al., 2015). Consequently, this shall be used in future development of this normalization tool.

\section{Limitations}

The primary limitation of this study is the method of simulating physical impairment. The torques exerted by patients in the healthy and constrained cases are quite different. While this does simulate the fact that patients with impairment tend to tire more quickly than their healthy counterparts, it is possible that fatigue may be a compounding factor on the results. The focus of this preliminary study, however, was to investigate whether the kinematics of the constrained participants could be normalized and thus kinetics were not considered. In future, EMG could be used to measure torque, and further studies will focus on incorporating kinetic data into the algorithm to give a fairer normalization of the abilities of impaired individuals.

There is also difficulty associated with accurately replicating a physical impairment. In reality, these are not modeled simply. However, the purpose of this study was to show that the kinematics can be normalized in the presence of a torque field that restricts motion. In this respect, the impairment can be treated as a black box, with the only important phenomenon being that it has an impact on kinematics. The use of biomechanical models could be considered for more accurately

\section{REFERENCES}

Abdollahi, F., Case Lazarro, E. D., Listenberger, M., Kenyon, R. V., Kovic, M., Bogey, R. A., et al. (2014). Error augmentation enhancing arm recovery in individuals with chronic stroke: a randomized crossover design. Neurorehabil. Neural Repair 28, 120-128. doi: 10.1177/1545968313498649

Bar-On, L., Desloovere, K., Molenaers, G., Harlaar, J., Kindt, T., and Aertbeliën, E. (2014). Identification of the neural component of torque during manuallyapplied spasticity assessments in children with cerebral palsy. Gait Posture 40, 345-351. doi: 10.1016/j.gaitpost.2014.04.207

Bar-On, L., Molenaers, G., Aertbeliën, E., Van Campenhout, A., Feys, H., Nuttin, B., et al. (2015). Spasticity and its contribution to hypertonia representing actual impairments; however, this was considered to be out of scope for a preliminary study.

The pilot sample size reduces the strength of the statistical outcomes. However, as a preliminary investigation into the feasibility of the algorithm, a small sample size was considered sufficient and has gleaned significant relevant information to provide interesting results and motivate further investigation into this work.

\section{CONCLUSIONS}

A new technique for investigating motor learning in physically impaired individuals has been proposed in an investigative pilot study focussing on wrist motion. Measurements of velocity as a function of position are used to derive subject-specific scaling factors that normalize the velocity of individuals to a "healthy" benchmark. Healthy participants were subjected to an artificial impairment that restricted both range of motion and velocity in the RU and FE axes of the wrist. The algorithms were statistically proven to restore velocity accurately. In a crossover study participants were able to complete the task with the same performance when the algorithm was applied to their constrained state as they did in the healthy state, with some evidence that motor learning had occurred. Overall, the results show promise for the further investigation of this technique in exploring motor learning in impaired individuals.

\section{ETHICS STATEMENT}

This research was supported by the Marsden Fund Council from Government funding, managed by the Royal Society of New Zealand.

\section{AUTHOR CONTRIBUTIONS}

Both authors contributed to identifying study motivation, study design and experimental protocol. Experiments were conducted by CJ.

\section{FUNDING}

This research was partially supported by the Marsden Fund Council from Government funding, managed by the Royal Society of New Zealand. in cerebral palsy. Biomed Res. Int. 2015:317047. doi: 10.1155/2015/ 317047

Barroso, P. N., Vecchio, S. D., Xavier, Y. R., Sesselmann, M., Araújo, P. A., and Pinotti, M. (2011). Improvement of hand function in children with cerebral palsy via an orthosis that provides wrist extension and thumb abduction. Clin. Biomechan. 26, 937-943. doi: 10.1016/j.clinbiomech.2011.05.006

Boudreau, S. A., Farina, D., and Falla, D. (2010). The role of motor learning and neuroplasticity in designing rehabilitation approaches for musculoskeletal pain disorders. Man. Ther. 15, 410-414. doi: 10.1016/j.math.2010. 05.008

Boyd, R. N., and Graham, H. K. (1999). Objective measurement of clinical findings in the use of botulinum toxin type $\mathrm{A}$ for the 
management of children with cerebral palsy. Euro. J. Neurol. 6, s23-s35. doi: 10.1111/j.1468-1331.1999.tb00031.x

Burtner, P. A., Leinwand, R., Sullivan, K. J., Goh, H.-T., and Kantak, S. S. (2014). Motor learning in children with hemiplegic cerebral palsy: feedback effects on skill acquisition. Develop. Med. Child Neurol. 56, 259-266. doi: $10.1111 / \mathrm{dmcn} .12364$

Cordella, F., Ciancio, A. L., Sacchetti, R., Davalli, A., Cutti, A., Guglielmelli, E., et al. (2016). Literature review on needs of upper limb prosthesis users. Front. Neurosci. 10:209. doi: 10.3389/fnins.2016.00209

Fattal-Valevski, A., Domenievitz, D., Giladi, N., Wientroub, S., and Hayek, S. (2008). Long-term effect of repeated injections of botulinum toxin in children with cerebral palsy: a prospective study. J. Children's Orthopaed. 2, 29-35. doi: 10.1007/s11832-007-0075-8

Fattal-Valevski, A., Sagi, L., and Domenievitz, D. (2010). Botulinum toxin a injections to the upper limbs in children with cerebral palsy: duration of effect. J. Child Neurol. 26, 166-170. doi: 10.1177/0883073810376446

Focke, A., Stockinger, C., Diepold, C., Taubert, M., and Stein, T. (2013). The influence of catch trials on the consolidation of motor memory in force field adaptation tasks. Front. Psychol. 4:479. doi: 10.3389/fpsyg.2013. 00479

Fujii, S., Lulic, T., and Chen, J. L. (2016). More feedback is better than less: learning a novel upper limb joint coordination pattern with augmented auditory feedback. Front. Neurosci. 10:251. doi: 10.3389/fnins.2016.00251

Haith, A. M., and Krakauer, J. W. (2013). Model-based and model-free mechanisms of human motor learning. Adv. Exp. Med. Biol. 782, 1-21. doi: 10.1007/978-1-4614-5465-6_1

Jarrett, C., and Mc Daid, A. (2017). Robust control of a cable-driven soft exoskeleton joint for intrinsic human-robot interaction. IEEE Trans. Neural Syst. Rehabil. Eng.

Jezernik, S., Colombo, G., and Morari, M. (2004). Automatic gait-pattern adaptation algorithms for rehabilitation with a 4-DOF robotic orthosis. IEEE Transac. Robot. Automat. 20, 574-582. doi: 10.1109/TRA.2004. 825515

Klingels, K., Feys, H., De Wit, L., Jaspers, E., Van de Winckel, A., Verbeke, G., et al. (2012). Arm and hand function in children with unilateral cerebral palsy: a one-year follow-up study. Euro. J. Paediat. Neurol. 16, 257-265. doi: 10.1016/j.ejpn.2011.08.001

Krakauer, J. W. (2006). Motor learning: its relevance to stroke recovery and neurorehabilitation. Curr. Opin. Neurol. 19, 84-90. doi: 10.1097/01.wco.0000200544.29915.cc

Krakauer, J. W., Ghilardi, M.-F., and Ghez, C. (1999). Independent learning of internal models for kinematic and dynamic control of reaching. Nat. Neurosci. 2, 1026-1031. doi: 10.1038/14826

Li, M. M., and Verma, B. (2015). “An improved RBF neural network approach to nonlinear curve fitting," in Advances in Computational Intelligence: 13th International Work-Conference on Artificial Neural Networks, IWANN 2015, Palma de Mallorca, Spain, June 10-12, 2015, eds I. Rojas, G. Joya, and A. Catala (Palma de Mallorca: Springer International Publishing), 262-275.

Lin, Y.-C., Lin, I.-L., Chou, T,-F. A., and Lee, H.-M. (2016). Quantitative evaluation for spasticity of calf muscle after botulinum toxin injection in patients with cerebral palsy: a pilot study. J. Neuroeng. Rehabil. 13, 1-11. doi: 10.1186/s12984-016-0135-8

Lipp, I., and Tomassini, V. (2015). Neuroplasticity and motor rehabilitation in Multiple Sclerosis. Front. Neurol. 6:59. doi: 10.3389/fneur.2015. 00059

Marchal-Crespo, L., Wolf, P., Gerig, N., Rauter, G., Jaeger, L., Vallery, H., et al. (2015). "The role of skill level and motor task characteristics on the effectiveness of robotic training: first results," 2015 IEEE International Conference on Rehabilitation Robotics (Singapore: ICORR).

McDaid, A. J. (2015). "Development of an Anatomical Wrist Therapy Exoskeleton (AW-TEx)," in 2015 IEEE International Conference on Rehabilitation Robotics (Singapore: ICORR).

Melendez-Calderon, A., Fisher, M., Tan, M., Burdet, E., and Patton, J. L. (2015). "Acquisition of motor skills in isometric conditions through synesthetic illusions of movement," in World Haptics Conference (WHC), 2015 (Chicago: IEEE).
Melendez-Calderon, A., Masia, L., Gassert, R., Sandini, G., and Burdet, E. (2011). Force field adaptation can be learned using vision in the absence of proprioceptive error. IEEE Trans. Neural Sys. Rehabil. Eng. 19, 298-306. doi: 10.1109/TNSRE.2011.2125990

Molenaers, G., Campenhout, A. V., Fagard, K., De Cat, J., and Desloovere, K. (2010). The use of botulinum toxin A in children with cerebral palsy, with a focus on the lower limb. J. Children Orthopaed. 4, 183-195. doi: 10.1007/s11832-010-0246-x

Molenaers, G., Schörkhuber, V., Fagard, K., Van Campenhout, A., De Cat, J., Pauwels, P., et al. (2009). Long-term use of botulinum toxin type A in children with cerebral palsy: treatment consistency. Euro. J. Paediat. Neurol. 13, 421-429. doi: 10.1016/j.ejpn.2008.07.008

Oboe, R., and Pilastro, D. (2014). "Non-linear adaptive impedance controller for rehabilitation purposes," in 2014 IEEE 13th International Workshop on Advanced Motion Control (Yokohama: AMC).

Ojakangas, C. L., and Ebner, T. J. (1991). Scaling of the metrics of visuallyguided arm movements during motor learning in primates. Exp. Brain Res. 85, 314-323. doi: 10.1007/BF00229409

Patton, J. L., Stoykov, M. E., Kovic, M., and Mussa-Ivaldi, F. A. (2006). Evaluation of robotic training forces that either enhance or reduce error in chronic hemiparetic stroke survivors. Exp. Brain Res. 168, 368-383. doi: 10.1007/s00221-005-0097-8

Paz, R., Nathan, C., Boraud, T., Bergman, H., and Vaadia, E. (2005). Acquisition and generalization of visuomotor transformations by nonhuman primates. Exp. Brain Res. 161, 209-219. doi: 10.1007/s00221-0042061-4

Pehlivan, A. U., Sergi, F., and O'Malley, M. K. (2015). A subject-adaptive controller for wrist robotic rehabilitation. IEEE/ASME Trans. Mechatron. 20, 1338-1350. doi: 10.1109/TMECH.2014.2340697

Rotella, M. F., Nisky, I., Koehler, M., Rinderknecht, M. D., Bastian, A. J., and Okamura, A. M. (2014). Learning and generalization in an isometric visuomotor task. J. Neurophysiol. 113, 1873-1884. doi: 10.1152/jn.002 55.2014

Sarlegna, F. R., Malfait, N., Bringoux, L., Bourdin, C., and Vercher, J.-L. (2010). Force-field adaptation without proprioception: can vision be used to model limb dynamics? Neuropsychologia 48, 60-67. doi: 10.1016/j.neuropsychologia.2009.08.011

Shadmehr, R., and Brashers-Krug, T. (1997). Functional stages in the formation of human long-term motor memory. J. Neurosci. 17, 409-419.

Smiley-Oyen, A. L., Worringham, C. J., and Cross, C. L. (2003). Motor learning processes in a movement-scaling task in olivopontocerebellar atrophy and Parkinson's disease. Exp. Brain Res. 152, 453-465. doi: 10.1007/s00221-003-1570-x

Stockinger, C., Focke, A., and Stein, T. (2014). Catch trials in force field learning influence adaptation and consolidation of human motor memory. Front. Hum. Neurosci. 8:231. doi: 10.3389/fnhum.2014. 00231

Tedroff, K., Granath, F., Forssberg, H., and Haglund-Akerlind, Y. (2009). Long-term effects of botulinum toxin A in children with cerebral palsy. Dev. Med. Child Neurol. 51, 120-127. doi: 10.1111/j.1469-8749.2008. 03189.x

Turner, D. L., Murguialday, A. R., Birbaumer, N., Hoffmann, U., and Luft, A. (2013). Neurophysiology of robot-mediated training and therapy: a perspective for future use in clinical populations. Front. Neurol. 4:184. doi: 10.3389/fneur.2013.00184

Valvano, J., and Rapport, M. J. (2006). Activity-focused motor interventions for children with neurological conditions. Infants Young Child. 19, 292-307. doi: 10.1097/00001163-200610000-00003

van Abswoude, F., Santos-Vieira, B., J., van der Kamp, and Steenbergen, B. (2015). The influence of errors during practice on motor learning in young individuals with cerebral palsy. Res. Dev. Disabil. 45-46, 353-364. doi: 10.1016/j.ridd.2015.08.008

Wallen, M. A., O'Flaherty, S. J., and Waugh, M. C. (2004). Functional outcomes of intramuscular botulinum toxin type a in the upper limbs of children with cerebral palsy: a phase II trial. Arch. Phys. Med. Rehabil. 85, 192-200. doi: 10.1016/j.apmr.2003.05.008

Wissel, J., Heinen, F., Schenkel, A., Doll, B., Ebersbach, G., Müller, J., et al. (1999). Botulinum toxin A in the management of spastic gait disorders in 
children and young adults with cerebral palsy: a randomized, double-blind study of high-dose versus low-dose treatment. Neuropediatrics 30, 120-124. doi: 10.1055/s-2007-973475

Wolbrecht, E. T., Chan, V., Reinkensmeyer, D. J., and Bobrow, J. E. (2008). Optimizing compliant, model-based robotic assistance to promote neurorehabilitation. IEEE Trans Neural Sys. Rehabilit. Eng. 16, 286-297. doi: 10.1109/TNSRE.2008.918389

Wulf, G., Chiviacowsky, S., Schiller, E., and Ávila, L. T. G. (2010). Frequent external-focus feedback enhances motor learning. Front. Psychol. 1:190. doi: $10.3389 /$ fpsyg. 2010.00190
Conflict of Interest Statement: The authors declare that the research was conducted in the absence of any commercial or financial relationships that could be construed as a potential conflict of interest.

Copyright (0) 2017 Jarrett and McDaid. This is an open-access article distributed under the terms of the Creative Commons Attribution License (CC BY). The use, distribution or reproduction in other forums is permitted, provided the original author(s) or licensor are credited and that the original publication in this journal is cited, in accordance with accepted academic practice. No use, distribution or reproduction is permitted which does not comply with these terms. 
OPEN ACCESS

Edited by:

Wenwei Yu,

Chiba University, Japan

Reviewed by:

Giuseppe D'Avenio, Istituto Superiore di Sanità, Italy

Hans-Eckhardt Schaefer,

University of Stuttgart, Germany

*Correspondence:

Nur Azah Hamzaid

azah.hamzaid@um.edu.my

Specialty section:

This article was submitted to

Neural Technology,

a section of the journal

Frontiers in Neuroscience

Received: 10 December 2016

Accepted: 05 April 2017

Published: 25 April 2017

Citation:

Jasni F, Hamzaid NA, Mohd Syah NE, Chung TY and Abu Osman NA (2017)

Analysis of Interrelationships among

Voluntary and Prosthetic Leg Joint Parameters Using Cyclograms.

Front. Neurosci. 11:230.

doi: 10.3389/fnins.2017.00230

\section{Analysis of Interrelationships among Voluntary and Prosthetic Leg Joint Parameters Using Cyclograms}

\author{
Farahiyah Jasni ${ }^{1,2}$, Nur Azah Hamzaid ${ }^{1 *}$, Nor Elleeiana Mohd Syah ${ }^{1}$, Tze Y. Chung $^{3}$ and \\ Noor Azuan Abu Osman ${ }^{1}$ \\ ${ }^{1}$ Department of Biomedical Engineering, Faculty of Engineering, University of Malaya, Kuala Lumpur, Malaysia, ${ }^{2}$ Department \\ of Mechatronics Engineering, Kulliyyah of Engineering, International Islamic University Malaysia, Selangor, Malaysia, \\ ${ }^{3}$ Department of Rehabilitation Medicine, Faculty of Medicine, University of Malaya, Kuala Lumpur, Malaysia
}

The walking mechanism of a prosthetic leg user is a tightly coordinated movement of several joints and limb segments. The interaction among the voluntary and mechanical joints and segments requires particular biomechanical insight. This study aims to analyze the inter-relationship between amputees' voluntary and mechanical coupled leg joints variables using cyclograms. From this analysis, the critical gait parameters in each gait phase were determined and analyzed if they contribute to a better powered prosthetic knee control design. To develop the cyclogram model, 20 healthy able-bodied subjects and 25 prosthesis and orthosis users (10 transtibial amputees, 5 transfemoral amputees, and 10 different pathological profiles of orthosis users) walked at their comfortable speed in a 3D motion analysis lab setting. The gait parameters (i.e., angle, moment and power for the ankle, knee and hip joints) were coupled to form 36 cyclograms relationship. The model was validated by quantifying the gait disparities of all the pathological walking by analyzing each cyclograms pairs using feed-forward neural network with backpropagation. Subsequently, the cyclogram pairs that contributed to the highest gait disparity of each gait phase were manipulated by replacing it with normal values and re-analyzed. The manipulated cyclograms relationship that showed highest improvement in terms of gait disparity calculation suggested that they are the most dominant parameters in powered-knee control. In case of transfemoral amputee walking, it was identified using this approach that at each gait sub-phase, the knee variables most responsible for closest to normal walking were: knee power during loading response and mid-stance, knee moment and knee angle during terminal stance phase, knee angle and knee power during pre-swing, knee angle at initial swing, and knee power at terminal swing. No variable was dominant during mid-swing phase implying natural pendulum effect of the lower limb between the initial and terminal swing phases. The outcome of this cyclogram adoption approach proposed an insight into the method of determining the causal effect of manipulating a particular joint's mechanical properties toward the joint behavior in an amputee's gait by determining the curve closeness, $\mathrm{C}$, of the modified cyclogram curve to the normal conventional curve, to enable quantitative judgment of the effect of changing a particular parameter in the prosthetic leg gait.

Keywords: transfemoral, biomechanics, cyclogram, prosthesis 


\section{INTRODUCTION}

Human walking is typically characterized by plotting kinematics and kinetics curves as a function of time or percentage of gait cycle. These kinematics and kinetics curves became the primary guideline in the prosthetic leg design that would mimic the normal gait. These were done by ensuring that the prosthesis produces user gait performance that is close to the normal singlevariable curve (Engeberg, 2013; Kutilek et al., 2013).

Although it is more common and convenient to use a single variable curve of kinematics or kinetics parameters as guideline in designing the control system for microprocessor based prosthetic leg, literatures proved that there are advantages of analyzing it as a pair. A number of relevant dynamic effects can be identified when pairs of kinematics and kinetic variables are examined together and correlations among them were concurrently assessed (Crenna and Frigo, 2011). These combinations of kinematics and kinetics variables, omitting the time variables from the two signals, create different cyclic trajectories known as cyclograms (Goswami, 1998). From the cyclic trajectories represented by the coupled variables, the dynamic variation of the gait can be easily visualized through the changes in the cyclograms pattern. In addition, the interaction between two co-existing parameter across the joints could be concurrently assessed. This is the foundation of applying the cyclograms concept to determine the most influential knee parameter in producing closest to normal and most efficient gait.

The use of cyclograms over a function of time has been used as a reference for designing a microprocessor-controlled prosthesis (Kutilek et al., 2013, 2014a,b). This is because, the fact that "locomotion is a tightly coordinated movement of several limb segments" can be more naturally grasped as the coupled variables of two or more joints rather than individual joint kinematics or kinetics. The cyclogram pattern was noted to be an extremely stable mechanism to identify gait behavior due to the use of cyclic traces of joint variables (Kutilek et al., 2014a). This was achieved based on the principal that a coordinated motion of a leg is to be perceived as an interaction between two or more limb segments rather than a phenomenon of isolated joint movements over time (Awai and Curt, 2014).

According to Pitkin, designing a better prosthetic leg does not revolve around the integration to mimic close-to-normal limb segments behavior (Pitkin, 2010). The design should take into account the amputees pathological condition (Pitkin, 2010). Therefore, there is a need to outline the basis for the prosthetic design that requires minimal modification to adjust with, if not all, most of the amputees' conditions.

The techniques that had been used to mathematically represent gait data and to analyze it include fuzzy system methods, multivariate statistical analysis, fractal dynamics, artificial intelligence (including neural networks), and wavelet methods (Arjunan and Kumar, 2010). Neural networks had been the most prevalent emerging non-traditional method applied to the analysis of gait data ( $\mathrm{Su}$ and $\mathrm{Wu}, 2000)$. The combination of neural network and cyclograms in a single study is rarely found in the literature, and none has been introduced for identifying the gait disparity in amputees. To date, neural network has been used to perform automated diagnosis of gait patterns represented by angle-angle cyclogram (Barton and Lees, 1997). In one study (Barton and Lees, 1997), once trained, the network can identify three different conditions-normal gait, a gait with unequal leg length and a gait with unequal leg weights at $83.3 \%$ success rate. The work of Kutilek and Farkasova that described the method of predicting the motion of lower extremities using neural network and had suggested that the predicted data may be useful for evaluation of human walking in physiotherapy practice based on angle-angle diagram (Kutilek and Farkasova, 2011). Their method was one of the earliest to apply neural network in a clinical practice for study of disorders or characteristics in motion function of human body, and also to be used in new design of lower limb prosthesis (Kutilek and Farkasova, 2011).

This study proposed a method of using the cyclograms as the cyclic representation of locomotion and two-variable interaction, to determine the most influential parameter in each gait subphases for the knee control design in transfemoral prosthesis. Using neural network, we analyzed the interaction between the users' gait coupled parameters holistically (of the joint itself and across two joints) toward the least error production from normal profile. The final parameter at each gait sub-phase was then determined by analyzing the effect of manipulating one parameter onto another parameter of the coupled variables at the most deviated cyclogram for different amputee's profile.

\section{METHODS}

This study consists of 3 phases. The first phase involved gait analysis and cyclogram model generation based on the leg joint parameters. Phase 2 verified the generated cyclogram models by determining the gait deviation at each sub phase throughout the gait cycle of participants with different pathological conditions. In the last phase, the modified cyclogram models were used to identify the most dominant parameter at each gait sub-phase in the case of transfemoral amputees.

\section{Phase 1: Gait Analysis and Generating Cyclograms}

In this section, the data collection procedure was explained in detail. Then, the generation of cyclogram pairs from the voluntary and mechanical joints' parameters was described.

\section{Data Collection and Gait Analysis Method}

Table 1 describes the details of the participants involved in this work.

The profile data for each of the transtibial, transfemoral and orthosis subjects and their respective pathological condition are presented in Table 2. All the subjects provided their written and informed consent by signing the consent form provided to them, to participate in this study. Approval for the gait analysis procedures was obtained from the University Malaya Medical Centre Ethics Review Board and this study confirmed with the regulatory standards.

The experimental procedures of this study were conducted in a motion analysis lab on a $4 \mathrm{~m}$ straight walkway. The lab was equipped with an optical three-dimensional motion capture 
TABLE 1 | Description of the subjects.

\begin{tabular}{|c|c|c|c|c|c|}
\hline Type of subjects & $\begin{array}{l}\text { No of } \\
\text { subjects }\end{array}$ & Mass (kg) & Height (cm) & Age (years) & Criteria \\
\hline $\begin{array}{l}\text { Normal healthy subjects (served as control } \\
\text { group) }\end{array}$ & 20 & $65.43 \pm 17.96$ & $161.4 \pm 10.25$ & $24 \pm 2.53$ & $\begin{array}{l}\text { - No pathological conditions } \\
\text { - No history of lower limb surgery } \\
\text { - No physiological disease } \\
\text { - Right limb dominant } \\
\text { - Can walk without assistance/any } \\
\text { upper extremity aids }\end{array}$ \\
\hline Transtibial amputees & 10 & $77.15 \pm 21.45$ & $168.45 \pm 9.95$ & $45.70 \pm 9.9$ & $\begin{array}{l}\text { - Unilateral amputee } \\
\text { - Has been wearing prosthesis for } \\
\text { more than } 6 \text { months. }\end{array}$ \\
\hline Transfemoral amputees & 5 & $70.60 \pm 19.07$ & $167.2 \pm 7.58$ & $34 \pm 7.56$ & \\
\hline $\begin{array}{l}\text { Orthosis wearer (represent prosthesis users } \\
\text { who do not have a perfect prosthetic device) }\end{array}$ & 10 & $67.40 \pm 17.31$ & $165.2 \pm 10.81$ & $35.40 \pm 12.9$ & $\begin{array}{l}\text { - Anatomical joints are still intact } \\
\text { - Able to walk without wearing orthosis }\end{array}$ \\
\hline
\end{tabular}

TABLE 2 | Profile data for transfemoral, transtibial and orthosis subjects.

\begin{tabular}{|c|c|c|c|}
\hline Subject code & Reason of amputation/wearing orthosis & Type of Prosthesis knee, locking, and foot/orthosis & Affected side \\
\hline TF1 & Osteosarcoma & Mechanical knee joint, Auto-lock system & $\mathrm{R}$ \\
\hline TF2 & Diabetes, infection & Quadrilateral socket, single axis knee joint, SACH foot & $\mathrm{R}$ \\
\hline TF3 & Trauma & Mechanical knee-joint, single-axis foot & $\mathrm{R}$ \\
\hline TF4 & Doctor carelessness during surgery & Hydraulic knee joint, auto-lock system Flex-foot & $\mathrm{R}$ \\
\hline TF5 & Trauma & Mechanical knee-joint, flex-foot & $\mathrm{R}$ \\
\hline T11 & Trauma & Pin lock, Flex-foot & $\mathrm{R}$ \\
\hline Т12 & Trauma & Shuttle lock, SACH foot & $\mathrm{R}$ \\
\hline T13 & Trauma & Pin lock, SACH foot & $\mathrm{L}$ \\
\hline ТT4 & Trauma & Shuttle lock, Flex-foot & $\mathrm{R}$ \\
\hline T15 & Trauma & Pin lock, flex-foot & $L$ \\
\hline TT6 & Trauma & Shuttle lock, flex-foot & $L$ \\
\hline T17 & Diabetes & Shuttle lock, Flex-foot & $\mathrm{R}$ \\
\hline Т18 & Trauma & Pin lock, SACH foot & $\mathrm{L}$ \\
\hline ТT9 & Trauma & Shuttle lock, Flex-foot & $\mathrm{R}$ \\
\hline T10 & Gangrene on 1st toe, Diabetes & Pin lock, Flex-foot & $\mathrm{R}$ \\
\hline OT1 & Congenital flexible pes planus & Custom-made shoe with arch insole & $\mathrm{B}$ \\
\hline OT2 & Limb length discrepancy $(1.2 \mathrm{~cm})$ & Custom-made insole & $\mathrm{L}$ \\
\hline OT3 & Diabetes, 1st metatarsal ray amputation & Diabetic shoe & $L$ \\
\hline OT4 & Inflammation at medial collateral ligament & Knee brace & $\mathrm{R}$ \\
\hline OT5 & Diabetes, callus at 5th metatarsal & Diabetic shoe with insole & B \\
\hline OT6 & Flexible Pes Planus & Arch insole & B \\
\hline OT7 & Diabetes, 2nd metatarsal ray amputation & Custom-made insole & $\mathrm{R}$ \\
\hline OT8 & Flexible pes Planus & Custom-made insole & B \\
\hline OT9 & Hallux valgus, present of bunion on 1st metatarsal & Hinged AFO & $\mathrm{L}$ \\
\hline OT10 & Charcot foot & Rigid AFO & $\mathrm{R}$ \\
\hline
\end{tabular}

R, Right; L, Left; B, Both; TT, Transtibial; TF, Transfemoral; OT, Orthotic; AFO, Ankle-foot Orthosis.

system, a 6-infrared camera Vicon Nexus 1.5 (Vicon, United Kingdom) mounted strategically around the room, working at $50 \mathrm{~Hz}$ and integrated with two force plates (Kistler Instruments, Switzerland) working at $200 \mathrm{~Hz}$, located midway to obtain synchronized kinematic and kinetic data within a capture volume of approximately $4 \times 4 \times 2 \mathrm{~m}$.
The subjects' bilateral leg length (from greater trochanter to lateral malleolus), ankle width and knee width were measured and recorded to the system software for automated calculation of mass, center of mass and moment of inertia. The knee width of residual limb was considered as similar with the sound limb for transfemoral subjects. The ankle width for both transfemoral and 
transtibial subject residual limb were also considered to be similar to the sound limb measurement. Ground reaction force was obtained from the force plates for kinetic automated calculation by the Vicon software to obtain joint power and moment. Sixteen reflective markers of $14 \mathrm{~mm}$ diameter were attached bilaterally based on Helen Hayes marker placement.

At least 20 trials were recorded for each subject and all the subjects were asked to rest in between the trial to prevent fatigue effect to the data collection. The gait data was analyzed with a conventional model Vicon PlugIn-Gait and filtered using second-order Butterworth filter. The ankle, knee and hip joint kinematics, moments and gait events as well as relevant markers' trajectories, Ground Reaction Force (GRF) and Center of Pressure (COP) data were imported into Matlab for extraction and further analysis. Linear interpolation was applied to the original data points to obtain data points for joint kinematics and kinetics data at every $2 \%$ of stride duration. The joint moment was computed by inverse dynamics, using subjects' measurements and anthropometric properties and normalized to body weight (Robertson, 2004).

The ankle dorsi-plantar flexion angles, knee and hip flexionextension angles were the kinematic data required in this study, while for kinetic analysis, all the three joints' sagittal moment and power were extracted.

\section{Cyclogram Models Generation}

A total of 36 relationships were obtained by pairing the variables of the joint itself and across the two joints pair. The relationships can be classified into 6 groups, namely:

i) angle-angle across the two joints pair (3 pairs),

ii) moment-moment across the two joints pair (3 pairs),

iii) power-power across the two joints pair (3 pairs), iv) angle-moment within the joint and across the two joints pair (9 pairs),

v) angle-power within the joint and across the two joints pair (9 pairs), and,

vi) moment-power within the joint and across the two joints pair (9 pairs).

Most of these relationships were not found in the literature except for angle-angle relationship across the two joints pair (Cavanagh and Grieve, 1973; Goswami, 1998) and moment-angle of the ankle joint (Crenna and Frigo, 2011; Wang et al., 2012).

The cyclogram relationships for the normal subjects were obtained by averaging parameters of 200 trials from 20 subjects due to the consistency of the gait sub-phase duration among the normal subjects. On the other hand, this averaging method does not apply to the transtibial, transfemoral, and orthosis subjects because of their irregularities of gait sub-phase duration in each trial. Thus, the relationships were obtained from each of the trial, for 10 trials for these amputee subjects. The amputee subjects' cyclograms in addition to its respective gait sub-phases from the trials would be input into the neural network in Phase 2 to predict the output of subject-based movement profiles and identify the gait deviation at each of the gait sub-phase.

From the normal cyclograms relationship obtained, it was found that the geometric shape of the cyclograms changed as the traveling direction of the gait sub-phases change between both legs. Thus, it was deduced that the interaction between the two variables were different between left and right leg. Primarily, this geometric difference was influenced by the limb dominance effect on the normal walking gait. The dominant limb was the preferred limb (leading limb) that the subjects used for mobilizing or propulsion; while in contrast, the nondominant limb is the non-preferred limb (trailing limb) that is used to support the actions, i.e., stability control. This support

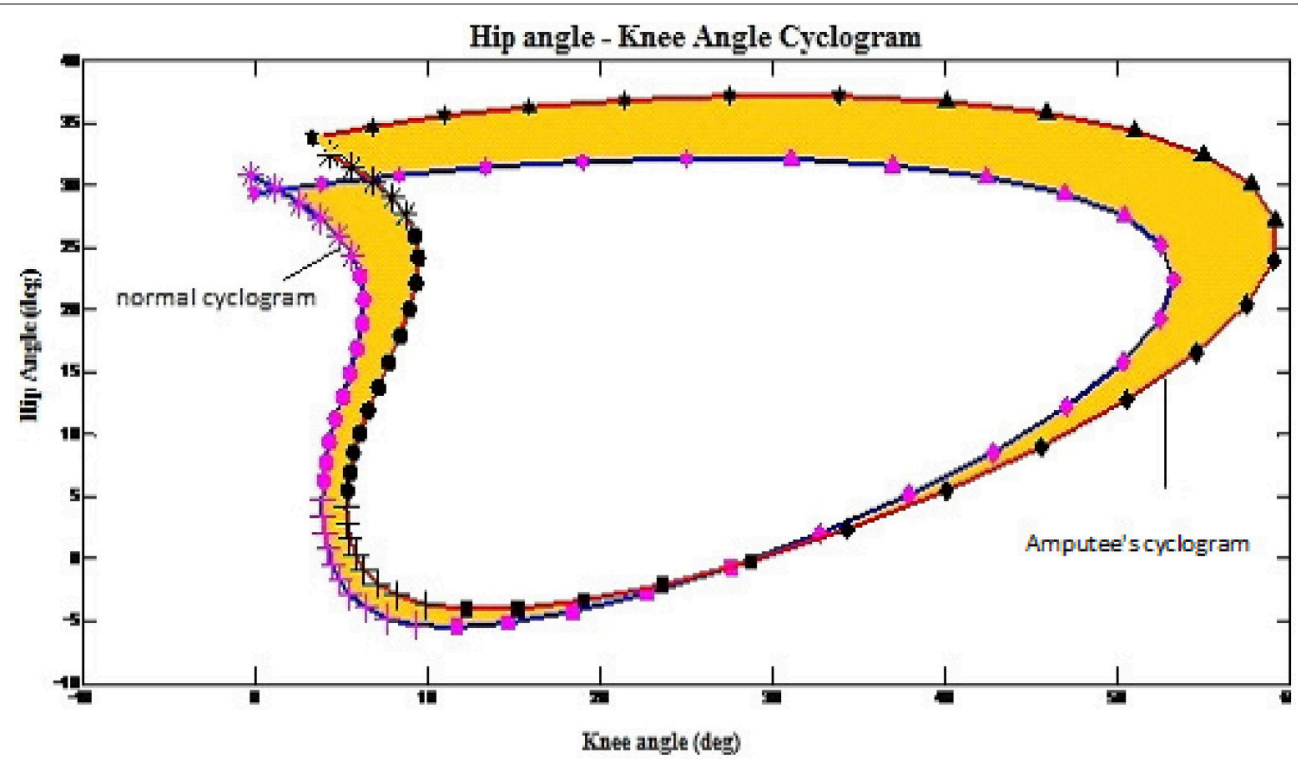

FIGURE 1 | Example on how the cyclograms being applied to obtain the gait disparity in amputees. The shaded area indicate disparity from norm. 


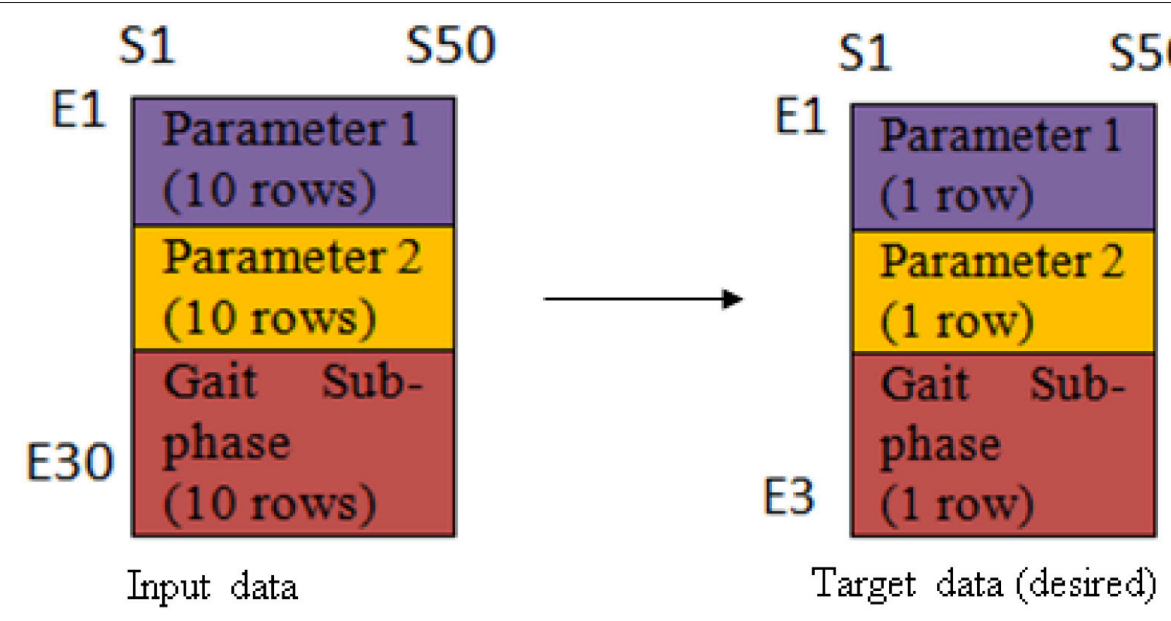

FIGURE 2 | Illustration of the arrangement of input data and target data for network training.

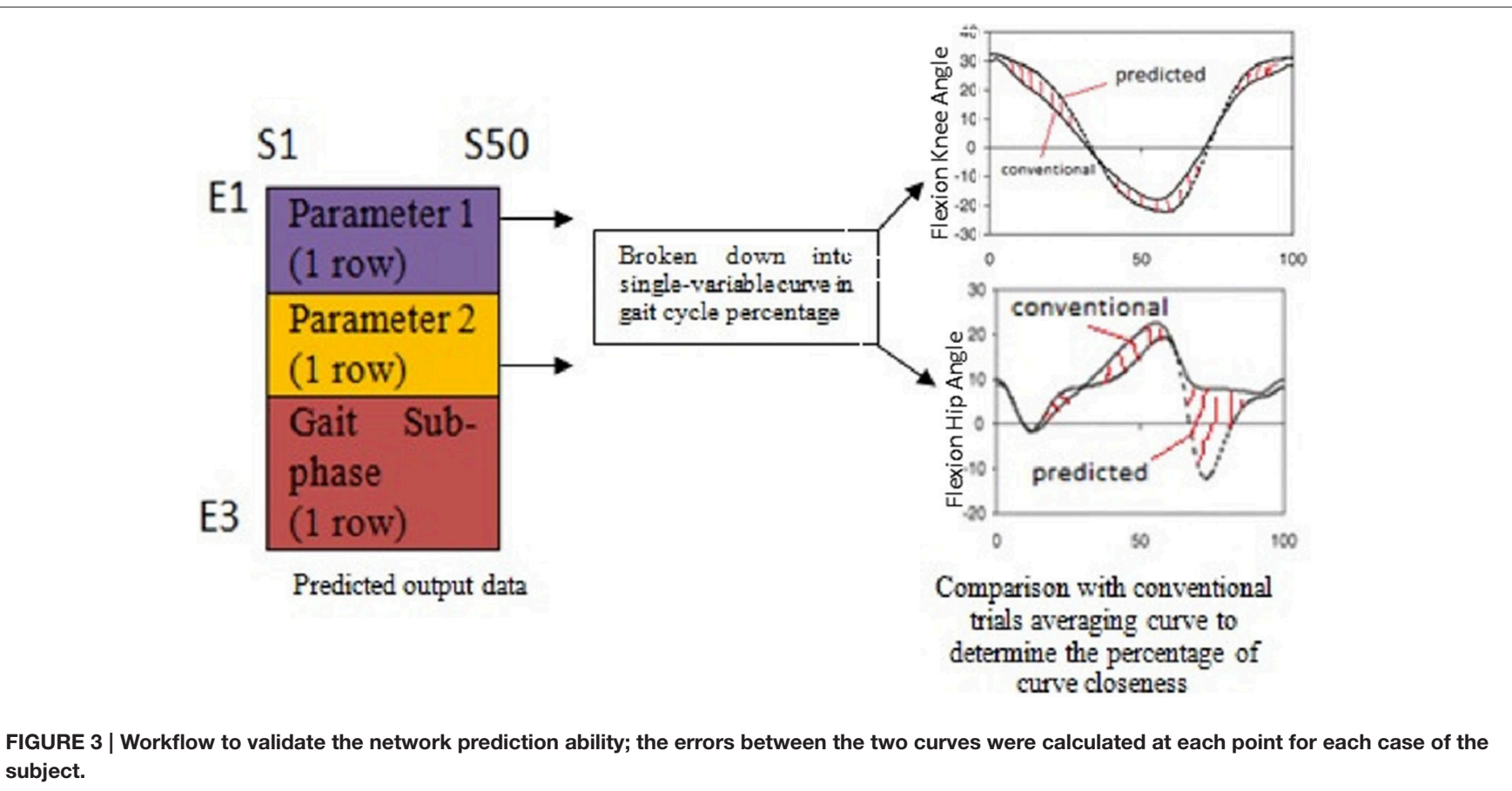

and mobility task performed by each leg was interpreted as gait asymmetry that is normal to occur even to healthy able-bodied subjects. All the normal subjects in this study were right limb dominant, therefore, right side served as the propulsion source during walking. The necessity of defining which limb contributes to propulsion or support task was important in this study in order to select the reference side of cyclograms that was identical to the functional role of the prosthetic and the intact side for amputees. It has been revealed in the study by Carpenter et al. that the transtibial amputees who undergone osteomyoplastic amputation (also known as Ertl) rely on the prosthetic side to support, while the intact side function to propel the body forward (Carpenter et al., 2012). This is further supported by the study that revealed the unilateral amputees experience increased asymmetry in their intact limb during loading and stance time due to the loss of ankle plantar-flexors which contribute to body propulsion and swing initiation (Liu et al., 2006). Therefore, the subsequent phases in this study make use of the cyclograms on the left side (non-dominant) to study the deviation that occur on the prosthetic side of the amputee.

\section{Phase 2: Using Cyclograms to Quantify Gait Disparity in Terms of Gait Sub-Phases}

All 36 cyclograms that were created in previous phase were used to determine the gait disparity of the amputees. Figure 1 illustrates the example on how the cyclograms was utilized to determine the gait disparity occurrence at each gait sub-phase for each of the subject. The orange-colored area represents the error 
between amputee's cyclogram and the normal cyclogram, i.e., the gait disparity. The higher the error as compared to the normal gait's cyclogram, the higher is the gait deviation of the subject.

At this stage, neural network was adopted as the tool to model the interaction between two parameters in a cyclogram, as well as to calculate the disparity between cyclograms. Hence, the feedforward network with backpropagation algorithm was employed. This network was chosen as it uses supervised learning rule during the training. The supervised learning rule provide an example for the network learning, and the network learn by comparing the predicted output with the desired output, and backpropagate the error to update the network weight/bias (i.e., the constant used to define the strength of the input in determining the output) (Beale et al., 2010). The neural network architecture was constructed in Matlab and the predicted outputs and errors obtained were used to quantify the disparity occurrence at each of the gait sub-phase.

The network consisted of 3 layers; input layer (30 neurons), hidden layer ( 7 neurons) and output layer ( 3 neurons). The relationship between the input data and the output data was found linear, thus linear regression functions were employed. The input and target data arrangement was illustrated as in Figure 2. The first 10 rows (E1-E10) represent the first cyclogram's variable for 10 trials, the next 10 rows are the second cyclogram's variable for 10 trials, and the last 10 rows are the gait sub-phases which annotated as 1 until 7 corresponding to loading response to terminal-swing for 10 trials. On the other hand, S1-S50 represents the samples of the variables series at an interval of $2 \%$ gait cycle and its respective gait sub-phase duration.

The network was first trained with data of 100 trials that was randomly chosen from 10 healthy subjects as network input and average normal data of 200 trials from 20 healthy subjects as the network target in order for the NN to learn the outcome of different paired variables interactions of normal subject as the reference. A total of $36 \mathrm{NN}$ were trained with 36 paired variables, i.e., cyclograms, respectively. During the training, default random division algorithm was used for the $\mathrm{NN}$ to divide the data randomly for training (60\%), validation (20\%), and testing (20\%). The network weight was reinitialized if all the following criteria for the network performance were not met, and only network that fulfilled the following criteria were kept for simulation with prosthetic cyclograms: (1) final mean square error $<0.9$ at the final iteration, (2) the test set error and validation set error has similar characteristics, (3) the regression for all set was $>0.9$.

Before simulating the trained network with amputee's cyclograms, the network was first tested and validated with 5 random normal subjects and 25 different cases of amputees and orthosis subjects' pathological conditions. The predicted output of cyclogram's pair from the simulation was split into two variables curve as a function of gait cycle percentage. These two curves were then compared with the curves that used the conventional trials averaging method. The errors between the two curves $\left(e_{p-c}\right)$ were calculated for each subject as illustrated in Figure 3.

The network model prediction ability was presented in terms of percentage of the closeness of the network predicted output curve as compared to the conventional trials averaging curve. The percentage of closeness was calculated as in Equation (1), with $C=100 \%$ indicates totally close to normal or a perfect match to normal.

$$
C=\left(1-e_{p-c}\right) \times 100
$$

where,

$$
C=\text { curves closeness }
$$

$e_{p-c}=$ Errors between predicted curve points and conventional curve points, with 1 being maximum (100\%) error or difference between predicted and conventional curve points, and 0 to be no error at all.

The validation percentage presented in Table 3 indicates that the model, as it was trained with the normal subjects' data, was as expected to have prediction ability of more than $90 \%$ with randomly-selected normal subjects. As for orthosis, transtibial, and transfemoral subjects, the percentage range indicates that the NN used has the ability to segregate the deviated data as a whole series of gait cycle and by the two interacting variables that might have contributed most to the gait deviation. The validation for these "pathological" subjects was expected to be $<90 \%$, with transfemoral subject having the greatest error (Table 3 ) due to the least voluntary control of residual limb. This error was used as the quantifying measure in this study.

\section{Phase 3: Using Manipulated Cyclograms to Identify the Dominant Parameter(s) in Each Gait Sub-Phases}

In this phase, the results obtained from the previous phase were used to assess the effect of manipulating one variable toward the other variable of the cyclograms. At each of the gait sub-phase for each amputee, the cyclograms relationship that resulted in the largest deviation was then being input back into the linear network model. A modification of the variable values at the particular sub-phase with the highest mean normalized error was done by inserting the normal value (i.e., from healthy subjects that is averaged using conventional trials averaging method described in Section Cyclogram Models Generation) at one of the paired variables as illustrated in Figure 4. The network model was simulated with the modified input data and the target output data was again being supplied. The error between the predicted output and the desired output was calculated again at each of the sub-phase. This process was repeated with the other variable of the pair. The results obtained were then compared to determine which parameter manipulation gives predicted output that was closer-to-normal cyclograms. This was the crucial identification

TABLE 3 | Percentage range of closeness between networks predicted output curve and conventional trials averaging curve.

\begin{tabular}{lcc}
\hline Subjects & $\begin{array}{c}\text { No. of } \\
\text { subjects }\end{array}$ & $\begin{array}{c}\text { Percentage range } \\
\text { (close-to-conventional-curve), C }\end{array}$ \\
\hline Normal & 5 & $91-99 \%$ \\
Orthosis & 10 & $75-87 \%$ \\
Transtibial & 10 & $73-81 \%$ \\
Transfemoral & 5 & $68-79 \%$
\end{tabular}


From Phase 2:

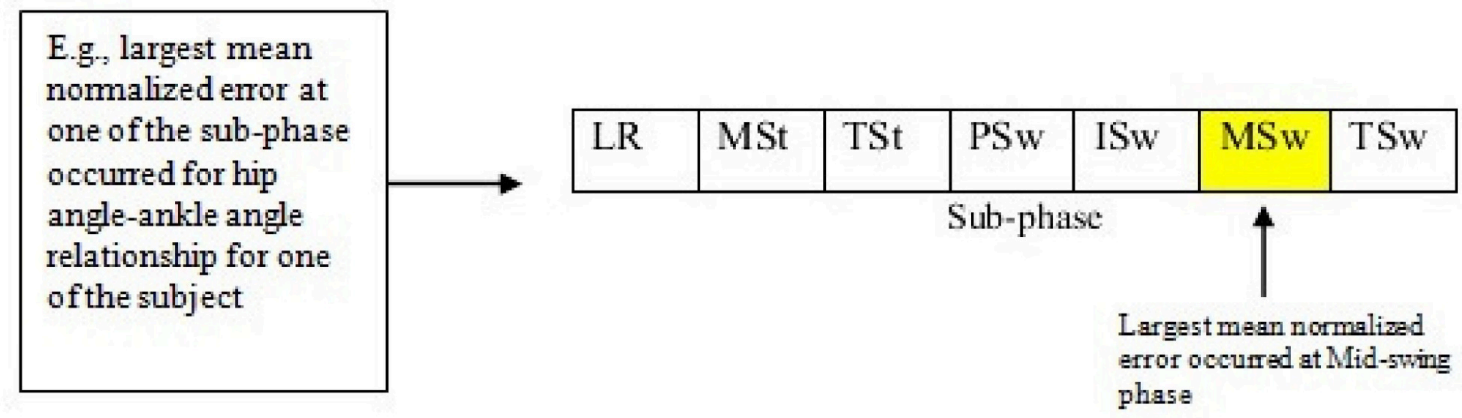

The hip angle - ankle angle relationship is trans ferred back into the $\mathrm{NN}$ in Phase 3

Phase 3:

\begin{tabular}{|l|l|l|l|l|l|l|l|l|}
\hline $\begin{array}{l}\text { H } \theta- \\
\text { A } \theta\end{array}$ & He & LR & MSt & TSt & PSw & ISw & MSw & TSw \\
\cline { 2 - 8 } & A $\theta$ & LR & MSt & TSt & PSw & ISw & MSw & TSw \\
\hline
\end{tabular}

\begin{tabular}{|l|l|l|l|l|l|l|l|l|}
\hline \multirow{2}{*}{$\begin{array}{l}\text { H } \theta- \\
\text { A }\end{array}$} & H $\theta$ & LR & MSt & TSt & PSw & ISw & MSw & TSw \\
\cline { 2 - 8 } & A $\theta$ & LR & MSt & TSt & PSw & ISw & MSw & TSw \\
\hline \multicolumn{10}{c}{} & †
\end{tabular}

Input backinto the $\mathrm{NN}$

Manipulated variable

The values are replaced with normal values at this

particular phase

FIGURE 4 | The workflow of transferring the results from Phase 2 and Phase 3.

step in determining the relevant parameter to be controlled at each of the gait sub-phase.

\section{RESULTS}

\section{Using Cyclograms to Quantify Gait Disparity in Terms of Gait Sub-Phases}

The cyclograms relationship that contributed to the highest mean normalized error at each gait sub-phase for each subject was summarized in Table 4. At loading response (LR), the kinematics-kinetics relationship shows high mean normalized error for each of the subject. The kinetics variable of the pair indicated in red color contributes to the highest mean normalized error, except for subjects TT3 (ankle angle, A $\theta$ ) and TT5 (knee angle, $\mathrm{K} \theta$ ).

At mid-stance (MSt) phase, the result indicated that the kinematics-kinetics relationship has the highest mean normalized error for each of the subject, except for TT5 (AM-HP), TT6 (AM-HP), and TT8 (AM-HP), which shows the kinetics-kinetics relationship. Nevertheless, the variable of the pairs that contributes the highest mean normalized error at this phase were all originated from the kinetics variable (knee power/hip power/hip moment). Except for TT5, TT6, TF4, and TF5 which shows that kinetics-kinetics relationship has high mean normalized error at terminal-stance (TSt), the rest showed that the kinematics-kinetics pair exhibits high mean normalized error. Similar with the MSt phase, the kinetic variable (knee power/hip power/hip moment) of the pairs has the highest mean normalized error in TSt phase compared to the kinematic variable.

Similar cyclograms' relationship profile as the aforementioned MSt and TSt were found for pre-swing (PSw), initial-swing (ISw) and mid-swing (MSw), whereby most of the relationships that yield the high mean normalized error were of kinematicskinetics pair except for TF4 (AM-HP) in pre-swing and TT2 (AM-HP) in ISw. As for terminal-swing (Tsw), 10 out of the 15 amputee subjects showed unanimously that the kinematics of knee angle variable yield the highest mean normalized error. This contradicted with the results of previous gait sub-phases in which kinetics variable (ankle moment/ankle power/knee moment/knee power/hip moment/hip power) of the pair contributed to the highest mean normalized error. 
TABLE 4 | Summary of the paired variables that contribute to the highest mean normalized error at respective gait sub-phase for each amputee subject.

\begin{tabular}{|c|c|c|c|c|c|c|c|}
\hline \multirow[t]{2}{*}{ Subjects } & \multicolumn{7}{|c|}{ Cyclograms relationship that contribute to highest mean normalized error } \\
\hline & LR & MSt & TSt & PSw & ISw & MSw & TSw \\
\hline T1 & $\begin{array}{l}\mathrm{K} \theta-\mathrm{AM} \\
\mathbf{( 1 7 . 7 2 )}\end{array}$ & $\begin{array}{l}\mathrm{H} \theta-\mathrm{HM} \\
\mathbf{( 1 0 . 8 8 )}\end{array}$ & $\begin{array}{l}\mathrm{H} \theta-\mathrm{HM} \\
\mathbf{( 1 1 5 . 1 6 )}\end{array}$ & $\begin{array}{l}A \theta-K P \\
(\mathbf{4 1 . 4 0 )}\end{array}$ & $\begin{array}{l}\mathrm{H} \theta-\mathrm{HP} \\
\mathbf{( 5 6 . 3 7 )}\end{array}$ & $\begin{array}{l}\mathrm{H} \theta-\mathrm{HP} \\
\mathbf{( 5 7 9 . 2 5 )}\end{array}$ & $\begin{array}{l}\mathrm{K} \theta-\mathrm{AM} \\
\mathbf{( 4 3 0 . 1 2 )}\end{array}$ \\
\hline Т12 & $\begin{array}{l}H \theta-K P \\
\mathbf{( 1 0 . 6 3 )}\end{array}$ & $\begin{array}{l}\mathrm{H} \theta-\mathrm{HM} \\
\mathbf{( 9 . 0 8 )}\end{array}$ & $\begin{array}{l}\mathrm{H} \theta-\mathrm{HM} \\
\mathbf{( 1 4 1 . 1 0 )}\end{array}$ & $\begin{array}{l}\text { AM-HP } \\
\mathbf{( 1 4 0 . 4 7 )}\end{array}$ & $\begin{array}{l}\text { AM-HP } \\
\mathbf{( 3 6 . 3 7 )}\end{array}$ & $\begin{array}{l}\mathrm{H} \theta-\mathrm{HP} \\
\mathbf{( 3 6 9 . 5 3 )}\end{array}$ & $\begin{array}{l}K \theta-K P \\
(\mathbf{2 4 5 . 6 0 )}\end{array}$ \\
\hline ТТ3 & $\begin{array}{l}\mathrm{A} \theta-\mathrm{HM} \\
\mathbf{( 5 1 . 6 9 )}\end{array}$ & $\begin{array}{l}A \theta-K P \\
\mathbf{( 5 0 . 3 1 )}\end{array}$ & $\begin{array}{l}\mathrm{H} \theta-\mathrm{HP} \\
\mathbf{( 1 5 6 . 7 8 )}\end{array}$ & $\begin{array}{l}A \theta-K P \\
\mathbf{( 3 8 . 6 7 )}\end{array}$ & $\begin{array}{l}A \theta-H P \\
\mathbf{( 2 2 3 . 3 0 )}\end{array}$ & $\begin{array}{l}A \theta-H P \\
\mathbf{( 3 6 7 . 8 1 )}\end{array}$ & $\begin{array}{l}\mathrm{K} \theta-\mathrm{AM} \\
\mathbf{( 7 0 6 . 8 8 )}\end{array}$ \\
\hline Tा4 & $\begin{array}{l}\mathrm{K} \theta-\mathrm{AP} \\
\mathbf{( 1 3 . 8 9 )}\end{array}$ & $\begin{array}{l}\mathrm{H} \theta-\mathrm{HM} \\
\mathbf{( 6 . 6 5 )}\end{array}$ & $\begin{array}{l}\mathrm{H} \theta-\mathrm{HM} \\
\mathbf{( 1 0 4 . 5 6 )}\end{array}$ & $\begin{array}{l}\mathrm{H} \theta-\mathrm{HP} \\
\mathbf{( 6 4 . 1 9 )}\end{array}$ & $\begin{array}{l}\mathrm{H} \theta-\mathrm{HM} \\
\mathbf{( 5 0 . 4 4 )}\end{array}$ & $\begin{array}{l}\mathrm{H} \theta-H P \\
\mathbf{( 4 5 5 . 8 8 )}\end{array}$ & $\begin{array}{l}\mathrm{K} \theta-\mathrm{AP} \\
\mathbf{( 1 9 1 . 6 5 )}\end{array}$ \\
\hline T15 & $\begin{array}{l}\mathrm{K} \theta-\mathrm{KM} \\
\mathbf{( 1 1 . 1 6 )}\end{array}$ & $\begin{array}{l}\text { AM-HP } \\
\mathbf{( 8 . 4 8 )}\end{array}$ & $\begin{array}{l}\text { AM-HP } \\
\mathbf{( 1 1 4 . 9 2 )}\end{array}$ & $\begin{array}{l}\mathrm{H} \theta-\mathrm{HM} \\
\mathbf{( 4 9 . 7 1 )}\end{array}$ & $\begin{array}{l}\mathrm{H} \theta-\mathrm{HP} \\
\mathbf{( 1 1 8 1 . 7 9 )}\end{array}$ & $\begin{array}{l}\mathrm{K} \theta-\mathrm{AP} \\
\mathbf{( 2 9 4 . 8 9 )}\end{array}$ & $\begin{array}{l}\mathrm{K} \theta-\mathrm{AM} \\
\mathbf{( 9 2 1 . 1 4 )}\end{array}$ \\
\hline Tा6 & $\begin{array}{l}\mathrm{H} \theta-\mathrm{KP} \\
\mathbf{( 1 0 . 7 0 )}\end{array}$ & $\begin{array}{l}\text { AM-HP } \\
(\mathbf{1 4 . 4 2})\end{array}$ & $\begin{array}{l}\text { AM-HP } \\
\mathbf{( 7 6 . 4 0 )}\end{array}$ & $\begin{array}{l}\mathrm{A} \theta-\mathrm{HP} \\
\mathbf{( 3 6 . 9 8 )}\end{array}$ & $\begin{array}{l}\mathrm{H} \theta-\mathrm{HP} \\
\mathbf{( 8 5 . 9 4 )}\end{array}$ & $\begin{array}{l}\mathrm{H} \theta-H \mathrm{P} \\
\mathbf{( 2 0 7 . 1 9 )}\end{array}$ & $\begin{array}{l}\mathrm{K} \theta-\mathrm{AP} \\
\mathbf{( 3 6 5 . 2 2 )}\end{array}$ \\
\hline T17 & $\begin{array}{l}\mathrm{H} \theta-\mathrm{HM} \\
\mathbf{( 8 . 8 5 )}\end{array}$ & $\begin{array}{l}\mathrm{H} \theta-\mathrm{HM} \\
\mathbf{( 1 1 . 5 9 )}\end{array}$ & $\begin{array}{l}\mathrm{H} \theta-\mathrm{HP} \\
\mathbf{( 1 5 6 . 9 6 )}\end{array}$ & $\begin{array}{l}\mathrm{K} \theta-\mathrm{AM} \\
\mathbf{( 2 6 . 0 4 )}\end{array}$ & $\begin{array}{l}\mathrm{H} \theta-\mathrm{HM} \\
\mathbf{( 8 5 . 0 1 )}\end{array}$ & $\begin{array}{l}\mathrm{H} \theta-\mathrm{HP} \\
\mathbf{( 3 6 0 . 3 5 )}\end{array}$ & $\begin{array}{l}\mathrm{K} \theta-\mathrm{AM} \\
\mathbf{( 2 5 5 . 8 7 )}\end{array}$ \\
\hline ТТ8 & $\begin{array}{l}\mathrm{H} \theta-\mathrm{HM} \\
\mathbf{( 1 3 . 3 7 )}\end{array}$ & $\begin{array}{l}\text { AM-HP } \\
\mathbf{( 1 5 . 4 6 )}\end{array}$ & $\begin{array}{l}\mathrm{H} \theta-\mathrm{HM} \\
\mathbf{( 7 3 . 9 8 )}\end{array}$ & $\begin{array}{l}\mathrm{H} \theta-\mathrm{HM} \\
\mathbf{( 1 0 . 2 4 )}\end{array}$ & $\begin{array}{l}\mathrm{K} \theta-\mathrm{AP} \\
\mathbf{( 7 3 . 8 5 )}\end{array}$ & $\begin{array}{l}\mathrm{H} \theta-\mathrm{HP} \\
\mathbf{( 5 2 3 . 1 8 )}\end{array}$ & $\begin{array}{l}\mathrm{K} \theta-\mathrm{AP} \\
\mathbf{( 2 2 4 . 1 2 )}\end{array}$ \\
\hline ТТ9 & $\begin{array}{l}A \theta-K P \\
(\mathbf{7 . 9 3 )}\end{array}$ & $\begin{array}{l}\mathrm{H} \theta-\mathrm{HP} \\
\mathbf{( 8 . 1 5 )}\end{array}$ & $\begin{array}{l}\mathrm{H} \theta-\mathrm{HP} \\
\mathbf{( 1 3 6 . 2 3 )}\end{array}$ & $\begin{array}{l}\mathrm{H} \theta-\mathrm{HM} \\
\mathbf{( 1 9 . 6 2 )}\end{array}$ & $\begin{array}{l}\mathrm{H} \theta-\mathrm{HM} \\
\mathbf{( 3 9 . 3 8 )}\end{array}$ & $\begin{array}{l}\mathrm{H} \theta-\mathrm{HP} \\
\mathbf{( 5 0 9 . 1 8 )}\end{array}$ & $\begin{array}{l}K \theta-A P \\
(\mathbf{1 8 6 . 9 1 )}\end{array}$ \\
\hline T10 & $\begin{array}{l}A \theta-K P \\
(\mathbf{9 . 4 3 )}\end{array}$ & $\begin{array}{l}\mathrm{H} \theta-\mathrm{HM} \\
\mathbf{( 9 . 4 5 )}\end{array}$ & $\begin{array}{l}\mathrm{H} \theta-\mathrm{HP} \\
\mathbf{( 1 2 5 . 6 6 )}\end{array}$ & $\begin{array}{l}\mathrm{K} \theta-\mathrm{AM} \\
\mathbf{( 2 2 . 2 4 )}\end{array}$ & $\begin{array}{l}\mathrm{K} \theta-\mathrm{AP} \\
\mathbf{( 6 9 . 5 7 )}\end{array}$ & $\begin{array}{l}\mathrm{H} \theta-\mathrm{HP} \\
\mathbf{( 4 0 4 . 9 8 )}\end{array}$ & $\begin{array}{l}\mathrm{K} \theta-\mathrm{AP} \\
\mathbf{( 3 4 9 . 9 2 )}\end{array}$ \\
\hline TF1 & $\begin{array}{l}\mathrm{K} \theta-\mathrm{AM} \\
\mathbf{( 1 6 . 1 1 )}\end{array}$ & $\begin{array}{l}\mathrm{H} \theta-\mathrm{HM} \\
\mathbf{( 9 . 5 0 )}\end{array}$ & $\begin{array}{l}\mathrm{H} \theta-\mathrm{HM} \\
\mathbf{( 2 2 5 . 6 6 )}\end{array}$ & $\begin{array}{l}\mathrm{H} \theta-\mathrm{HP} \\
\mathbf{( 4 3 . 7 4 )}\end{array}$ & $\begin{array}{l}\mathrm{K} \theta-\mathrm{AP} \\
\mathbf{( 1 2 7 . 4 3 )}\end{array}$ & $\begin{array}{l}\mathrm{H} \theta-\mathrm{HP} \\
\mathbf{( 5 2 5 . 0 5 )}\end{array}$ & $\begin{array}{l}\mathrm{H} \theta-\mathrm{K} \theta \\
\mathbf{( 4 0 2 . 2 0 )}\end{array}$ \\
\hline TF2 & $\begin{array}{l}\mathrm{K} \theta-\mathrm{AM} \\
\mathbf{( 1 5 . 1 7 )}\end{array}$ & $\begin{array}{l}\mathrm{H} \theta-\mathrm{HM} \\
\mathbf{( 8 . 3 3 )}\end{array}$ & $\begin{array}{l}\mathrm{H} \theta-\mathrm{HM} \\
\mathbf{( 1 3 2 . 9 6 )}\end{array}$ & $\begin{array}{l}\mathrm{H} \theta-\mathrm{HP} \\
\mathbf{( 5 1 . 4 4 )}\end{array}$ & $\begin{array}{l}\mathrm{H} \theta-\mathrm{HM} \\
\mathbf{( 8 7 . 0 0 )}\end{array}$ & $\begin{array}{l}\mathrm{H} \theta-\mathrm{HP} \\
\mathbf{( 5 7 0 . 2 0 )}\end{array}$ & $\begin{array}{l}\mathrm{H} \theta-\mathrm{K} \theta \\
\mathbf{( 4 9 9 . 6 6 )}\end{array}$ \\
\hline TF3 & $\begin{array}{l}\mathrm{H} \theta-\mathrm{HM} \\
\mathbf{( 1 3 . 4 6 )}\end{array}$ & $\begin{array}{l}\mathrm{H} \theta-\mathrm{HP} \\
\mathbf{( 2 6 . 8 3 )}\end{array}$ & $\begin{array}{l}\mathrm{H} \theta-\mathrm{HP} \\
\mathbf{( 4 0 0 . 5 3 )}\end{array}$ & $\begin{array}{l}\mathrm{H} \theta-\mathrm{HP} \\
\mathbf{( 1 4 3 . 4 8 )}\end{array}$ & $\begin{array}{l}\mathrm{K} \theta-\mathrm{AP} \\
\mathbf{( 7 0 . 9 8 )}\end{array}$ & $\begin{array}{l}\mathrm{H} \theta-\mathrm{HP} \\
\mathbf{( 5 2 5 . 0 0 )}\end{array}$ & $\begin{array}{l}\mathrm{H} \theta-\mathrm{K} \theta \\
\mathbf{( 4 8 9 . 0 7 )}\end{array}$ \\
\hline TF4 & $\begin{array}{l}\mathrm{K} \theta-\mathrm{AM} \\
\mathbf{( 1 2 . 7 1 )}\end{array}$ & $\begin{array}{l}\mathrm{H} \theta-\mathrm{HM} \\
\mathbf{( 9 . 7 1 )}\end{array}$ & $\begin{array}{l}\text { AM-HP } \\
\mathbf{( 2 3 0 . 0 8 )}\end{array}$ & $\begin{array}{l}\text { AM-HP } \\
\text { (87.39) }\end{array}$ & $\begin{array}{l}\mathrm{H} \theta-\mathrm{KM} \\
\mathbf{( 3 3 . 7 7 )}\end{array}$ & $\begin{array}{l}\mathrm{H} \theta-\mathrm{HP} \\
\mathbf{( 5 2 0 . 4 7 )}\end{array}$ & $\begin{array}{l}\mathrm{K} \theta-\mathrm{AP} \\
\mathbf{( 5 0 2 . 4 4 )}\end{array}$ \\
\hline TF5 & $\begin{array}{l}\mathrm{K} \theta-\mathrm{AM} \\
\mathbf{( 1 5 . 5 2 )}\end{array}$ & $\begin{array}{l}A \theta-K P \\
(6.71)\end{array}$ & $\begin{array}{l}\text { AM-KP } \\
\mathbf{( 1 2 . 9 2 )}\end{array}$ & $\begin{array}{l}\mathrm{H} \theta-\mathrm{HP} \\
\mathbf{( 1 3 3 . 4 0 )}\end{array}$ & $\begin{array}{l}\mathrm{H} \theta-\mathrm{HM} \\
\mathbf{( 3 8 . 5 0 )}\end{array}$ & $\begin{array}{l}\mathrm{H} \theta-\mathrm{HP} \\
\mathbf{( 3 2 9 . 2 1 )}\end{array}$ & $\begin{array}{l}\mathrm{K} \theta-\mathrm{AP} \\
\mathbf{( 4 3 2 . 5 4 )}\end{array}$ \\
\hline
\end{tabular}

A, Ankle; K, Knee; H, Hip; $\theta$, Angle; M, Moment; P, Power.

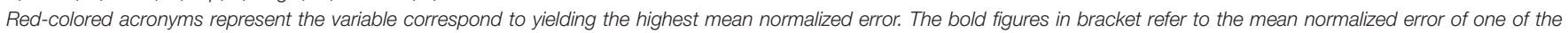
paired variable, $\bar{E}$.

\section{Using Manipulated Cyclograms to Identify the Most Dominant Parameter in Each Gait Sub-Phase}

The results of the manipulated variable that showed the lowest mean normalized error were translated as the variable that induced closest-to-normal cyclograms curve compared to the after-effect of manipulating the other parameters. The overall results were summarized as in Table 5. The results indicated that at the LR phase, when the knee power was manipulated, i.e., replaced with normal values, the mean normalized error was reduced at the phase and consequently the other phases along the gait cycle were also improved. At MSt phase, the result showed that manipulating ankle angle and knee power produced the lowest mean normalized error. The results obtained from NN showed that manipulating either one of these two parameters would yield the same result along the whole gait cycle. This indicated that modifying either one of the parameter is sufficient to produce close to normal gait profile during MSt. For TSt, ankle angle was the dominant parameter that demonstrated the lowest mean normalized error. While at PSw and ISw, hip power and hip angle, respectively produced the lowest mean normalized error.

Manipulation of any one of the parameters at MSw does not improve the sub-phase normalized errors. Observation at this phase indicated that the manipulation of parameter at this phase consequently caused the neighboring phases to yield a large mean normalized error. Therefore, no parameter was selected for manipulation at this sub-phase. Finally, for the TSw, knee power parameter was selected as it demonstrated the lowest mean normalized error when being manipulated.

\section{Identifying Dominant Knee Parameters for Potential Microcontroller Based Knee Application: A Case Study}

All the dominant parameters identified through this method were of either hip, knee or ankle parameters. However, in case of minimizing gait disparity through optimization of the knee parameters only, results related to hip and ankle parameters were revisited to identify the dominant knee parameters in its place. 
TABLE 5 | Manipulated parameter that yield the lowest mean normalized error, $\overline{\mathrm{E}}$.

\begin{tabular}{|c|c|c|c|c|c|c|c|}
\hline Gait Sub-phase & LR & MSt & TSt & PSw & ISw & MSw & TSw \\
\hline $\begin{array}{l}\text { a) Summary of the parameter that yield the } \\
\text { lowest mean normalized error }\end{array}$ & Knee Power & $\begin{array}{l}\text { Ankle Angle, } \\
\text { Knee Power }\end{array}$ & Ankle Angle & Hip Power & Hip Angle & - & Knee Power \\
\hline $\begin{array}{l}\text { b) Revised parameter at each of the gait } \\
\text { sub-phase specifically for prosthetic knee }\end{array}$ & Knee Power & Knee Power & $\begin{array}{l}\text { Knee Moment, } \\
\text { Knee Angle }\end{array}$ & $\begin{array}{l}\text { Knee Angle, } \\
\text { Knee Power }\end{array}$ & Knee Angle & - & Knee Power \\
\hline
\end{tabular}

MSw phase does not require any joint control.

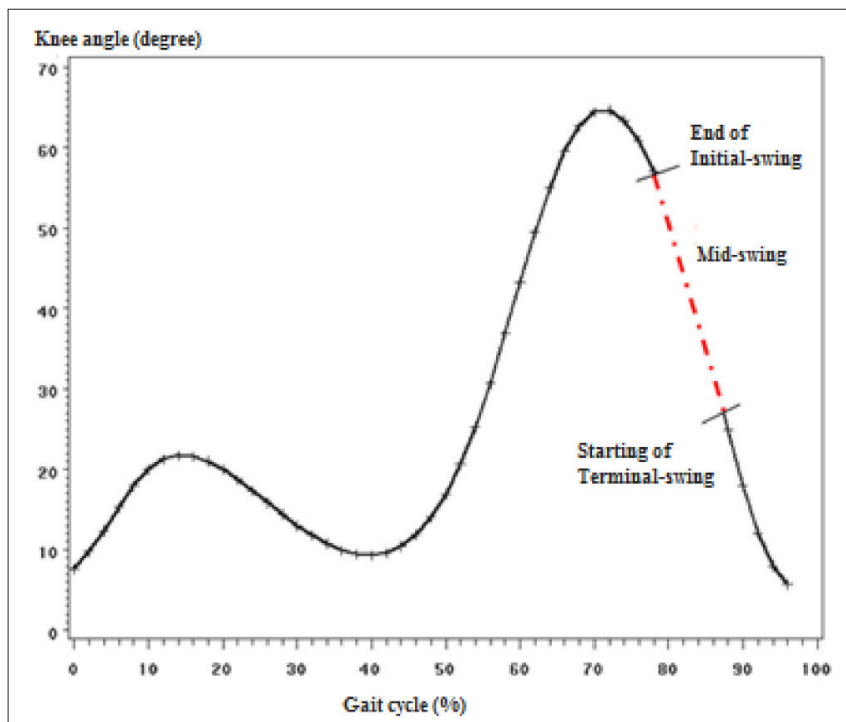

FIGURE 5 | Illustration of how the mid-swing phase connects the end of initial-swing with the start of terminal swing (knee angle curve).

This was done by referring to the values of mean normalized error at Phase 2 between hip and ankle variables paired with knee variables. The knee variables that yielded the least mean normalized error when paired with hip and ankle variables of the result as in Table 5(a) was extracted at that particular sub-phase. The revised result, i.e., for knee parameters only, is presented in Table 5(b).

\section{DISCUSSION AND CONCLUSION}

This study modeled all cyclograms of lower limb joint's gait variables holistically and analyzed it to identify the gait disparity among prostheses users. It also identified the most dominant knee parameter at each gait sub-phase for a prosthetic knee design.

The highlight of the finding was the results of MSw gait phase modified behavior. Manipulation of both parameters in the coupled pair that was found to have the largest mean normalized error in MSw phase did not improve the performance at that particular phase. Worse is, the manipulation of either parameter at the MSw phase caused the neighboring subphases (ISw and TSw) yield larger mean normalized error. This indicated biomechanical justification of the momentum and gravity influence during MSw. The prosthetic knee should be left independent to extend by the effect of momentum produced by its weight and the gravity at the mid-swing in order to connect the end of initial-swing phase with the beginning of terminalswing as illustrated in Figure 5. This concept was discussed as "ballistic synergy" concept introduced by Pitkin that allowed for the control simplification and reduces the power supplies demand when compared with robots for which all its degrees of freedom in motion are controlled during the entire gait (Pitkin, 2010). This would make the kinematics and kinetics match with normal human ballistic gait synergy.

In this study, the results shown that altering one of the dominant parameter that was identified using manipulate cyclograms method yielded to a better performance in most of the gait sub-phases. However, it might be oversimplified considering the complexity in the dynamics of human gait. Therefore, the findings will need to be further verified by performing experiment using a micro-controller knee on a patient and the effect in the gait of the patient is analyzed.

It has been recommended in a previous study that more prospective intervention studies that take into account the multifactorial nature on the amputees walking ability should be conducted (Van Velzen et al., 2006). Thus, we proposed the use of inter-relationship modification of two-variables interaction that co-exist during a gait cycle, especially variables across twojoints which provided an insight into the effect of controlling one variable onto another variable at other joints during walking for an amputee.

Finally, only the affected limb of each subject was thoroughly investigated and reported and the actual speed of each subject was not considered. Several adaptation strategies in terms of joint power or work in both the amputated and intact leg were demonstrated in patients with transfemoral amputation (Prinsen et al., 2011). Investigation on the bilateral control was only done to determine the difference in leading or trailing limb kinematic behavior but not in more detailed toward its cyclograms changes. Further investigation would offer a more precise understanding of each subject's compensatory movement.

\section{AUTHOR CONTRIBUTIONS}

FJ, NAH, and NMS developed the scientific content and context of the article. NMS performed the data collection of the study participants and analysis of the data. TC referred and pre-screened the study participants and contributed clinical insights into the article. NAO provided the funding, setup and 
facilities to perform the study and oversees the scientific content of the study. The final article was majorly written by FJ, NAH, and NMS.

\section{FUNDING}

This study was funded by Ministry of Higher Education (MOHE) of Malaysia; grant no: UM.C/HIR/MOHE/ENG/10 and the first author's research work is supported by Ministry of

\section{REFERENCES}

Arjunan, S. P., and Kumar, D. K. (2010). Decoding subtle forearm flexions using fractal features of surface electromyogram from single and multiple sensors. $J$. Neuroeng. Rehabil. 7:53. doi: 10.1186/1743-0003-7-53

Awai, L., and Curt, A. (2014). Intralimb coordination as a sensitive indicator of motor-control impairment after spinal cord injury. Front. Hum. Neurosci. 8:148. doi: 10.3389/fnhum.2014.00148

Barton, J. G., and Lees, A. (1997). An application of neural networks for distinguishing gait patterns on the basis of hip-knee joint angle diagrams. Gait Posture 5, 28-33. doi: 10.1016/S0966-6362(96)01070-3

Beale, M., Hagan, M., and Demuth, H. (2010). MATLAB: Neural Network Toolbox 7 User's Guide. Natick, MA: The MathWorks, Inc.

Carpenter, A. L., Smith, J. D., Christiansen, C. L., and Heise, G. D. (2012). "Weight bearing assymetries chanes in COP trajectories: Unilateral Ertl amputees vs. non-amputees," in 36th Annual Meeting of the American Society of Biomechanics, (Gainesville, FL).

Cavanagh, P., and Grieve, D. W. (1973). The graphical display of angular movement of the body. Br. J. Sports Med. 7, 129-133.

Crenna, P., and Frigo, C. (2011). Dynamics of the ankle joint analyzed through moment-angle loops during human walking: gender and age efffects. Hum. Mov. Sci. 30, 1185-1198. doi: 10.1016/j.humov.2011.02.009

Engeberg, E. D. (2013). A physiological basis for control of a prosthetic hand. Biomed. Signal Process Control 8, 6-15. doi: 10.1016/j.bspc.2012.06.003

Goswami, A. (1998). A new gait parameterization technique by means of cyclogram moments: application to human slope walking. Gait Posture 8, 15-36. doi: 10.1016/S0966-6362(98)00014-9

Kutilek, P., and Farkasova, B. (2011). Prediction of lower extremities' movement by angle-angle diagrams and neural netwoks. Acta Bioeng. Biomech. 13, 57-65.

Kutilek, P., Socha, V., Viteckova, S., and Svoboda, Z. (2014a). Quantification of gait asymmetry in patients with ankle foot orthoses based on hiphip cyclograms. Biocybern. Biomed. Eng. 34, 46-52. doi: 10.1016/j.bbe.2013. 10.001

Kutilek, P., Svoboda, Z., and Smrcka, P. (2014b). "Evaluation of bilateral asymmetry of the muscular forces using opensim software and bilateral cyclograms," in Mechatronics 2013, eds T. Březina and R. Jabloński (Cham: Springer International Publishing), 801-808.
Higher Education, Malaysia and International Islamic University Malaysia under SLAB/SLAI scholarship.

\section{ACKNOWLEDGMENTS}

The authors thank the subjects who participated in this study and the technical support staffs in the Motion Analysis Laboratory, Faculty of Engineering, University of Malaya.

Kutilek, P., Viteckova, S., Svoboda, Z., and Smrcka, P. (2013). Kinematic quantification of gait asymmetry in patients with peroneal nerve palsy based on bilateral cyclograms. J. Musculoskelet. Neuronal Interact. 13, 244-250.

Liu, M. Q., Anderson, F. C., Pandy, M. G., and Delp, S. L. (2006). Muscles that support the body also modulate forward progression during walking. $J$. Biomech. 39, 2623-2630. doi: 10.1016/j.jbiomech.2005.08.017

Pitkin, M. R. (2010). Biomechanics of Lower Limb PROTHESIS. New York, NY; Berlin; Heidelberg: Springer-Verlag.

Prinsen, E. C., Nederhand, M. J., and Rietman,. J. S. (2011). Adaptation strategies of the lower extremities of patients with a transtibial or transfemoral amputation during level walking: a systematic review. Arch. Phys. Med. Rehabil. 92, 1311-1325. doi: 10.1016/j.apmr.2011.01.017

Robertson, D. G. E. (2004). Research Methods in Biomechanics, Champaign, IL: Human Kinetics.

Su, F. C., and Wu, W. L. (2000). Design and testing of a genetic algorithm neural network in the assessment of git patterns. Med. Eng. Phys. 22, 67-74. doi: 10.1016/S1350-4533(00)00011-4

Van Velzen, J. M., Van Bennekom, C. A., Polomski, W., Slootman, J. R., Van der Woude, L. H., and Houdijk, H. (2006). Physical capacity and walking ability after lower limb amputation: a systematic review. Clin. Rehabil. 20, 999-1016. doi: $10.1177 / 0269215506070700$

Wang, R., Bronstrom, E. W., Esbjornsson, A., and Gutierrez-Farewik, E. M. (2012). Analytical decomposition can help to interpret ankle joint moment-angle relationship. J. Electromyogr. Kinesiol. 22, 566-574. doi: 10.1016/j.jelekin.2012.04.005

Conflict of Interest Statement: The authors declare that the research was conducted in the absence of any commercial or financial relationships that could be construed as a potential conflict of interest.

Copyright (c) 2017 Jasni, Hamzaid, Mohd Syah, Chung and Abu Osman. This is an open-access article distributed under the terms of the Creative Commons Attribution License (CC BY). The use, distribution or reproduction in other forums is permitted, provided the original author(s) or licensor are credited and that the original publication in this journal is cited, in accordance with accepted academic practice. No use, distribution or reproduction is permitted which does not comply with these terms. 


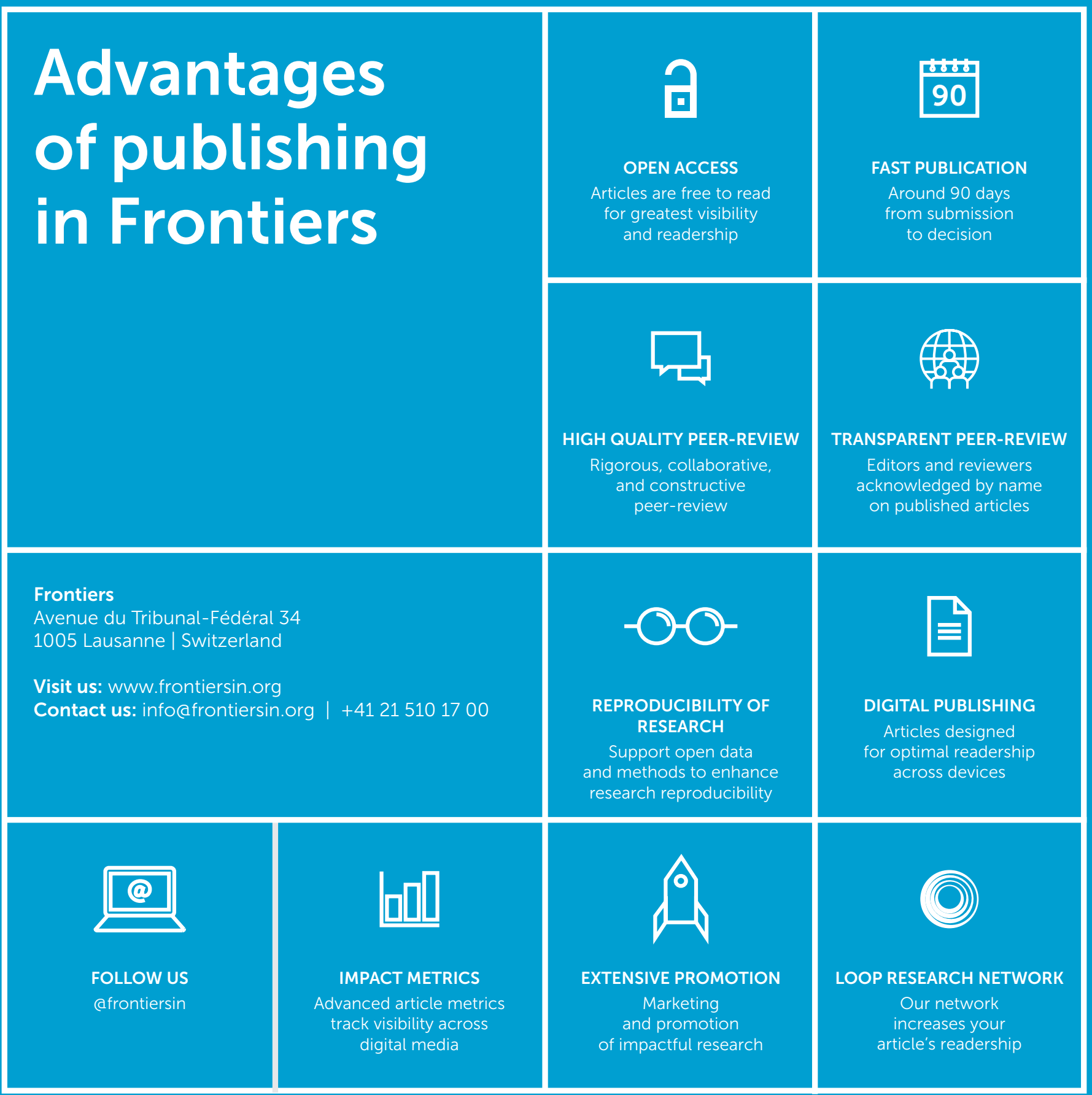

